\title{
NUCLEAR SCIENCE
}

\section{ANNUAL REPORT \\ 1980-1981}
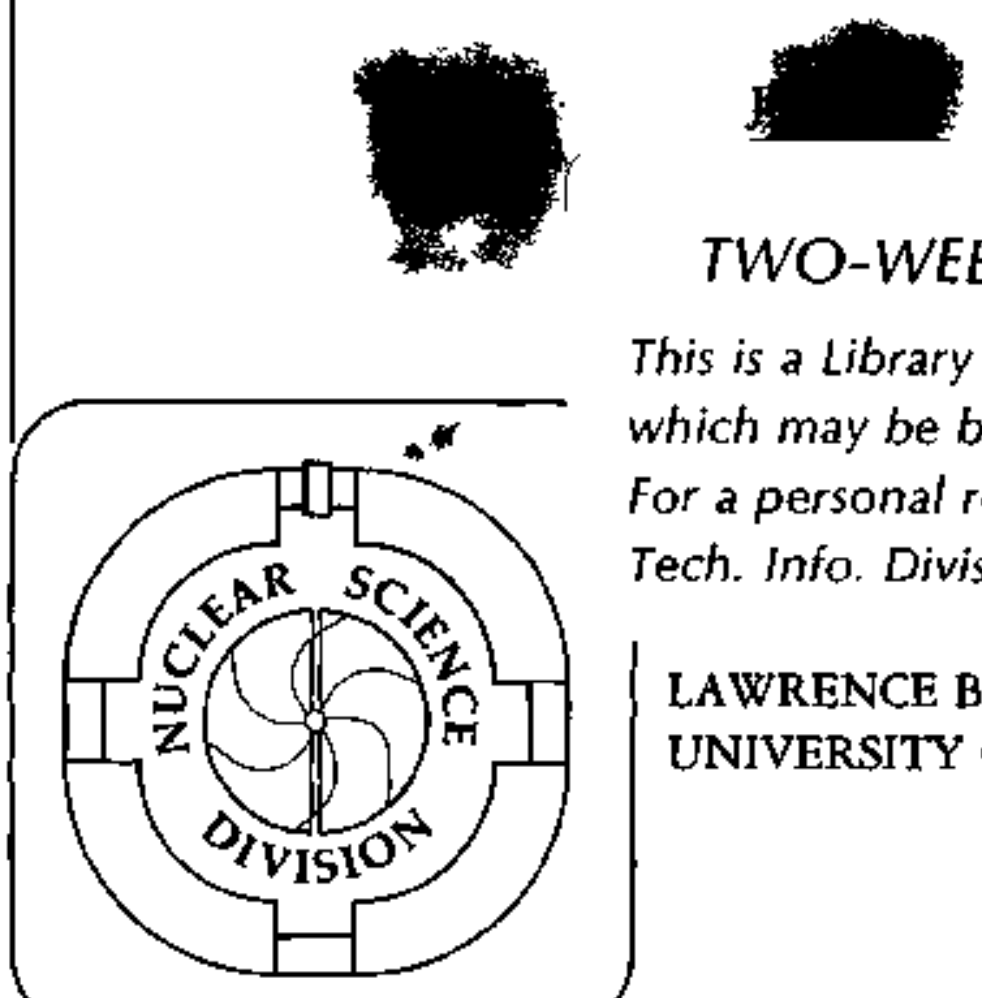

This is a Library Circulating Copy which may be borrowed for two weeks. For a personal retention copy, call Tech. Info. Division, Ext. 6782.

LAWRENCE BERKELEY LABORATORY UNIVERSITY OF CALIFORNIA 


\title{
NUCLEAR SCIENCE
}

\author{
Annual Report \\ for the period \\ July 1,1980 - June 30,1981 \\ J. Cerny \\ Division Head
}

E. M. Friedlander

Editor

\author{
Lawrence Berkeley Laboratory \\ University of California \\ Berkeley, California USA 94720
}

This work was supponted by the Director, Office of Energy Research, Office of High Energy and Nurdear Physics, Division of Nuclear Physirs and by the Office of Basic Energy Sciences, Division of Nuclear Sciences, of the U.S. Department of Energy under Contract No. DE-AC03-76SF00098. 


\section{Contents}

Introduction

J. Cern

\section{PART 1. Research Programs}

Erotic Nuclei and Nuclear Reactions

J. Cermy

Heavy Ion Resctions

B.G. Harvey and $R$ G. Stokstad

Nuclear Structure

R.M. Diamond, F. Stephens, and M.A. Deleplangue

Polarization Phenomena in Nuclear Physics

H.E. Conzett

Statistical Equilibrium in Deep-Inelastic Heavy Ion Reactions

L. G. Moretto

Heavy Element Research

$A$. Ghiorso and $J, M$. Nitschke

High Energy Nuclear Collisions

A.M. Poskanzer and H.H. Gusbrot

Relativistic Heavy Ion Physics H. H. Hecturan

Bevalac Research

H.G. Pkgh, L.S. Schroeder, A. Sandoval, and R Stock ..................................... I7

Light Particle Emission in High Energy Nuclear Collisions

S. Nagamiyo and H. Steiner

Heavy Ion Studies/Pion Studies

K.M. Gove and J.O. Rasmussen

Nucleus-Nucleus Collisions

P.B. Price

Nuclear Theory

N.K. Glendenning, M. Gyzlassy. W.D. Myers, J. Rendrup,

W.J. Swatecki

Heavy Iọn Superconducting Spectrontwter (HISS)

D. Greiner

Isotopes Project

J.M. Dainiki

\section{PART 2. Progress Reports}

\section{Experimental Research}

\section{A NUCLEAR STRUCTURE}

\section{Study of Exotic Noclei}

The Decay of ${ }^{8} \mathrm{He}$

T. Björnstod, H.À Gustafsson, B. Joneon, P.O. Larsson, V. Lindfors,

S. Matisson, G. Nyman, A.M. Poskanzer, H.L. Ravn, and D. Schard 
Delayed Neutron Emission Probabilities of ${ }^{9} \mathrm{Li}$ and ${ }^{11} \mathrm{Li}$

$T$ Bjornstad, H. A. Gustajsson, P,G. Hansen, B. Jorson,

V. Lindfors, S. Martsson, AM. Poskanzer and H.L. Rovn

Beta-Delayed Three-Neutron Radioactivity of " $\mathrm{Li}$

R.E. Azuma, T, Björnstad, H. A. Gussafsson, P.G. Hansen,

B. Jonson, S. Matisson G. Nyman A.M. Poskanzer, and H.L Ravn

Be13-Delayed Two-Neutron Emission from ${ }^{30,31,32} \mathbf{N a}_{\mathbf{a}}$

C. Detraz, M. Epherre, D. Guillemand, P.G. Hansen, B. Jonson,

R. Klapiseh, M. Langevin, S. Matrsson, F. Nawlin, G. Nyminn.

A.M. Poskanzer, H.L Ravm, M. de Saitr-Simon, K. Takahashi,

C. Thibetilt, and F. Towchard

The Measurement of $\beta$-Decay Lifetimes via Projectilc Fragmentation

M.J. Murpty. T.J.M. Symons, G.D. Westfall, and H.J. Crowford

$\beta$-Decay Energies and Masses of ${ }^{103-105} \mathrm{In}$

J.M. Wouters, H.M. Thierens, J. Alystb, M.D. Coble.

P.E. Houstein, RF. Parry, and Joseph Cerny

The e/ $\beta+$ Decay of ${ }^{145} \mathrm{Gd}$ : Resolution of Decay Branching

Ratio Anomalies and Evidence for Pronousced Structures in the

R. Decay Strength

R.B. Firestone, R.C. Pardo, R.A. Warner,

Wm. C. McHorris, and W.H. Kelly

Observation of Large Resonaces in the $\beta$-Desay of ${ }_{66}^{145} \mathrm{Od}_{\mathrm{g}}$ R.B. Firestone

Determination of the Half+Life and Absolute Gemma Intensities of ${ }^{|5|} \mathbf{G d}$

K.E. Gregorich, K.J. Moody, and G.T. Seaborg

The Decay of the ${ }^{155} \mathrm{~Tb}$ Compound Nucleus, Formed in the Reaction of ${ }^{27}$ we with ${ }^{133} \mathrm{Cs}$

$\boldsymbol{K}, J$. Moody and J.J. Hogat

Alpha Decay of Neutron Deficient Polonium and Bismuth Isotopes

$M$. Lino, $S$. Yashita, and $A$ Ghiorso

Search For Electron Capture-Delayed Fission in the $\mathbf{N}=126$

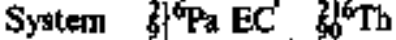

L.P. Somerville and M.J. Nurmio

Attempts to Identify the 1.5-Second Spontancous Fission Activity

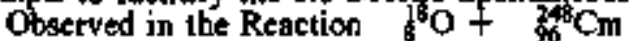

A. Ghiorsa, LP. Somerville. RM. McFarland.

R. Lougheed, and E.K. Huler

Spontaneous Fission of Rutherfordium Isotopes

LP. Somerville, J,M. Mischke, M.J. Nurmia, and $A$ Ghiorso

Production of Heavy Actinides from Interactions of ${ }^{20} \mathrm{Ne}$ and ${ }^{22} \mathrm{Ne}$ with ${ }^{248} \mathrm{Cm}$

D. Lee, H.V. Gunten, M. Nurmia, Y.-F. Lit,

G.T. Seaborg and D.C. Hoffman

Recoil Range Experiments in the Actinide Region

R.M. McFarland, LP. Somerville, M.J. Nurmia, and A Ghiorso

A Procedure for a Fast Separation of Berkelium and Cerium

Yuan-fong Liv, Cheng Luo, Hass R, vont Gunten,

and Glenn T. Seaborg

\section{High Angular Mang-itmm}

Study of High Energy Gamma Rays Enitted in the Deep-Inelastic Reactions ${ }^{161} \mathrm{Ta}+{ }^{136} \mathrm{Xe}(1150 \mathrm{MeV})$

J.O. Newton, J.E. Draper, KH. Lindenberger, R.M. Diamand,

C. Schück. F.S. Stephens, E.L. Dines, S. Shth, LG. Sobotho.

G.J. Wozniak, R.J. McDonald, D.J. Morrissey, A.J. Pacheco,

and $L$ G. Moretto 
Properties of Nuclei at Very High Spin

F.S. Slephens

Nuclear Structure at High Spins

F.S. Stephens

High-Spin Properties of ${ }^{164} \mathrm{Er}$ in the Multiple Band Crossing

Region From Coulomb Excitation and In-Bean Gamma-Ray Spectroscopy

M.W. Guidry, S.W. Yotes, I.Y. Lee, N.R Johnson, E. Eichler,

LL Riedinger, MW. Guidry, AC Kohler, D. Cline, R.S. Simon,

P.A. Butler, P. Colambanl. F.S. Stephens, R.M. Diamond.

R.M. Ronningen, RD. Hichwa, J.H. Hanilion, and E.L Robinson

Average g-Factors for High Sptn States in ${ }^{156} \mathrm{Dy}$

N. Rud, D. Ward, H.R Andrews, a fätusser, P. Taros,

J. Keinonten, M. Nejmer, R.M. Diamond, and F.S. Stephens

Alignment Effects in Correlation Spectra

C. Ellegeard, M.A. Deleplangue, O. Andersen, B. Herskind,

F. Stephers, R.M. Diamond, H. Kluge, C. Schück, and S. Shih

Otservation of Giant Dipole Resonances Buili on States of High

Energy and Spin

J.O Newton, B. Hershind, RM, Diamond, E.L. Dines, J.E. Droper,

K.H. Lindenberger, S. Shih, C Schück, and R.S. Slephens

\section{Polarization Studiess}

Polarization

Homer E. Conzelt

Polarization Tests of Time-Reversal Invariance: Past Failores

Homer E. Conzett

Deviation from the Polarization-Analyzing Power Equality and Implied Breakdown of Time-Reversal Invariance

H.E. Conzett, $P$. won Rassen, $F$. Hinterberger, R.J. Slobodriam,

C. Riowx, and R. Roy

Time Reversal and Charge Symmetry Studies in Single Nucleon Transfer

Reactions in the $A-5$ Systen

A.L Sagle, F.P. Brady, J.L Romero, B.E. Bonner,

N.S.P. King. M.W. MANatughton, and H.E. Conzet'

Polarization Effects in Light Noclei

H.E. Conzert

Search Ior Effect of Lomgitudinally Polarized Protons on Optically

Active Amino Acids

R.M. Lemmon, H.E. Conzett, and W.A. Bonner

4. Nucles Dats Sher Eralnation

Nuclear Data Sheets for A = 169 and A -193

V.S. Shirley

Nuclear Data Sheets for $\mathrm{A}=187$ and $\mathrm{A}=185$

Y.A Ellis-Akovali

Nuclear Data Shets for A = 189

$R$ B. Firestone

Nuclear Data Sheets for A - 188

B. Singh and D. Viggars

Nuclear Date Sheets for A $=191$

E. Browne

Evaluation of Nuclear Structure and Decay Data

C. Aflchat Lederer 


\section{B. NUCLEAR REACTION MECHANISMS WITH NONRELATIVISTIC HEAVY IONS}

\section{Curged Particle Barssion}

Alpha Particle Emisslon from Two Reactions lovolving Very Heavy loos

L.G. Sabotka, R.J. McDonald, G.J. Wozmiak, D.J. Morrissey,

A.J. Pacheco, and LG. Moretto

Energy Equilibration in Composite Nuclei at High Energy and Spin: Correlations between Evaporative ${ }^{1} \mathbf{H},{ }^{4} \mathbf{H e}$ and Fission

M.F. Rivet, D. Logon, J.M. Alexander, E. Duek,

M.S. Zisman, and M. Kaplan

Ditect and Evaporation-Like Emission of ${ }^{1} \mathrm{H}$ and ${ }^{4} \mathrm{He}$ jo

Both Fusion-Like and Inclastic Reactions of $340 \mathrm{MeV}{ }^{40} \mathrm{Ar}+{ }^{23 B} \mathrm{U}$

D. Logan, M. Kildir, M. Kaplan, M.S. Zismast.

D. Guerreat, J.M. Alexander, and LC. Var .

Prominent the Emission from the Composite Nucleus for $480 \mathrm{MeV}{ }^{56 \mathrm{Fe}}+\mathrm{Ag}$

D. Guerreau, D. Logan, M.S. Zisman, J.M. Alexander.

E. Datk, and $\boldsymbol{H}$. Kaplat

Comparison of Noncompound Neutron and Proton Emission in 160

Induced Reactions on ${ }^{23} \mathrm{U}$ at $310 \mathrm{MeV}$

J. Kasagl, S. Saini, T.C. Awes, A. Galonsity, C.K. Gelbke,

G. Poggi, D.X. Scotf, K.L. Wolf. and R.L Legrain

Light Particle Correlations in $20 \mathrm{MeV}$ per Nucleon ${ }^{16} \mathrm{O}$

Induced Reactions

C.K. Gelbke, W.G. Lynch, L.W. Rlchardson, M.B. Tsang,

and $R E$. Warner

Trends of Light Particle Spectra Observed in Nucleus-Nucleus Collisions

T.C. Awes, G. Poggi, S. Sarint, C.K. Gelbke.

R. Legrain, and $G$ D. Westfall

2. Angular Morentun Tramsfer

Q. Value and Z Dependence of Angular Momentum Transfer in Deeply lnelastic Scattering of ${ }^{56} \mathrm{Fe}+{ }^{200} \mathrm{Bi}$ M.S. Zismon, R.J. Puigh, $R$ Vandentosch, T.D. Thomas, and $L$ Nunclley

Total $\gamma$-Ray Energy Measurements for the Reaction

$136 \mathrm{Yb}+{ }^{165} \mathrm{Ho}$ at $8.5 \mathrm{MeV} / \mathrm{A}$

R.J. McDoncld. A.J. Pacheco, G.J. Wozniak, D.J. Marrissey,

LG. Sobotka, LG. Moretto, K. Lindenterger, C. Schück.

S. Shih, J.O. Newton, R.M. Diamond, and F.S. Stephens

Angular Mornentum, Statistical Equilibrium and Sequential Fission

in Very Asymmetric Systems

D.J. Morrissey. G.J. Wozniak, L.G. Sobotka, A.J. Pacheco,

C. C Hsw, R. McDondd, and L.G. Moretto

Fragment Spin Alignnent in Dexp-lnelastic Reactions

A,J. Pocheco, R.J. McDonald, G.J. Wozmiak. C.C. Hsu.

D.J. Morrissey, LG. Sobotka, S. Shih, R.M. Diamond,

F.S. Stephens, and LG. Moretto

The ${ }^{12} \mathrm{C}+{ }^{12} \mathrm{C}$ Reaction Cross Soction Between 70 and $290 \mathrm{MeV}$

Ottained from Elastic Scattering

A.J. Cole, W.D.M. Roe, M.E. Brondar, A. Dacal, B.G. Harvey.

R. Legrain, M.J. Murphy, and R.G. Stokstad

Isomer Ratio Measurements for the Reaction ${ }^{20} \mathrm{Si}\left({ }^{18} \mathrm{O}, \mathrm{p} 2 \mathrm{n}\right){ }^{44} \mathrm{Sc}^{4}{ }^{4} \mathrm{Sc}$

F. Groening, $K$. Aleklett, K.J. Moody, P.L. MfGaughey,

W. Loveland, and G.T. Seaborg 
Excitation Functions and Isomeric Ratio Measurements

for the Reaction ${ }^{41} \mathrm{~K}\left({ }^{6} \mathrm{~L}, \mathrm{p} 2 \mathrm{2n}\right){ }^{4} \mathrm{c}^{m}, \mathrm{St}^{8}$

H.D. Nguyeh, H.N Trinh, H.D. Le, K.J. Moody,

H. Groening, and G.T. Seaborg .

\section{Target and Projectile Fragmentation}

Transfer Versus Fragmentation for Reactions near $15 \mathrm{MeV} /$ Nucleom M.J. Murphy, B.G. Horvey. D.L. Hendrie, J. Mahoney,

W. Patg, and $\boldsymbol{X}$. Vant Bibber

Sinall Momentum Widths in Heavy lon Fragmentation at $20 \mathrm{McV} / \mathrm{amu}$ and Below

Bernerd G. Horvey

Coherent and Incoherent Processes in Projectile Breakup W.D. Rae, A.J. Cofe, A Dacal, $R$ Legrain, B.G. Harvey,

J. Mahomey, M.J. Murphy, R.C. Sioksted, and I. Tserruya

Observation of Sequential Breakup in the ${ }^{16} \mathrm{O}+{ }^{197} \mathrm{Au}$ Reaction at $21 \mathrm{~B} \mathrm{MeV}$ A.N. Bice, M.D. Cable, AC. Shotter, and Joseph Cerny

Observation of the Direct and Sequential Brealaup of ${ }^{7} \mathrm{Li}$ from ${ }^{12} \mathrm{C}$ and ${ }^{200} \mathrm{~Pb}$ Targets at $70 \mathrm{MeV}$ A.N. Bice, A.C. Shotter, J.M. Wouters, W.D. Ree, and Joseph Carny

Resonant Particle Production in the $172-\mathrm{MeV}{ }^{13} \mathrm{C}+{ }^{200} \mathrm{~Pb}$ Reaction A.N. Bice, M.D. Cable, AC. Shatter, and Joseph Carny

${ }^{12} \mathrm{C}$ Production in the ${ }^{12} \mathrm{C}+{ }^{208} \mathrm{~Pb}$ System at $230 \mathrm{MeV}$ A.N. Bice, AC. Shotrer, and Jareph Cerny

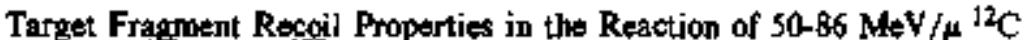
with ${ }^{18 \mathrm{~T}} \mathrm{Ta}$ and ${ }^{19} \mathrm{Au}$ W. Loveland, P.L McGaughey, K. Aleklett, K.J. Moody. P. Johnson, and G.T. Seaborg

Target Fragment Angular Distributions in the Reaction of $86 \mathrm{MeV} / \mu^{12} \mathrm{C}$ with ${ }^{1, \mu}$ and ${ }^{2}$ i $U$

$K$. Aleklett, W. Loveland, P.L. McGaughey, R H. Krats, Jr. Y. Morita, G.T. Seaborg. T. Lund, D. Molzakn, E. Hagebo, and I. Haldorsen

Target Fragmentation from $10-2100 \mathrm{MeV} / \mu$ W. Loveland, P.L. McGaughey, K. Aleklett, K.J. Moody, R.H. Kraus, Jr, R.M. McFariand, and G.T, Seabarg

\section{RELATIVISTIC HEAVY IONS}

\section{Fragmeatation Processes}

Energy Varjation of Target Fragment Mass and Charge Distributions in the Interaction of Relativistic Heavy Ions with Ta and $U$ W. Loveland, P. L. McGaughey, D.J. Morrissey, C. Oertel, L.L Nannelley, and G.T. Seabarg

Target Fragment Angular Distribuyions in the Reaction of 3.0 and $12.0 \mathrm{GVV}^{12} \mathrm{C}$ with ${ }^{19} \mathrm{Au}$ and ${ }^{238} \mathrm{U}$

Y. Morki, W. Loveland, P.L. McGaughey, and C.T. Senborg

Fragment-Spray Correlations in High Energy Heavy Ion Collisions A.I. Worwick, H.H. Gutbrad, S.B. Koufman, M.R Maier. J. Peter, H.G. Ritter, E.P. Steinberg. H. Sielzer, F. Weik. H. Wheman, and B.D. Wikins

Comparative Yjelds of Alkali Elements and Thalliurn from Uranium Irradiated with $\mathrm{GeV}$ Protons, ${ }^{3} \mathrm{He}$ and ${ }^{12} \mathrm{C}$ T. Björnstad, H.-A Gusiofsson, B. Jonson, O.C. Jonsson. V. Lindfors, S. Mattsson, A.M. Paskenzer, H.L Ravn, and D. Schardt 
A Compilation of World Data on "Anomalons" E.M. Friedlander and Y.J. Karant

Further Investigation of Projectile Fragmentation: the Shor Mean

Free Path Effect

E.M. Friedlander, R.W. Gimpel, H.H. Heckman, Y.J. Karam,

B. Judek and $E$. Canssatrge

Evidetece for Two Different Reaction Mochanisms in Relativistic Heavy lon Collisions

H.G. Bawmzardt, E.M. Friedlander and E. Schopper

Comment on the Energy Dependence of Target Excitation in RHI Collisions Harry H. Heckman

A Measurement of Deuteron-Deuteron Elastic Scattering at $5.75 \mathrm{GeV} / \mathrm{c}$ Edzar T. Whipple, V. Perez-Mender, A.L Sagle, $R L$ Talaga. F. Zarbakhsh. J.B. Carroll, G.J. Igo, J.B. McClelland, $M$. Bleszymst. and $\mathrm{K}$ Ganezer

\section{Pion Prodaction}

Productiçn of Pions and Light Fragments at Large Angles in High-Energy

Nuclear Collisions

S. Nagawiya, M-C. Lemaire, E. Moeller, S. Shmetzer,

G. Shapiro, H. Steiner, and I. Tanthata

Inclusive Single Negative Pion Poduction at Forward Angles from

Collisions of Light Relativistic Nuclei

E. Molller, W, Bruckner, L Anderson, S. Nagomiya,

S. Nissen-Meyer, L Schroeder, G. Shapiro, and H. Steiner

Pion Double Charge Exchange on ${ }^{4} \mathrm{He}$ and Meson Exchange Currents A. Stetz, LW. Swenson, J, Dowis, J. Käthe, RC. Minehart, R.R. Wirney, V. Perez-Mendez, A. Sogle. J. Carroll,

I. MeClellond, and $J$. Fawet!

Strong Coulomb Efiects on Pions Produced in Heavy lon Collisions J.P. Sulliwan, J.A. Bistirlich, H.R Bowmon, R. Bossingham, T. Buttke, K.M. Chowe, K.A. Frankel, C.J. Martoff.

J. Mftler. D.L Murphy. J.O Rasmussen, W.A. Zajc,

a. Hashimoso, M. Rolke, I. Peter, W. Benenson,

G.M. Crawley, E. Kashy, and J.A Nolen, Jr.

Pions Produced Near the Center-of-Mass Velocity in Heavy Ion Collisions

$K$ A Frankel. J.A Bustirlich, $R$ Bossingham, $H . R$ Bowman,

K.M. Crowe, C.J. Mariof, D.L Murphy, J.O. Rasmassen,

J.P. Suliwan, W.A. Zajc. J.P. Miller, O. Hoshimoto,

M. Koike, J. Peter, W. Benensan, G.M. Crawley, E. Koshy.

J.A. Nolen, Jr., and J. Quebert

- Temperatures, Sphericity and Throst for Central Collisions $\boldsymbol{R}$ Grockmatm, J.W. Hawris, A. Sondaval, H. Strobele. $\boldsymbol{R}$ Stack, M. Maler, RE. Renford, J. Miller, H.G. Pugh, M. Raff LS. Schroeder, F. Riess, $K$. Wolf, A Dacal, and $M$ E. Ortiz

Charged Particle Exclusive Analysis

R. Brackment, J.W. Hartis, A. Sondowad, H. Strabele.

$R$ Stack, M. Maler, RE Renford, J, Miller,

H.G. Pugh, M. Rafi. LS. Schroeder, F. Riess, K. Wolf,

A. Dacal, and M.E. Ortiz

Coherence and Self-Induced Transparency in High Energy Hadronic Collisions

G.N. Fowler, E.M. Friedlander, and R.M. Weiner

\section{Strange Puticle Productlon}

Kaon Prodoction in Relativistic Heary lon Collisions

S. Schnetzer, M. C Lemolre, $R$ Lombard, E. Moeller.

S. Nagasdya, G. Shoptro, H. Sieiner, and I. Tanihata 
Observation of Subthreshold $\mathrm{K}^{-}$Production in Relativistic

Nuclear Collisions

A. Shor, K Gonezer, J. CarrolI, G. Igo, J. Geaga.

S. Ahachi, A Sagle. T. Mulera, V. Perez-Mendez.

P. Lindsirom, F. Zarbakhsh, and D. Woodward

A Production Near Threshold in Central Nucleus-Nucleus Collisions

$\boldsymbol{R}$ Brockmann, J.W. Harris, A. Sandowal, H. Strabele.

R. Stack, M. Moler, R.E. Renford, J. Miller, H.G. Pugh,

$M$ Raff LS. Schroeder, F. Riess, $X$ Wolf,

A. Dacal, and M.E. Ortiz

\section{Corretution Stodles}

Measurement of Two-Particle Corcellations in $800 \mathrm{MeV}$ pA Collisions

I. Tanihata, Y, Miake, H. Hamegoki, S. Kadola

Y. Shida, R. Lomberd, E. Moeller, S. Nagamiya,

S. Schnetzer, and H. Steiner

Few Nucleop Interactions and Correlation Sudies

R.N. Thethoff, J.M. Engelage. J.V. Geaga, J.W. Horris.

P.N. Kirk, R.W. Kaontz, H.G. Pugh, G.R Roche, C.L. Ruiz,

and $L S$. Schroeder

Proton-Enitting Sources in Central Collisions of $\mathrm{Ar}+\mathrm{KCl}$ at $1.8 \mathrm{GeV} / \mathrm{N}$

A.L Sagle, F. Zarbakhsh, F. Brochard, T.A. Mulera,

V. Perez-Mendez, R. Talaga, I. Tanihata, J.B. Carroll,

K.S. Ganezer, G. Igo, J, Oosiens, D. Woodward, and R Sutter

Two-Pton Cortelations in Heavy Ion Collisions

J.A. Bistilich, R.J. Bossingham, H.R. Bowman, C.W. Canson,

K.M. Crowe, $X . A$ Frankel, O. Hashimoro, J.G. Ingersolt,

M. Koike, J.P. Kurck, C.J. Mfartoff. W.J. Macdonald,

J.P. Miller, D.L Murphy, J.O. Rasmussen, J.P. Sulfivan,

P. Trud, E. Yoo, and W.A. Zajc

Spece Structure of a Fireball and the Pion Ratius

T.P. Hoang, Bruce Cork. and H.J. Crowford

Two. Pion Correlations in Ceniral Collisions of $1.8 \mathrm{GeV} / \mathrm{u}^{40} \mathrm{Ar}$ on $\mathrm{KCl}$

R. Brockmanm, J.W. Harris, A. Sandoval, H. Strobele.

R. Stack, M. Majer, R.E. Renfordt, J. Miller, H.G. Pugh,

M. Reff, LS. Schroeder, F. Rless, K. Wolf. A. Dacal,

and M.E. Orriz

Correlations Between Cojncident Fragments in Relativistic Heavy-lon Reactions

E.P. Steinberg, M.S. Freedman, H.H. Gutbrod, D.J. Henderson

S.B. Koufman, M.R Haier, J. Peter, H.G. Riter,

H. Stelzer, A.J. Warwick, P. Weik, H. Weimon, and B.D. Wikins

\section{THEORETICAL RESEARCH}

Nuclear Deformation Energies

W.J. Swiatecki and $J$. Blocki

The Effect of Deformation and the Neutron Slin on

RMS Charge Radii

W.D. Myers and X.-H. Schmidr

Surface-Layer Corrections to the Level-Density

Formula for a Difuse Fermi Gas

W.J. Swiateckl and J. Toke

Schematic Calcalation of GT Strength in $N=47,49$ Isotones

S.G. Prussin, Z.M. Oliveira, and J. Randrup

Particle Angular Momentum Alignment Eflects in Deformed Nuclei

L.K. Peker, J.O. Rasmussen, and J.H. Kamilian 
Excitation of Shape-Vibrational Modes in Nuclei by Relativistic Heavy lons

J.O. Rasmossen, J.S. Blair and X.J. Qin

Calculation of Muon Final Probabilities after Muon-Induced

Fission in Four-State Basis

Ma Zhong-yn, Wh Xi-zhen, Zhang, Jing-shang, Zhwo Yi-zhong.

and J.O. Rosniwssen

Twa-Nucleon Transfer Reactions in Deformed Nuclei

Using Very Heavy Ions

M.W. Guldry, T.L. Nichols, RE. Nese, J.O. Rasmussen,

$L F$. Oliveira and $R$. Donangelo

Ground State Properties of Finite Nuclei In A Nonlinear

Relativistic Mean Field Theory

J. Boguta

Relativistic Quantum Field Theory of A-Hypernuclej

I. Boguta and S. Bohrmann

Density Dependence of the Single Particle Potential

in Nuclear Matter

J. Boguta

Dense Neutron Star Matter in the Nommal and Pion Condensed

State in a Relativistic Field Theory Constrained

by Bulk Nuclear Properties

N.K. Giendennting. B. Banerjee and M. Gyzlassy

The Energy-Depeodent Single Nucleon Potential in

a Relativistic Field Theory of Nuclear Matter

X. $-\boldsymbol{H}$. Miller

A Relativistic Quantum Field Theoretical Model for

Nuclear Slab Collision Dynamics

$\boldsymbol{X}$-H. Muller

Interacting Strtaming Nuclear Matter Systems in a

Relativistic Quantum Field Theory

$\boldsymbol{K}$. $\boldsymbol{H}$. Muller and S. Bohrmann

Theory of Nuclear Dynamics

W.J. Swatecki

The Cut hing of two Colliding Nuclei

G. Fof

Fusion-Fission, Molecular-Capture And Symmetric Fragmentation

In 3D-TDHF Calcalation of the Reaction: ${ }^{23} \mathrm{U}$,

${ }^{2} \mathrm{~Pb}(4,6-8,0 \mathrm{MeV} / \mathrm{u})^{64} \mathrm{Ni},{ }^{2} \mathrm{Ca}^{\mathrm{P}+\mathrm{C}}$

H. Stö́cker. RY. Cusson, H.J. Lustig. A. Gobbl,

$J$ Hohe JA Morubn and W. Gelner

Quantum Treattnent of Charge Equilibration in Fission

W.D. Myers, G. Martizourayis, and J. Rondrup

Correlated Mass and Charge Transporn Induced by

Statistical Nucleon Exchange in Dampod Nuclear Reactions

W.U. Sethröder, J.R. Huizenga and J. Ravdrup

Correlaled Charge and Mags Distributions from

Reactions of ${ }^{56} \mathrm{Fe}$ with ${ }^{58} \mathrm{Ni}$ and ${ }^{64} \mathrm{Ni}$

H.C. Brift, B.H. Erkktla, A Gavan, Y. Potin, R.H. Stokes,

M.P. Webb, P.R. Christensen, O. Honsen, S. Pontoppldan,

F. Videbaek, RL Fergluson, F. Plasil, G.R. Young.

M. Blont, and J. Randrup

Transport of Angular Momentum in Dampad Naclear Reactions Jorgen Randrup 
Equilibrium Statistical Treatment of the Spin

Fluctuations Generated in Deep-Inelastic Reactions

A.J. Pacheco, R.P. Schmint and LG. Moretto

Systemutics of Angular Momentum Transfer in

Intermediate Energy Heavy-lon Reactions

C.C. Hsu, D.J. Morrissey, LW. Richardson.

G.J. Wozsink, and LG. Moretto

Shell Efects on the Angular-Momentum Transfer in Heavy-Ion Reactions

LG. Maretto and A.J. Pacheco

The Influence of Fluctuations on the Correlation

between Exit-Channel Kinetic Energy and

Entrance-Channel Angular Momenium Tor Heavy

Ion Collisions

L.G. Moretto and LG. Sobotka

On the Use of Isomer Ratios in ${ }^{4}$ Sc for Predicting

Spin Populations in Figh Energy Heavy Ion Nuclear Reactions

H. Groening, K.J. Moody and G.T. Seaborg

Pre-Equilibrium Nucleen Jets in TDHF Calculations

of Mediunt-Energy, Heavy-Ion Colljigions

H. Stocker, R.Y. Custon, J.A Morthn, and W. Greiner

Explosion-Evaporation Model for Fragment Production

in Intermpodiate-Enetgy Nudear Collisions

George Fol and Jorgen Rondinp

Pion, Light Fraguient, and Entropy Production in Nuclear Collisions H. Stöcker

Pion Production Irom Heavy Ion Collisions at $90.380 \mathrm{MkV} / \mathrm{N}$

P. Hecking

Mieroseopic Theory of Deuteron Formation

M. Gyulatsy, E. Remler, asd X Frankel

Coolomb Final State Interactions M. Gyulassy and S.X. Kauffmant

Formulation of the Coulomb Effects of Spectator Fragments on Pions from Heavy Ion Collisions Hafez M.A. Rodt. J.O. Rasmussen, J.P. Sullivan,

K.A. Frankel, and $O$ Hashimoto

Dynamics va Symmetrization in Hadron Interferometry

M. Gyulassy

The Quast-Elastic Component in High-Energy Nuclear Collistons

Bernd Schürmonn and Jorgen Rondrup

Consequences of Impact Parameter Restrictions in

Relativistic Nuclear Collisions

Sieffen Bohnownt

Recent Results of Statistical Model Calculations

For Relativistic Nuclear Collísions

Siefjen Bohrntann and Järn Kroll

$20 I$

Cascade Calculations of Relativistic Nuclear Collisions

J.D. Stevextort

Kaon Rescattering in Relativistic Nuclear Collisions

J. Randrup

Central Collisions of Heavy Ions - Indication for Fluid Dynamical Behapior?

H Siöker, C. Riedel, G. Buchwald, LP. Csernai,

G. Groebner, W. Greimer, J.A. Marukn, P. Subramantion.

$\boldsymbol{Y}$. Yariv, K Frankel, M. Gyulassy. J. Stephenson,

D. Strottman, J.R. Nix, B. Schïrmatn 
Two Particle Correlations Caused by Collective

Fluid - Dynamical Flow

H. Stacker, L.P. Csemai, G. Buchwald, G. Graebner,

J.A Maruhn and W. Greiner

Jets of Nuclear Matter from High Energy Heavy Ion Collisions

H. Stöcker, L.P. Csernai, G. Graebner, G. Buchwaid,

H. Kruse, RY. Cussom J.A. Maruhn, and W. Greiner

The Bounce-Of Effect as a Barometer for Hot.

Dense Matter in High Energy Nuclear Collisions

Hortt Stöcker and Bernd Muller

Probing Dense Nuclear Matter via Nuclear Collisions

H. Stöcker. M. Gyulassy. and J. Boguta

Optical Model Solutjons for Pions in Nuclear Matter

P. Hecking

Finite Temperature Pion Condensation

N.K. Glendenuing and A. Lumbroso

Pion Condensation Threshold in Nuclear Matter and Thermal $\Delta$-Isobars

P. Hecking

Test for Pion Condensation in Nuclear Collistons

M. Gythossy

Significance of Temperature Messurement in Relatjvistic

Nuclear Collisions

H. Stöcker, AA, Oglablin, W. Greiner

On the Sigaificance of Temperature Measurements of Noclear Fireballs

Nomian K. Glendenntrig

Nuclear Physies from Subhadronic Structure-a Toy Model

Herbert M. Ruck

Percolation versus Gibbs Equilibrium - the Phase

Transition from Hadron Matter to Quark Matter

Horst Stöcker

Strange Baryon Fraction from Quark-Gluon Plasma

N. $K$ Glendenming, $M$. Gywlassy, $R$ Anishetty,

$P$. Koehter, and L MeLerron

Nor-Linear Vacuum Polarization in Strong Fields

$M$, Gyulassy

\section{INSTRUMENTATION}

\section{A. Spectrounters}

HISS Detector Systems

Hank Crowford

Monitoring the HISS Magnet

$F$. Bleser and C. McParland

Program Needs for a High-B Field Facility Jor Visual Detectors

at the Bevalac

Harry H. Heckman

TASS - Two-Arm Spectrorneter System

G.R. Roche, J.M. Engelage, J.V. Geaga, J,W, Harris,

P.N. Kirk R.W. Koontz, H.G. Pugh, C.L. Ruiz,

LS. Schroeder, and RN. Treuhaft 
Streamer Chamber Instrumentation

$\boldsymbol{R}$ Brockmont, J.W, Horrts, A. Sondovai, H. Strobele,

R. Stock, M. Moler, RE. Renfordt, J. Mfiler, H.G. Pugh,

M. Raff, LS. Schroeder, F. Aless, $K$. Whif, A. Dacal,

and M.E. Otiz

Use of a Directly Digitized CCD Camera

$R$ Brockmann, J.W. Harris, A Sandoval, H. Strabele,

R. Stock, M. Moier, R.E. Retfordt, J. Miller, H.G. Pugh,

M. Raff, LS.S. Schroeder, F. RJess, $\mathbb{K}$ Wolf, A. Dacal,

and M.E. Ottiz

Image Intensifier Canneras for Event Recording at the Streamer Chamber

R. Brocknenti, J.W. Horris, A. Sandoval, H. Strobele, R Stack,

M. Mater, RE. Renfordt, J. MHler, HG. Pugh, M. Raff.

L.S. Schroeder, F. Riess, K. Wolf. A. Docal.

and M.E. Ortiz

The Plastic Ball Spectrometer

A. Baden, H.H. Gutbrod, H. Lähner, M.R Maier.

AM. Poskanzer, H. Riedesel, H.G. Rister, H. Spieler.

A.I. Worwick, F, Weik, H. Wieman, and K.L. Wolf

New Developments at OASIS

J.M. Nitschte and J.D. Mollioris

A Versatile, High Temperature, Higk Efficiency lon Source for the On-Line Isotope Separator OASIS

J.M. Nisschke

\section{B. Detectors}

A Glow Memory Chamber for Uste in the Measusement of High Multiplicity Events

M.A Elola, T.A. Mulera, V. Ferez-Mendez,

and P.E. Wiedenbeck

Developunent of a Calorinteter for Reiativistic Heavy Ions

J.D. Stevenson, J. Mortints and P.B. Price

A Lead-Glass Gamma-Ray Detector for Relativistic Heavy Ion Collisions M.P. Budionsky. S.P. Ahlen, and G. Tarie

Response of Organic and Inorganic Scintillators to Relativistic

Heavy lons

M.H. Solamon and S.P. Ablen

Calculation of the Relativistic Bloch Correlation to Stopping Power

S.P. Ahlen

A Search for New Nuclei Far Irom the Valley or Stability

J.D. Sievenson, $P, B$. Price and J. Musser

Radiation Damage in Si(Au) Surface Barrier Detectors Produced by Energetic Heavy Iơns LG. Sobatke, G.J. Wozhak. R.J. McDonald, and LG. Moretto

Developatent of a Beta Detector and Computer Analysis Systetl for the On-Line Mass Seperator, RAMA

J.M. Wouters, H.M. Thierens, M.D. Cable, R.F. Purry, and Joseph Cemy 


\section{Enrisions and Plestic Track Detectors}

Dernonstration of a Detector with Unprecedented Charge Resolution

S.P. Ahlen, P.B. Price, G. Tarie, and M. Tincknell

Identification and Inaging of Nuclear Particles Using Etched Solids

S.P. Ahlen, P.B. Price, and G. Torle

Applications of Nuclear Track-Recording Solids to Higb-Energy Phenoment

P.B. Price

On the Possibility of Detecting Fractionaly Charged

Relativistic Projectile Fragments in Nucles Emulation

Harry H. Heckmat

ICAMS: A System for Computer Assisted Nuclear Microscopy

E.M. Friedlander, H.H. Heckman, Y.J. Karant

Study of Highly Ionizing Particles at Mountain Altitude

X. Kinashisa and P.B. Price

\section{Conputers}

The Design of MIDAS-A Modular Interactive Data Analysis System Oreve Maples, Willam Rathbun, Danie! Weaver.

and John Meng

Development of a MIDAS Prototype

Greve Maples, William Rathbun, Dariel Weaver,

and Jahn Meng

The Utilization of Parallel Processors in a Data Analysis Environment

Creve Moples, Daniel Weaver, WII Ran Rathon,

and John Meng

A Fast, Time-Sliced, Multiple Data Bus Suructure for Overlapping

$\mathrm{I} / \mathrm{O}$ and $\mathrm{CPU}$ Operations

W. Rothbun, C. Maples, J. Meng, and D. Weaver

Multidimensional Numerical Integration

R.W. Koontz

DATACQ, A New Data Acquisition Program

Friedemann Weik

An X-Y Histogramming Package Added to MLLTI

Friedemant Weik

Data Acquisition at the HISS Facility

Charles McPoriand

Data Acquisition Setop for the PJastic Ball (488H)

Friedemann Wetk

\section{ACCELERATORS}

88-Inch Cyclotron Operation

D.J. Clark, C.M. Lymeis, D. Elo, L. Glasgow, R. Lam,

T. Most, $R$ Muller, P. Tons, and J. Welch 
SuperHILAC Operations and Restarch

M.S. Zismon and R M. Diamond

\section{PART 3. Appendices}

Thesis Abstract ................................................................................. 267

Nuclear Sclente Division Seminars ............................................................... 267

Nuclear Theory Meetings ........................................................................... 270

Papers Published and LBL Reports Issued 1980-81 ...................................... 272

Atthor Index .......................................................................................... 283 


\section{Introduction}

This annual report describes the scientific research carried oul within the Nuclear Science Division between July 1, 1980 and June 30, 1981. The principel activity of the division ontinues to be the experimental and theoretical investigation of the interaction of heavy ions with target nuclei. Complementary research programs in light-ion nuclear science, in nuclear data evaluations, and in the development of advanced instrumentation are also carried ont.

Heayy ion research at the 88-Inch Cyclotron and the SuperHILAC focuses on spectroscopy and the properties of nuclear reactions. Currently the mechanism of nucleusnucleus collisions (decply inelastic scattering) and the nature of the angular momentum transfer during collision

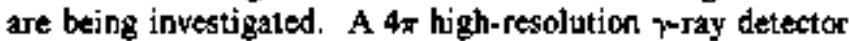
is being constructed to aid in the study (at both accelerators) of muclei excited to states of extrenely high angular momentum (60-70 h). The on-line isotope separator. recently completed at the SuperHILAC, is being used in searches for new isotopes throughout the periodic table. In order to keep pace with the ever more sophislicated multipararieter data acquisition techniques, a modular system for high speed interactive data analysis is under test

At the Bevalac, emphasis is on production of very high nuclear densities and temperatures by high-energy, head-on collisions. Recent research with relativistic heavy ions at the Bevalac has emphasized projectile and target fragmentation studies, with increasing focus on high-multiplicity events. This work is guided by close interactions with the nuclear theory group. The uranium beam capability (in FY 1982) and the recent completion of major new experimental facilities-the Heavy-Ion Spectrometer System (HISS) and the Plastic Ball/Wall detector system-will lurther this research. In addition, the Low Energy Beam Line is being brought into full operation to permit spectroscopic studies in the important transitional 30 to $200 \mathrm{MeV}$ per nucleon energy range.

All three accelerators support strong outside user programs, including both domestic and foreign cooperative efiorss. In particular, major collaborations with our German and Japanese colleagues continue to flourish at the Bevalac.

One hundred twenty six reports and journal artiches were published by division members. Direclly affiliated with the division throughoul this period wore 5 faculty sentior scientists, 18 staff sentior scientists, 26 staf' scientists, 1 divisional fellow, 12 posidoctoral fellows, 18 graduate students, and 26 1echnical, adminisirative, and clerical support staff. Clase to 50 additional graduate students from Berkeley and other universities make use of LBL's nuclear science facilities for much or all of their Ph.D. thesis work. Nearly 70 scientists from other institutions, both domestic and foreign, visited the division, bringing with them the diversity of ideas and talents upon which our research thrives.

During this period, Claude Lyneis joined the division as a staf scientist, responsible for the operation of the 88Inch Cydotron and for the development of cyclotron improvements. He came to LBL from the High Energy Physics Laboralory at Stanford University. Miklos Gyur lassy and Jorgen Randrup were promoted to senior stafi scientist positions. Both were previously Divisional Fellows; both are theoreticians and are instrumental in guiding the Bevalac research program.

Close to 1000 scientists from throughout the world gathered in Zellerbach Auditorium on the U.C. Berkeley Campus for the International Conference on Nuclear Physics, August 2430, 1980. Conterence arrangements were coordinated by members of the Nuclear Science Division: Bernard G. Harvey, program chairman, and Richerd $M$. Diamond and John $\boldsymbol{O}$. Rasmussen, conference secretaries. In addition to the approximately 100 invited and contributed talks, the poster sessions were extremely successful, and both types of presentations led to many stimulating discussions.

In order to meet heavy-ion physics needs beyond the 1980s, the division is collaborating with the Accelerator and Fusion Research Division to develop the concept of the Variable.Energy-Nuclear Synchmotron (VENUS). In addition 10 fixed-target operation at energies of up to $20 \mathrm{GeV} /$ nucleon, VENUS would be capable of collidingbeam operation equivalent to that of a $!-\mathrm{TeV} /$ nucleon fixed-target accelerator. It is expected that at such energy densities, new degrees of freedom of the nucleus will be excited and a quark-gluon plasma will be produced.

As part of the VENUS planning process, the sth High Energy Heavy Ion Study was held at LBL, May 18-22, 1981. The program commitles included Lee Schroeder, chairman, David Hendrie, Howel Pugh, Hans Gutbrod and Jorgen Randrup. The meeting brought logether a wide spectrum of physicists ( $\sim 150$ from the U.S., Europe, and Japan) to discuss the value of heavy ion collision studies in terms of conventional nuclear physics and in the production of new and exotic states of matter. The publisbed procedings will sterve as a valuable research document for the field in general and for the VENUS project in particular. 
PART 1. RESEARCH PROGRAMS

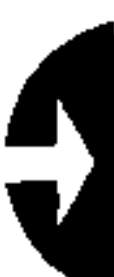




\section{Exotic Nuclei and Nuclear Reactions}

Joseph Cerny

\author{
A. Bice \\ M. Cable \\ R. Parry
}

\author{
H. Thierens \\ J. Wouters
}

This gromp has been actively investigating nuclei far from the valley of stability. Studies of this type yield experimental information testing various theoretical models predieting ground state mass excesses and energy level schemes. Several techniques have been developed for studying nuclei produced in low-yield reactions. Current experiments are concentrated in two regions of the chart of the nuclides, one near the double shell closure for nuclei with $N=Z=50$ and the other in the mass $=20$ region exploring nuclei which are predicted to define the proton drip line.

The experimental loot used to study isotopes near the mass 100 double shell closure has been the on-line mass separator, RAMA (an acronym for recoil atom mass analyzer) employed at the 88-Inch Cyclotron. This apparatus consists of a helium jet to transport recoil nuclei that are produced in a target chamber by both light- and heavy-ion bombardment to a hollow calhode ion source. Isotopes are then extracted lrom this ion source and magnetically analyzed. The mass of interest is collected on the RAMA focal plane by a tape transport sysiem that shuttles the activity to a $\beta-\gamma$ detection station. The decays of 102. 105 In have been observed following their production via $110-\mathrm{MeV}{ }^{14} \mathrm{~N}$ bombardment of $\mathrm{Mo}$ largets. Knowledge of the mass excesses of the daughter Cd nuclei and the measurement of the $\mathrm{Q}_{\beta}$ values for the ${ }^{103-10 S}$ In decays have yielded mass-excess values for these indium isolopes. Comparison of these results with various theoretical predictions bas produced interesting disagroements with the expected model mass surlaces. Future studies with RAMA are planned that investigate somewhat lighter isolopes (e.g., Se) at or near the $\mathrm{N}=\mathrm{Z}$ line.

A rocent developotent in this program of studying nuclei far from stability is an allempt to observe the decays of the (predicted) is-delayed proton precursors ${ }^{22} \mathrm{~A}(4 n+2$ mass series, $\left.T_{Z}=-2\right)$ and ${ }^{23} \mathrm{Si}(4 n+3$ mass series, $T_{\mathrm{Z}}=-5 / 2$ ). The experimental technique employed u1ilizes a minimal length He-jet to transport these short lived nuclides from the target region to a collection point in front of a solid state detector telescope. Observation of the existence of these isotopes will comprise a substantial test of theoretical predietions of the stability of proton-rich nuclei in this light mass region.

In recent years, this group's restarch interests and eflorts have also included developing techniques to detect unbound resonant states as nuclear reaction products. With these detection capabilities, it has been possible to extract valuable spectroscopic information from transfer reactions such as $\left({ }^{12} \mathrm{C},{ }^{8} \mathrm{Be}\right)$. $\left({ }^{4} \mathrm{He},{ }^{2} \mathrm{He}\right)$ and $\left({ }^{3} \mathrm{He},{ }^{4} \mathrm{He}\right.$ $\left.\left(0^{+}, 20.1 \mathrm{MeV}\right)\right]$. Recent studies have utilized similar techniques to investigate light heavy-ion reaction mechanisms in the range $10-20 \mathrm{MeV} /$ aucleon. In particular, the importance of sequential decay processes in projectile frasmentation reactions has been under investigation with ${ }^{7} \mathbf{L}$, ${ }^{12} \mathrm{C}_{+}{ }^{13} \mathrm{C}$, and ${ }^{16} \mathrm{O}$ projectiles. Such studies could prowe useful in verifying theories pertaining to the mechanisms intolwed in fragmentation teactions and fast light-particle production. 


\title{
Heavy Ion Reactions
}

\author{
B.G. Havey and R G. Stokstad
}

\author{
J. Mahoney \\ M.J. Marphy \\ W.D. Rae \\ C. Albiston* \\ Lowrence Berkeley Leboratory
}

\author{
$R$ Legrath \\ Saciay, France \\ J. Cole \\ Grenoble, fronce
}

This grovp is concerned primarily with heavy ion reac tions in the so-called transition tegion. This is a region of bernbarding energy in which the phenomena characteristic of low bombarding energies, fusion and nucleon transfer. are expected to diminish as the bombarding entergy increases and processes such as projectile fragmentation and nucleon-nucleon induced collisions dominate. There are several specific experiments under this nmbrella which address this goal.

The most general or inclusive property of a nucdear reaction is the total crass section ${ }_{T}$, which is comprised of nuclear elastic scattering and the lotal reaction cross sectjon $\sigma_{\mathrm{R}}$. Remarkably little was known about the behavior of ${ }^{\circ} \mathrm{K}$ and $\sigma_{\mathrm{T}}$ in the transition region for projectiles heavier than belium Ore knew only that, at energies less than $10 \mathrm{MeV} /$ nucleon (Lab), $\sigma_{\mathrm{R}}$ rises with $\mathrm{E}$ and at energies $>800 \mathrm{MeV} /$ nucleon $\sigma_{\mathrm{R}}$ is much smaller. Measurements for the ${ }^{12} \mathrm{C}+{ }^{12} \mathrm{C}$ system ${ }_{\mathrm{R}}$ mpleted by this group during the past year have localized the region in which $\sigma_{\mathrm{R}}(\mathrm{E})$ reaches a maximurn at $-15 \mathrm{MeV} / \mathrm{v}$. This agress rather well with the predictions of a microscopic calculation by DeVries et al. based on the known free nucleon-huctoon cross section. However, further work over a wider tange of energies will be needed to undersiand how the respective roles of "mean field" and "nucleonnutecteon" mechanisms are exenpilified in the total reaction cross section.

Peripheral reactions may be divided soughly into 1) those producing a two body final state in which the mass missing from the projectile is eaptured by the tariget, or 2) fragmentation or break-up reactions. Because of its $4 \pi$ efficiency, the hybrid streamer chamber loealed at the 88-Inch Cyclotron makes possible the identification and separation of these two processes. Measurements of the reaction $\{16.5 \mathrm{MeV} / \mu)^{16} \mathrm{O}+\mathrm{Cs}_{\text {l }}$ have recently been analyzed and, at least in terms of the relative yjelds of transfer reactions producing the elements $\mathrm{N}, \mathrm{C}$, and $\mathrm{B}$, are explained in terms of Wilczynski's model for incomplete fusion. The next step is to compare the energy dependence of the magnitude and width of the quasj-elastic yields of these reaction products separately for transfer and fragmentation events. Because of the large amount of scanning that would be associated with a strtames chamber measure ment, a $4 \pi$ plastic scintillator array is being developed. The information geined from the streamer chamber is of great value in the design of the conuter experiment. The ability to aoquire data quickly should enable a detailed study of the projectile, target, and energy dependence of these widthe, the yalues of which have recently been shown to approach the limiting value, $\sigma_{0} \approx 85 \mathrm{MeV} / \mathrm{c}_{1}$ more slowly than previously thought.

Fragmentation or break-up processes are best studied by coincidence experiments in which the relative kinetic energy of the delected particles is accurately determined. To do this requires a high angulas resolution which can only be attained, without lass of solid angle, by using position sensitive detectors. Posjtion senșitive $(x-y) \Delta E-E$ telescopes have been used to study the reaclions $160+$ $\left({ }^{12} \mathrm{C}_{+}{ }^{13} \mathrm{C}^{28} \mathrm{Si}\right) \rightarrow \mathrm{HI}+\alpha$. The resolution in the spectrum of relative kinetic energy of the $\mathrm{Hl}+$ a system was - $130 \mathrm{keV}$. This enabled the clear identification of break-up processes proceeding 1hrough discrete stales of ${ }^{16} \mathrm{O}^{\circ}$ (and other excited nuclei as well). By studying the particular states that were excited it was passible to identify al Jeast two mochanisms in the breakap of ${ }^{16} \mathrm{O}$ into ${ }^{12} \mathrm{C}+\alpha$. The first is a coherenl process that excites the projectile to states whose struclure is related to that of the ground state via a multipale operator. This mechanism is important only for the lower $Q$ values while the second meshanism, an incolherent process in which the $\alpha$ and ${ }^{12} \mathrm{C}$ constituents are assumed to interact independently with the target, is present for very negative $Q$ values as well.

A parl of the group's effort involves collaboration with outside users. Physicisis from Michigan State University, Oak Ridge National Laboratory, the University of Mexics, and the Jagelonian University in Poland have worked with this group at the 88- Inch Cyelotron duting the pas1 year. Studies of the direct reactions, rusion-evaporation, and fusion-fission of light nuclear systems such as $\mathrm{N}+\mathrm{C}$. $\mathbf{N}+\mathbf{B}$, and $\mathbf{L i}+\mathbf{C a}$ have begun.

The instrumentation at the 88-Inch Cyclotron used by the group includes the smali hybrid streamer chamber, a newly developed time of flight spectrometer with a onemeter long, posilion sensitive ionizauion chamber, and solid-stale, position-sensilive $\Delta \mathrm{E}-\mathrm{E}$ telescopes. A most recent addition is a 60-inch diameter scatlering chamber buik by the Jagellonian Universily in Cracow, Poland. This chamber replaces the outdated 36-inch chamber and will be available to all users.

Footnote

Graduate student 


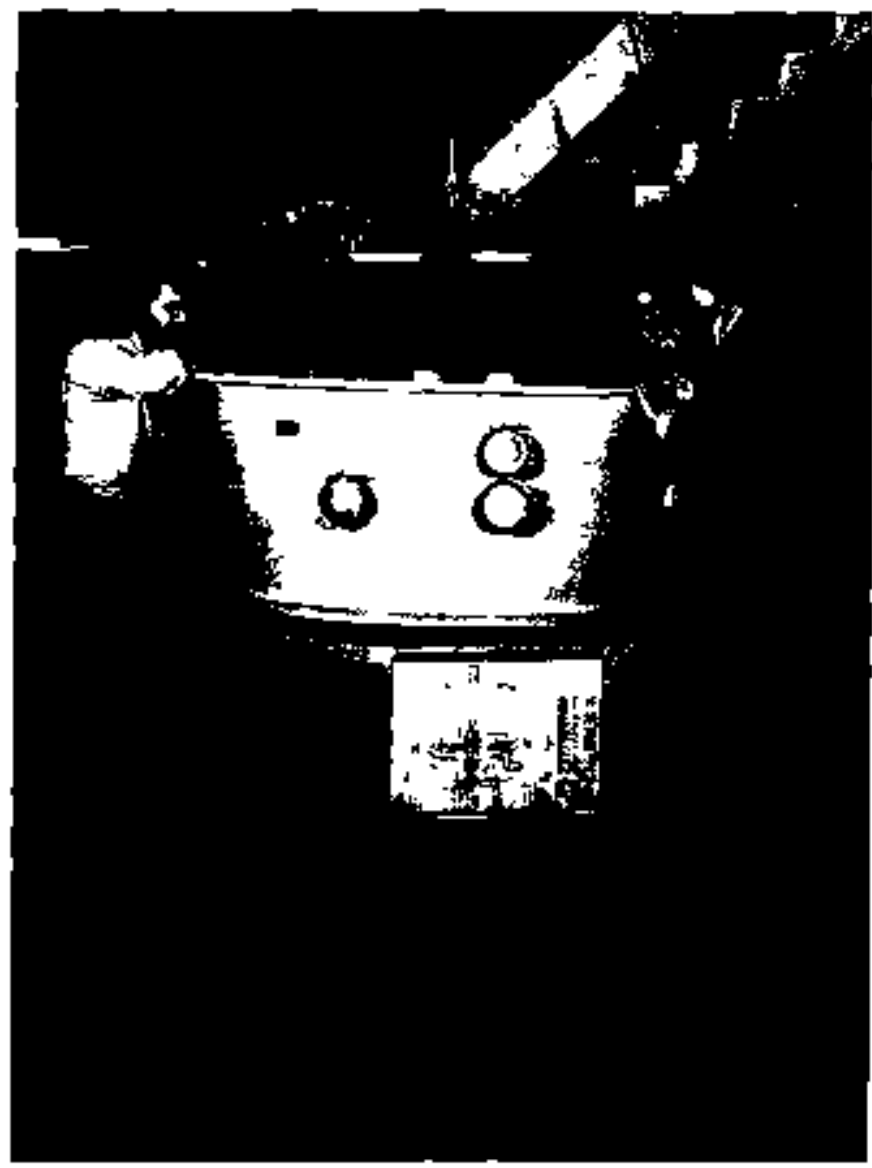

The newly acquired 60-inch diameter scattering chamber built in Poland by the Jagellontan Universly. Carolyn AJbiston and Martin Murphy are shown inspeeting the chamber.

(CBB 819-8447) 


\title{
Nuclear Structure
}

\author{
RM. Diamond, F. Stephens, M.A. Deleplangue
}

\author{
J.E. Draper \\ E.L Dines \\ U.C. Davis \\ K.L. Lindenberger \\ HM, Berlin, West Gernany \\ C. Schück \\ CSinsid, orsay. Frace \\ J. Burde \\ Hebrew Uhiwersity, Istael
}

\author{
J.O. Newton \\ Aushaliton Notional University, Conberra \\ S.H. Shih \\ Shanghal Instituite of Nuclear Resedrch \\ Peoples Republit of Ohina \\ A. Machianelli \\ Buesos Aires. Argentimat
}

This group is interested in studying and understanding a number of aspects of nucleat structure. For some years the principal effort has been isvestigation of nuclei at bigh angular momentum. Nuclei around the middle of the periodic table have spins near $70 \mathrm{~h}$ before the Coriolis and centrifugal forses disrupt them, causing fission. The chance to study nuclei under onditions where these famjliar forces become major factors in determining the nuclear structure is attractive, particularly sines the ${ }^{40} \mathrm{Ar}$ projectiles available from the LBL 88-Inch Cyclotron can easily bring the full $70 \mathrm{~h}$ into a compound nuclear system. We have learned that nuclei have both oollective and single particle features at these high spins. They usually rotate in a rather classical manner; but, because the system is finite (- 100 particles), there are strong irregularities "backbends" the to just one or two particles realigning their angular momentum. In such nuclei, a single ${ }^{(} i_{13 / 2}$ or $\mathrm{j}_{15 / 2}$ ) particle can carry angular monentum as high as $10 \%$ of the maximum the system can hold. The interplay of these two modes of motion is one of the fascinating aspects of studies of high spin states. Howtever, such studies are complicated by the fact that the $\gamma$-ray spectrum from states above $-30 \%$ bas been thus far unresolvable. The popolation in this region is spread over too many levels. Several techniques have been developed to study auch unresolved spectra: arrays of detectors, large (sum-energy) crystals, and currently the development of $4 \pi$ detector systems.

Most recent]y, our group has been collaborating with Herskind et al. from Copenhagen to study the correlations of $\gamma$-ray energies in these unresolved spectra. These twodimensional correlation spectra sbow structures up to the highest spins, which give information about the average behavior of both the collective and the single-particle motion. Nevertheless, it is becoming increasingly clear that the full elucidation of the physics cones only with the full resolution of the spectrum We are now bujlding a system consisting of an $\sim 4 \pi$ bismuth germanate inner spherical shell with apertures to $\approx 20$ Compton-suppressed intrinsic germanium detectors. This system will combine the spin selection of the inner ball with the high resolving power of Ge-Ge coinciderces, and promises to give delailed information to much higher spins.
The newest study this group has undertaken, and at present one of the most exciting, is the observation of the giant dipole resonance (GDR) in the deexcitation $\gamma$-Jay cascades from heavy-ion fusion and deep inclastic reaction produets. States in a nucleus $15 \mathrm{MeV}$ or more abowe the yrasi line are thought to contain adruixtures of the GDR based on states - $15 \mathrm{MeV}$ lower in energy (this is sometimes called Brink's hypothesis). When excited, all such states have the possibility to emil a GDR El $\gamma$ ray in competition with the more usual netution or particle emission. After particle evaporation down to an energy less than a neutron binding energy abow the yrast line. the nucleus decays to the ground state by a cascade of up to $25.30 \gamma$ rays. In such an onresolved spectrum, the transitions above $\sim 2 \mathrm{MeV}$ fall of exponentially in intensity with increasing $\gamma$-ray entgy (Lhe statistical region) and are very weak above $8 \mathrm{MeV}$. We have observed shoulders at 10-18 MeV in such detxcitation spectes from heavy ion fusion reaction products and have interpreted them as the GDR decays of the highly excited states above the main $\gamma$ ray cascades. The use of our tolal-energy $\gamma$-ray spectrometer was instrumental in eliminating $\gamma$ rays of other origin, and at the morkent cosmic rays are our limiting background. The information from such spectra is of a new type and potentially very interesting. It appears likely that one can measure the energy, yield, width, and shape of the GDR component of the statistical $\gamma$-ray spectrum. The strength of the resontance is related to jts collectivity, and its width, to the more detailed features of the nudear dynamics. The present measurements open the possibility of studying these as a function of excitation energy (tenperalure) and spin. The gross structure of the GDR is related to the shape of the nucleus; in deformed nuclei with two or three distinct radii, the GDR is split into two or three components. Thus, the observation of only the general struclure of the resonance peak sbould provide information on the nuclear shape, again as a function of temperature and spin. Because such studies can be made on many nuclei, the observation of GDR effects in nuclear deexcitation $\gamma$-ray spectra seems likely to provide a new and general method to study nuclear dynamics far from the ground stale. 
In addition to these two main efforts, the group pursues its angular momentum studies to higher values than the compound system can hold by studying "deep inelastic collisions" in collaboration with the Moretto group. Here, reaction channels with angular momenta of several hundred $h$ can be isolated and studied. We also study other aspects of nuclear structure and shapes in eollaboration with several outside groups: Clíne et al. from Rochester, Ward et al. from Chalk River, and Johnson \&l al. from Oak Ridge. These studies primarily concern Coulomb excita tion with very heavy joms ( ${ }^{136} \mathrm{Xe}$ and ${ }^{208} \mathrm{~Pb}$ ) produced by the SuperHILAC, and curtently include Coulomb excitation studies of transition nuclei such as $\mathrm{Ph}, \mathrm{O}, \mathrm{Ru}$, and
Pd; work on deformed nuclei; Doppler-shift lifetime measurements; and g-factor measurements by means of the transient field

Our group is also launching studies of particle transfer reactions betwen very beavy systems. lnteresting questions arise concerning how the orientation of deformed nuclei at contact affects the transfer probabilities. One can also learn how much the pairing correlations enhance the Iransfer of pairs of nucleons. The only limitation of investigations such as these is the arajlability of accelerator time and manpower. 


\section{Polarization Phenomena in Nuclear Physics}

\author{
H.E. Conzent \\ R.M. Larimer \\ Lowrence Berkeley Laboratory
}

\author{
E.J. Ludwig \\ Universily' of Narth Carodina \\ P. von Rossen \\ Chiversity of Bonn, West Getmotny
}

This group's research uses the polarized beams from the 88-Inch Cyclotron and is concerned with spinpolarization effects in nuclear scattering and reactions. This research particularly addresses such fundamental questions as parity violation by the weak interaction com. ponent in pp scaltering, time-reversal invariance in reactions, charge symmetry of the nucleon-nucleon interaction, and the three nucleon problem.

A most important feature of polarization effects is their ability to often display uniquely the operation of a basic symmetry property of the nuclear inleraction. Our group is pursuing several fundamentally important experiments of this nature:

I. We have prepered a longitudinally polarized proton beam and the experimental apparatus for a major experiment on the basic nucleon-nucleon interaction, i.e., a determination of the weak interaction parity nonconserving part of this interaction from a measurement of the longitudinal analyzing-power component in pp scattering at $50 \mathrm{MeV}$. Calculations predict a maximum value $\left(3 \times 10^{-7}\right)$ at $50 \mathrm{MeV}$. The group uses one of the three existing polarized-bean facilities in the world with the capability to do the experiment at that energy.

2. We have been engaged in a collaborative experiment al TRIUMF to determine the level of validity of charge symrnetry in the nucleon-nucleon interaction, which implies an equality of the neutron and proton polarizations in np scattering. The experiment is designed to check this equality to an accuracy of $10^{-3}$. The one experimental sun that has taken place in pp scattering, verifies that this accuracy is passible.

3. We have started a collaborative experimenl with a group from Laval University to 1est the principle of timereversal invaciance in nuclear reactions. This principle resul1s in the polarization-aralyzing power equality, i.e.. the polarization in a reaction $A(a, b) B$ is equal to the analyzing power in the inverse reaction with polarized projectile $B(b, a) A$ Polarizations have been measured at
Laval in several ( ${ }^{3} \mathrm{He}, \mathrm{p}$ ) reactions, and measurements of the analyzing powers in the corresponding $\left(p^{3} \mathrm{He}\right.$ ) inverse reactions have been made at $\mathrm{LBL}$. Previous checks of time-feversal invariance in nuctear reactions have been limited to tesis in elastic proton scattering.

Substantial differences between the polarization and the analyzing power have been measured. If such differences are confirmed in additional experiments underway, it would be firm evidence for the violation of the fundamental postulate of time-reversal invariance of the nuclear interaction. In view of these results, a survey of the past polarization 1esis of time-reversal invariance was made. Surprisingly. it was found that all such lests were inadequate, due either 10 lack of precision or lack of sensitivity to a violation of time-reversal invariance. Therefore. further experiments are being undertaken in order to provide significant tests of this important principle.

Polarization observables are often unique probes that can answer specific questions concerning nuclear structure. As examples, this group is planning measurements of the transfer of spin in $(p, n)$ and $(d, p)$ retactions. The $(p, n)$ measurements can give explicit information on the nuclear collective modes excited, which is of great curren! interest, while the $(d, p)$ measurements may provide a direct determination of a very elusive quantity, the deuteron D-stale probability, which is fundamenta] to the most basic nuclear system.

In future work, we plan 10 continue 10 focus on experiments with polarized particles which examine the operation of the basic symmetries of the nuclear interaction, i.e. parity conservation in pp scattering, charge symmelry in np scattering, and time-reversal invariance in nuclear reactions. The increased polarized beam intensities of 2-3 microamperes, provided by our FY 1980 Acceleralor Improvement Project. will be particulaty important to these experiments because they require high statistical accuracy and overall precision. We will also continue studies of the consequences of particle identity and charge symmetry in nuclear reactions. 


\title{
Statistical Equilibrium in Deep-Inelastic Heavy Ion Reactions
}

\author{
LG. Moretto
}

\author{
J. Hunter \\ R.J. McDonald \\ D.J. Morrissey \\ C.G. Sobotko \\ A.J. Pacheco" \\ G.J Wozniak \\ Lantence Berkedtl Labororory
}

\author{
$\mathrm{C.CH} \mathrm{H}_{\mathrm{u}}$ \\ Betfing. Propites Repubitc of Chat
}

During the last several years, thiș group hats been investigating the short-lived dinuclear system formed in heavy ion collisions. In perticular, we have been studying the tranşfer, alignment and partitioning of angular momentum within the dinuclear system. The experimental investigations utilize heavy ion beams of $5-50 \mathrm{MeV} / \mathrm{A}$ from the 88. Inch Cyclotron, the SuperHILAC, and the low-energy line of the Bevalec.

In a parallel effort, our group has been studying the theoretical implications of fuctuations in deep inelastic heary ion collisions. The relevance of the statistical equilibrium limit to the descriplion of substantially replaxed degrees of freedom has been assessed. The effects of fuctuations have been considered for (I) the correlation between the entrance-channel angular momentum and the exit-channel kinetic energy. (2) the sharing of the dissipated kinetic energy between the two fragments, and (3) the alignment of the fragment angular momentum.

Our investigations indicate that statistical fuctuations play a major role and that the statistical equilibrium limit seens to have been reached in a number of instances. For mass-symmetric and near-symmetric heavy systems, continuum $\gamma$-ray angular distributions indicale that the fragment spin alignment is destroyed at large Q-values by random spin luctuations. In very mass-asymmetric systems, the statistical excitation of most angular momentum bearing modes is strongly suppressed; this results in a preferential dealignment of the spin crenting a substantial in-plant anisolropy of sequential fission fragments. This anisolropy was observed at large Q-values for the ${ }^{20} \mathrm{Ne}+197 \mathrm{Au}$ and ${ }^{20} \mathrm{Nk}+{ }^{230} \mathrm{U}$ systems. Further investigations of fuclua. tions are also being undertaken with a total $\gamma$-ray energy spectrometer.

An important question regarding heavy ion oollisions is how the reaclion mechanism evolyes from the statistical equilibrium, observed in the low energy regime, to the completely nonequilibrium processes observed in the relativistic regime. In order to explore the intermediate regime, the angular momentum transferred to the heasy target-like Iragment was followed in the reaction ${ }^{20} \mathrm{Ne}+{ }^{181} \mathrm{Ta}$ in the energy range of 7.5 to $42 \mathrm{MeV} / \mathrm{A}$ Comparisons of the measured intrinsic spin with that expected from the missing linear momentum indicale that, at bombarding energies of $17 \mathrm{MeV} / \mathrm{A}$ and above, the ${ }^{20} \mathrm{Ne}+{ }^{181} \mathrm{Ta}$ reaction has lost is "Lwo-body" character.

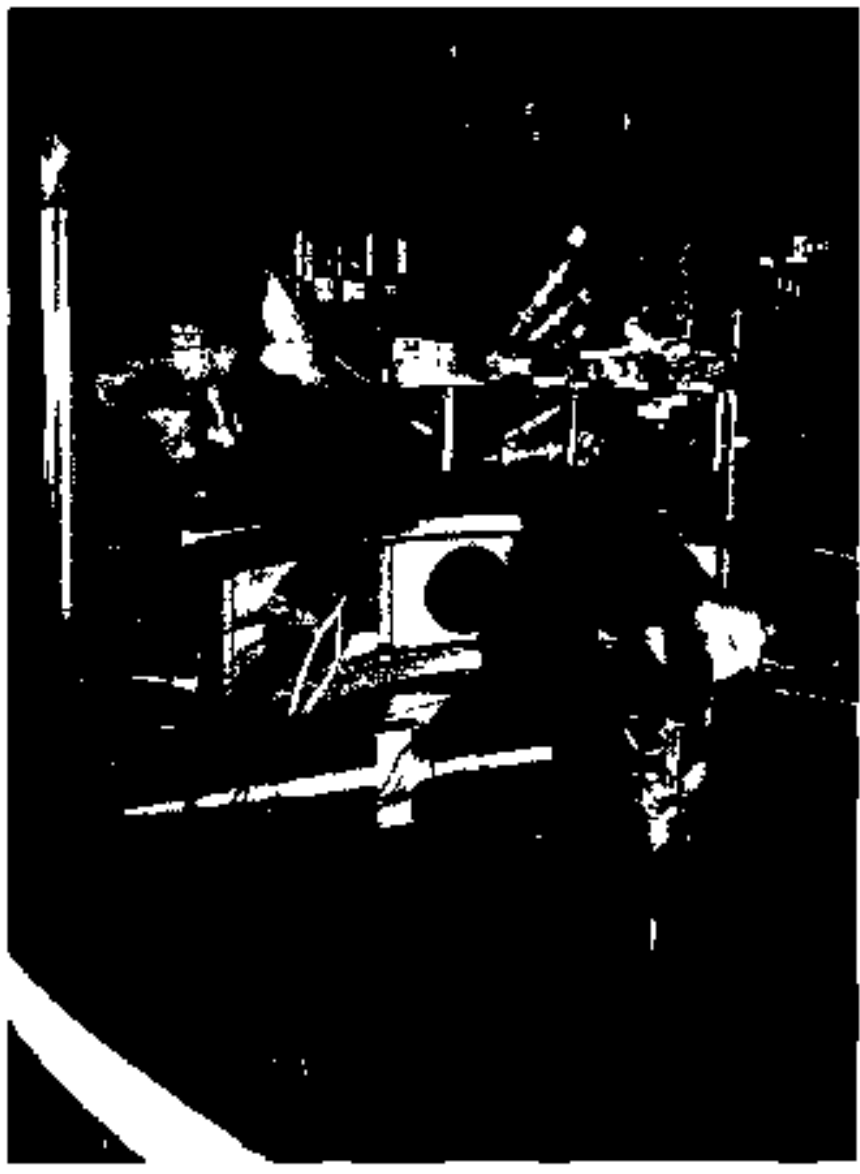

Scattering chamber and detector sctup for sequential fission iraginent angular distribution measurements. The beam enters the scatlering thamber on the left through the brass tube and strikes a ${ }^{\prime \prime}$ Au target in the eenter. To the right of Dave Morrissey's hand is a Z-telescope that is used to delect the projectile residues and define a reaction plane. Note the raised lid and the attached array of silicon fission fragment detectors.

\section{Footmote}

"Graduate students 


\section{Heavy Element Research}

A Ghiorso and J.M. Nitschke

\author{
M.J. Nurmia \\ L.P. Somerville \\ S. Yashila \\ Lamence Berkefey Labarasory \\ D.C. Hofimann \\ Los Aldmos National Letharatory \\ M. Leino \\ Chiversisy of Helsinki, Finland
}

\author{
E.K. Hulet \\ R.W. Lougheed \\ J.F. Widd \\ Lawrence Livermave Noridotal Labonatory \\ J.D. Molitoris \\ Sranford Universioy
}

The Heavy Element Research group is engaged in synthesizing new nuclides and determining the radioactive properties or known species. Important in these efforts, graduate sudents are trained in the advanced methods of nuclear physics. The group performs experiments, both at the 88. Inch Cyclotion and the SuperHILAC, and at times collaborates with other American laborationies in experimenls at GSI, Germany.

Research at the 88-Inch Cyclotron is focused primarily on the production of neutron-rich nuclides made from various target malerials from plutoniurn to californium with high intensily light ion beams. Three analysis methods are being employed: (1) the MG system to determine the E1 vs E2 characteristics of the itagments of short-lived spontaneous fission (SF) emitters; (2) the kilometer-long tape system to detect very short-lived SF nuelides in the presence of high intensity, longer-lived SF backgrounds; and (3) the differential range-measuring system for determination of nucleas reaction mechanisms by the direct analysis of the alpha and SF decay characteristics of recoil atoms lodged in stacks of thin foils.

The group's eflorts al the SuperHILAC are centered around two large instruments: SASSY, a gas-filled magnetic spectrometer, and OASIS, the on-line isotope separator. SASSY (Small Angle Separator SYstem) is used for the analysis of fusion or near-fusion recoil products with half-lives as shor as a microsecond. The system has an acceptance angle of about two degrets in the beam direction and is able to completely suppress the heavy ion beam while transmitling a vety high fraction of the recoils within its acceptance angle. The heavy recoils are brought to a focus four melers downstream from the target al a focal plane where two types of detectors can be used-a solid slate detector array for recoils, alphas, and SF, or a gas-filled ion chamber for recoils alone. Two avalanche counters with ultra-thin windows intercept the recoil path to provide START-STOP signals for velocity (TOF) measurements. Using the crystal detector array we have studied neutron-deficient alpha emitters in the Bi-Pa region, both to learn about the perlormance and limitations of SASSY, and to supplement existing information about a number of nuclides.

The on-line isotope separator at the SuperHILAC has turned out to be a viable specimen and it has therefore betn given a suitable name: OASIS (On-line Apparatus for SuperHILAC Isotope Separation). It has proven its versatility with the separalion of more than twenty different ele. ments so far. On-line study of the separated isotopes has, however, been restricted to particle spectroscopy or off-line $\gamma$ measurements because of the large background inside of the cave. This problem will be alleviated by an isotope transfer line which is now being built. It will gujde the beam of a single separated isotope to a room on the second floor of Building $7 l$ where it will be oollected by a tape system. The radiation background in this room has been measured to be very low and will allow sensitive $\beta, \gamma$, and neutron-measurements. The tape sysiem (based on a surplus IBM 729) facilitates the study of short-lived isotopes far from the line of $\theta$-stability in the presence of unwanted long-lived isobars of $\beta \pm$ decay chains. The range of elements which can be separated with OASIS hes been greatly enlarged with improvernents of the plasmet jon source.

A modification of the source now perrnits the study of isotopes produced in deep inelastic collisions. With this reaction mechanism it is possibte to reach parts of the neutron-rich side of the nuclide chart which are not easily accessible by other means. By using neutron-rich projectiles from the upgraded SuperHILAC, we will be able to complement research which has in the past been limited to fission products and high energy fragmentation. The other side of the yalley of $\beta$-stability up to the proton drip line can be approsebed with compound nucleus reactions of neutron-deficient targets and projectiles. A special ion source has been built for this kind of reaction which also allows the use of radioastive targets.

Our collaboration with GSI was continued with a second ${ }^{238} \mathrm{U}+{ }^{248} \mathrm{Cm}$ experiment this year. Previous experience had shown that the ${ }^{238} \mathrm{U}$ beam caused serious degradations of the metallic ${ }^{248} \mathrm{Cm}$ targets. An improve: ment in the targel cooling, however, yielded an increase of nearly two orders of magnitude in the ${ }^{238} \mathrm{U}$ beam integral. This will push, in our present experiment, the detection limit for heavy actinides and superheavy elements thell below the $10^{-33} \mathrm{~cm}^{2}(1 \mathrm{nb})$ mark for half-lives between several hours and several years. The perfection of our ircadiation techniques will also enable us in the future to reach shorter half-lives with chemical and physical on-line techniques. 


\section{High Energy Nuclear Collisions}

\section{A.M. Poskanzer}

Lowretace Berkeley Labordtory

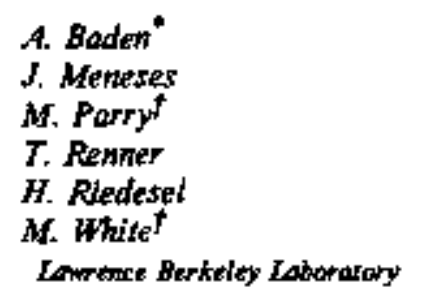

A. Wolf

Argone Nationd Laboratory

\author{
H.H. Gutbrod' \\ GS, Darnstad, Wav Germeny
}

H.G. Ritter

H. Spieler

R. Stock

F. Welk

H. Wieman

GSI. Durmstat, West Germsthy
This group studies central collisions of relativistic heary ions with the aim of learning aboul nuclear matler al high temperature and density. The group is a continuing collaboration between GSI and LBL

In 1980. an experiment was performed to sludy the correlations between slow and fest tragments. For the slow fragments, en array of 40 detectors consisting of silicon counters, gas $\Delta \mathrm{E}$ counters, and avalanche counters was used. For the fast fragments, the 80 -eounter multiplicity array and the Plastic Wall were used. The analysis focuses in one part on the $\phi$ correlation between the fast and slow fragments, to be used as a tool to study reaction dynamics. The correlation between two slow fragments, on the other hand, reveals novel features in the breakup of the nucleus.

The most importanl project this year was the construction of the Plastic Ball. This is an attempt to make a much more exclusive measurement of relativistic heavy ion interactions to search for collective phetomena.

The Pastic Ball covers $96 \%$ of $4 \pi$ wilh 815 modules, each consisting of a $\mathrm{CaF}_{2} \Delta \mathrm{E}$ detector and a plastic scintillator $\mathbf{E}$ detector. The two scintillators are observed by the same photomultiplier tube, and the signals are separated electronicaliy by taking advantage of their different decay characteristies. In addition, positive pions are identified by their $\pi^{+} \rightarrow \mu^{+} \rightarrow \mathrm{e}^{+}$decty, as we have done previously. The small forward angle region is covered by the Plastic Wall placed $6 \mathrm{~m}$ downstream. The Wall consists of 177 plastic scintillators and identifies particles by their time of flight and energy loss. In the first experiments, the Plastic Wall provides also the trigger for the reaction. The Plastic Ball was completed in time for the very successful datetaking run in June 1981.

Footnotes

Graduate studen

†Undergraduate student 


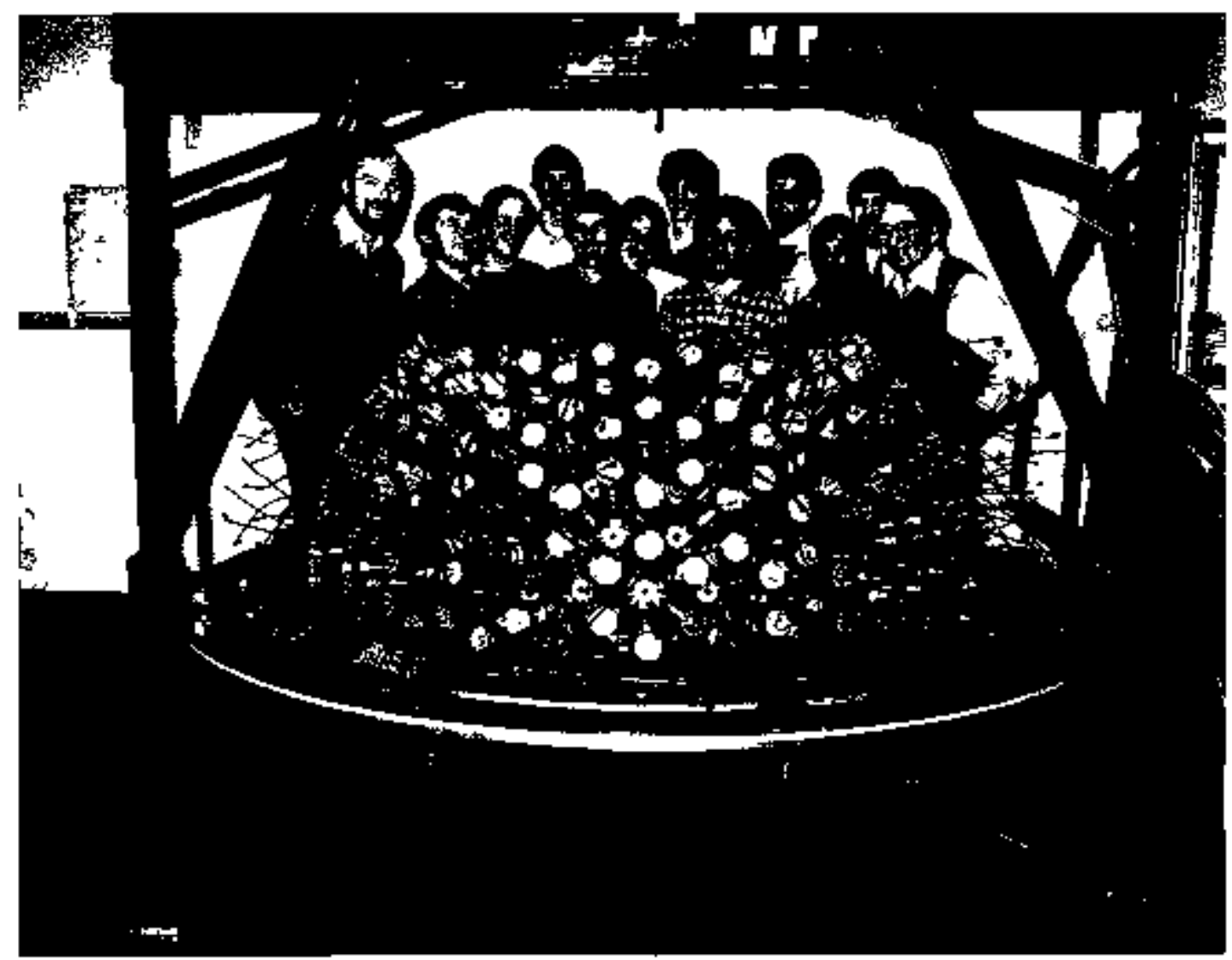

One half of the Plastic Ball in the process of being stacked. From left to right: H. Gutbrod, H. Wieman, H. Riedesel, M. Perry, H.-G. Ritter, A Warwick, H. Loehner, A. Baden, M. Maier, M. While, J. Meneses, and A. Poskanzer.

(CBB $811-837)$ 


\section{Relativistic Heavy Ion Physics}

H.H. Herkman

E. Friedlander
Y. Karant
M. Bloomer
J. DeLara
R. Gimpel
M. Heckman
S. Janssen
J. Sturia
H. Yer
Lowrence Berketey Laboratory

This group's experimental program in relativistic heavy ion physics at the Bevalac has focused on two impottant aspects of projectile fragmentation during FY 198 l. These are, first, the interaction properties of relativistic projectile fragments of $\sim 2 \mathrm{~A} \mathrm{GeV}{ }^{6} \mathrm{O}$ and ${ }^{56} \mathrm{Fe}$ and the evidence therefrom for "anomalons." As an integral part of this particular program, we undertook the development of an interactive computer assisted mcasuring system (ICAMS) to improve the rate and seope of data acquisition. Second is the electromingnetic dissociation of beam nuclei by interactions wilh the viftual photons of the nuciear Coulomb field in high- $Z$ targets (i.t., the Weizsācker-Williams process), this investigation being performed with 1.7 A GeV- ${ }^{18} \mathrm{O}$.

In the field of nuclear interactions at energies comparable to those accessible in the VENUS project, a col]aborative effort with the Universities of Exeter, England, and Marburg, West Germany, has produced the firsl direct evidence for coherent pion emission in proton-nucleus collisions along with the theoretical treatment of the suppression of nuclear cascading via self-induced transparency of nuclear matter to the colerent pulse produced in the first nucleor-nucleon encounter in the nucleus.

During collaboration with the University of Frankfurt/M (West Germany), evidence has been obtained for two kinds of reaction mechanisms in nucleus-nucleus collisions induced by Bevalac beams in nuclear emulsions. Both in the target and in the projectile frame, a subset of events could be ragged which appear to display a higltemperature $(-40 \mathrm{MeV})$ thermodynamical behavior.

\section{Projectile Fragmentation and the Evidence for "Anomatons"}

In collaboration with B. Judek of the National Research Council of Canada, we have compieted two independent nuclear emulsion experiments using Bevalac beams of ${ }^{16} \mathrm{O}$ and ${ }^{56} \mathrm{Fe}$ at $-2 \mathrm{~A}$ GeV. We find, with $>99.7 \%$ confidence, that the reaction mean-free paths of projectile fragments, $3 \leq \mathrm{Z} \leq 26$, are shorter for a few centimeters after their emission than at larger distances or shoner than predicted from experiments on bearn nuclei. Our results can be summarized as follows:

\author{
D. Otson \\ Lawresce Livermove Narional Labourarory \\ E. Ganssauge \\ Lhiwersify of Marburg. West Germany \\ B. Judek \\ Natianat Resedich Cowncti, Oitawa. Camada
}

1. Dver the first few centimeters after emerging from a muclear interaclion (or about $10^{-11}$ sec proper time), the projectile fragments exhibit significantly shorter mean-Iree paths than those derived from "normal" beams of the stame charge $\mathrm{Z}$,

2. At larger distances from the enission point the mean-free paths revert to "nomality," and

3. The data are not compatible with a homagencouss lowering of the mean-free path and require the presence among projectile fragments of at least one component with an unexpectedly high reaction cross section.

These effects are interpretable under the simplest assumption that a small fraction $(-6 \%)$ of the projectile Iragments are anomalons with a mean free path $\lambda_{\mathrm{a}} \simeq 2.5$ $\mathrm{cm}$, independent of charge. Such a mean-free path corresponds to a cross section 5 to 10 times larger than geometric for the fragment considered. The extent of these diflerences between the primary, secondary, and later generation fragments have not been, and perhaps cannot be, explained within the framework of conventional nuclear physies.

Bevolac Experiment $559 \mathrm{H}$. This experiment on the fragmentation reactions of ${ }^{96} \mathrm{Fe}$ at $1.8 \mathrm{~A} \mathrm{GeV}$ was carcied out in two run periods during this fiscal year. The experiment extends our studies on the evidences for anomalons and in wolved the irradiation of emulsion detectors to a variety of bearis, including ${ }^{3} \mathrm{He},{ }^{6} \mathrm{Li},{ }^{1} \mathrm{C}_{+}$and ${ }^{40} \mathrm{Ar}$, in addition to ${ }^{56} \mathrm{Fe}$, the principal beam under study. Experimert $559 \mathrm{H}$ also included a series of emulsion exposures to measure the lifetime of the anomalon effect. All irradiations were sucessiul and the scanning and measuring of the emulsions are in progress.

\section{Electromagnetic Dissociation of Relativistic ${ }^{18} \mathrm{O}$ Nuclei}

Bevalac Experfmewt $350 \mathrm{H}$. This experiment, carried out in collaboration with D.L. Olson and B.L. Berman (LLNL), investigated the electromagnetic fragmentation of I.7 A CeV ${ }^{18} \mathrm{O}$ nuclei via interactions with the nuclear Coulomb field of target nuclei. Measured in this experiment were the electromagretic contribution to the single- 
proton and the one- and two-neutron removal fragmentation cross sections for ${ }^{18} \mathrm{O}$ in a variety of targets, be throwgh $\mathrm{U}$. It was found that the electromagnetic fragmentation cross sections deduced from the experiment for these particular reactions agroe both in magnitude and $Z_{\text {larget }}$ dependence with the predictions of the classical relativistic virtual. photon theory (the Weizsäcker and Williams process) which incorporates experimentally measured photonuclear cross sections. An important result 10 come from this experiment is that the factorization of the cross sections for nuclear processes (into beam/fragment and target dependent terms) is valid to an accuracy better than $4 \%$, a quantily consistent with the known systematic uncertainlies in the experiment.

Bevalac Experiment $517 \mathrm{H}$. This experiment will use the HISS facility to investigate the electromagnetic dissociation of $2.1 \mathrm{~A} \mathrm{GeV} 16 \mathrm{O}$ in the reaction $160 \mathrm{O}+\mathrm{U} \rightarrow \mathrm{U}+$ ${ }^{15} \mathrm{~N}+\mathrm{p}$. In preparation of Experiment 514H, and as part of the overall HISS program, we have supported the lollowing activities:

I. Specification of the technical criteria for the measurement of the magnetic field of HISS,

2. Bringing into operation of a computer code that expresses the field in parametric form, and

3. Development of a rapid computer conversion from trajectory information (i.e., drift-chamber coordinates) to the mourenta and production vectors of reaction products that are transmitted through HISS.

Assistance was given to Experiment $513 \mathrm{H}$, the first prototype experiment carried out with HISS, and information was gained on the problems related to the detection of p. ${ }^{15} \mathrm{~N}$ coincidences and, by use of a $\mathrm{Nal}$ detector, the feasibility of measuring the energy of $\gamma$ rays from the decay of projectile fragments.

\section{Isotopic Measurements of Cosmic Ray Nuclej}

Data from the LBL-UCSSL (HKH) instrument aboard the ISEE-3 spacecraft launched 12 August 1978 continues to be of excellent quality. The experiment continues to suppor1 systematic studies of the nuclear composition of the cosmic-ray source. Such data provide the basic input for theoretical models of stellar nucleosynthesis and the evolution of the composition of matter in the galaxy.

\section{Future Directions}

With the sucessful emulsion irradiations obtained from Bevalac Experiment 559H, we shall pursue a vigorous scianning/measuring program in order to improve the statistical significance of our observations on the anomalon component annong relativisis projectile fragments. These measurements, and their ultimate analysis, will enabie us to study with improved statistics the following aspects of the experiment: (1) the interaction properties of $Z=2$ Jragments, (2) lifelime estimates, (3) eflects in higher order generations of the extranudear cascade, (4) the semi-inclusive properties of the collision parameters, and (5) the dependence on projectile nucleus. Our aim is to enhance the subset(s) of fragmentation events responsible for the effect, which can provide insights into the pertinent production mechanisms.

A new generation of experiments in heavy ion physics will begin in 1982 when the Bevalac will initiate acceleration of all nuclei up 10 and including ${ }^{238} \mathrm{U}$. With nuclear 1rack emulsions we shall reoord and analyze all ionization tracks of nudei, $Z \Rightarrow 1$ 10 92, produced in the interactions of ${ }^{238} \mathrm{U}$ in matter. Planned experiments will be able 10 give information on:

1. Projectile-related phenornena

a Charge and multiplicily distributions of projectile fragments

b. Angular distribution of projectile fragments; P of nuclei and nuclear clusters

c. Shower production; $\pi$ production

d. Fission of U via nuclear and electromagnetic interactions

e. Inleractions of laler generalion projectile fragments

2. Target-related phenomena in uranium collisions

a. Target fragmentation; charge, multiplicities and energy spectra, $E \neq 30 \mathrm{~A} \mathrm{McV}$

b. Fragments in mid-rapidity region.

In support of our future program of rescarch we plan to extend ICAMS to include additional measuring stations and automatic charge measurements, and to develop a $0.3-$ $0.5 \mathrm{MG}$ pulsed magnetic field to permit direct rigidity measurements in nuclear emulsion and other high-spatialresolution visual detectors. 


\section{Bevalac Research}

\author{
H.G. Pugh \\ L.S. Schroeder \\ Lowrence Berkeley Ioboratary
}

TASS:

$R$ Koontz

$\boldsymbol{R}$ Treuhafi

Lawrence Berketldy Labaratary

J.W. Harris

OSI, Darmstodt, West Germany

J. Engelage
P.N. Kirk
G. Krebs
C. Ruiz
Lowitiane Stote Linversity

G. Rache

Germonl-Farrond, Frates

J. Geaga

UC. tos Angeies

\author{
A. Sandoval \\ R. Slock \\ GSI, Darmstad. West Germony
}

\author{
STREAMER CHAMEER: \\ J.P. Drannigan \\ E.H. McConn \\ I.W. Muskovich \\ L.M. Tinay \\ Lowente Berketey Laboratory
}

\author{
R. Brockmant \\ J.W. Harris \\ J. Miller falso LAL) \\ M. Raff (also LBLC) \\ H. Siraebele \\ M. Majer idiso Litiversily of Mavburg! \\ $R E$. Renfordit (aiso Undwersity of Nowburg) \\ F. Reiss iolso bhiversily of woticht \\ OSi, Darmulad, West Germany \\ K.L. Wolf \\ Arganne National Labaratory
}

\author{
M. Brandan \\ A. Dacal \\ A. Menchaca-Rocha \\ M. Ortiz \\ University of Wexico
}

This group operates two complementary research instruments in relativistic beavy ion research al the Bevalac TASS is a double am spectrometer, used in collaboration with the Loutsiana State University. In contrast to this small solid angle, few-particle coordinate measuring. fully electronic instrument, the Streamer

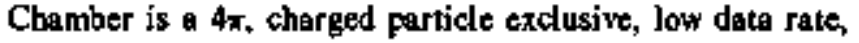
visual data storage device. It is run in a GSI-LBL collaboration. In addition, the groups of $K$. Wolf at ANL and A. Dacal at the University of Mekico have made major commitments to this effort.

\section{TASS}

The Two-Arm Spectromeler System (TASS) consists of two fully rotable magnets, with accompanying wire chambers and scintillation hodoscopes for partica identification. The first nun occurred in July 1980. The device has sinte been expanded by the addition of further wire chambers. The main activities on TASS have been:

444H. This experiment involved measuring the angular and momentum correlations between high energy particles $\pi^{+}$,p.d enitted in the forward and backward hemispheres. The majority of the running involved $2.1 \mathrm{GeV}$ protoncarbon interactions. Particle production at backward angles is of considerable interest since jt is either strictly forbjdden (e.g., nucleon scattering) or severely constrained (e.g., pion production, where $\mathrm{P}_{\max } \leqslant 300 \mathrm{MeV} / \mathrm{c}$ ) in "free" N-N collisions. Thus, the observation of correlations between forward and backward particle emission pro. vides a towl to probe possible exotic or cooperalive production mechanisms, that is, study the short-range behavior of nucleons in nuclear matter. The data are being analyzed, with particular attention being given to that portion of the data that is sensitive to the incident proton scattering from two or more nucleon correlations in the target nucleus. The initial phase of studies was finished in early 1981.

559H. This experiment looks into the possible differences of $\pi^{-}$and $\pi^{+}$yield distributions in the central region and op to the projectile fragmentation domain. The planned ron is ${ }^{40} \mathrm{Ca}+{ }^{40} \mathrm{Ca}$ at $1.05 \mathrm{GeV} / \mathrm{A}$ where only Coulonb effects can be responsible for any diflerence observed. A multiplicity detector system was added to TASS, in order to separate peripheral and central events for the sake of a more conclusive comparison with theorits of the dynamical Coulomb effects. The purposes of this experiment are to cover the full phase space at high statistics, to ascertain the possible efiects of the Coulonb field on low energy pion production and to help settle controversy between other previous experiments. A parasitic run at 670 and 
$800 \mathrm{MeV} / \mathrm{A}$ with $\mathrm{Ne}$ on $\mathrm{NaF}$ in the spring of 1981 was successful: these data are now being analyzed.

Future research with TASS will inwolve measurements of $\pi \pi$ and $\pi \mathrm{N}$ correlations to ascerlain the contributions of both meson and baryon resonances to the pion spectrum observed in heavy ion collisıons.

Finally. we feet that a study of di-lepton production $\left(\mathrm{e}^{+} \mathrm{e}^{-}\right.$or $\left.\mu^{+} \mu^{-}\right)$might be a very sensitive lest to determıne if exolic slales of nuclear matter are formed in central collisions. Being weakly interacting, the leptons will nol suffer the latge attenuation that pions, nutleons, and. to some extent, kaons experience in traversing the nuclear medium from their creation point in the collision process. Tes! runs have been made, in collaboration with J. Carrod and L Madansky, and the erroup has helped organize a workshop, held at LBL on 26-27 May 1981 to sludy the experimental and theoretical feasibility of observing a transition to the quark-gluon plasma by this means. The primary problems are experimentally those of obtaining sulficient acceptance and theoretically those of distinguisting the effect from "uninteresting" backgrounds such as $\pi^{+} \pi^{-}$annihilation in the nuclear environment. A further meeting of this workshop is planned prior to a final report.

\section{Streamer Chamber}

This group, with members from LBL, GSI Darmstadt, Universily of Marburg, ANL, and University of Mexico, is using the LBL streamer chamber, which, with its $4 \pi$ charged particle exclusive information, enables one 10 gather simulianeously dala aboul particle production, wo particle correlations, and colleclive fealures of the reaction mechanism as reflecled in the particle/momentum flux patterns of the events. Instrumentation advances have been made concerning internal metallic targess, chamber gas omposition, Marx generalor oulput, new triggering schemes, inage intensifer cameras, and direck digitalization of pictures by use of CCD cameras. Data are still taken on conventional film, and measuring is done withoul computer automatization. We are, however, adapting a semi-automatic PEPR measuring system and considering the possibility of recording our data entirely with CCD cameras.

Recent work by the group has included the following experimerts:

400 H. This experimert used a proton bear to investigate the systematies of backward-going baryons. Complementing a corresponding TASS study, these streamer chatmber data provide a look at other fealures of the everts, relevant to the backward emission mechanism. The data are now being analyzed and discussed in terms of isobar excitation and absorption or decay.

407H. This experiment measured $\mathrm{Ar}+\mathrm{KCl}$ reactions between 0.4 and $1.8 \mathrm{GeV} / \mathrm{A}$ in central and minimus bias Irigger configurations. From these data, a study of $\pi^{-}$and charged particle mulliplicity systematics was first completed. ' Analysis is under way of negative pion momentum fux and temperature distributions. of two $\pi^{-}$corfelations (Hanbury-Brown, Twiss elfect), and of global features of the full event. In order to speed up the ful] reconstruction of events (also applied to central $\mathrm{Ar}+\mathrm{Pb}$ data at $1,0 \mathrm{GeV} / \mathrm{A}$ ), the semi-automatic PEPR measuring facility at the University of Hejdetberg has been adapted to 3-view, $35 \mathrm{~mm}$ film antalysis. A further aspect of $40 \mathrm{H}$ dala is $A$ production in central Ar $+\mathrm{KCl}$ collisions at $1.8 \mathrm{GeV} / \mathrm{A}$. A preliminary study of $A<p_{\perp}>\left\langle p_{1}\right\rangle$, and production eross section, as well as 1 polarization, was completed. ${ }^{2}$

564H. Study of ${ }^{40} \mathrm{Ca}$ reactions with $\mathrm{KCl},{ }^{40} \mathrm{Ca}$ and $\mathrm{Pb}$ at $2.1 \mathrm{GeV} / \mathrm{A}_{\text {, }}$ іл central and inelastic Irigger mode. The $\sim 100$ hour central $\mathrm{Ca}+\mathrm{Ca}$ run at 2.1 GeV/A was aimed specifically at beller a production statistics in order to anvestigate the spectra and, in particular, the 1 polariza. tion. These collisions should produce the highest energy density of nuclear matter presently atlainable al the Bevalac. The data will also be analyzed for all the other 40] H topics, mentioned abowe. For example, it will be interesting to compare $\pi^{-}$production in $\mathrm{Ar}+\mathrm{KCl}$ wilh that of the isospin zero system $\mathrm{Ca}+\mathrm{Ca}$. Furthermore, in $\mathrm{Ca}+\mathrm{Ca}$ we should have $\mathrm{Cn}_{\mathbf{r}}>=\mathrm{Cn}_{-}>$. it will thus be mosl appropriate in order to test cur $x^{4}$ identification capabilities

This experiment used metallic targets of $\mathrm{Ca}$ and $\mathrm{Pb}$, replacing $\mathrm{KCl}$ and $\mathrm{Pb}_{3} \mathrm{O}_{4}$ targets. These targets were movnted inside $\mathrm{SF}_{6}$-filled plastic boxes with thin Mylar wndows, in order to avoid discharges across the target. In a parasitic run with a $1.05 \mathrm{GeV} / \Lambda \mathrm{Ca}$ beam, very thin $(50)$ and $100 \mathrm{mg} / \mathrm{cm}^{2}$ ) metallic $\mathrm{Pb}$ targets were employed 10 push down the lower proton identificalion limit to about $10 \mathrm{MeV}$. The CCD camera was also tested in these runs.

557H. This experiment addresses the reaction rnechanists at the lowest Bevalac entergies of $30-90 \mathrm{MeV} / \mathrm{A}$, trying to cross the gap to low erergy studies in $\mathrm{Ar}+\mathrm{BaJ}_{2}$ and $\mathrm{Ar}+\mathrm{KCl}$ reactions. A slack of seven thin $\mathrm{Bal}_{2}$ largets was developed to oblain a sufficient number of event triggers while keeping the targets thin enough to let heavy recoil nuclei emerge irom them. This experiment was Irying to address the problem of wide range in streamer brilliance, from fast protons to slow $Z>10$ recoil nuclci, by use of image intensifies cameras. Along with this the operating electric field strength could be lowered. thus reducing the flare and spark-breakthrough abundances in the chamber. These data are antlyzed on the ALICE system al ANL obtaining particle identification from track profile measurements.

In 1981, the group will finish the analysis of $400 \mathrm{H}$, $40 \mathrm{IH}$, and continue on 564H. 557H. Eariy in 1982, the rurning of $564 \mathrm{H}, 557 \mathrm{H}$ will be finished, and a new sludy of reactions with heavier beams from the up-graded Bevalac, such as $X_{e}+X_{e}$, is planned.

The streamer chamber will be adapted to the higher multiplicities by an external $4 \pi$ detector system measuring and points and $\mathrm{dE} / \mathrm{dx}$ of each track. The latter will scrve for $\mathrm{p}, \mathrm{d}, \mathrm{l}, \mathrm{He}$, and $\mathrm{p}, \mathrm{r}^{+}$differentjation, a problem which must be overcome to make possible meaningiul analysis of 
global features of fully reconstsucted events, A new online compuler will be introduced with this $4 \pi$ system. Further advances in piesure slorage and semi-automatic measuring will be sought.

\section{References}

1 A. Sandoval et al., Phys. Rev. Lett 45, 874, 1980.

2 J.W Harris et al., Phys, Rev, Lett, 47, 229, 1981. 


\title{
Light Particle Emission in High Energy Nuclear Collisions
}

\author{
S. Nagamiya and H. Steiner
}

\author{
S. Schnetzer \\ Lawreme Berkeley Laboralosy \\ H. Hanugaki \\ S. Kadola \\ I. Tonthata \\ INS, Japon
}

\author{
R. Lombard \\ DPhNME, CEN, Saclay, Fratee \\ Y. Miake \\ Osake Utwiversity, Jopan \\ E. Moeller \\ Freie Untuersitât Berlin, West Germaty
}

The main emphasis of our research program is the study of the reaction mechanisms involved in high-energy nuclear collisions. This experimental program includes both inclusive crosss-section measurements and particle correlation studies. Mosl of the measutements involve the detection and identificalion of relatively light particles $\left(\pi^{ \pm}, K^{+}, \mathbf{p}, \mathrm{d}, \alpha_{1} \ldots\right)$ over a wide kinematic domain. We are particularly interested in studying processes which are kinematically -inaccessible in the collisions between Iwo Tree nucleons, and thereby probing short-distance effocts in nuclei. We are also searching for any unusus effects which might result when two massive nuclei interact at high energy, especially in small-impact-parameter collisions. Most of these experiments are performed under the INS (University of Tokyo)-LBL collaboration.

In Expetiment 471H, kaon production has been measured with 2.1 GeV/A Ne, 2.1 GeV/N d, and $2.1 \mathrm{GeV}$ proton beams on various nuclear targets. The inclusive cross section with the Ne beam shows a much stronger targetmass dependence than that with the $\mathbf{d}$ or $\mathbf{p}$ beams; this result suggests that collective effects are important in kaon produetion. The charged-particle multiplicity associated with katn production has been measuted as well as the multiplicities associaled with producing pions and protons. The data indicate that the associated multiplicity is slightly larger when a kaon is detected than in the cast of either pions or protons.

The data-1aking part of Experiment $472 \mathrm{H}$ has been finished. In this experiment, the goal is to measute various two-particle correlations, e.g., p-p, p-d,... Meastrements have been made using prolons of 400 and $800 \mathrm{MeV}$ and $\mathrm{Ne}$ of $4(X)$ and $800 \mathrm{MeV} / \mathrm{A}$. New insight into the production mechanism responsible for backscattered protons in $800 \mathrm{MeV} p+A$ collisions has been obtained by studying forward-backward correlations. The data suggest that the scallering of incident protons on a two-nucleon cluster is the dominant production mechanism for backward protons. Analysis of other data ate in progress.

The analysis of experiment $205 \mathrm{H}$ has been completed and (wo papers are presently being prepared for publica. tion. In this experiment, the inclusive spectra of pions, protons, deuterons, tritons, ${ }^{3} \mathrm{He},{ }^{4} \mathrm{He},{ }^{6} \mathrm{He}$, and ${ }^{8} \mathrm{He}$ have been measured at small production angles $\left(0 \leq \theta_{1} \leq 12^{\circ}\right)$ with $p, d, \alpha$ and $C$ besms at $1.05 \mathrm{GeV} / \mathrm{A}$ and $2.10 \mathrm{GeV} / \mathrm{A}$ bornbarding energy on various targets. Upon comparing our pion results with various model predictions, we find that none of the existing models reproduce al] of the data well in a quantitative sense and some are not even close. For light projectiles and targets, the hard scattering models (e.g., Schmidi and Bunkertbecler, etc.) seem to do as well as any of the calculations and better than most.

The preparation of Experiment SI2H, in which largetransverse-momentum particles near kinematical limit will be measured, has been finished. The HISS magnetic spectrometer will be used in conjunction with time-of.fight and $\mathrm{dE} / \mathrm{dx}$ measurements. The detection system involves large atrays of scinlillation counters, Iwo large drift chambers, and several multiwire proportional chambers. The major data taking is scheduled for spring of 1982 . 


\title{
Heavy Ion Studies/Pion Studies
}

\author{
K. M. Crowe and J.O. Rosmussent
}

\author{
J.A. Bistitich \\ R. Bossingham \\ H.R Bowman \\ K.A. Frankel \\ J. Kurck" \\ D.L. Nutphy* \\ J. Ridout ${ }^{t}$ \\ W. Schimmerlins \\ J.P. Stilivan" \\ E. YOot \\ W.A. Zaje \\ Lemence Berkeley Laboratory
}

\author{
O. Hashimoto \\ INS. Jopat \\ W.J. MacDonald \\ Lhipersity of Alberto \\ J. Peter \\ Osay. France \\ M. Salomon \\ University of British Cofumbia \\ P. Truoet \\ SIN. Switzeriand
}

The Berkeley research activities of this group center mainly around the study of charged pions or correlated pairs of particles produced in high-energy heavy-ion colli. sions al the Bevalac. The tochnical approach inwolves a large ( -1.5 meter long) magnetic spectrometer (JANUS) and combinations of fast scintillators and wite chambers interfaced with computer data systems. One major part of this work is the deternination of the cross sections, with an exploration of the angular dependence, for pion production in beavy ion reactions. The existence of both positively and negatively charged pions facilitates determinations of simple Coulomb effects and, thereforc, the charge density evolution in heavy ion collisions by observation of the $\boldsymbol{x}^{+} / \mathbf{r}^{-}$ratio as a lunction of pion energy, bombarding energy, and target-projectile charges. In fact, we have found that pions produced with low energy in the projectile frame have large म $^{-} / \mathrm{x}^{+}$ratios due to the Coulomb fields of the fragments. Systematic observations of the sharp anomaly are being made to compare with various models lor production. The effects due to esoteric mechanisms, i.c., condensates, etc, are expected to modily the conventional modes; and these phenomens are being explored.

We are also studying the production cross section for low energy $\pi^{-}$and $\pi^{+}$at $90^{\circ}$ in the center of nesss, where an enhancemient in the $\pi^{+}$production has been observed. We want to ascertain that this $90^{\circ}$ peak does disappear at low energy and to atiempt to measure the threshold for its appearance. The group has underlaken thick target studies in order to evaluate the practicalisy of heavy ion production of useful $x^{-}$bearns.

The second major part of the work is an experiment to look at the Hanbury-Brown-Twiss effect for like-charged pions made by heavy ion collisions in events where many pions are produced. We look at the production of a pair of closely related pions,++ or,-- at the Lab angle $45^{\circ}$.
In the past ten years, such exotic phenomena as pion condensation, pionic lasing and quark matter have been predicted as possible for nuclear matter in a heavy ion collision. Gyulassy, Koonin, and others have pointed out that one possible way to see the effect of pion instabilities in heavy $\mathrm{i}$ on colljsions is to look for the correlation of like pions in the siluation when the relative momentum is exirapolated to zero.

More quantitatively, one studies the correlation function $C_{2}\left(k_{1}, k_{2}\right\}$ for two pions. For the case of two pions, $C_{\gamma}(k, k)=0$ for coherent sources. Highly coherent pions will have a different distribution in relative moments from that expected for chaotically produced pions, and the range of coherence depends on the size of the interaction region. This technique of pion interferometry has been employed to measure the size and lifetime of the pion source formed in the collision of $1.8 \mathrm{GeV} / \mathrm{A}{ }^{40} \mathrm{Ar}+\mathrm{KCl}$. The values oblained provide useful ponstraints on models detailing the pion production mechanism. The Bose-Einstein entranoement for low relative monentum was observed.

We have also conducted measurements of neutronproton pairs produced in the $\mathrm{Ne}+\mathrm{U}$ reactions. Such measurements give information on exciled (virtual) state deuteron production and on spin correlations associated with pion condensation. Preliminary results show an enhancement near zero nop relative momentum. Future work will improve on resolution and statistics for the virtual deuterons and for wide-angle n-p correlations measuring quasj-free scattering and collective-flow processes. Cluster production provides the main information on entropy production in relativistic heavy ion collisions.

Fulure work will utilize the experimental techniques and apparatus we have developed. The availability of Bevalac beams of the heavier elements ( $\mathrm{A} u, \mathrm{~Pb}$, or $\mathrm{U}$ ) will
Foof notes
"Graduate student
tUndergraduale student 
call for new pion spectroscopic measurements to search for evidence of highly compressed nuclear matter effects, such as pion condensation, quark matter, etc. The $\pi^{+} / \pi^{-}$ratios will again be exploited for heavier beams to measure the dynanjic charge eyolution in relativistic heayy ion collisions.

The advent of $\mathrm{Xe}, \mathrm{Pb}, \mathrm{U}$, and other hcavy beams at the Bevalac provides new opportunities in atomic physics. Our earliest Bevalac work involved measuring $K x$-ray' production cross sections. We plan not only rormal survey experiments but also more exotic single-crystal experiments looking for coherence eflecls in $x$-ray emission.

Various members of the group are ifvolved in collaborative work centered at other laboratories: pion or mulon experiments at LAMPF; theoretical studies on muon fission with collaborators in Chira; theoretical nuclear studies by the Classical Limit S-Matrix Method with collaborators at the Universities of Tennessee, Brazil, and Kuwait; and hybrid plaslic and emulsion studies of relativistic heavy ions wilh collaborators in Nagoya and Cairo. 


\title{
Nucleus-Nucleus Collisions
}

\author{
P.B. Price
}

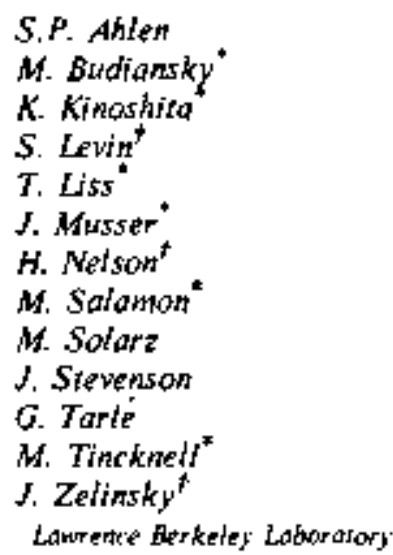

Al] of this group's offices and equipment are located in Birge Hall on the UCB campus, which makes it easy to attract rew students, but restricts 10 some extent interaction with Nuclear Science Division stafT. Current research falls into the following five principal areas.

1. Relativislic heavy ion tesearch (DOE support)

a. Production of heavy ion fragments, includ. ing exotic neutron-rich nuclides near the dripline.

b. Production of high-energy gamma rays.

c. Search Ior Les-Wick matter.

d. High-order effects in electrodynamics of s]owing heavy ions.

c. Response of various detectors to heavy ions.

f. Hadron calorimetry for nucleus-nucleus collísiòns.

g. Detailed properties of secondary nuclei with anomalously shor interaction lengths ("anomalons").

2. Search for highly ionizing particles in $\mathrm{e}^{\boldsymbol{+}} \mathrm{e}^{-}$ annihilation (PEP.2 experiment, supported by NSF)

3. Cosmic ray astrophysics (NASA support)

a. Relative abundances of isotopes of very heavy cosmic rays.

b. Abundances of cosmjc rays in the vicinity of uranium and beyond.

c. Design of future, large-area detectors for long-duration space exposures.

d. Sudy of highly ionizing particles with anomalously long interaction lengibs at mountain altitudes, including a search for grand-unification magnetic monopoles and quark globs.

Footnotes

*Graduate students

${ }^{\dagger}$ Undergraduate students
D. O'Sutivivan

Dublis Institute for Advanced Si wolies

Iretand

F. Sauli

CERN, Geneva, Swisztriatd e. Design of an experiment to detect anti-iron nuclei in cosmic rays at a level $3 \times 10^{-7}$ of iron.

4. New detectors (NASA and Calspace support)

a. CR.39 with charge resolution $a_{Z}<0.1$ e.

b. Streamer chamber for imaging Cerenkov light cone from heavy nucleus.

5. Neutron dosimetry (DOE support)

a. Thin-film dosimeter for $10-200 \mathrm{keV}$ neutrons.

Doctors Ahlen, Stevenson, and Tarlé play a major role in conceiving new experiments and in training students. There is much overlap among the various projects. Detec. tors devised to solve one problem frequently make possible the solution of all or part of another problem. A result obtained with plastic detectors may stimulate the construction of an electronic system to do a second-peneration experiment. Expertise gained in resolving isolopes in a balloon-bome cosmic ray experimenl may be used at PEP or the next-generation neutron-rich auclide experiment. In all these projects, both post-graduate and undergraduate students play an active part.

Among the experiments planned Tor the next two years at the Bevalac are a high-energy gamma ray experiment with $10^{2}$ times bigher collecting powter, delection of many new nuclides delineating the neution dripline, hadron calorimetry with large solid angle, search for Lee-Wick matter in $\mathrm{U}-\mathrm{U}$ collisions, measurement of higher-order electrodynamios in $\mathrm{dE} / \mathrm{dx}$ and Cecenkow radiation by jons up to $\mathrm{V}$, detailed studies of anomalons with detectors capable of resolving noninlegral charge, and determination of the response of plastic track detectors to relativistic ions up to $\mathrm{U}$ (the latter being of crucial importance for the proof that cosmic rays come from freshly synthesized supernova debris). 


\section{Nuclear Theory}

\author{
$N K$ Glendenming \\ $M$ Grulassy \\ WD Myers \\ $\checkmark$ Randrup \\ WJ Swateck? \\ J Boguta \\ $G$ Far \\ M Redisch \\ $H$ Ruck \\ $V$ Ruck \\ Lowrence Bertidet Laborason,
}

\author{
$S$ Bohrmann \\ MPt Hetdediterg West Germant \\ $P$ Hecking \\ Regersburs West Germony \\ A Lumbroso \\ CEN Socioy France \\ KH Muller \\ THD Darmstad West German) \\ H Stocker
WSi Darmstadt West Germany
}

The nuclear theory program encompasses the general structure of static muclel (including neutron stars), nuclear dynamucal propertics as probed in nuclear collisıons at various cnergies, and the manifestation of the basic quark degrees of freedom

General nuclear structure is being studied by macros. copic as well as microsoppe tools ln particular, a non. linear relativisuc mean-field theory has been found to possess remarkable predictıve power for a varıety of nuclear propertles Due to its simplicity and consistency, this model is a versatile tool for exploring more exotic nuclear properties. $e \mathrm{~g}$. the structure of hypernuclet The good agreement with much more complicated many-body calculations suggests that the low-density properties of nuclear matter are determıned by a few simple properties, such as the compressibility at saluration The theory also implies that knowledge of the free nucleon-nucleon interaction does not suffice to understand the nuclear many-body properties Separate studies have focused on generalızıng felativistic mean-field theory to the study of tıme-dependent problems While practically equivalent to time-dependent HartreeFock calculations at low energy, the relativistic mean field model proyides a natural inclusion of the mesonic degrees of freedom which become significant at higher energues A relativistic field theory has also been used to study derise neutron matter in the normal and pion condensed phases

Recently, a program to apply nuclear theory to astro. physies has been unitiated For example, the role of deformations on slowing down pulsars is being studied, and beta stable reutron star massts and the temperature dependence of the equation of state has been computed

The dynarmical properties of maderately excited nuclear systems continules to be an important area of research The general macroscopic theory of nuclear dynarmucs has been further developed, and favorable confrontalion with experiment has been made, in particular regardıng the "extra push" required to achevc the temporary coalescence of two colliding nucleı

In the area of damped nuclear reactions, the study of the charge equilıbratıon phenomenon has been a central theme during the past year In parucular, confrontations of a previously formulated transport theory with various sets of data have demonstrated that the observed isotopic and isobarsc fragment distributions can be guantitatively well understoxd to result from the statistical exchenge of individual nucleons between the two reacting nucles, thus elıminatıng the apparent need for a collective chargeequilibration model to explain the data lin wew of this success, work has been donc to involue the angularmomentum carrying degrees of freedom to further test the model

As the bombarding energy is raised, the binary idealı. zation becornes increasingly inadequate and multi-fragment final states grow predominant The fragment production mechanisms have been studied by various approaches Sorne have focused on the production of a specific fragment type, such as nucleon jets appeaning as a result of the kinematical boost of transferred nucleons due to the nuclear motion, pions resultıng from nuclear collisions at a few hundred $\mathrm{MeV} / \mathrm{n}$, and the production of deuterons within the framework of the intranuclear cascade descriptron Other studies have attempted a comprehensıve description of fragment production where all final states are treated on an equal fooling In relation to current attempls to probe the entropy production in a nuclear collision, it has boen found that the crentual deexcitation of unstable ejectiles plays an important role in modifying the observed distributions relative to those immedialely after the collision process

The area of high-energy nuclear collısions is a major parl of the theory program and several directions have been pursued simultaneously, e 8 , the development of analytical models for elucidating the consiraunts of geometry and phase space on the observables, the employment of nuclear hydrodyramics for studylng nuclear flow phenomena and their possible detection, and intranuclear cascade studres of the equslibration process Special topics which have been addressed are $\pi$ and pp correlations, $\pi$ and $\mathrm{K}$ production, multıplicity distributions, composite frágment formation, and Coulomb final state interactions 
In regard to collective flow searches, an extensive comparison of model calculations with high-multiplicily triggered central data has indicated a qualitative failure in the cascade modcls, while, on the other hand, hydrodynam ics appears to reproduce the observed forward suppression of protons. The existence of hydrodynamical phenomena would be very encouraging for our hopes to eventually deduce the high-density nuclear equation of state.

Further wrork has been done on various aspects of the pionic degree of frecdom in nuclear matter: the propagation of the pion through matter has been studied within an optical model, pion condensation al finite temperatures has been given special attention, and a new experimental signature for pion condensation bas been proposed.
An important modern frontier is associated with nuclear collisions at ultrarelativistic energies where the ficlds of nuclear, particle and astrophysics have common ground. We are pursuing problems in this area. In a schematic quantum chromodynamic model the properties of single hadrons, their interactions, and many-particle states have been sludied, yielding insight into the oecurrence of quark confinement and nuclear saturation. The phase transition from hadron to quark matler. which is expected to occur at sufficiently high energy density, has been expiored: The onset of the phase transition has been studied in different models and the use of the strange baryon fraction as a signature for a quark phase has been discussed. 


\title{
Heavy Ion Superconducting Spectrometer (HISS)
}

\author{
HISS GROUP A
}

\author{
E. Beleal \\ M. Bnorson \\ D. Greiner \\ P. Lindsirom \\ M. Symons \\ R. Wada \\ Lowrence Berkeley Laboratory \\ F. Bieser \\ H. Crawford \\ I. Flores \\ C. AcParland \\ U.C. Spdice Sciences Laborotory
}
G. Barsch
P. Brady
C. Castanoda
M. Johnson
J. Romero
UC. Davis

The HiSS facility rook its first data in June 1981 just before the Bevalac Improvement shutdown beginning Juse 29,1981 . For much of the time it appesered that it would be impossible to get data this year. Beginning in December 1980, physicists from LBL, UC Davis, the instiIute Jor Nuclear Studies (Tokyo), and LSU overcame the budgel and manpower limitations slowing delector con. struction and assembled the Phase I detectors. Another difficulty was a leak in the magnet vacuum system which made operations very difficult. However, we decided to altempl to run at a reduced field of $20 \mathrm{kG}$ and continue with the experiments. This gamble peid off, end steady operation at $20 \mathrm{kG}$ was achieved in June 1981.

The major essential detector systems were the drift chambers and the time-of-flight wall. Both these devices were brought to completion in early June 1981 with Doug Greiner leading the effort on the drift chambers and Roy Wada the-time-of-flight wall. Magnet control and testing headed by Fred Bieser achizved stable running conditions in mid-June 1981 .

The magnel and detector systems would be of little use without the necessary sofiware. In the area of magnet monitoring, Chuck McParland (SSL), and bis team put logether a system that collected and achieved data from the magnet sensors; this enabled diagnostic plots to be made using the MULTI programs on the VAX 11-780 compuler. This tool proved essential in understanding the difficulties of running the magnel and to devising successful operating procedures.

\author{
J. Carrol \\ U.C Los Angeles \\ B. Berman \\ D. Ofsen \\ Lowrence Livermate National Laborafory \\ H. Sarth \\ OSI, Damstod, West Germaty \\ J. Engelage \\ Loustana State thiversity \\ S. Kadora \\ INS, Jopan
}

The data was collected in the PDP 11.45 front ont computer and transmitted via a shared tisk to the VAX 11-780 for on-line diagnostics. Peter Lindstrom led the work on the front end compulers; Chuck McParland's leath did the data transmission; Hank Crawford (SSL) and a large group of physicists provided the necessary diagnostic programs in the VAX 11-780.

The payoff for all this hard work came on the last weekend of Bevalac running, when several hours of thata were recorded from the first HISS experiment, which sirmiltaneously measurad all the projectile fragments from a $1.9 \mathrm{GeV} / \mathrm{A}{ }^{12} \mathrm{C}$ beam and several targets. With this multiparticle data, we will be able for the first time to examine the energy and momentum transfer to the excited projectile nuclevs, which will give crucial information about the reaction mechanism. The data also permit fragment spectra to be searched for resonance formation and the existence of long lived states that have been reported recently from emulsion data.

We still have many things to do this year. Data taken in June is being eagerly analyzed, and we have starled to improve and document the results of the rush to get data.

In FY 1982, we will finish consiruction of the facility and complete the five Phase 1 HISS experiments. Building the Phase II detectors will also be a major task for this year. After final analysis of the performance of the Phase I delectors, the designs will be finalized and the construction begun. 


\section{Isotopes Project}

\author{
J.M. Dajikj \\ E. Browne \\ R.B. Firestone \\ C.M. Lederey \\ V.S. Shirley \\ Lawence Berkeley Lotatatory
}

\author{
Y.A. Ellis-Akovali \\ Ouk Ridge Nationst Lahoratary
}

The Isotopes Project compiles and evaluares nuclear structure and decay data, and develops compilation methodology. Much of the group's effort is directed to the evaluation of experimental data, based on the knowledge of experimental methods, the researchers themselves, and the relationships and constraints imposed upon the data by nuclear theory and systematics. Considerable effort is also devoted to the selection and presentation of data in formats most convenient for users.

From 1940 to 1978, the Project's main objective was the production of the Table of Isoropes. Since publication of the seventh (and last) edition in 1978, the group has coordinated its nuclear data evaluation effort with that of the other data centers via the national and international nuclear data oetworks. The group is curtenty responsible for the evaluation of mass chains $A=146-152$ and $A=163-194$. Al evaluated data ace entered into the international Evaluated Nuclear Structure Data File (ENSDF) and published in Nuclear Dat a Sheets.

During the past year the evaluation of nuclear structure data for all nuclei with mass $A=191$ was publisbed in Nucleor Dato Sheets; evaluations of $A=193$, $A=188$, and $A=185$ were accepted for publication, and the evaluations of $A=169, A=187, A=189$, and $A=190$ were completed and submitied for revlew. Updated evaluations of threc additional mass chains $(A=181, A=174$ and $A=192$ ) are in progress.

The Project continues to develop data handling and analysis techniques. A tecent example is the restructuring of the Table of Isotopes level-gcheme fle into a computer. searchable data base. It is the most up-to-date file of nuclear structure data available and is readily accessible for horizontal compilations and systematic studies of nuclear properties.

Programming tools to aid the evaluation effort have also been developed. The physics analysis programs, written by the Nuclear Data Project (ORNL) for data in ENSDF formats, bave been adapted and extended into an interactive package for use on the CDC- 7600 computer. The resulting program, PACK enables immediate calculation of such quantitio as internal onversion coefficients, level energy fits, and $\log$ ft valuos and level feedings in radioactive decay. It also percuits manipulation of the data to give, for example, energy-ordered y-ray listings and allows on-line editing.

The Table of Nuclear Moments, a compilation of nuclear magnetic and quadrupole moments published by the Isotopes Project, has been the only such table maintained on a contimuing basis. The most recent version was publisbed as an appendix to the Table of Isotopes. User response has encouraged plans for expanding the amount of data for presentation in the next edition.

The seventh edition of the Table of Isolopes wntinues to be the most up-10-date general reference source available for nuclear data. Sales through April 1981 totaled 6,668 copies (3,269 clothbound and 3,399 paperback). Nearly all 7,000 copies of the Nuclear Wallet Cards, produced in 1979 by the Isotopes Project on behalf of the U.S. Nuclear Data Network have been distributed to the user corntrunity.

In addition to the evaluation effort, the Isotopes Project will produce, on behalf of the U.S. Nuclear Data Network (NDN), a Radioactivity Handhook for applied users. The purpose of the Hardbook is to prowide a compilation of recommended decay data that is detailed enough for use in sophisticated applications, but organized clearly so as to be usable in routine applications. The handbook will be produced at four-year intervals, beginning in 1983. Reoommended decay data will be taken froxt the current version of ENSDF, without updating. Additional calculations and evaluation will be performed to puovide recommended data for atomic radiations and conversion alectrons. Each mass chain will be referenced to its most recent evaluation in Naclear Dato Sheets as the source for further details and the references to the ariginal papers.

Nearly 6,000 copies of a Handboak sample illustrating the contents and format proposed by the group have been distributed to otber data centers and members of professional societies. The suggestions and comments obtained are being considered in the development of the ingl for. mat.

Work is proceeding on the computer codes needed to produce the handboak. This involves retrieval of the desired data from ENSDF and their presentation in the final publication formats. The first step is to modify the data in ENSDF 30 that each decay data set will ontain "best" values for the $\gamma$-ray and level properties independent of the decay parent. Nonuijornities and holes in the ENSDF data are being corrected as work proceds. The existing Jevel-scheme graphics program (used for the Table of (sotopes) has been modified to handle data in ENSDF formats.

Promoting the science of nuclear data evaluation and providing assistance to the user community are important 
aspects of the Project's role. With regard to the former, Project nembers are organizing the First Annual Conference on Nuclear Data Evaluation, to be held in conjunction with the fall meeting of the APS Division of Nuclear Physics at Asilomar (October 28-30, 1981). Periodic exchanges of both information and scientisis with other data centers (both foreign and domestic) also enhance the evaluation effort. The Project continues to process approximately two user requests per month. Most require a brief data search, a lelter, or a telephone response. Major requests are now referred to the National Nuclear Data Center at BNL General use of the Project's exiensive library, which contains comprehensive data files and major auclear physics journals, is encouraged. 
PART 2. PROGRESS REPORTS

I. EXPERIMENTAL RESEARCH 
$+$ . 


\title{
A. NUCLEAR STRUCTURE
}

\section{Study of Exotic Nuclei}

\section{The Decay of ${ }^{8} \mathbf{H e}^{*}$}

\author{
T. Björnstad, ${ }^{\dagger}$ H.A Gustajsson, ${ }^{\dagger}$ B. Jonson, P.O. Larsson, ${ }^{\dagger}$ V. Lindforst ${ }^{\dagger}$ \\ S. Mansson, G. Myman ${ }_{r}^{\ddagger}$ A.M. Poskanzer. H.L. Ravn ${ }^{\dagger}$ and D. Schard ${ }^{\dagger}$
}

The heaviest isotope of the element helium ( $Z$ - 2) has mass nurnber 8 and is the nucleus with the highest neutron to proton ratio available. It was first definitely identified with a counter telescope technique as the third fragment in ${ }^{252} \mathrm{Cf}$ fission, and the firsi study of its decay was made ${ }^{2}$ in 1965 . In the latter experiment the ${ }^{8} \mathrm{He}$ activity was produced by high-energy protons in a target of cotton fibers. The ${ }^{8} \mathrm{He}$ that difused out of the target was purified by gas phase chemistry and transported to a counting chamber. By means of neuiron counting the half-jife was determined 10 be $122 \pm 2 \mathrm{~ms}$. The state in ${ }^{8} \mathrm{Li}$ at $98 ! \mathrm{keV}$ with $\mathrm{I}=1$ is the only state below the neutron separation energy that could be fed in allowed beta-decay. The gammin conission from this state, observed with a NaJ detector, established its parity as positive. The delayed neutron branch, $P_{n}$, was determined to be $(12 \pm 1)^{\text {we }}$ from the ratio of the neutron to gamms count rales. Aspects of the decay have been verifiod but no new information has been contributed to the original decay schems.

In this paper we report on a new series of measure ments on the ${ }^{8} \mathrm{He}$ decay. The half-life and the betadelayed neutron branch have bees remeasured and energy specira of neutrons and gamma rays were recorded. A starch for the possible beta-delayed charged-particle decay mode, involving the break-up of ${ }^{\mathrm{L}} \mathrm{Li} *$ into $a+1+n$, has been made.

The ${ }^{8} \mathrm{He}$ activity was produced at the ISOLDE facility through Iragmentation reactions induced either by $600 \mathrm{MeV}$ proton or $910 \mathrm{MeV}^{3} \mathrm{He}$ beans from the CERN Synchro-Cyclotron. The production target consisted of a $47.0 \mathrm{~g} / \mathrm{cm}^{2}$ uranium carbide matrix. A cooled transfer tube estrecting the target to the plasma ion source assured chemical purity of the on-line mass separated ion beam

The ${ }^{8} \mathrm{He}$ was directed to a low-background counting area where the $60 \mathrm{keV}$ ions were deposited in an aluminized mylar tape. A beta detector, consisting of a $1 \mathrm{~mm}$ thick $24 \times 26 \mathrm{~mm}$ plastic scintillator, mounted on a $30 \mathrm{mIm}$ diameter phototube, was placed bebind the tape at the collection position. The tape was moved periodically in otder to remove the daughter activity. The beta-detection efficiency was determined on-line to be $(40 \pm 3) \%$ in a beta-gamma coineidesce measurement on the nuclide ${ }^{23} \mathrm{Ne}$. A coaxial $\mathrm{Go}(\mathrm{Li})$ crystal was placed close to the collection point and a fast coincidence technique was used for the beta-gamma coincidence measurements.
For the $\mathrm{P}_{\mathrm{n}}$-value measurements a $4 \pi$ neutron counter, consisting of twelve ${ }^{3}$ He proportional counters imbedded in paraffig-wax, was slid over the beta detector so that the collectica position was in the center of the counter. The neutron detection efficiency was determined to be $(20 \pm 1)$ 菏 with a sample of uranivis A possibie source of systematic error is the energy dependence of the neutron detection efficiency, which has not been determined experimentally. Since the energy spectra of ${ }^{8} \mathrm{He}$ and the fission neutron spectrum of ${ }^{23}{ }^{\mathrm{U}} \mathrm{U}$ are similar, the systematic error is considered to be small. The beta-neutron coincidence method used for the determination of the $\mathrm{P}_{\mathrm{n}}$ value involved the technique ${ }^{3}$ for measuring the time delay between the beta particle errission and the detection of a neulton.

The neutron energy spectrum was measured with a ${ }^{3} \mathrm{He}$ filled bigb resolution spectrometer. For the charged particle measurenents the ${ }^{8} \mathrm{He}$ ions were collected in a $\mathrm{Ni}$ foil in front of a $100 \mu \mathrm{m}$ thick surface-barrier $\$ \mathrm{Si}$ detector.

The half-life of ${ }^{8} \mathrm{He}$ was measured with the $4 \pi$ neutron counter to be $117.5 \pm 1.5$ ms. Combining this new value of the half-life with the one published earlier ${ }^{2}$, we recommended a best value of $119 \pm 1 \mathrm{~ms}$.

The measurements of the $P_{n}$ value were done both with a saturated ${ }^{8} \mathrm{He}$ source, and with periodic removal of the activity. When oounting the bela activity in the saturation pode, one has to take into account the contribution from the $0.84 \mathrm{~s}^{8} \mathrm{Li}$ daughter which is in equilibrium with ${ }^{8} \mathrm{He}$. If $\mathrm{P}_{\mathrm{y}}$ denotes the probability of beta decay 10 the $0.98 \mathrm{MeV}$ siate in ${ }^{8} \mathrm{Li}$, then the beta count rate is proportional to $1+P_{y^{\prime}}$ This is equa! to $2-P_{n}$ since one can assume thal $\mathrm{P}_{n}+\mathrm{P}_{\gamma}=1$. In the short collection mode, where the activity was collected and counted during a 100 ms time interval followed by an interruption of $100 \mathrm{~ms}$, when the tape was moved, the bete count rate was calculated to be proportional to $1+0.03 \mathrm{P}_{\gamma}$

The beta-nevtron coincidence measurements gives a $P_{n}$ value of $(15 \pm 1)$ \% This value is based on four measurements in saturation mode and one in the short collection. mode. The error comes mainly from the uncertainty in the neutron detection efficiency. Nine sets of simultanecus beta neutron singles measurements give a somethat larget $P_{n}$ value $(18 \pm 2) \%$ where the larger error is due to the uncestainty in the beta deteclion efficiency. Another method to obiain $P_{n}$ is to use the ratio of neutron to gamme count rates, both normelized to their respective 
bela count rates. This method again gives $(18 \pm 2)^{\%}$ even though the beta detection efficiency cancels oul of the calculation. From all these measusements we adopt a value For $P_{n}$ equal to $(16 \pm 1)$ 爱 This is somewhat larger than the earljer publishod value, ${ }^{2}$ but we believe that the present techniques, especially the $\hat{\beta}$-n coineidence method, offer the best available determination of $P_{n}$.

The gamma energy spectrum showed, in addition to the $981 \mathrm{keV}$ line, a brosder peak at $478 \mathrm{ke} V$ from the deexcitation of the first excited state in ${ }^{7} \mathrm{Li}$ populated atter neutron emission. The larger width of the $478 \mathrm{keV}$ peak is presurtably a doppler broadening caused by the neutron emission recoil. The ratio of the probability of emission of the $478 \mathrm{keV}$ ganmina to $P_{\gamma}$ was found to be $0.060 \pm 0.003$. Using our adopted valued of $P_{n}$, this shows that the frac tion of neutrons emitted to this state amounts to $(32 \pm 3)$ \%

The neutron energy spectrum showed no significant structure in the spectrum, as expocted, since the knows neuiron emitting states in ${ }^{8} \mathrm{Li}$ are brosd. The spectral shapes measured with and witboul lead around the source position were the same, which sbows that high energy beta particles do not contribute to the spectrum.

The $Q_{B}$ ralue of ${ }^{8} \mathrm{He}$ is $10.649 \mathrm{MeV}$ and the thresholds for the break up of ${ }^{8} \mathrm{~L}$ into $a+1+n$ and ${ }^{5} \mathrm{He}+\mathrm{t}$ are at 4.499 and $5.39 \mathrm{MeV}$, respectively. It was therefore considered possible to detect beta feedings to states above these energies from the observation of betadelayed triton emission. The measured charged particle spectrum was, however, severely interfered with both by alpha particles from the 'Lt daughters and by beta particles from ${ }^{8} \mathrm{He}$ ilself. As a result only upper limíts can be sel at $10 \%$ per decay for charged particles with an energy below $1 \mathrm{MeV}$ and $1 \%$ per decay with an energy above I MeV. These are not very useful limits as one expects mainly low-energy alphas and tritons from the possible ${ }^{8} \mathrm{He}$ delayed charged particle decay mode.

It has earlier been assumed that the main part of the emitted neutrons originates in the $3.21 \mathrm{MeV}$ stale. In order to try to detect neutron emission from higher excited stales in ${ }^{8} L$ we have calculated the shape of the neutron energy spectrom and compared it with our measured spectrum. The ealculated spectrum follows the train trend of the experimental spectrum but there is some additional intensity in the experimental spectrum at the lowest energy and at aboul $1.8 \mathrm{MeV}$ which cannol be explained from neutron emission from any known state in ${ }^{8} \mathbf{L}$. It is interesting to speculate whether part of the intensity at the lowest energy could be due to $\alpha+t+n$ break-up. Assuming that al] the additional intensity up to $\mathrm{l} \mathrm{MeV}$ arises from this decay mode get an upper limit for this decay brathch of $2 \%$ We suggest that the $3.21 \mathrm{MeV}$ state, which has spin equal to I, has posilive parity, and that the $5.4 \mathrm{MeV}$ state could be either $0^{+}$or $\mathrm{I}^{+}$.

\section{Footnotes and References}

'Condensed from Nucl. Phys. A 366, 461 (1981).

tCERN-ISOLDE, Geneva, Switzerland.

FDepartment of Physics, Chalmers University of Technology, Göteborg, Sweden.

1. S.W, Cosper, J. Cemy, and R.C. Gatti, Phys, Rev. Let. 154, 1193 (1967).

2. A.M. Poskanzer, R.A Esterlund, and R. McPherson, Phys. Rev. Lett. 15, 1030 (1965).

3. T. Björnstad et al., Nud. Phys. A 359, I (1981).

\title{
Delayed Neutron Emission Probabilities of ${ }^{9} \mathrm{Li}$ and ${ }^{11} \mathbf{L i}^{*}$
}

\author{
T. Björnstad, ${ }^{\dagger}$ H.A Gustefsson. ${ }^{\dagger}$ P.G. Hansent ${ }^{\dagger}$ B. Jonson, ${ }^{\dagger} V$. Lindfors. ${ }^{\dagger}$ \\ S. MatLsson, A.M. Paskanzer and H.L Rovat
}

Because of the inherent difficulty of neutron detoction, the known $P_{n}$ value of an accessible nucleus is often used to determine the ratio of the efficiencies of the neutron and $\beta$-detectors. For example, the $P_{\text {alues }}$ for ${ }^{11} \mathrm{Li}$, and many $\mathrm{Na}, \mathrm{Rb}$ and $\mathrm{Cs}$ isotopes $1,2^{\mathrm{n}}$ have been deduced by using the adopted value ${ }^{3}$ for ${ }^{9} 1 \mathrm{li}(35 \pm 5)$ \% by Chen et al. ${ }^{4}$ Our new measurement of the ${ }^{2} i P_{n}$ value gives $(50 \pm 4) \%$ and increases the other results ourrespondingly. In facl, it was the $\gamma$-measurement of ${ }^{11} \mathrm{Li}$ rtported here, which proved to be inconsistent with the published $P_{n}$ value and prompted us to remeasure the $P_{n}$ of ${ }^{9} L i$. The $P_{n}$ value of ${ }^{3} \mathbf{L i}$ is of particular interest because of the recent discoveries that it is a $a$-delayed two-neutron and threeneutron emitleer.
The lithium isotopes were produced by the $900 \mathrm{MeV}$ ${ }^{3} \mathrm{He}$ beam from the CERN $\$ \mathrm{C}$ irradiating uranium carbide or tantalum targels in combination with a thermal ion source at the ISOLDE facility. The $60 \mathrm{keV}$ ion beam from the separator was deposiled on a movable aluminized mylar tape. The beta deteclor which consisted of a 1 mun thick $24 \times 16$ mm plastic scintillalor (NE 102) mounted on a $30-\mathrm{mm}$ photolube was placed in a metal cylinder right behind the tape at the collection position.

The $4 \pi$ neutron counter consisted of eight ${ }^{3} \mathrm{He}$ proportional counters imbedded in paraffin wax in concentrical geometry around the cylinder containing the bela detector. The neutron detection efficiency was determined with a sample of ${ }^{238} \mathrm{U}$ to be $(14 \pm 1) \%$. The variation of the 
neutron detection efficiency witb energy, which bas not been measured, is in the present work the largest source of systematic error. The $r$-detector was a coexial $G e(L)$ crystal.

Since the residence time of a neutron in the paraffin moderated detector is exponentially distributed with a mean lifetiste of about $100 \mu \mathrm{s}$, the neutron- $\beta$ coincidence measurements were performed by starting a clock with the $\beta$ signal and stopping it with the neutron signal. The data were fitted to an exponential plus a constant value where the latter accounted for the random background at the low coincidence rates of about $10 \mathrm{~s}^{-1}$ used here. From $\hat{p-r}$ coincidence measurements on ${ }^{26} \mathrm{Na}$, the beta detection efficiency was determined to be $(40 \pm 3)$ 货

From two $\beta$-neutron coincidence measurements on ${ }^{9} \mathrm{l}$ the $P_{n}$ value was determined to be $(50 \pm 4)$ \% The sta tistical uncertainty is four times smalle; the error shown comes minty from the uncertainty in the efficiency of the neutron detector. Ten other sets of singles measurements were made, which with the determined \&-detection efficiency also yielded a $P_{n}$ value of $50 \%$ with an equally small statistical error, but with the uncertainty in the $\beta$ detection efficiency giving a larger systematic error. Table $]$ presents the $P_{n}$ values measured by Roeckl et al. 1,2 renormalized to a ${ }^{n} \mathrm{Li} \mathrm{P}_{\mathrm{n}}$ value of $50 \%$ instead of 35 资

Since the ground state of ${ }^{9} \mathrm{Be}$ is the only non-neutron emitting state, the p-decay branch to the ground state is thus also $(50 \pm 4) \%$

Tabte 1. Remormalized $\mathrm{P}_{\mathrm{n}}$ values (\%)

\begin{tabular}{lc}
\hline \hline${ }^{11} \mathrm{~L}^{\mathrm{a}}$ & $87 \pm 10$ \\
${ }^{27} \mathrm{Na}^{\mathrm{a}}$ & $0.11 \pm 0.04$ \\
${ }^{28} \mathrm{Na}^{\mathrm{a}}$ & $0.8 \pm 0.2$ \\
${ }^{29} \mathrm{Na}^{\mathrm{a}}$ & $22 \pm 3$ \\
${ }^{30} \mathrm{Na}^{\mathrm{a}}$ & $47 \pm 5$ \\
${ }^{31} \mathrm{Na}^{\mathrm{a}}$ & $43 \pm 11$ \\
${ }^{93} \mathrm{Rb}^{\mathrm{b}}$ & $1.8 \pm 0.2$ \\
${ }^{94} \mathrm{Rb}^{\mathrm{b}}$ & $12.1 \pm 1.3$ \\
${ }^{95} \mathrm{Rb}^{\mathrm{b}}$ & $12.2 \pm 1.3$ \\
${ }^{96} \mathrm{Rb}^{\mathrm{b}}$ & $19 \pm 2$ \\
${ }^{97} \mathrm{Rb}^{\mathrm{b}}$ & $39 \pm 4$ \\
${ }^{98} \mathrm{Rb}^{b}$ & $19 \pm 3$ \\
${ }^{145} \mathrm{Cs}^{\mathrm{b}}$ & $17 \pm 2$ \\
${ }^{146} \mathrm{Cs}^{\mathrm{b}}$ & $20 \pm 2$ \\
\hline
\end{tabular}

${ }^{\text {a}}$ From Ref. 1.

brom Ref. 2.
For ${ }^{11} \mathbf{L}$, the balf-life was redetermined by neutron measurements 10 be $8.83 \pm 0.12 \mathrm{~ms}$. Combining this with the previous result ${ }^{1}$ of $8.5 \pm 0.2 \mathrm{~ms}$, we recommend a best value of $8.7 \pm 0.1 \mathrm{~ms}$.

When counting the $\beta$ 's of $" \mathrm{~L} \mathrm{j}$ in the saturation mode one bas to take into account the contribution from the $14 \mathrm{~s}$ "Be daughter in equilibrium. Assuming that the " $\mathrm{Li}$ decay to the ${ }^{11} \mathrm{Be}$ ground state is negligible, then all the II Be yield must come from $\beta$-decay to the $320 \mathrm{keV}$ state, which is the only excited neutron bound state. If the probability of beta decay to this state is denoted $\mathbf{P}_{\gamma^{3}}$ then the $\beta$ count rate in the saturation should be proportional to $1+P_{\gamma}$ Taking this into account the $\theta-\gamma$ coincidence metsurement gave a value for $P_{\gamma}$ equal to $(9.2 \pm 0.7) \%$. The "'Be $\gamma$-ray at $2.12 \mathrm{MeV}$ was also visible. From this the "Be decays per " $\mathrm{Li}$ decay in saturation came to $(9 \pm 2) \%$, in agreement with $P_{\gamma}$ From this one can calculate that the one standard deviation upper limit on $\mathbf{P}_{\mathbf{g}}$. is $2 \%$ The $3.368 \mathrm{MeV} \gamma$-ray from the frst excited state of ${ }^{10} \mathrm{~B}$ e populated following neutron emission was seen with a probsability of $(35 \pm 3)$ \%

For the ${ }^{11} \mathrm{lj} \beta$-neutron coincidence measurement, the tape was moved every two seconds (with interruption of wounting) in order to reduce the contribution of the ${ }^{11} \mathrm{Be}$ daughter to an almosl negligible amount. The resulis gave a value for $P_{\text {equal }}$ to $(96 \pm 8)$, of which the statistical error was $5 \%$ and the rest of the error came from the uncertainty in the neutron detection efficiency. Six sets of singles measurements were also performed. The average value of $\mathrm{P}_{\mathrm{n}}$ was $93 \%$ with aboul the same statistical error as in the coincidence measurement, but with a larger systematic error. We adopt a best $P_{n}$ value of ( $95 \pm 8$ ) The renormalized value from the measurements of Roeckl ef al. 'given in Table 1 agroes with this new value.

Previously ${ }^{1}{ }^{\prime} \mathrm{Li}$ had been reported ${ }^{5}$ to be a multiple neutron emitter. The relative probabilities of two-neutron and three-neutron emission were given to be ${ }^{6}$

$$
\begin{aligned}
& P_{2 n} / P_{l n}=0.048 \pm 0.005 \\
& P_{3 n} / P_{1 n}=0.022 \pm 0.002
\end{aligned}
$$

From this one obtains:

$$
P_{n} / P_{10}=1+2 P_{2 n} / P_{1 n}+3 P_{3 n} / P_{1 p}=1.16 \pm 0.01
$$

By assuming that the ${ }^{8} \mathrm{Li}+\mathrm{t}$ disintegration mode as well as the ground-state $\beta$-decay are negligible one can state that:

$$
1-P_{\gamma}=P_{1 n}+P_{2 n}+P_{3 n}
$$

Using the values for two- and three-neutron emission given in Eq. (I)

$$
\left(I-P_{\gamma}\right) / P_{l n}=1.07 \pm 0.005
$$


Combining this with Eq. (2) one finds that the relationship between $P_{n}$ and $P_{\gamma}$ is given by:

$$
P_{n} /\left(1-P_{Y}\right)-1.08 \pm 0.01 \text {. }
$$

Our results presented above give for this ratio the value $1.05 \pm 0.08$, which shows consistency between our neutron and $r$ measurements.

With these assumptions, one may calculate a more accurate value for $P_{n}$ from the measured value of $P_{\gamma}$ by using Eq. (4). This gives a 11 Li $P_{n}$ value of $(98 \pm \hat{h})$, and by using Eqs. (2) and (1), the following multi-neutron emission probabilities are obtained:

$$
\begin{aligned}
& P_{1 \mathrm{n}}=(85 \pm 1) \%, \\
& P_{\text {1屯 }}=(4.1 \pm 0.4) \% \\
& P_{3 \mathrm{n}}=(1.9 \pm 0.2) \%,
\end{aligned}
$$

From the intensity of the $3.3 \mathrm{MeV} \gamma$-ray in ${ }^{10} \mathrm{Be}$ and the $\mathrm{P}_{\mathrm{In}}$ value the fraction of one neutron emission leading to excited states of ${ }^{10} \mathrm{Be}$ becones $(41 \pm 4)$ 䍕.
Foothotes and References

Condensed from Nucl. Phys. A 359, 1 (1981).

${ }^{\dagger}$ CERN-ISOLDE, CERN, CH-1211 Geneva 23, Switzerland.

tinstitule of Physics, University of Asthos, Dennark.

1. E. Roeckl, P.F. Ditıner, C. Détraz, R. Klapisch, C. Thibault and C. Rigaud, Phys. Rev. C 10, 1181 (1974),

2. E. Roeckl, P.F. Dittner, R. Klapisch, C. Thibault, C. Rigaud and R. Priets, Nuct. Phys. A 222, 621 (1974).

3. F. Ajzenberg-Selove, Nuc]. Phys. A 320, J (1979).

4. Y.S. Chen, T.A. Tombrello and R.W. Kavanagh, Nuct. Phys. A 146, 136 (1970).

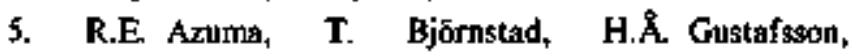
P.G. Hansen, B. Jonson, S. Mattsson, G. Nyman, A.M. Poskanzer and H.L. Ravn, Phys. Lett. B 96, 31 (1980).

6. Note that the probability of emission of $i$ neutrons is denoted $P_{\text {in }}$ so that the usual $P_{n}$ value is given by $P_{n}=\Sigma_{j} P_{i n}$

\title{
Beta-Delayed Three-Neutron Radioactivity of ${ }^{11} \mathrm{Li}^{*}$
}

\author{
RE. Azuma, ${ }^{\dagger}$ T. Bjärnstad, ${ }^{\ddagger}$ H.A. Gustafsson, ${ }^{\ddagger}$ P.G. Hansen,' B. Jonson, ${ }^{\ddagger}$ \\ S. Mattsson, ${ }^{\ddagger}$ G. Nyman, A.M. Poskanzer, and H.L. Ravn
}

Beta-delayed emission of two neutrons has recently been observed in the decay of ${ }^{11} \mathrm{Li}$ (Ref. 1) and of the sodium isctopes ${ }^{36-32}$ Na (Ref. 2). In the present paper the extension is reported of the neutron time-correlation techniques used in the previous work. With this method a new radioactive decay mode, beta-delayed three-nentron enission, has been observed in ${ }^{\prime} \mathrm{L}$ in an intensity $P_{3 n}=(1.8 \pm 0.2) \%$

The isotope ${ }^{11} \mathrm{lj}$ with a half-life of $8.6 \pm 0.2 \mathrm{~ms}$ is ideally suited for a search for $3 n$ crission. It has a $Q_{\beta}$ value of $20.7 \mathrm{MeV}$ (Ref. 3) and the thresholds for breakup into three neutrons lie at $8.888 \mathrm{MeV}$ (residual two ${ }^{4} \mathrm{Hc}$ ) and at $8.979 \mathrm{MeV}$ (residual ${ }^{8} \mathrm{Be}$ ).

The radioactivity was produced by bombarding a target of uraniom carbide at $2000^{\circ} \mathrm{C}$ with a $0.2-2 \mu \mathrm{A}$ proton beam of $600 \mathrm{MeV}$ from the CERN synchrocyclotron. The 1 li radioactivity, was separated on-line in the ISOLDE facility and directed as an for beam to the centre of a paraffin-filled $4 \pi$ neutron counter.

The neutron crunter ${ }^{1,2}$ now was equipped with a total of $12{ }^{3} \mathrm{He}$ tubes, which increased the efficiency to

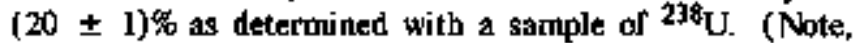
however, that the neutron detection effieiency is expected to depend appreciably on the neutron eneTgy.) The residence time in the detector, as determined from beta-neutron coincidences on ${ }^{9} \mathrm{~L}$ was exponentially distributed with a mean of $89 \pm 1$ as. The neutron counters were connected in parallel and fed into a microprocessor unit that allowed the arrival times of individual neutrons to be read by a "flying clock" with a precision of 1 ws.

The rate of triple tyents and the histograms (Fig. I) laken on-line with strongly reduced proton beam intensity clearly indicated the presence of 1rue triple events. The presence of random triple correlations due to the combination of single and double events, however, necessitated a utore detajled analysis.

As the evaluation is very sensitive to the precision with which the correttion for randoms can be carried out. the analysis was checked in several ways. (i) Randon data taken with a $\gamma$-source and with ${ }^{5} \mathrm{~L} i$ neutrons were analyzed and it was found that the corrections for random events were accurate to typically (3-7) rate in the "Li experiments was varied from $7.9 \mathrm{c} / \mathrm{s}$ to $202.8 \mathrm{c} / \mathrm{s}$ without any appreciable change in the results. This is a very bensitive test as the random events vary with the square or the cube of the rate. (iii) The analysis time 8 was raried between 79.8 and $456 \mu$, again without any detectable effect (iv) The time distributions shown in Fig. I were calculated from the results of the data analysis and evidently agree exactly with experiment.

From the analysis we propose as adopted values

$$
\begin{aligned}
& P_{2 n} / p_{1 n}=(4.8 \pm 0.5) \times 10^{-2}, \\
& P_{5 n} / p_{1 n}=(2.2 \pm 0.2) \times 10^{-2}
\end{aligned}
$$




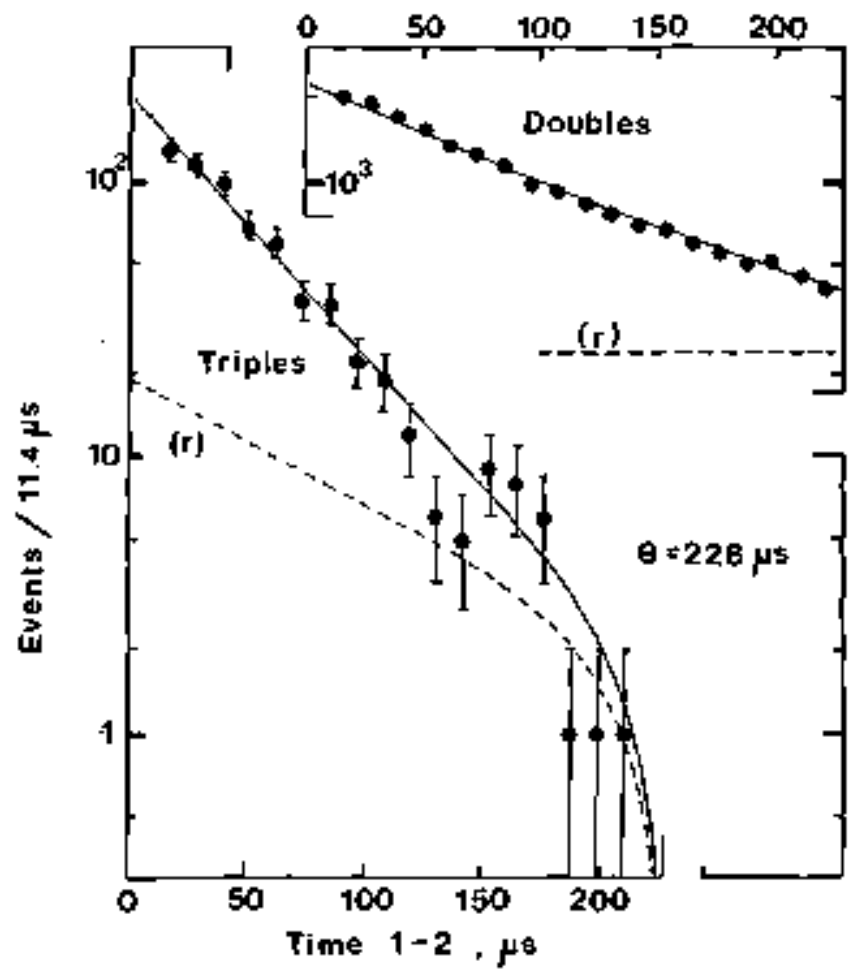

Fig. 1. Distribution of the time interval between the first and the second neutrons for ewents registered as doubles and triples with a corrtation time $\theta=228 \mu \mathrm{s}$. The data are from a $12 \mathrm{~h}$ run with an average neutron count rate of $22.9 \mathrm{c} / \mathrm{s}$. The theoretical curves showing the total number of everts athd the contribution from candom events ( $r$ ) do nol represent a fit: they have been calculated on an absolute scale. Note that the triples events clearly show the $\mathrm{e}^{-2 \mathrm{t}}$ dependence expected theoretically. (XBL 817-10857)
The $p_{\text {in }}$ values may be put on an absolute scale by means of the total probability for neutron emission $\mathrm{P}_{\mathbf{1}}=\sum_{-1}^{\infty}$ i $\mathrm{p}_{\mathrm{in}}$. We use here the value $\mathrm{p}_{\mathrm{n}}=(95 \pm 8)^{\circ}{ }^{5}$ From this one finds for ${ }^{11} \mathrm{Li}$

$$
\begin{aligned}
& p_{1 \mathrm{~A}}=(82 \pm 7) \%, \quad P_{2 \mathrm{~s}}=(3.9 \pm 0.5) \% \\
& p_{\text {到 }}=(1.8 \pm 0.2) \%
\end{aligned}
$$

The experiment represents the first observation of a new radjoactive decay mode: $\beta$-delayed three-neutron emission. It is furtbermore noteworthy that the residual nucleus itself is unstable so that the process detected in the present work effectively is a break-up into the five nuclear particies $2 \alpha+3 n$.

\section{Foothotes and References}

"Condensed from Phys. Let1. B 96, 31 (1980).

'Department of Physies, University of Toronto, Toronto. Ontario, Canada.

tCERN-ISOLDE CERN, CH-1211 Geneva 23, Suitzerland.

Institute of Physics, University of Aarhus, DK-8000 Aarhus, Dennark.

Depariment of Physics, Chalmers Institute of Technology, S-41296 Göteborg. Sweden.

I. RE. Azums et al, Phys. Rev. Lett. 43, 1652 (1979).

2. C. Détraz at al., Phys. Lett. B 94, 307 (1980).

3. C. Thibautt et al., Phys. Rev. C 12, 644 (1975).

4. F. Ajzenberg-Selove and C.L. Busch, Nuci. Phys. A 336, l ( 1980).

5. T, Björnstad et al., Nucl. Physs, A 359, 1 (1981).

\title{
Beta-Delayed Two-Neutron Emission from ${ }^{30,31,32} \mathrm{Na}^{*}$
}

\author{
C. Detraz, ${ }^{\dagger}$ M. Epherre, D. Guillemaud, ${ }^{\dagger}$ P.G. Hansen, ${ }^{5}$ B. Jonson, ${ }^{\$} \boldsymbol{R}$. Klapisch, \\ M. Langevin," S. Mattsson, F. Nawlin, G. Nyman ${ }^{\dagger}$ A.M. Poskanzer, H.L. Ravn, \\ M. de Salnt-Simon, K. Takahashi,' C. Thibawit, and F, Touchard ${ }^{\ddagger}$
}

Beta-delayed emission of two neutrons has recenty been detected for the first time ${ }^{1}$ in the decay of $8.6 \mathrm{~ms}$ ${ }^{1} \mathrm{Li}$. This decay mode is also to be expected in the heavier elements and especially in those with odd $Z$, as may be seen from an inspection of theoretical mass tables. The element sodium $(Z=\mid 1)$ is a particularly fawourable elese, as its neutron-rich isotopes can be produced in high yields through mass separation of fragmentation products from reactions with high-energy protons. We report here the application of two differenl experimental methods, $n-n$ time correlations with a $4 \pi$ teutron counter and $\gamma$ spectroscopic identification of daughter products with mass
A -2 , for demonstrating that the $\beta^{-}$decays of the isotopes ${ }^{30-32} \mathrm{Na}$ are accompanied by $2 \mathrm{n}$-emission.

One series of experiments was based on the n-n time correlation method, which has already been discussed in some detail in previous work. ${ }^{1}$ The radioactivity was produced by bombarding a target of uranium-carbide at $2000^{\circ} \mathrm{C}$ with $600 \mathrm{MeV}$ protons (rom the CERN synchrocycloron. Afler mass separation in the ISOLDE separator, the isolope of interest was directed to the centre of a $60 \mathrm{~cm}$ long parafîn-filled cylinder containing eight ${ }^{3} \mathrm{He}$ proportionsl counters. With the detectors connected in parallel, a search was made by time correlation techniques 
for pairs of neutron events with the chacacteristic mean time of $120 \mu$ for residence in the delector.

The experimental time correlation spectra for ${ }^{30,32} \mathrm{Na}$ are shown in Fig. I. The intensity of the correlated events relative to the total singles neutron counts determines the proportion of 2 a-events when allowance is made for randoms, correlated background, deadtime losses and, of course, the efficiency of the neutron detector. The latier was determined with neutrons arising from ${ }^{239} \mathrm{U}$ spontane. ops fission.

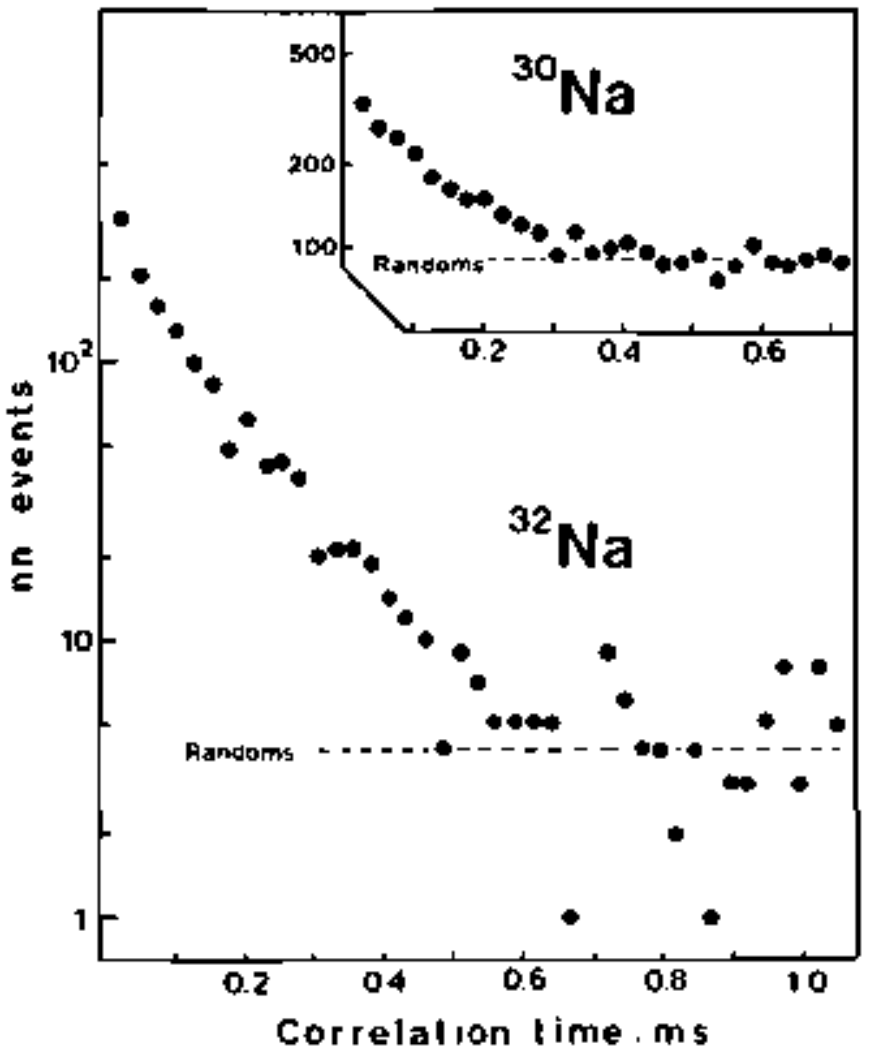

Fig. I. Messured n-n time correlations for the isotopes ${ }^{30} \mathrm{Na}$ and ${ }^{32} \mathrm{Na}$. The measuring time was 14.3 and $7,4 \mathrm{~h}$, respectively. For the ${ }^{30} \mathrm{Na}$ measurement, the initial beam intensity was reduced by a factor of about $\mathbf{3 0}$ in order to improve the ratio of true to randorn events. The dashed lines indicale the mean contributions of random $n-n$ correJations.
The second series of experiments used the $24 \mathrm{GeV}$ proton beam from the CERN synchrotron to bombard an iridium target at high temperature. After separation in the mass spectrocinter ${ }^{2}$, the beam of interest was directed to the end of a thin Al collecting tube viewed by a $\mathrm{Ge}(\mathrm{L}$ ) delector. The presence of one- and two-neutron decay processes is revealed by the appearance of $\gamma$-rays belonging to lower mass chains than the one collected. Absolute intensities wete obtained from the $r$-spectra, from monjtoring the f-radioactivity during the collection and from wounting of the $\mathrm{Na}$ ions before and after the collection. The presence of mass-28 lines in the $\gamma$-spectrum of a samtple produced in a long collection at mass 30 was shown.

For the case of ${ }^{32} \mathrm{Na}$, the r-spectra measured on-line showed contributions (rom the decays of ${ }^{32} \mathrm{Na}_{1},{ }^{31} \mathrm{Al}$ and ${ }^{30} \mathrm{Mg}$, so that the relative contributions of zero-, one- and two-neutron emission could be deduced. At mass 31 no rrays above background were observed. ${ }^{30-32} \mathrm{Na}$ are $\beta$ delayed two-neutron emitters and the intensities measured by the two methods agree well. The final values ${ }^{3}$ that we have adopted for $\mathrm{p}_{2 \mathrm{p}}$ are ${ }^{30} \mathrm{Na}(1.2 \pm 0.2)$ \% ${ }^{3}{ }^{31} \mathrm{Na}$ $(0.70 \pm 0.25) \%$ and ${ }^{32} \mathrm{Na}(5.1 \pm 1.8) \%$

The experiments demonstrale that $\beta$-delayed twoneutron emission is not a rare process far from stability; in fact, for ${ }^{32} \mathrm{Na}$ it accounts for half of all neutrons emitted. It is thetefore important to lake this mechanism into account in the calculation of yields in the astrophysical rprocess.

\section{Footnotes and References}

'Condensed from Phys. Rev. Lett. B 94, 307 ( 1960)

IInstitute de Physique Nucleaire, F-91406 Orsay. France.

*Laboratoire Rene Bernas, CSNSM, F-91406 Orsay, France.

The ISOLDE Collaboration, CERN, CH-1211 Geneva, Switzerland.

Institut Jur Kemphysik, Technische Hochschule, D-6100 Darmstadi, Federal Republic of Gertrany.

I. RE Azuma el al., Phys, Rev. Lett. 43, 1652 (J979).

2. C. Thibault et al., Phys. Rev. C 12, 644 (1975).

3. The probability per $\beta$-decay for a process leading to the emission of $\mathrm{j}$ neutrons is demoted $\mathrm{p}_{20}$, so that $\Sigma_{p_{0}}=1$. The usual total branching ratio for $\beta$-delayed neutrom emission is $p_{n}=\Sigma j p_{i n}$. 


\title{
The Measurement of $\beta$-Decay Lifetimes via Projectile Fragmentation"
}

\author{
M.J. Murphy, T.J.M. Symons, G.D. Westfall, and H.J. Crawfordt
}

It has recently been shown 1,2 that heavy ion projectile fragmentation produces a brosd spectrum of isotopes extending far from the valley of is stability. This discovery has enabled the developrnent of a significant new technique to measure the pidecay lifetimes of large numbers of very neutron-rich nuclei in a single experiment. The technique utilizes a multi-element position-sensitive $\$$ i( $(\mathrm{Li})$ telescope to stop and identify each projestile fragnent; the same detector array is then used with higher electronic gain to observe the delayed electron from the embedded fragment's decay. The correlation sbserved between fragment and $\beta$ is established by interrupting the beam upon detection of a particular isotope, and then recording the elapsed time to the first $\beta$-decay event originating at the position of the stopped fragment. Deflection of the fragments through a magnetic spectrometer greatly rectuces background by selecting isotopes of a specifie rigidity.

In the first application of this method, the fragmentation of ${ }^{40}$ Ar at $280 \mathrm{McV} / \mathrm{n}$ was used to measure several known and new s-decay lifetimes in the range from $40 \mathrm{mrec}$ to $600 \mathrm{msec}$. The half-lives are extracted by first averaging the multiple $\Delta \mathrm{E}-\mathrm{E}$ measurements provided by the six-element telescope to cotain mass resclution in the particle-identification spectrum for each element. An example of the resulting spectrum for aluminum isotopes of a particular rigidity is shown in Fig. 1. Gates are then set around each mass peak, and a spectrum of the elapsed time between the fragment and $\beta^{-}$events is made. The $\left.{ }^{3}\right|_{A}$ and ${ }^{32} \mathrm{Al}$ peaks in Fig. I yielded the two half-life curves shown in Fig. 2 . The observed ${ }^{31} \mathrm{Al}$ half-life of $620 \mathrm{~ms}$ agrees well with the tabulated value of $640 \mathrm{~ms}$. The halflife of ${ }^{32} \mathrm{Al}$ was previously unknown; we obtain a value of $40 \mathrm{mis}$.

The success of the initial run indicates that, with the higher intensities and heavier besms which will becoms available from the upgraded Bevalac, this technique can make a major contribution to the study of $\beta$ decay systerratics, with applietions to nuclear structure and to astropbysics.

\section{Footnotes and References}

"Condensed from the Proceedings of the Sth High Energy Heavy lon Study (1981).

tSpace Sciences Laboratory, University of California, Berkeley, CA 94720.

1. T.J.M. Symons et al., Phys. Rev. Lett. 42, 40 (1979).

2. G.D. Westfall et al., Phys. Rev. Lett. 43, 1859 (1979).

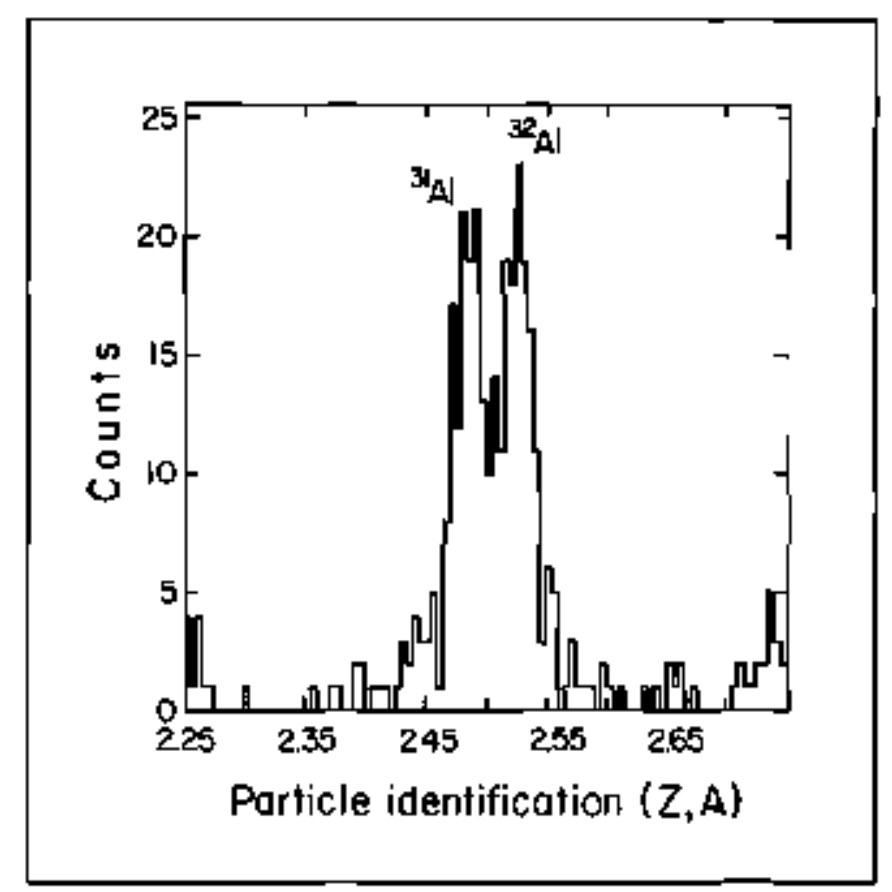

Fig. 1. Neutron-rich aluminum isotopes observed in the fragmentation of $285 \mathrm{MeV} / \mathrm{n}^{40}{ }_{\mathrm{Ar}}$.

(XBL $816-927$ )
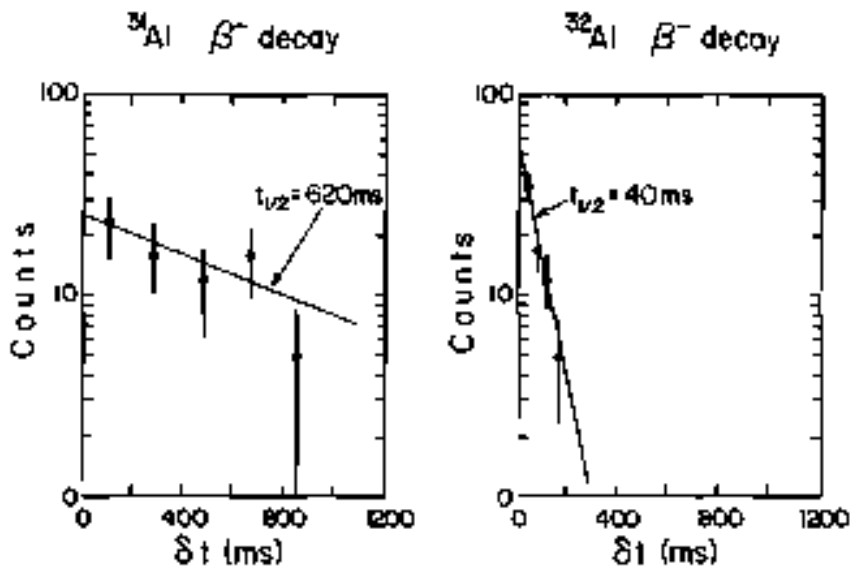

Fig. 2 -decay lifetime curves associated with the ${ }^{31} \mathrm{Al}$ and ${ }^{32} \mathrm{~A}$ fragiment peaks shown in Fig. 1 . (XBL 816-926) 


\title{
$\beta$-Decay Energies and Masses of ${ }^{103-105}$ In
}

\author{
J.M. Wowters, H.M. Thierens,* J. Aysto, M.D. Cable, \\ P.E. Hawstein, ${ }^{\ddagger}$ R.F. Parry, and Joseph Cerny
}

The study of the nuclidic mass surface in the vicinity of the doubly magic ntcleus $10_{S \mathrm{~S}}$ is of fundamental interest in providing information on the strength of the shell closure when $Z=N=50$. A comparison of measured mass excesses with currently available model mass predictions can determine the accuracy with which the various models include the effects resulting from shell closures. As a further step in the extension of the known mass surface we bave measured the decay energies of 103-105 In using $\beta-\gamma$ coincidence spectroscopy following online mass separations. The decay of ${ }^{102}$ In was also observed ${ }^{l}$ but with inadequale statistics up to now to determine an accurate endpoint energy.

Heavy ion beams of ${ }^{12} \mathrm{C},{ }^{14} \mathrm{~N}$ and ${ }^{16} \mathrm{O}$ from the 88Inch Cyclotron were dirscted onto various targets to produce indium isotopes via $(\mathrm{Hl}, \times n)$ and (HI,pxn) reactions. The recoils were transferred via a heliurr-jet to the RAMA on-line mass separator, where they were mass analyzed. The mass separated recoils were collected on a mylar tape and transported to the detector station for $\beta$ - $\gamma$ coincidence spectroscopy. A separate contribution to this annual report gives a detiiled description of the tape transport system, the delector station, and the procedure used for analyzing the forspectra to obtain p-endpoints.

Table 1 presents a summary of the $Q_{\text {EC }}$ determinations and Fig. 1 presents the Fermi-Kurie plot of ${ }^{103} \mathrm{In}$ as an exarople spectrum. Figure 2 shows a comparison of the predictions of the known indium masses with selected representatives of the different mass theories that are available. Those masses calculated acoording to the mass relations of the Garvey-Kelson ${ }^{4}$ type and those calculated from the shell model formula of Liran-Zeldes agree very well with the experimental results from ${ }^{106}$ In to ${ }^{123}$ In (Fir. 2a). For each of these mass models the root-mean-square deviation of theory from experistent for these nuclides is less than $200 \mathrm{keV}$. In the neutron-deficient side of figure $2 \mathrm{a}$ at ${ }^{103}$ In, a strong deviation of about $\mathrm{M} \mathrm{MeV}$ of the experimen1al value from the predictions of Liran-Zeldes and the

Table 1. Summary of $\mathrm{Q}_{\mathrm{EC}}$ delemininations.

\begin{tabular}{cccc}
\hline & & \multicolumn{2}{c}{$\mathrm{Q}_{\mathrm{EC}}(\mathrm{MeV})$} \\
\cline { 3 - 4 } Nuclide & Gate & This Hork & Literature \\
\hline${ }^{109} \mathrm{In}$ & 188 & $5.38 \pm 0.13$ & $5.9 \pm 0.5$ (Ref. 2) \\
${ }^{104} \mathrm{In}$ & 658,834 & $7.42 \pm 0.14$ & $7.1 \pm 0.2$ (Ref. 3) \\
${ }^{105}$ In & 131 & $5.14 \pm 0.13$ & \\
\hline \hline
\end{tabular}

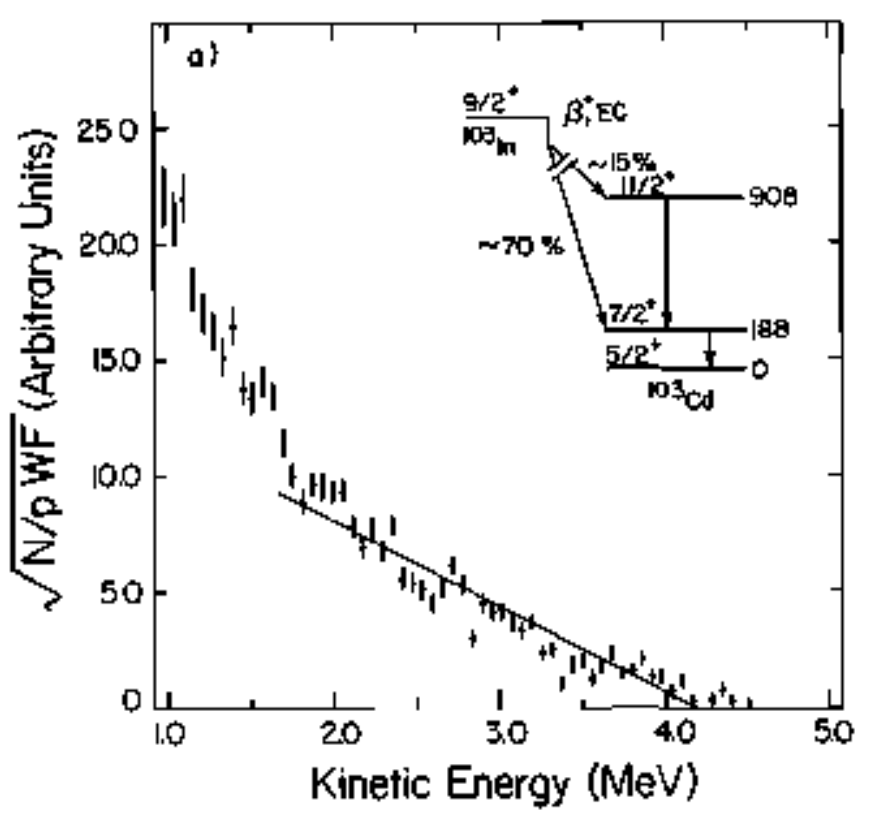

Fig. 1. Fermi-Kurie plot and partial decay scheme for ${ }_{103}^{10}$. (XBL 8111-1592)

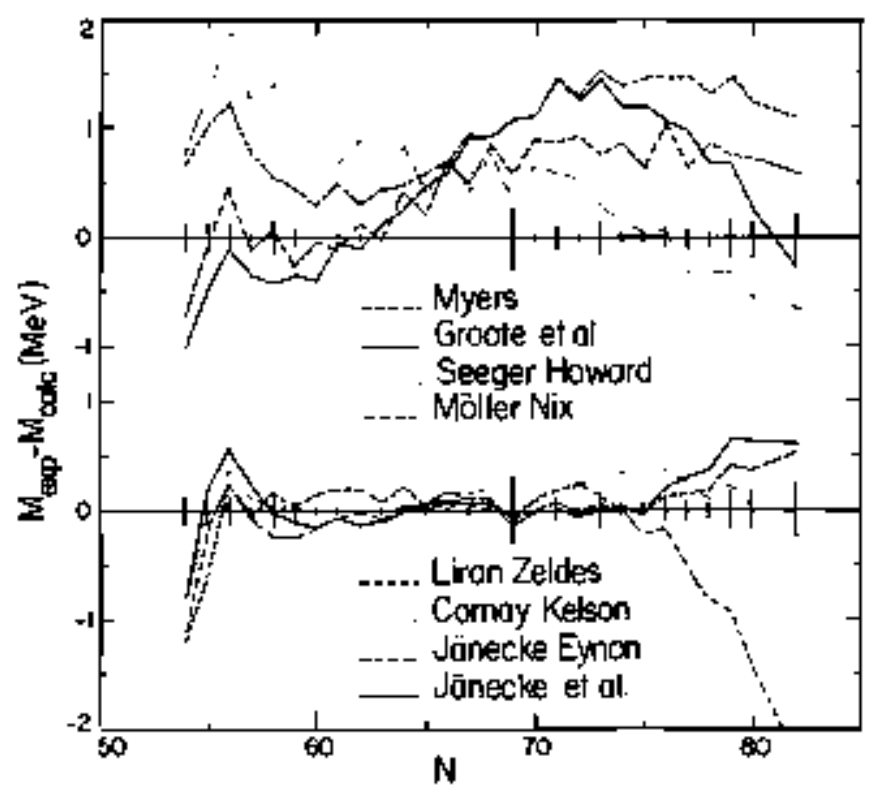

Fig. 2. A conparison of known indium masses with the predictions of some selected mass equations.

(XBL 819-1350) 
different Garvey-Kelson type mass formulas suddenly appears. The predictions of those liquid drop mode]s considered here differ more from the experimentally observed mass behavior than the results of calculations based on the Garvey-Kelson relations (ste Fig. 2b). For the models of Myers, ${ }^{4}$ Groote et al., ${ }^{4}$ Seeger-Howard ${ }^{4}$ and Moller-Nix, ${ }^{5}$ the root-mean-square deviation from all the indium mass data is $1070,830,780$ and $630 \mathrm{keV}$, respectively. As was noted for those mass models displayed in Fig. 2a, a sudden change in the systematic differences between the experjmental and calculated masses also sets in for ${ }^{103}$ In in the comparison with the liquid drop model predictions shown in Fìg. $2 \mathbf{b}$.

In conclusion, according to our results, ${ }^{103}$ [n is about $1 \mathrm{MeV}$ more bound than predicted by the Liran-Zeldes and the different Garvey-Kelson type mass formulas. Other investigations of the mass surface near ${ }^{100} \mathrm{Sn}$ will show whether the observed deviation from the sysiematics for
${ }^{103}$ In might have any possible relationship with the nearby double-shell closue.

\section{Footnoles and References}

"Present address: University of Gent, Belgium

†Permanent address: University of Jyväskylä, Finland

FFermanent address: Brookhayen National Laboratory, U.S.A.

I. R. Beraud, J. Tréberne, A Charvel, R. Duffeait, J. Genevey, A Gizon, J. Gizon and M. Meyer, Z. Physik A 299,279 (1981).

2. G. Lhersonneav, G. Dumont, K Cornelis, M Huyse, and J. Yerplancke, Phys. Rev, C 18, 2688 (1978).

3. H. Huang, B.P. Pathak and J.K.P. Lee, Can. J. Phys. 56. 936 (1978).

4. At. Data Nucl. Data Tables 17, 41 (1976).

5. P. Mßller and J. Nix, Report LA-UR-80-1996.

\title{
The $\epsilon / \beta^{+}$Decay of ${ }^{145} \mathrm{Gd}$ : Resolution of Decay Branching Ratio Anomalies and Evidence for Pronounced Structures in the $\beta$-Decay Strength ${ }^{*}$
}

\author{
R.B. Firestome, R.C. Pardo, R.A. Warner ${ }^{\dagger}$ \\ WmC. MeHarris, and W.H. Kelly $f^{f}$
}

The $\epsilon / \beta^{*}$ decay of ${ }^{145}$ Gd has been investigated in Breat detail. We have phaced I36 levels deexcited by $326 \gamma$ rays in ${ }^{145} \mathrm{Eu}$. In addition, $\epsilon / \beta^{*}$ decay branching ratios, the decay energy, and the half life have been aceurately measured by various coincidence and $\gamma$-ray singles techniques. This work is the culmination of over 10 years of experimental effort by our group.

Previously reported $\epsilon / \beta^{+}$decay branching satio anomalies have been eljminated in these experiments. ${ }^{1}$

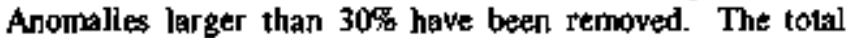
ground state decay energy was measured by $\beta-\gamma$ coincidence techniques to be $5.07 \pm 0.06 \mathrm{MeV}$. A new halflife of $23.0 \pm 0.4 \mathrm{~m}$ was determined, eliminating a preexisting discrepancy in seported values.

The decay of ${ }^{145} \mathrm{Gd}$ offers a unique opportunity to investigale the nature of bela decay to regions of high level density. Here $>98 \%$ of the total decay intensity is placed and the microsopic detail of the decay is observed. Previous authors have suggested that these experiments would not be possible due to the large number of very weak iransitions. ${ }^{2}$ In fact, simple nuclear strnctures were observed to dominate the decay concentrating the intensity into relalively few important channels. These siructures are discussed in a separate report. A part of the total ${ }^{145}$ Cd level scheme is shown in Fig. 1.

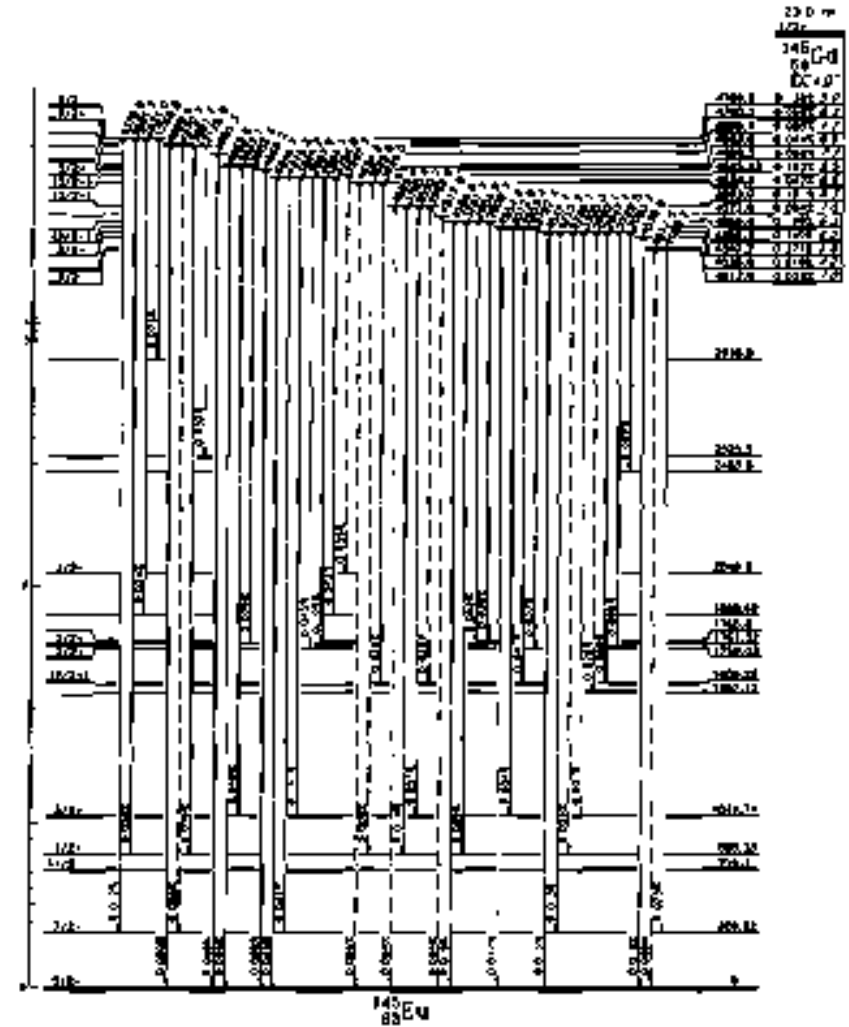

Fig. 1. Portion of the level scheme for the decay of 23.0 min ${ }^{145} \mathrm{Gd}$ to levels of bigh excitation in ${ }^{145} \mathrm{Eu}$. (XBL $813-8490$ ) 


\section{Foolnotes and References}

'Condensed from LBL 12424, Physicel Review C 25, (1982).

National Superconducting Cyclotron Laboratory and Departments of Chemistry and Physics, Michigan State
University, East Lansing, MI 48824

1. R.B. Firestone, R.A. Warmer, Wm C. MeHarris, and W.H. Kelly, Phys, Rev, Lett. 33, 30 (1974); 35, 713 (1975).

2. J.C. Hardy, L.C. Carraz, B. Jonson, and P.G. Hansen, Phys. Lett. B 71, 307 (1977).

\section{Observation of Large Resonances in the $\beta$-Decay of ${ }_{64}^{145} \mathrm{Gd}_{81}^{*}$}

\section{R.B. Firestone}

The $\epsilon / \beta+$ decay of ${ }^{145} \mathrm{Gd}$ has been studied in detail and is further discussed in another report. This decay proceseds to a region of high level density in ${ }^{145} \mathrm{Eu}$. It has been suggested that sweh docay should exhibit to pronounced structure and would be impossible to investigate completely. I That prediction was not borne out by these experiments as is indicated by the $\beta$-decay strength distribution, $S_{8}=$ s-inlensity/ft, which is ploted in Fig. 1 . Three resonances at $1.8,2.6$, and $4.5 \mathrm{MeV}$ were observed.

${ }^{145} \mathrm{Gd}$ decay is renarkable because the valence protons outside the $\mathrm{Z}=50$ closed shell occupy $\mathrm{rl}_{5 / 2}$ and $\pi_{g_{7 / 2}}$

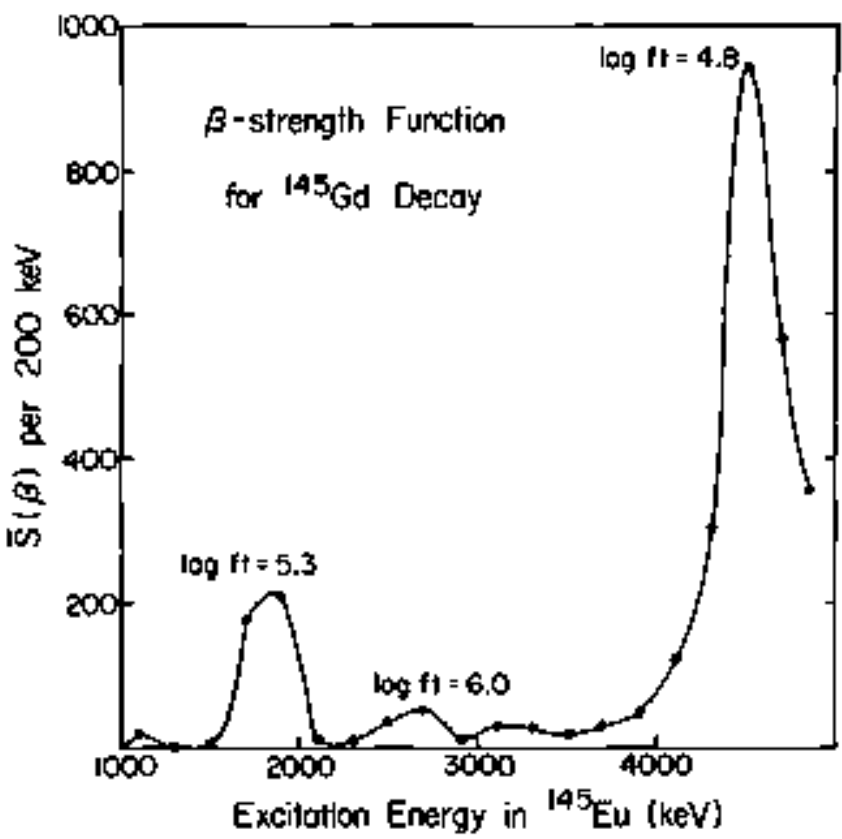

Fig. 1. The $\beta$-strength function $\bar{S}(\beta)$ (per $200 \mathrm{keV}$ ) plorted as a function of excitation energy in ${ }^{145} \mathrm{Eu}$. Here we define $\bar{S}(\beta)=b(E) / \mathrm{ft}$ where $\mathrm{b}(\mathrm{E})$ is the intensity fraction per 200-keV interval of excitation and $f$ is the standard $\beta$ decay rate to that energy domain of the daughter. Browd resonances at $1.8,2.6$, and $4.5 \mathrm{MeV}$ arc observed, and the effective logit's to these resonances are sbown. (XBL 803-8699) shell model oxbitats while only a $\nu \mathrm{s}_{1 / 2}$ hole in the neutron core can be populated by decay. Thus no strong low-lying single particle transitions are expected or obseryed. The resonances at 1.8 and $2.6 \mathrm{MeV}$ can be interpreted as the decay from $\left(\pi \mathrm{d}_{5 / 2}\right)^{2}$ pairs to collective $\pi \mathrm{d}_{5 / 2} \times$ $\left(2_{t}^{+}+2_{2}^{+}\right)$configurations. This is confirmed by energy systematics, decay rates, and $\uparrow$-ray deexcitation patterns when compared with decay of ${ }^{144} \mathrm{Eu}$ to the ${ }^{144}$ mn corc (Fig. 2).

The strong resonance at $4.5 \mathrm{MeV}$ probably corresponds to decay across the $\mathrm{N}=82$ shell energy gap.

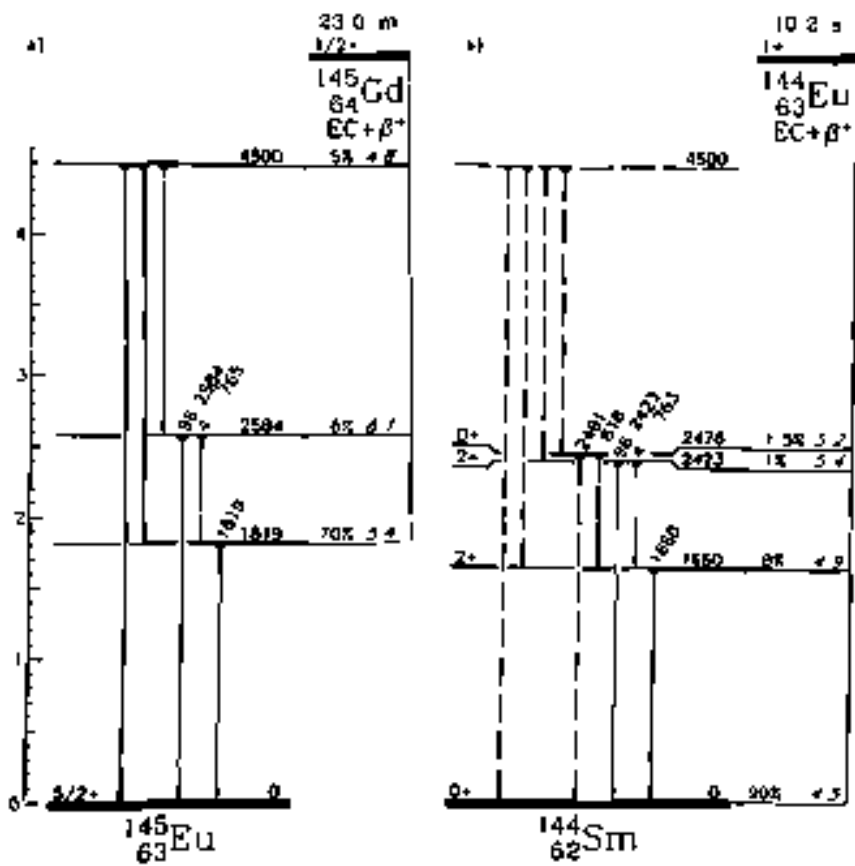

Fig. 2. Simplified decay scheme for ${ }^{145} \mathrm{Gd}$ (a) is compared with the decay of the ${ }^{44} \mathrm{Eu}$ core. Note the close agreement between the entergy separation and decay intensity pattem of the $2^{+}{ }^{144}$ sm core levels and the ( $\mathrm{md}_{5 / 2} \times 2^{+}$) levels in ${ }^{145} \mathrm{Eu}$. Although the logft's do nol compare so well, additional unobserved decay to high energy states in ${ }^{14} \mathrm{Sm}$ can improwe that comparison substantially. 
Calculations of $\nu f_{7 / 2}, \Delta i_{13 / 2}$, and $\Delta h_{9 / 2}$ orbitals lie at 3.9, 4.9. and $5.3 \mathrm{MeV}$, respectively. Assuming significant $\left(\pi \mathrm{h}_{11 / 2}\right)^{2}$ pair oceupancy, the decay to $\left(\pi \mathrm{h}_{1 \mathrm{l} / 2} \times \mathrm{vh}_{9 / 2} \times\right.$ uS $1 / 2$ 3-quasiparticle states should be very fast. The $\pi h_{11 / 2} \rightarrow v h_{9 / 2} \beta$-decay from ${ }^{147} \mathrm{~Tb}$ has been observed to procesd with a low logit. Admixtures of other orbitals with the $\pi h_{9 / 2}$ orbital will scatier some of its strength to lower energies and a loss of spherical symmetry will further depress that state providing reasontable agretment with the energy of the oberved resonance. Indered the increased $\beta$ strength begins at $3.9 \mathrm{MeV}$, exactly where the shell gap is predicted.

Further studies are planned to investigate the decay to higher $Z, N=82$ nucleit. For ${ }^{147} \mathrm{~Tb}$, the proton becomes unbound at about $1.9 \mathrm{MeV}$ so the higher energy part of the highest resonance might be observed to proton decay. Heavier nuclei should predominantly decay through this bigh-energy channel and can be characterized by the domtinant pidelayed proton decay mode.

\section{Footnotes and References}

'See LBL-12424 and the Proceedings of the International Conference on Nuclear Physics, August 24-30, 1980, Berkeley. CA, p. 201.

I. J.C. Hardy, L.C. Carroz, B. Jonson, and P.G. Hansen, Pbys. Lett. B 71, 307 (1977).

\title{
Determination of the Half-Life and Absolute Gamma Intensities of ${ }^{151} \mathbf{G d}$
}

\author{
K.E. Gregorich, KJ. Moody, and G.T. Seaborg
} by the

A ${ }^{151}$ Gd source was produced at the 88-Inch Cyclotron

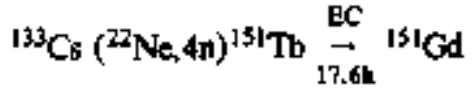

reaction at a bombarding energy of $94 \mathrm{MeV}$. The half-life of the eloctron capture decay of ${ }^{151}$ Gd has botn determined to be $129.1 \pm 1.2$ days, which is more accurate than the previously best known value of $120 \pm 20$ days.' The absolute intensities of the gamma rays acoornpanying this decay were also determined for the first time.

A target consisting of $600 \mu \mathrm{g} / \mathrm{cm}^{2}$ layer of $\mathrm{CsCl}$ on a $0.10 \mathrm{~mm}$ thick gold backing was irradiated with 500 electrical namoamperes of ${ }^{22} \mathrm{Ne}+4$ for 8.5 hours. ${ }^{151} \mathrm{~Tb}$ is formed in the reaction with a cross section of roughly 400 mb. Terbium was chemically separated from the target as follows: The target was dissolved in $3 \mathrm{ml}$ aqua regia and the gold was extracted into diethyl ether three times. Residual gold was removed from the aqueous portion by heating it to dryness and kassing through a $2 \mathrm{~mm} \times 5 \mathrm{~cm}$ anjon column filled with Dowex $1 \times 8$ anion resin in $2 M \mathrm{HCl}$. The eluant was taken to dryness and the activity was picked up in 1 drop of 0.1 M HNO. A terbium fraction, free from gadolinium was obtained by loading this on a $2 \mathrm{~mm} \times 4$ con exchange column filled with Aminex $Q$ 150-S cation resin which had been prepared with $I M$ $\mathrm{NH}_{4} \mathrm{C}$. The terbium fraction was isolated by eluting at room temperature with $0.5 M$-hydroxyisobutyric acid, which had been adjusted to a pH of 3.30 with $\mathrm{NH}_{4} \mathrm{OH}$. The lack of any lines in the gamma-cay spectra due to a ${ }^{153}$ Gd tracer indicate an upper limit on the Gd contamination in the $\mathrm{Tb}$ fraction to be 01 \% Other nuclides present in the ${ }^{151} \mathrm{~Tb}$ fraction were ${ }^{149,}{ }^{150,}{ }^{152} \mathrm{~Tb},{ }^{160} \mathrm{~Tb}$ tracer and smal] amounts of some first row transition metals with +3 oxidation states.

The decay of the ${ }^{131} \mathrm{~Tb}$ and the growth and decay of the ${ }^{151}$ Gd daughter were measured with a $60 \mathrm{~cm}^{3} \mathrm{Ge}(\mathrm{li})$ detector with a 4096 channel analyzer. After collecting spectra over a period of 180 days, the data were fed into the SAMPO code ${ }^{2}$ which locales the peaks and gives them a Gaussian fil wilh exponential tails. SAMPO also assigns a time to tach spectrum and corrects for detector efficiency. The resultant decay curves for the ${ }^{151}$ Tb lines were given weighted least squares single component fits; the results are shown in Table 1, giving a half-life $17.609 \pm 0.014$ hours. This is an improvement on the previous best value of 17.6 \pm 0.1 hours. These fits, along with the known branching ratios for ${ }^{151} \mathrm{~Tb}$ gamula lines, gave the ${ }^{[5]} \mathrm{Tb}$ decay rate al the time of chemical separation from $G d$ to be $6.24 \pm$ $0.29 \times 10^{6} \mathrm{DPM}$.

Table 1. Half-lives of ${ }^{151} \mathrm{~Tb}$ and ${ }^{151} \mathrm{Gd}$.

\begin{tabular}{cccc}
\hline$E_{\gamma}(\mathrm{keV})$ & $\mathrm{t}(\mathrm{h})$ & $E_{\gamma}(\mathrm{keV})$ & $\mathrm{l}(\mathrm{d})$ \\
\hline 108 & $17.599 \pm 0.026$ & 154 & $129.39 \pm 1.99$ \\
180 & $17.589 \pm 0.030$ & 175 & $127.91 \pm 2.01$ \\
252 & $17.600 \pm 0.034$ & 243 & $130.56 \pm 2.45$ \\
287 & $17.644 \pm 0.034$ & & \\
444 & $17.639 \pm 0.042$ & & \\
\hline Combined: & $17.609 \pm 0.014$ & Combined: & $129.1 \pm 1.2$ \\
\hline \hline
\end{tabular}


The SAMPO results for the ${ }^{151} \mathrm{Gd}$ lines were given a weigbied least squares fil for a 17.609-hour growth and subsequent decay. The balf-life of the ${ }^{151} \mathrm{Gd}$, as deter+ mined from the three most intense lines at 154,175 , and $243 \mathrm{keV}$, is $129.3 \pm 1.2$ days (sce Table 1). These growth and decay fits for the ${ }^{151} \mathrm{Od}$ lines also gave activities at the time of chemical separation of the To fraction of the "paresnts" of the ${ }^{41} \mathrm{Gd}$ lines. The parent activily corresponds to the activity a ${ }^{151} \mathrm{~Tb}$ line would have if it had the same absolute intensity as the ${ }^{151} \mathrm{Gd}$ line in question. Since the absolute intensities of the $\mathrm{real}^{151} \mathrm{To}$ lines are known, the absolute intensities of the ${ }^{51}$ Gd lines could now be calculated. The results are presented in Table 2.

\section{References}

I. N.M. Anton'eva et al., Bull. Acad. Sti. USSR 22, 135 (1958)

2. J.J. Roulti, and S.G. Prussin, Nucl. Instr. Methods 72 , $125(1960)$

3. Y.Y. Chu, E.M. Franz, G. Friedlander, Phys. Rey, C 1, $1826(1970)$.

4. J.W. Ford. A.V. Ramayya, Nucl. Phys. A 146, 397 (1970).
Table 2 Relative and absolute intensities of the gamma-Jays from the decay of ${ }^{\mid{ }^{S 1}} \mathrm{Gd}$.

\begin{tabular}{|c|c|c|c|}
\hline $\mathrm{E}_{\gamma}$ & $\begin{array}{l}\text { Relative } \\
\text { intensities } \\
\text { (Ref. 4) }\end{array}$ & $\begin{array}{c}\text { Relative } \\
\text { intensities } \\
\text { (this work) }\end{array}$ & $\begin{array}{l}\text { Absolvie } \\
\text { intensities } \\
\text { (第) }\end{array}$ \\
\hline 21.5 & $46.2(19)$ & & $2.78(20)$ \\
\hline ] 16.5 & $1.29(6)$ & $1.45(4)$ & $0.087(6)$ \\
\hline 110.5 & $0.12(1)$ & & $0.0072(21)$ \\
\hline 153.6 & $100.0(30)$ & 100.0 & $6.01(35)$ \\
\hline 174.6 & $48.3(15)$ & 49.6 & $2.98(17)$ \\
\hline 196.5 & $0.4 \&(2)$ & & $0.0288(22)$ \\
\hline 222.2 & $0.009(3)$ & & $0.00054(18)$ \\
\hline 239.0 & $2.31(11)$ & & $0.139(11)$ \\
\hline 243.2 & 89.9્( & $9] .7(29)$ & $5.5 J(33)$ \\
\hline 260.4 & 0.84 & & $0.0565(38)$ \\
\hline 286.0 & $1.35(4)$ & $1.51(5)$ & $0.091(6)$ \\
\hline 307.4 & $16.5(5)$ & $16.7(4)$ & $1.00(6)$ \\
\hline 328.3 & $1.32(5)$ & $1.48(4)$ & $0.088(6)$ \\
\hline 332.1 & 0.17 & & (14) \\
\hline 353.5 & 2.04 & $2.29(7)$ & 0.1 \\
\hline 394.8 & $0.06(3)$ & & $0.0036(18)$ \\
\hline 416.3 & $0.012(3)$ & & $0.00072(18)$ \\
\hline
\end{tabular}

\title{
The Decay of the ${ }^{155} \mathrm{~Tb}$ Compound Nucleus, Formed in the Reaction of ${ }^{22} \mathrm{Ne}$ with ${ }^{133} \mathrm{Cs}$
}

\author{
K.J. Moody and J.J. Hogan*
}

The cross sections for several evaporation products from the decay of the rotational compound nucleus ${ }^{155} \mathrm{~Tb}$ have been determined as a function of projectile energy in the reaction of ${ }^{22} \mathrm{Ne}$ with ${ }^{133} \mathrm{Cs}$. The purpose of our study is to examine the dependence of the decay of the compound nucleus on the amount of angular momentum brought in by the heavy ion. This particular reaction offers two interesting sets of evaporation products: The ${ }^{133} \mathrm{Cs}\left({ }^{22} \mathrm{Ne}, \mathrm{xn}\right)$ 135-x Tb products (for $x=3,5,6,7$, and 8 ) consist of two well-characterized isomeric states, each with known garrma abundances and long enough half-lives to make each pair useful in radiochemical studies. The ${ }^{13{ }^{3}} \mathrm{Cs}\left({ }^{22} \mathrm{Ne}\right.$, axn $)$ $151-\pi$ Eu products (for $x=3,4,5$, and 6 ) have been studied with similar techniques in (compound nucleus, $x$ ) studies by Hogan ${ }^{1}$ and by Lee and Markowitz. ${ }^{2}$

The ${ }^{22} \mathrm{Ne}$ beams used in this study were produced at the 88-Inch Cyclotron. Intensities of as much as two electrict microamperes were delivered to our targets at six energies from 94 to $146 \mathrm{MeV}$. The targets consisted of $600 \mathrm{mg} / \mathrm{cm}^{2}$ layers of $\mathrm{CsCl}$ vaculurt-sublimaled onlo thick target backings. The low melting point of CoCl required that the target backings be directly cooled by water. Two irradiations were performed at each energy. In the first bombardments, targets mounted on gold were irradiated for five to ten minutes and then rapidly transported to the counting facilities in Building 70, where bey were placed whole in front of a $60 \mathrm{~cm}^{3} \mathrm{Ge}(\mathrm{Li})$ detector. The time from the end of the irradiation to the beginning of the first counting interyal varied between three to five minutes, enabling us to see the gamma rays from the decay of the high-spin $\mathrm{Tb}$ isomers with balf-lives as short as 100 seoonds $\left({ }^{147} \mathrm{~Tb}\right.$ ). The second bombardments were performed with largets on nickel backjogs. After irradiations from two to four bours in length, the targets were dissolved in $\mathrm{HNO}_{3}$, a rare-earth tracer was added, then $\mathrm{LaF}_{3}$ was precipitaled, which carried the activities of interest. This precipitale was filtered out on nitrocellulose paper, washed with $\mathrm{HCl} / \mathrm{HF}$ and water, then was mounted for counting for the gamma-rays arising from the decay of the low-spin To isomers and the Eu isotopes. Cross sections were obtained from decay curve analysis and have been corrected for feeding during the bombardments.

The experimental results are shown in Figs. 1 and 3 , along with a fit to the data generated by the computer code ALERT. ${ }^{3}$ The evaporation of charged particles is strongly affected by changes in the nuclear temperature, and the best fit to the ( ${ }^{2} \mathrm{Ne}$, ann) data shown was abtained wilh a level density parameter of $\mathrm{A} / 12$. Two things are immedi- 


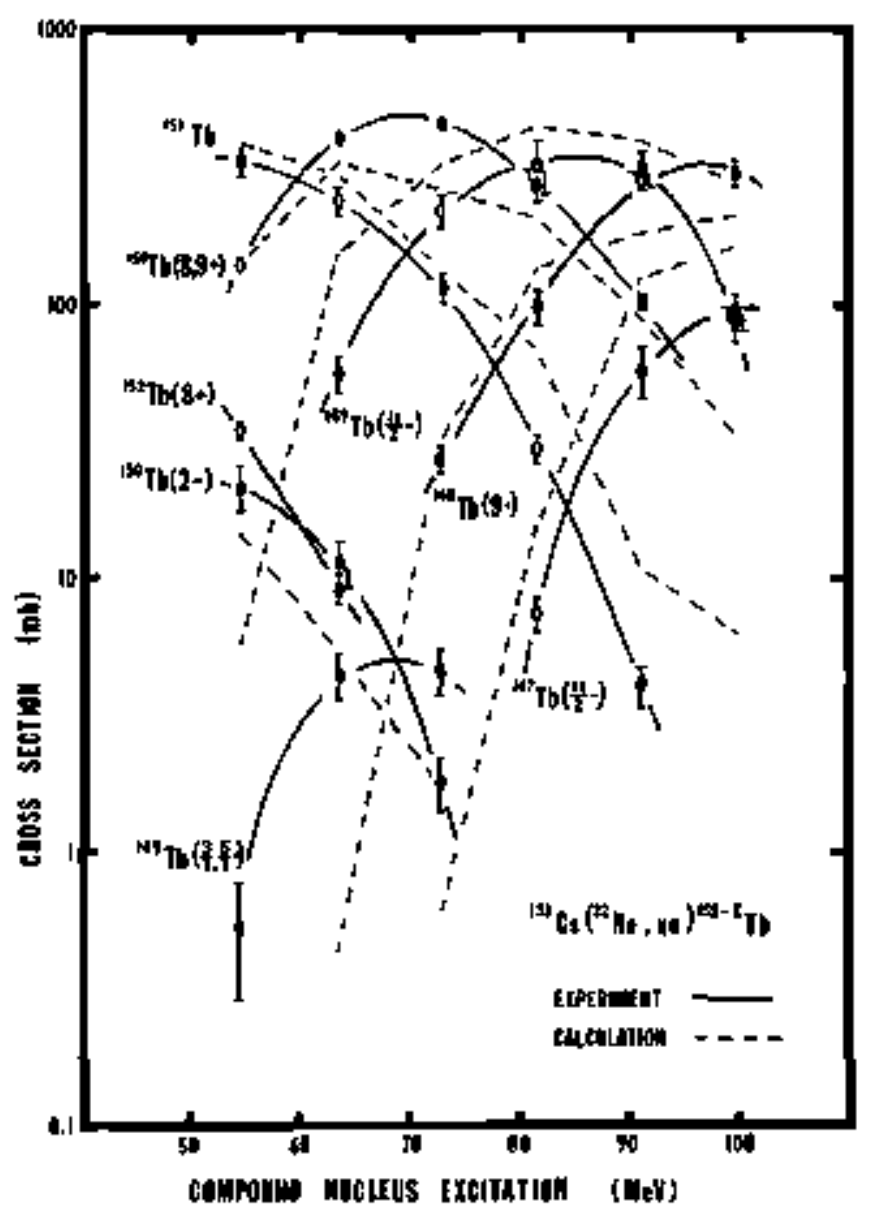

Fig. 1. Cross section distributions as a function of compound nucleus excitation energy for the ${ }^{33} \mathrm{Cs}\left({ }^{22} \mathrm{Ne}, \mathrm{xn}\right)$ 155-x $\mathrm{Tb}$ reaction ( $\mathrm{x}-3,4,5,6,7$, and 8 ).

(XBL 818-11355)

ately striking in the data: The low-spin isomers of the Tb isotopes are populated only very weakly and the cross sections of the $\left({ }^{22} \mathrm{Ne}, \alpha \times n\right)$ products show structure at the highest energies. The preferential population of the highspin states in the terbiums comes about because the rootmean-square values of angular motnentum in the compound nucleus vary from 28 to 56 to over the energy range siudied, and the resultant yrast cascade would largely end up in the high-spin isomers. The structure in the $\left({ }^{21} \mathrm{Ne}, \alpha \times n\right)$ product cross sections is probably due to the contribution of the evaporation of 2 protons and $x+2$ neutrons.

The authors would like to acknowledge B.Y. Jacak for ber help with the irradiations, Dr. H. Groening for his

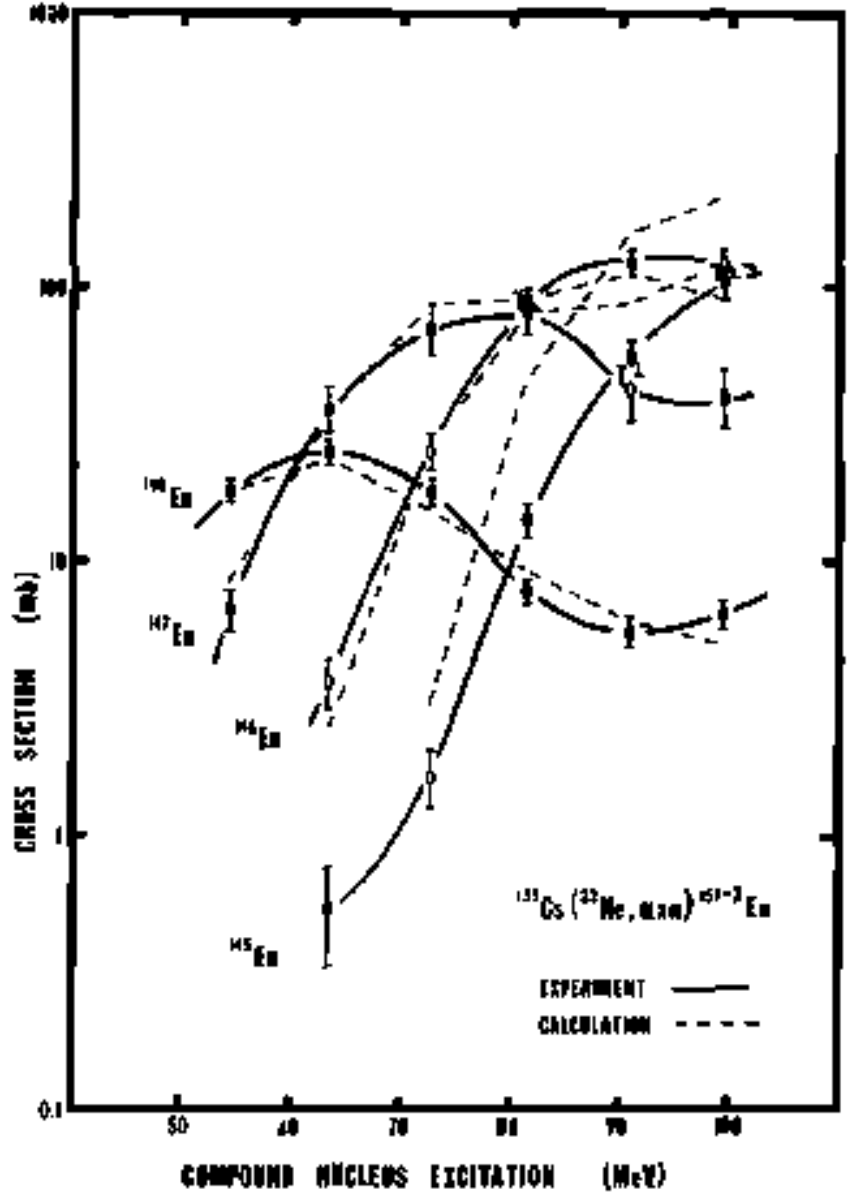

Fig. 2. Cross section distributions as a function of compound nucleus excitation energy for the ${ }^{133} \mathrm{Cs}\left({ }^{22} \mathrm{Ne}\right.$,oxn) ${ }^{151-x}$ Ev reaction $(x=3,4,5$, and 6$)$.

(XBL 818-11354)

assistance with the modeling. Dr. M. Blann for the use of his computer code and for many helpful discussions, and Dr. G.T. Seaborg for his interest and support.

\section{Foolnoles and References}

"Penmanent Address: Department of Chenristry, MeGill University, Montreal Quebec, Canada.

1. J.J. Hogan, LBL-97]1 (1980), p. 74.

2. D.M. Lot and S.S. Markowitz, LBL-2366 (1974), p. 34.

3. M. Blann, Phys, Rev. C 21, 1770 (1980). 


\title{
Alpha Decay of Neutron Deficient Polonium and Bismuth Isotopes
}

\author{
M. Leina, S. Yashita, and A. Ghlorso
}

We have been using SASSY, our gas-filled on-line mass separator, to study neutron-deficient alpha-emittíng isotopes in the $\mathrm{Pb}$-Ac region made in fusion reactions by heavy-ion bombardment A number of known nuclides have been identified and a new isotope, ${ }^{192} \mathrm{Po}_{0}$ and an isomeric state in ${ }^{191}$ Bi have been found.

In SASSY, the separation of fusion products from the bombarding heavy-ion beam is achieved by a 1-torr belium-filled magnetic system consisting of a dipole followed by a quadrupale. Downstream from the quadrupole are two pentane-filled parallel-plate position-sensitive aralanche counters that prowide information on the trajec tories and energy losses of recoil nuclei as well as a determination of their time-of-fight between the two counters. The recoils are implanted into an array of ten $13 \times 22$ mon $\$$ surface-barrier detectors to give an indieation of their kinetic energy and an aceurate measure of any subsequent alpha decay. An approximate mass can be determined from the recoil velocity and energy measurements but the calculation is severely limiled in accuracy by the energy determination. Nuclide identificalion is thus determined by alpha-alpha genetic relationships where possible, by cross bombardments, and by excitation functions. Short half-lives are determined by measuring the time between arrival of an implanted recoil and subsequent alphe particle delay.
We have produced polonium isotopes in the reactions ${ }^{n a t} \mathrm{Ce}\left({ }^{56} \mathrm{Fe}, \mathrm{xn}\right) \mathrm{Po}$ and ${ }^{14}{ }^{\mathrm{Pr}} \mathrm{Pr}\left({ }^{56} \mathrm{Fe}, \mathrm{pxn}\right){ }^{166-\pi} \mathrm{Po}$, and bismuth isotopes in the reactions nat $\left.\mathrm{Ce}^{56} \mathrm{Ft}, \mathrm{pxn}\right) \mathrm{Bi}$ and $\left.{ }^{141} \operatorname{Pr}^{56} \mathrm{Fe}, \alpha \times f\right)^{193-x} \mathrm{Bj}$. The excitation Nunctions for ${ }^{192-196} \mathrm{Po}$ and $191-193 \mathrm{Bi}$, including their isomers, and the half-life of ${ }^{1 / 2}{ }_{\mathrm{Po}}$ were determined using the ${ }^{{ }^{6}} \mathrm{Fe}+{ }^{\text {mal }} \mathrm{Ce}$ reaction. The results of the alpha particle measurements are shown in Table 1.

In Fig. 1 show an alpha spectrum measured in one of the detectors in the bombardment of nat $\mathrm{Ce}$ with 254 $\mathrm{MeV}{ }^{{ }^{6} 6} \mathrm{Fe}$ particles. Excitation functions shown in Fig. 2 were used to make nuclide assignments. The new alpha particle activity at $7.17 \mathrm{MeV}$ was found to have a half-life of $34 \pm 3 \mathrm{~ms}$, in good agrecment with the value of $30 \mathrm{~ms}$ calculated from the formula of Taagepera and Nurmia ${ }^{1}$ Our value for the ${ }^{192} \mathrm{Po}$ alpha energy, $7.17 \pm 0.02 \mathrm{MeV}$, is compatible with the systematios of Liran and Zeldes.

The bombarding energits used in these experiments were not high enough to allow the determination of the excitation Nunctions for the $6.32 \cdot \mathrm{MeV}, 6.63-\mathrm{MeV}$, and $6.86-\mathrm{MeV}$ activities that have been assigned to ${ }^{191} \mathrm{Bi}$ in previous investigations. ${ }^{2}$ However, the threshold energies to produce al] three activities were found to be similar and the relative yields were within a faclor of two from those reported in Ref. 1, so we conclude that these activities probably all belong to Bi with mass number 191. On the

Table 1. Resuls of alpha decay measurements

\begin{tabular}{|c|c|c|c|c|}
\hline \multirow[b]{2}{*}{ Isotope } & \multicolumn{2}{|c|}{ Present Work } & \multicolumn{2}{|c|}{ Ref. 2, Ref. 3} \\
\hline & $\begin{array}{c}\mathrm{E}_{t} \\
(\mathrm{MeV})\end{array}$ & $\begin{array}{l}\mathrm{T}_{\mathrm{lf} \mathbf{2}} \\
(\mathrm{ms})\end{array}$ & $\begin{array}{c}E_{\alpha} \\
(\mathrm{MeV})\end{array}$ & $\begin{array}{l}T_{1 / 2} \\
\text { (ms) }\end{array}$ \\
\hline${ }^{194} \mathrm{Po}_{\mathrm{O}}$ & $6.84^{\mathrm{a}}$ & $410 \pm 30$ & $6.84^{b}$ & $700 \pm 100^{b}$ \\
\hline${ }^{193} \mathrm{Po}$ & $6.94 \pm 0.02$ & $360 \pm 50$ & $6.94^{b}$ & $450 \pm 150^{b}$ \\
\hline${ }^{193 m \mathrm{mo}}$ & $7.00 \pm 0.02$ & $260 \pm 20$ & $6.995^{b}$ & $420 \pm 100^{b}$ \\
\hline${ }^{192} \mathrm{Po}$ & $7.17 \pm 0.02$ & $34 \pm 3$ & $7.12^{b}$ & $\ldots$ \\
\hline $19 \mid \mathrm{m} \mathrm{Bi}$ & $6.86 \pm 0.02$ & $150 \pm 15$ & $6.86 \pm 0.02^{\mathrm{c}}$ & $20000 \pm 15000^{\circ}$ \\
\hline
\end{tabular}

"Value used for intermal alibration.

Ref. 3.

'Rer. 2. 


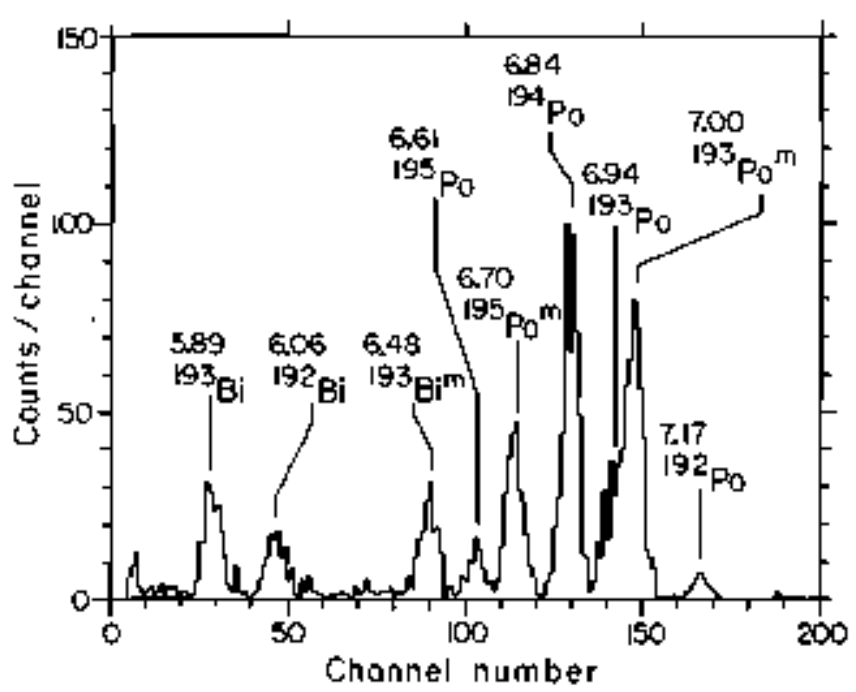

Fig. 1. Alpha particle energy spectrum of the nuclides produced in the reaction ${ }^{56} \mathrm{Fe}+{ }^{a a t} \mathrm{Ce}\left(\mathrm{E}_{\mathrm{tah}}=\right.$ $254 \mathrm{MeV}$ ).

(XBL $812-186$ Y)

other hand, our half-life of $150 \pm 15$ ms for the 6.86MeV activity differs greatly from the value $20 \pm 15 \mathrm{~s}$ reported in Ref. 2. Our upper limit for the intensity of the component in the 6.86-MeV peak with $\mathrm{T}_{1 / 2}>5 \mathrm{~s}$ is approximately $50 \%$ of the intensity of the $6.63 \mathrm{MVV}$ peak. In Ref. 2, the relative intensities of the $6.63+\mathrm{MeV}$ and $6.86 \cdot \mathrm{MeV}$ peals were reported to be $30 \%$ and $70 \%$ respec tively. In this work, the relative intensities were $25 \%$ and $75 \%$. We were unable to determine the half-life of the 6.63- MeV activity accurately in this experiment, but it is on the order of seconds.

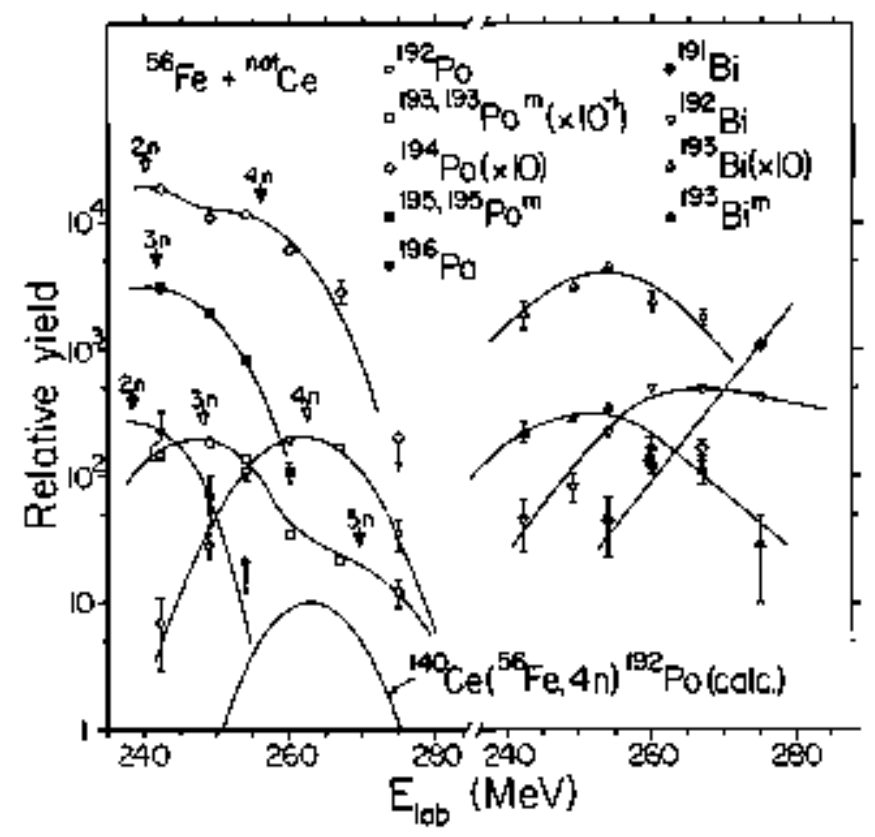

Fig. 2. Relative yields of $\mathbf{P o}$ and $\mathbf{B i}$ isotopes produced in the reaction ${ }^{56} \mathrm{Fe}+{ }^{\text {mit }} \mathrm{Ce}$. The open and solid arrows show the calculated locations of the $x$ n curves for ${ }^{56} \mathrm{Fe}+$ ${ }^{140} \mathrm{Ce}(89 \%)$ and ${ }^{5} \mathrm{Fe}+{ }^{142} \mathrm{Ce}(11 \%)$ respectively. The calculated excitation function is not to scale.

(XBL 812-18BY)

\section{References}

1. R Tangepera and M. Nurmia, Ann. Acad Sci. Finn. A V1 No. 78 (196]).

2. Y. LeBeyec, M. Lefort, J. Livet, N.T. Porile, and A. Siivola, Phys. Rev, C 9, 1091 (1974).

3. S. Della Negra, B. Lagarde, and Y. LeBeyec, J. Phys. 38, L-393 (1977).

\title{
Search For Electron Capture-Delayed Fission in the $\mathrm{N}=126$ System ${ }_{91}^{216} \mathrm{~Pa} \underset{\mathrm{EC}}{\longrightarrow}{ }_{90}^{216} \mathrm{Th}$
}

\author{
LP. Somerville and M.J. Nurmia
}

Several neutron-deficient isotopes have been discovered with half-lives in the minute range and which give rise to spontaneous fission (SF). The hypothesis of electron capture-delayed fission has been suggested as the means of decay for these nuclides. According to this hypothesis, a nucleus, e.g., ${ }^{2}$ fsAm (2.6 min), decays by electron capture to a series of levels in the daughter nucleus ${ }^{2}{ }_{4}^{4} \mathrm{Pu}$, which are near the top of the fession barrier. Fission can then take place readily from these levels.

The system ${ }^{216} \mathrm{~Pa} \stackrel{\mathrm{EC}}{\rightarrow}{ }^{2} \mathrm{G} 6 \mathrm{Th}$ appeared to be an attractive candidate for the lightest koown spontaneously fissioning nuclems. Due to the 126 neutron shell in ${ }^{216} \mathrm{Th}$, the
Q value for electron capture was expected to be quite high, approximately $7.6 \mathrm{MeV},{ }^{1} \mathrm{~A}$ high Q vaine would allow levels near the top of the fission barrier to be readily populated in some fraction of the electron capture decays, as long as the bejght of the fission barrier for ${ }_{96}^{216} \mathrm{~Tb}$ is less that $7.6 \mathrm{MeV}$. The half-life for ${ }_{91} \mathrm{Pl}_{\mathrm{Pa}}$ was known to be $170+100 \mathrm{~ms}$ based on alpha particle decay measurements by Schmidi et al. ${ }^{2}$ The eloctron capture branch for ${ }^{216} \mathrm{~Pa}$, however, was unknown.

We searched for SF events from ${ }^{22} \mathrm{~g}_{1} \mathrm{~Pa}$ in the following reactions (Table 1). Cross-section upper limits for $\mathrm{SF}$ decay and upper limits for $\mathrm{SF}$ relative to the calculated 
Table 1.

\begin{tabular}{|c|c|c|}
\hline Reaction & $\begin{array}{l}\text { Upper limit } \\
\text { for SF (nb) }\end{array}$ & $\sigma^{S P} / \sigma^{\omega / \varepsilon}$ (JORPLE) \\
\hline $129-137-\mathrm{MeV} 2{ }_{12}^{2} \mathrm{Mg}+{ }_{7197} \mathrm{Av}$ & $\leq 0.4$ & $\leq 10^{-6}$ \\
\hline $140-146-\mathrm{MeV}$ & $\leq 0.6$ & $\leq 8 \times 10^{-5}$ \\
\hline \multirow[t]{3}{*}{$\leq 185-\mathrm{MeV}{ }_{17}^{37} \mathrm{Cl}+$} & 513 & $55 \times 10^{-4}$ \\
\hline & 57 & 50.03 \\
\hline & $\leq 6$ & $\leq 3 \times 10^{-5}$ \\
\hline
\end{tabular}

production cross section using the JORPLE code are quoled. The cross sections calculated by the JORPLE code are likely to be overestimated, however.

The fission batrier for ${ }_{90}^{216} \mathrm{Th}$ is calculated by W.D. Myers to be $-10.5 \mathrm{MeV}$ using the droplet model. ${ }^{1}$ Since his calculation does not include shell cocroctions to the saddle mass, the true fission barrier might be even higher and is likely to exceed the Q value for electron capture of ${ }^{216} \mathrm{~Pa}(7.6 \mathrm{MeV})$. Thus, even if ${ }^{216} \mathrm{~Pa}$ has a sizeable electron capture branch to excited levels in ${ }_{90}^{2 ! 5} \mathrm{Th}$. these levels are oot likely to decay by fission since they would be at least $\sim 2.9 \mathrm{MeV}$ below the estimated fission barrier and, consequently, have a fission probability of $\sim 10^{-8}$. Although sub-barrier fission following electron capture has been suggested by Yu. Ts. Oganessian ${ }^{3}$ for ${ }^{230} \mathrm{Am} \rightarrow{ }_{934}^{230} \mathrm{Pu} \rightarrow$, the high estimated fission barrier is a possible explanation for the lack of SF events from ${ }_{9}^{2} \mathrm{P}$ P in our experiments.

\section{References}

J. W.D. Myers, Droplet Model of Alomic Nuclei, Plenum Publishing Co. (1977).

2 K.H. Schmidt et al., Nucl. Plys, A 318, 253 (1979).

3. Yu. Ts. Ogantessian, Symposium on Classical and Quantum Mochanical Aspects of Heayy Ion Collisions, Heidelberg, p.122, Aug. 1974. Proceedings published by Springer Verlag (1975).

\title{
Attempts to Identify the 1.5-Second Spontaneous Fission Activity Observed in the Reaction ${ }_{8}^{18} \mathrm{O}+{ }_{96}^{248} \mathrm{Cm}$
}

\author{
A. Ghiorso, L.P. Somerville, R.M. McFarland, R. Lougheed, and E.K. Hulet
}

In two previous annual reports, ${ }^{1,2}$ a 1.5 -second sponlancous fission (SF) activity was reporled as a product of the reaction $95 \mathrm{MeV} 18 \mathrm{~g}+{ }^{24} \mathrm{~g} / \mathrm{Cm}$ and found to have a half-life, totil kinetic energy of fission, and fission mass distribution very close to some properties reported for ${ }_{000}^{39} \mathrm{Fm}$ in the reaction $16 \mathrm{MeV} \mathrm{t}+{ }^{360} \mathrm{Fm}^{3}$ The eross section for production of the 1,5-s SF activity was measured to the $16 \mathrm{gb}$ in the reaction $95-\mathrm{MeV} 18 \mathrm{O}+248 \mathrm{Cm}$. The excitation function was found to be quite broad, as expected for a noncompound nucleus product.

If our 1.5-s SF activity is indeed ${ }_{100}^{299} \mathrm{Fm}$, it would be produced in an exotic "lpe transfer reaction. Previous attempts to observe a ${ }_{d}^{1} \mathrm{Be}$ transfer under more faworable conditions using ${ }^{18} \mathrm{O}$ ions borbarding targets with lower atomic number have yielded surprisingly low cross sections of $\sim 10 \mathrm{nb}$ in the reaction $110 \mathrm{MeV} 18 \mathrm{O}+20 \mathrm{sb}$ (Rer. 4) and $\leq 16 \pm 10 \mathrm{nb}$ in the reaction $124 \mathrm{MeV}$ $1 \mathrm{~g} \mathrm{O}+{ }^{23 \mathrm{~S}_{0}} \mathrm{Th}{ }^{5}$ We considered the possibility that the liBe transfer products might be produced with excitation entergies below the hssion barrier, which might explain why the cross sections using targets of lower atomic number than ${ }^{24}{ }_{90} \mathrm{Cm}$ are not higher.

Recently we bombarded a ${ }^{245} \mathrm{Cm}$ target ${ }^{6}$ with ${ }^{18} \mathrm{O}$ ions at $93 \mathrm{MeV}$ and $99 \mathrm{MeV}$ in search of the ${ }_{4}^{1} \mathrm{Be}$ transfer 
product ${ }_{106}^{25} \mathrm{Fm}$. The energy of $93 \mathrm{MeV}$ was chosen 10 give the same excitation energy as in the system $95 \mathrm{MeV}$ ${ }^{18} \mathrm{O}+{ }_{9}^{24} \mathrm{Cm}$, in whjch a cross section of $16 \mathrm{mb}$ was measured for 1.5-s SF activity. Ten solid state detectors reoorded alpha particles and SF events from a series of ten $100 \mu \mathrm{g} / \mathrm{cm}^{2}$ aluminum catcher foils placed over the detectors after the end of the bombardment. Only upper limits of $2 \mathrm{nb}$ at $93 \mathrm{MeV}$ and $2 \mathrm{nb}$ at $99 \mathrm{MeV}$ could be blished for the llbe transfer product ${ }_{4}^{256} \mathrm{Fm}$ in the reaction $18 \mathrm{~g}+{ }_{96}^{145} \mathrm{Cm}$. Table ] shows, however, that copious quantíties of other alpha particle-emitting nuctides were produced with cross sections comparable to the analogous products in the reaction ${ }_{8}^{18} \mathrm{O}+{ }_{96}^{24} \mathrm{Cm}$.

One possible conclusion is that the 1.5-s SF activity produed in the reaction $95 \mathrm{MeV} 18 \mathrm{O}+{ }_{94}^{48 \mathrm{Cm}}$ is probably not ${ }^{250} \mathrm{Fm}$ Also, either the I.5-s SF activity is another twin nuclews to $259 \mathrm{Fm}$ with respect to half-life, total kinetic energy of fission, and fission mass distribution, or it is the same nucleus produced in the original reaction $1+{ }_{60}^{57} \mathrm{Fm}$, but different from ${ }_{100}^{259} \mathrm{Fm}$

\section{Referentes}

1. Nuclear Science Division Annual Report, 1978-79, LBL-9711 (1980), p.63.

2. Nuclear Science Dirison Annual Repor, 1979.80, L.BL- 11588 (1981), p. 85.

3. E.K Hulet el al., Phys, Rov. C 21, 966 (1980).

4. A Ghiorso el al. 1979-1980 Nuclear Science Anmual Report, LBL-11588, p.85.

5. R.M. MeFartand et al., Second Chemical Congress of the North American Continent, Aug. 24-29, 1980, Las Vepas, Nevada

6. The ${ }_{96}^{245} \mathrm{Cm}$ Material was oblained by milking Jrom ${ }^{209} \mathrm{Cf}$ that had been grown from the utrapure ${ }^{249} \mathrm{Bk}$.

7. D. Lee et al., private communication (1980).

Table i.

\begin{tabular}{|c|c|c|c|c|}
\hline \multirow[t]{2}{*}{ Pickup Particle } & \multicolumn{2}{|c|}{$93 \mathrm{MeV} 18 \mathrm{O}+{ }_{965}^{245} \mathrm{Con}$} & \multicolumn{2}{|c|}{$97 \mathrm{MeV}{ }_{8}^{18} \mathrm{O}+{ }_{9}^{248} \mathrm{Cm}^{4}$} \\
\hline & nuclìde & $\sigma(\mu \mathrm{b})$ & $\sigma(\mu \mathrm{b})$ & nuclide \\
\hline$+2 p-2 n$ & ${ }_{96}^{2} \mathrm{Cf}$ & -. & 45 & ${ }^{2488} \mathrm{Cf}$ \\
\hline$+2 p-n$ & ${ }_{96}^{26} \mathrm{Cf}$ & 50 & 280 & ${ }^{248} \mathrm{Cr}$ \\
\hline $2 p$ & $348 \mathrm{Cr}$ & - & 850 & ${ }^{256} \mathrm{Cf}$ \\
\hline${ }^{3} \mathrm{He}$ & ${ }^{24} 8 \mathrm{Cr}$ & 3000 & 980 & 닝의 \\
\hline$\alpha$ & ${ }^{249} \mathrm{Cr}$ & $\ddot{n}$ & $\begin{array}{l}280 \\
\sigma(n b)\end{array}$ & ${ }_{92}^{2 x} \mathrm{Cf}$ \\
\hline$q_{B e}$ & ${ }_{000}^{251} \mathrm{Fm}$ & - & 2800 & ${ }^{234} \mathrm{Fm}$ \\
\hline $7 \mathrm{Be}$ & ${ }_{1002}^{232} \mathrm{Fm}$ & 1800 & 700 & ${ }_{100}^{25} \mathrm{Fm}$ \\
\hline${ }_{4}^{8} \mathrm{Be}$ & ${ }_{100}^{253} \mathrm{Fm}$ & 800 & 300 & ${ }^{356} \mathbf{6 0 m}$ \\
\hline${ }_{4}^{1} \mathrm{Be}$ & 3665 & $\leq 2$ & $\begin{array}{c}16 ? \\
(95 \mathrm{MeV})\end{array}$ & 㫊 $\mathrm{Fm}$ \\
\hline
\end{tabular}




\title{
Spontaneous Fission of Rutherfordium Isotopes
}

\author{
L.P. Somerville, J.M. Nitschke, M.J. Nurmia, and A. Ghiorso
}

In previous issues of this annual report ${ }^{1,2}$ Spontaneovs Fission (SF) activities with half-lives of $21 \mathrm{mb}, 53 \mathrm{~ms}$, $1.4 \mathrm{~s} ;$ and $4,8 \mathrm{~s}$ were reported in the reactions $16 \mathrm{O}+$

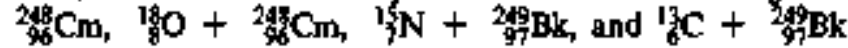
using the recoil tape transport system Our goal js to identify these new SF activities and, thus, gain an understanding of the SF half-life systematics for the heaviest known elements.

A passible assignrment for the 21-ms SF activity is $260 \mathrm{Rf}$. Half-lives determined by the maximum likelihood method and production cross sections have now been measured in the following reactions. (The errors indicated are statistical only; no estimate of possible systematic errors has been made.)

Absence of the 21-ms SF activity in the reactions $16 \mathrm{O}+{ }_{944 \mathrm{Pu}}{ }^{18} \mathrm{O} \mathrm{O}+{ }^{244} \mathrm{Pu}$, and ${ }^{15} \mathrm{~N}+{ }_{96}^{248} \mathrm{Cm}$ suggests that it is probably an isolope of rutherfordium with A $>259$. A 9-nb production cross section for a possible $19.3-\mathrm{ms}{ }_{104}^{260} \mathrm{Rr}$ in the reaction ${ }^{249} \mathrm{Cf}\left({ }_{8}^{18} \mathrm{O}, \alpha 3 \mathrm{n}\right){ }_{104}^{260} \mathrm{Rf}$ is consistent with kown cross sections of $\sim 20 \mathrm{nb}$ for the reaction ${ }^{248} \mathrm{Cm}\left({ }_{8}^{8} \mathrm{O}, \alpha 3 \mathrm{n}\right){ }^{2} \mathrm{r}_{02} \mathrm{No},{ }^{3}$ considering the effect of fission competition, and $\sim 0.5 \mathrm{nb}$ for the reaction

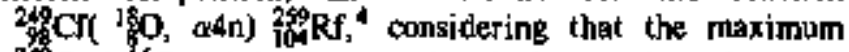
${ }_{96}^{24} \mathrm{Cm}(16 \mathrm{O}, a 3 \mathrm{n})$ cross section is larger than the maximum of the ${ }_{90}^{248} \mathrm{Cm}\left({ }_{6}^{16} \mathrm{O}, 04 \mathrm{n}\right)$ cross section. ${ }^{5}$ In the reastion $100-\mathrm{MeV} \mathrm{g}_{8} \mathrm{O}+{ }^{248} \mathrm{Cm}$ a half-life slightly shorter than the others listed in Table $I$ and a cross section of $\sim 9 \mathrm{nb}, \sim 50$ times the calculaled cross section to produce ${ }_{100} \mathrm{R}$, present the only possible inconsistencies for the assignitient of ${ }_{1040}^{260} \mathrm{Rf}$. The large cross section, shorter half-life, and broad excitation function for the SF activity produced in the reaction $109-\mathrm{MeV}{ }_{\mathrm{g}}^{\mathrm{B}} \mathrm{O}+{ }^{24} \mathrm{C} \mathrm{Cm}$ suggest that this is a different SF activity from the one produced in the other reactions listed in Table I. However, we cannot. experimentally rule out the remote passibility that the $\left({ }^{8} 0,6 n\right)$ reaction cross section is enhanced by some as yet unknown mechanism and that the half-life is truly 202] ins.

The 53-ms SF activity produced in the reactions 89 - to

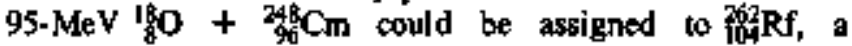
deduction based on a narrow excitation function similar to the calculated one and data from other cross bombard. ments. In a preliminary study of the reaction 112.7 * $\mathrm{MeV}{ }_{10}^{2} \mathrm{Ne}+{ }_{94}^{4} \mathrm{Pu} 77 \mathrm{SF}$ events were observed with a half-life of $50 \pm 16 \mathrm{~ms}$ and a production cross soction of $=1 \mathrm{nb}$. This is elose to the $1.5 \mathrm{nb}$ calculated to produce Jit $_{0}^{2} \mathrm{Rf}$, which would be consistent with our previous assignment for this activity.

Assignments of $2602 \mathrm{Rf}$ to the $20-$ to $21-m s$ and ${ }_{104}^{26} \mathrm{Rf}$ to the 52-ms SF activities would confirm the theoretical predictions of Randrup et al. ${ }^{6}$ which suggesi a change in SF half-life systematics at $Z=104$, as shown in Fig. J, due to the disappearance of the second hump in a double. bumped fission barrier.

In the reaction $93-\mathrm{MeV} 18 \mathrm{O} O+245 \mathrm{Cm}$, a search for

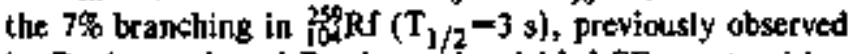
by Druin et al. and Bemis et al., yielded $\mathrm{SF}$ events with a half-life of $3.4 \pm 1.7 \mathrm{~s}$ corresponding to $8 \pm 3 \%$ of the

Table 1 .

\begin{tabular}{|c|c|c|c|}
\hline \multirow[b]{2}{*}{ Reaction } & \multirow[b]{2}{*}{ Half-]ife } & \multicolumn{2}{|c|}{ Cross Sections } \\
\hline & & Experimental & Calculated Jor ${ }_{1004}^{260} \mathrm{Rf}$ \\
\hline $80 \mathrm{MeV}{ }_{15}^{1} \mathrm{~N}+{ }_{9 j}^{24 \mathrm{Bk}} \rightarrow{ }_{[04}^{26 \mathrm{Rf}^{*}}$ & $19.8 \pm 1.2 \mathrm{~ms}$ & $14 \mathrm{nb}$ & $10 \mathrm{nb}$ \\
\hline $92-\mathrm{MeV}{ }_{6}^{160}+{ }_{96}^{248} \mathrm{Cm} \rightarrow{ }_{104}^{26 \mathrm{R}^{*}}$ & $21.0 \pm 1.1 \mathrm{~ms}$ & $6 \mathrm{nb}$ & $4.4 \mathrm{nb}$ \\
\hline $109-\mathrm{MeV}{ }_{8}^{18} \mathrm{O}+{ }_{96}^{248 \mathrm{Cm}} \rightarrow{ }_{106}^{26} \mathrm{R}^{*}$ & $17.1 \pm 2.2 \mathrm{~ms}$ & 9 nb & $0.2 \mathrm{ab}$ \\
\hline 96-MeV ${ }_{858}^{18} \mathrm{O}+{ }_{96}^{24} \mathrm{Cf} \rightarrow{ }^{263} \mathrm{J06}$ & $19.3 \pm 1.4 \mathrm{~ms}$ & $9 \mathrm{nb}$ & .. \\
\hline
\end{tabular}




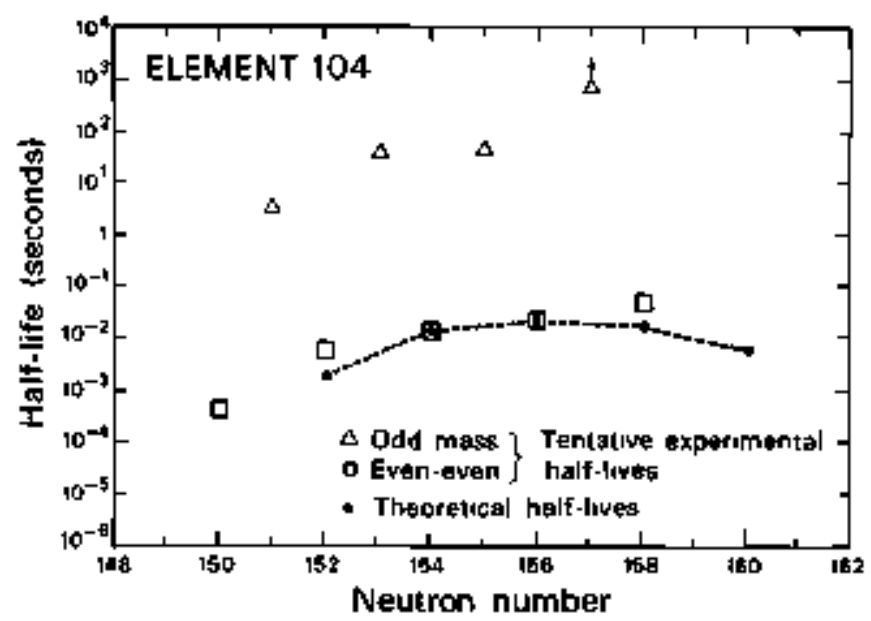

Fig. 1. Dotted lines connecting small solid circle points show the theoretically predicted partial half-lives of element 104 nuclei. ${ }^{6}$ The experimental values correspond to $\Delta$ for odd isotopes and $\square$ for even isotopes. None of these are known with ertainty.
(XBL 82]-60) 3.6-nb calculated total cross section for 23992 R. SF branching ratjos of possibly $7 \%$ in $25 \%$ RS (3 s) and $45 \pm 20 \%$ in ${ }_{105}^{25} R f(1.4 \mathrm{~s})$ suggest that 250 f $R f(4.8 \mathrm{~s}) \mathrm{might}$ also have a measurable SF branch and that the odd isotopes of element 104 may have lower hindrance factors than odd isotopes of lighter elements. This effect can also be understood in terms of a disappearance of the second barrier at element 104. ${ }^{6}$

\section{References}

I. Nuclear Science Division Annual Report, 1978-79, LBL-971। (1980), p.63.

2. Nuclear Science Division Annual Repart, 1979-80, LBL- 1588 (1981), p. 85 .

3. R. Silva et al., Nucl. Phys. A 216, 97 (1973).

4. A. Ghiorso et al., Phys. Rev. Lett. 33, 1490 (1974),

5. A. Ghiorso, Proceedings of the Robert A. Welch Foundation Conf. on Chemical Research XIV; Houston Texas; p. 107, W. Milligen, editor (1970).

6. J. Randrup el al., Phys. Rev. C 13, 299 (1976); Nuel. Phys, A 217, 221 (1973).

\title{
Production of Heavy Actinides from Interactions of ${ }^{20} \mathrm{Ne}$ and ${ }^{22} \mathrm{Ne}$ with ${ }^{248} \mathrm{Cm}$
}

\author{
D. Lee, H.V. Gunten," M. Nurmia, Y.-F. Liu, \\ G.T. Saabarg and D.C. Hofmant
}

Systematic studies of the transfer of many nucleons from heavy projectiles to heavy actinide targets are continuing. Measurements for the produclion of heavy actinides in bombardments of ${ }^{248} \mathrm{Cm}$ with ${ }^{20} \mathrm{Ne}$ and ${ }^{22} \mathrm{Ne}$ ions at energies near the Coulomb barrier bave now been completed (See Fig. 1). The experimental procedures and results for ${ }^{16} \mathrm{O}$ and ${ }^{18} \mathrm{O}$ were reported in the previous ennual report. ${ }^{1}$

Comparisons of the maximum yields for each element are given in Table 1. In general, the maximum yield for a given element occurs about two mass units heavier fot ${ }^{18} \mathrm{O}$ and ${ }^{22} \mathrm{Ne}$ than for ${ }^{16} \mathrm{O}$ and ${ }^{20} \mathrm{Ne}$ respectively, reflecting the neutron excess of the projectile.

The dependeace of the yields on the energy of the pro jectiles is now being investigated and ircadiations with ${ }^{18} \mathrm{O}$ energies on targets of 97, 103, III, and $120 \mathrm{MeV}$ have been performed. Preliminary analyses of the data show different exergy dependences for production of the various actinlde isotopes. These excitation functions appear to be

Fig. 1. lsotopic distributions measured for $115 \cdot \mathrm{MeV} 2 \mathrm{~N}$ te and 116-MeV ${ }^{22} \mathrm{Ne}$ bombardments of ${ }^{248} \mathrm{Cm}$. ${ }^{20} \mathrm{Ne}$ data are open symbols; ${ }^{22} \mathrm{Ne}$ data are solid symbols.

(XBL $814-644)$

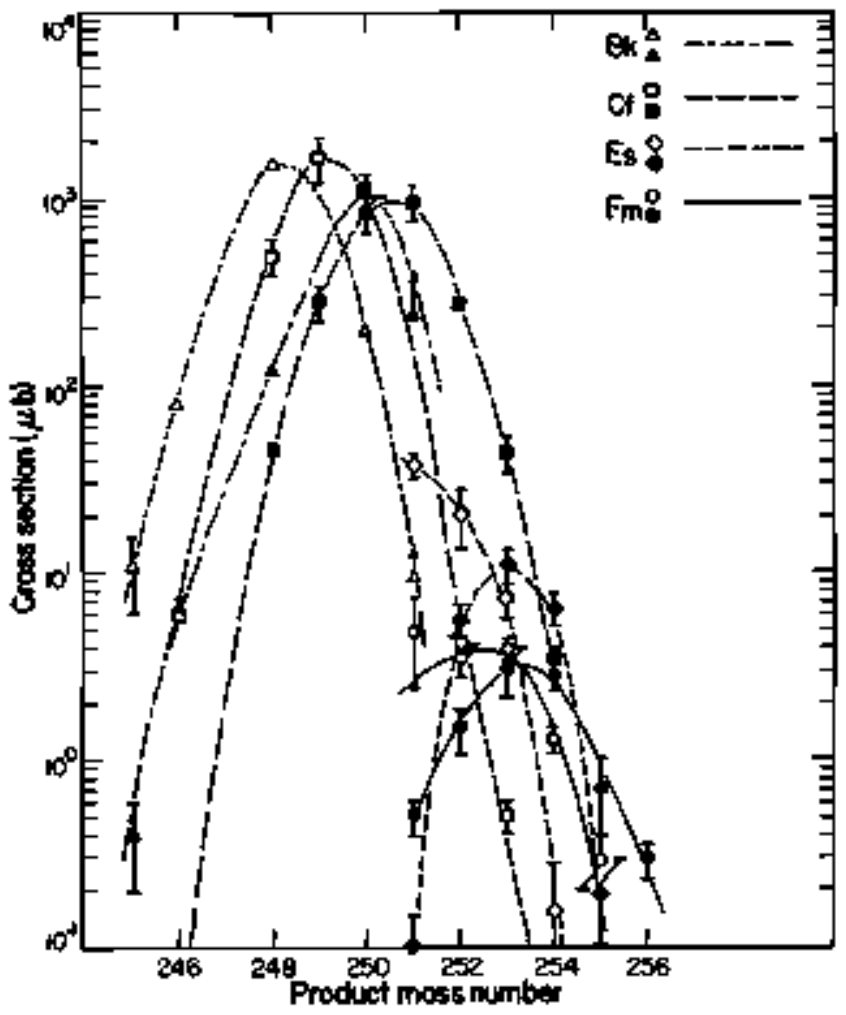


Table 1. Comparison of position and magnitude of maximum yields for $16,18 \mathrm{O}$ and $20,22 \mathrm{Ne}$ bombardments of ${ }^{248} \mathrm{Cm}$.

\begin{tabular}{|c|c|c|c|c|}
\hline & ${ }^{16} 0$ & 180 & ${ }^{20} \mathrm{Ne}$ & ${ }^{22} \mathrm{Ne}$ \\
\hline $\mathbf{E}_{\mathrm{c}, \mathrm{m}}(\mathrm{MeV})$ & 92 & 90 & 106 & 106 \\
\hline $\mathbf{E}_{\text {Coulomb }}(\mathrm{MeV})$ & 89 & 88 & 108 & 108 \\
\hline $\begin{array}{l}\mathbf{B k} \\
\boldsymbol{\mu b}\end{array}$ & $\begin{array}{l}248 \mathrm{~m} \\
1600\end{array}$ & $\begin{array}{r}250 \\
1100\end{array}$ & $\begin{array}{l}248 \mathrm{~m} \\
695\end{array}$ & $\begin{array}{r}-249 \\
-60\end{array}$ \\
\hline $\begin{array}{l}\mathrm{Cl} \\
\mu \mathrm{b}\end{array}$ & $\begin{array}{r}249 \\
1700\end{array}$ & $\sim=1000$ & $\begin{array}{r}249 \\
2670\end{array}$ & $\begin{array}{r}251 \\
1040\end{array}$ \\
\hline $\begin{array}{l}\text { Es } \\
\mu b\end{array}$ & $\begin{array}{r}251 \\
38\end{array}$ & $\begin{array}{r}253 \\
11\end{array}$ & $\begin{array}{l}251 \\
100\end{array}$ & $\begin{array}{r}253 \\
6\end{array}$ \\
\hline $\begin{array}{l}\mathrm{Fm} \\
\mu b\end{array}$ & $\begin{array}{r}-252 \\
-44\end{array}$ & $\begin{array}{c}253.5 \\
3\end{array}$ & $\begin{array}{r}252 \\
16\end{array}$ & $\begin{array}{r}254 \\
3\end{array}$ \\
\hline
\end{tabular}

consistent with calculations based on energy balance considerations for the appropriate transfer reactions, assuming the energy of the projectile in excess of the Coulomb barrier is apportioned to the target according to the fraction of the projectile mass transferred.

\section{Footnotes and Reference}

"University of Bern, CH-3000 Bern and Eidgenössisches Institut för Reaktorforschung, CH-5305 Wirenlinger, Swizerland.

'Peloing University, 100871 Beijing, The Peoples Republic of China.

${ }^{\ddagger}$ Los Alamos Scientific Laboratory, Los Alamos, NM 87545 .

I. D. Lee, et al., Nuclear Science Division Annual Report, LBL-9711 (1980).

\title{
Recoil Range Experiments in the Actinide Region
}

\author{
R.M. McForland, LP. Somerville, M.J. Nurmia, and A. Ghiorso
}

One of our mejor projects is the experimental study of the mechanism of production of nuclides of $\mathrm{Z}_{n} \mathrm{~A}$ between those of the target and the compound mucleus in reactions between light heavy ions $(\sim 10 \leq \mathrm{A} \leq 40)$ and actinide targets.

One branch of this study is a series of experiments to measure the recoil range distributions and cross sections of product actinides using a variety of projectiles and targets. The angular distribution of a nuclide produced via transfer reaches a maximum at the grazing angle. Its ringe parallel to the bearm axis is shorter than that for a compound nucleus evaporation residue. Therefore, recoil ranges ean be used to differentiate between these two mochanisms. They also give rough values for the peak and width of an angular distribution. ${ }^{1}$ The cross sections will be compared with those predicted from Wilczynsk's quasi-elastic transfer model, ${ }^{2}$ with correction for fission and particle evaporation by the primary heavy fragment.

All bombardments were done at the 88-Inch Cyclotron. Table I surmarizes experiments done to date. Targets are actinide fuorides or oxides deposited upor 2.5 $\mathrm{mg} / \mathrm{em}^{2}$ Be foil by vacuum deposition ${ }^{3}$ of electrospraying, respectively. Beam intensities are -1 to 2 electrical microamperes.

The beam traverses a $1.85 \mathrm{mg} / \mathrm{cm}^{2}$ Havar window, a $0.45 \mathrm{mg} / \mathrm{cm}^{2} \mathrm{~N}_{2}$ cooling gas, and the $\mathrm{Be}$ backing before striking the target. Recoiling nuclej are caught in a stack of ten $100 \mathrm{\mu g} / \mathrm{cm}^{2}$ Al foils. After the bornbardment each foil is placed over a 1 inch diameter $\$$ i $(A u)$ surface barnier
alpha-SF detector. The detoctors are inlerfaced with a oomputer. Pulse height analysis generates alpha spectra.

The decay of alpha and SF activity in each foil is followed for several months. Nuclides are identified through $\mathrm{E}_{\alpha}$ and $\mathrm{t}_{1 / 2}$. Table 2 shows decay properties of nuclites consistently produced in these bombardments.

The possibility of exotic, neutron-rich nucleon transfers from ${ }^{18} \mathrm{O}$ to an actinide nucleus has been debated since the production of an $\$ F$ activity from ${ }^{248} \mathrm{Cm}$ with decay properties very like those reported for ${ }^{259} \mathrm{Fm}^{4}$ The reponted cross section was 15 nb. ${ }^{5}$ Some rocoil range bombardments were designed to probe neutron-rich transfers using the $2.64 \mathrm{~h} \mathrm{SF}$ decay of ${ }^{256} \mathrm{Fm}$. In the actinide region, no such transfers have been observed with cross sections greater than a few nanobarns. The remainder of the data are now being analyzed.

\section{Rejerences}

I. R.J. Otto, G.T. Seaborg, and M.M. Fowler, Phys. Rey. C 17, 1071 (1978).

2. J. Wilezynski, et al., Ptys. Rev. Let1. 45, 606 (1980).

3. Fluoride largets provided by E.K. Hulet and R. Lougheed of Lawrence Livermore Laboratory.

4. D.C. Hoffman, et al., Nuclear Science Division Annual Report, LBL-9711, p. 66 (1980).

5. D.C. Hoffman, "Fission PToperties of Very Heavy Actinides " IAEA 4th Symp. Phys. and Chem. of Fission, Julich, Federal Republic of Germany, May, 1979; IAEA $\mathrm{Sm}-241 / \mathrm{B}] 4$. 
Table 1. Sumnary of bombardments,

\begin{tabular}{|c|c|c|c|c|c|}
\hline Expt & Projectilc & Target & $\begin{array}{c}\mathrm{E}_{1 \mathrm{ab}}(\mathrm{MeV}) \\
\text { on target }\end{array}$ & $\begin{array}{l}\text { Sought } \\
\text { exotic transier } \\
\text { product }\end{array}$ & $\begin{array}{l}\text { Nucleon } \\
\text { Iransfer }\end{array}$ \\
\hline $\mathrm{M}$ & ${ }^{18} \mathrm{O}$ & ${ }^{233} \mathrm{U}$ & 95.7 & & \\
\hline $\mathbf{N}$ & ${ }^{18} \mathrm{O}$ & ${ }^{233} \mathrm{U}$ & 98.7 & & \\
\hline 0 & 180 & ${ }^{244} \mathrm{Pu}$ & 98.7 & $\begin{array}{l}256 \text { Es (growth } \\
\text { of } 256 \mathrm{Fm} \text { ) }\end{array}$ & $5 p 7 \mathrm{a} " 12 \mathrm{~B}^{* *}$ \\
\hline $\mathbf{P}$ & 160 & ${ }^{233} \mathrm{U}$ & 96.4 & & \\
\hline $\mathrm{Q}$ & 160 & ${ }^{244} \mathrm{Pu}$ & 102.1 & & \\
\hline $\mathbf{R}$ & ${ }^{15} \mathrm{~N}$ & ${ }^{233} \mathrm{U}$ & 98.7 & & \\
\hline $\mathbf{S}$ & ${ }^{15} \mathrm{~N}$ & ${ }^{244} \mathrm{Pu}$ & 98.7 & & \\
\hline $\mathbf{T}$ & ${ }^{18} \mathrm{O}$ & ${ }^{245} \mathrm{Cm}$ & 93.0 & ${ }^{256} \mathrm{Fm}$ & 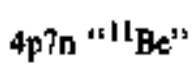 \\
\hline U & 180 & ${ }^{245} \mathrm{Cm}$ & 99.6 & ${ }^{256} \mathrm{Fm}$ & $4 p^{7 n}$ "11 Be" \\
\hline v & ${ }^{18} \mathrm{O}$ & ${ }^{245} \mathrm{Cm}$ & 99.6 & ${ }^{256} \mathrm{Fm}$ & " \\
\hline $\mathbf{w}$ & 180 & ${ }^{245} \mathrm{Cm}$ & 94.0 & ${ }^{256} \mathrm{Fm}$ & $4 \mathrm{p}_{\mathrm{n}} \cdot 11 \mathrm{Be}$ \\
\hline$x$ & 180 & ${ }^{244} \mathrm{Pu}$ & 95.7 & & \\
\hline $\mathbf{Z}$ & ${ }^{18} \mathrm{O}$ & ${ }^{249} \mathrm{Cf}$ & 111. & & \\
\hline$A A$ & $18_{0}$ & ${ }^{246} \mathrm{Cm}$ & 95.7 & ${ }^{256} \mathrm{Fm}$ & $4 p^{6 n} \cdot{ }^{-10} \mathrm{Be}$ \\
\hline$A C$ & 180 & ${ }^{246} \mathrm{Cm}$ & 87.2 & ${ }^{256} \mathrm{Fm}$ & 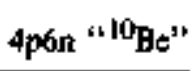 \\
\hline
\end{tabular}

Table 2. Consistently observed actinide alpha-emitters.

\begin{tabular}{lccc}
\hline Nuelide & Decosy Mode & $\mathrm{t}_{1 / 2}$ & $\mathrm{E}_{\alpha}$ \\
\hline${ }^{242} \mathrm{Cm}$ & $\alpha$ & $162.8 \mathrm{~d}$ & 6.113 \\
& & & 6.070 \\
${ }^{246} \mathrm{Cm}$ & $\alpha$ & $35.7 \mathrm{~h}$ & 6.758 \\
& & & 6.7190 \\
${ }^{246} \mathrm{Cf}$ & $\alpha$ & $333 \mathrm{~d}$ & 6.26 \\
${ }^{252} \mathrm{Fm}^{\mathrm{a}}$ & $\alpha$ & $25.4 \mathrm{~h}$ & 7.04 \\
${ }^{254} \mathrm{Fm}$ & $\alpha$ & $3.240 \mathrm{~h}$ & 7.187 \\
& & & 7.145 \\
${ }^{255} \mathrm{Fm}^{\mathrm{a}}$ & $\alpha$ & $20.1 \mathrm{~h}$ & 7.022 \\
& & & 6.963 \\
\hline
\end{tabular}

anot well resolved. 


\title{
A Procedure for a Fast Separation of Berkelium and Cerium*
}

\author{
Yyan-fang Liu, ${ }^{\dagger}$ Cheng Luo, ${ }^{\ddagger}$ Hans $R$, won Guten, and Glenn T. Seaborg
}

Berkelium and cerium ean be casily separated from the rest of the actinides and lanthanides using their oxidation/reduction properties. However, a separation of the two elements from each other is more difficult to achieve and generally gujte time consuming. In an attempt to measure decay properties of short-lived $B k$ jsotopes produced in heavy jon reactions, it was mandatory to develop a procedure for a fast separation of the two elements. The melbods described in the literature are either too time consuming ${ }^{1}$ or could not be reproduced in our laboratory. ${ }^{2,3}$

The procedure developed in our work combines the separation of $\mathrm{BK}$ and $\mathrm{C} C$ from other actinides, lanthanides, and fission products with a subsequent fractionation of the two elements using high pressure liquid chronatographic (HPLC) techniques.

Berkelium and oerium were oxidized with $0.1 \mathrm{M} \mathrm{CrO}_{3}$ in $4 \mathrm{M} \mathrm{HNO}_{3}$ to the $4+$ state, extracted with 0.15M 2diethylhexylorthophosphoric acid (HDEHP) in heptane. reduced to the $3+$ state with $\mathrm{H}_{2} \mathrm{O}_{2}$, back-extracted into $10 \mathrm{M} \mathrm{HNO}_{3}$ " and this solution was evaporated to drymess. The residue dissolved in $0.1 \mathrm{ml}$ of $0.5 \mathrm{M} \mathrm{HNO}_{3}$ and was injected into the cation exchange colum of an HPLC-system (ALTEX-BECKMAN, Model 110A). The precision glass column ( $J(0) \times 2 \mathrm{~mm}$ ) was filled with the resin in the Na-form (Benson Co., BC-X]2, 7.10 $\mathrm{mm}$ ) and was beated with a water jacket to a temperature of $82^{\circ} \mathrm{C}$. After the injection of $\mathrm{Bk} / \mathrm{Ce}$, the elution was started using $0.5 \mathrm{M}$ ammoniem alpha-hydroxyisobutyrate at $\mathrm{pH} 4.2$ for the berkelium fraction (1-110 drops; I drop $=15 \mu \mathrm{l})$, with a change of the eluant to $\mathrm{pH} 4.4$ for the cerium fraction (111-200 drops). A flow rate of $0.5 \mathrm{ml} / \mathrm{min}$ (resulting in a pressure of $\sim 300 \mathrm{psi}$ ) was employed. A typical elution curve is shown in Fig. 1. A very good and clean separation of the two elements is obtained, the elution peaks being quite sharp and having only little tailing The separation of Bk on the HPLC-solumn takes only about 4 minutes from the injection to the completion of the eletion.

Footnotes and References

"Condensed from LBL 12909.

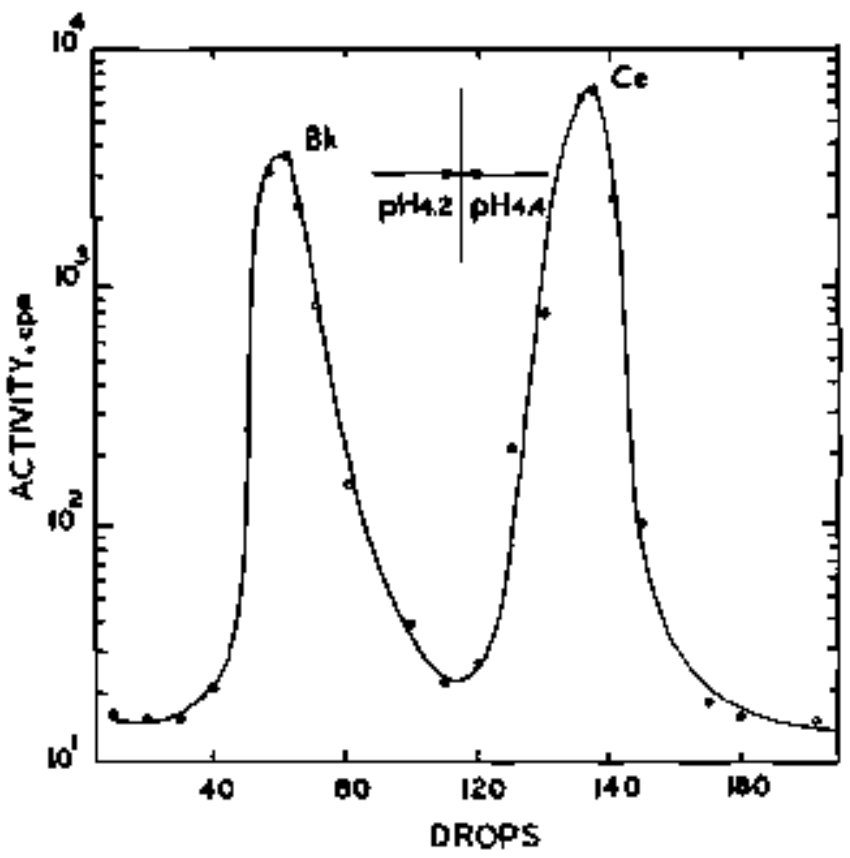

Fig. J. Elution curve of $\mathrm{Bk}$ and $\mathrm{Ce}$ using $0.5 \mathrm{M}$ ammonium alpha-hydroxyisobutyrate.

(XBL 814-9447)

${ }^{\dagger}$ On leave from Peking University, lo0s7] Beijing, The People's Repeblic of China.

Eon leave from the Institute of Atornic Energy, P.O. Box 275, Beijing, The People's Republic of China.

IOn leave from the Universily of Bern, $\mathrm{CH}-3000$ Bern and Eidgenössisches 1nstilute tür Reaktorforschung. CH-5305 Wirtenlingen, Switzerland.

1. D.C. Hofiman, et al., LA-1721, 4th Ed., 251 (1975).

2. F.L. Moore, Anal, Chem. 41, 1658 (1969).

3. V.N. Kosyakov, E.G. Chudinov, and I.K Sbvetsow, Proceedings of the Moscow Symposium on the Chemistry of the Transwarnium Elements, 99 (1976).

4. K.E. Williams and G.T. Seaborg. Phys. Rev, C 19. 1794 (1979). 


\title{
2. High Angular Momentum
}

\section{Study of High Energy Gamma Rays Emitted in the Deep-Inelastic Reactions ${ }^{181} \mathrm{Ta}+{ }^{136} \mathrm{Xe}(1150 \mathrm{MeV})$}

\author{
J.O. Newfon, J.E. Draper, K.H. Lindenberger, R.M. Diamond, C. Schück, \\ F.S. Stephens, E.L. Dimes, S. Shih, LG. Sobotka, G.J. Wozmiak, \\ R.J. McDonald, D.J. Morrissey. A.J. Pacheco, and L.G. Morefto
}

In a recent paper ${ }^{1}$ we reporled on the observation of gamma rays from giant dipole resonances (GDR) built on states of high entrgy and spin. Such measurements have the potential for providing new information on the properties of nuclei at high temperatures ( $T$ ) and large angular monenta (I). For exsmple, from observation of structure in the GDR rtgion, one may learn about the dependence of nuclear deformation on $T$ and $I$. In addition, one may be able to learn about the GDR itself by examining its energy ( $\left.E_{G}\right)$, strength, and widith as a function of $T$ and $I$.

Rocently, we have employed the deep inelastic reaction ${ }^{181} \mathrm{~T}_{\mathrm{a}}+{ }^{136} \mathrm{Xe}(1150 \mathrm{MeV})$ at the SuperHILAC to explore the Q-value dependence of gianl dipole transitions. The insert in Fig. I shows a schematic diagram of the experimental epparalus. By gating on different Q-value regions (see Fig. 1). the coincident gamma-ray spectra can be studied a function of $\mathbf{T}$. These spectra are shown in Fig. 2. A strong increase is observed in the yield of high

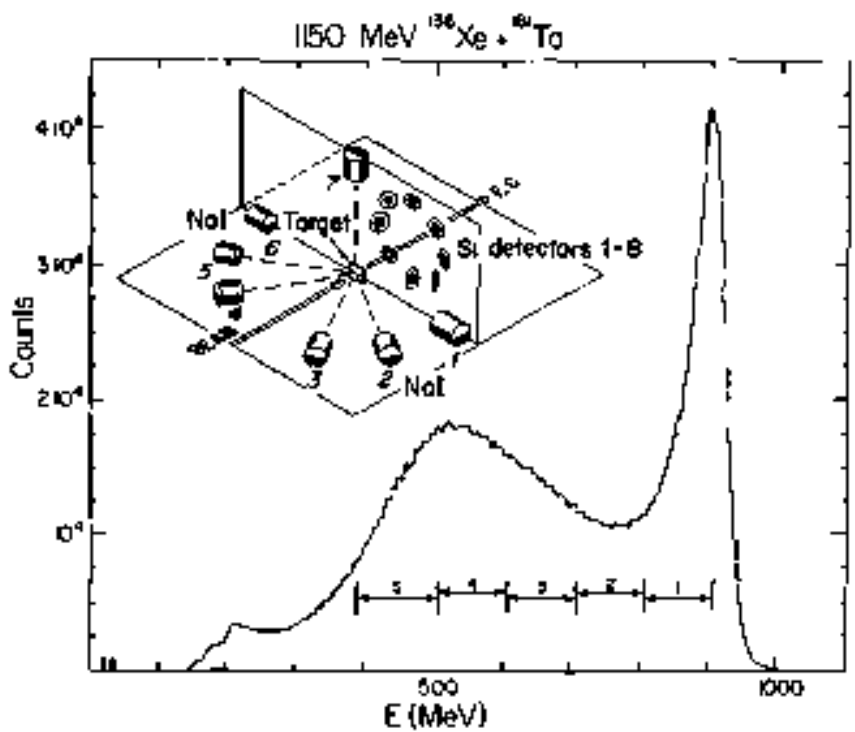

Fig. 1. The insert is a schematic of the experimental confoguration, depicting the seven $5 \times 6$ in. $\mathrm{Nal}$ and eight Si solid-state detectors. The main figure shows the heavy. ion energy spectra and the five Q-value windows.

(XBL 816-1017)

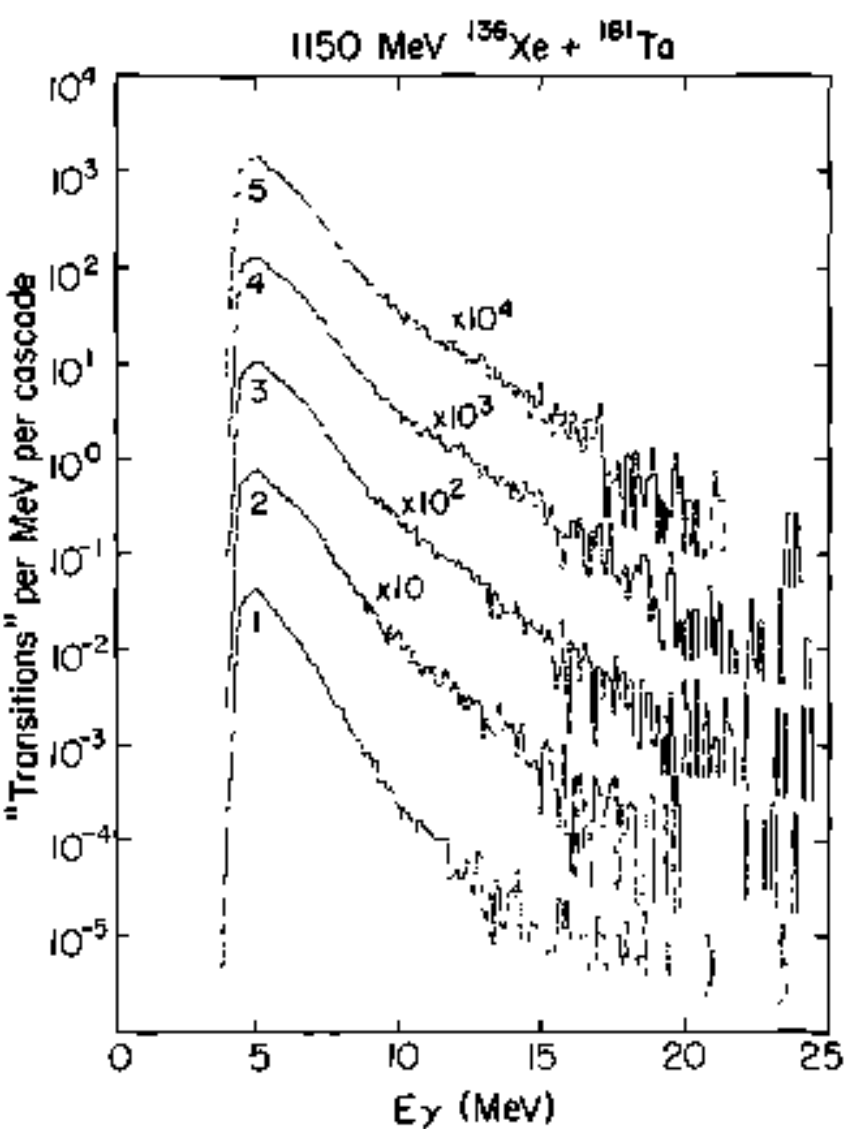

Fig. 2. Coincident gamma-ray energy spectra for the five Q-value bins shown in Fig. 1.

(XBL 8I6-326])

energy $\gamma$ rays in the region of the GDR $\left(E_{\gamma} \simeq 14 \mathrm{MeV}\right)$ as one gates on more negative $Q$-values or bigher temperatures. Most of this increase occurs between Q-value regions $I$ and 3 followed by a leveling of of the yield for the largest $Q$ values.

Work is presently in progres to determine whether these specira are in agreegnent with statistical model calculations where the GDR strength function has been included for El gammin decay.

\section{Reference}

I. J.O. Newton te al., Phys. Rev. Lett. 46, 1383 (198i). 


\title{
Properties of Nuclei at Very High Spin*
}

\author{
F.S. Siephens
}

Our understanding of the structure of high-spin states has developed rapidly in the last few years, and a rather satisfying perspective is now emerging. I will discuss $\gamma$ ray stcudies because at present they give the most detailed information about niaclear structure at these spin values.

The first question has to do with the amount of angular momentut that one can get into $y$-ray esscades. By far the best method presently known to produce high-spin states is through heavy-ion fusion reactions. The maximum angular momentum resulting in $\gamma$ emission following such reactions is shown in Fig. I. Above and to the right of the curye in Fig. I, the angular momentom is lost through fission, whereas above and to the left it is removed by particle (largely a) evaporation. Below the line, $\gamma$ emission dominates, deexciting the nucleus to its ground state. It is apparent that the highest angular momentum that decays through $\gamma$ emission is about $70 h$ for masses around 150 . If these nucled were produced cold, only aboust is to $20 \mathrm{~h}$ more could be retained. Thus we can study most of the possib]e range.

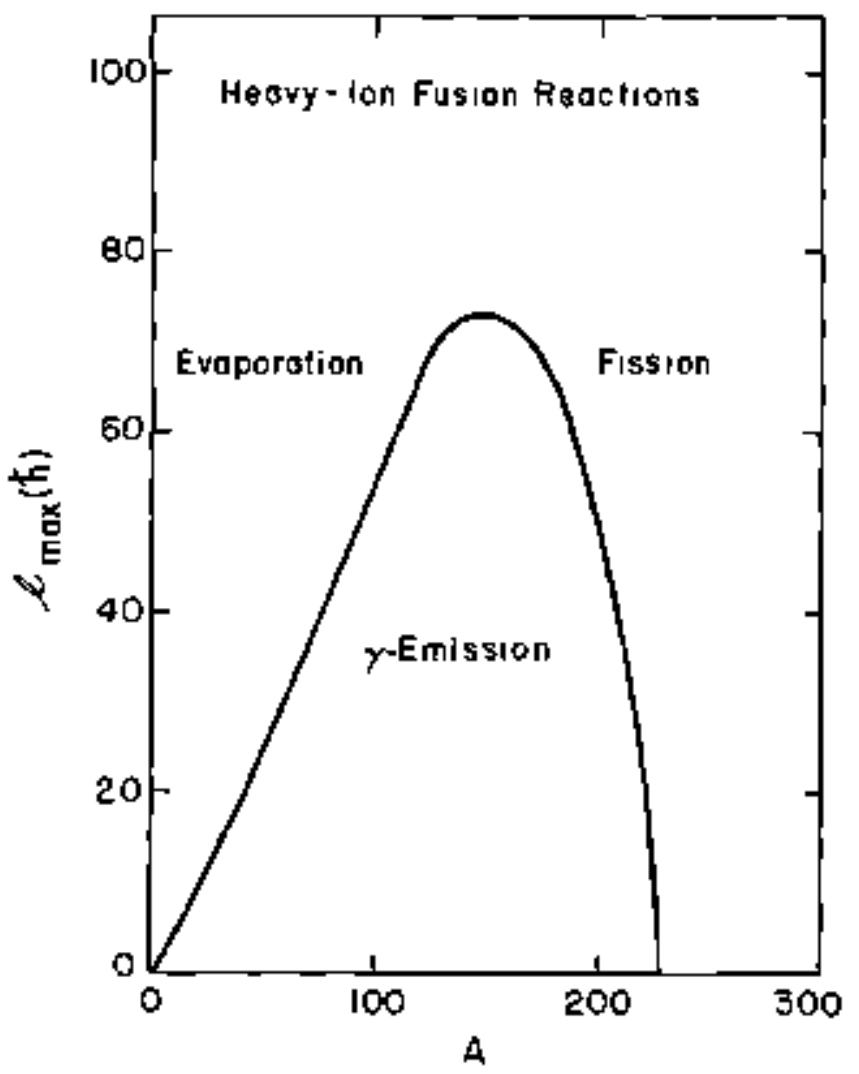

Fig. 1. The line indicates the limiting angular momentum for a nucleus (of mass A) between $\gamma$ ray emission (below) and either fission (above, right) or particle evaporation (above, left).
To understand the structure of nuclei at such spins, consider a classical rigid rotor. It is not clear that nuclei behave this way, though at high spins, where the pairing correlations are quenched, most people believe this is approximately true. Two limiting models for nuclear behavior suggest that this is reasonable.' The moment of inertia of a rigid ellipsoid (having constant volurne) compared with that of a rigid sphere is shown in Fig. 2 as a function of shape $(\gamma)$ for two deformation valuts. The value $\approx=0.3$ corresponds roughly to that found in deformed rare-earth nuclei, whereas $t=0.6$ corresponds to an axis ratio of about 2: l-the lergest that one could hope to see in these (or probably any) nuclei. It is clear in Fig. 2 that there is a favored region of large moments of inertia, extending from an oblate shape rotating about its symutry axis $\left(\gamma-60^{\circ}\right)$ to a prolate shape rotating about a perpendicular axis $\left(\gamma-0^{\circ}\right)$. To see just how significant this favoring is, I have put an energy scale on the right side of Fig. 2 for the case of mass 160 and spin 60 h.

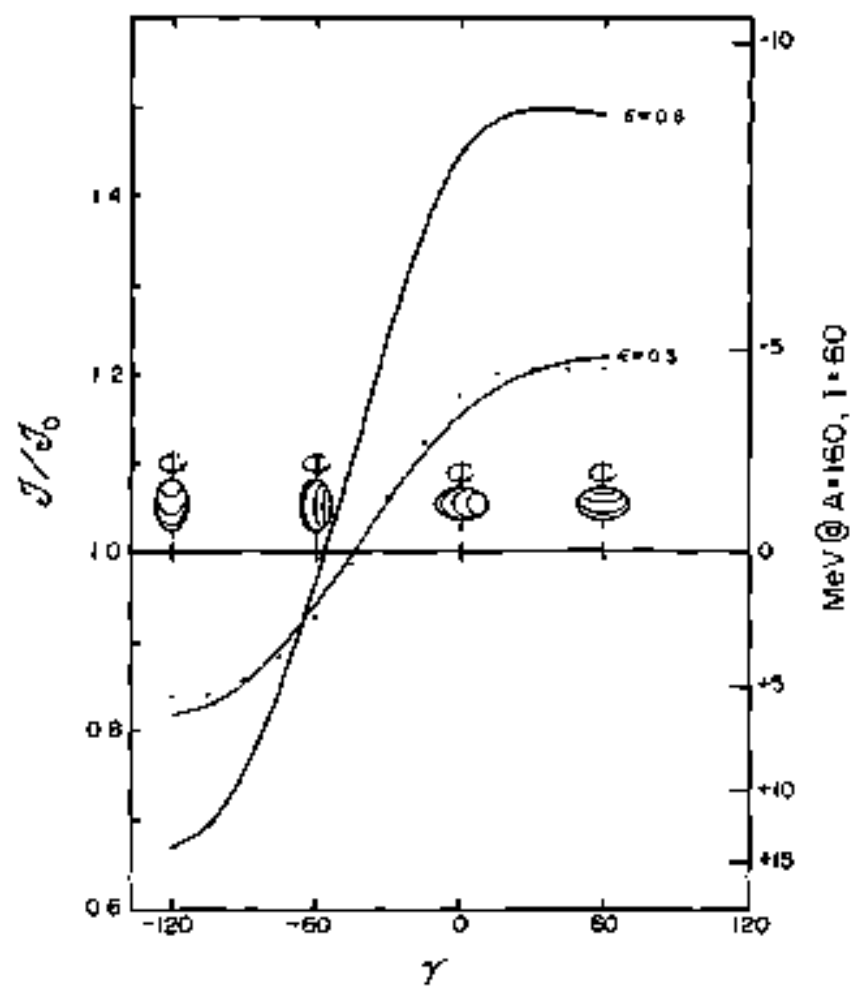

Fig. 2. Ratio of the montent of inertia of a rigid ellipsoid to that of a rigid sphere ws. the shape parameter $\gamma$ for two values of the deformation $c=0.3$ and $E=0.6$. The right-hand scale gives the difference in rolational energy in $\mathrm{MeV}$ for a mucleus with $\mathrm{A}=160$ and $\mathrm{I}-60$. The dols give the differences in total liquid-drop energy (rotation + surface + Coulonb) for the nucleus.

(XBL B08-11414) 
These shapes are favored by about $10 \mathrm{MeV}$ over the others. Since the average shell effects are only around $3 \mathrm{MeV}$, these moment-of-inertia effects should dominate at high spins and restrict the nuclei to the above shape region. It is interesting that the shape dependence of the surface and Coulomb energy terms in the liquid drop model is small, and the full LDM energy, given by the dots of Fig. 2, is rematkably near to that oblained from the morments of inertia alone-simply geometry.

There is a further aspect of these shapes that is important. The rotation of a nucleus about an axis perpendicular to the symmetry axis is a collective rotation wilh smooch bands and strongly enhanced $\mathrm{E}_{2}$ iransitions connecting the levels. There are many beautiful examples of such rotors in the regions around mass 160 and 250 . On the other hand a quantal system like the nucleus cannot rotste around a symmetry axis. This degree of freedon is contained in the single-particle motions. Thus a nucleus of $y$ $=60^{\circ}$ builds up its angular momentum by aligning one or more individual nucleons with the symmetry axis. This is completely noncollective and is much like the case of spherical closed-shell nuclei. It seems that around mass 150 some objate nuclei exist, ${ }^{2}$ with such noncollective states ronning up to around $40 \mathrm{~h}$. We have found that nuclei at the highest spins lie between these limits $\left(\gamma=0^{\circ}\right.$ and $60^{\circ}$ ), displaying some colleclive and some noncollective properties.

\section{Footnole and References}

"Presented at the International Conference on Nuclear Physici, Berkeley, CA, August 1980.

1. A. Bohr and B.R Mottelson, Nuclear Structure, vol. 2 (Benjanin, Reading Mass., 1975).

2. T.L. Khow, R.K. Snither, B. Hazs, O. Hausser, H.R. Andrews, D. Horn, and D. Ward, Phys. Rev. Lett. 41, 1027 (1978)

\title{
Nuclear Structure at High Spins*
}

\author{
F.S. Stephens
}

Nuclei are composed of a small (but not too small) number of nucleons. As a result they display both collective and single-particle (non-collective) features. For example, in the rare-earth and actinite regions, the low. lying rotational bands represent an almost pure collective motion, with energies following the $\mathrm{l}(\mathrm{I}+\mathrm{l})$ rotor formula to within a percent or two, and E2 transition probabilities nearly 200 times larger than a single proton would have. On the other hand, near the closed shells, the energy levels are almost completely deternined by the motion of a single nucleon. Mast nuclear levels display both collective and non-collective features, and high-spin states are no exceptjon. The present objective is to understand the kinds of mixtures of colltctive and single particle motion that are important in nuclei at the highest spins. Our ideas about such states have undergone important developments recently that now make possible a reasonably sjonple description of this subject.

I will start with a collective rotational nucleus, and couple to this first one and then more single particles. The rotational angular momentum is necessarily perpendicular to the nuclear symunetry axis, and the particle angular momentum, $\mathbf{j}$, can couple either along the symmetry axis or along the rotation axis. The former situation is that considered by Bohr and the projection of $j$ along the symmetry axis, alled $\Omega$, is a constant of the motion. In this case the collective angular momentum, $R$, and the projection of $j$ along the rotation axis are not constants of the motion. In the latter situation, the projection of $\mathbf{j}$ along the rotation axis, cailed $j_{3}$, is sharp, and here $R$ and $\Omega$ are not constants of the motion. It seems rather clear that a perpendicular relationship between $R$ and $\mathbf{j}$ will be much less favorable for producing low-energy high-spin states than a parallel one. This is borne out by the fact that as the nucleus rotates there is a Coriolis for $\infty$ which tends to align $j$ with the rotation axis. The back-bending phenomenon, and a number of other related effects, are now known to be connected with such "rotation-aligned" states. I want to try to trace how the inclusion of such states can effect a smooth transition between fully collective and fully nonollective regions of nuclear behavior.

In the upper left portion of Fig. 1 a complete collective behavior is illastrated. The nuxcleus is taken to be prolate, as indicated by the small $\beta, \gamma$ plot, and ach intrinsic state (angular momentum along the symmetry axis is ignored, implying $K=0$ ) has a collective rotational band corresponding to rotation about the axis perpendicular to the symmetry axis. The tolal angular mernentom is just that of the collective motion, $\omega \theta$. In such bands the energy is given by parabolas centered on the $y$-axis and displacod vertically by a band-head energy. The $\gamma$-ray energy in such a band is related to the slope of this parabola. The difference between successive $\gamma+$ ray energies is related to the curvalure of the parabolas, and is independent of spin for a perfect rotor.

In the upper right portion of Fig. I, a small amount of single-particle angular momentum aligned along the rotation axis, $j_{\mathrm{a}}$, has been added. The orbits of these particles are roughly in the plane perpendicular to the rotation axis, and will cause a bulge in the otherwise prolate nucleus. Thus the nucleus necessarily becomes slightly tri-axial as indicatod in the small $\beta, \gamma$ plot The total angulas mornentern is now the sum of the collective part, $\Delta \theta_{\text {coll }}$, and a single particle part, $\mathrm{\Sigma}_{\mathrm{a}}$. The energy of the bands is given by parabolas whose horizontal displacement from the 


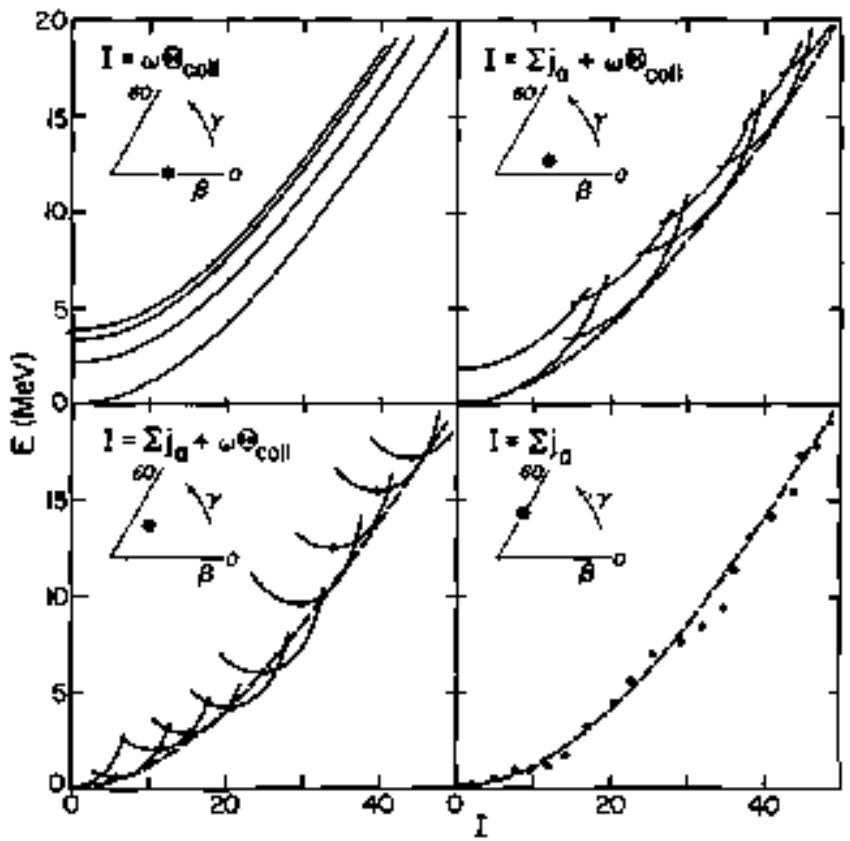

Fig. 1. \$cheraatic excitation energy vs, spin plots for various relative amounts of collective angulat momentum and single-particle rotation-aligned angular momentum. Bandhead (pure single-particle) energies are shown in the lower two panels. The solid curves correspond to real bands, whereas the dashed curve is the envelope of the real bands.

(XBL 804-544)

$y-a x i s$ is $j_{2}$ and whose vertical displacement is still the band head energy. The salid lines in Fig. 1 represent these bands. Since the aligned angular morientum is usually not known, one generally ignores it and uses the usual rotational formula. This corresponds to the envelope curve (dashod) in Fig. 1. The average slope, and thus $\mathbf{E}_{\gamma}(\mathrm{I})$, are the same for this envelope and for the populated portion (near the envelope) of the real bands, so that one cannot distinguish the real band structure this way. However, the curvature of the real bands in the upper right part of Fig. 1 is larger than that of the envelope. This curvature is still related to differences between r-tay energies, provided they ate two (cosrelated) r-rays within the same band. There are now experiments sensitive to this curvature.
In the lower left part of Fig. 1, the alignment process is assumed to continue. The nucleus is moving toward an oblate shape as more particles align and thereby move in roughly circular orbits perpenticular to the rotation axis. The total angular momentum is now mostly aligned, $\Sigma_{\mathrm{a}^{2}}$, with only a modeat collective contribution. The band head entergies are indicated as dots, and they have moved out tather close to the envelope line. As sketched (somewhat arbitrarily), there is only an average of 6 or $8 \mathrm{~h}$ in the bands at the spins where they are likely to be populaied (along the envelope). The band beads were not indicated in the previous panel (upper right) where they were sather far from the envelope line-15.20h on the averagecorresponding to a considerably larger collective contribution to the total angular momentum. The curvature of the bands is much larger now since the shape is becoming more oblate, and the rotation axis will then become a symmetry axis. Another way to view this is that most of the reasonably high-j particles are aligned and thus no longer contribute to the collective morsent of inertia. These bands show a much higher rate of crossing, and although the slope behaves regularly, the detailed band structure will be quite irregulat. It is worth emphasizing that the $\gamma$ ray energies alone cannot tistinguish among these first three panels; one must look at the $\gamma$-ray energy cocrelations.

Finally, in the lower right part of Fig. 1, the nucleus has aequired an axially symmetric oblate shape-the rotation axis has become the symmetry axis and collective rotations cannot exist about this axis. The band hesds now scatter around the envelope line and ase purely singleparticle states. At $\beta=0$ these would be the usual spherical shel]-model states, but reasonably large $\beta$ values may also occur. Such states are observed in several regions and will be discussed later by Khoo and others. We have thus followed the motion from collective to non-collective in a continuous way by aligning more and more particles.

There is good evidence that nuclei do exist with behavior like that shown in the first, second, and fourth penels. I believe we now have the experimental tocks to determine whether nuclei at the very highest spins fall into this sequence, and if so, where. The next few years should thus be exciting ones in the study of very high-spin states.

\section{Foolmote}

"Presented at the International Conference on Nuclear Behavior at High Angular Momentum, Sirasbourg, France, April 22-24, 1980. 


\title{
High-Spin Properties of ${ }^{164} \mathrm{Er}$ in the Multiple Band Crossing Region From Coulomb Excitation and In-Beam Gamma-Ray Spectroscopy"
}

\author{
S.W. Yotes, t‡ I.Y. Lee, $\$ 5$ N.R Johnson, E. Etchler, $\$$ L.L Rledinger, $\neq .1$

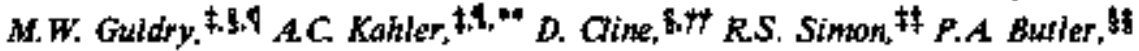 \\ P. Colambani, I F.S. Stephens, R.M. Diamond, R.M. Ronningen'19.**" \\ R.D. Hickwa."11.tH J.H. Hamilion, 11 and E.L. Robinrontt
}

\begin{abstract}
High-spin states in ${ }^{164} \mathrm{Er}$ have been studied by using the ${ }^{150} \mathrm{Nd}\left({ }^{8} 0,4 n \gamma\right)$ and ${ }^{164} \mathrm{Dy}(\alpha, 4 n \gamma)$ reactions and by multiple Coulomb excitation with ${ }^{136} \mathrm{Xe}$ ions. Several positjwe-parity bands are excited, and two negative-parity bands have betn identified. The previously reported backbending behavio of the yrast sequence is demonstrated to result from the intersection of the ground band (sten to spin $22^{+}$) with an even-spin "superband" observed from spins $12^{+}$to $24^{+}$. The odd-spin yrast superband and the second lowest even-spin superband also have been observed. Al] the superbands have moments of inertia appreciably larger than the ground band. The observed intersection of these multiple superbands with the $y$ band produces different backbendigt behavior and a stagigering of the energies of the odd- and even-spin members of the $y$ band. $B(E 2)$ values for transitions below and through the band intersection regions of both the ground band and $\gamma$ bands have been deduced from multiple Coulomb excitation yields. In addition, lifetimes bave been measured for the ground band by the Doppler-broadened lineshape technique. The level epergies and B(E2) values are consistent with weak interaction matrix elements ( $\simeq 45 \mathrm{keV}$ ) between the intersecting bands. The B(E2) values of the unperturbed band are found to obey the simple rigid-rotor relationship. The $7^{-}$negative-parity band exhibis a nor: $\mathrm{mal}$ rotational sequence, while the $5^{-}$band exhibjts a large odd-even staggering and a larger mament of inertig. Twoquasiparticle-plus-rotor model calculations indicate sirong rotation alignment and reproduce the observed properties of
\end{abstract}

the high-spin states for both the positive and negativeperily bands in ${ }^{164} \mathrm{Er}$.

\section{Footnotes}

"Condensed from Phys, Rev, C 21, 2366 (1980).

tUniversity of Keniucky, Lexington, KY $\mathbf{4 0 5 0 6}$

Foak Ridge National Laboratory, Oak Ridge, TN 37830

SLBL

'Deceased

University of Tennessec, Knoxville, TN 379J6

"Present address: Cyclotron lnstitute, Texas AkM University, College Station, Texas 77843.

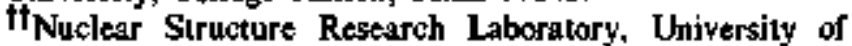
Rochester, Rochesier, NY 14\$2]

\#Fresent address: Gesellschaft für Schwerionenforschung. Darmstadt, Germany.

S5isesent address: Deparment of Physics, University of Ljverpool, P.O. Box 147, Liverpool 169 3BX, United Kingdom.

1 Present address: Eludes et Productions, Sctulumberget, 25 Rue de la Cavee, 92142 Clamart, France.

19 Vanderbili University, Nashville, TN 37235

*" Present address: Cyclotron Laboratory, Michigan State University, Enst Lansing, Michigan 48824.

1 Present address: Department of Radjology, Medical Physies, University Hospitals, Madison, Wisconsin 53706.

\# University of Alabarma in Birmningham, Birmingham, Alabarma 35294

\section{Average g-Factors for High Spin States in ${ }^{156} \mathrm{Dy}^{*}$}

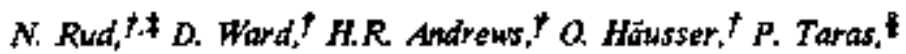 \\ J. Kejnoner, 1, 1 . Neiman, R.M. Diamond. and F.S. Stephens
}

The intitial deexcitation process of nuclei formed at high spin involves a large number of possible decay paths which give rise to a quasi continuous $\gamma$-ray spectrum formed from many unresolved overlapping transitions. Nevertheless, it has been possible to determine certain average properties of the enjitting states sucb as, average monents of inertia and collectivity. In the presept experiments, we have mensured average magnetic moments for states lying on and above the yrast line in the nucleus ${ }^{156} \mathrm{Dy}$ populated in the reaction ${ }^{24} \mathrm{Mg}(136,4 \mathrm{n}){ }^{156} \mathrm{Dy}$ at various bombarding energies. The $156 \mathrm{Dy}$ recoiled through a layer of magnetized jron durting which time the aligned states precessed in the intenst magnetic field experienced by ions moving rapidly in a ferrornagnet. After about 1 ps the recoils left the iron and enterted a copper layer, where the magnetic field was zero so that no further precession 
could occur. However, the precession accurrulaled in the iron was passed down through further $\gamma$-ray cascades resulting in a cumulative rotation of the $\gamma$-ray angular distribution pattern for the discrete transitions at low spin. We shall refer to these low spin states as "probe" states.

The ${ }^{136} \mathrm{Xe}$ beam was obtained from the Berkeley SuperHILAC. The precession measurements were performed with 1 wo $\mathrm{Ge}(\mathrm{L} \mathrm{i})$ detectors placed $7.5 \mathrm{~cm}$ from the target and set $60^{\circ}$ on either side of the beam direction. Spectra were routed acoording to the field direction, which was reversed every two minutes. The effect of procession on the probe states (the $2^{+}, 4^{+}, 6^{+}$, and $8^{+}$states) was derived in the usual way from appropriate ratios of their observed photopeak intensities in the two $\gamma$-ray detectors for the two field directions. The precession angles themselves were obtained by using the $\gamma$-ray angular distribution soefficients measured previously.

The centroid of the initial spin distribution, $\hat{\mathbf{j}}$, was assumed to be $\bar{J}=2(\langle M\rangle-1.5)$ with a $F W H M$ of 14h. The results are summarized in Fig. $I$, where it can

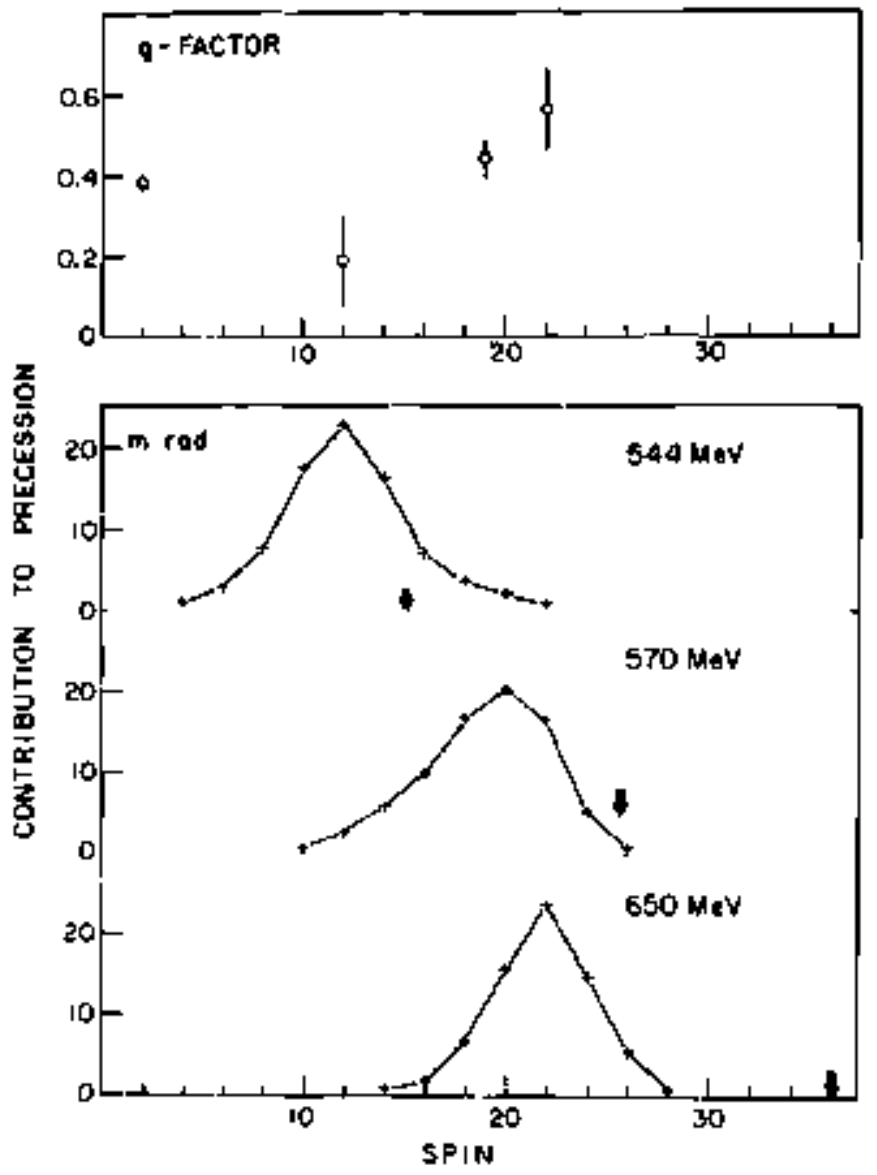

be seen that the centroid of the spin region contributing to the precession was appreciably lower than that of the entry distribution. This we because the higher spin stales haying the shortest lifetimes were not exposed to the full action time of the field, and further, there was appreciable decay during the period spent in the arget and bufier layers (from! $=0$ to $\sim 0.5 \mathrm{ps}$ ).

For average entry spin $\overline{\mathrm{j}}=15 \mathrm{~h}$ there uould be a strong population of the states in the vicinity of the crossing at spin 16 of the ground band by a band believed to have a neutron-aligned character, and predicted to have near zero g-factor at spin 12-16). In comparing our results with calculations, it must be remembered that states above the yrast line were also sampled; nevertheless, some reduction of the g-factor would be expected for this spin region Irom the predorsinance of neutron aligned states there, and this is indicated by the dala. At the higher entry spins, the average $\mathrm{g}$-factor has recovered to or may even exceed the $2^{+}$value. This behavior is expected from the break. down of proton pairing and the oceurrence of aligned proton bands which should become yrast for $\bar{J}>26 \%$. The present rise at spins as low as $J=20 \mathrm{~h}$ may result from the population of aligned proton bands lying above the (neutron aligned) yrast line.

\section{Footnotes}

Condensed from Phys. Lett. B 101 (1,2), 35 (1981).

†Alomic Energy of Cajada Limited, Chalk River Nuclear laboratories, Chalk River, Ontario, Canada KoJlJo F Visiting scientist from Aarhus University, Denmark Laboratoire de Physique Nueléaire, Université de Montréal, Montréal, P.Q. Canada

'Queen's Uhiversity, Kingsion, Ontario, Canada IPermanent address: University or Helsinki. Finland

Fig. I. Sumunary of average g-factors in ${ }^{156} \mathrm{Dy}$. The lop panel shows the average $g$-factor plotted at the centroid of stales contributing to the precession. The $2^{+}$value was oblained from systematics of $2^{+} \mathrm{g}^{+}$-factors in Dy isotopes and the $\mathrm{N}=\$ 0$ isotones. The lower panels show the contributions made by the states of spin J (not necessarily yrast) to the tolal precession for each entry spin distribution whose centroids are indicated by the arrows.

(XBL 819-1783) 


\title{
Alignment Effects in Correlation Spectra ${ }^{*}$
}

\author{
C. Ellegaard, ${ }^{\dagger}$ M.A. Deleplanque, ${ }^{t, \neq}$ O. Andersen ${ }^{\dagger}$ \\ B. Herskind, F. Stephens, R.M. Diamond, H. Rluge, C. Schick,' and S. Shih?
}

In the deformed rare earth region, nuclei with spins as high as -70 h may be populated by heavy-ion fusion reactions, For the $\gamma$-ray depopulation of such nucdei, discreteline $\gamma$-ray spectroscopy can follow the decay to spins as high as $32 \hbar$. Above this limit the $\gamma$-ray spectrum is unresolved and thus appear as a continuum. Many measurements have shown that this unresolved $\gamma$-ray spectrum consists of statistical $\gamma$ rays, which cool the sudteus towards the yrast line and give rise to an exponentially decreasing bigh-energy tail, and of a lower energy bump, which is composed of yrast-like transitions that remove spin and contain most of the nuclear structure information.

Recent experiments have shown that it is passible to observe structure in this continuum region. These experiments are based on $\gamma$ - $\gamma$ coincidence measurements, and the strueture is brought sut by measuring correlations amoing the $\gamma$-ray energies. These correlations arise from regularities of the $\gamma$ cascades that result Irom certain features of the nuclear structure. The angular momentum carried by the nucleus can be generated either from the oolective motion of the nucleus as a whole or from the motion of individual particles. Collective sotation of the pucleus is indicated in the two-dimensional $\gamma \gamma$ energy plots by a val ley along the diagonal jndicating an absence of two transitions of the same entergy-a property of rotational bandsand by flanking ridges whose spacing is related to the band moment of inertia. There is also evidence in these nuclei that angular momentum is carried by single-particle motion; specifically, bands involving unpaired high-j particles whose angular monentum becomes aligned with the rotation axis above a certain spin. Such bands generally cross other (less aligned) bands as the spin increases. These band crossings, which occur in many bands at a few specific frequencies, lead to marked deviations from the regular rotational pattetn. It is important to recognize clearly the observable features associated with this latter behavior.

A backbend in a cascade is characterized by several (two, three, or even four) $\gamma$ rays of roughly the same energy. An upbend is the clearest example of such behavior and seems to be common at high spins, as illustsated by the known second backbends. It has been bhown.' that these features appear as bridges across the valley or peaks in the valley of the correlation spectra. In such cases the $\gamma$ ray transitions in coincidence with the bridge or peak transitions should also be enhanced. If there is more than one backbend in a cascade, there will be a further enhancernent of the intensity at the intersection of the two beckbend energies. Thus one might expect to see a "square" patters in the correlation spectra, i.e., intersecting horizontal and vertical stripes made up of pints having higher than average intensity.

In this experiment $156,157,158 \mathrm{Er}$ nuclej were populated at high spin with the ${ }^{12}{ }^{3} \mathrm{~S}\left({ }^{40} \mathrm{Ar}, \mathrm{xn}\right)$ reactions with $x=6$. 5 and 4. The $\gamma$-rays were detected in five $G e(L i)$ detectors placed at $\pm 150, \pm 130$ and $90^{\circ}$. A sum spectrometer was placed around the target and $G(L)$ detectors. Since peak-to-total ratio for the $\mathrm{Ge}(\mathrm{Li})$ detectors used was only I0 to 15\% a method for subtracting uncorrelated events (Compton and background transitions) from the data was used. The method is an improvement over the one एeviously described. ${ }^{1}$

The result of this proceduse is shown in Fig. 1. This plat corresponds to a high slice in the total energy spectrum of the sum spectrometer and thus populates highest spins 50 , producjing a spectrum which is roughly fat from about $1300 \mathrm{keV}$ down. The low energy lines from the first backbend in the An channel are present in this spectrum, though they are weak and not so clearly seen on this low-resolution plot. The dominant feature is the strong line at $1300 \mathrm{keV}$ (Ch 49). It has strong intersections with the low-energy an lines, the second backbend (Ch 22) and a $1060 \mathrm{keV}$ line (Ch 32). Thus it definitely belongs to the 4n channel. It is impressive that the population at these spins, $\geqslant 50 \%$, fow strongly through the $1300 \mathrm{keV}$ backbend; whereas wen it is fed at lower spins this is almost completely missed. The sum-spectrometer thus provides a powerful method for studying those correlations involyed in deexciting particular spin regions.

It seems clear that the enhanced rows and columns in correlation plots like Fig. 1 contain information about the single-particle aspects of nuclei at very high angular momentum. They very probably signal the alignment of the angulat momenturn of the major high-j orbitals. While we have not tried to make specific assignments here, the general method presented for extracting this information from correlation spectra seens clesir and offers exciting possibilities for determining the important single-particle components of high-spin states throughout the periodic table. 

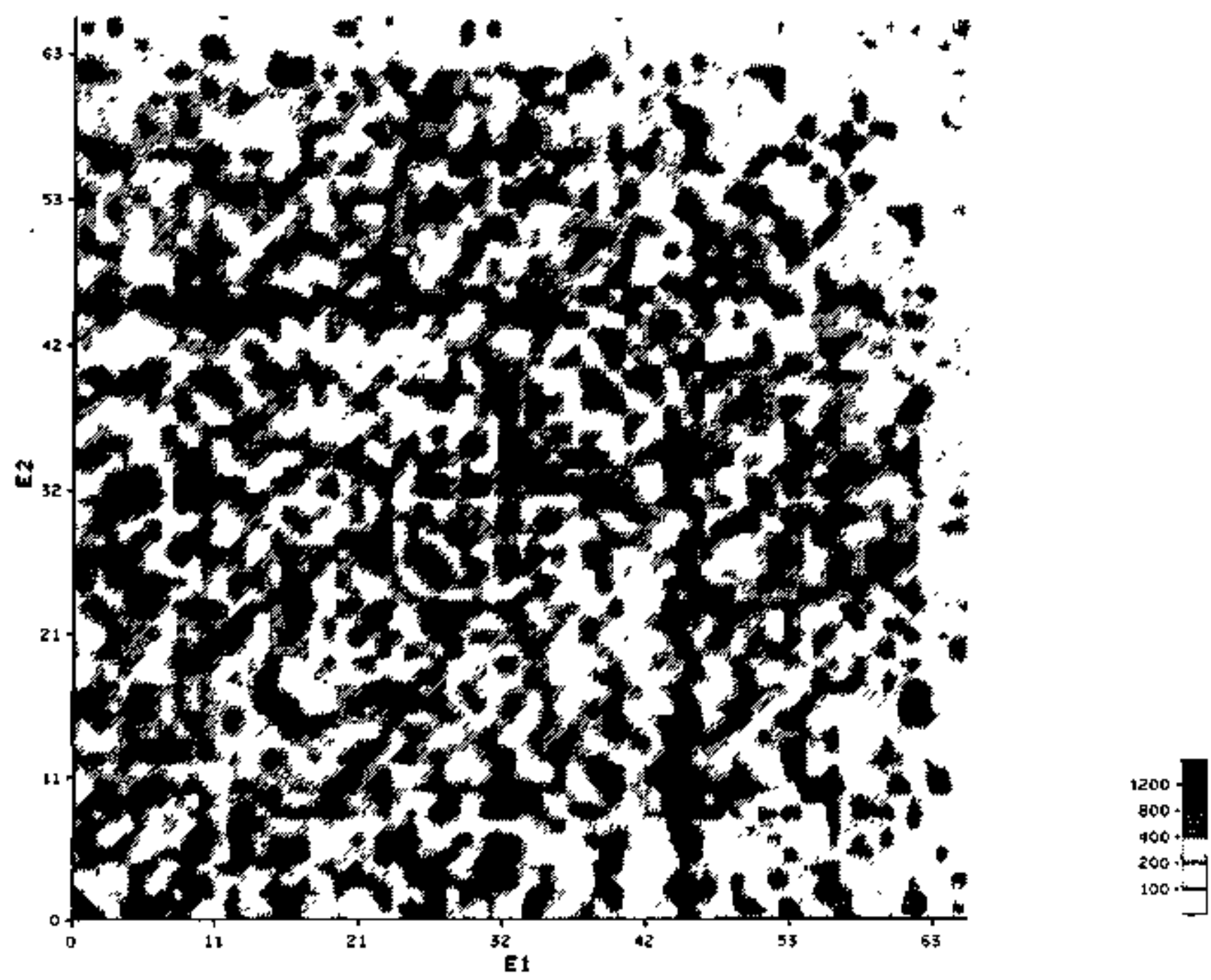

\section{SUMCORZ HIGH 16ERR-DIV-EFF $040020 \mathrm{KCH}$}

Fig. 1. Gamma-energy correlation spectra from the $122_{\mathrm{Sn}}\left({ }^{40} \mathrm{Ar}, \mathrm{xn}\right)$ reaction after 20 iterations ( corresponds to the highest-energy slice in the sum spectrometer and thus the bighest spins. Channel zero of this plot is at $0.4 \mathrm{MeV}$ and there are $0.020 \mathrm{MeV}$ per channel.

(XBB 808-9845)

Footmotes and References

"Submitted to Phys. Rev, Leth.

${ }^{\dagger}$ The Niels Bohr Institute, University of Copenhagen, Copenhagen, Denmark

IPermanent address: Institut de Physique Nucléaire, B.P. no. 1, 91406 Orsay, France.

Permanent address: Hahn-Meitner Institut, Ber|in, Germany.
'Permanent address: Centre de Spectrometrie Nucleaire el de Spectronetrie de Masse, Orsay, France.

Termanent address: Shanghai Instituie of Nudear Research Shanghai, Poople's Republic of China.

1. M.A. Deleplanque, F.S. Stephens, O. Andersen, J.D. Garrett, B. Herskind, R.M. Diamond, C. Ellegaard, D.B. Fossan, D.L. Hillis, H. KJuge, M. Neiman, C.P. Roulet, S. Shib, and R.S. Simon, Phys. Rev, Lett. 45, 172 (1980). 


\title{
Observation of Giant Dipole Resonances Built on States of High Energy and Spin*
}

\author{
J.O. Newton, ${ }^{\dagger}$ B. Herskind. ${ }^{\ddagger}$ R.M. Diamond, E.L. Oines. ${ }^{p}$ J.E. Draper. ${ }^{\ddagger}$ \\ K.H. Lindenberger,'S. Shih,' C. Schück, "* and F.S. Stephens
}

Stuties of the GDR have beten restricted mostly to coberent excitation from nuclear ground states which excites only the giant resonances built on them. Brink, however, has proposed that every state in a nucleus has a GDR associated with it. A consequence of this idea is that the strength functions for electric dipole transitions from every state would bave a Lorentzian-like shspe as a function of $\gamma$-ray energy $E_{\gamma^{\prime}}$ with a magniturde determined from the El sum rule. Such a variation of strength with $E_{\text {a }}$ would affect the shape of the spectrum of $\gamma$-rays emitted from a highly excited nucleus, particularly in the vicinity of $\mathrm{E}_{\gamma}=\mathrm{E}_{\mathrm{G}}$, the energy of the GDR We have observed this effect in the statistical rays following heavy-kot fusion reactions.

The present experiments make use of a sumspectrometer-multiplicity technique that selects the $\gamma$-rays from moderately high-spin $(\sim 20-65 h)$ states produced in heavy-ikn compound nucleus reactions. The sum spectron. eter consists of two $33 \mathrm{~cm}$ diameter by $20 \mathrm{~cm}$ thick Nal detectors facing the target $2.5 \mathrm{~cm}$ above and below the bearn axis, each subdivided into four elements. Eight NaI $(12.7 \times 15.2 \mathrm{~cm})$ delectors were placed $50 \mathrm{~cm}$ from the target at angles of $\pm 160^{\circ}, \pm 100^{\circ}, \pm 80^{\circ},-135^{\circ}$ and $-45^{\circ}$ and were shielded lrom ench other and the beam slits by $5 \mathrm{~cm}$ of lead. Targets $\left(-1 \mathrm{mg} / \mathrm{cm}^{2}\right)$ of ${ }^{\mathrm{B}} \mathrm{St}$, $110 \mathrm{Pd}$, and $124 \mathrm{Sn}$ were bonbarded with $-10 \mathrm{nA}$ of $170 \mathrm{MeV}{ }^{40} \mathrm{Ar}$ ions from the LBL 88-Inch Cyclotron. Spectra from the eight NaI detectors, associated with three regions of sum spectrometer energy $E_{s}$ with the range $\sim 10-40 \mathrm{MeV}$, were added. Spectra for the ${ }^{82} \mathrm{Se}$ case are shown in Fig. 1. In the energy interval from $-2-8 \mathrm{MeV}$, the spectra show an exponentially falling tail, composed of the statistical transitions deexciting the product nuclej after neutron evaporation. All spectra rise considerably higher than this exponential at Energies above $\sim 10 \mathrm{MeV}$, indicating a different source of $\gamma$-rays. Beyond $\sim 20 \mathrm{MeV}$ the spectra are flat and probably due to cosmic rays.

The reason for the steep slopes in Fig. 1 is that the level densities for the final states, to which the transition probabilities are proportional, vary approximately exponentially with $E_{x}$ (and thus as $\exp \left(-E_{\gamma} / T\right)$ ). A rough way to see the shape of the $\gamma$-ray strength functions is to remove the level density dependence by multiplying by $\exp \left(\mathbf{E}_{/} / \mathbf{T}_{\varepsilon}\right)$, where $\mathrm{T}_{\varepsilon}$ is an effective $\mathrm{T}$. For the less interesting region with $\mathbf{E}_{\mathrm{r}} \approx 8 \mathrm{MeV}, \mathrm{T}_{\varepsilon} \simeq 1 \mathrm{MeV}$. Above $10 \mathrm{MeV}$ the curves are flatter, indicating that these $\gamma$-rays are emitted at much higher $T_{\text {' We have somewhat }}$ arbitrarily taken $T_{e}=1.43 \mathrm{MeV}$ for ${ }^{164} \mathrm{Er}^{1}$ ( ${ }^{124} \mathrm{Sin}$ target), and adjusted the others for the expected mass dependence:

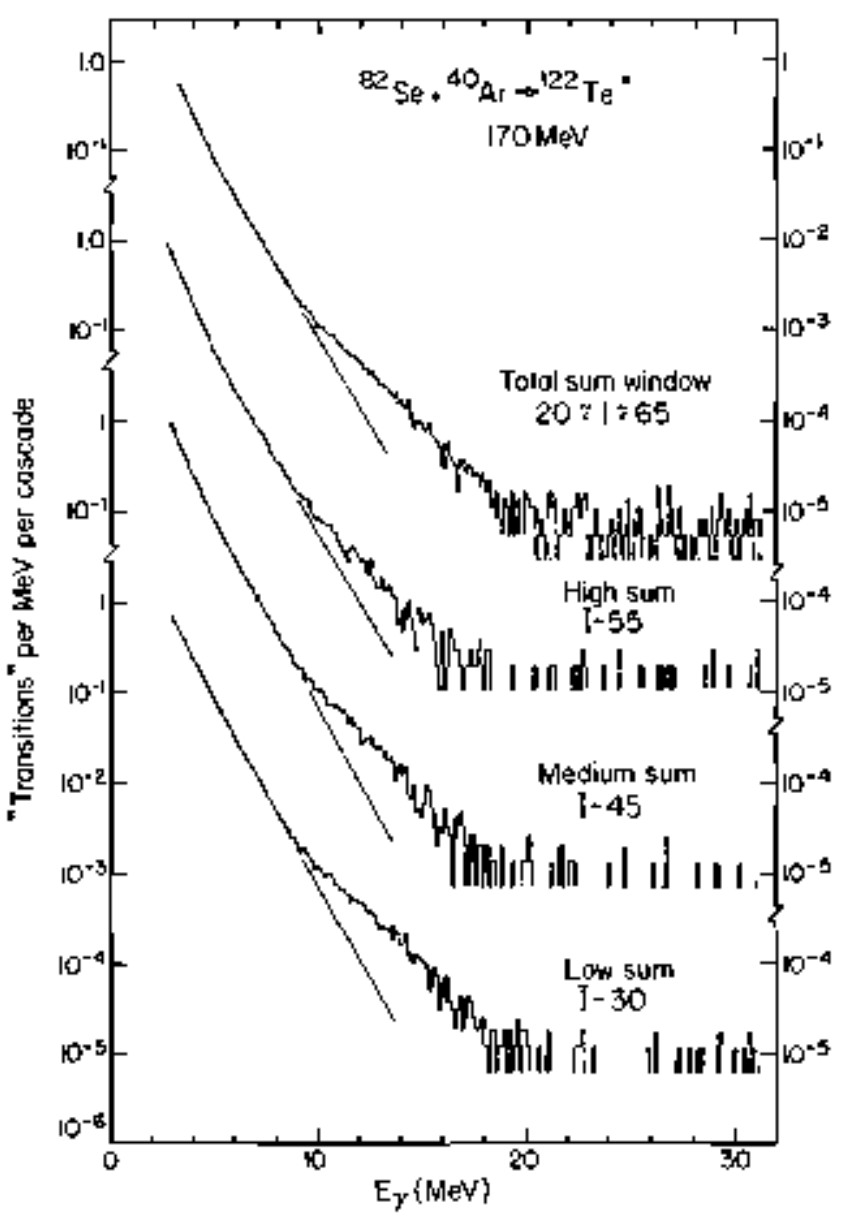

Fig. 1. Nal spectra corresponding to $E=10-40 \mathrm{MeV}$ and three windows within this range for the ${ }^{82} \mathrm{Se}+\phi_{\mathrm{Ar}}$ system. The sloping lines show exponential extrapolations of the lower $\mathrm{E}_{\gamma}$ parts of the spectra. The shapes of the true $\gamma$-ray spectra are not expected to differ greatly from these, and hence the ordinate in "transitions per $\mathrm{MeV}$ " should be approximately correct,

(XBL 812-268)

$\mathbf{T} \propto \mathrm{A}^{-1 / 2}$. The data from the total sum window (with the flat high-energy background subtracted) multiplied by these exponentials are shown in Fig. 2. The peaked struetures have maxima ( $-14 \mathrm{MeV}$ ) and widths similar to those for the GDR based on ground stales and strongly suggest GDR strength functions. In addition, the bump becons higher in energy as the target mass decreases, as would be expected for the GDR $\left(\mathrm{E}_{\mathrm{g}} \propto \mathrm{A}^{-1 / 3}\right)$. These measurements demonstrate that one can study the GDR in the $\gamma$ ray deexcitation spectra following beary-ion fusion reactions. 


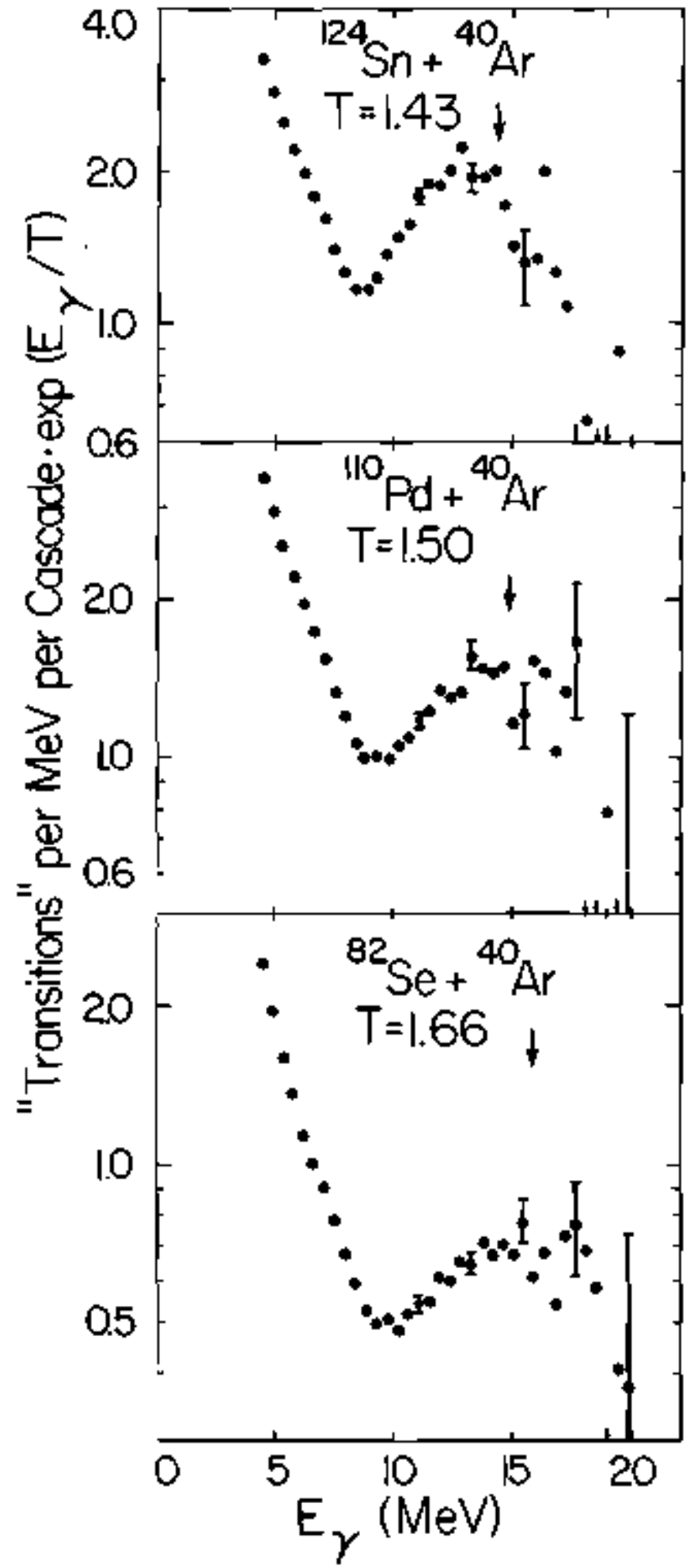

Fig. 2. Background subtracted total-window spectra multiplied by $\exp \left(\mathrm{E}_{\gamma} / \mathrm{T}_{e}\right)$. Arrows indicate $\mathrm{E}_{\gamma}=$ $78 / \mathrm{A}^{1 / 3} \mathrm{MeV}$, the centroid of the ground state GDR.

(XBL BI]-7I)

\section{Foothotes and Reference}

"Condensed Irom Phys. Rev. Lett 46, 1383 (1981).

TPermanent address: Australian National University, Carberra, Australia.

IPermanent address: Niels Bohr Institute, University of Copenhagen, Copenhagen, Denmark.

SPermanent address: University of California, Davis.

Permanenl address: Hahr-Meitner Institute, Berlin, W. Germany.

Permantent address: Shanghai Institute of Nuclear Research, Shanghaj, China.

"Permanent address: Centre de Spectrometrie Nueleatire et de Spectrometrie de Masse, Orsay, France.

l. D.M. Brink, doctoral thesis, University of Oxford (1955). 


\title{
3. Polarization Studies
}

\author{
Polarization*
}

Homer E. Conzett

A polarized beam (ot target) of particles is one in which the particle spins are preferentially oriented, as contrasted with the random distribution of spin directions in an unpolarized beant.

The polarization of an assembly of spin- $1 / 2(\hbar)$ partcles is given by the vector $p$ with components

$$
A \equiv\left\langle\sigma_{f}\right\rangle=\mathrm{N}_{+}-\mathrm{N}_{-}, \quad \mathrm{i}=x, y, z \text {. }
$$

The expoctation value of the spin-1/2 operator $g$ is averaged over the spin states of all the particles; and for spins along ( + ) and opposite to ( - ) a specific quantization axis, $N_{+}$and $N_{-}$are the fractional number of particles is each of the too spin states.

Preferential orientations in spit-1 assemblies are characterized by both vector, $\mathrm{P}_{\mathrm{i}}$, and tensor, $\mathrm{P}_{\mathrm{ij}}$, polariza tion components:

$$
\begin{aligned}
& P_{1}=\left\langle S_{i}>-N_{+}-N_{-}\right. \\
& P_{i j}=3 / 2\left\langle S_{i} S_{j}+S_{j} S_{j}>-2 s_{i j}=1-3 N_{j}\right.
\end{aligned}
$$

$S$ is the spin- 1 operator and $N_{+}, N_{o}$ and $N_{-}$are the frac. tional magnetic substrate populations, again referred to a given quantization direction. It is seen that the less tamiliar lentsor component is a measure of the difference of $\mathrm{N}_{\text {o }}$ ftom the unpolerized value $\mathrm{J} / 3$. The photon earries a spin of 1, but since the electromagnetic field is transverse to the direction ( $z$ ) of propagation, the photon spin substale $\left(\mathrm{m}_{\mathrm{z}}\right)$ populations are limited to $\mathrm{N}_{+}$and $\mathrm{N}_{-}$, corresponding to right and left circular polarizations, sespectively. Since $\mathrm{N}_{0}$ - 0 , photon beams are, in a strict sense, always tensor polarized.

Ion sources of polarized spin-1/2 and spin-1 nucleas particles have been developed, and this makes it possible to provide energetic polarized beams at various accelerators, principally Van de Graaf machines, cyclotrons and synchrotrons. These beams are crucial for experiments to identify spin-dependent effects in nuclear interactions.

\section{Foatnate and Bibliography}

"Encyclopedia of Physics, eds. R.G. Lerner and G.L. Trigg (Addison-Wesley Publ. Co., Reading, Mass., (]981).

I. L. Wolfenstein, Ainn. Rev, Nucl. Sci. 6, 43 (1956).

2. S.E. Dardem, in Polarization Ptenomena in Nuclear Reactions, H.H. Barschall and W. Haeberli, eds. (University of Wisconsin Press, Madison, Wisconsin, 1971), p.39.

3. W. Haeberi, in Nuclear Spectroscopy and Reactions, Part A. J. Cerny, ed. (Academic Press, New York, 1974), p. [5].

4. G.G. Oblsen, Rep. Prog. Phys, 35, 717 (1972).

5. M. Simonius, Lecture Notes in Physics 30, D. Fick, ed. (Springer Yerlag. New York, 1974), p. 38.

6. B.A. Robson, The Theory of Polarization Phenoment (Clarendon Press, Oxford, 1974).

\section{Polarization Tests of Time-Reversal Invariance: Past Failures}

\author{
Homer $E$. Conzett
}

An important test of time-reversal invariance (TRI) is provided by the comparison of the polarization (P) and the apalyzing power (A) measured in a nuclear reaction and its inverse, since the P-A equality follows directly from TRI. ${ }^{1}$

The most accurate of these P-A tesis have been made in $\mathrm{p}+{ }^{3} \mathrm{He}$ (Ref. 2) and $\mathrm{p}+{ }^{13} \mathrm{C}$ (Ref. 3). It is necessary to scatter from a nonzero spin nuclews, otherwise parity conservation alone ensures that $\mathbf{P}=\mathbf{A}$. We have found that neither of these compatisons was accurate enough to provide a significant test of TRI, because the equality between $P$ and $A$ depends on the equality of the two possible spin-flip probabilities. And it is now known from measurements of the depolarization in p-nucleus elastic scattering that the spin*flip probabilities are very small, leading to $P-A=0$ even if the probabilities are not equal as required by TRI. Specifically, in terms of the spin-dependent cross sections,

$$
P=\left(\sigma^{++}+\sigma^{-+}-\sigma^{+-}-\sigma^{--}\right) / 2 \sigma
$$


and

$$
A=\left(\sigma^{++}+\sigma^{+-}-\sigma^{-+}-\sigma^{--}\right) / 2 \sigma,
$$

where $a^{-+}$is the cross section for the scattering of a proton from an initial negative spin-state to a final positive spin-state, and

$$
\sigma=\left(\sigma^{++}+\sigma^{+-}+\sigma^{-+} \sigma^{--}\right) / 2 .
$$

The positive $(+\mathbf{+ y})$ direction is along $\mathbf{k}_{\mathbf{j}} \times \mathbf{k}_{\mathbf{r}}$. Thus,

$$
\mathrm{P}-\mathrm{A}=\left(\sigma^{-+}-\sigma^{+-}\right) / \sigma_{\text {, }}
$$

and $\sigma^{-+}=\sigma^{+-}$under IRI. Defining the spin-flip asymmetry as

$$
\Delta S=\left(\sigma^{-+}-\sigma^{+-}\right) /\left(\sigma^{++}+\sigma^{+-}\right) .
$$

its absolute limits are $-1 \leq \Delta S \leq 1$, but TRI requires that $\Delta S=0$. Since the depolarization parameter is given by

$$
\mathrm{D}=\mathbf{1}-2 \mathrm{~S} \text {, }
$$

with the (total) spin-flip probability

$$
\mathrm{S}=\left(\sigma^{+-}+\sigma^{-+}\right) / 2 \sigma,
$$

measurements of D provide determinations of S. I1 follows, then, from Eqs. (1)-(4) that

$$
P-A=(1-D) \Delta S \text {. }
$$

Thus, even though $\Delta S$, which is the real measure of timsreversal vialation, may be significaatly different from zero, a small value of the factor $(I-D)$ would make the P-A comparison quite insensitive to this violation. This is, in fact, just the case in these $\mathrm{p}^{3} \mathrm{He}$ and $\mathrm{p}^{12} \mathrm{C}$ experiments. From the measurement of $1-D=0.05 \pm 0.03$ clo6e to the energy and angle of the $\mathrm{p}^{3} \mathrm{He}$ experiment and an estjmate of $I-D \leq 0.06 \pm 0.02$ at the energy and angle of the $\mathrm{p}^{13} \mathrm{C}$ experiment, Eq. (9) gives $(\mathrm{P}-\mathrm{A}) \leq 0.017$ and 0.02 , respectively, for a value of the spin-fip asymmetry $\Delta S=1 / 3$, which would constitute a clear and substantial violation of TRI. These P - A values are essentially as small as the experimental errors in these P-A comperisons, so no tests of TRI were really made.

It is immediately obvious from this discussion that tests of TRI in elastic scattering. using the P.A equality, should be made through measurements where the spin-flip probability is expected or known to be large. Even better. more stringent and conclusive tests are provided by $\mathrm{P}$-A comparisons in a reaction and its inverse, since the testing of TRI is not then limied to the spin-6lp cross sections.

Similarly, it follows that tests of TRI in the basic nucleon-pucloon interaction, via comparisons of $P$ and $A$ in $p-p$ and/or n-p scatlering, should be made at energies and angles for which the quantity $(1-D)$ is maximized. Since spin-exchange forces are well-known components of the nucleon-nucteon interaction, spin-filp probabilities are generally substantial, so there should be little difficulty in satisfying this criterion. For example, in one report ${ }^{5}$ that includes both P-A comparisons and measurements of D in p-p scattering at $142 \mathrm{MeV}$, values of ( $-D$ ) range between 0.7 and 1.2 . The $(\mathrm{P}-\mathrm{A})$ valutes are generally consistent with zero within the experimental errors of several percent, although differences of 0.04 to 0.08 are listed. However, as $\mathrm{Bryan}^{6}$ bas noted, the Pp system, because of identical-particle symmetry, has reduced sensitivity for the testing of TRI. That is, $T$ asymmetry can only show up in the coupled states of $\mathrm{j}^{\mathrm{P}}=2^{-}, 4^{-}$, etc. Then, in a model ${ }^{6}$ where the nucleon-nucleon $T$ asymmetry is due to the excbange of the $A_{1}(1070 \mathrm{MeV})$ meson and is of short range, the angular monentum barrier drastically inhibits the obseryable effects of TRI violation in the Pp system at the energies (up to about $600 \mathrm{MeV}$ ) that have been investigated to date.

Now that it is realized that essentially all previous polarization tests of TRI have either been nontests or tests of very limited sensitivity, it is clear that significant tests of this importani fundamental postulate should soon be pursued.

\section{References}

1. R.J. Blin-Stoyle, Proc. Phys. Soc. A 65, 452 (1952); G.R. Sarchler, Nucl. Phys. 8, 65 (1958); L. C. Biedenharn, Nucl. Phys. 10, 620 (1959).

2. D.G. McDonald, W. Haeberli, and L.W. Morrow, Phys. Rev. 133, B] [78 (1964).

3. E.E. Gross tt al., Phys. Rev. Lett 21, 1476 (1968).

4. W.G. Weitkamp el al., Nucl. Phys. A 311, 29 (1978).

5. C.F. Hwang et al., Phys. Rev. 119, 352 (1960).

6. R.A. Bryan, Phys. Rev. C 12, 1968 (1975). 


\title{
Deviations from the Polarization-Analyzing Power Equality and Implied Breakdown of Time-Reversal Invariance*
}

\author{
H.E. Conzetf. P. von Rossen, ${ }^{\dagger} F$. Hinterberger, ${ }^{\dagger} R J_{+}$Slobodrian ${ }^{\ddagger}$ \\ C. Rioux, and $R$ Roy $\ddagger$
}

We are reporting on the first lest that compares the polarization (P) and the analyzing power (A) from measurements in a muclear reaction and its inverse. We find an astonishingly large P - A difference. The clear implication is that time-reversal inyariance (TRI) is broken in some component of the nuclear interaction, since the polarization-analyzing power oquality follows firestly from TRJ. ${ }^{1}$ Thus, in view of the fundamental position that the P-A theoren has held in spin-polarization physics, both in theory and experiment, we would be very surprised if our results are not viewed with some skepticism.

The reactions chosen for the P-A comparisons were the two-nucleon transfers ${ }^{7} \mathrm{Li}\left({ }^{3} \mathrm{He}, \mathrm{p}\right){ }^{9} \mathrm{Be}\left({ }^{3} \mathrm{He}, \mathrm{p}\right){ }^{11} \mathrm{~B}$, with 14 $\mathrm{MeV}$ incident ${ }^{3} \mathrm{He}$ ions, and their inverses studied at the same CM energies. The Q-values are large, implying considerable mass, energy, and momentum reartangement. The experiments were initiated by the laval grous through the measurements of the proton polarizations in he $\left({ }^{3} \mathrm{He}, \overrightarrow{\mathrm{p}}\right)$ reactions, and results have already been published. ${ }^{2}$ The analyzing powers in the inverse $\left(\overline{\mathrm{p}}^{3} \mathrm{He}\right)$ reactions were measured at Berkeley. The $\left({ }^{3} \mathrm{He}, \vec{p}\right)$ proton polatizatfons were measured with a pair of Si polarimeters. placed at equal left-right reaction angles. The Si polarimeter combines the bigh scattering efficiency of a thick analyzer with the good energy resolution obtained by adding the $\Delta E$ pulse from the analyzer detector to each of the $E$ pulses from the left and right stopping detectors.

In Fig. $I$ are shown our $P$ and $A$ measurements in the ${ }^{9} \mathrm{Be}\left({ }^{3} \mathrm{He}, \overline{\mathrm{p}}\right){ }^{11} \mathrm{~B}$ reaction. There is eviderce for a decrease in $P$ as the energy bite is increased, which is not unexpected. In any event, the smallest energy bite for the $P$ measurement is nearest to the energy bite sampled in the $A$ measurement (also indicated), and for this we find the largest P - A difference. In Fig. 2 is shown an excitation function of $\mathrm{A}$ at $37^{\circ}$ lab, which is near the peak of $\mathrm{A}$ shown in Fig. 1. Ower an energy span of some $800 \mathrm{keV}$, about $400 \mathrm{keV}$ on either side of our original energy, we find a smooth variation of $A$ Thus, there are no sharp increases in $A$ that could move its value closer to $P$ with a sriall shift in the energy, Comparably large $P$ - A differences were found, also, in the ${ }^{7} \mathrm{Li}\left({ }^{3} \mathrm{He}, \mathrm{p}\right){ }^{9} \mathrm{Be}$ reac. tion.

In summary, we bave found large differences between $\mathrm{P}$ in the ${ }^{7} \mathrm{Li}\left({ }^{3} \mathrm{He}, \mathrm{p}\right){ }^{9} \mathrm{Be}$ and ${ }^{9} \mathrm{Be}\left({ }^{3} \mathrm{He}, \mathrm{p}\right){ }^{11} \mathrm{~B}$ reactions and $A$ in their inverse processes. Since such an inexuality belween $P$ (in a reaction) and $A$ (in its inverse) directly implies a breakdown of 'TRI, it follows that this is clear

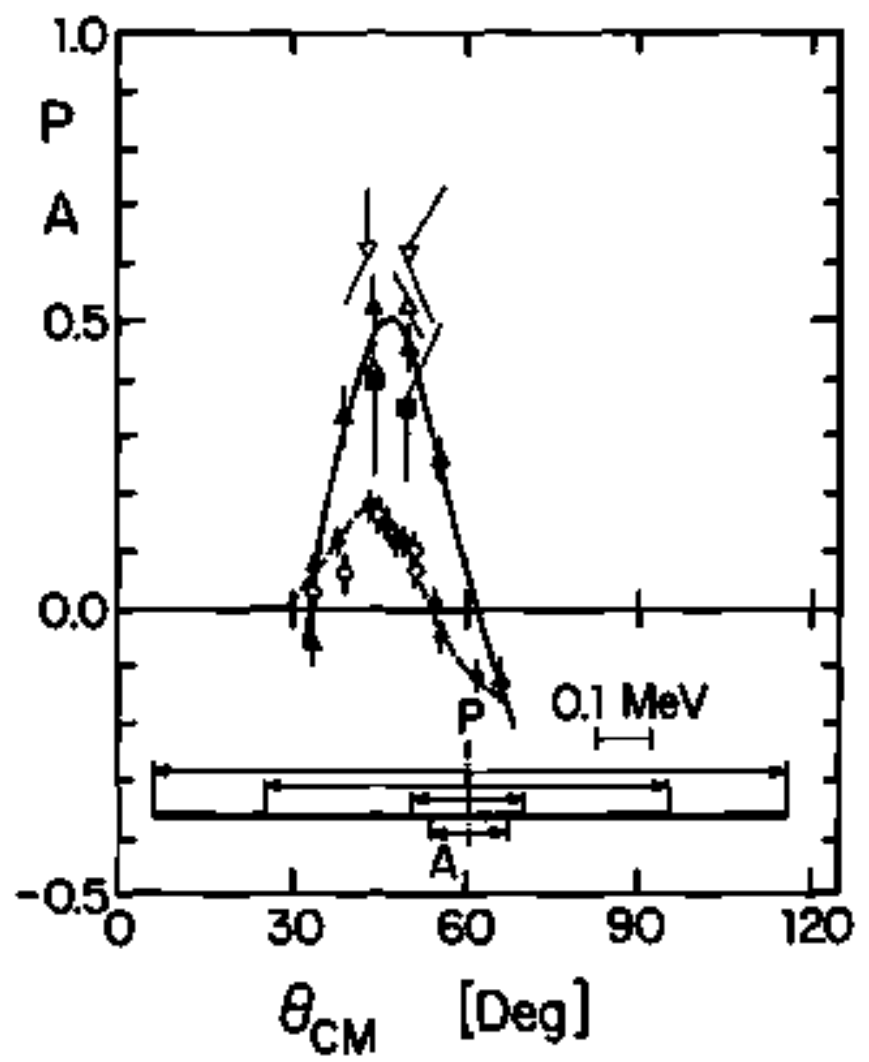

Fig. 1. The open and clased circles represent two separate measurements of $A$ The solid triangles are the original P resulis with the intermediale energy bite (due to target tbickness and beam energy-widh) indicated by an arrow on the energy scale near the botton of the jigure. The open triangles are later checks of the orjginal data. The solid squares ase measurements made with a completely independent polarimeter at Berketley and with the largest energy bite indicaled. The inverted open triangles are measurements with the smallest entergy bite.

(XBL $807-10786$ )

evidence that the interaction of nuclear particles is not time-reversal invariani. Clearly, many more experiments are necessary to explore in detail the TRI-breaking interactions. 


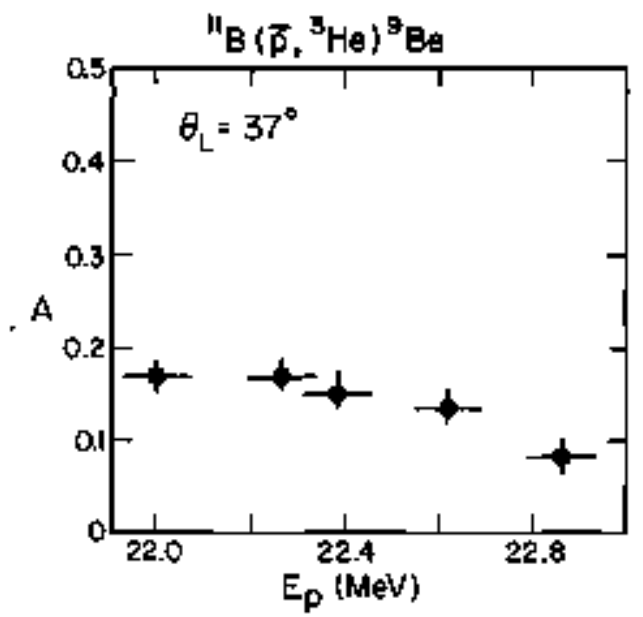

Fig. 2. Excitation function of $\mathrm{A}\left(\theta_{1}=37^{\circ}\right)$ in the ${ }^{\circ} \mathrm{Be}(\overrightarrow{\mathrm{p}}$, $\left.{ }^{3} \mathrm{He}\right){ }^{1 \mathrm{~B}}$ reaction.

\section{Foornotes and References}

"Condensed from LBL 1 1576, invited paper, High-Energy Physics wjth Polarized Beams and Polarized Targets, eds. C. Joseph and J. Sofer, Experientia Suppl. 38 (Birkhäuser Verlag, Basel, (981), p. 364.

†Institur für Strahlen und Kernpbysik, Universität Bonn, D-5300 Bonn, Germany.

'Laboratoire de Physjque Nucleaire, Université Laval, Québec GIK7P4, Canada.

1. R.J. Blin-Stoyle, Proc. Phys. Soc. A 65, 452 (1952); G.R. Satchiter, Nuci. Plyss, 8, 65 (I\$58); L.C. Biedenharn, Nucl. Phys. 10, 620 (1959).

2. M lrshad et al., Nucl. Phys. A 265, 349 (1976); M. Irshad et al., Nucl. Ptys. A 286, 483 (1977).

\title{
Time Reversal and Charge Symmetry Studies in Single Nucleon Transfer Reactions in the A $=5$ System ${ }^{*}$
}

\author{
A.L. Sagle, F.P. Brady, J.L. Romero, ${ }^{\dagger}$ B.E. Bonner, \\ N.S.P. King, ${ }^{\ddagger} M . W$. MeNaughron, ${ }^{\ddagger}$ and H.E. Conzet!
}

Time-reversal invariance (TRI) and charge symmetry (CS) are such important fundamental postulates in the description of nuclear interactions that any significant test of their experimental consequences should be pursued. Very recent reports of spin-polarization experiments in some nuclear reactions show results which differ substantially from those expected on the basis of TRI and CS, so it is clear that additional tesis of these symmetry principles are most appropriate.

Concerning TRI, Slobodrian et al. ${ }^{1}$ reported on comparisons of the polarization (P), in the ${ }^{7} \mathrm{Li}\left({ }^{3} \mathrm{He}, \overrightarrow{\mathrm{p}}\right)^{9} \mathrm{Be}$ and ${ }^{5} \mathrm{Li}\left({ }^{3} \mathrm{He}, \overrightarrow{\mathrm{p}}\right){ }^{11} \mathrm{~B}$ reactions, with the analyzing-power (A) in the inverse reactions. Large differences between $\mathbf{P}$ and $\mathbf{A}$ were found. Since the P-A equality follows directly from TRJ, ${ }^{2}$ conffirmation of this result of corroborative $\mathbf{P}-\mathrm{A}$ differences in other reactions would, indeed, constitute firm evidence for violation of TRI. Additionally, Conzett ${ }^{3}$ has examined all of the previous polatization tests of TRI in nuclear scattering and reactions, and he shows that almost all of them are really nontests of TRI and that the remaining ones are inadequate due either to lack of precision or lack of sensitivity to a violation of TRI. In view of these developments, it is now particularly important that new lests of TRl be mide.

With respect to charge symmetry, a simjlar $P_{n}-A$ equality, which follows from the combination of TR and $C S$, has been established in ( $p, n)$ transitions between the mirror nuclear slates of an isospin doublet. ${ }^{4}$ Here, $P_{n}$ is the neutron polarization in the $(\mathrm{p}, \overline{\mathrm{n}})$ transition and $\mathrm{A}_{\mathrm{n}}$ is the proton analyzing power in the $(\bar{p}, n)$ transition with palarized protons. This, bowever, is not an exact equality since the Coulomb force breaks the charge synunetry. Early $\mathbf{P}_{\mathbf{n}}$ - A differences were reduced to equalities by later, more precise measurements, 3,6 but surprisingly large differences were found ${ }^{6}$ in the ${ }^{15} \mathrm{~N}(\mathrm{p}, \mathrm{n}){ }^{15}{ }_{0}$ reaction for proton energies in the region of 5 to $9 \mathrm{MeV}$, where resonance structure is prevalent. Thus, tests of CS in nuclear reactions, as well as TRl, are now of particular interest.

We report here exiensive use of the P.A equality in metsurecrents of single nucleon transfer reactions in the mass-5 system. In one sel of measurements this theorem is checked by comparing the andyzing power of ${ }^{4} \mathrm{He}(\bar{\beta}, \mathrm{d})^{3} \mathrm{He}$ with the polarization of ${ }^{3} \mathrm{He}(\mathrm{d}, \overrightarrow{\mathrm{p}}){ }^{4} \mathrm{He}$ at equivalenl centerof-mass energies and angles. In a second experiment an absolute measurement is made of the neutron polarization in a double scattering experiment with the reactions $T(d, \vec{n})^{4} \mathrm{He}$ and ${ }^{4} \mathrm{He}(\overrightarrow{\mathrm{n}}, \mathbf{d}) \mathrm{T}$. The analyzing powess of the charge-symmetric reactions ${ }^{4} \mathrm{He}(\overrightarrow{\mathrm{p}}, \mathrm{d}){ }^{3} \mathrm{He}$ and ${ }^{4} \mathrm{He}\left(\overrightarrow{\mathrm{n}}_{1}, \mathrm{~d}\right) \mathrm{T}$ are then compared and found to be in agreement.

In the first experiment the analyzing powers wese measured for the reaction ${ }^{4} \mathrm{He}(\bar{p}, \mathrm{~d}){ }^{3} \mathrm{He}$ at $32,40,50$, and $52.5 \mathrm{MeV}$ at the Lawrence Berkeley Laboratory using polarized protons from the 88-Inch Cyclotron. In Fig. 1 


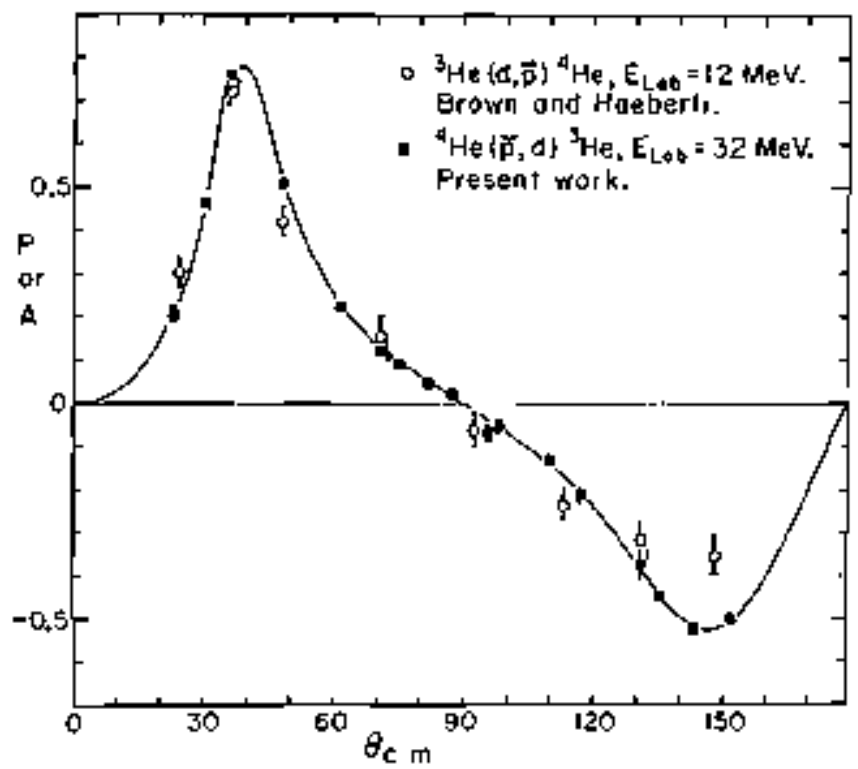

Fig. 1. Analyzing power angular distribution for the ${ }^{4} \mathrm{He}(\overrightarrow{\mathrm{p}}, \mathrm{d}){ }^{3} \mathrm{He}$ reaction (solid squares) compared with the polarization measurements of the ${ }^{3} \mathrm{He}(\mathrm{d}, \overrightarrow{\mathrm{p}})^{4} \mathrm{He}$ reaction ${ }^{7}$ at the same (compound nucleus) center-of-mass energy. The plotled data of Ref. 7 do not include an estimated 5 to $10 \%$ uncertainty in the analyzing power of their polarimeter. The curve is a Legendre polynomial fil to the "He $(\overrightarrow{\mathrm{p}}, \mathrm{d})^{\mathrm{j}} \mathrm{He}$ data.

(XBL B19-1782)

we eompare the analyzing power for this reaction at $\mathrm{E}_{\mathrm{p}}=$ $32 \mathrm{MeV}$ with earlier measurements ${ }^{\top}$ of the proton polanzation in the inverse reaction ${ }^{3} \mathrm{He}\left({ }_{(1, \vec{p})}{ }^{4} \mathrm{He}\right.$ at $12 \mathrm{MeV}$. These energies are equivalent in the center of mass because of the large reaction $Q$ value $(18.6 \mathrm{MeV})$. Although there is general agreement between $P$ and $A$, the datum for ${ }^{3} \mathrm{He}(\mathrm{d}, \mathrm{p})^{4} \mathrm{He}$ at ${ }^{8} \mathrm{~cm} .=140^{4}$ is higher than the rend of the ${ }^{4} \mathrm{He}(\vec{p}, d)^{3} \mathrm{Hc}$ data. This disagreement, however, may not be significant because of the large uncertainty in the value of the $\mathrm{p}^{12} \mathrm{C}$ analyzing power used by Brown and Haeberli. ${ }^{7}$

In the second experiment an absolute measurement of the polarization was made with the $50 \mathrm{MeV}$ polarized neutron facility at the Crocker Nuclear Laboratory 76-lnch Cyclosron by using a double scattering technique. The polarized neuirons produced at $\theta_{L a b}=29.7^{\circ}$ from the reaction $T(d, \vec{n})^{4}$ He impinged on a liquid-nitrogen-cooled ${ }^{4} \mathrm{He}$ gas cell. Simultaneous left-right measurements were then made of the deuterons from the reaction ${ }^{4} \mathrm{He}(\overrightarrow{\mathrm{n}}, \mathrm{d}) \mathrm{T}$ at $\theta_{\text {Lab }}=25^{\circ}$ with a pair of plastic scintillation counter telescopes. At these angles, the center-of-mass entergies and angles are the same for these inverse reactions and the polarization of the neutrons produced in the first teaction is equal to the neutron analyzing power of the second reaction from the P-A equality. The measured result was $f=0.231 \pm 0.015$, and use this value to extract the analyzing power $\left(\epsilon=\mathrm{P}^{2}=\mathrm{A}^{2}\right)$ of $0.480 \pm 0.016$.

The datum for the analyzing power at $\theta_{\text {Lab }}=25^{\circ}$ is also a measurement of the $50 \mathrm{MeV}$ neutron beam polarization if the P-A equality is valid. We have verified this equality at forward angles and at a lower energy for the charge sysnrnetric reaction ${ }^{3} \mathrm{He}(d, \vec{p})^{4} \mathrm{He}$ and its inverse. When we use the absolute measurement of the neutron polarization, the angular distributions for ${ }^{4} \mathrm{He}(\vec{n}, d) \mathrm{T}$ and ${ }^{4} \mathrm{He}(\bar{\beta}, \mathrm{d}){ }^{3} \mathrm{He}$ at $50 \mathrm{MeV}$ agree quite well.

We thus conclude that our set of measurements for these (nucleon,d) reactions are all consistent (within experimental errors) with time-reversal invariance and charge symmetry.

\section{Footnotes and References}

"Condensed from LBL-13232.

Crocker Nuclear Laboratory and Departument of Physics, University of California, Davis, CA 95616.

Foos Alamos National Laboratory, Los Alamos, NM 87545. J. R.J. Slobodrian, C. Rioux, R. Roy, H.E Conzett, P. Conzett, P. von Rossen, and F. Hinterberger, preprint; H.E Conzett, Polarization Phenoment in Nucleor Physics-1980, AIP Conf. Proc No. 69, ed. G.G. Ohlsen et al. (Ann. Inst. of Phys., New York, 1981), p. 1422.

2. R.J. Blin-Stoyle, Proc. Phys Soc. A 65, 452 (1952); G.R. Satchler, Nucl. Pbys. 8, 65 (1958); L.C. Biedenharn, Nucl. Phys. 10, 620 (1959).

3. H.E. Conzett, Ref. 1, p. 1452 .

4. H.E. Conzett, Phys. Lett. B 51, 445 (1974); L.G. Arnold, Proceedings of the Fourth International Sympasium on Polarization Phenamena in Nuclest Reactions, eds. W. Grítblet and V. König, Experientia Supp'. 25 (Birkhäuser Verlag, Basel, 1976), p. 503; Bull. Ann. Phys. Soc 22, 588 (1977).

5. T.R. Donoghue, et al., Phys. Rev. Lett. 37, 981 (1976).

6. R.C. Byrd et al. Bull. Am. Phys. Soc. 22, 587 (1977); R.L. Walter and R.C. Byrd, The $(p, n)$ Reaction and the Nucleon-Nucleon Forte, ed. C.G. Goodman at al. (Plenum, New York, 1980), p. 469; R.C. Byrd and R.L. Walter, Polarization Phenonena in Muclear Physics-1980, AlP Conf. Proc. No. 69, ed. G.G. Otlsen et al. (Am. Inst. of Phys., New York, 1981), p. 1475.

7. R.I. Brown and W. Haeberli, Phys. Rev. 130, 1163 (1963). 


\title{
Polarization Effects in Light Nuclei ${ }^{*}$
}

\author{
H.E. Conset!
}

This article is the rapporteur's report, prepared by the author, of the meeting on Podarization Phenomena in Nuclear Physics, August I]-15, 1980 at Santa Fe, New Mexico. The discussion during the session on Polarization Effocts in Light Nuclel was limited to the following topics:

1. The deuteron D-state.

2. The three-nucleon system.

3. Polarization vs analyzing-power in the ${ }^{15} \mathrm{~N}(p, n){ }^{15} \mathrm{O}$ reaction.

\section{Deuteron D-State}

The D-state of the deuteron received the most discussion, probably because there bas been a flurry of activity concerning it, both theoretical and experimental, during the past 1wo years. In separate papers, Arrado and Firar suggested that the D-state probability, $\mathrm{P}_{\mathrm{D} \text { e }}$ is not really accessible to experimental determination. At about the same time, Amado, Locher, and Simonius showed that $\nu_{D}$ the asymptotic $D$ to $\$$-state ratio of the deuteron wavefunction, was experimentally determinable. This ratio is defined as:

$$
\rho_{D}=\left[\mathrm{U}_{2}(\mathrm{r}) / \mathrm{U}_{0}(\mathrm{r})\right]_{\mathbf{r}-\infty},
$$

where $U_{2}(r)$ and $U_{0}(r)$ are the deuteron $D$ and S-state radjal wavefunctions, respectively. Although their first preseription for the determination of $\rho_{\mathrm{D}}$ had to be changed a bit, the method is very clear and difect. First, measure the differential cross section and the tensor anlyzing. power component $\mathrm{T}_{22}(\theta)$ in elastic $-\mathrm{p}$ scattering. Then, construet the lunction

$$
f(z)=k^{2} \phi(z) T_{22}(z)\left(z-z_{p}\right)^{2} /\left(1-z^{2}\right),
$$

where $z=\cos \theta$. Next, extrapolate $\Upsilon(z)$ to the nucleon exchange pole at $z_{p}=-\left(5 / 4+9 B / 4 E_{d}\right)$, with $B$ and $E_{d}$ the deuteron binding energy and the deuteron lab energy, respectively. Then one deduces op directly from

$$
f\left(z_{p}\right)=-0.054 z_{D}
$$

Since $\rho_{\mathrm{D}}$ must be independent of $\mathrm{E}_{\mathrm{d}}$, the incident deuleron energy, it is important to do the experiment over a range of $E_{d}$ in order to evaluate the consistency of the extrapolation procedure. The Zurich group reported very consistent results from measurements at ten energies between $E_{d}=5$ and $45.4 \mathrm{MeV}$. Their final result is

$$
\rho_{\mathrm{D}}=0.0259 \pm 0.0007 \text {, }
$$

where the error includes both statistical and datanormalization uncertainties. This value is in excellent agreement with the earlier Berkeley result

$$
\rho_{\mathrm{D}}=0.0263 \pm 0.0013
$$

In a completely different experiment, the Wisconsin group have determined $\rho_{D}$ from measurements of the tensor analyzing powers in the ${ }^{206} \mathrm{~Pb}(\overrightarrow{\mathrm{d}}, \mathrm{p})^{209} \mathrm{~Pb}$ stripping reaction at sub-Coulomb energies. In the DWBA calculations these analyzing powers scale directly with $\rho_{\mathrm{D}}$, and the calculated fits to these dats have yielded

$$
\rho_{\mathrm{D}}=0.02649 \pm 0.00043
$$

at their latest value. This is some $14 \%$ larger than their original (1975) value, and I assume that data taken at lower energies and mare contrplete DWBA calculations are responsible for the change. Clearly, Eqs. (3) (4) and (5) are in complete agreement. I would, however, caution that once the experimental uncertainties are reduced to the level of (3) and (5), one must be concerned with the "theoreti. cal" uncertainties of the extrapolation procedure, on one hand, and of the DWBA calculation, on the other.

\section{Three-Nucleon System}

The discussion of the three-nucleon system centered on the present state of agreernent or disagreement between experimental results and results calculated with the Faddeev equations (principally by Doleschall). Recent meassurements of deuteron break-up cross sections in the ${ }^{2} \mathrm{H}(\mathrm{p}, 2 \mathrm{p}) \mathrm{n}$ reaction at $26 \mathrm{MeV}$ were reproduced very well by the calculations. Doleschall discussed the major outstanding disagreement in elastic nucleon-deuteron scallering. The cross sections and the nucleon and deuteron analyzing powers have been measured at several energies up to $E_{N}=23 \mathrm{MeV}$. The major djscrepancy between experiment and theory is found with the nuclecen antyzing power $\mathrm{A},(\theta, \mathrm{E})$. Above $\mathrm{E}_{\mathrm{N}}=10 \mathrm{MeV}$ there is a clear and increasing difference with increased energy, which certainly cannot be repaired by including the Coulomb inleraction in the alculations. The usual concern is that not enough NN partial waves are included, but a calculation which included F-waves gave essentially the same result for $A_{1}(\theta)$ as the calculation which onjtted them. All is not lost, however, since the ${ }^{3} \mathbf{P}_{0,1,2}$ waves and the ${ }^{3} \mathbf{S}_{1}-$ ${ }^{3} D_{1}$ mixing parameter $\epsilon_{1}$ ase still not firmly pinned down by the lower energy NN scaltering data. In fact, high precision $\overrightarrow{p p}$ analyzing power data reported here are not fitted by the most up-to-date NN potentials. Some adjustment of the ${ }^{3} \mathbf{P}$-waves is very likely necessary and $\mathbf{j t}$ is known that 
the $\vec{N} d A_{y}(\theta)$ is quite sensitive 10 these. So, between that and $t_{1}$ there may still be enough flexibility to bring experiment and calculation into agreement for the $\overline{\mathrm{N}} \mathbf{d}$ analyzing powers.

$$
\text { P-A in }{ }^{15} \mathrm{~N}(\mathrm{p}, \mathrm{n}){ }^{15} \mathrm{O}
$$

There was a brief discussion of the polarization ws analyzing power in the ${ }^{15} \mathrm{~N}(\mathrm{p}, n)^{15} 0$ reaction, specifically

$$
P \text {, in }{ }^{15} \mathrm{~N}(\mathrm{p}, \mathbf{n})^{150}
$$

and

$$
A_{p} \text { in }{ }^{15} \mathrm{~N}(\overrightarrow{\mathrm{p}}, \mathrm{n})^{15} \mathrm{O} \text {. }
$$

Their difference is given by

$$
\mathbf{P}-\mathbf{A}=\left(\sigma^{-+}-\sigma^{+-}\right) / \sigma \text {. }
$$

with $\sigma^{-+}$the cross section for the nucleon transverse spin flip from down to up and $\sigma$ the unpolarized cross section.
Since $P-A=0$ follows from time-reversal invariance and charge symmetry, the Iarge $\mathrm{P}$ - A difterences found in this reaction for $\mathrm{E}_{\mathrm{p}}=5109 \mathrm{MeV}$ were quile unexpected and exciting. As was detailed in Philpott's talk earlier in this conference, these difierences are very nicely explained qualitatively via microscopic shell-model calculations which include the necessary non-central nucteon-nucleon interac. tion and isospin mixing. It is noteworthy that almast 25 years ago Wilkinson selected this ${ }^{15} \mathrm{~N}(\mathrm{p}, \mathrm{n}){ }^{15} \mathrm{O}$ reaction is a prime candidate for isospin mixing at the excitation energies spanned in this experiment. Even though either timereversal violation or charge-symmetry breaking could lead to $\mathrm{P}-\mathrm{A} \neq \mathrm{O}$, in view of the very satisfying explanstion in terms of isospin mixing I would be the last person at this conference to even suggest that time-reversal violation might, also, be involved.

\section{Foornote}

'Condensed from Rapporteur's Report in Polarization Phenomeno in Nuclear Physics-Ig80, eds. G.G. Otisen et al., AlP Conf. Proc. No. 69 (American Institute of Physics, New York, 1981).

\title{
Search for Effect of Longitudinally Polarized Protons on Optically Active Amino Acids"
}

\author{
R.M. Lemmon, H.E. Conzett, and W.A. Bomert
}

Many efforts have been madel to find an experimental demonstration of the hypothesis of Vester and Ulbricht ${ }^{2}$ To explain the fact that prodein amino acids are (with soute eninor exceptions in bacteria) entirely formed from the $\mathrm{L}$ optical isomers, this hypotbesis postulates that the excess of "anti-parallel" polarized beta particles from natural radionuclides, i.e., parity violation, gave a slight preference for the genesis of L-amino acids over D-amino acids on the prebiological Earth. This preference would arise from the betas' interactions in such a way as to selectively favor the formation of $L$-amino and/or promote the selective decomposition of D-amino acid,

Experimental work on the Vester-Ulbricht (V-U) hypothesis has included studies on a great variety of optically active organic componnds that have been irradiated with betas ${ }^{3}$ and positrons ${ }^{4}$ from radionuclides and with accelerated, polarized electrons ${ }^{5}$ and muons. ${ }^{6}$ The results have been mixed, but il ean be said at once that no report of a claimed selective oplical isomer-polarized particle interaction bas been successfully duplicated in another laboratory. The V-U hypotbesis is still considered a reasonable one, bui, without doubt, it still lacks refroducible experimental confirmation.

Another promising approach to test the V-U hypothesis is through the use of polarized protons. Sucb protons are available with the equipment recently installed on the Lawrence Berkeley Laboratory's sector-focused 8BInch Cyclotion. The polarized beam from the cyclotron has its spin-polarization axis aligned along the cyclotron magnetic field direction (vertical). The external beam first passes along the axis of a solenoid magnetic field, which precesses the spin direction through $90^{\circ}$ into the horizontal plane, although still transverse to the beam direction. A dipole magnet than bends the bean through an angle of $47.7^{\circ}$, and, at the same time, precesses the spin axis into the beam direction. Thus, the vertical polarization (up or down) of the proton beam from the cyclotron is converted to longitudinal polarization (positive is in the direction of the beam's propagation, end negative is in the reverse direction). The beams incident upon the samples were typically of about $10 \mathrm{MeV}$ and were limited to $10-14$ nanoamps. The degree of polarization for both proton spin directions was approximately $80 \%$.

We have carried ont six irmadialions with polarized protons. Each irtadiation was done on a pair of DL-leucine disks, each about $\mathrm{I}$ cm in diameter, about 0.1 cm thick, and weighing $110-130 \mathrm{mg}$. Leucine was the amino acid of choice because it gives an excellent separation of its D- and L-optical isoners on the optically active gas chromatographic tumn. The analyses of the irradiated samples are shown in Table $\mathrm{I}$. 
Table I. Percent of optical isomers remaining in DL-leucine irradiated with longitudinally polarized protons.

\begin{tabular}{crccc}
\hline Expt. & Beam $^{\mathrm{a}}$ & \% D & \% L & $(+)^{\mathrm{f}}$ \\
\hline 1 & A & 50.12 & 49.88 & $\cdots$ \\
& PT & 49.99 & 50.01 & $\cdots$ \\
2 & A & $50.14^{\mathrm{b}}$ & $49.86^{\mathrm{b}}$ & 0.04 \\
& PT & $50.01^{\mathrm{c}}$ & $49.99^{\mathrm{c}}$ & 0.13 \\
3 & A & 50.11 & 49.89 &.- \\
& PT & 50.12 & 49.88 & -- \\
4 & A & $50.20^{\mathrm{b}}$ & $49.80^{\mathrm{b}}$ & 0.11 \\
& PT & $50.20^{\mathrm{d}}$ & $49.80^{\mathrm{d}}$ & 0.14 \\
5 & A & $49.98^{\mathrm{d}}$ & $50.02^{\mathrm{d}}$ & 0.11 \\
& PT & $49.96^{\mathrm{d}}$ & $50.04^{\mathrm{d}}$ & 0.15 \\
6 & A & $50.23^{\mathrm{e}}$ & $49.77^{\mathrm{2}}$ & 0.19 \\
& PT & $50.12^{\mathrm{d}}$ & $49.88^{\mathrm{d}}$ & 0.06 \\
\hline \hline
\end{tabular}

${ }^{A} \mathrm{~A}=$ =beam absorbed in sample;

PI = beain passed litrough sample.

b Average of 2 GLC analyses.

Average of 4 GLC analyses.

Average of 3 GLC analyses.

Average of 7 GLC analyses.

IStandard deviation of replicate analyses.
It is apparent from Table I that we have not detected any asymmetsic degradation of BL- leucine with longitudinally polarized protons, even at $\$ 0 \%$ gross degradation.

\section{Footnotes and References}

"Condensed from LBL-12243; Otigins of Life, in press.

'Department of Chemistry, Slanford University, Stanford, CA 94305

1. D.C. Walker, ed., Origins of Optical Activity in Nature. Elsevier, Amsterdam, $197 \%$.

2. T.L.V. Uibricht and F. Vester, Tet. 18, 629 (1962).

3. W.A. Boriner and R.M Lemmon, Bioorg. Chem. 7, 175- 187 (1978),

4. Y.C. Jean and H.J. Ache, J. Phys. Chem. 81, I157 (1977).

S. W.A. Bonner et al,, Isratel J. Chem. 15, 89 (1976/77);

L.A. Hodige et al., Nature 280, 250 (1979).

6. R.M. Lemunon et al. Nature 252, 692 (1974). 


\title{
4. Nuclear Data Sheet Evaluation
}

\section{Nuclear Data Sheets for $A=169$ and $A=193$}

\author{
V.S. Shinley
}

Data pertaining to the levels of all nuclei with mass $A=169$, as populated both by radicactive decay and in nuclear reactions, were evaluated for publication. ${ }^{1}$ First, individual level schemes were compiled for each decay mode or reaction for each nucleus. Then a summary set of "adopred" level properjes, including best energies, halflife values, nuclear motments, spin-parity assignnents, modes of deexcitation, and relationships to expectations from theory, was derived for each nucleus.

The evaluation/compilation process was straightforward and manageable for all of the $A \Rightarrow 169$ nuclei except for ${ }^{169} \mathrm{Yb}$. With 60 levels and $400 \gamma$ rays involwed in the 169 Yb levels populated in ${ }^{169} \mathrm{Lu}$ decay, and slightly fewer, but many overlapping, levels and $\gamma$ rays involved in the pepulation by thermal neutron capture, the ${ }^{16 \%}$ Yb evaluatiom process was overwhelmingly complex. Fhysics and mathernatic computer programs, designed to aid in the evaluation process, made it possible to sort oul the data and arrive at a more somplete level scheme for each mode of population than was possible in a study of tither mode alone. For example, $\gamma$-ray branchings from levels weakly populated in ${ }^{169}$ Ly decay could be deduced from the data for the same, more strongly populated Jevels in thermal neutron capture, and vice versa. The resulting level properties summarized in the "adopted" levels for ${ }^{169}$ Yb wete as consistent and complete as the data alowed.

Data for $\mathrm{A}=193$ were similarly evaluated and prepared for publication. ${ }^{2}$ Unlike the case with A - 169 , though, the evaluation of the A - 193 data was strajghtforward for every A $=193$ nucleus.

\section{References}

I. V.S. Shirley, Nuclear Data Sheets, submitted for publication, 1981.

2. V.S. Shírley, Nuclear Data Sheets, accepted for publication, 1981 .

\section{Nuclear Data Sheets for $A=187$ and $A=185$}

\author{
Y.A. Ellis-Akovali
}

The available experimental data pertaining to the structure of nuclei with mass numbers $A=187$ and A - 185 (Refs. I and 2 respectively) have been evaluated. The best data for decays and reactions, as well as the adopted properties of levels and gamunas deexciting the levels in each mucleus are presented Inconsistencies, discrepancies, and areas which need further study are noted Argurnents for adopted spin, parity and configuration assigmutents are also included.
Footnote and References

"Nuclear Data Project, Oak Ridge National Laboratory, Oak Ridge, TN 37830.

1. Y.A. Ellis-Akovali, Nuclear Data Sheets, submitted for publication, 1981.

2. Y.A Ellis-Akovali, Nuclear Data Sheets, accepted for publication, 1981 . 


\title{
Nuclear Data Sheets for A $=189$
}

\author{
R. B. Firestone
}

Radioactive decay and reaction data for nuclei with mass A = 189 have been evaluater. All data published through Seplember 1980 were covered. Some outstanding diserepancies in the literature were resolved, and new information has been inferred.

For ${ }^{189} \mathrm{~W} \beta^{-}$-decay, the available $\gamma$-ray data were combined with ${ }^{189} \mathrm{Re}$ reaction data to produce the first partial level scheme from that decay. Data for ${ }^{189} \mathrm{O}$ s levels were compiled with only minor revisions, except for the level at $233.53 \mathrm{keV}$; this level was previфusly assigned spin $5 / 2-$ but is morc consistent with spir $3 / 2-$ due to an apparent MI + E2 $\gamma$-ray feeding a levtl of known spin $1 / 2-$. The levels of ${ }^{185} \mathrm{Ir}$ still sufter from considerable ambiguity due to conflicting results in the literature, especially for reaction $\gamma$-ray data. Some $\gamma$ rays were placed without proper regard for intensity balances. The level at $2332.3 \mathrm{keV}$ was originally suggested as $25 / 2+$ and $27 / 2-$ by separate authors. 1,2 Re-analysis of the delayed $\gamma$-ray iptensity date ${ }^{3}$ in this evaluation allowed unambiguous determination of the critical conversion coefficients for $\gamma$ rays deexciting that level. Transitions from the 2332.3. $\mathrm{keV}$ level were determined to feed levels of $23 / 2+$ by EJ, 23/2- by Ml, and 21/2+ by $M / 2$ multipolarity. Thus, a new spin $25 / 2-$ was adopled in disagreement with the previous authors (Refs. I and 2).
Decay data for ${ }^{189} \mathrm{Pt}$ were less ambiguous but incornplete. It was necessary to estimate some $\gamma$-ray intensities on the basis of published spectra becanse values were not reported. Also, numerous multiple $\gamma$ ray assignments in the decay data were eliminated. ${ }^{89} \mathrm{Au}$ decay data were too incomplete to calculate logft values for the decay scheme. The reaction $\gamma$ ray data were not entirely consistent between authors, and discrepancies in the ordering of a rotational band built on the $440 \mathrm{~ms}$ isomer at $2678.1 \mathrm{keV}$ could not be resolved. The relative intensities of a critical unresolved $r$-ray doublet at $198.6 \mathrm{keV}$ were determined in this avaluation from the associated $\gamma$ ray intensity balances from the level scheme. Only minimal data exist for levels in ${ }^{189} \mathrm{Hg} .{ }^{189} \mathrm{Tl}$, ${ }^{189} \mathrm{~Pb}$, and ${ }^{189} \mathrm{Bi}$.

The status of data for mass $A=189$ remains inadequate. Insufficient and conflicting results abound for these da1a, and most $\mathrm{A}=189$ nuclei are still poorly characterized. The unavailability of convenient targets for production of most of these nuclei has retarded interest in producing then by light-ion reactions, but with heavier beams becoming available, mass $\mathrm{A}=189$ should deserve further interest.

References

I. P. Kemnitz et al., Nucl. Phys. A 245, 22] (1975).

2. S. Andre et al., Nucl. Phys, A 243, 229 (1975).

\section{Nuclear Data Sheets for $A=188^{*}$}

\author{
B. Singht and D. Viggars F $^{\dagger}$
}

Experimental data pertaining to the nuclear structure and decay of all nuclei with mass number $A=188$ have been evaluated. Level schemes from radiogetive decay and reaction studies are presented for each nucleus. An adopted level scheme is also included for each nucleus; it contains both adopted level and ganma propertics.

\section{Foothotes}

B. Singh and D. Viggars, Nuclear Data Shoets, accepted for publication, 1981 .

'Kuwait Institute for Scientific Research, Shuwaik, Kuwait.

†Physics Department, Kuwait University, Shuwaik, Kuwait. 


\title{
Nuclear Data Sheets for A $=191$
}

\author{
E. Browne
}

Experimental data for all nuclei with mass number A - I9l have been compiled and evaluated from over I70 reference papers. Level schemes Irom radioactive decay and reaction work, as well as other adopted nuclear properties, have been included. The literaure cut-off date is September 1979.'

A serious inconsistency in the level scheme of ${ }^{191} \mathbf{P t}$ from the electron-cupture decay of 191 Au led the evaluator to reinterpret the experimental data given in Ref. 2. Figure I shows some low-lying levels and comnecting transitions in ${ }^{191} \mathrm{Pt}$ from Ref. 3. The predominantly E] mullipolerity assigned to the $24,39 \mathrm{keV}$ transition in Ref. 2 on the basis of the conversion-electron subshell ratios $L_{1} / L_{2}=1.7 \pm 0.4$ and $L_{2} / L_{3}=0.65 \pm 0.15$ is not consistent with the placement of this transition between two even-parity states as suggested in Ref. 3. However, it is evident that, because of the extremely large values for the E2 conversion cofficients $\alpha\left(\mathrm{L}_{2}\right)$ and $\alpha\left(\mathrm{L}_{3}\right)(1390$ and

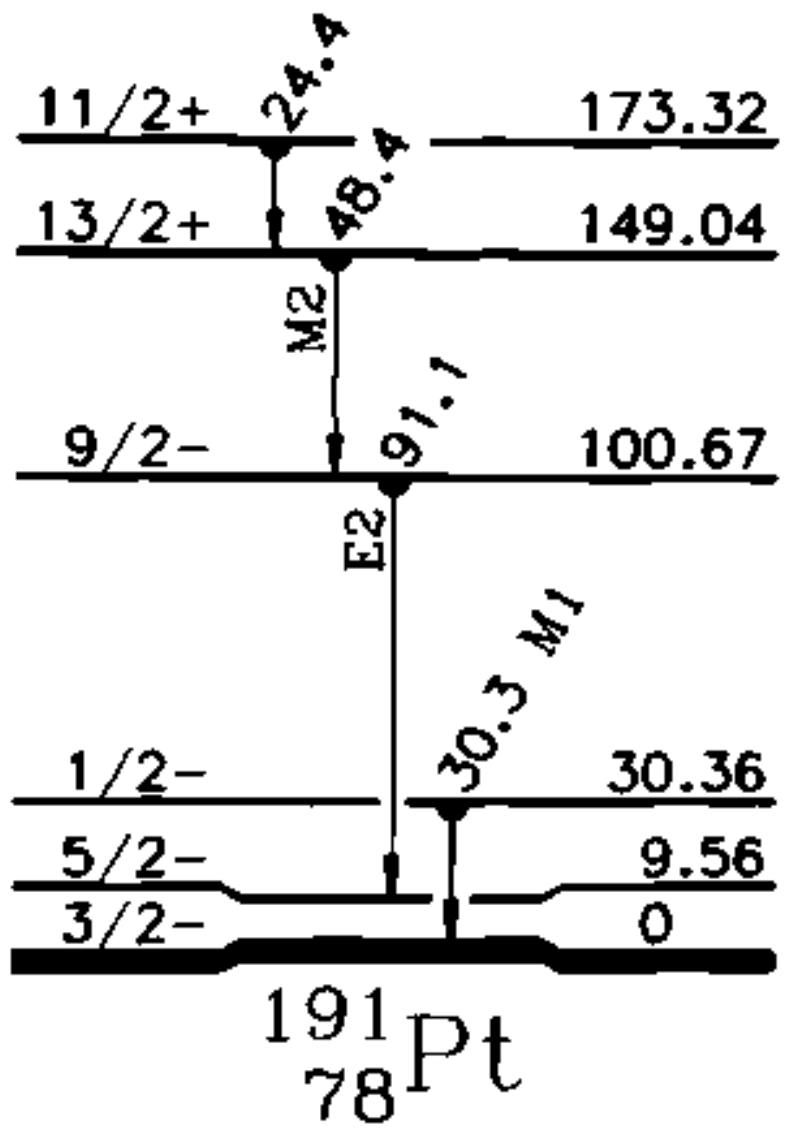

Fig. 1, Some low-lying ${ }^{191} \mathrm{Pt}$ levels from level scheme reported in Ref, 3.
1630 respectively) compared to $\mathrm{M} 1$ (5.4 and 0.59 respectively), the subshell ratio $L_{2} / L_{3}$ for an $M 1+E_{2}$ transition is not sensitive to the amount of E2 (above 2\%) mixed with Ml. The experimental subshell ratio $\mathrm{L}_{2} / \mathrm{L}_{3}=$ $0.65 \pm 0.15$ is essentially consistent within two standard deviations with the value of 1.0 expected from the $L_{1} / L_{2}$ experimental subshell ratio for an $\mathrm{M1}+2 \% \mathrm{E} 2$ tranșition. This multipolarity is consistent with the placement of the $24.39 \mathrm{keV}$ transition on the level scheme given in Ref. 3 .

Another inconsistency is shown is Table J, where the experimental conversion coefficients of the 30.27 and $87.55-\mathrm{keV}$ transitions in Ref. 3 are in total disagreement with the theoretical ones ${ }^{4}$ calculated on the basis of the assigned multipolarities. Either the photon intensities or the conversion-electron intensities, or both, are incorrect for these transitions. The intensity balance through the $149 \mathrm{keV}\left(\mathrm{J}_{\pi}=13 / 2+\right)$ level calculated using the eloctron inlensities given in Ref. 2 for the 24.27. and 48.37-keV transitions sbows essentially no electron-capture population to this level. This indicates that the electron intensities for these low-energy transitions are correct. Therefore, the electron intensities of the 30.27- and 87.5-keV transitions were assured to be correct, and their photon intensities recalculated using thecretical converșion coefficients. ${ }^{4}$ A new measurement of these photon intensities to confirm this interpretation would be very useful.

Table I. Comparison of Experimental and Theoretical LConversion Coefficients in $191 \mathrm{pt}$.

\begin{tabular}{|c|c|c|c|}
\hline $\begin{array}{c}\text { Energy } \\
(\mathrm{keV})\end{array}$ & $\begin{array}{l}\text { Exp. L-cons, }{ }^{a} \\
\text { coeff. }\end{array}$ & $\begin{array}{c}\text { Theor. L-conv. } \\
\text { coeff. }\end{array}$ & $\begin{array}{l}\text { Mutipolarity } \\
\text { assigned in Ref. } 3\end{array}$ \\
\hline 30.27 & 1.7 & $31(\mathrm{MI})$ & M1 \\
\hline 87.55 & 0.52 & $\begin{array}{l}1.4(\mathrm{M} 1) \\
5.9\left(\mathrm{E}_{2}\right)\end{array}$ & $\mathrm{M} 1 / \mathrm{E}^{2}$ \\
\hline
\end{tabular}

From Ret. 3.

brom Ref. 4.

\section{References}

1. E. Browne, Nucl. Data Sbeets 30, 653 (1980).

2. A Johansson et al., Nucl. Phys. A 98, 278 (1967).

3. M. Pijparinen et al., Nucl. Phys. A 265, 253 (1976).

4. R.S. Hager and E.C Seltzer, Nuct. Data A 4, 1 (1968). 


\title{
Eraluation of Nuclear Structure and Decay Data
}

\author{
C. Michael Lederer
}

${ }^{190} \mathrm{O}$ s has been restudied recently via the reaction ${ }^{189} \mathrm{OS}(\mathrm{n}, \gamma){ }^{1}$ and by Coulomb excitation with heavy ions. ${ }^{2}$ A summary diagram for this much-studied nucleus is shown as Fig. 1. The format of this diagram, preparted for use by the author, has several advantages over the ladder diagrams used for publication in the Nuclear Data Sheets. It shows explicttly which adopted level or levels correspand to a level observed in a particular reaction or decay scheme. it also shows the known $\gamma$-decay branches of the levels in an abbreviated form.

A recent finding ${ }^{3}$ of some imierest is the small magnitude and negative sign of the g-factor of the 24. J-ns isomer in ${ }^{19} \mathrm{H}_{\mathrm{g}}$ (Fig. 2). The measured value, $\mathrm{g}=$ $-0.21 \pm 0.02$, supports the assigntient of the isomer as a member of the $\left(\mathrm{ri}_{\mathrm{H} / 2}\right)^{2}$ band, whereas the $2596.8 \mathrm{keV}$ $\left(10^{+}\right)$state, previously assumed to be the isomeric stale, had been assigned the configuration $\left(\pi \mathrm{L}_{11 / 2}\right)^{2}$ in order to explain jts hindered $\gamma$ decay. ${ }^{4,5}$ An $\mathrm{h}_{\mathrm{L} 1 / 2}$ assignment for the isomer is inconsistent with the measufed $\mathrm{g}$-factor. It is thought that the isomer lies abowe the $10^{+}$state and decays to it by a low-energy, unobserved transition; further measurements to confirm and clarify this point are recommended.

\section{Footnote and References}

*To be published in Nuclear Data Sbcets (1981).

1. RF. Casten el al., Nuct. Phys. A 316, 61 (1979).

2. Douglas Cline, private communication (Oelober 1980 ); C.Y. Wu et al. Bull. Am. Phys. Soc. 25, 605, KF/2 (1980).

3. S.A. Hjorth et al., Phys. Rev, Lett 45, 878 (1980).

4. RM. Lieder et al., Nuct. Phys. A 248, 317 (1975).

5. T. Inamura et al., J. Phys, Soc. (Japan) 32, l 163 (1972).

Level energies are adopted volues that take into occount meosurements of the decay of the ${ }^{190} \mathrm{TI}$ isomer's otso. It is not known whether the 3015.8-keV stote decays to the $2596.8 \mathrm{keV}$ state (as shown), or to the 24.1-ns isomeric stote.

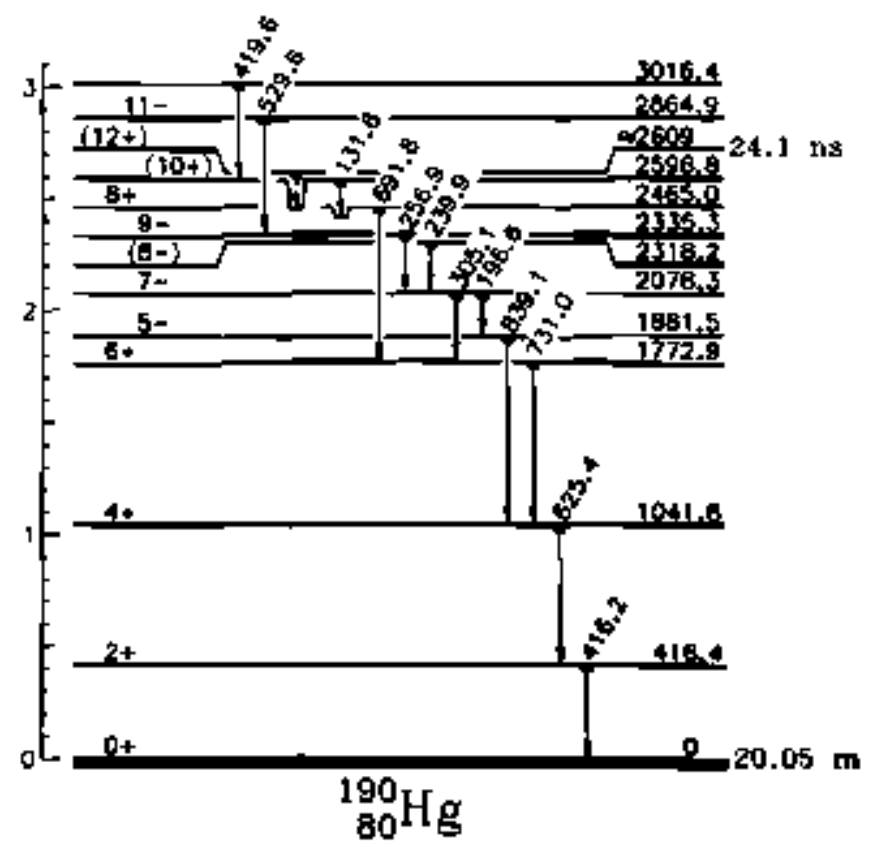

Fig. 2. Levels of ${ }^{190} \mathrm{Hg}$ observed jn (heavy ion, $x \mathrm{n}$ ) reactions. A recent measurement of the 8 -factor of the 24. J-ns state supports the assignment of a $\left(v_{13 / 2}\right)^{2}$ configuration wo the isomer, in contrast to previous interpretations, and requires the assumption of a low-energy, unobserved transition from the isomer.

(XBL 827-888) 


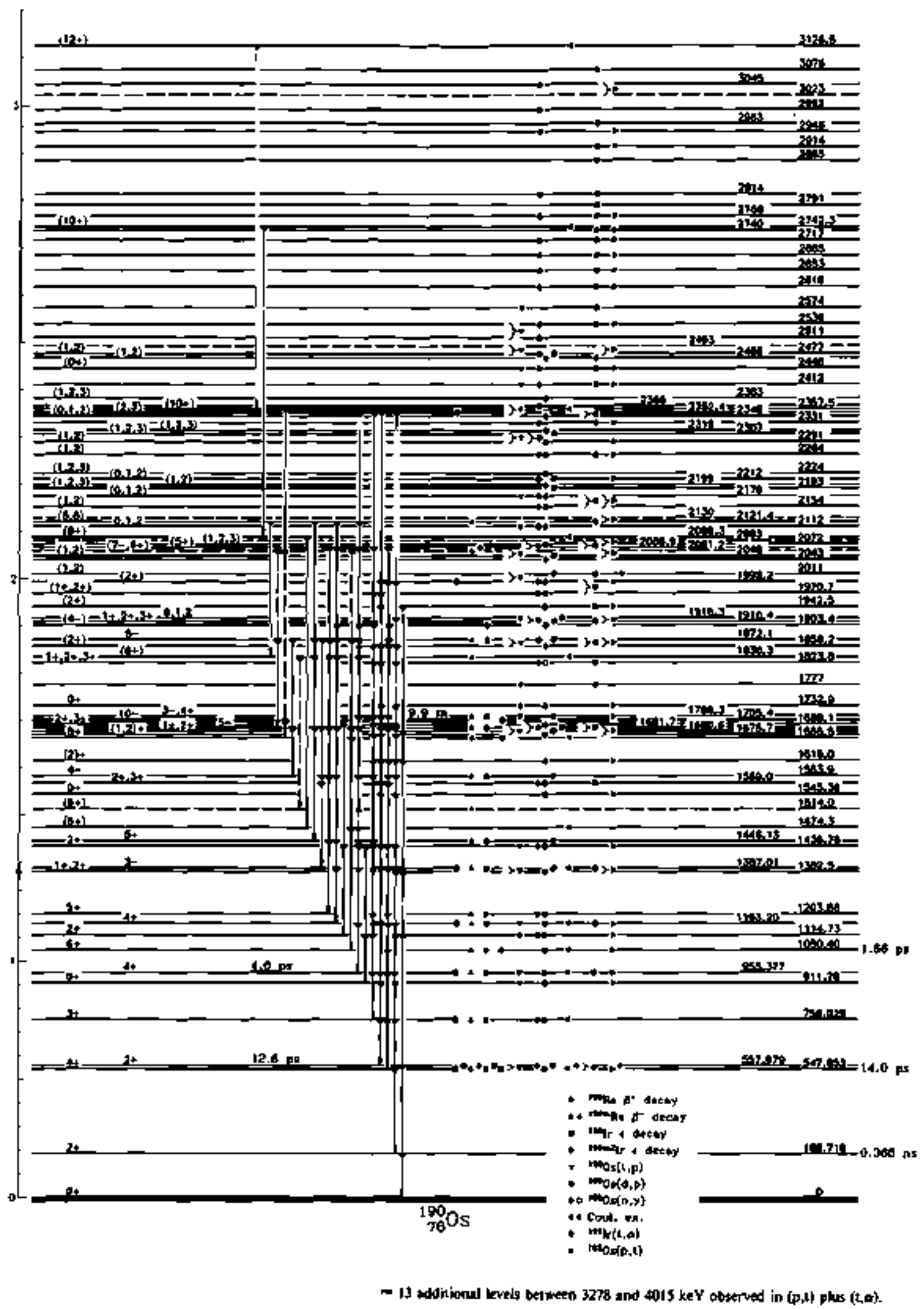

Fie. 1. Adopted levels of ${ }^{190} \mathrm{O}$, showing which decay parents and nuclear reactions populate each of the levels. (All parents and reactions also populate the ground and first excited states.) 


\title{
B. NUCLEAR REACTION MECHANISMS WITH NONRELATIVISTIC HEAVY IONS
}

\author{
1. Charged Particle Emission
}

\section{Apha Particle Emission from Two Reactions Involving Very Heavy Ions}

\author{
L.G. Sobotka, R.J. McDonald, GJ. Wozniak, D.J. Morrissey,
}

A.J. Pacheco, and LG. Moretto

There is some question as to whether light particle emission associaled with deep inelastic processes in heavy ion reactions can be explained by evaporation from the fully acselerated fragments ${ }^{1,2}$ or if some pre-scission ${ }^{3}$ or noneraporative mechanism is present. We have attempted to answer this question for two near symmetric beavy systems: ${ }^{n a t} \mathrm{Ag}+{ }^{84} \mathrm{Kr}(664 \mathrm{MEV})$ and ${ }^{181} \mathrm{Ta}+{ }^{165} \mathrm{Ho}$ (1354 MeV). By using coineidence lectiniques (beavy ion-light particle) involving five light-particle telescopes, we have measured coincidence light particle energy spectra over a large range of angles.

In order to address the question stated above, one can plot a vector diagram for the reaction and superimpose the extracted root mean square velocity, $\left\langle\mathrm{V}^{2}>1 / 2\right.$, for the $\alpha$ partictes. This has been done in Figs. 1 and 2 where we have plotted the $\left\langle V^{2}\right\rangle^{1 / 2}$ for the detected projectile-like fragment (gated on the DI events), the calculated velocity of the undetected fragment, and the measured velocity of the $\alpha$-particles. The full rings indicate the loci of expected a-particle velocities from three different rest frames.

One can clearly see from Fig. 1 that the trend in the data does nol agree with evaporation from a source moving with the system center-of-mass velocity. On the other hand, the data follow the prediction for evaporation from the target-like fragmint quite well. The systematic overprediction of $\left\langle\mathrm{V}^{2}>>^{1 / 2}\right.$ is probably due to an overestimate of the Conlomb barrier. Additional support for Iragtrent emission is seen in the energy spectra at the most forward angles, which show two distinct peaks. The associated $c V^{2}>1 / 2$ for both pealks clearly show that the lower energy peak corresponds to evaporation from the projectile-like fragment, while the higher energy peak is due to evaporation from the target-like fragment.

The same conclusion-that the bulk of the o-particles can be explained by evaporation from the target-like fragment-is reached after examining the data for the

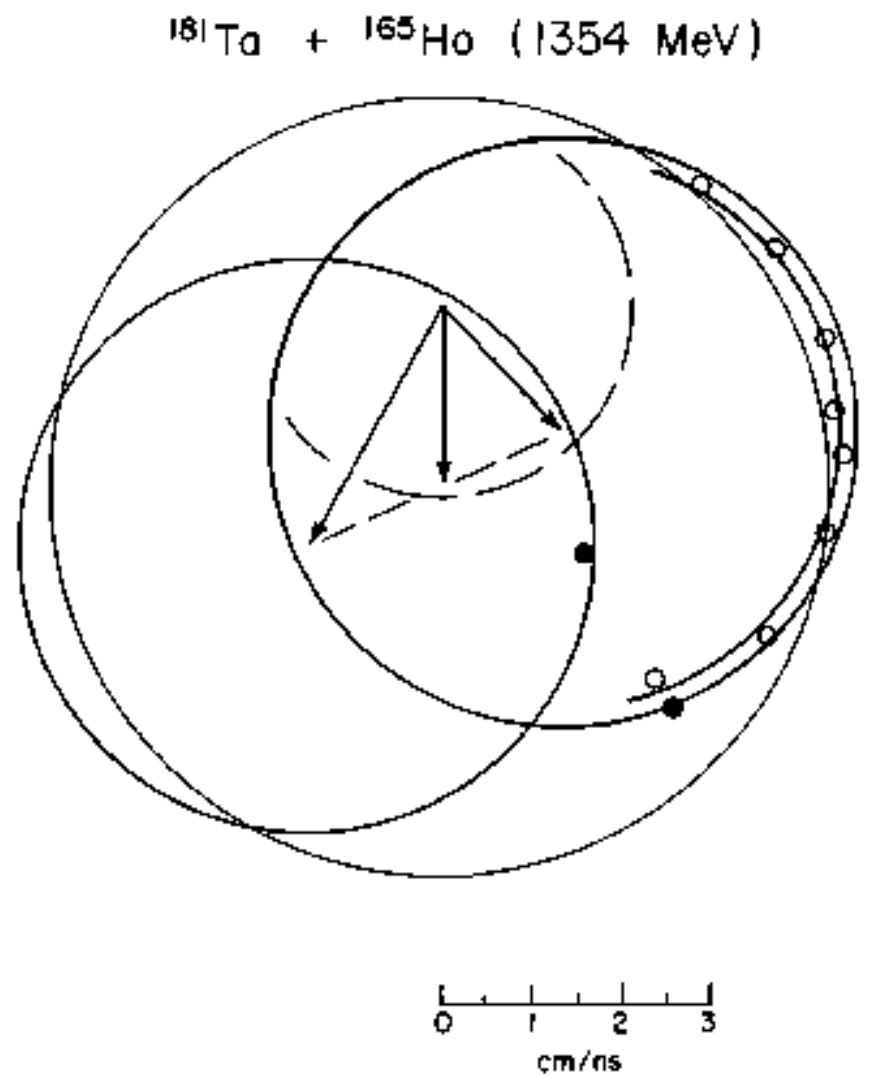

Fig. 1. Velocity diagram for the ${ }^{181} \mathrm{Ta}+{ }^{165} \mathrm{Ho}$ (1354 MeV) system. Open circles are velocities extracted from $\alpha$-perticle energy spectra. Solid circles indicate velocities of the two separate peaks in the most forward data. Large full rings indicate loci of expected o-particle velocities from the three different rest frames. For target-like fragment, locus of velocities for a $10 \%$ reduction in expected average emission energy is indicated by a partial ring. Detection threshold is shown as a dashed are.

(XBL 818-2452) 


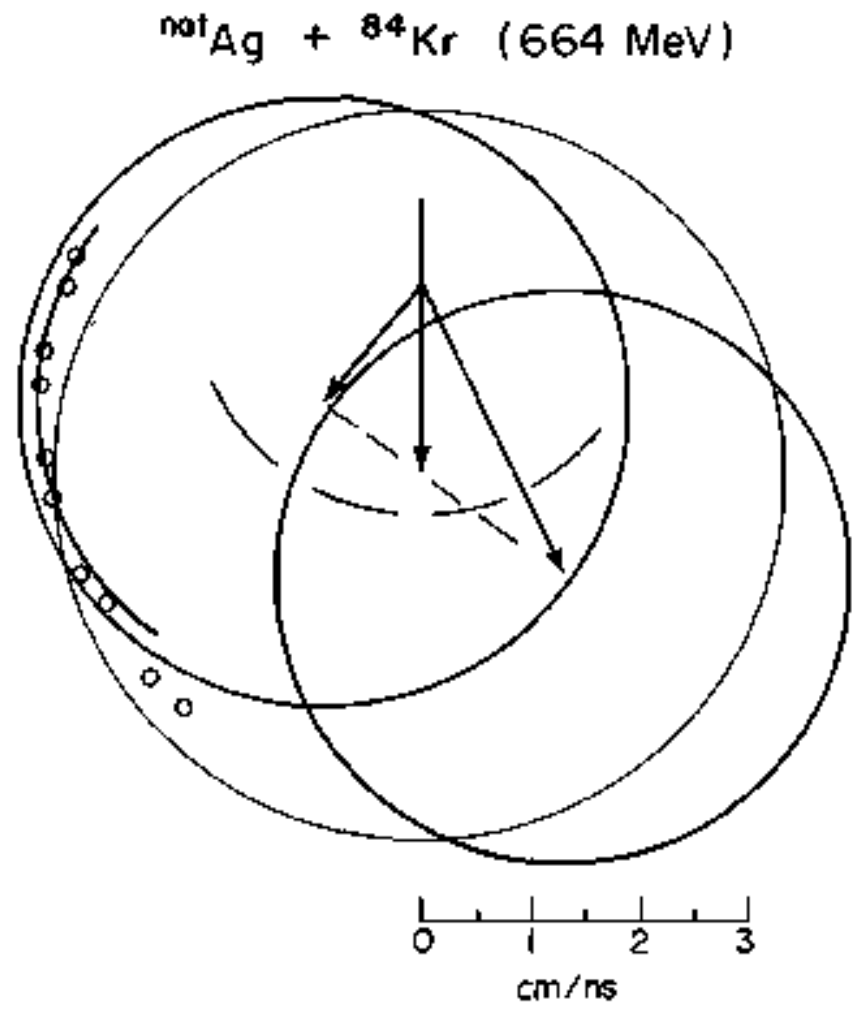

Fig. 2. Velocity diagrasn for the ${ }^{\text {nat }} \mathrm{Ag}+{ }^{B 4} \mathrm{Kr}$ (664 MeV) system; symbols same as Fig. 1.

(XBL 81\&24SI)
${ }^{\text {mat }} \mathrm{Ag}+{ }^{84} \mathrm{Kr}$ system shown in Fig. 2. Again, the trend of the data, except for the two most forward points, agroes with expectations for evaporation from the larget-like fragment The energy spectra for these angles exhibit a bighenergy component that is not explained by evaporation from either fragment. The claim of fragrnent exaporation is further supported by the lack of an angular dependence of the spectral shape when examined in the rest frame of the target recoil.

References

I. R. Babinet et al., Z Physik A 295, 153 (1980).

2. L.G. Sobotka et al., Phys. Rev. Lett. 46, 887 (1981).

3. D. Logan et al., Phys. Rev. C 22, 1080 (1980).

\title{
Energy Equilibration in Composite Nuclei at High Energy and Spin: Correlations between Evaporative ${ }^{1} \mathbf{H},{ }^{4} \mathbf{H e}$ and Fission
}

\author{
M.F. Rivet, D. Logan' J.M. Alexander," E. Dvek," M.S. Zisman, and M. Kaplant
}

Studjes' bave recently indicated that the dominant emission source of light charged particles in 200 to $340 \mathrm{MeV}{ }^{40} \mathrm{Ar}$ induced reactions with ${ }^{116 \mathrm{Sn}},{ }^{164} \mathrm{Dy}$, and ${ }^{197} \mathrm{All}$ is from the composites prior to seission. The arguments that led to this conclusion were based on the following observations:

1. The measured singles spectra were much narrower than those expected of evaporation from the thermally equilibrated fission fragments.

2. Similarly, the measured coincidence spectra for both in- and out-of-plane correlations with fission were quite difierent in shape, and moreover did not follow the trend in angular distribution expected for fission parentage. Here, as in the above case, the comparisons were made with simulated spectra generated by Monte Carlo calculntions.

3. The observed ratio of proton to alpha multiplicities suggested much larger than expected spins for fission parentage. (The measured values of $\ell_{\text {crit }}$ along with the sticking model allow a first-order estimation of the maximum spin for fission.)

4. The backward angle singles energy spectra in the center-of-mass system indicated emission barriers and temperatures consistent with exaporation from a thermally equilibrated composite.

In the present work, we have extended these measursments by recording the backward angle out-of-plane correlations between fission and ${ }^{1} \mathrm{H}$ and ${ }^{4} \mathrm{He}$ for the systems of $340 \mathrm{MeV} \mathrm{Ar}+\mathrm{Sn}_{4} \mathrm{Sm}$ and Au. It has been shown ${ }^{2}$ that these correlations may be expected to follow a distribution that gotes as exp $\left(\hat{\beta}_{2} \sin ^{2} \phi_{\text {con }}^{*}\right)$ where $\phi_{c \text { ti }}^{*}$ is the angle with respect to the spin vector of the emitter. For our systems, we have assumed emission from the composites and also that their spin vectors are wejl approximated by the normal to the plant determined by the fission detector and the beam axis. 
The above formalism has been extended ${ }^{3}$ to allow an iterative procedure between the back angle singles spectra and the measured $\beta_{2}$ parameter to infer some properties of the composites. However, this prosedure has merit only to the extent that the out-of-plane correlations, when integrated over light-particle and hovy-particle space, agree with the measured singles symuntric cross sections. This comparison is indicated in Fig. 1 lor alphas in the systerine of $\mathrm{Ar}+\mathrm{Sm}$ and $\mathrm{Ar}+\mathrm{Au}$. On the top are shown the center-of-mass singles angular distributions, the fits to the component symmetric aboul $90^{\circ} \mathrm{c.m}$. (solid lines), and the inferred cross sections $\sigma_{\text {exay }}$. The out-of-plane correlations are shown on the bottonf for each system, with the two coincidence geometries shows as insets. Indicated are the appropriate $\beta_{2}$ values as weil as the predicted total cross sections in coincidence with fission, $\sigma_{\text {dxinc }}$ As may be evident, a exinc agrees very well with $\sigma_{\text {suap }}$ for the Au system and is approximetely $80 \%$ for the Str system For the latter case, the small discrepancy is apparently due to eyaporation from the composites that survive fission. Thus, for the An system and, with slightly less confidence, the Sm system, the procedure alluded to above may be used to infer some properties of the composites. This was not the case for the Sn system, where only $20 \%$ of the singles events were found to be in coincidence with fossion.

The results of this analysis allow first-order estimites of the barrier to emission B, the temperature $\mathbf{T}^{\prime}$, the level parameter $\mathrm{A} / \mathrm{a}$, and the roo mean square spin $\mathrm{J}_{\text {fms }}$. These are shown in Table $I$ for alphas and protons for the indicated systems. Also entered in this table are the average center-of-mass energy in singles $\langle\langle<\rangle\rangle$, the expected emission barriers from fusion systematics, ${ }^{4}$ and the meas-

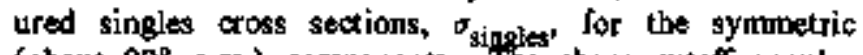
(about $90^{\circ} \mathrm{sm}$ ) compontents. The sharp cutoff angular momenta implied by the messured cross sections for evaporation residues and fusion are shown as $t_{E R}$ and $\ell_{\text {crit }}$

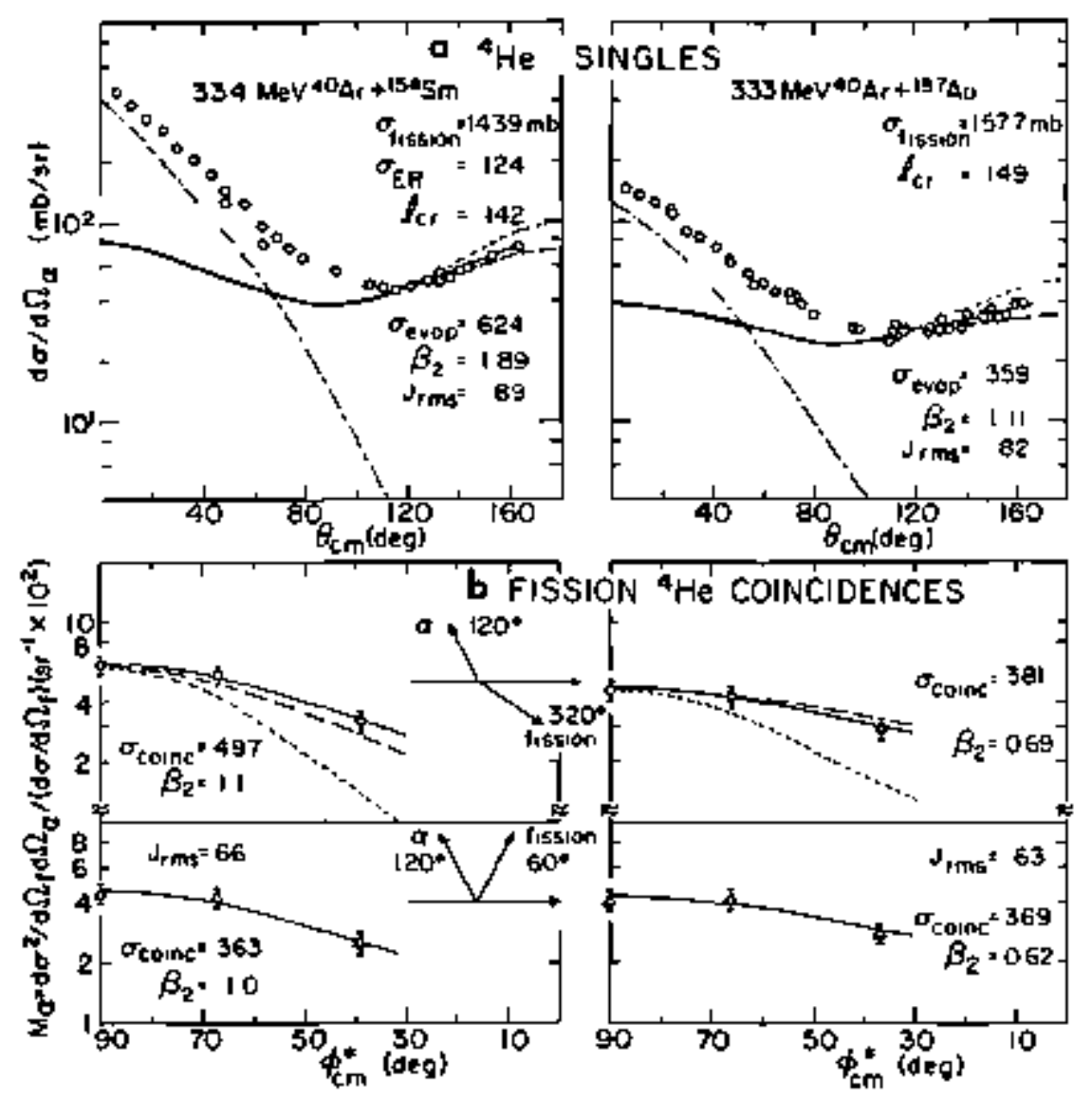

Fig. 1. (a) Measured angular distributions for ${ }^{4} \mathrm{He}$ (points) in the singles mode from Ref. I. The decomposition into evaporative and direct components is shown: solid line shows least squares fit of Eq. (12) of Ref. 3; dot-dash line shows djfference between data points and solid line. Also given are the related cross sections, $\ell_{\text {ctil }}{ }^{*} \beta_{2}$ and $J_{\text {tms }}$ values as taken from the singles data (see Ref. 1). Long and short dashed curves were drawn from Eq. (12) of Ref, 3 to indieate mínimura and maximum aceptable anjsotropies. (b) Out-of-plane correlations are shown from this work with solid lines indicating least-squares fits to Eq. (6) of Rer. 3. Also shows ate the resulting values of $\beta_{2}$ and the corresponding integrated coincidence cooss sections $\sigma_{\text {cainc }}$ Long and short dasshed curves were drawn fron Eq. (6) of Ref. 3 with minimum and maximum values of $\beta_{2}$ considered acceptable for the singles data as shown above.

(XBL 818-11284) 
Table L. Quantities derived from singles and coincidsnce data for reactions of $333 \mathrm{MeV} 40_{\mathrm{Ar}}+\mathrm{X}$

\begin{tabular}{|c|c|c|c|c|c|c|}
\hline & \multicolumn{2}{|c|}{${ }^{197}$ All } & \multicolumn{2}{|c|}{$1345 m$} & \multicolumn{2}{|c|}{$\operatorname{lig} \mathrm{sn}$} \\
\hline & ${ }^{4} \mathrm{He}$ & ${ }^{\prime} \mathbf{H}$ & ${ }^{4} \mathrm{He}$ & ${ }^{1} \mathbf{H}$ & ${ }^{4} \mathrm{He}$ & ${ }^{\mathrm{I}} \mathrm{H}$ \\
\hline \multicolumn{7}{|c|}{ Results from studies in singles mode } \\
\hline$T(M e V)^{a}$ & $2.54(+.1)$ & $2.06 \pm 0.1$ & $2.63 \pm 0.10$ & $2.30 \pm 0.15$ & $3.22 \pm 0.15$ & $2.44 \pm 0.08$ \\
\hline $\mathrm{A} / \mathrm{a}(\mathrm{MeV})^{\mathrm{b}}$ & 9.75 & 6.4 & 7.1 & 5.4 & 8.7 & 5.0 \\
\hline$\langle<s>>(\mathrm{MeV})$ & $24.0 \pm 0.3$ & $12.3 \pm 0.3$ & $22.1 \pm 0.1$ & $11.8 \pm 0.1$ & $21.4 \pm 0.3$ & $10.8 \pm 0.1$ \\
\hline $\mathrm{B}(\mathrm{MeV})^{\mathrm{c}}$ & $17.4 \pm 0.4$ & $7.3 \pm 0.2$ & $14.7 \pm 0.1$ & $6.2 \pm 0.3$ & 11] \pm 1.3$. & $5.4 \pm 0.1$ \\
\hline $\mathrm{B}_{\text {fusion }}(\mathrm{MeV})^{\mathrm{d}}$ & 22,35 & 12.0 & 19.2 & 10.4 & 17.14 & 9.35 \\
\hline$g_{2}^{c}$ & $1.11 \pm 0.58$ & $0.98 \pm 0.69$ & $1.89 \pm 0.55$ & $0.78 \pm 0.39$ & $1.70 \pm 0.70$ & $0.95 \pm 0.82$ \\
\hline $\mathrm{J}_{\mathrm{rms}} \mathrm{f} \cdot \mathrm{B}$ & 82 & 140 & 89 & 115 & 66 & 100 \\
\hline$\sigma_{\text {cosinc }}(m b)^{I_{1} B}$ & 252 & 145 & 287 & 348 & 232 & 356 \\
\hline$\sigma_{\text {singles }}(m b)^{l}$ & $359 \pm 54$ & $386 \pm 58$ & $624 \pm 94$ & $703 \pm 106$ & $1158 \pm 174$ & $1697 \pm 254$ \\
\hline$\ell_{\mathrm{ER}^{\mathrm{q}}}$ & \multicolumn{2}{|c|}{0} & \multicolumn{2}{|c|}{39} & \multicolumn{2}{|c|}{68} \\
\hline$\ell_{\text {erit }}$ & \multicolumn{2}{|c|}{149} & \multicolumn{2}{|c|}{142} & \multicolumn{2}{|c|}{112} \\
\hline \multicolumn{7}{|c|}{ Results from coincidence studies } \\
\hline \multicolumn{7}{|c|}{$\theta_{\mathrm{G}}=60^{\circ} \quad \theta_{\mathrm{S}}=120^{\circ}$} \\
\hline$\beta_{2}^{h}$ & $0.62 \pm 0.11$ & $0.09 \pm 0.07$ & $1.0 \pm 0.1$ & $0.26 \pm 0.13$ & $0.6 \pm 0.6$ & $0.57 \pm 0.16$ \\
\hline$J_{\mathrm{fms}}^{i}$ & 61 & 42 & 65 & 66 & 39 & 77 \\
\hline$\sigma_{\text {coine }}{ }^{i}$ & 369 & 351 & 363 & 400 & 117 & 368 \\
\hline \multicolumn{7}{|c|}{$\theta_{G}=320^{\circ} \theta_{S}=120^{\circ}$} \\
\hline$\beta_{2}^{h}$ & $0.69 \pm 0.07$ & $0.11 \pm 0.11$ & $1.1 \pm 0.1$ & $0.22 \pm 0.12$ & $2.3 \pm 0.2$ & $0.30 \pm 0.15$ \\
\hline $\mathrm{j}_{\mathrm{rms}} \dot{j}$ & 65 & 47 & 68 & 6] & 77 & $\$ 6$ \\
\hline${ }^{\theta} \operatorname{cosinc}^{i}$ & 381 & 363 & 497 & 447 & 194 & 393 \\
\hline
\end{tabular}

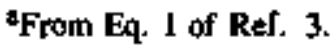

bFrom Egs. 13, 14 of Rer. 3.

'From $\left\langle\langle\right.$ दe $>\rangle$, Es, $9-11$ and $\beta_{2}$ from fits of Eq. 6 (see Ref, 3) to the coincidence data.

'From Ref. 4,

EFrom fit of Eq. 12 (Ref. 3) to singles date.

From $\beta_{2}$ for singles data (e above).

From Rel. 1.

hFrom fits of Eq. 6 (Rel. 3) to the coincidence data.

iFrom $\beta_{2}$ for coincidence data (h above). 
respectively. Under the section labeled coincidence studies are shown the measured $a_{2}$ values, the portion of the singles cross sections in coincidence with fission, $\sigma_{\text {coinc }}$ and the inferred values of $\mathrm{I}$.ms'

The values of the temperatures and the level density parameters are in agreement with those found in lighter, less energetic systems. However, the inferred envission barriers we find are of the order of $20-30 \%$ lower than expected from fusion systematics," implying that some deformation may exist for these composites prior to emission. The values of $\mathrm{J}_{\mathrm{rms}}$ deduced are of the order of $60-70$ h. This is surprising in that, for composites of such high excitation energy and spin, models such as the rotating liquid drops suggest that first step de-ercitation should be dominated by fission because of the near-zero fission barriers. Overall, we are led to conclude that these composites are characterized by quite large multiplicities for equili- brated alpha and proton emission. We further conclude that the competition between light charged particle emis. sion and fission is not completely governed by the phase space equilibrium statistical mode.

Footmotes and References

"State University of New York at Stomy Brook, Stony Brook, NY 11794.

†Carnegie-Mellon University, Pittsburgh, PA 15213.

l. D. Logan et al., Phys. Rev. C 22, 1080 (1980).

2. T. Doessing (unpublished).

3. M.F. Rivet, D. Logan, J.M. Alexander, E. Duek, MS. Zisman, and M. Kaplan (subrnitted to Phys. Rev.).

4. MA McMaban and J.M. Alexander, Phys. Rev. C 21, 1261 ( 1980$)$.

5. S. Cohen, F. Plasil and W. Swiatecki, Antn. Phys. (N.Y.) 82, 557 (1974).

\title{
Direct and Eraporation-Like Emission of ${ }^{1} \mathbf{H}$ and ${ }^{4} \mathrm{He}$ in Both Fusion-Like and Inelastic Reactions of $340 \mathrm{MeV}{ }^{40} \mathrm{Ar}+{ }^{238} \mathrm{U}$
}

\author{
D. Logan * M. Kildir. ${ }^{\dagger}$ M. Koplan." M.S. Zisman, \\ D. Guerreau, ${ }^{\ddagger}$ J.M. Alexander, and LC. Vaz
}

Reactions between oomplex nuclei generally lead to substantial emission of $\mathrm{H}$ and $\mathrm{He}$. Evaporative emission has been observed from compound or composite nuclei ${ }^{1}$ as well as from strongly damped reaction products after scission. ${ }^{2}$ Pre-equilibrium emission has also been reported, but its nature cannot yet be said to be well understood, ${ }^{3}$

A simple and powerful technique for associating $\mathrm{H} / \mathrm{He}$ emission with a fusionlike reaction is provided by a triple-coincidence requirement ${ }^{4}$ between two fission fragments and the $H$ or $H e$. A small folding angle $\left(\theta_{f}\right)$ between fission fragments prowides a clear signature for the large momentum transfer of a fusion-like collision. ${ }^{4}$ A quasi-elastic or deeply inelastic collision will generally impart a much smaller momentum to the target, bui still enough excitation energy for it to sequentially undergo fission. This smaller velocity leads to a distinctly larger folding angle. ${ }^{3}$ We have measured coincident fissionfragment pairs, produced in the $340 \mathrm{MeV}{ }^{40} \mathrm{Ar}+{ }^{218} \mathrm{U}$ reaction, by means of a gas ionization telescope (GT) and a two-dimensional, position-sensitive avalanche detector (PSAD) with full apertures of $2^{\text {t }}$ and $25^{\circ}$, respectively. ${ }^{6}$ Two GTs were used simultanequsly 80 that a range of $118^{\circ}$ $<\theta_{\mathrm{p}}<168^{\circ}$ was observed, sufficient to separate the deeply inelastic and fusion-like events. A second coincidence was required between one of the GT's and any of four three-element Si solid state telescopes (SST) that identified $\mathrm{H}$ or $\mathrm{He}$.
The angular dependence of the differemial multiplicities for ${ }^{1} \mathrm{H}$ and ${ }^{4} \mathrm{He}$ are shown in Fig. 1. Each has a nearly isotropic component (in- and out-of-plane) aft of $\approx$ $100^{\circ}$ and a significant forward-peaked comporient. The angle-inlegrated multiplicities, $M$, for the isotropic components are also indicated in Fig. I. Typical energy distributions for ${ }^{4} \mathrm{He}$ at $\theta_{\mathrm{e} . \mathrm{m} .} \approx 110^{\circ}$ (coincident with fusionlike collisions) show evaporative behavior and the spectra are very similar in- and out-of-plane. At $\theta_{\text {c. ra }} \simeq 28^{\circ}$, again in coincidence with fusion collisions, añ additional bigh-energy component is abundanily present in-plane but more weakly out-of-plane; at the same angle, alptas coincident with sequential fission show a dominant bigh-energy component which again exhibits a decrease as one moves out-of-plane.

A simpte test for the dominant source of emission can be obtained from the angular dependence of the observed mean velocities as shown for ${ }^{4} \mathrm{He}$ in Fig. 2. The vector diagram shows mean velocities for the detected fission fragments as arrows and those expected for evaportative ${ }^{4} \mathrm{He}$ as circles, dashed for the fragments and solid for the composile nucleus. Pọints indicating measured mean velocities are most consistent with the solid circle representing evaporation from the composite system, and do not follow the trends of the dashed circles. We find similar behavior for the ' $\mathrm{H}$ emission as well. Therefore, we conclude that the composite nueleus is the dominant emission source for the 


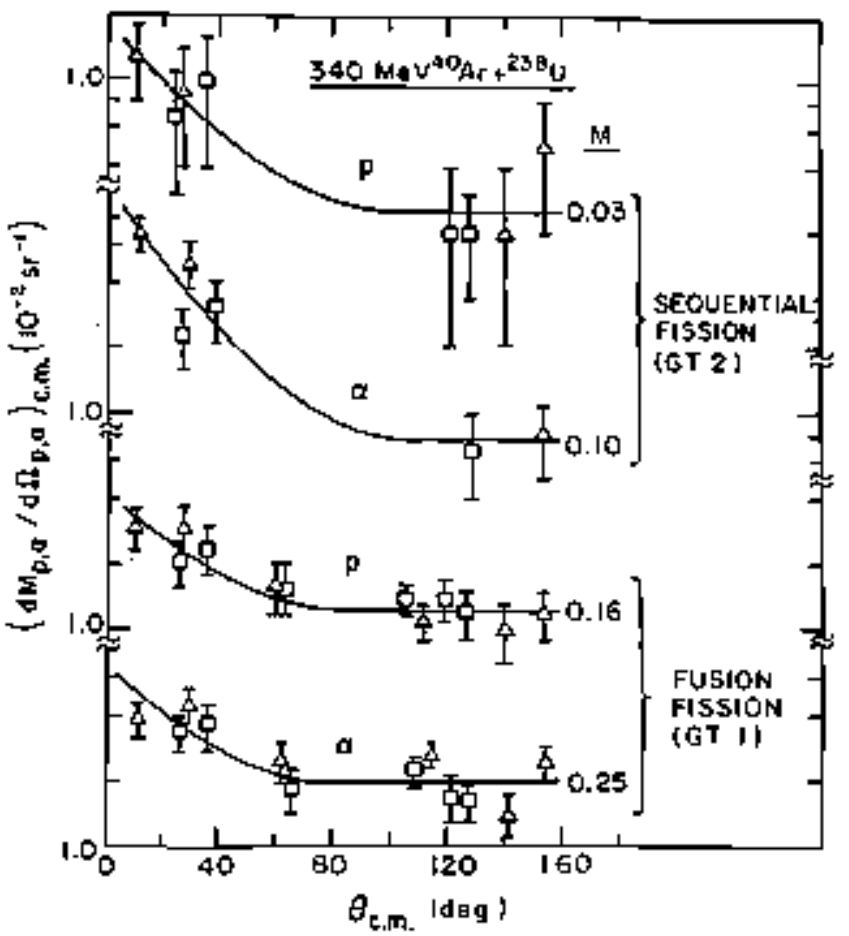

Fig. 1. Differential multipljcities ( $\left.\mathrm{d} \mathrm{M}_{\mathrm{p}, \alpha} / \mathrm{d} \Omega_{\mathrm{p}, \mathrm{e}}\right)_{\mathrm{c}-\mathrm{m} .}$ vs center-or-mass angle $\theta_{\mathrm{cm}}$, for ${ }^{1} \mathrm{H}$ and ${ }^{4} \mathrm{He}$ in friple coincidence with fissjon-Iragment pairs registered in the PSAD and GT 1 or GT 2 . Triangles represent measurements in the reaction plane, and squares correspond to out-of-plane detectors $\left(\phi_{1}=21^{\circ}\right.$ at the more forward angles and $\phi_{\text {Lab }}=55^{\circ}$ at the more backward angles). Angleintegrated multiplicities $M$ are indicated for the isotropic component of each curve.

(XBL 818-11285)

low energy components of both ${ }^{4} \mathrm{He}$ and ${ }^{l_{H}} \mathrm{~F}$ For $\mathrm{H} / \mathrm{He}$ in coincidence with sequential fission, the number of events recorded is more limited, and the vector diagram is more complicated. Here, we must also consider the projectilelike and target-like fragments as potential sources of evaporative emission. As in the fusion-fission case, the meas. ured mean velocities for $\mathrm{H} / \mathrm{He}$ do not follow the trends expected for evaporation from fission tragments. Furthermore, although it cannot be completely excluded, evapora. tion from projectile-like fragments is not a dominant emission source.

ln summary, our data show evidence for forward angle emissions, characterized by strong forward peaking and bigher than evaporatjve velocities with a preference for the reaction plane. This direct emission appears to be more abundant for the dexply inelastic than for the fusion-like collisions. These features, along with the sizcable multiplicities for ${ }^{4} \mathrm{He}$ as well as ${ }^{1} \mathrm{H}$ emission, seem to be evidence for angular monentum as a primary driving force. The nearly isotropic components have afl the appearances of a classic evaparation mechanism. Those from fusion-like collisions must arise from a composite system of $Z=110$, and are expected to have spins up $10 \ell=130$ but no barrier 10 fission (for $\mathrm{J} \geq 50$ ). ${ }^{7}$ The integrated multiplicities for ${ }^{1} \mathrm{H}$ and ${ }^{4} \mathrm{He}$ evaporation for these fusion collisions are

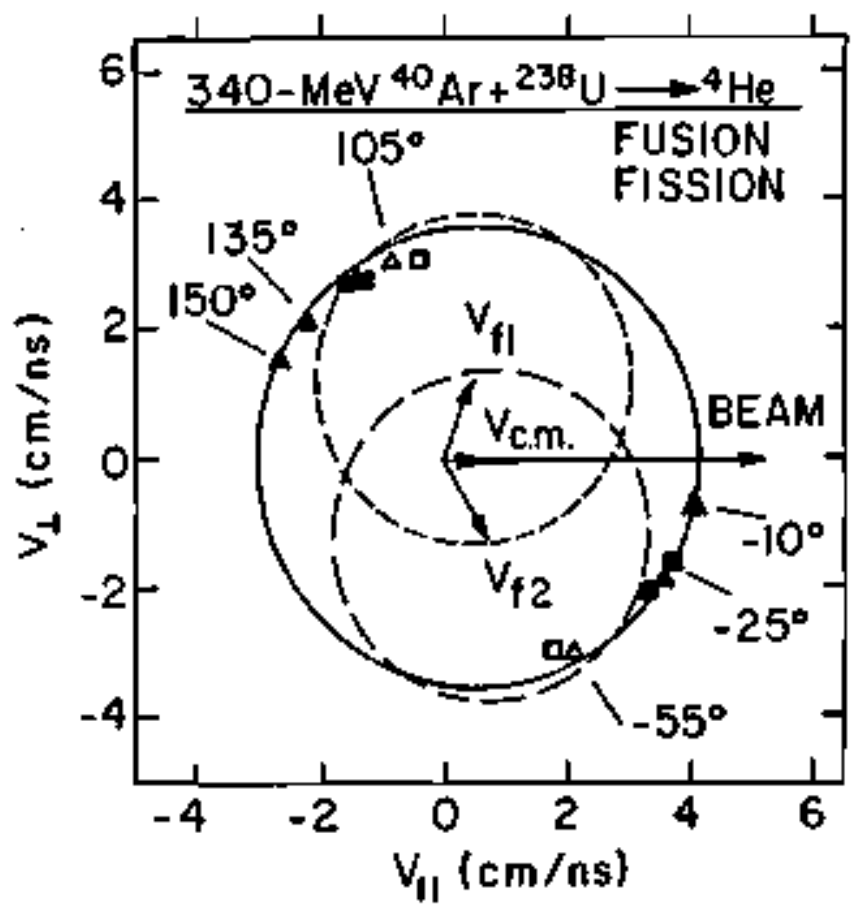

Fig. 2. Vector diagram for ${ }^{4} \mathrm{He}$ emitted in fusion-fiasion. Mean ${ }^{4} \mathrm{He}$ velocities observed in-plane are given by trjangles and out-of-plane by squares. Short and long dashed circles correspond to expected mean velocities for evapora tion Irom the fully accelerated fission fragments (for GT 1 or $V_{f}$ at $70^{\circ}$ ). Large solid circle is for evaporation from the composite system Open points at about $-55^{\circ}$ and $105^{\circ}$ were measured in coincidence with GT $I$ or $V_{\text {fI }}$ at $40^{\circ}$ rather than at $70^{\circ}$ as shown here.

(XBL, 81811286)

rather high, $\simeq 0.2$, and are comparable to those from the much lighter systems ${ }^{2}{ }_{37}^{37} \mathrm{Bk},{ }_{84}^{204} \mathrm{Po}$, and ${ }^{19}{ }_{0}^{4} \mathrm{Hg}$. The lack of a strong $Z$ dependence for this $\mathrm{H} / \mathrm{He}$ evaporation clearly indicates that dynamics rather than phase space must be controlling the choice of exil channel. ' These emission processes are particularly exciting because they may provide the most direct view yet available of a composite system predicted not to have an equilibrium shape.

\section{Footnotes and References}

'Carnegie-Mellon Unjversity, Pittsburgh, Pennsylvania 15213.

'Department of Chemistry, Middle East Technical University, Ankara, Turkey.

'Laboratoire de Chimit Nueléaire, B.P. No. 1, 91406 Orsay, France.

State University of New York at Stony Brook, Stony Brook, New York 11794.

1. D. Logan el al., Phys. Rev. C 22, 104 (1980) and Phys. Rev, C 22, 1080 (1980); M.F. Rivet et al. (submitted to Phys. Rev. C.).

2. See, for example, $R$, Babjint et al, Z. Phys. A 295, I953 (1980). 
3. Procedings of the International Symposium on Continuwm Spectrc of Heavy Ion Reoctions, San Antonio, Texas (1979), Harwood Acadenic Publishers (New York).

4. B.B. Back at al., Phys. Rev, C 22, 1927 (1980) and

references therein.
5. T, Sikkeland, Phys. Lett. B 27, 277 (1968).

6. M.M. Fowler and R.C. Jared, Nucl. 1nstruments and Metbods 24, 34I (1975); Y. Eyal and H. Stelzer, Ibid. I55, 157 ( 1978 ).

7. S. Cohen et al., Ann. Phys. (N.Y.).82, 557 (1974).

\title{
Prominent ${ }^{4}$ He Emission from the Composite Nucleus for $480 \mathrm{MeV}{ }^{56} \mathrm{Fe}+\mathrm{Ag}$
}

\author{
D. Guerreau, ${ }^{*}$ D. Logan, M.S. Zisman, J.M. Alexander, \\ E. Duekt and $M$. Kaplant
}

Reently, varions studies of heavy ion reactions have identified an evaporative component for ${ }^{4} \mathrm{He}$ emission and have used it to determine the root mean square spin of the enitters. ${ }^{1}$ To search for the process of emission of exaporation-like a particles from a very short-lived composite nucleus, we have chosen the reaction $4 \$ 0 \mathrm{MeV}{ }^{56} \mathrm{Fe}+$ Ag. We have measured a particles in coincidence with beavy fragments in a geometrical configuration selected to test for evaporative emission from all three sources, the composite system, fission fragments, or deeply inelastic fragments. Four three-member Si solid-state detector telescopes (SST) were used to identify the ${ }^{4} \mathrm{He}$ particles, and a ges ionization telescope (GT) was used to measure $Z$ and energy of the lighter of the two heavy fragments. A GT was placed at $50^{\circ}$ or $30^{\circ}\left(\theta_{c, m}-95^{\circ}\right)$ to emphasize detec tion of fission-like and strongly-damped reaction products well aft of the grazing angle, 25\%. The natural $\mathrm{Ag}$ tatget $\left(670 \mu \mathrm{g} / \mathrm{cm}^{2}\right)$ was itradiated with ${ }^{56} \mathrm{Fe}$ from the SuperHILAC. Details of the experimental technicques have been described elsewhere. ${ }^{2}$

Measured coincidence spectra are most readjly discussed in terms of invariant cross sections ${ }^{1,3}\left(d^{3} \sigma / p_{\alpha} c\right.$ $\mathrm{d} \Omega_{\mathrm{f}} \mathrm{d} \Omega_{\alpha} \mathrm{dE} \mathrm{E}_{\alpha}$ ); these are plotted vs the velocity in the frames of the average mowing fragnents in Fig. I. Dashed curves are drawn with empirically estimated shapes for evapora. tion from these fragments. ${ }^{3,4}$ As these evaporative components must be essentially isolropic (in-plane), ${ }^{3}$ we have set their upper limits by reference to the SST at $75^{\circ}$ (emphasizing emission from the lighter of the two heary fragments) and $15^{\circ}$ and $-15^{\circ}$ (emphasizing emission from the complimentary heavy fragments). From this analysis, we estimate molltipliétites of 0.09 and 0.2 (upper limits) for emission ftom light and heavy fragments respectively. It is clear from Fig. I that the bulk of a-particle emission (in the coincidence mode) cannot be described by evaporation from the fragments. This resuit is in strong contrast 10 the results from Rei. 6 for ${ }^{\mathrm{B} 4} \mathrm{Kr}+\mathrm{Ag}$; possibly the angular range explored in that study did not allow the measurement of emission characteristics from the composite system

To what extent can the additional $\alpha$ enission be ascribed to "quasi-evaporation" from the composite nucleus? The average "He velocities and energies are rather close to those expected for such evaporation, and the observed c.m. spectra for $V_{\alpha} \geq 3 \mathrm{~cm} / \mathrm{ns}$ (see Fig. 2a) are very similar to the shape expected for exaporation from a nucleus of $Z=73$. However, the differences in the spectra for $+15^{\circ}$ and $-15^{\circ}$ (for $V_{\alpha}<3 \mathrm{~cm} / \mathrm{ns}$ ) indicate significant emission lrom the fragments. In Fig. $2 b$ and 2c. we compare the laboratory spectra to the sum of the evaporation components calculated with multiplicities taken from Figs. 1 and 2a. We can see the pecessity to include prominent emission from the omposite nucleus itself in addition to that from the fully accelerated fragments. We note that, even for the composite system, there seems to be a rough confocmity of the spectra to evaporative behavior. Angular integration for such an evaporative component gives a multiplicity of 0.44 .

Integrated cross sections are listed in Table I and compared with corresponding quantities from the reaction $340 \mathrm{MeV}{ }^{40} \mathrm{Ar}+\mathrm{Ag}$. For the ${ }^{56} \mathrm{Fe}$ reaction, more $\alpha$ par* ticles are emitted both from the fragments and from the composite nucleus. The former enhancement is surely expected with increasing fragment spin, ${ }^{1}$ but the latter enhancement is less obvious. Whereas the composite sys. tem was a negligible source of ${ }^{4} \mathrm{He}$ emission for $280 \mathrm{MeV}$ ${ }^{40} \mathrm{Ar}^{\prime}+{ }^{5 \mathrm{Q}} \mathrm{Ni},{ }_{\mathrm{i}}$ is roughly comparable to the fragments in the reaction Ar + $\mathrm{Ag}$ (see Table 1); and, finally, it is the dominant source for the present Fe + Ag system. It seems likely that the entrance channel spin provides the driving force for this inerease. However, one expeets the lifetime of the composite system to dectease with increasing spin, and therefore it is amazing to find so much evaporation-like emission from composite systerns with such high spins. Whether or not emission from the tjps of a strongly deformed system can account for this o-particle emission remeins an open question at present. 


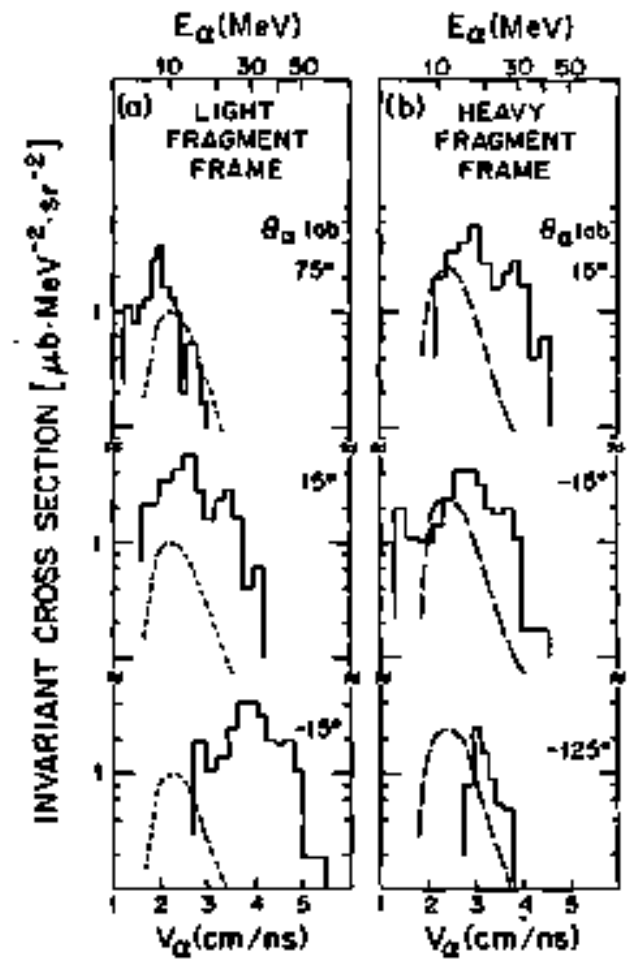

Fig. 1. The invariant cross section $\left(d^{3} \sigma / \alpha \Omega_{p} d \Omega_{\alpha} d E_{\alpha} p_{\alpha} c\right)$ vs mean velocity in the delected light fragment frame (a) and in that of its heavy complement (b). Histograms show the experinental data; dashed lines give empirically estimated shapes for evaporation spectra. These shapes are normalized to maximunt aliowable magnitude (for the light frag* ment at $75^{\circ}$ and the beavy forgment at $15^{\circ}$ and $-15^{\circ}$ ).

(XBL 818.11282)

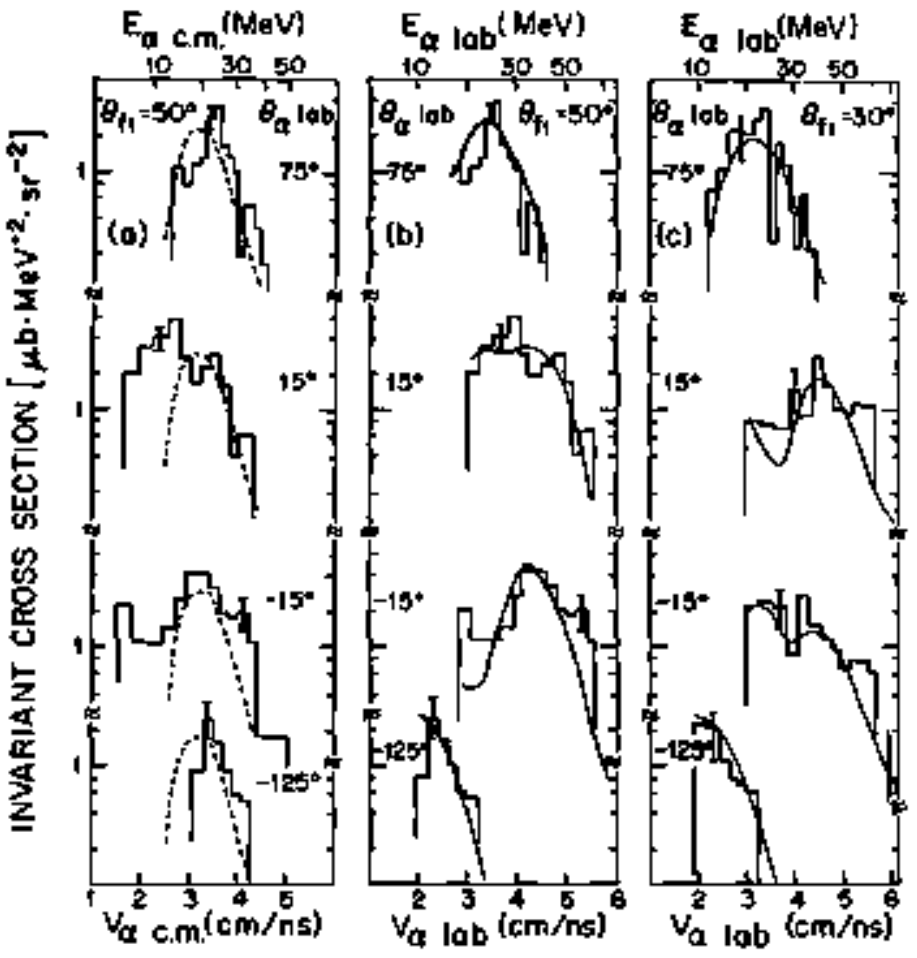

Fig. 2. Invariant cross section ws (a) c.m. velocity and (b) and (c) laboratory velocity for the GT angles $50^{\circ}$ and $30^{\circ}$. The dot-dashed curves were drawn in (a) for the composite system. The solid curves in (b) and (c) represent the sum of all three evaporative corraxintents.

(XBL 818-) 1283) 
Table $I$. Cross sections (mb) and $\ell$ values for two reactions Af (natural) $+\mathrm{X}(8.5 \mathrm{McV} / \mathrm{u})$.

\begin{tabular}{|c|c|c|}
\hline & ${ }^{40} \mathrm{Ar}$ & ${ }^{56} \mathrm{Fe}$ \\
\hline$E^{*}(\mathrm{MeV})$ & 195 & 205 \\
\hline$\sigma_{E R}(m b)$ & $455 \pm 50^{2}$ & $-290^{h}$ \\
\hline$\ell_{\mathrm{ER}}(h)$ & $(71)^{\mathrm{a}}$ & $(71)^{\mathbf{b}}$ \\
\hline "Fission-like" & $800 \pm 80^{\circ}$ & $1480 \pm 17 a^{d, e}$ \\
\hline$\sigma_{\text {sum }}\left(\varepsilon_{\text {sum }}\right)$ & $1255(118)$ & $\sim 1770(\sim 175)$ \\
\hline$\sigma_{R}\left(\varepsilon_{\max }\right)$ & $2704^{b}(172)$ & $2694^{b}(217)$ \\
\hline $\begin{array}{l}\text { Symmetric } \alpha \text { 's from the } \\
\text { composite in coincidence } \\
\text { with fragments (their } \\
\text { multiplicity) }\end{array}$ & $\begin{array}{c}125 \pm 35^{\prime} \\
(0.16)\end{array}$ & $\begin{array}{c}650 \pm 100^{d} \\
(0.44)\end{array}$ \\
\hline $\begin{array}{l}\alpha \text { s evaporated } \\
\text { from fragments } \\
\text { (their multiplicity) }\end{array}$ & $\begin{array}{c}155 \pm 40^{f} \\
(0.2)\end{array}$ & $\begin{array}{c}430 \pm 65^{d} \\
(0.29)\end{array}$ \\
\hline
\end{tabular}

"H.C. Britt et al., Phys. Rev, C 13, 1483 (1976).

Estimated from systematios.

'Rer. 2.

dDetermined in this work

$\circ \mathrm{dr} / \mathrm{d} \Omega$ for $18<\mathbf{Z}<38$ at $50^{\circ}$ integrated by assuming $1 / \sin \theta$ angular distribution.

${ }^{5}$ Fragment detector at $\theta_{\text {c. }}=90^{\circ}$ where only fission-like fragments were observed; values from Ref. 2.
Foormotes and References

"Laboratoire de Chimí Nucleaire, Institut de Physique Nucléaire, B.P. No. I, 91406, Orsay, France.

'Carnegie-Mellon University, Pittsburgh, Pennsylvania 15213.

'State University of New York at Stony Brook, Stony Brook, NY 11794.

I. D. Guerreau and $R$ Babinet, J. de Physique (Paris) C 10,217 (1980) and references therein.

2. D. Logan et al., Phys. Rev, C 22, 1080 (1980);

G. Catchen et al., Phys. Rev, C 21, 940 (1980);

M.F. Rivet et al. (submitted to Phys. Rev, C, 1981);

D. Goerreau et al. (to be published).

3. R. Babinet tet al., Z. Phys. A 29, 153 (1980).

4. Systematic studies of evaporation spectra $\$$ how that spectral shapes can be approximated by the standard formula $P\left(E_{\alpha}\right) \propto\left(E_{\alpha}-B\right) \exp -\left[E_{\alpha} / T\right]$. The empirical paramelers ( $B$ and $\mathbf{T}$ ) have been taken from the data given in Re[s. 1 and 5. Mean evaporation velocities can be estimated with relative uncertainties of less than $3 \%$

5. W. Kuhn et al., Z. Phys. A 298, 95 (1980).

6. LG. Sobotka et al., Phys. Rev. Lett. 46, 887 (1981), and LBL 11840 (1981).

\title{
Comparison of Noncompound Neutron and Proton Emission in ${ }^{16} 0$ Induced Reactions on ${ }^{238} \mathrm{U}$ at $310 \mathrm{MeV}$
}

\author{
J. Karagi, "S. Saim," T.C. Awes," A. Galonsky." \\ C.K. Gelbke, G. Poggi, D.K. Scott, K.L Wolf, and RL Legrain
}

In the last few years, it has toen established that collisions between complex auclei at non-relativistic energies may lead to the emission of energetic light particle which cannot be explained in terms of evaporation from completely equilibrated nuclei. Assuming that protons and neutrons have similar spectral shapes, it has been shown ${ }^{1,2}$ that the energy spectra of deuterons and tritons may be related to the energy spectra of protons by a simple coalescence relation. However, a direct comperison of noncompound proton and neutron cross sections has not yet been performed. To make such a comparison, we used the LBL 88-Inch Cyclotron to investigate the reaction ${ }^{23} \mathrm{U} U\left({ }^{16} \mathrm{O}, \mathrm{pf}\right)$ and ${ }^{233} \mathrm{U}\left({ }^{16} \mathrm{O}, \mathrm{nf}\right)$ at $310 \mathrm{MeV}$ incident energy.
The differential neutron multiplicities per fission event, $d^{2} \mathrm{~N} / \mathrm{N}, \mathrm{dEd}$, are shown in Fig. $\mathrm{d}$. At low energies, the spectra are dominated by a low temperature component that is consistent with the statistical evaporation of neytrons from equilitrated target residues and fission fragments. The high energy regions of the neutron spectra exhibil characteristics that are qualitatively similar to those observed for the emission of energetic charged particles: with increasing scattering angle the cross sections decrease and the energy spectra beome steeper.

To facilitate the comparison of proton and neutron cross sections, we have decomposed each neutron spectrum into two components by fitting the energy spectra with the following function: 


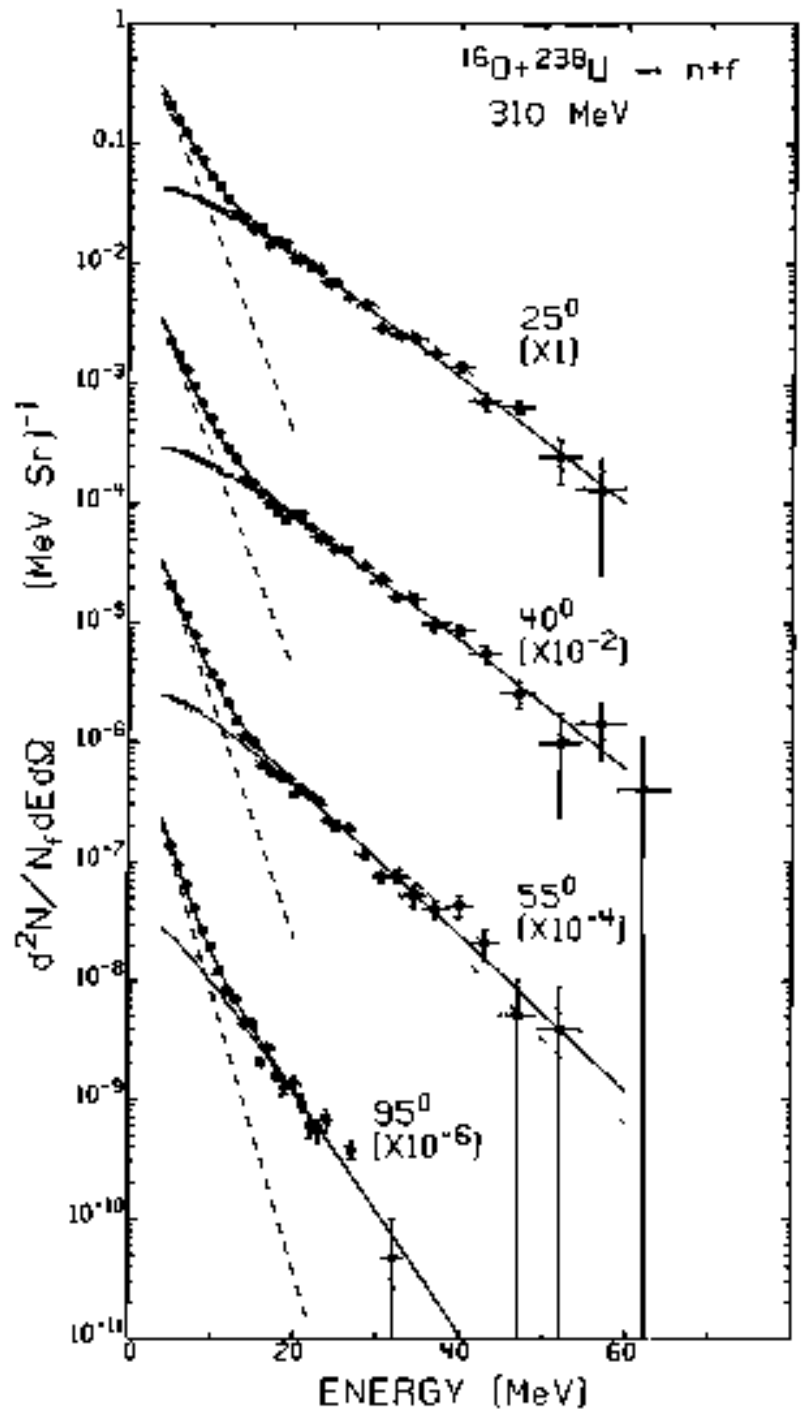

Fig. 1. Differential neutron multiplicities per hission event measured for the reaction ${ }^{238} \mathrm{U}\left({ }^{16} \mathrm{O}, \mathrm{nN}\right)$ at $310 \mathrm{MeV}$. Solid and dashed lines show the decomposition jnto oquilibrium and non-equilibrium components, respectively. Dotted lines indicate the estimated errors within which the bigh energy regions of the neutron spectra are established. These limits are used for the comparison with the proton spectra in Fig. 2.

$$
\frac{d^{2} N(E)}{N_{p} d E d \Omega}=N_{0} e^{-E / T_{0}}+N_{1} \sqrt{E} e^{-E / T_{l}} .
$$

The first term, which omits an energy factor, adequately represents the low energy component, and the second term describes the high energy component which we associate with preequilibrium processes; $\mathrm{N}_{0}, \mathrm{~T}_{0}, \mathrm{~N}_{\mathrm{l}}, \mathrm{T}_{1}$ are adjustable parameters. This decomposition is shown in Fig. 1 for the parameters given in Table 1.

The proton cross sections measured in this experiment are compared to the corresponding neutron eross seclions in Fig. 2. The shapes of the proton and neutron spectra exhibil significant differences. At lower energies (E E $30 \mathrm{MeV}$ ) the neutron cross sections exceed the proton cross sections. However, the neutron cross section drops below the proton cross section at higher energies (E $\geq 30 \mathrm{MeV}$ ). a result which conflicts with the simple expectation ${ }^{3}$ that the neutron-to-proton eroes section ratio should scale with the neutron-to-proton ratio of the emitting system (be it projectile, target, or the composite system).

The differences between the low energy part of the proton and neutron spectra are most likely due to barrier penetration effects. To illustrate this point. we have transformed the neutron spectra of Fig. 2 a according to the relation

$$
\frac{d^{2} N^{(D)}(E)}{N_{f} d E d \Omega}=\frac{\sigma_{R}^{(p)}(E)}{\sigma_{R}^{(t)}(E)} \frac{d^{2} N^{(n)}(E)}{N_{f} d E d \Omega} .
$$

In Eq. (2), the inverse reaction cross sections are cal. culated from the optical model by using the potentials of Ref. 4. The resulting cross sections are shown by the shaded areas in Fig. 2(b). Reasonable agreement with the proton cross sections is obtained in the low exergy region. The high energy part of the spectrum is, however, only slightly modified $\left(\sigma_{R}{ }^{(p)} \simeq \sigma_{R}{ }^{(t)}\right.$ at high energies) and, as a consequence, the proton-to-neutron ratio at high energies is not affecled by this procedure.

The coalescence relation referred to above was derived $^{2}$ by making the simplifying assumption that the cross sections for preequilibrium proton and neutron emis* sion are related by ${ }^{2,5}$

Table 1. Parameters of Eq. (1) used for the decomposition of equilibrium and nonequilibrium components shợn in Fig. I.

\begin{tabular}{ccccc}
\hline$\theta_{\mathrm{n}}$ & $\mathrm{N}_{0}(\mathrm{MeV} \cdot \mathrm{sr})^{-\mathrm{l}}$ & $\mathrm{T}_{0}(\mathrm{MeV})$ & $\mathrm{N}_{\mathrm{l}}\left(\mathrm{MkV}^{3 / 2} \cdot \mathrm{sr}\right)^{-\mathrm{l}}$ & $\mathrm{T}_{\mathrm{l}}(\mathrm{MeV})$ \\
\hline $25^{\circ}$ & 1.35 & 2.45 & 0.036 & 7.60 \\
$40^{\circ}$ & 1.85 & 2.35 & 0.025 & 7.4 \\
$55^{\circ}$ & 2.00 & 2.20 & 0.024 & 6.2 \\
$95^{\circ}$ & 1.78 & 1.85 & 0.038 & 4.0 \\
\hline
\end{tabular}




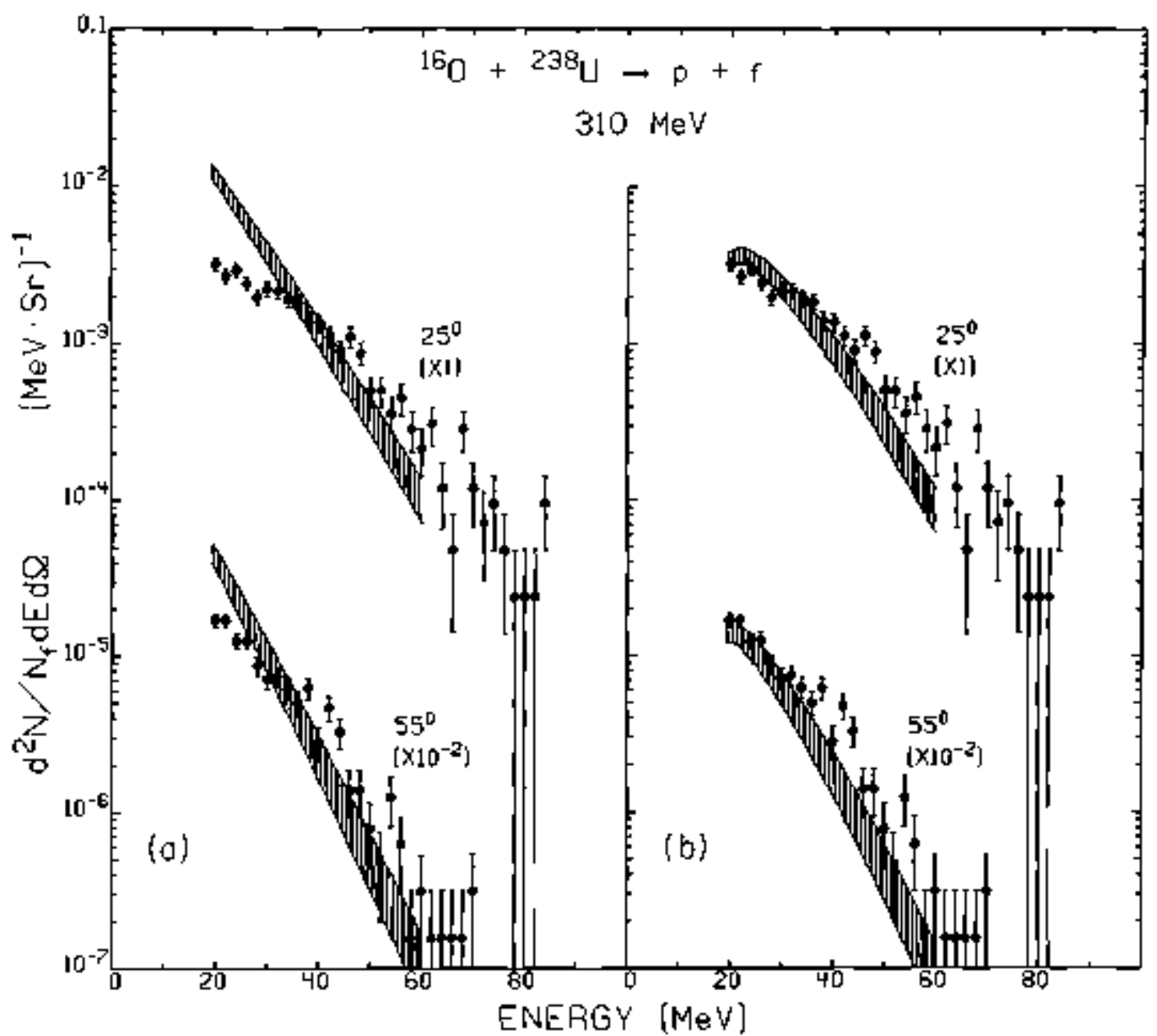

Fig. 2. Differential proton multiplicities per fission event measures for the reaction ${ }^{238} \mathrm{U}\left({ }^{16} \mathrm{O}, \mathrm{n}\right)$ at $310 \mathrm{MeV}$. (solid points) Shaded areas represent the medsured preeguilibrium neutron multiplicities (part a) and their predicted transformation into proton multiplicilies according to Eq. (2) of the text (part b).

(XBL 8] II- ]2353)

$$
\frac{d^{2} \sigma_{a}(E)}{d E d \Omega}=\frac{N}{Z} \frac{d^{2} \sigma_{p}\left(E+E_{c}\right)}{d E d \Omega}
$$

In Eg. (3), $N / Z$ is the neutron-to-proton ratio of the compound nucleus. The energy shift $\mathrm{E}_{\mathrm{q}}$ was introduced to correct for the final state Coulonb repulsion from the target nucleus. The physical picture behind Eq. (3) is that protons and neutrons bave identical kinetic energy distributions at the nuclear surface, where the observed complex particles are most likely to originate. The Coulonb feld of the target nucleus merely displaces the energy spectrum of charged particles by the amount $\mathrm{E}_{c}$ It is clear from $\mathrm{Fig}$. 2(a) that $\mathrm{Eg}$. (3) is inconsistent with our observations. The shapes of the neutron spoctra predicted from the proton cross sections would differ significantly trom the messured neuiron spectra. The coalescence calculations are quite sensitive to this low energy region; as a conse- quence, the deuteron cross sections are not given by the product of proton and neutron cross sections as determined in this experiment. Possible alternative explanations 6 for the formation of composite light particles remain an interesting subject for future investigations.

\section{Footnoles and References}

"Michigan State University.

†Argenne National Laboratory.

I. T.C. Awes th al., Phys. Rev. Lett. 46, 513 (1980).

2. T.C. Awes te al., Phys. Rev. C 24, 89 (1981).

3. W.W. Morison et al. Phys. Lett. B 93, 379 (1980).

4. F.D. Becchetti, Jr., and G.W. Greenlees, Phys. Rev. $182,1190(1969)$.

5. M. Gyulassy and S.K. Kaufimann, Nucl. Phys. A 362, 503 (1985).

6. E. Hachenberg, H.C. Chiang and J. Hufner, Phys. Lett. B 97,183 (1980). 


\title{
Light Particle Correlations in $20 \mathrm{MeV}$ per Nucleon 160 Induced Reactions
}

\author{
C.K. Gelbke," W.G. Lynch," LW. Richardson, M.B. Tsang," and R.E. Warnert
}

The attainment of local thermal equilibrium in heavy ion induced reactions is an important assumption underlying many theoretical models. Until now, light particle correlations testing the thermalization assumption have been measured only at relativistic energies $(E / A=400$ and $800 \mathrm{MeV}$ ) where the existence of a non-thermal knockout component was demonstrated. In this article, we discuss the first such measurement at non-relativistic energies.

Aluminum and gold targets of 1.6 and $10 \mathrm{mg} / \mathrm{cm}^{2}$ thickenes were bombarded with $310 \mathrm{MeV}{ }^{16} \mathrm{O}^{6+}$ ions from the LBL 8B-Inch Cyclotron. Single and coincident protons and iteuterons were detected wich four $\triangle E$,E clescopes consisting of solid state silicon- $\Delta \mathrm{E}$ and $\mathrm{NaI}$ (T) -E detectors. The telescopes werte mounled in a plane at the angles of $-110^{\circ},-30^{\circ},+30^{\circ}$, and $+75^{\circ}$ with respect to the beam axis, subiending solid angles of $62,49,49$, and $62 \mathrm{msr}$, respectively.

We present our results in terme of the correlation function $\sigma_{12} /\left(\sigma_{1} \sigma_{2}\right)$ where

$$
\sigma_{12}=\sigma_{0} \int_{\Delta E_{1}} d E_{1} \int d E_{2} \frac{d^{4} \sigma\left(\theta_{1}, E_{1}, \theta_{2}, E_{2}\right)}{d E_{1} d E_{2}},
$$

and

$$
\sigma_{k}=\int_{d E_{k}} d E_{k} \frac{d^{2} \sigma\left(\theta_{k}, E_{k}\right)}{d E_{k} d \Omega_{k}} ; k=1,2
$$

The constant $\sigma_{0}$ was arbitrarily fixed by requiring $\sigma_{12} /\left(\sigma_{1} \sigma_{2}\right)=1$ for the proton-proton correlation corresponding to the variables $\theta_{1}=-30^{\circ}, \theta_{2}=+30^{\circ}$, $\Delta E_{1}=20-40 \mathrm{MeV}$, and $\Delta \mathrm{E}_{2}=20-100 \mathrm{MeV}$.

Our experimental results are shown in Fig. 1. The first light particle is defined to be the one deteeted at $\theta_{1}=$ $-30^{\circ}$. The range of enersy integration for the second particle was fixed at $\Delta E_{2}=20-100 \mathrm{MeV}$ tor $\theta_{2}=+30^{\circ}$ and $\Delta \mathrm{E}_{2}=10-100 \mathrm{MeV}$ for $\theta_{2}= \pm 110^{\circ}, \pm 75^{\circ}$. The eror bars shown in the figure are purely statistical; sysematic errors are believed to be on the order of $5 \%$

The correlation function exhibits sizable variations which are more pronounced for the Al target than for the Au target. It usually has a minimum value for the forward envission of the second light particle. For the Al target, a pronounced left-right asyminetry is observed, corresponding to an entanced probabitity for cojncident emission of the two light particles on opposite sides of the beam.

For the case of thermal envission from an infinite ensemble, the correlation function is constant. However, for nuclear reaclions, finite particle number effeets might not be negligible and variations in the correlation function could be imposed by conservation laws. We have performed simple alculations to investigate the correlations that might be imposed by energy and momentum conservation for a source of A nucleons that emits light particles with a Gaussian momentum distribution. The Galileaninvariant cross section is then given by

$$
\frac{d^{3} \sigma}{d p^{3}}\left\langle\vec{p}, \vec{\nabla}_{00} T, m\right)=\varnothing(2 \pi m T)^{-3 / 2} \exp \left[-\frac{\left(\vec{p}-m \vec{F}_{0}\right)^{2}}{2 m \bar{T}}\right](3)
$$

where $C$ is a normalization constant, $m$ is the mass of the emitted particle, $T$ is the temperature parameter and $\vec{v}_{0}$ is the velocity of the source in the laboralory.

The tetection of a light particle of laboratory momentum $\overrightarrow{\mathbf{p}}_{1}$ and mass $\mathrm{m}_{1}$ changes the values of both the $\mathrm{kem}$ perature and the velocity of the remaining ensemble. Denoting $\vec{v}_{0}^{\prime}$ and $T$ ' as the new souree velocity and temperature respectively, the coincidence cross section for the emission of particle 1 of momenturn $\vec{p}_{1}$ followed by the emission of particle 2 of momentum $\overrightarrow{\mathrm{p}}_{2}$ is then proportional to the product

$$
\begin{aligned}
\mathbf{P}\left(\overrightarrow{\mathrm{p}}_{1}, \overrightarrow{\mathrm{p}}_{2}\right)= & \frac{\mathrm{d}^{3} \sigma}{\mathrm{dp}_{1}^{3}}\left(\overrightarrow{\mathrm{p}}_{1}, \overrightarrow{\mathrm{v}}_{0}, \mathrm{~T}_{2} \mathrm{~m}_{1}\right) \\
& \times \frac{\mathrm{d}^{3} \sigma}{\mathrm{dp}_{2}{ }^{3}}\left(\overrightarrow{\mathrm{p}}_{2}, \overrightarrow{\mathrm{v}}_{0}^{\prime}, \mathbf{T}^{\prime}, \mathrm{m}_{2}\right) .
\end{aligned}
$$

Since, experimentally, we cannot distinguish the reverse time sequence, the cross section for the emission of particles 1 and 2 is given by

$$
\frac{\mathrm{d}^{6} \sigma}{\mathrm{d}_{\mathrm{P}} \mathrm{P}^{3} \mathrm{~d} \mathrm{P}_{2}^{3}}=\mathrm{C}_{0}\left[\mathrm{P}\left(\overrightarrow{\mathrm{P}}_{2}, \overrightarrow{\mathrm{P}}_{1}\right)+\mathrm{P}\left(\overrightarrow{\mathrm{P}}_{1}, \overrightarrow{\mathrm{P}}_{2}\right)\right]
$$

where $C_{0}$ is a norualization constant.

The results of these calculations are compared to the experimental proton-proton corretations for the AI target in Fig. 2. The source parameters of $v_{0}=0.085 \mathrm{c}$ and $\mathrm{T}=$ $6.25 \mathrm{MeV}$ for the Al target were obtained from fits to the singles cross sections. The calculations were arbitrarily normalized to the data al a point which is obvious in the figures. To exhibit the effect of the number of source nucleons on the predicted correlation several values of $A$ have been used for the calculations. In addition, one calculation is shown for the case of $T=T^{\prime \prime}=T^{\prime \prime}$ (which violates energy onservation for the ensemble but still exhibits the coostraints due to momentum conservation). 


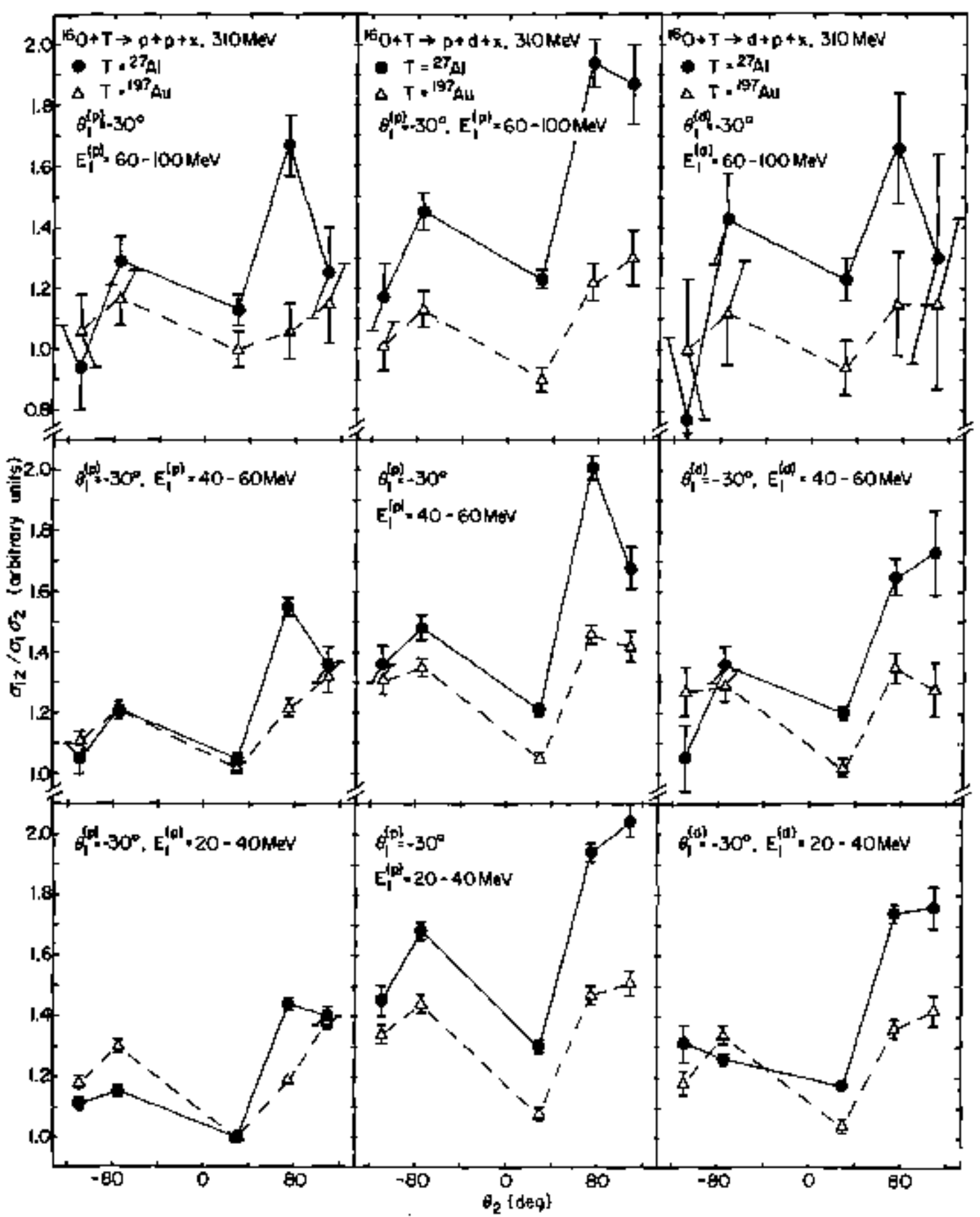

Fig. 1. Experitrental light particle correlations for $20 \mathrm{MeV} / \mathrm{A}{ }^{16} 0$ ions incident on $\mathrm{Al}$ and Au targets.

(XBL $8111-12327$ )

For the Al target (Fig, 2) the magnitude of the observed left-right asymmetries are comparable to those already predicted for the maximum number of participating nucleons, $A=43$. This comparison of calculation with experiment cannot quantitatively determine A since neither the small values of the correlation at $\theta_{2}=+30^{\circ}$ nor the large values at $\theta_{2}=+75^{\circ}$ can be explained by the calculations in a consistent way.
These discrepancies are interesting subjects for future studies with more refined models. Of particular interest is the determination of the influence of impact parameter averaging on the correlation function, and whether the small values of the correlation function at $\theta_{2}=+30^{\circ} \mathrm{can}$ be caused by the presence of low multiplicity peripheral collisions in the singles spectrum at lorward angles. Or comparable importance is the calculation of the direct 


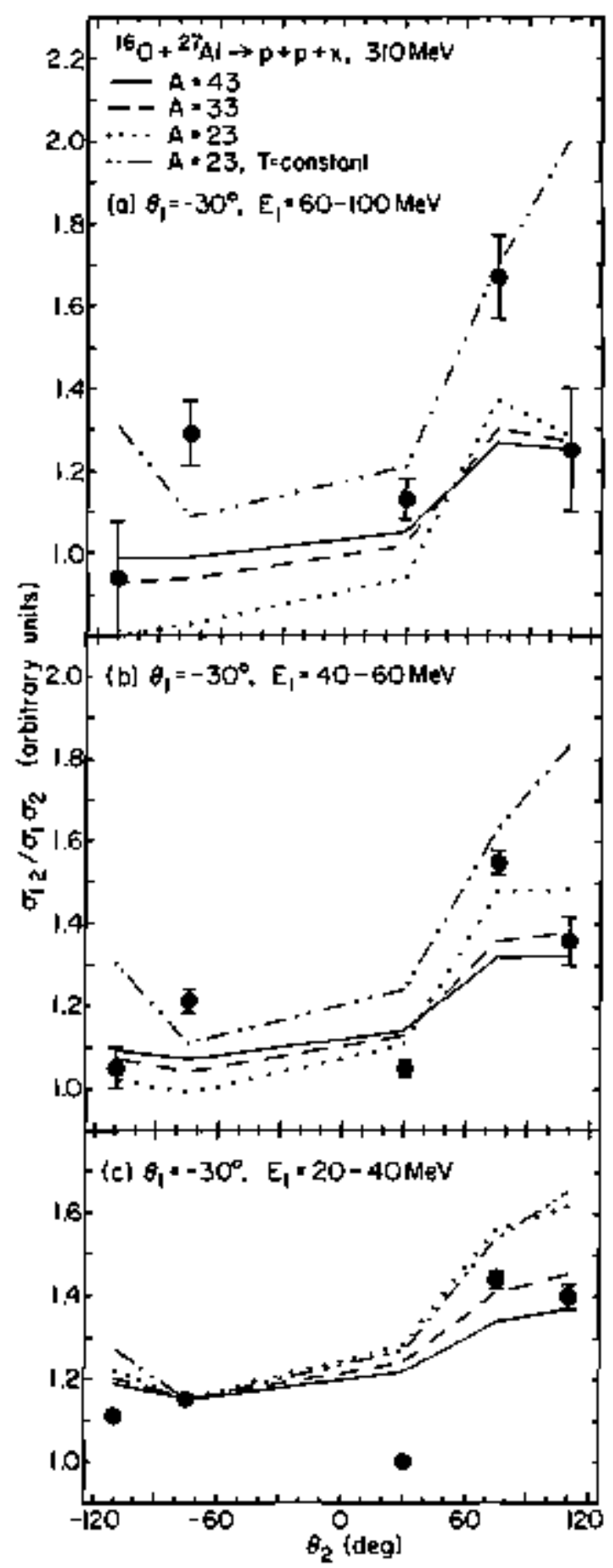

Fig. 2 Comparisons of measured proton-proton correlations with the predictions of the simple thermal mode! deseribed in the text.

(XBL, BI11-12328)

knockout contribution to the carrelation as a lunction of the Largel mass. Such a component, if present, would be expected to be more important for lighter target projectile combinations and for relative proton laboratory angles close to $90^{\circ}$. This could possibly be relaled to the enhencement in the correlation at $\theta_{2}=+75 \%$ for the Al target. Moreower, rescattering and absorptive effects (sha. dowing). especially for heavy target projectile combina. tions, and possible hydrodyoamic effects are of potential interest.

\section{Foothotes}

*National Superconducting Cyclotron Laboratory, Michigan State University, Easi Lansing, MI 48824.

Toberlin College, Oberlin, $\mathrm{OH} 44074$ and National Supercondueting Cyclotron Laboratory. 


\title{
Trends of Light Particle Spectra Observed in Nucleus-Nucleus Collisions
}

\author{
T.C Awes, ${ }^{*}$ G Poggi, S. Saini," C.K. Gelbke, R. Legrain, and G.D. Westfall
}

Information about the early stage of heary-ion induced reactions may be obtained from the detection of energetic light particles which cannot be associated with evaporation from the compound nucleus. At low energies ( $\mathrm{E} / \mathrm{A} \subseteq 20 \mathrm{MeV}$ ), recent experiments bave quinly used coincidence techniques to study detajled aspects of the reaction mechanisms producing energetic light particles. However, few single particle inclusive measurements bave been published in this energy domain, and little information is available about the dependence of non-compound light particle emission on projectile, target, and beam energy. We report here some of the results oblained from a survey of reactions on $A \mathbf{J}, \mathbf{Z r}$, and Au targets using beams of 140,215 , and $310 \mathrm{MeV}$ energy from the LBL 88-Inch Cyclotron.
As an example of the general featores of the energy spectra that were observed in the present experiment, Fig. I shows the results for the ${ }^{197} \mathrm{Au}\left({ }^{16} \mathrm{O}, \mathrm{p}\right)$ reaction for three incident energits. The energy spectra are smooth structureless đistributions which, at forward angles, exiend to about four times the incident energy per nucleon. With incrasing detection angle, the cross sections decrease, and the slopes of the energy spectra become steeper.

The cross sections can be described in terms of a Maxwellian distribution observed in a rest frame that moves with a velocity intermediate between target and projectile (see solid curves in Fig. I.) Correcting for the Coulomb repulsion from the target nucleus, one obtains non-relatjvistically ${ }^{1,2}$

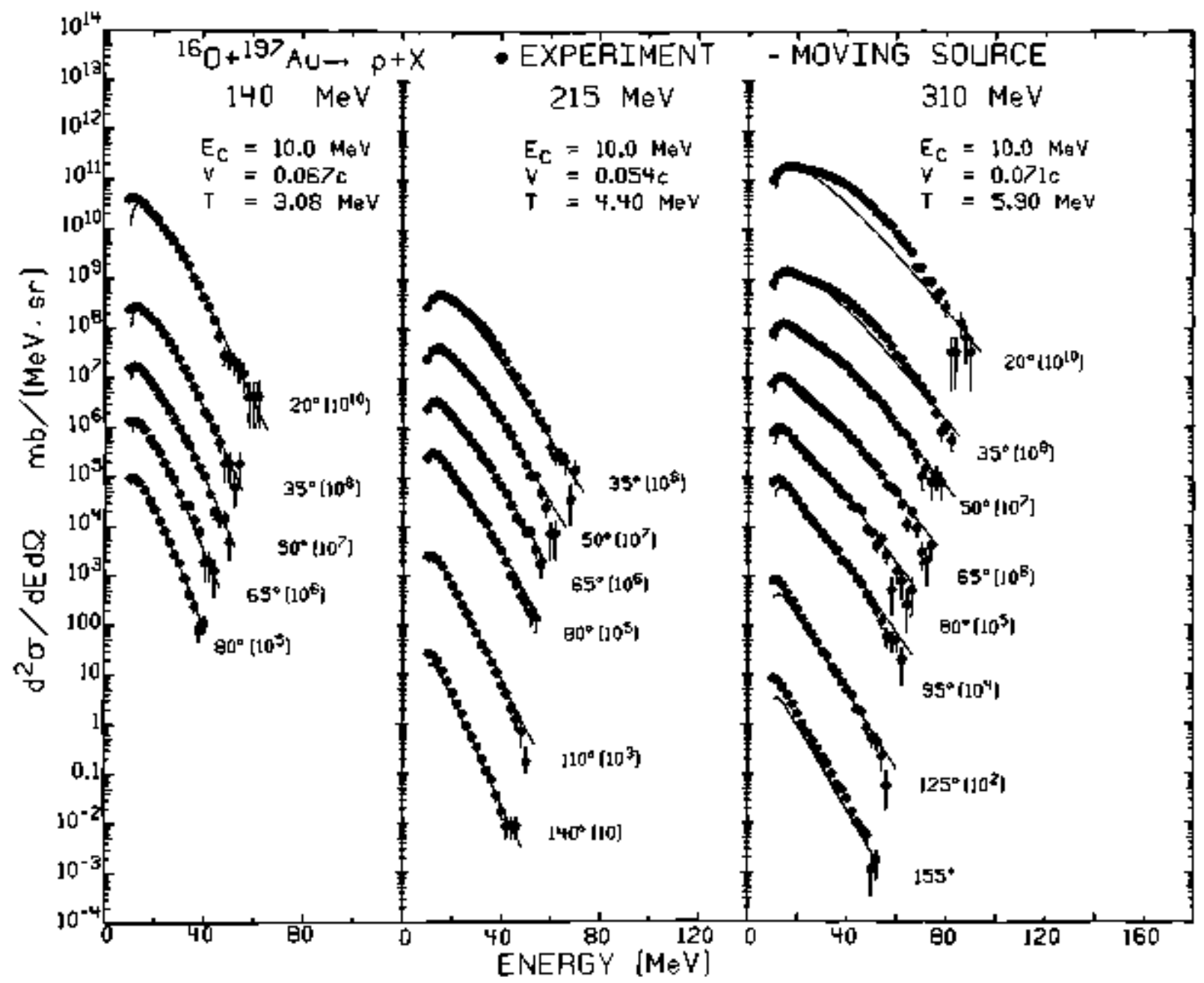

Fig. I. Energy spectra of protons detected in the reaction ${ }^{19}{ }^{A u}\left({ }^{16} \mathrm{O}, \mathrm{p}\right)$ at incident energies of 140, 215, and $310 \mathrm{MeV}$. The carves hare been ealculated with Eq. (1) of the text.

(XBL 8111-12354) 


$$
\begin{aligned}
\frac{d^{2} \theta}{d E d \Omega} & =N_{0}\left(E-E_{0}\right)^{1 / 2} \\
\exp & {\left[-\left[E-E_{c}+E_{1}-2 E^{1 / 2}\left(E-E_{2}\right)^{1 / 2} \cos \theta\right] / T\right\} . }
\end{aligned}
$$

Here, $\mathrm{E}_{\mathrm{c}}$ is the kinetic energy gained by the Coulomb repulsion from the target, $E_{1}=1 / 2 v^{2}$ is the kinetic energy of a particle al rest in the moving frame, $T$ is the temperature, and $\mathrm{N}_{0}$ is a normalization constant. The suc cessful application of this parameterization should not be interpreted as evidence for a hot gas of nucleons separated from the target nucleus. ${ }^{1,2}$ Instead, it indicates the randorsization of light particle velocities in a rest frame that is different from the compound nucleus rest frame. In the prestent analysis, the values of $\mathrm{E}_{\mathrm{c}}=0,5$, and $10 \mathrm{MeV}$ were used for the $\mathrm{Al}, \mathrm{Zr}_{\text {r }}$ and Au targets, respectively.

The temperature parameters determined in this experiment are shown in Fig. 2, where they are plotted as a function of $\left(E-V_{C}\right) / A$, the beam energy per nucleon above the Coulomb barrier. For a given type of light particle, the apparept temperatures are approximately proportional to $[(E-V) / A]^{1 / 2}$ or, equivalently, to the relative velocity of projectile and target at the point of contact (see insert. Fig. 2). Such a dependence is not expected from contpound nucleus emission. It rather suggests more rapid processes such as knockout or, perhaps, the formation of a thermalized subset of nucleons consisting of about equal numbers of target and projectile nucleons. The deduced values of $T$ are systematically larger for deuterons and tritons than for protons. Al present, it is not clear whether this effect oould be due to different contributions to the spectra from mort equilibrated processes such as compound nudeus ovaporation.

The paramelerization used to describe our data is similar to the thermal models 3,4 that have been used to describe light particle spectra from relativistic heavy-ion collisions. In order to compare the trends observed at low energies to the ones at relativistic energies, we hawe deter. mined the temperature parameters for the reaction ${ }^{20} \mathrm{Ne}+$ $\mathrm{NaF} \rightarrow \mathrm{p}$ at energies of $\mathrm{E} / \mathrm{A}=400$ and $800 \mathrm{MeV}^{5}$ by using the relativistic gemeralization of Eq. ( I):

$$
\begin{gathered}
\frac{E_{d^{2} \sigma}}{\rho^{2} d p d n}=\mathrm{N}_{0} \gamma(E-\beta p \cos \theta) \\
\exp (-\gamma(E-\beta p \cos \theta) / T) .
\end{gathered}
$$

where $\beta$ is the velocity of the source (c $=1), \gamma=(1-$ $\left.\beta^{2}\right)^{-1 / 2}$, and $E=\left(p^{2}+m^{2}\right)^{1 / 2}$. To minimize the contri. bution from projectile fragmentation and knockout. ${ }^{5}$ we have testricted the data to large transverse momenta $(\theta \geq$ $45^{\circ}$ ) for the determination of T. Despite the simplicity of the parameterization, the fits are of acceptable quality. The resulting temperature parameters are included in Fig. 2.

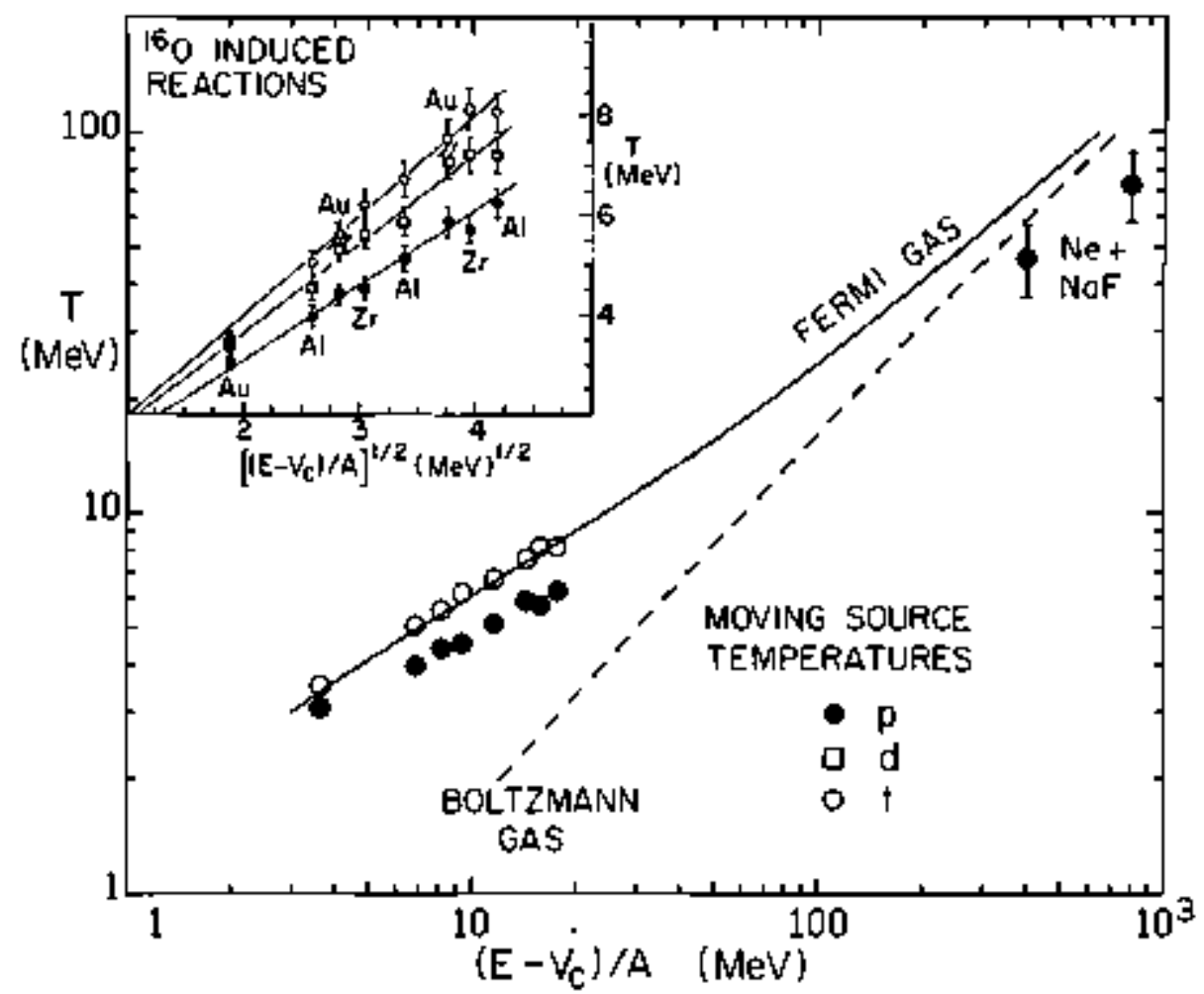

Fig. 2. Temperature parameters fot the proton, deuteron, and triton spectra in ${ }^{16} \mathrm{O}$ induced reactions on targets of Al, $\mathrm{Zr}$, and Au at incident energies of 140,215 , and $310 \mathrm{MAV}$ and for the reaction $\mathrm{Ne}+\mathrm{NaF} \rightarrow \mathrm{p}$ at 400 and $800 \mathrm{MeV} / \mathrm{A}$. The solid and dashed curves are explained in the text.

(XBL 8111-12355) 
As is obvious from Fig. 2, the trends observed at low energies ean be connected smothly to the observations at high entergies. For orientation, the solid and dashed curves have been calculated for relativistic Fermi and Boltzmann gases consisting of equal nucleon contributions from target and projectile.

The general trend of the experimental temperature parameters is seen to follow the one depieted by the Fermi gas carve. Although the observed trends suggest the thermalization of a subst of nucleons, investigations should be made to determine if alternative approaches such as single or multiple nucleon scattering models or hydroctynantrical models would predict similar trends.

\section{Foothole and References}

"Michigan State University.

1. T.C. Awes et al., Phys. Rev. Lett. 45, 513 (1980).

2. T.C. Awes te al., Phys. Rev. C 24, 89 (1981).

3. G.D. Westfall et al., Phys. Rev. Leti. 37, 1202 (1976).

4. J. Gosset, J.I. Kapusta, and G.D. Westall, Phys. Rev. C 18, 844 (1978).

5. S. Nagarnya et al., LBL 12123, to be published; and private comerenicatión. 


\title{
2. Angular Momentum Transfer
}

\section{Q-Value and Z Dependence of Angular Momentum Transfer in Deeply Inelastic Scattering of ${ }^{56} \mathrm{Fe}+{ }^{209} \mathrm{Bi}$}

\author{
M.S. Zismam, R.J. Puigh, R. Yandenbosck, ${ }^{*}$ T.D. Thomas, ${ }^{\dagger}$ and L. Nutnelleyt
}

In the last several years our group at the SuperHLAC has been interested in studying the angular mornentum transfer, and its alignment, in the depply inelastic scattering (DIS) process. ${ }^{1-4}$ The technique we have developed to accomplish our goal is described in detail in Ref. 2; it involves measurement of the in-plane and out-of-plane sequential fission angular corrtlations of a heavy target which is excited by a DIS process.

Subsequent to the publication' of our ${ }^{86} \mathrm{Kr}+{ }^{209} \mathrm{Bj}$ data a similar experiment was performed by Specht, ${ }^{5}$ but using reverse kinematics, i.e., a ${ }^{2088} \mathrm{~Pb}$ beam bombarded targets of ${ }^{90} \mathrm{Zr}$ and ${ }^{58} \mathrm{Ni}$. In contrast to our results, how ever, they reported that there was wo evidence for an inplane anisctropy in their data. Given this discrepancy, we have re-examined some of the techniques used to analyze our (kinematically incomplete) experiments. ${ }^{1-3}$

First of all, we have looked jinto the approximation, made in previous analyses, that the laboratory to centerof-mass Jacobians can be property estimated by using "average" values corresponding to a symmelric mass split of the fissioning ${ }^{209}$ Bi system. We have done this by carrying out Monte Carlo calculations of the fission decay using a code provided by M.F. Rivet. ${ }^{6}$ The ende allows us to investigate the effects of a (presumed) syurnetric mass split having a standard deviation of $25 \%$ and an energy spread of 8\% After first ensuring that the code properly reproduced our "analytic" Jacobians in the limil of zero mass and entergy widths, we find corrections of the magnitude stown in Fig. 1 for the ${ }^{56} \mathrm{Fe}+{ }^{209} \mathrm{Bi}$ case. These corrections do not appear to be of primary concern for the $\mathrm{Fe}+\mathrm{Bi}$ system, since they are quite small over the angut lar range for which we have data. We note, howewer, that the corrections are in such a direction as to reduce the anisotropy compared with that obtained using the analytic Jacobians, that is, the forward angle cross sections are reduced somewhat while these at more backward angles are increased compared with the average.

It turns out, however, that the size of this correction depends strongly on the velocity of the recoiling target-like nucleus. Thus, for the ${ }^{86} \mathrm{Kr}+{ }^{209} \mathrm{Bi}$ system studied earlier, the corrections are larger. Fortunately, however, our $\mathrm{Kr}+\mathrm{Bi}$ data were measured at fairly forward angles $\left(<61^{\circ}\right.$ ) so the corrections will not be more than about 15-20\% (Agein, the sense of the correction is to reduce, but not eliminate, the reported ${ }^{1,2}$ anisotropy.) Because of the experimental technique employed, the Specht dalas require no such correction.

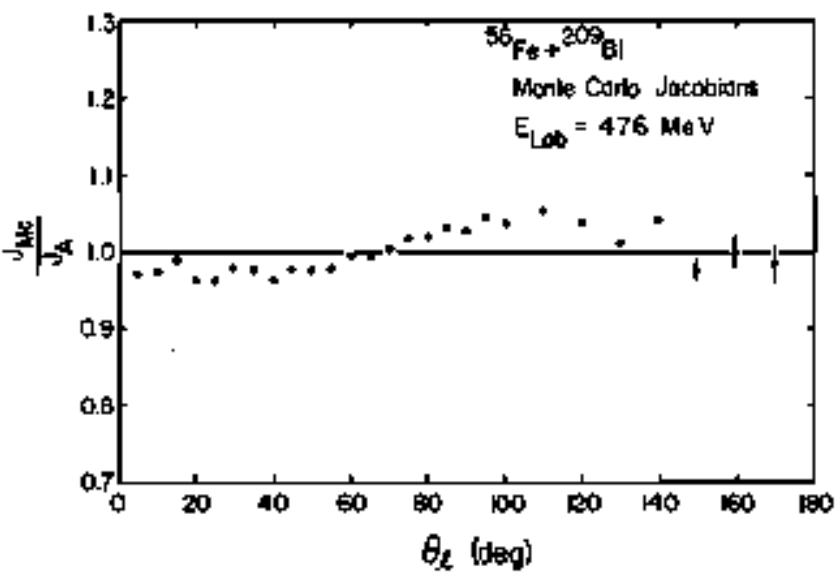

Fig. 1. Ratio of Monte Carlo Jacobians (including the finite mass distribution) to analytic Jecobians (assuming enission of two equal mass fragments) for ${ }^{56} \mathrm{Fe}+{ }^{209} \mathrm{Bi}$.

(XBL 813.472)

Another aspect of the data analysis has to do with the location of the symmetry axis. Based on the obseryed peaking along the recoil direction, Vandenbosch ${ }^{7}$ has developed a model in which the Fermi motion of the transferred nucleons generates a random component of angular momentum in the plane perpendicular to the line of centers belween the collision partners. This leads to a symmetry axis along the recoil direction, i.e, $0^{\circ}$ in our coordinate system. In fact, bowever, the correspondence between the recoil axis and the symmetry axis is strictly true only for the elastic scattering case. A shift in the symmetry axis will influence (but probably only slightly) our determination of the random component $\mathrm{j}_{2}$. More importantly, it could modify to some extent the angular distribution reported by Specht ${ }^{5}$ since their data were reflected around $\theta_{\mathrm{t}-\mathrm{m}}=0^{\mathrm{o}}$; reflection around a displaced axis will tend to reduce the apperent anisotropy in the data.

On the assumption that the random component of angular momentum is indeed generated in the plane perpendicular to the line of enters, we have estimated the location of the symmetry axis by making use of a trajectory calculation code, due to Randrup ${ }^{8}$ which employs a onebody dissipation mechanism including a neck degree of freedom By following the trajectory corresponding to a particular energy loss, we determine the location of the line of centers between the interacting nuclei at the point when 
belf of the total number of exchanges has taken place. In our picture, this direction corresponds to the (laboratory) symmetry axis for that energy loss. For both the Fe $+\mathrm{Bi}$ and $\mathrm{Kr}+\mathrm{Bi}$ systems of interest here, we get results very nearly identical to what one would predict from the simple picture in which the owerall trajectory is viewed as a single coulomb orbit. In this latter ease the normal to the line of centers at the halfway point in the orbit is at $\theta_{c . m a} / 2$ and the laboratory symmetry angle is given by

$$
\theta_{\mathrm{sym}}=\frac{\theta_{\mathrm{cm}}-\pi}{2} .
$$

Athough we bave not yet incorporated this refinement into ouf fitting code, the predicted location of the symmetry axis generally seems to agres well with the data points.

If we compare our results for $\mathrm{Fe}+\mathrm{Bi}$ with the $\mathrm{Pb}+$ $\mathrm{Ni}$ in-plane correlation of Specht ${ }^{5}$ we see (Fig. 2) that the shapes of the two data sets agree reasonably well except for the normalization. Interestingly, a French group has recently repeated the experiment on the ${ }^{58} \mathrm{Ni}+200 \mathrm{~Pb}$ system at $7.5 \mathrm{MeV} / \mathrm{A}$, but without asing "reverse" kinemat. ics. In agreement with our resuits, their preliminary data indicate a rather large in-plane anisolropy, peaked more or-less along the resoil direction. Thus, the disctepancy still appears not to be completely understood.

\section{Footnotes and References}

*University of Washington, Seattle, WA 98195 .

tOregon State University, Corvallis, OR 97331 .

†Chemeketa Community College, Salem, OR 97308.

1. P. Dyer et al., Phys. Rev. Lett. 39, 392 (1977).

2. P. Dyer et al., Nucl. Phys. A 322, 205 (1979).

3. R.J. Puigh et al., Phys. Lett. B 86, 24 (1979),

4. R.J. Puigh et al., Nucl. Phys. A 336, 279 (1980).

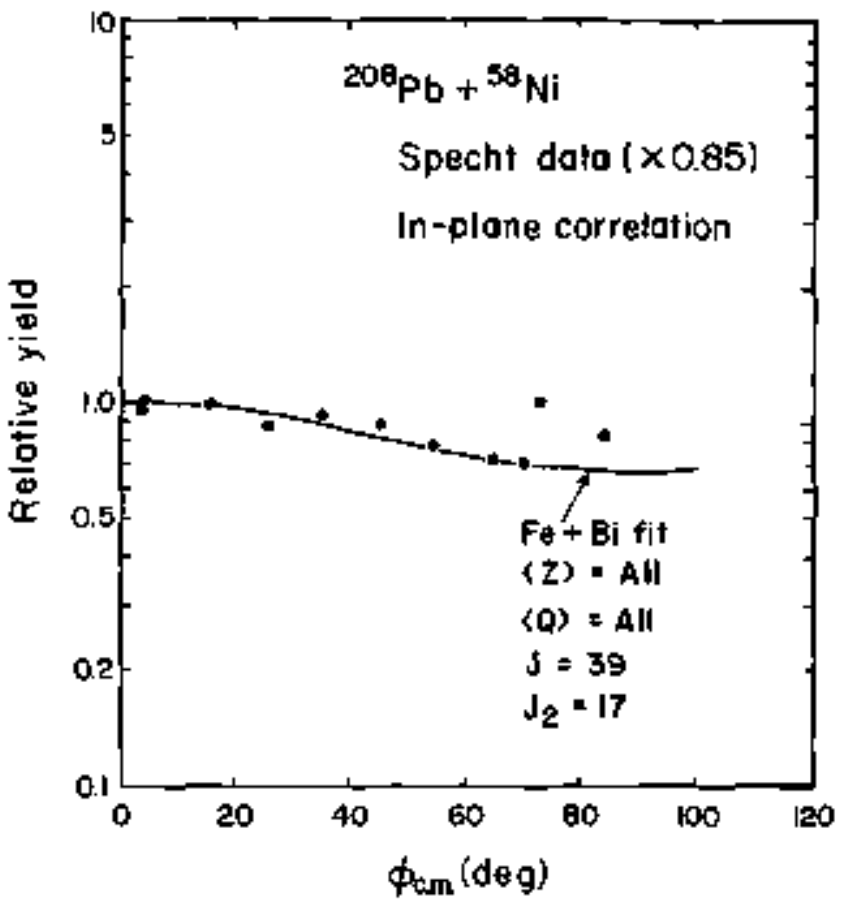

Fig. 2 Renormalized in-plane angular correlation from the ${ }^{208} \mathrm{~Pb}+{ }^{38} \mathrm{Ni}$ reaction (Ref. 5) compared with the best fit curve lor our ${ }^{56} \mathrm{Fe}+{ }^{20 \%} \mathrm{Bi}$ data.

(XBL $813-474$ )

5. H.J. Specht, Invited Talk at lni. Conf. on Nuct. Interactions, Canberra, Australia, 1978; D.V. Harrach et al., Phys. Rev. Lẹt. 42, 1728 (1979).

6. M.F. Rivet, privale communication.

7. R. Vandenbosch, Phys. Rev, C 20, I7l (1979).

8. J. Randrup, (unpublished).

9. C. Le Bron et al., preprint, 1980 .

\title{
Total $\boldsymbol{\gamma}$-Ray Energy Measurements for the Reaction ${ }^{176} \mathrm{Yb}+{ }^{165} \mathrm{Ho}$ at 8.5 MeV/A
}

\author{
R. McDanald, A.J. Pacheco," G.J. Woznlak, D.J. Morrissey, L.G. Sobotka, \\ L. G. Moretfo, H. Lindenberger, C. Schück, ${ }^{\ddagger} S$. Shih, \\ J.O. Newron.' R.M. Diamond, and F.S. Stephens
}

The correlation between fragment spin and $\gamma$-ray multiplicity has been used extensively to investipate angular mamentum transler in both compound-nucleus and drepinelastic reaclions. Since rotational energy is proportionel to spin, a similar correlation should exist between the total $\gamma$-ray energy emitted from the reaction products and the transferred nuclear spin. This latter technique has been used to investigate high-spin states formed by sompoundnucleus reactions. In this paper, we report the first measurement of tọtal $\gamma$-ray energy for a deep-inelestic resction.
The reaction ${ }^{176} \mathrm{Yb}+{ }^{165} \mathrm{Ho}$ was chosen since it had previously been siudied via continuum $\gamma$-say mulliplicity and anisotropy techniques.

The rationale behind the sum-crystal technique is that nuclear rotational energy is given by $E_{s}=l(I+\$) / 20$ where $\theta$ is the nuclear spin and $I$ is the moment of inertia. In reality, this picture is complicated by the emission of statistical $\uparrow$ rays and neutrons which deposit energy in the sum crystal along with the "rotational" $\gamma$-rays. Fluctuations plus the $<1002$ efficiency of the sum crystal also 
increases the width of the distribution above that expected from the distribution of spins alone.

Two particle detectors were placed at $28^{\circ}$ to the beam direction. Scattering into evther of these detectors defined a reaction plane. A $5 \times 6$ inch Nal deloctor was placed in this reaction plane at $90^{\circ}$ to the beam An identical detector was placed perpendicular to this direction, also at $90^{\circ}$ to the beam. This detector looked through a bole in the sum erystal. The upper and lower halves of the sum crystal were separated by 2 inches.

The $5 \times 6$ inch NaI detectors were used to measure the $\gamma$-ray multiplicity and anisotropy for the reaction ${ }^{176} \mathrm{Yb}$ $+{ }^{165} \mathrm{Ho}$ at $8.5 \mathrm{MeV} / \mathrm{A}$ over the range of $\mathrm{Q}$-values from 0 to $-400 \mathrm{MeV}$. Almost all of these particle- $\gamma$ colncidence events were accompanied by sum-energy data.

Figure 1 shows the resulting data for the average $\gamma$ ray energy and the standard deviation of its distribution as a function of Q-value. Only "arbitrary energy units" are used in Fig. 1 due to difficulties in callbrating the sum spectrometer.' Note how the total enetgy increase with increasing Q.yalut, similar to the well known inerease of the $\gamma$-ray multiplicity with energy damping. Besides uncertainties in the energy calibration, the sum energy contaiss significant energy due to neutrons (up to 30 neutrons are enitted per teaction) and statistical $\gamma$-rays.

Figure 2 a shows the $\gamma$ ray multiplicity both with and without gates on the sum energy. Similarly, Fig. 2(b)

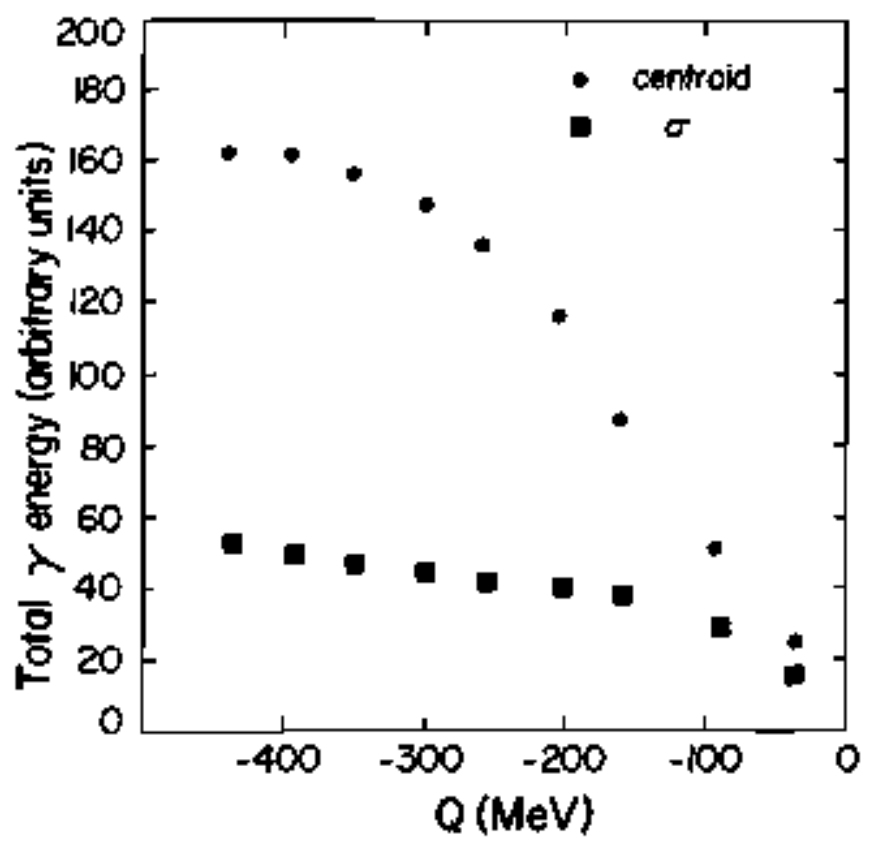

Fig. 1. Centroid and standard deviation of the sum-energy distribution.

(XFL 818-2444)

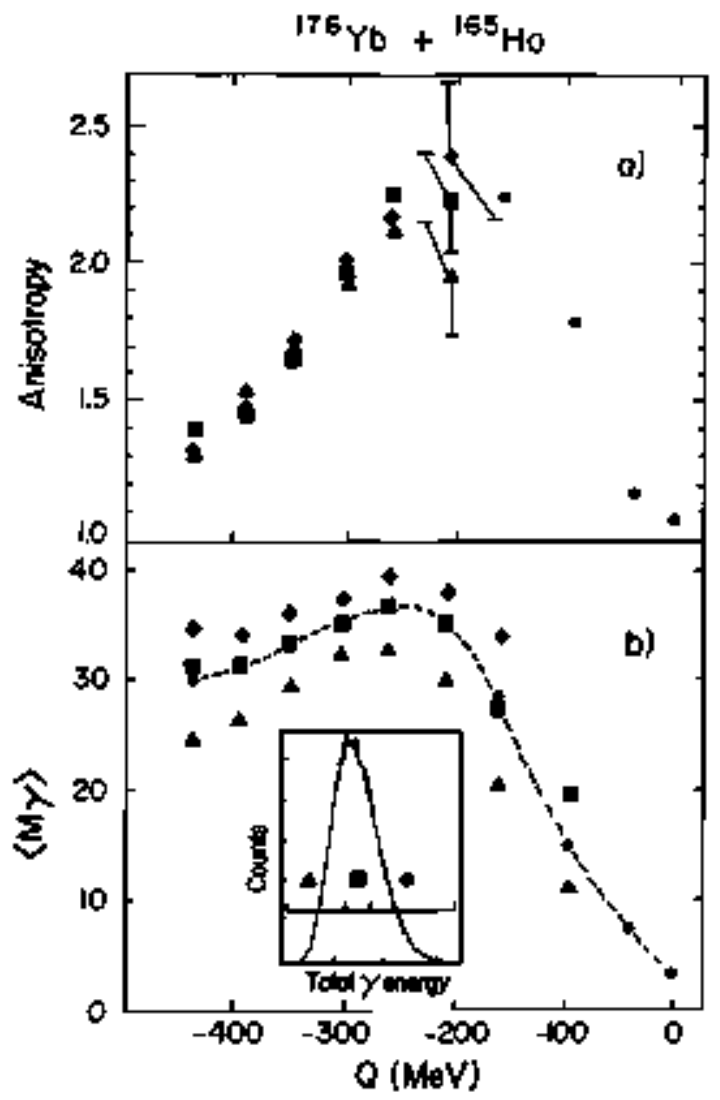

Fig. 2. (a) Continuum $\gamma$-ray anisotropy and (b) $\gamma$-ray multiplicity with and without gates on sum-E shown as an insert.

(XBL. 818-2443)

shows the continuum $\gamma$-ray anisotropy both with and without sum-energy gates.

At present, significant uncertainties are associated with a quantitative interpretation of the sum-energy data. However, the trends in multiplicity and anisotropy are as expected from the simple theory given above. A gate on higher than average sum energy is associated with larger than average $\left\langle M_{\gamma}\right\rangle$ (and therefore spin) and higherthan-average spin events end to exhibit a slightly higher anisotropy.

\section{Foothotes and References}

"Comisión Nacional de Energía Atómica, Argentina.

THahn-Meitner Institute, Beflin, Germany.

tC.S.N.S.M, Orsay. France.

SShanghai Institute of Nuclear Research, People's Republic of China.

Australian National Unjversity. Canberra.

1. F. Folkmonn et al., Nucl. Phys. A 361, 242 (1981). 


\title{
Angular Momentum, Statistical Equilibrium and Sequential Fission in Very Asymmetric Systems"
}

\author{
D.J. Morrissey. ${ }^{\dagger}$ G.J. Wozniok, LG. Sobotka, A.J. Pacheco ${ }^{\ddagger}$ \\ C.C. Hsw, R.J. McDonald, and LG. Moretto
}

The messurement of the internal spin and its alignment for reaction partners from deep-inelastic heavy-ion collisions (DIC) has become an importani tool for studying the process of angular momentum transfer. The measuroment of fluetuations in the spin components prowides a test of whether statistical equilibrium of angular momentum bearing modes of the dinuclear complex is achieved in a DIC.' Evidence for statistical equilibrium has been observed in symmetric reactions which have been studjed by means of y-ray angular distributions. In the very asymmetric ${ }^{20} \mathrm{Ne}+{ }^{197} \mathrm{Au}$ and ${ }^{278} \mathrm{U}$ systems the statistical excitation of a number of angular momentum bearing modes is strongly suppressed. In particular, a large difference in the rrornents of inerlia of the two reaction partners will increase the amount of energy necessary to excite any mode in which the small fragment is forced to rotate (wriggling, bending and twisting). Excitation of the only surviving mode (tilting) predicts a minimum in the angular distribution of sequential fission fragments along the line-ofcenters, ${ }^{2}$ Thus very asymmetric reaction systems should prowide an excellent test of the excitation of selected normal modes and of the statistical model in general.

A bean of $252-\mathrm{MeV}{ }^{20} \mathrm{Ne}$ from the 88-Inch Cyclotron was incident on targets of et ther $915 \mu \mathrm{g} / \mathrm{cm}^{2}{ }^{197} \mathrm{Au}$ or 922 $\mu \mathrm{g} / \mathrm{cm}^{2}{ }^{238} \mathrm{UF}_{4}$. Projectile-like products were detected in a solid state telescope (11 $\mu \mathrm{m} \Delta \mathrm{E}, 300 \mu \mathrm{m} \mathrm{E}$, do $=3 \mathrm{msr}$ ) fixed at $-30^{\circ}$. This angle is slightly behind of right at the classical grazing angles (26 and $30^{\circ}$, respectively). Fission fragments were detected in coincidence on the opposite side of the beam in an array of ten single element surfacebarrier detectors (dn $=9 \mathrm{msr} /$ detector), five in-plane and five out-of-plane. This array was moved to cover the angular region between $+30^{\circ}$ atrd $+160^{\circ}$ in-plane as well as between $0^{\circ}$ and $75^{\circ}$ oul-of-plane along and perpendicular to the laboratory recoil axis. Fission fragments were unambiguously identified in a two-dimensional map of hission fragment energy ws time. The dala were transformed event by event into the rest frame of the recoiling target nucleus using the energy, charge and angle of the light product and the energy and angles $(\theta, \phi)$ of the fission fragment. The measured angular distributions are presented in Figs. I and 2 for the ${ }^{20} \mathrm{Ne}+{ }^{238} \mathrm{U}$ and ${ }^{197} \mathrm{Au}$ systems as a function of Q-value. The data have been integrated over the fission fragment energy and the atomic number of projectile residues (6 $\leq Z \leq 14)$. The direction $\Phi^{\mathrm{H}}=\mathbf{0}$ was arbittarily chosen to coincide with the laboralory rocoil angle calculated as a function of $Q$ (negative angles lie belween the recoil direction and the beam). In order to ensure that evaporation was not biasing the kinematics, the data was also gated on $9 \leq Z \leq 10$. This gating does not alter the results, it only decreases the

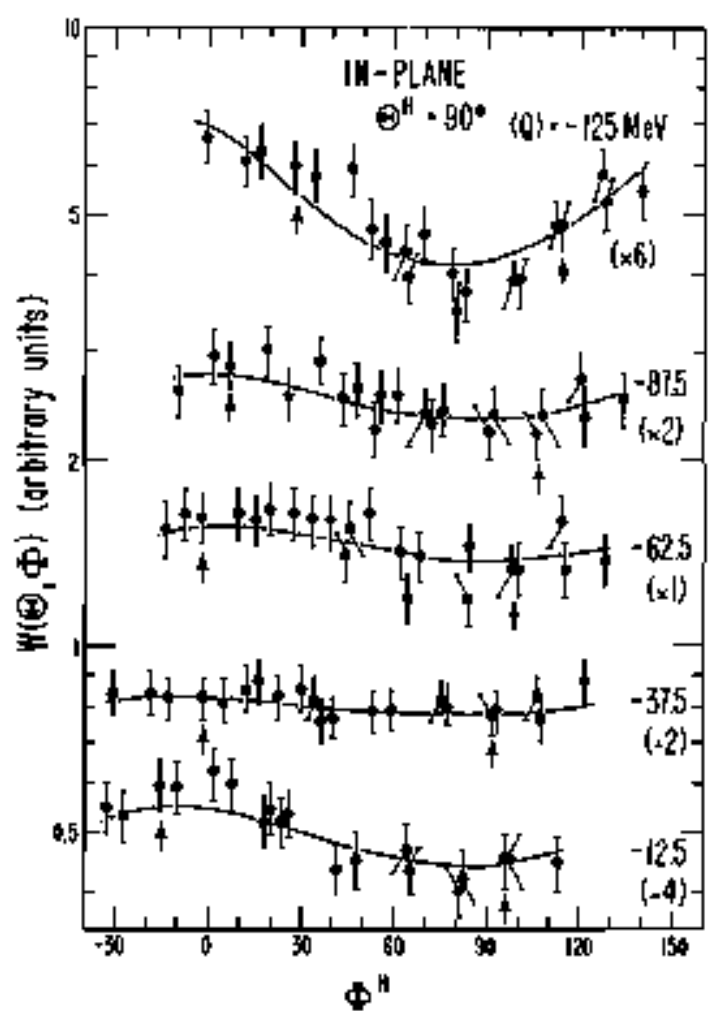

Fig. 1. The in-plane angular distributions of sequential fission fragments in the rest frame of the heavy fragment (H) are shown as a function of reaction Q-value for the ${ }^{30} \mathrm{Ne}+{ }^{238} \mathrm{U}$ system. The arrows indicate the in-plane angles at which ont-of-plane measurements were made.

(XBL 813-2180)

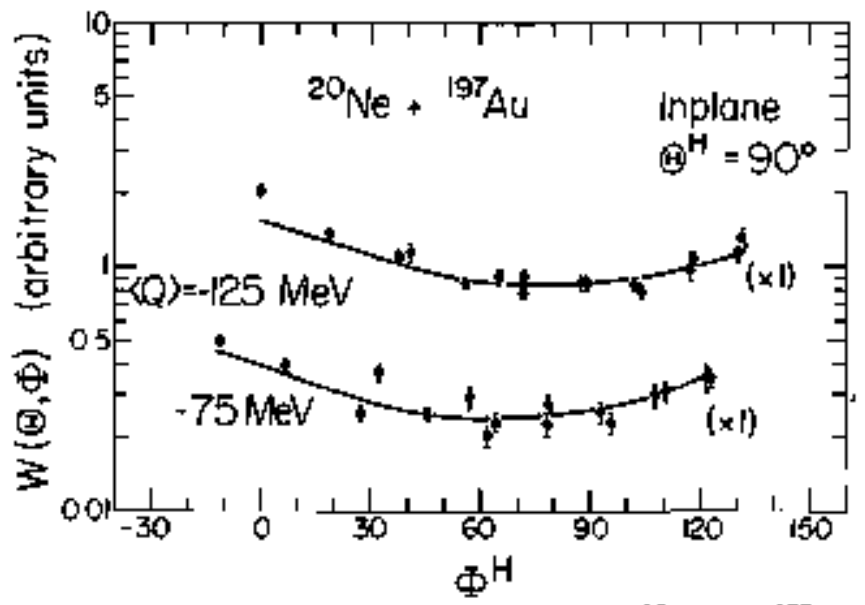

Fig. 2. Similar to Fig. I, except for the ${ }^{20} \mathrm{Ne}+{ }^{197} \mathrm{Au}$ system.

(XAL 817-2365) 
statistical accuracy.

In order to extract quantitative values for the spin polarization of the heavy fragment we have fit the angular distribution data to the generalized function of the statistical model of Broglia et al. ${ }^{3}$ In this description the three cartesian widths $\sigma_{x}, \sigma_{y}$ and $\sigma_{z}$ (defined such that $x$ lies along the line-of-centers) appear explicitly along with the projection of the spin on the alignment axis, $I_{2}$, and the projection of 1 on the fission separation axis, $K_{0}$. Finally, one must determine the direction of the line-of-centers of the intermediate complex with respect to the traditional reference direction, the laboratory recoil angle. The appropriate choice for the line-of-centers in a equilibrium statistical model is the line connecting the centers of the two fragments at scission when the diroctions and magnitudes of thermally induced spin components are frozen in. An extimate of the direction of the line-of-centers can be made by tracing the projectile-like product backward along the coulomb trajectory that corresponds to the average orbital angular momenlum, $\mathrm{l}_{\text {our }}$, for each bin. These estimates are consistent with the shift in the pasitions of the minime seen in Figs. I and 2. The results of reduced chisquared fitting of the function to the data are shown by the solid curves in Figs. 1 and 2 .
From the results of the fitting we find that the statistical model predictions are in good agreement with all of the out-of-plane angular distributions. For the more stringent test afforded by the in-plane angular distributions, this model overpredicts the anisotropy, except for the most negative $Q$ value where the agreentent is good. These trends agree with our expectations that the statistical model represents the long-time limit which is attained in collisions with the largest energy-losses.

\section{Footnotes and References}

"Condensed from LBL-12181, submitted to Z. Phys.

†Permanent address: Department of Chemistry, Michigan State University, East Lansing, Michigan 48824.

\$Permentent address: Comisión Nacional de Energía Atómica, Buenos Aires, Argentina.

SPermament address: Institute of Atomic Energy, Beijing, People's Republic of Ching.

1. L.G. Moretto and R.P. Schmit, Phys. Rev. C 21, 204 (1980).

2. R.P. Schmitl and AJ. Pacheco, to be published. Sec also contribution to this annual report.

3. R.A Broglia et al., Phys. Rev. Lett. 43, 1649 (1979).

\title{
Fragment Spin Alignment in Deep-Inelastic Reactions
}

\author{
A.J. Pacheco," R.J. McDanald, G.J. Wozniak, C.C. Hsu,' D.J. Morrissey, \\ LG. Sobotka, S. Shih ${ }^{\ddagger}$ R.M. Diamond, F.S. Stephens, and LG. Moretto
}

Recently, the megnitude and aligntment of the spin transferced to the fragments in the system ${ }^{165} \mathrm{Ho}+{ }^{165} \mathrm{Ho}_{\mathrm{O}}$ at $8.5 \mathrm{MeV} / \mathrm{amo}$ were studied using continuum $\gamma$-ray multiplicity and anisotropy techsiques. ${ }^{1}$ Results were in substantial agreement with the predictions of an equilibrium statistical model. ${ }^{2}$ In the present work we report preliminary results from a simular investigation carried out in three diderent reactions: ${ }^{1 / 6} \mathrm{Yb}+{ }^{165} \mathrm{Ho}_{0}{ }^{148} \mathrm{Sm}+{ }^{165} \mathrm{Ho}_{\mathrm{O}}$ and ${ }^{\mathrm{Ant}} \mathrm{AB}+{ }^{165} \mathrm{Ho}$, all at $8.5 \mathrm{MeV} / \mathrm{amu}$.

The purpose of the experiment was to investigate the extent to which the measurement of continuum $\gamma$-rays in different systems represents a useful technique in the atudy of angular-momentum transfer processes. The targets used cover a wide region of the chart of nuclides and therejore exhibit well differentiated nuclear properties. The neutron-rich ${ }^{176} \mathrm{Yb}$ nuclens lies in the region of the good rotors, and the expected evaporation of a large number of neutrons per fragment in these reactions still leaves the residual fragments in the rotational region. On the other hand all the isotopes in ${ }^{\text {nal }} \mathrm{Ag}$ are well removed from this region of good rotors. Finally, the ${ }^{148} \mathrm{Sm}$ nucleus, only 4 neutrons away from the $\mathbf{N}=82$ closed shell, represents an intermediate situation between these two regimes. It is also our purpase to utilize the data from the various systems to further test the validity of the equilibrium model.

The experimental set-up was similar to that used in Rer. I. Three surface-barriter Si delectors were used for heavy-particle detection. These counters logether with the beam direction determined two mutvally perpendicular reaction planes. Three $5 \times 6$ inch NaI crystals for $\gamma$-ray detection were arsanged so that each of then gave at least one in-plane and one $90^{\circ}$-out-of-plane measurement when obserwed in cojncidence with the various Si detectors. This arrangernent provided a high redundancy that helped in the identification and correction of possible systematic errors. In addition an array of eight $3 \times 3$ inch Nal detectors (multiplicity filter) was used, and the number of detectors furing per event was recorded.

For each of the throe targets average multiplicities and anisotropies were calculated as a function of $Q$-value. The average "in-plane" and "out-of-plane" multiplicity was calculated as proportional to the ratio between particle- $\gamma$ coincidences and particle singles, using all the combinations between each $5 \times 6$ inch Nal detector and each $\mathrm{Si}$ detector. Corrections for angular distributions were made in order to calculate the average multiplicity 
$\left\langle\mathrm{M}_{\gamma}\right\rangle$. The anisotropies were calculated as the ratio between in-plane and out-of-plane multiplicities.

Figure I shows the dependence of $\left\langle\mathrm{M}_{\gamma}>(9)^{\circ}\right)$ on $Q$ for the three systems. There is a characteristic rise in the quasi-elastic region followed by a saturation or even a slight decrease at the most inelastic events. The maximum value reached steadily decreasts when going from the ${ }^{176} \mathrm{Y}$ b to the nal Ag target. This trend is expected from changes in both the maximum total angular morrentim in the system and the moments of inertia of the Iragments. In all three targets the selection on 2-and-higher-jold coincidences in the multiplicity array produces a systematic increase of $c M_{>}>$throughout the whole Q-value range. This effect illustrates the correspondence between the independent measurements of high-fold $\gamma$-ray coincidences and $\left\langle M_{\gamma}>\right.$.

Figure 2 show $\gamma$-ray anisotropies as a function of $\mathbf{Q}$ value. For the throe targets ont observes a rise at the quasielastic and a fall throughout the deep-inelastic region.

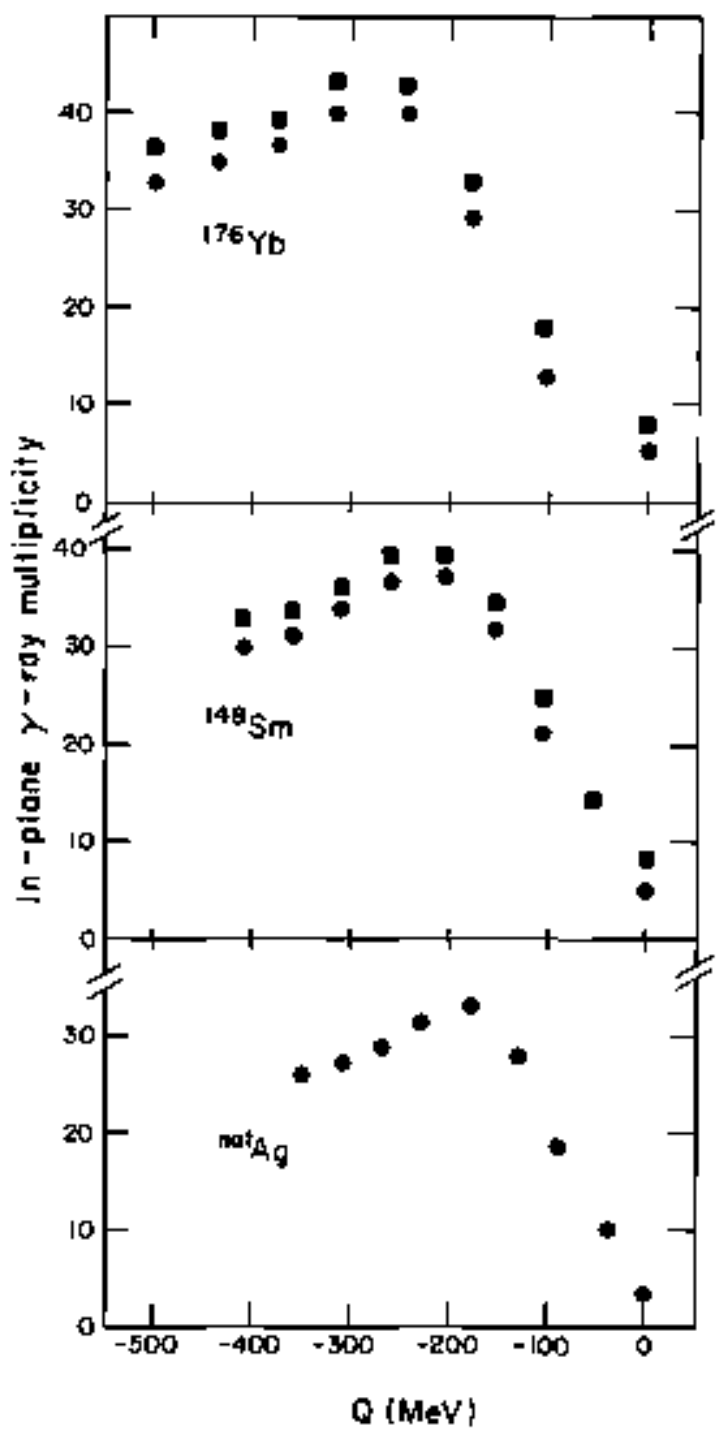

Fig. 1. In-plane multipicity as a function of $Q$ value with (squares) and without (circles) gates on bigh-fold coincidences.

(XBL 818-2448)

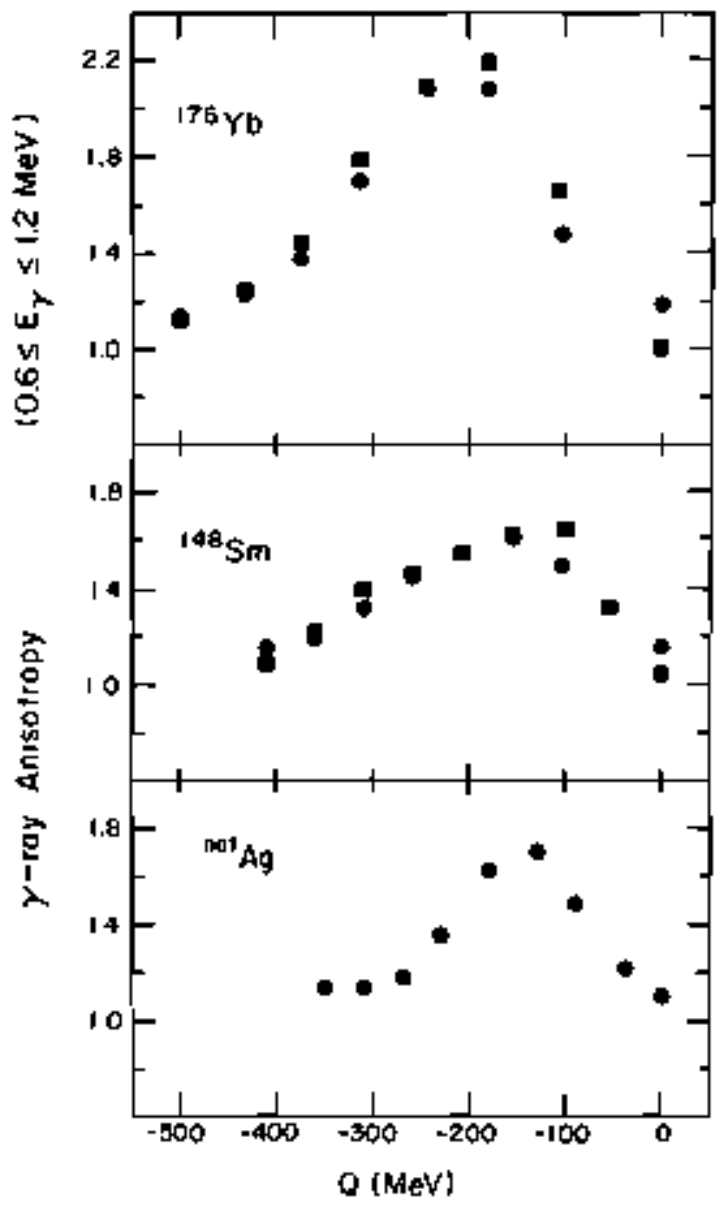

Fig. 2. Anisotropy as a function of Q-value with (squares) and without (circles) gates on high-fold coincidences.

(XBL 818-2449)

There is a notictable difference in the maximum value reached when the ${ }^{176} \mathrm{Yb}$ data are compared to those of the other two targets. However one can tentatively conclude that even in the ${ }^{\mathrm{nat}} \mathrm{Ag}+{ }^{165} \mathrm{Ho}$ reaction the predominant $\gamma$-decay occur via stretched E2 transitions, and therefore the observed anisotropies are correlated to the spin alignment. Finally one observes that gating on 2-and-higherfold coincidences does not alter substantially the anisotropies. This result may be ascribed to the faet that the geometrical acrangement of the multiplicity filier was not optimized to detect the in-plane-peaked quadropole radiation.

A more quantitative interpretation of these data in terms of the angular-momentum-transfer mechanisto and the comparison with the equilibriug model are underway.

\section{Footmotes and References}

"Comisión Nacional de Energía Atómica, Buenos Aires, Argentína.

'Institute of Atomic Energy, Beijing, People's Republic of China.

tShanghai Institute of Nuctear Research, People's Republjc of Ching.

1. G.J. Wozniak et al., Phys. Rev. Lett- 45, 1080 ( 1980).

2. L.G. Moretto and R.P. Schmith Phys, Rev. C21, 204 (1980). 


\title{
The ${ }^{12} \mathrm{C}+{ }^{12} \mathrm{C}$ Reaction Cross Section Between 70 and $290 \mathrm{MeV}$ Obtained From Elastic Scattering ${ }^{*}$
}

\author{
A.J. Cole, W.D.M. Rae, M.E. Brantant' A Datal, B.G. Harvey. \\ R. Legrain ${ }^{\ddagger}$ M.J. Murphy, and R.G. Stoksiad
}

The variation of the nuclear reaction cross section ${ }^{t} \mathrm{R}(\mathrm{E})$ has been investigated rectently by DeVries and collaborators. 1,2 They sugegest that the nuclear transparency increases quite rapidly at onergies above $10 \mathrm{MeV} / \mathrm{A}$ even for strongly absorbed projectiles such as a particles and that this increase seens to corselate wikh the known fall-of of the nucleon.mucleon cross section ${ }^{3,4}$ suggesting the Aominance of the latter in determining on . In particular their calculations prodict that for ${ }^{12} \mathrm{C}+\mathrm{H}^{2} \mathrm{C}$ scttering of and $\sigma_{T}$ reach a maximum at $E_{a m}=100 \mathrm{MeV}$.

Altbough some data for ${ }^{12} \mathrm{C}+{ }^{12} \mathrm{C}$ exist at higher 5,6 and lower 7,8 energies, there is a serious lack of experimental data on $\sigma_{\mathrm{R}}$ for beavy ions in this energy region. Thus toeasurements of the elastic seattering of ${ }^{12} \mathrm{C}+{ }^{12} \mathrm{C}$ were undertaken between 120 and $290 \mathrm{MeV}$.

The experiments were carried out using $4^{+}$and $5^{+}$ beams of ${ }^{12} \mathrm{C}$ from the LBL 88-Inch Cyclotron. Measure ments were made in $0.25^{\circ}$ steps in the forward angle region $\left(2^{\circ}-15^{\circ}\right.$ lab) which is of critical importance in the determination of $\sigma_{R}$ and $a_{T}$ Since an accurate knowledge of the scattering angle is essential for the accurate determination of $\sigma_{R}$ and $\sigma_{T}$, two sets of four litbium-drifted silicon detec tors of $3 \mathrm{~mm}$ thickosss were located on moveable arms at angles to the left and right of the beam direction. The targets Here made of natural carbon of thicknesses berween 275 and $1000 \mu \mathrm{g} / \mathrm{cm}^{2}$ deter mined to $\pm 5 \%$ by $\alpha$ energy loss measurements. Data were raken at 10 energies between $121.6 \mathrm{MeV}$ and $287.8 \mathrm{MeV}$.

Final differential cross sections were obtained by averaging results from left and right detectors, thereby eliminating to first order effects of beam movement and nisalignmest. The angular error of the data was essentíally due to the precjsion with which the angles of the detectors could be set and was estimated to be $0.125^{\circ}$. An additionsl measusement at $289 \mathrm{MeV}$ was carried oul using two position-sensitive detectors and a strip target. The angular aecuracy in this case was $\pm 0.07^{\circ}$.

Several of the resultant differential cross sections are shown in Fig. 1. The results are plotted against momentum transfer rather than c.m. angle to emphasize that the positions of the most forward angle minima are almost energy independent and that the depths of the minima evolve smogthly over the energy range investigaled.

A phase shift paramelerization was used in a computer program that optimized the parameters using a nonlinear least squares fitting procedure. The ability of this parameterization to reproduce known values of $\sigma_{R}$ and $\sigma_{T}$ was determined by fitling theoretical "data" between $5^{b}$ and $25^{\circ}$ (cm) tenterated from an optical model calculation. Values of $\sigma_{R}$ and $\sigma_{T}$ so deduced agreed to within $1.5 \%$ with

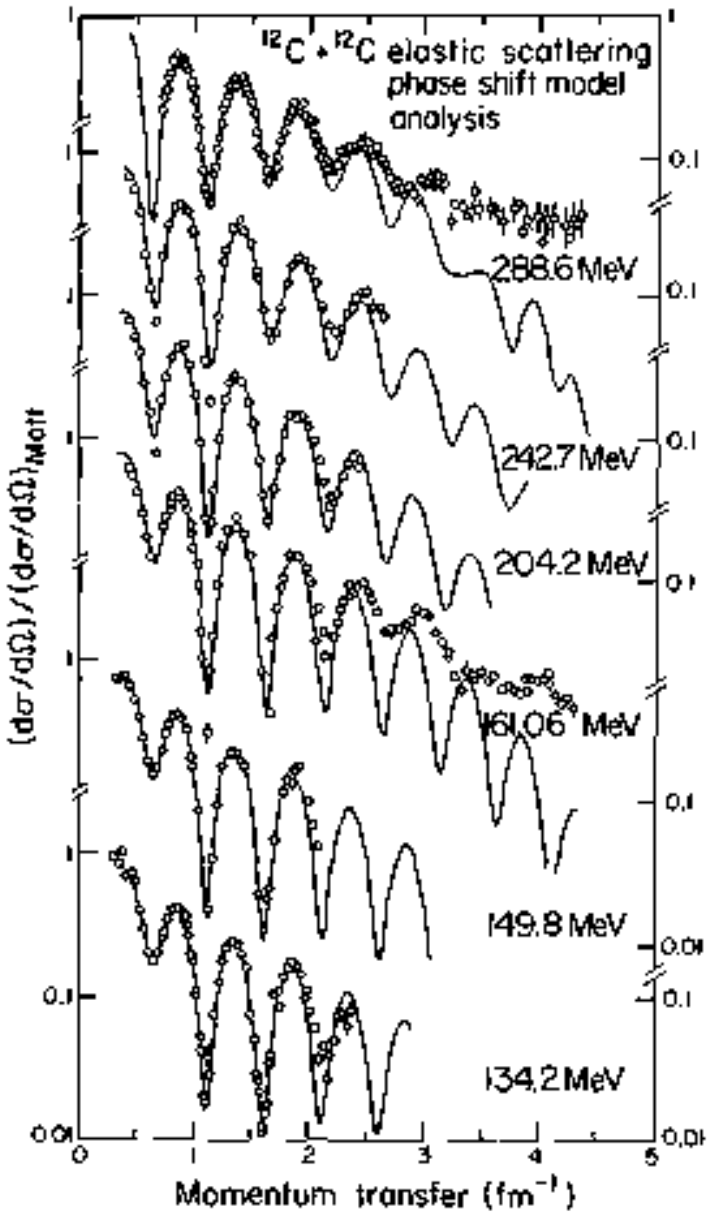

Fig. 1. Elastic seattering cross sections as a function of momentum transfer. The smoth lines are the results of calculations using the parameterized phaste shift model.

(XBL $817.2416 A)$

the optical model values. Values ablained from the analysis of the experimental data are displayed in Fig. 2. The above analysis was also applied to publisbed data ${ }^{8}$ between 70 and $127 \mathrm{MeV}$ (Lab) and the results are included in Fig. 2.

To investigate the model dependence of the deduced values of $\sigma_{R}$ and $\sigma_{T}$, our data were also fitied using the standard 6 parameter optical model. It should be made clear that in these fits emphasis was placed on obtaining the best description of the forward angIt diffraction structure and not on the determination of a suroothly enersydependent optical model parameter set. The deduced 


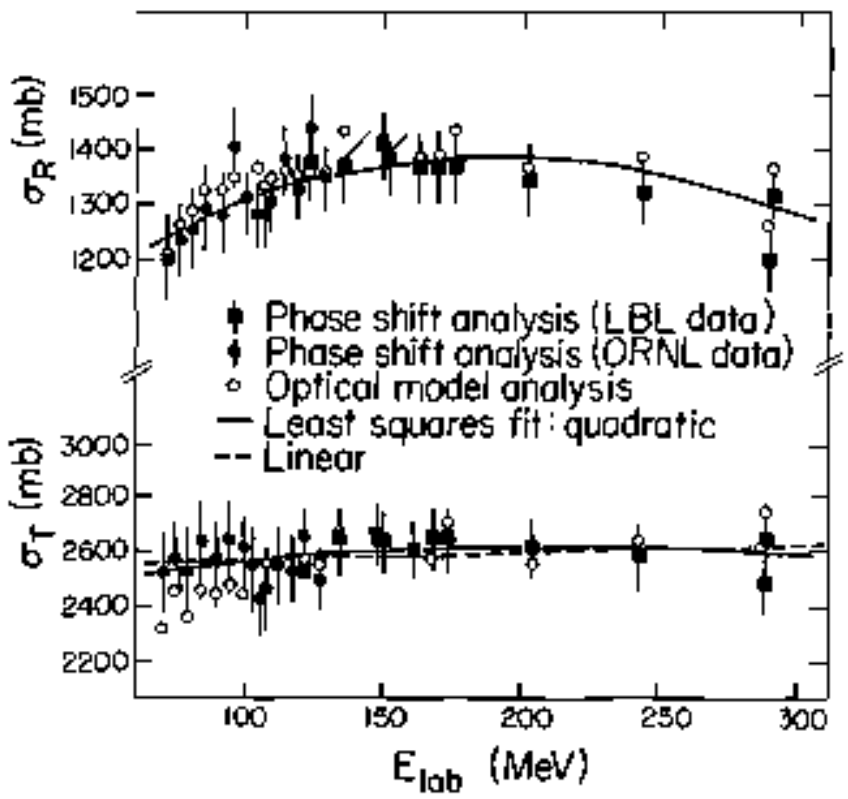

Fig. 2. Deduced values of $\sigma_{\mathrm{R}}$ and $\sigma_{\mathrm{T}}$ from parameterized phase shift analysis shown with least squares quadralic fit for $\sigma_{\mathrm{R}}(\mathrm{E})$ and least squares linear and quadratic fits for $\sigma_{\mathrm{T}}(\mathrm{E})$. Results of optical model fits are also given.

(XBL $817-2417$ )

values of $\sigma_{R}$, although slightly higher (sez Fig. 2), exhibit the same trend with energy as those obtained from the phase shift model. The values of $\sigma_{\mathrm{T}}$ are also very similar for the two models except at the lousest energies.

In Fig. 3 our results for $a_{\mathrm{B}}$ are compared with the calculations of DiGiacomo of al. ${ }^{2}$ and those for $\sigma_{\mathrm{T}}$ with that of Peng et al. ${ }^{4}$ The calculation for $\sigma_{T}$ disagres somewhat with our data, which do not exbibit the sharp rise and fall of the predicted values. In fact our data are consjstent with a constant value $\left(a_{T}=2570 \mathrm{mb}\right.$ ) over the energy range studies. The agreement for $\sigma_{R}$ is more satisfactory. In particular it does appear that the reaction cross section reaches a maximum at $\mathrm{E}_{\mathrm{cm}}=100 \mathrm{MeV}$.

In conclusion our experiments indicate a maximum in $\sigma_{R}(E)$ at $E_{c m} \simeq 100 \mathrm{MeV}$ as predicted in Refs. I and 2 . However, the role of the energy dependence of the nucleon-mucleon force in producing such a maximum remains uncertain. Accurate measurements of $\sigma_{R}$ and ${ }^{t} T$ over a wider range of energits will undoubtedly be of use in understanding the respective roles of "mean field" and "nucleon-mucleon" aspects of the problem Further theoretical work in the low and mediom energy region near the maximum of $\sigma_{R}(E)$ would also be valuable.

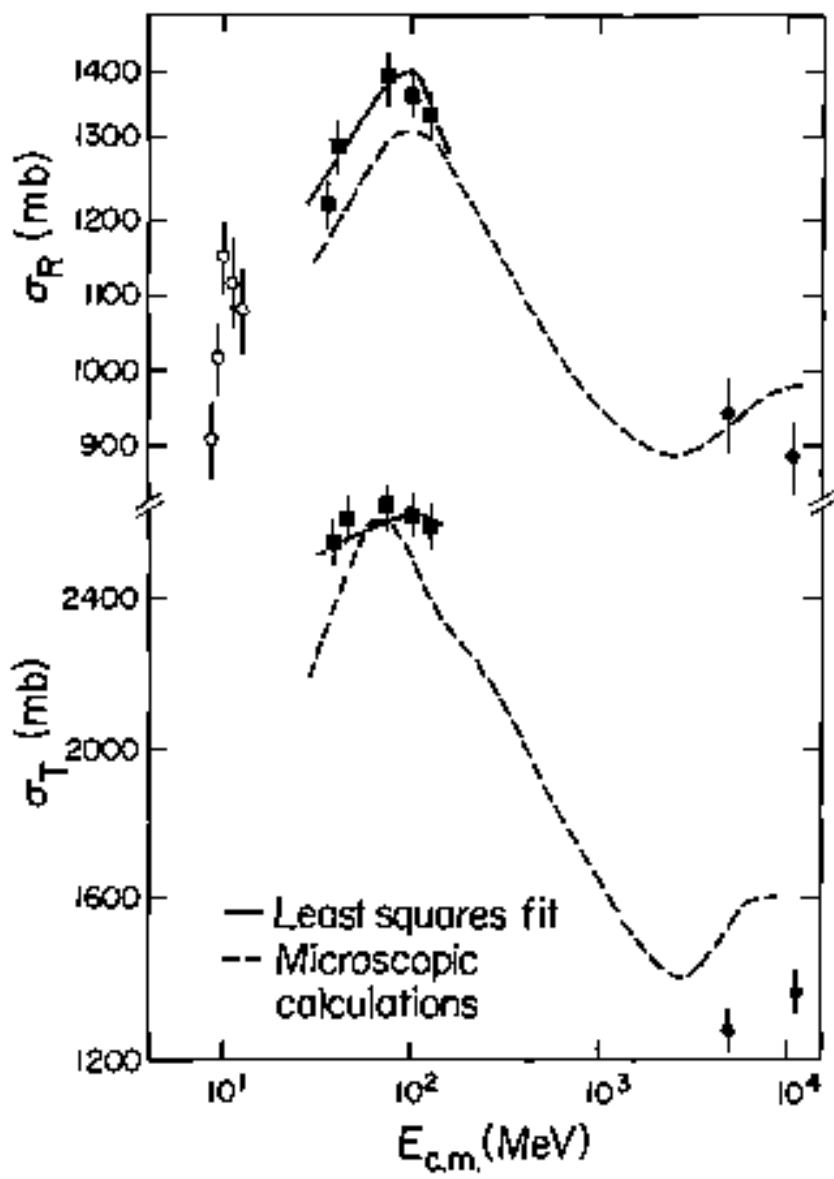

Fig. 3. Least squares quadratic 6ts from Fig. 2 shown with microscopic alculations for $a_{R}$ (Ref. 2) and $\sigma_{T}$ (Ref. 4), Representative data points from the present work (squares) as well as low energy data taken from Ref. 7 (open circles) and high entergy data from Ref. 6 (filled cirdes) are given in the figure.

(XBL 817-2418)

\section{Foomotes and References}

Condensed from LBL-13011.

†Permanent addres: Instituto de Fisjec, Universidad National Autonoma de Mexico, Mexico 20 DF.

Fermanent address: DPbN-BE, CEN-Saclay, PB no. 2. 91190 Gíf-sur* Yvett, France.

1. R.M. DeVries and J,C. Peng, Phys, Rev. Lett. 43, 1373 (1979); R.M DeVries and J.C. Peng. Phys. Rev. C 22, $1055(1980)$.

2. N.J. DiGiacomo, private comrnunjcation.

3. N.J. DiGiacono et al. Phys. Lett B 101, 383 (1981).

4. J.C. Peng, et al., Phys. Lett. B 98, 244 (1981).

5. M. Buenterd et al., Phys. Lett. B 102, 242 (1981).

6. T. Jaros et al., Phys. Rev. C 18, 2273 (1978).

7. W. Treu et al., Phys. Rev. C 22, 2462 (1980).

8. R.G. Stokstad et al, Phys. Rev. C 20, 655 (1979), 


\title{
Isomer Ratio Measurements for the Reaction ${ }^{29} \mathrm{Si}\left({ }^{18} \mathrm{O}, \mathrm{p} 2 \mathrm{n}\right){ }^{44} \mathrm{Sc}^{\mathrm{m}},{ }^{44} \mathrm{Sc}^{\mathrm{g}}$
}

\author{
H. Groening, K. Aleklett. ${ }^{\dagger}$ K.J. Moady, P.L. McGaughey, \\ W. Loveland $d^{\ddagger}$ and G.T. Seaborg
}

The isouxeric ratio dependence on projectile binttis energy for the reaction $\left.{ }^{29} \mathrm{Si}^{18} \mathrm{O}, \mathrm{p}_{2 \mathrm{n}}\right){ }^{44} \mathrm{Sc}^{\mathrm{m}}$. ${ }^{44} \mathrm{Sc}$, was previously reporied in the 1978-1979 LBL Nuclear Science Annual Report. ${ }^{1}$ We have attempted to fit the experimental data with essentially one parameter, that is, the ratio of dipole to quadrupole gamurs ray strengths. Our calculations were divided into two parts. It the first pert, we have used M. Blann's evaporation oode "ALERT"2 to calculate population distributions in the E-J plane for ${ }^{44} \mathrm{Sc}$. In the second parl, we have taken the portion of the ${ }^{44} \mathrm{Sc}^{*}$ population lying between the yrast line and a line roughly one neutron binding energy above it and calculated the fractions of such populations decaying to the isomeric and ground states, respectively. The best fit obtained in the calculation was for a quadrupole to dipolt gamma ray strengths ratio $\left|M_{10}(E 2)\right|^{2} /\left|M_{1}(E 1, M I)\right|^{2}=0.03$, where $M_{i f}(\sigma \lambda)$ is the reduced matrix element for radiation of multipolarity $\lambda$; o represents the nature of the radiation, magnetic of electric; and $|\mathrm{M}(\mathrm{El}, \mathrm{M})|^{2}$ is the sum of $\left|\mathrm{M}_{\mathrm{j}}(\mathrm{E})\right|^{2}$ and $\left|\mathrm{M}_{\mathrm{u}}(\mathrm{Ml})\right|^{2}$. Figure $\mathrm{l}$ shows sorne of the fits to the experimental data. The gamma ray strengths ratio deduced in this work agrees substantially will with the one estimated from measured gamma ray strength averages in the literature ${ }^{3}(-0.02)$. Reducing the number of discrete levels results in an increase of the isomeric ratio.

Footnotes and References

"On leave from Universidad Simón Bolivar, Caracas, Yenezoela.

†Present address: Sudsvik Science Res. Lab. S-611 82, Nyköping, Sweden.

FPresent address: Radiation Center, Oregon State Univ., Corvallis, OR 97330.

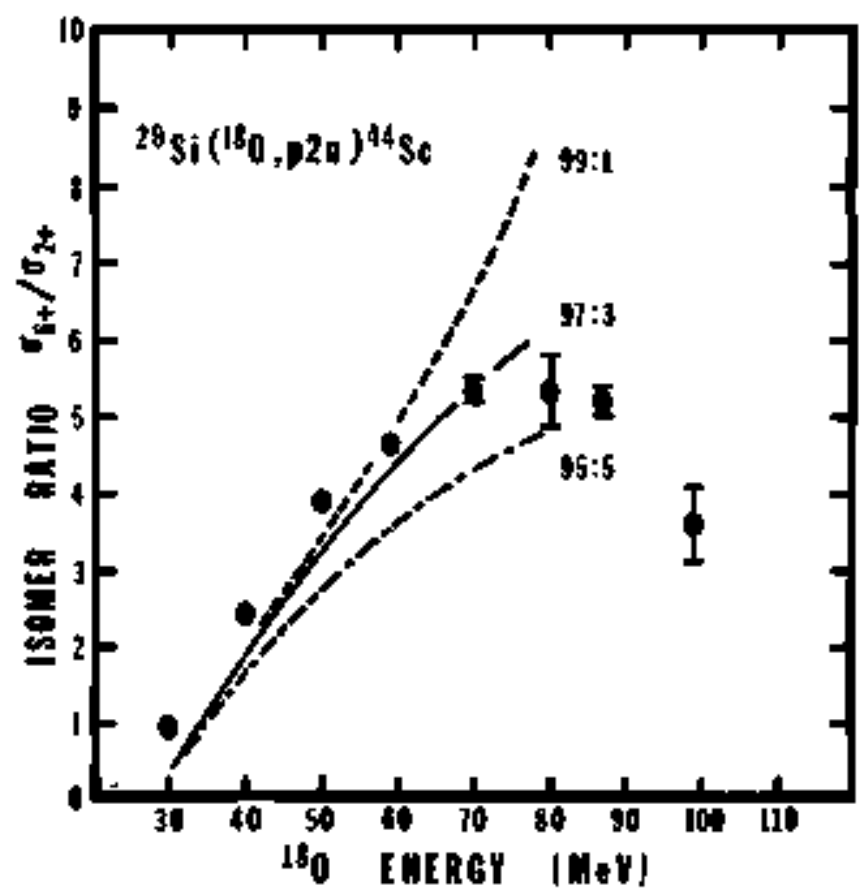

Fig. I. Isomer ratios and calculated fits for the reaction ${ }^{29} \mathrm{Si}\left({ }^{18} \mathrm{O}, \mathrm{p}^{2} \mathrm{n}\right){ }^{44} \mathrm{Sc}^{\mathrm{m}}$, ${ }^{44} \mathrm{~S} \mathrm{c}^{\mathrm{b}}$, using different values of dipole to quadrupole gamma- ray strengths ratio.

(XBL 818-11296)

1. K. Aleklett et al., Lawrence Berkeley Laboratory Report LBL-9711, p. 77 (19B0).

2. M. Blann, Phys. Rev. C 21, 1770 (1980).

3. P.M. Endt, Alomic Data and Nuclear Data Tables 23, 3 (1979).

\section{Excitation Functions and Isomeric Ratio Measurements for the Reaction ${ }^{41} \mathbf{K}$ ( ${ }^{6} \mathrm{Li}, \mathrm{p} 2 \mathrm{n}$ ) ${ }^{44} \mathrm{Se}^{\mathrm{m}},{ }^{44} \mathrm{Seg}^{\mathrm{g}}$}

H.D. Ngyyen, H.N. Trinh, H.D. Le, K,J. Moody, H. Groening," and G.T. Seabarg

In a previous report, ${ }^{\prime}$ isomeric ratios in ${ }^{44}$ Se resulting from the ${ }^{41} \mathrm{~K}(\alpha, n)$ and ${ }^{2} \mathrm{Si}\left({ }^{18} \mathrm{O}, \mathrm{p} 2 \mathrm{n}\right)$ reactions were related to specific patterns in the average spins and energies of the" previous to mosi of its gamma ray dexcitation. The purpose of this work is to determine additional points in the $\langle E\rangle-<1>$ plante for low values of the average angular momentum in ${ }^{45} \mathrm{Sc}$ that will result in corresponding ${ }^{44} \mathrm{Sc}$ isomer ratios. With this in mind, we have measured excitation functions and isomeric ratios of the reaction ${ }^{41} \mathrm{~K}\left({ }^{6} \mathrm{Li}, \mathrm{p}^{2} \mathrm{n}\right){ }^{44} \mathrm{~S}^{\mathrm{m}},{ }^{44} \mathrm{~s} \mathrm{c}^{\mathrm{b}}$. The excitation function has been determined for ${ }^{6} \mathrm{Li}$ energies of 14.4 , 23.4, 32.0, and 4l.5 MeV, while the isomeric ratios have 
been deduced tor entrgies of $14.4,23.4,32.0,37.0,41.5$, and $50.0 \mathrm{MeV}$.

Tatgets cansisting of a $500 \mu \mathrm{BB} / \mathrm{cm}^{2}$ layer of enriched (99\%) ${ }^{4} \mathrm{KCl}$ on a backing of $0.25-\mathrm{mm}$ Ta were irradiated with as much as a particle-nicro-ampere of ${ }^{6} \mathrm{Li}$ at the 88Inch Cyclotton. With ${ }^{6} \mathrm{~L}$ at higher energies, activation of the Ta backing was severe, and chemistry was performed to separate scandium from the other reaction praducts. Figure 1 shows the isomeric ratio as a function of ${ }^{6} \mathbf{L i}$ kinetic energy for the reaction ${ }^{41} \mathrm{~K}\left({ }^{6} \mathrm{Li}, \mathrm{p} 2 \mathrm{n}\right){ }^{44} \mathrm{Se}^{\mathrm{m}},{ }^{44} \mathrm{Sc}^{5}$. The increase in isomeric ratio with ${ }^{6} \mathrm{Li}$ energy cannot be explained through compound nucleus theory. As Fig. I shows, experimental ratios increase much slower than predicted by this mechanism. We hope to infer the initial $\langle\mathrm{E}\rangle-\langle\mathrm{J}\rangle$ distributions in ${ }^{4}{ }^{4} \mathrm{Sc} *$ and ${ }^{45} \mathrm{Sc}^{*}$ by accounting properly for the direct component in this reaction.

\section{Foothote and Reference}

On leave from Universidad Simón Bolfvar, Caracas, Venezoela.

1. H. Groening, KJ. Moody and G.T. Seaborg, in this Annual Report.

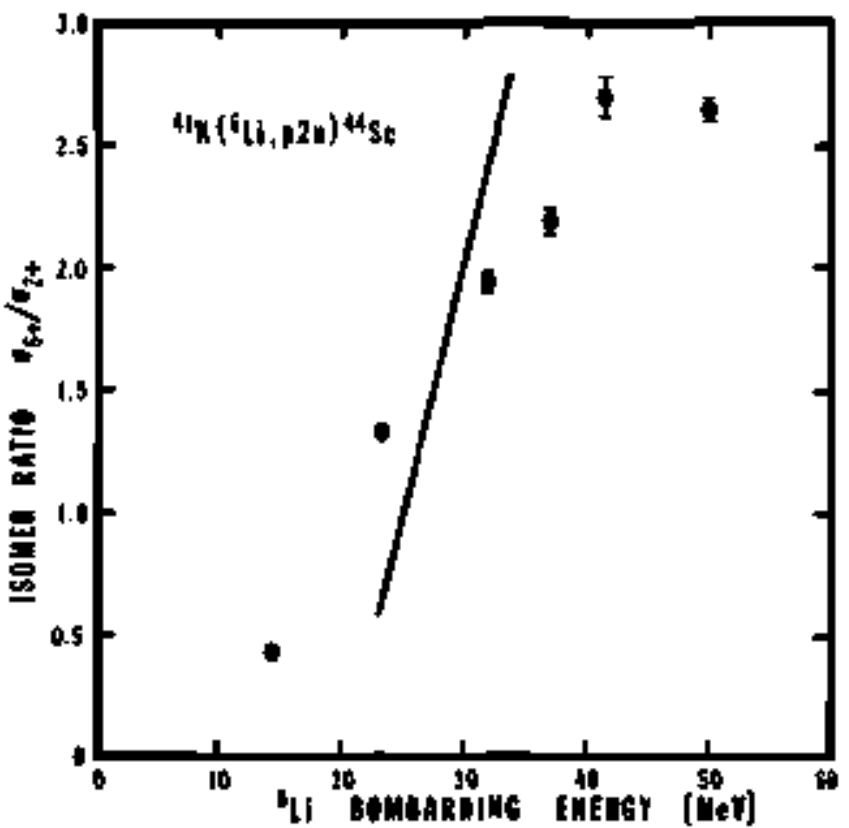

Fig. 1. Isomeric ratio as a function of projectile kinetic energy for the reaction ${ }^{41} \mathbf{K}\left({ }^{6} \mathrm{Li}, \mathrm{p}^{2} \mathrm{n}\right){ }^{44} \mathrm{Sc}^{\mathrm{m}}{ }^{4}{ }^{44} \mathrm{So}^{8}$. Solid line is calculated isomeric ratio, assuming that the reaction proceeds only through compound nucleus formation.

(XBL 818-11295) 


\title{
3. Target and Projectile Fragmentation
}

\section{Transfer Versus Fragmentation for Reactions Near $15 \mathrm{MeV} /$ Nucleon"}

\author{
M.J. Murphy, B.G. Harvey, D.L Hendrie, J. Mahoney, W. Pang, and K Van Bibbert
}

Somewhere betweet 10 and $100 \mathrm{MeV} /$ Nucleon, peripheral heary jon reactions change from being mainly transfer-like events to being mainly fragmentation-like events. In the same energy range, the intrinsic momentum width $\sigma_{0}$ of the inclusive heavy ion spectra for peripheral reactions changes from a characteristic low entrgy value to a limiting high energy widtb. It appears that these transitions are well underway at $15 \mathrm{MEV} /$ nucleon. $^{2}$

In an effort to find the explanation for the transition from transfer to fragmentation and its (possible) comec tion with the change in inclusive momentum widths, we have been studying the spectra of heayy ion ejectiles as a function of charged particle mulipicity for the reaction of $16.5 \mathrm{MeV} /$ mucleon $16 \mathrm{O}+\mathrm{CsL}$. The first measurements hawe been made at the 88-Inch Cyclotron using a streamer chamber triggered by forward-angle Si(L) particle telescopes. The telescopes identify the charge, mass, and energy of a particular heavy ion jectile, and the streamer chamber records the total charged-particle multiplicity of the event. This enables a separation of complete transferlike events from higher multiplicity (fragmentation and incorpplete transfer) events.

Our first results show two important features. First, the relative probability of transferring a cluster of mass $A$ decreases as $A$ increases, in a way closely consistent with Wilezynski's model of transfer ${ }^{3}$ (Fig. 1). Second, as the ejectile mass decreases, the relative probability of hightr multiplicity events increases, i.e., there is a trade of between transfer and fragmentation (Fig. 2).

The next research step is an analysis of the ejectile momentum widths as a function of final state multiplicity. This will show if the change in intrinsic widths with increasing energy is due to a basic difference in the characteristic widths for transfer and for fragmentation. The strearner chamber system places rather too severe a limit on the statistics for a definitive study of the reaction width (this will be made using solid state detectors), but it is an invaluable device for studying the basic multiplicities and topology of reactions in this energy range.

\section{Footmotes and Rererences}

Condensed from the Proceedings of the Sth High Energy Heavy Ion Study, Lawrence Berkeley Laboratory Report, LBL 12652 ( 1981 ).

†Stanford University.

1. D.K Seott. The Current Experimental situation in Heavy-Ion Reactions. Lawrence Berkeley Laboratory Reporl LBL-7727 (1978).

2. M.J. Morphy et al., Praceedings of the 5th High Energy Heavy Ion Sfudy. Lawrence Berkeley Laboratory Report LBL-12652 (1981).

3. J. Wilczynski et al., Phys. Rev. Lett. 45, 606 (1980).
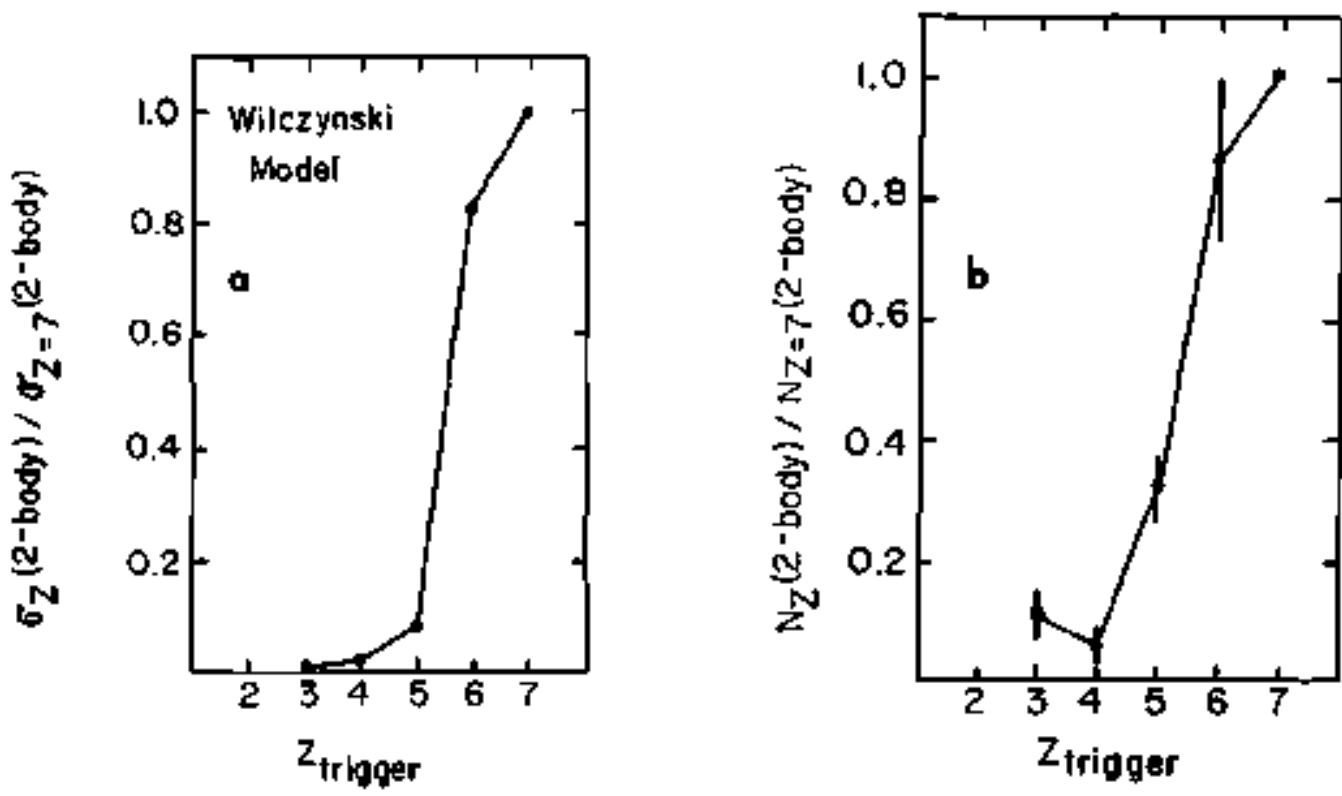

Fig. 1. A comparison of calculated (a) and observed (b) cross sections for transfer in the reaction ${ }^{60} \mathrm{O}+\mathrm{CsI}$ at $16.5 \mathrm{MeV} / \mathrm{n}$, as a function of cluster charge and nomalized to $\mathrm{z}=1$ clusters.

[(a) XBL 819-1278; (b) XBL 819-127]] 
104

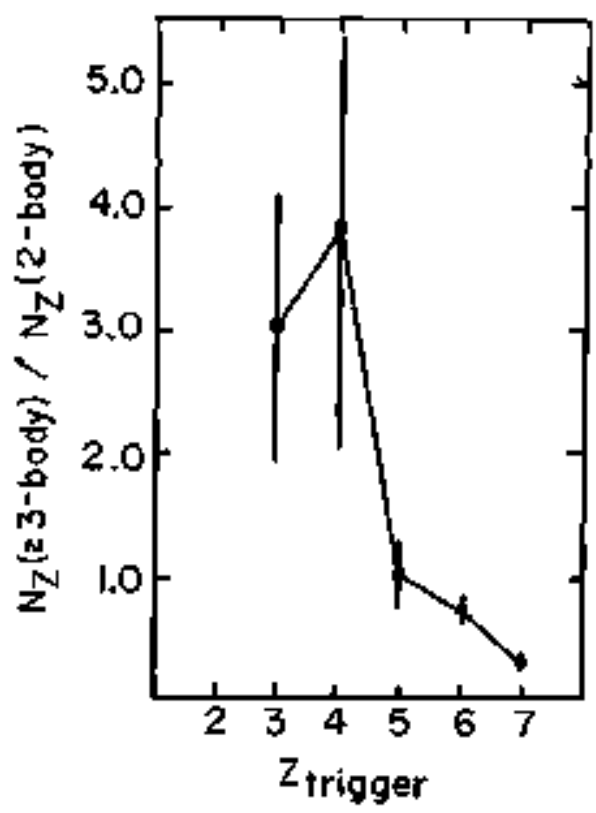

Fig. 2 The relative probability of transfer-like and fragmentation-like events for ${ }^{16} \mathrm{O}+\mathrm{CsL}$, as a function of ejectile charge.

(XAL $819-1279$ )

\title{
Snall Momentum Widths in Heavy Ion Fragmentation at $20 \mathrm{MeV} / \mathrm{amu}$ and Below*
}

\author{
Bernard G. Harvey
}

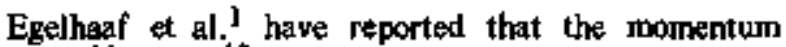
widths of ${ }^{16} \mathrm{O}$ and ${ }^{12} \mathrm{C}$ produced by the fragmentation of ${ }^{20} \mathrm{Ne}$ on a ${ }^{19}$ All target are substantially smaller than the value published by Gelbke to al, ${ }^{2}$ for the fragmentation of 160 by a $\mathrm{Pb}$ target. In Ref. 1, several reasons for the apparent discrepancy are given, one of them being that Gelbike et al. measured the momentum specire for eleutents whereas Egelhaaf th al. measured thern for individual isotopes. Moreover, Egelhas et al. extracted the momenium width by fitting a Gaussian only to the high-momenturn side of each peak. The peak shapes in both experiments are approximately Gaussian on the high-momentum side and down to half-maximmm on the low-momenturn side.

In reality, the monenturn width reported by Gelike et al. ${ }^{2}$ is not a fit to the spectrum of any single element. It was adjusted to make an approximate fit to alI the elsments from Li to $\mathrm{N}$ that were observed in the experiment. A Gaussian least squares fit just to the $C$ pealk in fact yields a wjdth that is substantially smaller than the global value for all obstrved elements.

Scott et al, ${ }^{3}$ have used the data of Ref. 2 and addjtional data to measure the spectra of indjuidual isotopes produced by the fragmentation or incomplete fustion of ${ }^{16} \mathrm{O}$ at several energies on $\mathrm{Pb}$ and $\mathrm{Au}$ targets. Making a least squares fit to these unpublished data yields a width $\sigma$ which can be related to the reduced width $\sigma_{0}$ by:

$$
\sigma^{2}=\sigma_{0}^{2} m_{\gamma}\left(m_{p}-m_{l}\right) /\left(m_{p}-1\right),
$$

where $m_{p}$ and $m_{f}$ are the projectile and otserved fragment masses. ${ }^{4,5}$ The results for ${ }^{12} \mathrm{C}$ are shown in Table 1 and compared with the results of Ref, 1 .

Table 1 shows that the reduced widths for ${ }^{16} \mathrm{O} \rightarrow{ }^{12} \mathrm{C}$ are much smaller than the "global fil" value of $80 \mathrm{MeV} / \mathrm{c}$ of Ref. 2. There is no serious diserepancy between the two experiments.

Soott ${ }^{6}$ used the results of Ref. 2 to suggest that $\sigma_{0}$ attains a limiting value of about $86 \mathrm{MeV} / \mathrm{c}$ at a beam energy of only $10 \mathrm{MeV} / a m u$ above the Coulomb barrier. The results of Egelhaaf et al. and the present reanalysis of the unpublished data of $\$$ cott et al. ${ }^{3}$ show that, at least for the reactions ${ }^{16} \mathrm{O} \rightarrow{ }^{12} \mathrm{C}$ and ${ }^{20} \mathrm{Ne} \rightarrow{ }^{16} \mathrm{O}$, ${ }^{12} \mathrm{C}$ the limiting value is approached much more slowly than was previously thought. In the fragmentation of $92.5 \mathrm{MeV} / \mathrm{amm}{ }^{16} \mathrm{O}$ by ${ }^{157} \mathrm{Au}$, though, of averaged $80 \mathrm{MeV} / \mathrm{c}$ for all observed fragments. ${ }^{7}$ The ewolution of $\phi_{0}$ for beam energies between $20 \mathrm{MeV} / \mathrm{amu}$ and $90 \mathrm{MeV} / \mathrm{amu}$ remains to be explored. 
Table 1. Reduced widths of fragmentation spectra.

\begin{tabular}{|c|c|c|c|c|}
\hline \multirow{2}{*}{$\begin{array}{c}\text { Projectile } \\
\text { Energy } \\
\text { (MeV/amu) }\end{array}$} & \multicolumn{2}{|c|}{${ }^{16} \mathrm{O}-{ }^{12} \mathrm{C}$} & \multirow{2}{*}{$\begin{array}{c}{ }^{20} \mathrm{Ne} \rightarrow{ }^{15} \mathrm{O} \\
\sigma_{0}^{\mathrm{c}} \\
(\mathrm{MeV} / \mathrm{c})\end{array}$} & \multirow{2}{*}{$\begin{array}{c}{ }^{20} \mathrm{~N}-{ }^{12} \mathrm{C} \\
\sigma_{0}^{\mathrm{c}} \\
(\mathrm{MeV} / \mathrm{c})\end{array}$} \\
\hline & $\begin{array}{c}\sigma_{0}^{a} \\
(\mathrm{MeV} / \mathrm{c})\end{array}$ & $\begin{array}{c}\sigma_{0}^{b} \\
(\mathrm{MeV} / \mathrm{c})\end{array}$ & & \\
\hline 19.7 & 50 & 58 & 35 & 42 \\
\hline 15.6 & 43 & 50 & 35 & 41 \\
\hline 13.6 & 33 & 40 & 34 & 40 \\
\hline 8.75 & 32 & 34 & $2 I$ & 31 \\
\hline $\begin{array}{l}\mathrm{a}_{\sigma_{0}} \text { obtained } \\
\text { of Ref. } 3 \text {. } \\
\mathrm{b}_{\sigma_{0}} \text { obtained } \\
\text { on low-mome } \\
c_{\sigma_{0}} \text { from Fig. }\end{array}$ & $\begin{array}{l}\text { om fit to } \\
\text { um side } \\
\text { of Ref. }\end{array}$ & $\begin{array}{l}\text { all deta do } \\
\text { of peak. D } \\
1 \text {. }\end{array}$ & $\begin{array}{l}\text { wh to one-h } \\
\text { ata of Ref. }\end{array}$ & ak. Data \\
\hline
\end{tabular}

Foothote and References

"Condensed from LBL-12787.

1. Ch. Egel hataf et al., Phys. Rey. Leit. 46, 813 (1981).

2. C.K. Gelbke te al., Phys. Lett. B 70, 415 (1977).

3. D.K. Seott et al., LBL-7729 (June 1978), unpublished.

4. A.S. Goldhaber, Phys. Lett. B 53, 306 (1974),

5. D.E. Greiner et al., Phys. Rev. Lett. 35, 152 (1975).

6. D.K Soott, Proceedings of the Interntional Conference on Nuclear Physics, Berkeley. Aug. 1980, Vod. 2, p. $375 c$.

7. K. Van Bibber et al., Phys. Rev, Lett. 43, 840 (1979).

\title{
Coherent and Incoherent Processes In Projectile Breakup*
}

\author{
W.D. Rae, A.J. Cole, A. Dacal't R. Legrain ${ }^{\ddagger}$ B.G. Harvey, \\ J. Mahoney, M.J. Murphy, R.G. Stokstad, and I. Tserruya
}

There has been much interest recently in the field of heavy ion breakup and fragmentation reaclions. The simplest case that can be studied is binary breakou where only two projectile fragments ate produced. Binary breakup has been classified as either sequential or direct. ${ }^{1}$ Sequential breakup is associated with dectiy from excited states of the projectile, while direct breakup describes those events for which the relative kinetic energy of the two fragments is not necessarily correlated with an excited state of the projectile.

Our recent analysis ${ }^{2}$ of the ground state $(Q=$ $-7.16 \mathrm{MeV}$ ) breakup of $140 \mathrm{MeV} 16 \mathrm{O} \rightarrow{ }^{12} \mathrm{C}+\alpha$ on a ${ }^{12} \mathrm{C}$ target revealed only sequential processes. We have continued these studies, and our new data suggest a classification different from that implied by the terms "sequential" and "direct" because we observe at least two mechapisms leading to sharp states in 160 which decay sequentially.

Although the experiment and analysis were similar in principle to those described in Ref. 2, the introduction of position-sensitive detectors made possible the nse of large solid angles without loss of angular resolvtion ( $\leqslant 0.2^{\circ}$ Lab). In the analysis of the data for each coincident event, two quantities were determined the sum $\mathrm{E}_{\mathrm{T}}=\mathbf{E}_{\alpha}+$ $E_{12 c^{*}}$ which is related to the reaction $Q_{3}$-value ${ }_{1}$ and $E_{\text {refal }}$. the relative kinetic energy of the $\alpha$ and the ${ }^{12} \mathrm{C}$ in their center-of-mass system.
The spectrum of $\mathrm{E}_{\mathrm{T}}$ and the spectra of $\mathrm{E}_{\mathrm{Teal}}$ for different values of $\mathrm{E}_{\mathrm{T}}$ are $\mathrm{shown}$ in Figs. I and 2. For the highest $\mathrm{E}_{\mathrm{T}}$ bin, which corresponds to $\mathrm{Q}_{3}=-7.16 \mathrm{MeV}$, states at the following energies in 160 are clearly observed: $9.88\left(2^{+}\right), 10.36\left(4^{+}\right), 11.06\left(4^{+}\right), 11.32\left(2^{+}\right), 12.41$ $\left(1^{-}\right), 13.10\left(2^{+}\right), 14.00\left(0^{+}\right)$, and $15.60 \mathrm{MeV}\left(3^{-}\right)$. For $Q_{3}=-11.60 \mathrm{MeV}$, an additional strong peak is observed which probably corresponds to a state at $17,14 \mathrm{MeV}\left(2^{+}\right)$ which decayed to $\alpha+{ }^{12} \mathrm{C}(4.44 \mathrm{MeV})$.

There is a marked qualitative change in the spectra of $E_{\text {rel }}$ for values of $Q_{3}<-11.6 \mathrm{MeV}$ : only the states at $9.85,10.35$, and $11.10 \mathrm{MeV}$ and a broad background extending beyond $\mathrm{E}_{\mathrm{Tel}}=14 \mathrm{MeV}$ are observed. Significantly, these three states are the ooly states cont sistently observed in our ditis which ste populated in $\alpha$ transfer reactions on ${ }^{12} \mathrm{C}$. ${ }^{3}$ Furthermore, with the exception of the state at $15.60 \mathrm{MeV}$, all other strong states observed for low $\left|Q_{3}\right|$-values are excited strongly in ${ }^{1}{ }^{6} \mathrm{O}\left(\alpha, \alpha^{\prime}\right)$. ${ }^{16} \mathrm{O}\left(\mathrm{e}, e^{\prime}\right)$, or ${ }^{15} \mathrm{~N}(p, \gamma)$ reactions.

The mechanisms which excite the background and the states at $9.85,10.35$, and $11.10 \mathrm{MeV}$ appear to be different from that which excites the collective states. Collective excitation of the projectile involves the colerent response of the whole projectile to the field of the target nucleus; thus, we term the excitation and decay of these states "colvent breakup." 

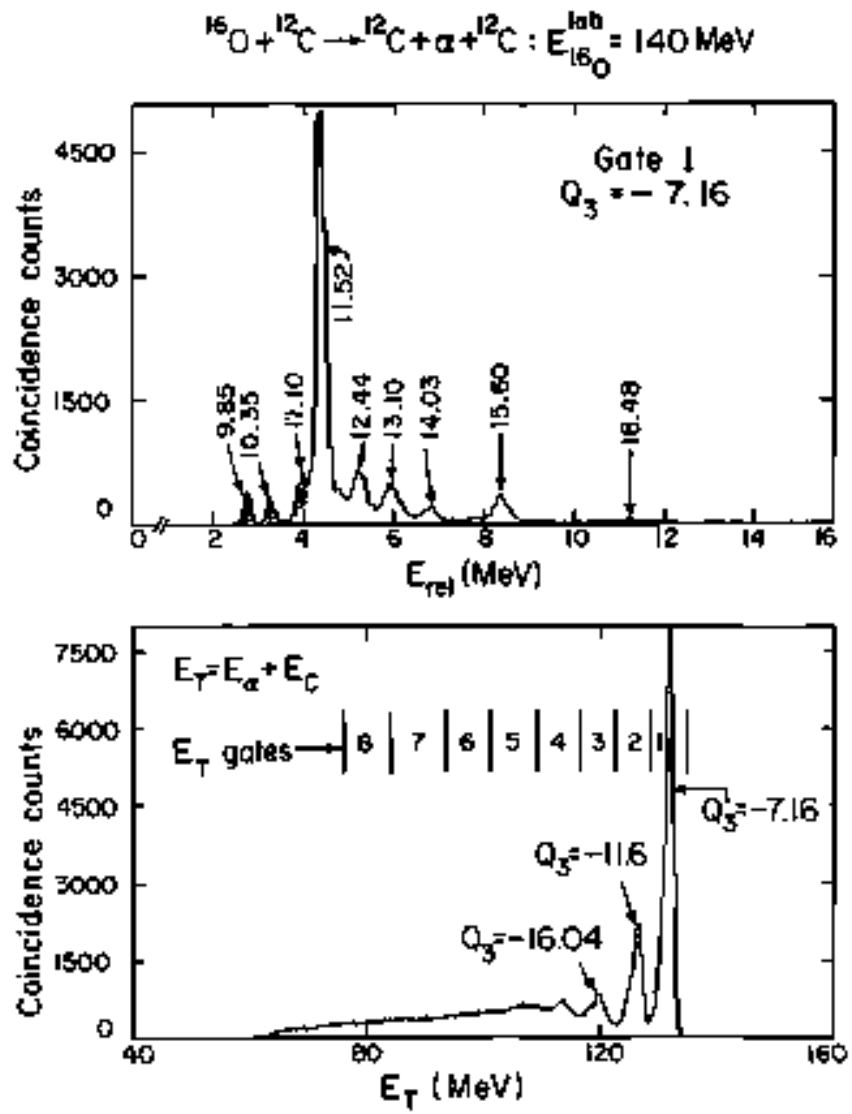

Fig. 1. $\mathrm{E}_{\mathrm{T}}=\mathrm{E}_{\alpha}+\mathrm{E}_{1 z_{c}}$ and $\mathrm{E}_{\text {tel }}$ spectra (as defiged in text) for $Q=-7,16 \mathrm{MeV}$. Excitation energies refer to $16 \mathrm{O}$ and are in $\mathrm{MeV}$.

(XBL 814-655)

On the other hand, the three states which are also observed in o-transfer reactions are those which might be expected to be observed in a quasi-free scattering of the $\alpha$ and ${ }^{12} \mathrm{C}$ constituents of $16^{6} \mathrm{O}$ to the continuum via the individual interaction of either constituent with the target. Since the $\alpha$ and ${ }^{12} \mathrm{C}$ constituents are assumed to interact independently with the target, we can class this mechanism as an inooherent breakup process.

In conclusion, our experimental data reveals the presence of at least two mechanisms in the breakup of ${ }^{16} \mathrm{O}$ and ${ }^{12} \mathrm{C}+a:$ (1) a coherent process which excites the projectile to states whose structure is related 10 that of the ground state via multipole operator, and (2) incoherent processes. The incoherent amplitudes contain resonant contributions at relative energies corresponding to specific excited states of the projectile, as well as possible nonresofiant contribution. We speculate that the dominant incoherent process is the quasi-free scattering of the $\alpha$ or ${ }^{12} \mathrm{C}$ constituenis of the projectile by the target, viz, the direct breakup of the ${ }^{16} \mathrm{O}$ projectile.

\section{Foatmotes and References}

*Condensed from Lawreace Berkeley Laboratory Report LBL 12568 (1981).

TUniversidad Nacional Autonoma de Instituto de Física, Mexio 20, D. F.

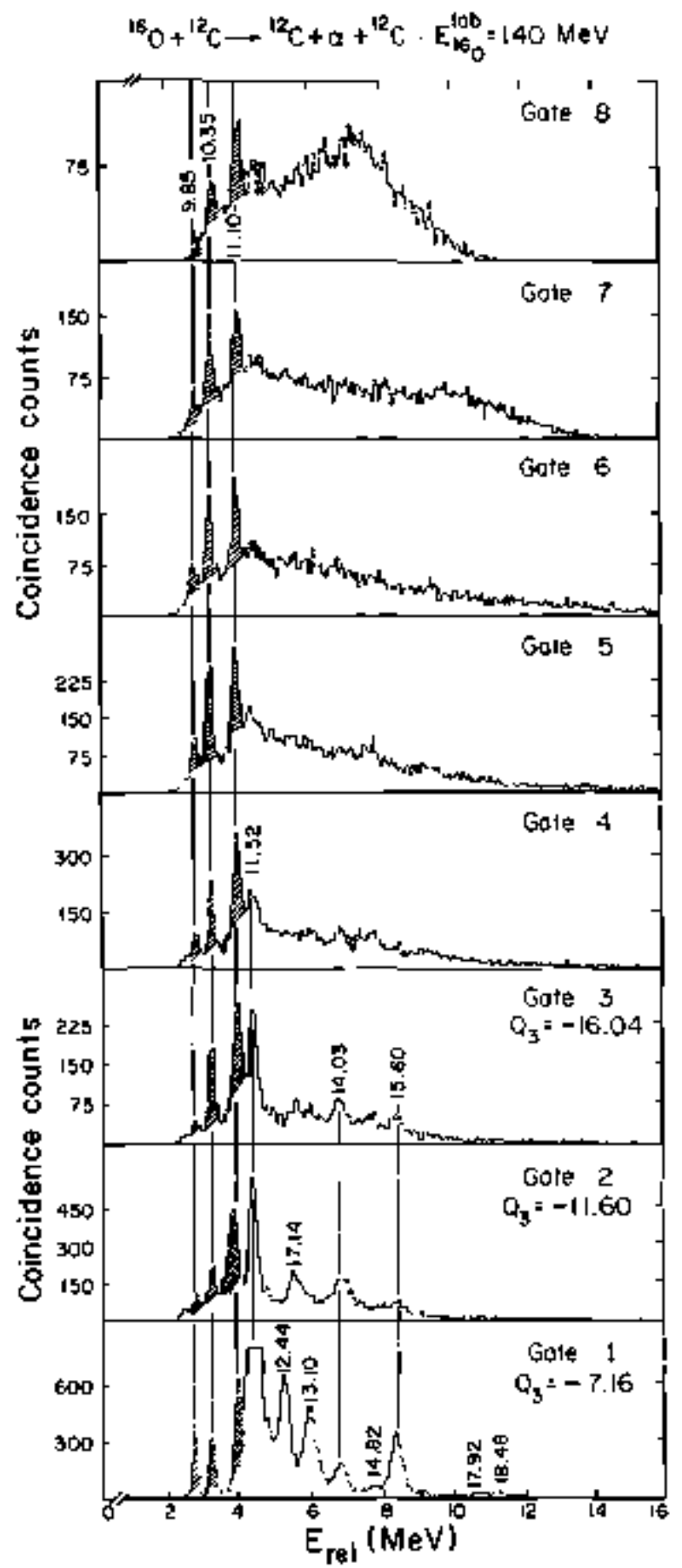

Fig. 2. $E_{\text {rol }}$ spectra for various $\mathrm{E}_{\text {gates. There is a }}$ detection threshold at $\mathrm{E}_{\mathrm{ref}}=2.5 \mathrm{MeV}$. The data have not betn adjusted for coincidence efficiency which is a strong function of $\mathrm{E}_{\mathrm{Ted}}$ but depends weakly on $\mathrm{E}_{\mathrm{T}}$ (XBL 814-654)

IDipartement de Physique Nucléaire/Basses Energies, Centre d'Etudes Nucleaires de Saclay, F-91190 Gif-5ur* Yvette, France.

Department of Physics, Weizmen Institute of Sciences, Rehowot, Israei.

I. A.C. Shotter et al., Phys. Rev. Let1. 46, 12 (1981).

2. W.D. Rae et al., Phys. Rev. Lett. 45, 884 (1980).

3. P.T. Debevec et al., Phys. Rev, C 9, 2451 (1974). 


\title{
Observation of Sequential Breakup in the $160+$ ${ }^{197}$ Au Reaction at $218 \mathrm{MeV}$
}

\author{
A.N. Bice, M.D. Cable, AC Shotter," and Joseph Cerny
}

Angular correlations between fast o-particles and gutgoing heavy reaction products for the system $160+197$ All and ${ }^{16} \mathrm{O}+{ }^{206} \mathrm{~Pb}$ bave previously been studied at the bortbarding energies of $140 \mathrm{MeV}$ and $310 \mathrm{MeV} .{ }^{1}$ Although a detailed understanding of the reaction mechanism responsible for the observed fast $\alpha$-particle production was nol achieved, Gelbke et al. ' inferred that the $\alpha$-particles could not qriginate from the sequential decay of an ejectile. In contrasi, our simple Monte Carlo simulations indicated that the data of Gelbke el al. were consistent with a sequential decay process and, hence, that this type of reaction mechanism should be considered further. Therefowe, an initial investigative study of the ${ }^{16} \mathrm{O}+{ }^{19}$ All system at $218 \mathrm{MeV}$ was performed. It should be noted that Binj et al. ${ }^{2}$ recently reported that most of the $\alpha$-particles observed in the ${ }^{16} \mathrm{O}+{ }^{197} \mathrm{Au}$ system at $310 \mathrm{MeV}$ were eritted from projectile-like fragments. Also Rae et al. ${ }^{3}$ reported a sirong $160^{\circ}$ sequential decay channel in the ${ }^{160}+{ }^{12} \mathrm{C}$ sysiem at $140 \mathrm{MeV}$.

A $218 \mathrm{MeV} 16 \mathrm{O}$ beam produced by the 88-Inch Cyclotron was used to bombard targets of ${ }^{19} \mathrm{Au}$ and ${ }^{208} \mathrm{~Pb}$. The data taken with each target are qualitatively the same. A triple telescope systern (as described in the contribution ${ }^{412} \mathrm{C}$ * production in the ${ }^{12} \mathrm{C}+{ }^{208} \mathrm{~Pb}$ system at $230 \mathrm{MeV}, "$ Bice et al., in this Annual Report) was used to record particle-particle coincidences. Since the three particle telescopes are located in close proximity, the observation of the decay of ${ }^{16} \mathrm{O}$ states near the breakup threshold is facilitated. Figure I(a) shows a summed energy spectrum of $\alpha+{ }^{12} \mathrm{C}$ coincidences taken at $19^{\circ}$ in the laboratory with the closest spaced two counter configuration (center-to-center counter separation of $5.9^{\circ}$ ). Strong transitions to the ground state and low-lying states of 197 All are evident as well as a significant number of $a+{ }^{12} \mathrm{C}$ evens with more negative production Q-values. Figures $\mathrm{l}(\mathrm{b})$ and $\mathrm{l}(\mathrm{c})$ show projected spectra onto the ${ }^{12} \mathrm{C}$ energy axis of $\alpha+{ }^{12} \mathrm{C}$ coincident events that sumuried to an energy falling within the gates indicated in Fig. 1(a). The peaks in $\mathrm{Fig}$. 1(b) are representative of ${ }^{16} \mathrm{O}^{*}$ sequential decay into the $\alpha+{ }^{12} \mathrm{C}$ channel and resemble the data observed by Rae at al. ${ }^{3}$ for the ${ }^{15} \mathrm{O}+{ }^{12} \mathrm{C}$ system. The projected spectrum (Fig. 1 (c)) that results from $\alpha+{ }^{12} \mathrm{C}$ events with more negative $Q$-values does not display any clear peaks in the "relative energy" spectrum and suggests that other processes thas sequential decay are involved in this reaction. Taken together, Figs. I(b) and $l(c)$ indicate the need for further consideration and measurement of the relative contributions of sequential and nonsequential processes to fast $\alpha$-particle production and $\alpha$-heavy ion correlation measurements.

In addition to the observation of the $\alpha+{ }^{12} \mathrm{C}$ chan nel, other particle-particle coincidences were investigater
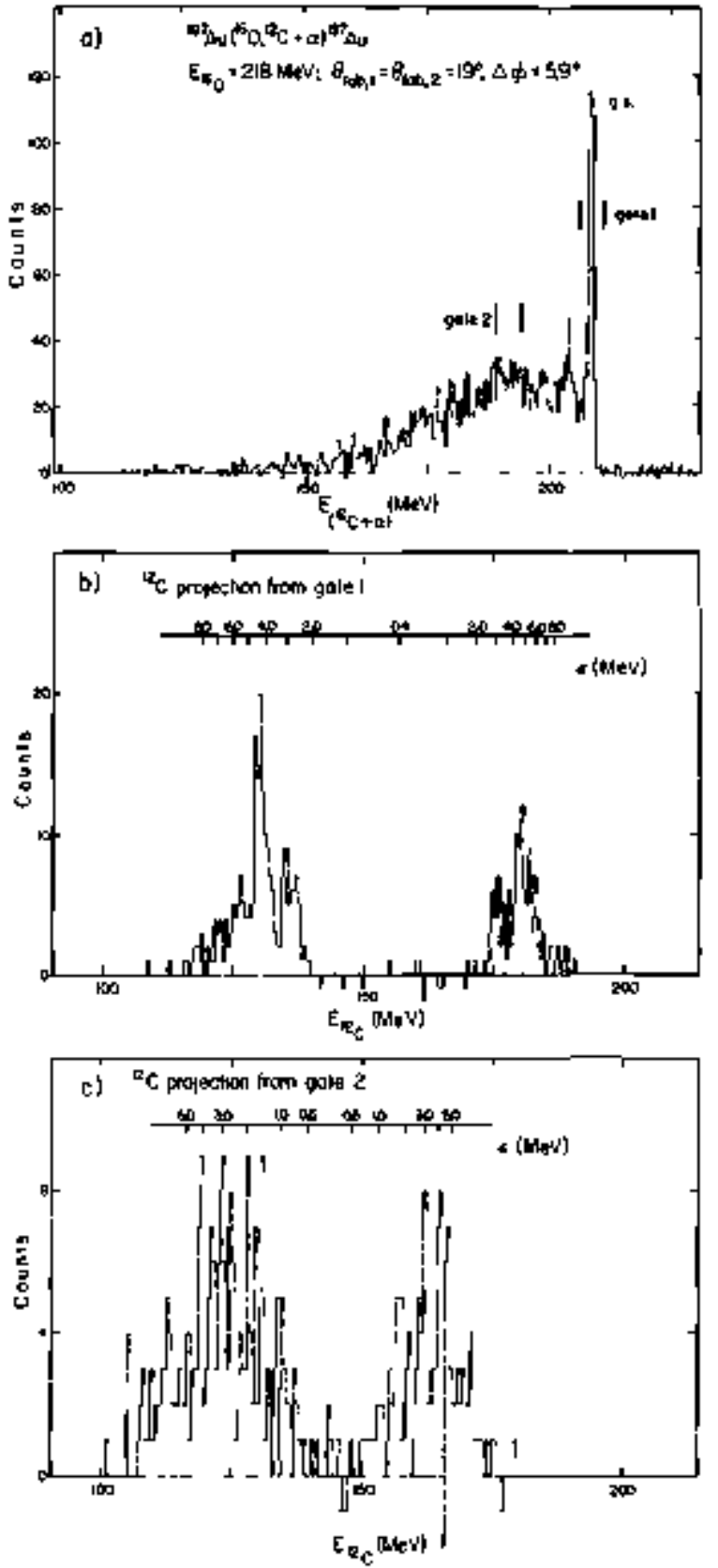

Fig. 1. (a) Energy spectrum obtained from the $\left.{ }^{197} \mathrm{Auf}^{160} \mathrm{O},{ }^{12 \mathrm{C}}+\alpha\right)$ reaction at $\theta_{1 \mathrm{~b}}=19^{\circ}$ with an ${ }^{160}$ bombarding energy of $218 \mathrm{MeV}$. The 1wo particle telescopes were separated center-to-center by $5.9^{\circ}$. (b) Projected energy spectrum of ${ }^{12} \mathrm{C}$ nuclei in one telescope in coincidence with ${ }^{4} \mathrm{He}$ nuclei such that their summed energy is located within the gate 1 indicated in (a). (c) Same as (b) but for gate 2 .

(XBL 816-944) 
for this system. The owerwhelming mijocity of all particle-particle coincidences consisted of $\alpha-\alpha$ events. Closer examination indicated most $\alpha-\alpha$ oincidences were due to the decay of a ${ }^{8} \mathrm{Ee}(\mathrm{g}-\mathrm{s}$.$) or an excited$ ${ }^{8} \mathrm{Be}(2.94 \mathrm{MeV})$ nucleus. The mechanisan for the large production of ${ }^{8} \mathrm{Be}$ is not known and would provide an interesting area of study for the future. Indeed, it is of interest to see if the singles fast $\alpha$-particles and ${ }^{8} \mathrm{Be}$ noclei produced in the ${ }^{16} \mathrm{O}+{ }^{19}$ Au system at $218 \mathrm{MeV}$ are explainable in terms of current incomplete fusion models.

Foothote and References

"Physics Department, University of Edinburgh, Edinburgh EH93.JZ, United Kingüm.

1. C.K. Gelbke et al., Phys. Lett. B 71, 83 (1977).

2. M. Bini et al., Phys. Rev. C 22, 1945 (1980).

3. W.D. Rae et al., Physs. Rev. Lett. 45, 884 (1980).

\title{
Observation of the Direct and Sequential Breakup of ${ }^{7 \mathrm{~L}}$ from ${ }^{12} \mathrm{C}$ and ${ }^{208} \mathrm{~Pb}$ Targets at $70 \mathrm{MeV}^{*}$
}

\author{
A.N. Bice, A.C. Shotter, J.M. Wouters, W.D. Rae, and Joseph Cerny
}

The reaction mechanisms involved in projectile dissociation in the field of a target nucleus are currently attracting substantial interest. For projectile entrgies befow the Coulomb barrier, the projectile may be excited by the electromagnetic field between the incident channel nucled. If the excited states are abowe the threshold for particle emission and have small entrgy widths, the projectile will in most instances sequentially break up beyond the influence of the target's nuclear field. In contrast, at high energies $(-100 \mathrm{MeV} / \mathrm{A})$ a projectile incident on the peripheral region of the target nucleus might be expected to undergo a rapid fragmentation in the surface field of the nucleos. ${ }^{\text {' }}$

For a projectile with an intermediate energy, it should be possible to identify a transition from a sequential to a rapid nonsequential process. A sujtable projectile is ${ }^{7} \mathrm{~L}$, in which the first $\alpha$ decaying state is at $4.63 \mathrm{MeV}, 2.16 \mathrm{MkV}$ above the $\alpha+t$ breakup threshold. Thus, direct ' $L i$ breakup events with $\alpha-t$ relative energies less than $\sim 2.1 \mathrm{MeV}$ can clearly be distinguished from all possible sequential breakup events.

Presented briefly here are results of an investigation of $70 \mathrm{MeV}{ }^{7} \mathrm{Li} \rightarrow a+t$ breakup on ${ }^{12} \mathrm{C}$ and ${ }^{208} \mathrm{~Pb}$ targets. The breakup fragments were detected by two particle telescopes in close vertical geometry. Summed energy spectra of $a+\mathrm{t}$ ofincidence events obtained with this system from a ${ }^{12} \mathrm{C}$ target yielded throe peaks, corresponding to those breakup events which leave the ${ }^{12} \mathrm{C}$ target in the g.s. or excited to the $4.4 \mathrm{MeV}_{+} 2+$ or $9.6 \mathrm{MeV}, 3-$ states. For the ${ }^{208} \mathrm{~Pb}$ target the majority of breakup events into the $\alpha+t$ channel left the target in its ground state.

Futber interpretation of the character of these breakup events was obtained from the $t$ projected spectra. Figure 1(a) shows such a spectrum corresponding to the ${ }^{12} \mathrm{C}(\mathrm{g}, \mathrm{s}, \mathrm{)}$ transition. The two peaks of Fig. I(a) correspond to the two binematically allowed 1 energies (as indicated by the arrows) from the sequential breakup of ${ }^{7} \mathrm{Li}^{*}(4,63 \mathrm{MeV})$. If direct breakup had occurred in the field of the target nucleus, the relative entrgy, $\epsilon$, between the $\alpha$ and $t$ would $n o$ longer be restricted to a definite value corresponding to ${ }^{7} \mathrm{Lj}^{*}$ sequential docay; but, instead, the value of $f$ could vary over some continuous distribution related to the momentum distribution of the fragments in the projectile ground state. ${ }^{2}$ In Fig. I(a), there is little or no evidence of such a distribution of $\epsilon$.

Two projected spectra corresponding to the ${ }^{208} \mathrm{~Pb}$ (g.s.) transition for the lab angles of $32^{\circ}$ and $18^{\circ}$ are shown in Figs. 1(b) and I(c), respectively. The arrows again indjcate the triton emergy limits for the sequential breakup of ${ }^{3} \mathrm{U}^{\prime}(4.63 \mathrm{MeV}$. It is apparent that Figs. I(a) and $\mathrm{l}$ (b) are similar, showing little evidence for direct breakip. However, data taken at moce forward angles (Fig. 1(c)) include events with a continuous distribution of $\mathrm{\epsilon}$ that cannot arise from discrete states of ${ }^{7} \mathrm{Li}$. This is taken as evidence of direct breakup.

Angular distributions for the sequential and the direct components were measured and showed distinct differences. At this time, however, it is only possible to speculate as to the mechanisms involved in direct projectile brealup. Whatever the precise mechanisms are, it will be of great interest to determine how the direct and sequential breakup components vary with incident energy, especially in the region 10-100 $\mathrm{MeV} / \mathrm{A}$, because this could lead to a much greater understanding of fragmentation processes in general.

\section{Foothotes and References}

"Condensed from Lawrence Berkeley Laboratory Report LBL-11458, Phys. Rev. Lett, 46, 12 (1981).

Thysics Department, Unjversity of Edinburgh EH93JZ, United Kingdom.

1. A.S. Goldhaber and H.H. Heckman, Ann, Rev. Nucl. Sci. 28, I61 (1978).

2. R. Shyam, G. Băur, F. Rờsel and D. Trautmanan, Phys. Rev. C 19, 1246 ( 1979 ). 
109

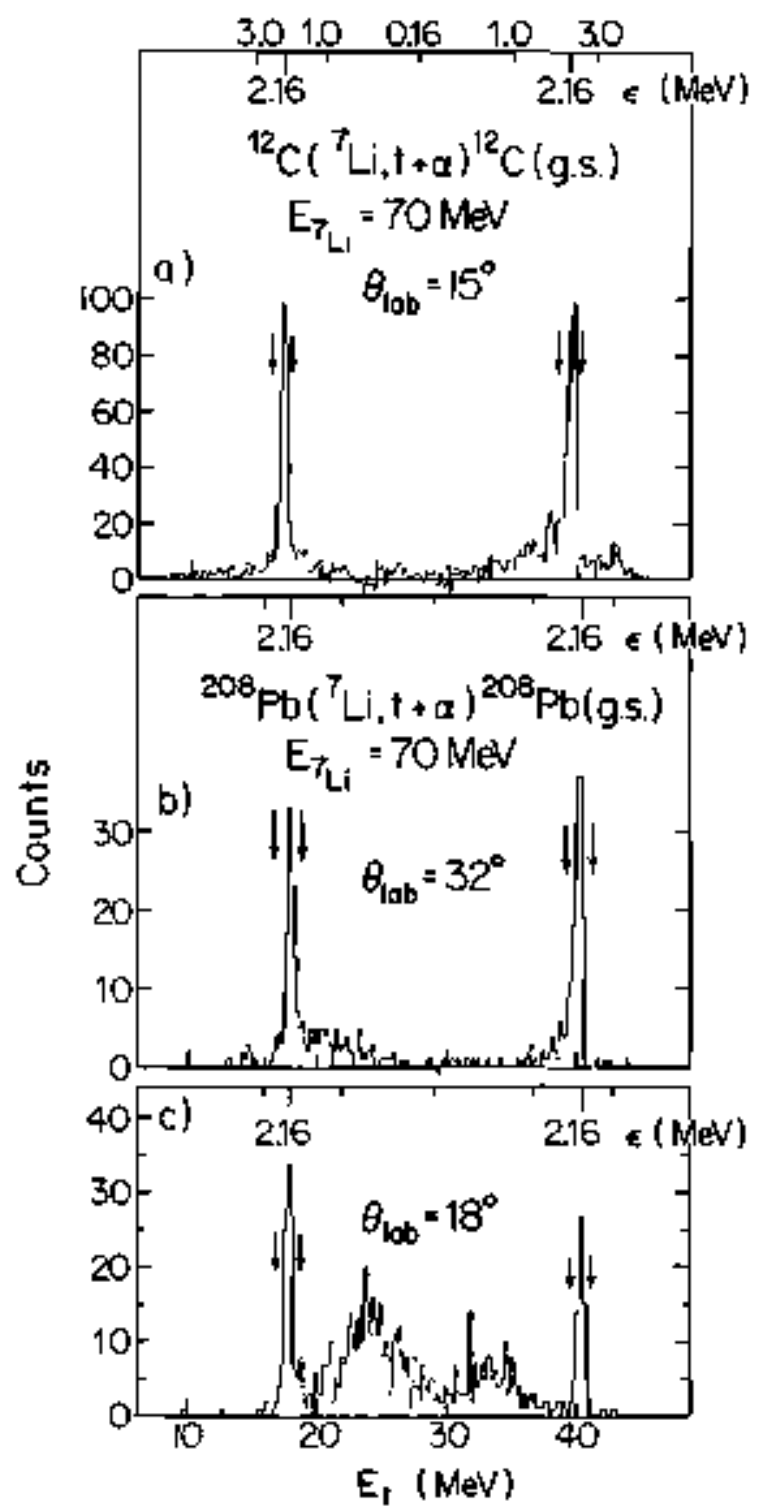

Fig. 1. (a) The triton energy spectrum for coincident $\alpha+t$ events in which the residual ${ }^{12} \mathrm{C}$ nucleus remsined in its ground state. The detector system was located at $\theta_{12 b}=15^{\circ}$. (b) The triton energy spectrum for coincident $\alpha+t$ events in which the residual ${ }^{208} \mathrm{~Pb}$ nucleus remained in its ground state. The detector system was located at $\hat{\theta}_{1 \mathrm{ab}}=32^{\circ}$. (c) As for (b) but with the detector system at ${ }_{\mathrm{Lab}}-\mathrm{t} 8^{d}$.

(XBL 809-188t) 


\title{
Resonant Particle Production in the 172-MeV ${ }^{13} \mathrm{C}+{ }^{208} \mathrm{~Pb}$ Reaction
}

\author{
A.N. Bice, M.D. Cable, A.C. Shotter, and Joseph Cerny
}

In a previous breakup reaction study of the ${ }^{12} \mathrm{C}+$ ${ }^{208} \mathrm{~Pb}$ system at $187 \mathrm{MeV}$ bombarding energy, it was noled' that there was a relatively sirong neutron pickup channe], which yitjded ${ }^{13} \mathrm{C}$ slates unbound with respect to alpha decay. Two possible reaction mechanisms for the production of ${ }^{13} \mathrm{C}^{*}$ are: (I) a direct neytron transfer to unbound levels in ${ }^{13} \mathrm{C}$ and (2) a two-step process such as ${ }^{2018} \mathrm{~Pb}\left[{ }^{13} \mathrm{C}^{13} \mathrm{C}(\mathrm{g} . \mathrm{s}).\right]\left[{ }^{13} \mathrm{C},{ }^{13} \mathrm{C}^{+}\right]^{208} \mathrm{~Pb}(\mathrm{~g} s$.$) . If this latter$ process [inwolving the ${ }^{13} \mathrm{C}$ (g.s.)] were dominant, then one might expect that the reaction ${ }^{206} \mathrm{~Pb}\left({ }^{13} \mathrm{C},{ }^{13} \mathrm{C}^{*} \rightarrow \alpha+\right.$ $\left.{ }^{9} \mathrm{Be}\right)^{208} \mathrm{~Pb}$ (g.s.) would exbibit a relatively large yield in reactions conducted at a similar bombarding energy. To investigate this mechanism as well as to provide an interesting comparison to the ${ }^{12} \mathrm{C}+{ }^{2068} \mathrm{~Pb}$ systerth an initial survey was made of the ${ }^{208} \mathrm{~Pb}\left({ }^{13} \mathrm{C},{ }^{13} \mathrm{C}^{*}\right)$ reactions at $172 \mathrm{MeV}$.

A $1.5 \mathrm{mg} / \mathrm{cm}^{2}{ }^{206} \mathrm{~Pb}$ target was bombarded with a $172 \mathrm{MeV}{ }^{13} \mathrm{C}$ beam from the 88-]nch Cyclotron. The production of resonant unbound reaction products was observed by detecting the decay products in coincidence with a "piggyback" telescope system This system was comprised of three particle telescopes stacked vertically with respect to the scattering plane (which bisected the post separating two of the telescopes). This system allowed three combinations of particle-particle coincidences to be observed at each laboratory scattering angle.

The yield of $\alpha+{ }^{9} \mathrm{~B} e$ coincidences between $15^{\circ}$ and $25^{\circ}$ in the laboralory system was observed to be small, a factor of $15-20$ less than that observed in the ${ }^{206} \mathrm{~Pb}\left({ }^{12} \mathrm{C}\right.$, ${ }^{13} \mathrm{C}^{*}$ ) reaction at $187 \mathrm{MeV}$. Indeed, only two strong reaction chamnels were observed for this system- $\left({ }^{13} \mathrm{C}, \alpha\right)$ and $\left({ }^{13} \mathrm{C}, a+\alpha\right)$. Figure $\mathrm{l}$ (a) shows a ${ }^{2018} \mathrm{~Pb}\left({ }^{13} \mathrm{C}, \alpha+\alpha\right)$ coincidence spectrum obtained by the two telescope combination, which has an average angular separation of $\Delta \psi \boldsymbol{Z}$ 5.9\%. A rather broad peak near bean velocity is seen in the coincident spectrum as was obserwed in the ${ }^{12} \mathrm{C}+$ ${ }^{2008} \mathrm{~Pb}$ system. Figure $\mathrm{l}$ (b) shows a projected spectrum of the ${ }^{4} \mathrm{He}$ energy in one telescope from $\alpha+\alpha$ eoincident events located within the gate indicated in Fig. 1(a). The majority of $\alpha_{1}+\alpha_{2}$ events have $\mathrm{E}_{\mathrm{a}_{\mathrm{b}}} \simeq \mathrm{E}_{\alpha_{2}}$ as expecied from the decay of a ${ }^{B} \mathrm{Be}(\mathrm{B}$.s.). The other two detector combinations for particle-particle coincidences, which are separated by angles larger than a ${ }^{8} \mathrm{Be}$ (B.s.) decay cone width, recorded very few $\alpha+\alpha$ events. Evidently most $\alpha+\alpha$ coincidences do arise from the decay of a ${ }^{8} \mathrm{Be}$.

Given the large production cross section of ${ }^{\mathrm{B}} \mathrm{Be}$ (at $\theta_{\text {Lab }}=15^{\circ}, \mathrm{d} \sigma / \mathrm{d} \Omega=360 \mathrm{mb} / \mathrm{sc}$ ), it is interesting to deduce what fraction of the singles alpha spectrum arises from decaying ${ }^{8} \mathrm{Be}$ nuclei. Integrating ower Fig. L(a) and folding in the ${ }^{8} \mathrm{Be}$ detection efficiency, it is found that approximately $55 \%$ of the singles alphas at $15^{\circ}$ must arise from the decay of "Be nuclei.

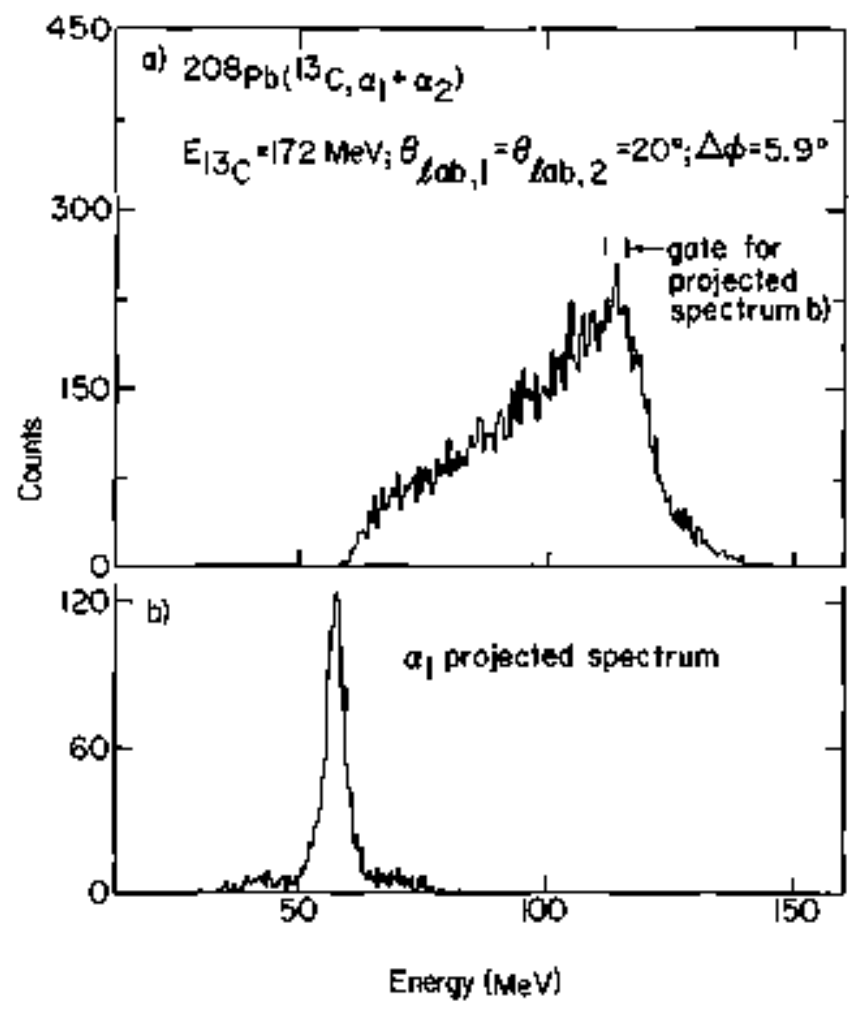

Fig. l. (a) Energy spectrum obxained from the ${ }^{20} \mathrm{~Pb}\left({ }^{13} \mathrm{C}, \alpha+\alpha\right)$ reaction at $\theta_{\text {Lab }}=20^{\circ}$. The two particle telescopes were separated center-lo-center by $5.9^{\circ}$, (b) Projected energy spectrom of "He nuclei in one telescope in coincidence with ${ }^{4} \mathrm{He}$ nuclej in the other telescope such that their summed energy is located within the gate indicated in (a).

(XBL \&1\&-11233)

Of future interest is the determination of the mechanism responsible for the copious $a$ and ${ }^{8} \mathrm{Be}$ production in this reaction. The similarity between the ${ }^{13} \mathrm{C}+{ }^{208} \mathrm{~Pb}$ and ${ }^{12} \mathrm{C}+{ }^{200} \mathrm{~Pb}$ systerns suggesis that it also would be interesting to extend this comparison to a variety of other bombarding energies.

\section{Footnote and Reference}

"Physics Department, University of Edinburgh, Edinburgh EH93JZ, United Kingdom.

1. A.N. Bice, A.C. Shotter, and J. Cerny. Lawrence Berkeley Laboratory Nuclear Science Division Annual Report 1979-1980, LBL-11588, p. 69. 


\title{
${ }^{12} \mathrm{C} *$ Production in the ${ }^{12} \mathrm{C}+{ }^{208} \mathrm{~Pb}$ System at $230 \mathrm{MeV}$
}

\author{
A.N. Bice, A.C. Shotter," and Joseph Cerny
}

The reaction mechanism involved in the production of fast o-particles in heavy-ion collisions is a subject of study that has only recently been under intense investigation. Interestingly, the observation of such fast $\alpha$-particles was made as early as 1961 by Britt and Quinton ' from heavyion reactions at entergies of about $10 \mathrm{MeV} / \mathrm{A}$. Recent studies have concentrated in part on whether such fast alpha particles are produced when a massive fragment of the projectile is transferred to and retained by the target nucleus. Recently, Siwek-Wilczynsks et al. ${ }^{2}$ proposed a model of incomplete fusion reactions based partially on their study of the ${ }^{12} \mathrm{C}+{ }^{160} \mathrm{Gd}$ system at bombarding energies of 90 $200 \mathrm{MeV}$. Their measurements and incomplete fusion model calculations suggest that, for the ${ }^{12} \mathrm{C}+{ }^{160} \mathrm{Gd}$ systern, $\sim 40 \%$ of the observed singles o-particles result from the massive transier reactions $\left({ }^{12} \mathrm{C}, \alpha\right)$ and $\left({ }^{12} \mathrm{C}, 2 \alpha\right)$. The remaining $-60 \%$ or more of the singles a particle cross section, which amounts to several hundred millibarns, is suggested to arise from 30 -particle breakup. However, direct measurement of the 3o-particle breakup cross stetion, which includes the sequential processes ${ }^{12} \mathrm{C}^{*} \rightarrow 3 \alpha$, were not performed by Siwek-Wilczynska et al.

We have previously reported ${ }^{3}$ measurements of ${ }^{12} \mathrm{C}$ breakop for a very similar system, the ${ }^{12} \mathrm{C}+{ }^{2018} \mathrm{~Pb}$ systern, at 132 and $187 \mathrm{MeV}$. Our moasurements have recently betn extended to a bombarding energy of $230 \mathrm{MeV}$ with a beam produced at the 88-Inch Cyclotron. As before, two counter telescopes, arranged in a close vertical geometry with respect to the normal scattering plane, were employed to detect $3 \alpha$ events. In addition, for this experiment, a third telescope was located above the other 1wo particle telescopes, thereby providing three possible telescope-telescope coincidences and vertical angular separations at each laboratory angle. For this titiple telescope system, the minimum vertical angular separation of two telescopes was $1.5^{\circ}$ and the maximum was $20^{\circ}$.

Simular to the results of the $132 \mathrm{MeV}$ and $187 \mathrm{MeV}$ bombarding energy measurements, Jittle evidence for direct $3 a-p a r t i c l e ~ b r e a k u p$ was obtained at $230 \mathrm{MeV}$ with this detector configuration. The majority of the $\alpha+2 a$ coircident events resulted from the sequential decay of ${ }^{12} C$, primarily from its $7.6 \mathrm{MeV}, \mathrm{O}^{+}$state. The angular distributions for the sequential breakup of ${ }^{12} \mathrm{C}$ into the $a+{ }^{8} \mathrm{Be}$ channel, where the target remains vnexcited, is shown in Fig. $\mathrm{l}(\mathrm{a})$ for the three $\sqrt{2} \mathrm{C}$ bormbarding energies. The total eross sections, obtained by integrating the angular distributions in Fig. I(a), are shown in Fig. l(b).

From the data displayed in Fig. I(b) and estimates of ${ }^{12} \mathrm{C}$ " production for more negative $Q$-valuts, it is possible to conclude that ${ }^{12} \mathrm{C}^{*}$ production and subsequent decay is not a significant source of fast alpha particles for this system Furthermore, if the $3 \alpha$ production is as likely as
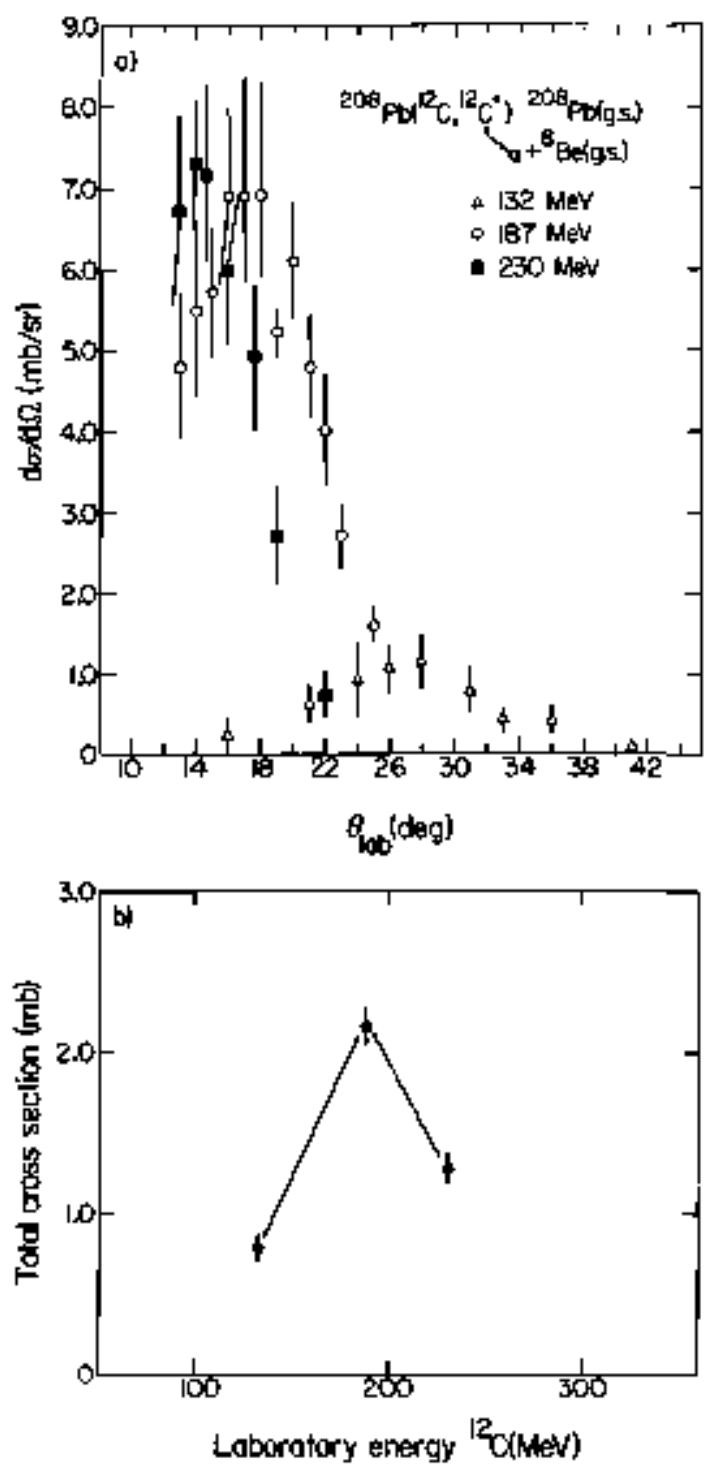

Fig. 1. (a) The measured differential cross section for the ${ }^{20 \mathrm{HS}} \mathrm{Pb}\left({ }^{12} \mathrm{C}, \alpha^{\mathrm{B}} \mathrm{Be}\right){ }^{2 \mathrm{~B}} \mathrm{~Pb}(\mathrm{~g}, \mathrm{~s})$ reaction at 132,187 and $230 \mathrm{MeV}$. (b) The tolal cross section for the reaction ${ }^{20} \mathrm{~Pb}\left({ }^{12} \mathrm{C}, \mathrm{a}^{\mathrm{B} e}\right)^{208} \mathrm{~Pb}(\mathrm{~g} . \mathrm{s}$.$) .$

(XBL 818-1158)

Wiczynske at al. suggest, we can conclude that the three breakup a's are not locused in as narrow a cone as they would be, for instance, if they originated from a sequential ${ }^{12} \mathrm{C}^{*}$ decay. Clearly, more detailed knowledge of the laboratory distribution of a's from the proposed ${ }^{12} \mathrm{C} \rightarrow 3 \alpha$ breakup reaction is needed. 


\section{Footnote and References}

"Physics Department, University of Edinburgb, Edinburgh EH93JZ, United Kingdorn.

1. H.C. Britt and A.R. Quintom, Phys. Rev. 124, 877 (1961).
2. K Siwek-Wilczynska et al., Phys. Rev, Leth 42, 1599 (1979) K. Siwek-Wilczynska et al., Nucl. Phys. A 330, 150 (1979).

3. A.C. Shoter, AN, Bice, and Joseph Cerny, Nuctear Science Division Annual Report for 1979.1980, LBL11588, p. 73.

\title{
Target Fragment Recoil Properties in the Reaction of 50-86. MeV// ${ }^{12} \mathrm{C}$ with ${ }^{181} \mathrm{Ta}$ and ${ }^{197} \mathrm{Au}$
}

\author{
W. Loveland, P.L McGaughey, $K$ Aleklett, ${ }^{\dagger}, J$. Moody, \\ P. Johnson," and G.T. Seaborg
}

As a guide to assist in the understanding of the chenges that accur in nucleus-nucleus collisions as the pro. jectile energy increases from the low energy (드 $10 \mathrm{MeV} / \mu)$ to the bigh energy regimes $(250 \mathrm{MeV} / \mu)$, we have undertaken a program of measurement of the target fragmentation systematiss throughoul this energy range. Earlier, we reporled the results of such measurements for the interaction of $7,4,13.0,17.1$, and $19.7 \mathrm{MeV} / \mu{ }^{16} \mathrm{O}$ with ${ }^{197} \mathrm{Au}^{1}$ More recently, we have made measuremnents of the average target fragment momenta and energies using the thick-target thick+catcher reooil technique, which has been described previously ${ }^{2}$ for the reactions of 50 and $86 \mathrm{MeV} / \mu{ }^{12} \mathrm{C}$ with ${ }^{197} \mathrm{Au}$, and for 75,80 and $86 \mathrm{MeV} / \mu^{12} \mathrm{C}$ with ${ }^{181} \mathrm{Ta}$. These experiments were performed using the $86 \mathrm{MeV} / \mu{ }^{12} \mathrm{C}$ provided by the CERN SC synchrocyclotron. The beam energy was degraded using $\mathrm{AJ}$ absorbers to supply the three lower energies.

A range-weighted measure of the extent of forward peaking of the fragment anguler distributions is the F/B ratio, the ratio of the fraction of target fragments recoiling forward (F) from a thick target to the fraction of fragments recoiling backward (B). The variation of this $\mathrm{F} / \mathrm{B}$ ratio with target fragment mass number $A$, shown in Fig. l, behaves similarly for the interaction of $86 \mathrm{MeV} / \mu$ ${ }^{12} \mathrm{C}$ with both ${ }^{181} \mathrm{Ta}$ and ${ }^{197} \mathrm{Au}$. The magnitude of the $\mathrm{F} / \mathrm{B}$ ratios is extremely large and far exceds any values obtained from proton induced reactions using similar targets. Furthermore, the observed F/B values greatly exceed those seen for similar products produced in the interaction of $7.419 .7 \mathrm{MeV} / \mu{ }^{16} \mathrm{O}$ with ${ }^{197} \mathrm{Au}$. The changes in the recoril properties in the projectile energy range of 50 to $86 \mathrm{MeV} / \mu$ are modest and dependent on the atornic number and mass of the rarget fragment. The interpreta. lion of these data in terms of the two step vector model to deduce average fragment energies and momenta is discussed in the repori entíled "Target Fragmentation from [0-2] $00 \mathrm{MeV} / \mu^{\prime \prime}$ by Loveland et al. in this Annual Report.

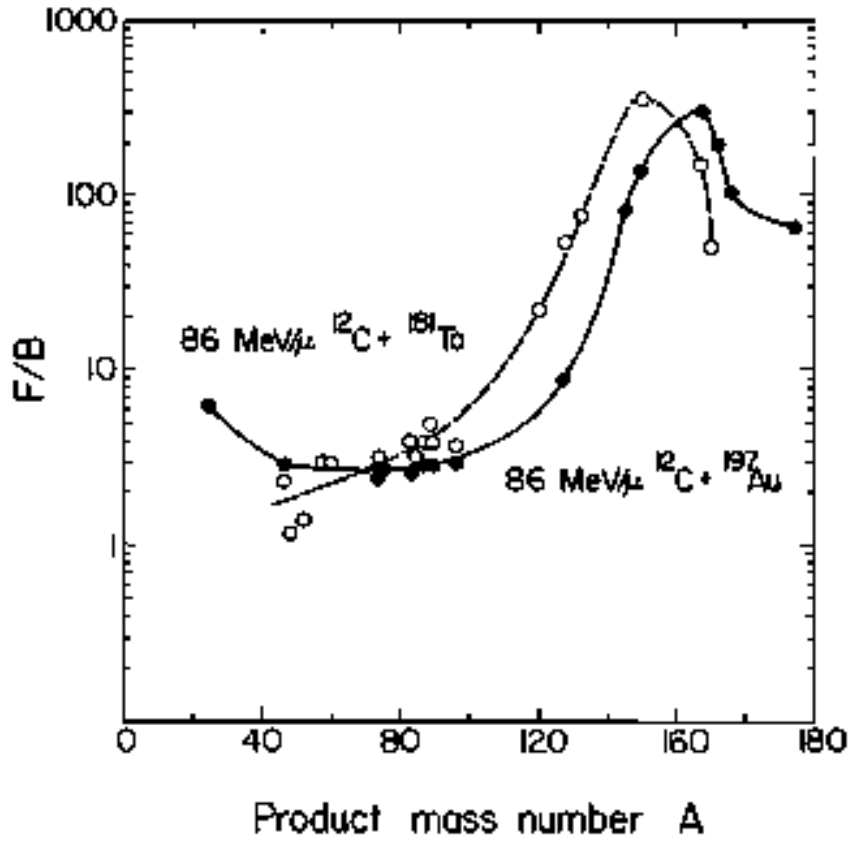

Fig. I. Variation of the $\mathrm{F} / \mathrm{B}$ ratio as a runction of target fragment mass number $A$ for the interaction of $86 \mathrm{MeV} / \mu$ ${ }^{12} \mathrm{C}$ with ${ }^{181} \mathrm{Ta}$ and ${ }^{1 m_{\mathrm{Au}}}$.

(XBL 818-1125)

\section{Footnotes and References}

"Radiation Center, Oregon State University, Corvallis, OR 97331.

${ }^{\dagger}$ Studsvik Science Research Laboratory, S-61l 82 Nyköping. Sweden.

1. W. Loveland et al., Oregon State University Report DOE/ER/70035-1, September 1980.

2. W. Loveland et al., Phys. Rev, C 23, 253 (1981). 


\title{
Target Fragment Angular Distributions in the Reaction of $86 \mathrm{MeV} / \mu{ }^{12} \mathrm{C}$ with ${ }^{197} \mathrm{All}$ and ${ }^{238_{\mathrm{U}}}$
}

\author{
K. Aleklett, W. Loveland, P.L MCGaughey, R.H. Krass, Jr, ${ }^{\dagger}$ \\ Y. Morita, G.T. Seaborg. T. Lund,,$^{\ddagger}$ D. Molzahn, ${ }^{\ddagger}$ E. Hagebo, ${ }^{\ddagger}$ and I. Haldorgen
}

The direct measurement of target fragment angular distributions and energy spectra enables one to experimentally characterize in a model independent manner the kinematics of the nucleus-nucleus collision that produced the fragments. The availability of high intensity energetic heavy ion bearns from the CERN SC synchrocyclotron has made it possible for the first time to measure these quantities for intermediate energy nucleus-nucleus collisions. We report herein the preliminary results of the first messurements of target fragment angular distributions from the interaction of $86 \mathrm{MeV} / \mu{ }^{12} \mathrm{C}$ with ${ }^{197} \mathrm{Aur}$ and ${ }^{298} \mathrm{U}$.

Thin targets of ${ }^{197} \mathrm{Au}$ and ${ }^{238} \mathrm{U}\left(\sim 750 \mu \mathrm{g} / \mathrm{cm}^{2} \mathrm{UF}_{4}\right.$ - $300 \mathrm{\mu g} / \mathrm{cm}^{2} \mathrm{Au}$ ) were placed at the center of cylindrical scattering chambers and were irradiated separately with an $86 \mathrm{MeV} / \mu{ }^{12} \mathrm{C}$ beam from the CERN SC synchrocyclotron. The irradiations lasted $\sim$ IS hours with an average beam flux of 3 to $5 \times 10^{11}{ }^{12} \mathrm{C}$ ions per second The beam spot diameter was less than $10 \mathrm{~mm}^{2}$. Fragments emerging from the targets were stopped in catcher foils that were laler cut into nine or ten sections corresponding to particular angutar intervals. The radionuclides present in each foil were assayed by off-line gamma-ray speciros. copy. The solid angle subtended by each catcher foil was calculated analytically.

In Fig. I, we show the angular distributions of typical fragments from the $86 \mathrm{MeV} / \mu{ }^{12} \mathrm{C}$ reaction with ${ }^{197} \mathrm{Au}$. Note the enormous magnitude of the forward peaking of the heaviest Iragments and the inilial tendency for the degree of forward peaking to increase as the target fragment mass decreases. Further decreases in fragment mass result in less forward peaking of the angular distributions, while the lightest fragments show more forward peaking.

For the reaction of $86 \mathrm{MeV} / \mu{ }^{12} \mathrm{C}$ with ${ }^{238} \mathrm{U}$, we thirty fragnents whose angular distributions were measured can be placed into four representative classes. In Fig. 2, the distributions for one fragment from each of the four classes are given. The neutron-rich fission fragments have isotropic angular distributions indicating that little or no monentum transfer occurred before fission. The neutron-deficient products of similar mass are more forward peaked, as are the light fragments. The greatest degree of forward peaking accurs for the heavy fragnents. This paltern is similat to that observed for the interaction of $4.8 \mathrm{GeV}{ }^{12} \mathrm{C}$ with ${ }^{238} \mathrm{U}$. ' Fragments from the ${ }^{238} \mathrm{U}$ target have a much smaller degree of peaking compared to the corresponding ${ }^{197} \mathrm{Aw}$ fragments.

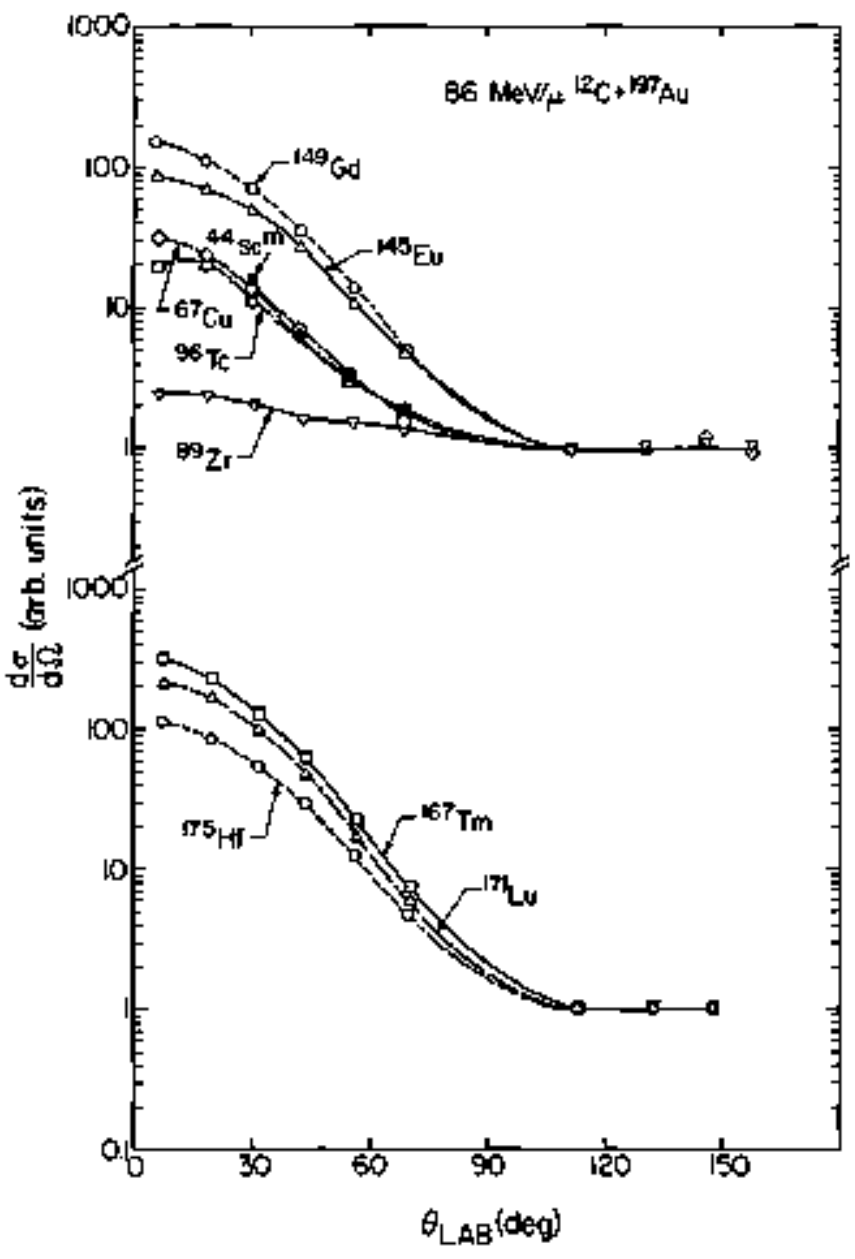

Fig. I. Selected target ftagnent angular distributions from the jniteraction of $86 \mathrm{MeV} / \mu{ }^{12} \mathrm{C}$ with ${ }^{193} \mathrm{Au}$.

(XBL $818-1122)$

Comparisons of these resplts with one another and with current models of bigh entergy nudeus-mucleus collisions are in progress and should yield interesting results. We hope to complement these experimental meastrements by determining the target Iragnent energy spectra using difierential range techniques at the CERN SC synchrocyclotron. 


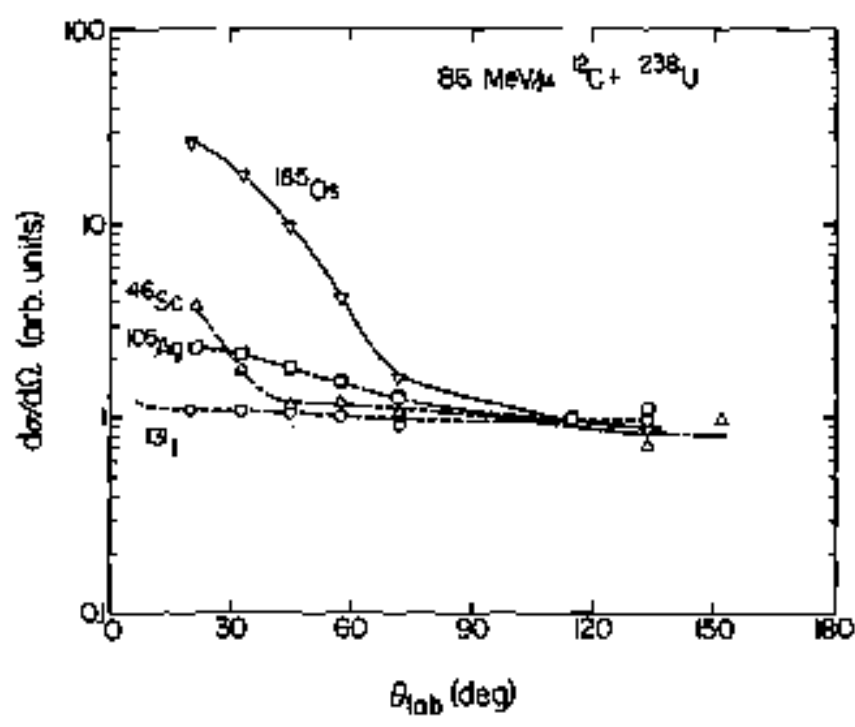

Fig. 2. Representalive target fragment angular distributións fơ a typical neutron-rich fission 5 ragment $\left({ }^{131} I\right)$, a neutron deficient fragment $\left({ }^{105} \mathrm{Ag}\right)$, a ligbt fragment $\left({ }^{46} \mathrm{Sc}\right)$, and a beavy $\int \mathrm{rag} m e n t\left({ }^{185} \mathrm{Os}\right)$ from the interaction of $86 \mathrm{MeV} / \mu{ }^{12} \mathrm{C}$ with ${ }^{238} \mathrm{U}$.
Foormotes and Reference

"Studsvik Science Research Laboratory, S-61] 82 Nyköping, Sweden.

'Radiation Center, Oregon State University, Corvallis, OR 97331 .

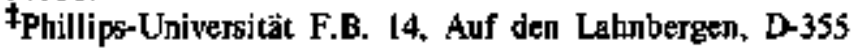
Marburg/Lahn, West Germany.

SDepartment of Nuclear Chenistry, University of Oslo, Oslo, Norway.

1. W. Loveland at al., Lawrence Berkeley Laboratory Report LBL-11658 (1980).

\title{
Target Fragmentation from 10-2100 $\mathrm{MeV} / \mu$
}

\author{
W. Loweland," P.L. McGaughey, K. Aleklett, ${ }^{\dagger}$ K.J. Moady, \\ R.H. Kraus, Jr." R.M. McFarland, and C.T. Seaborg
}

In recent years, there has been increasing interest in studying how the mechanisms in nuclear heavy-ion reactions change as the projectile energy increases from low energies $(-10 \mathrm{MeV} / \mu)$ to relativistic energies (2100 MeV/ $/ \mu$ ). We thought that it would be valuable to make radiochemical measurements of target fragment average energies and momenta to help characterize the changes in target fragmentation ower this projectile energy regime. Acoordingly, in this paper we compare the results of sludies of target fragnentation in the reaction of ${ }^{12} \mathrm{C},{ }^{16} \mathrm{O}$, and $20_{\mathrm{Ne}}$ at seven difterent projectile energies from 13$2100 \mathrm{MeV} / \mu$. We report new dala from studies of reactions induced by projectiles whose energies ranged from $13-86 \mathrm{MeV} / \mu$, showing unusually large momentum transfers to the target fragments. It is interesting to compare our data with that of Kaufman et al., who studied the interaction of $400-2100 \mathrm{MeV} / \mu{ }^{12} \mathrm{C}$ with ${ }^{197} \mathrm{Au} .{ }^{1}$ The overall thrust of the data requiras significant revision of our ideas concerning the energy evolution of nuclear heavy ion reactions.

We studied the interaction of 13 and $19 \mathrm{MeV} / \mu / 6 \mathrm{O}$ with ${ }^{197} \mathrm{Au}$, using the LBL 88-Inch Cyclotron, and the interaction of 50 and $86 \mathrm{MeV} / \mu{ }^{12} \mathrm{C}$ with ${ }^{197} \mathrm{Au}$, using the CERN SC synchrocyclotron. The $50 \mathrm{MeV} / \mu$ beam was obtained by degrading the primary $86 \mathrm{MeV} / \mu$ beam with an aluminum absorber. The thick-target thick-eatcher recoil properties of the fragments were measured, from which we deduced the average fragment momenta. The experimental techniques bave been described previously. ${ }^{2}$

A range-weighted measure of the extent of forward peaking of the fragment angular distributions is the F/B ratio, the ratio of the fraction of fragments recoiling forward (F) from a thick target to the fraction recoiling backward (B). The $F / B$ ratios for four typical target fragments produced by the reaction of energetic heavy ions with ${ }^{19}$ Aul, shown in Fig. I, increase with total projectile kinetic energy until $\sim \mathrm{I} \mathrm{GeV}$ and then decrease with further increases in projectile energy. This behavior is quantitatively similar to that observed in the interaction of energetic prolons with ${ }^{197} \mathrm{Au}^{3}$ and has been qualitatively explained as a consequence of time dilation. ${ }^{4}$ If similar mechanism(s) are operating in both heavy-ion and proton induced reactions, this explanation must be incorrect șince at these energies ( $50-86 \mathrm{MeV} / \mu$ ) time dilation eflects are negligible for heavy ions.

The two step vector model $]^{5}$ was used to deduce values for $P_{1}$, the longitudinal component of the monentum transferred to the target fragment precursor during the initial projectile torget interaction. As an aid to understanding the variation of $P_{11}$ with fuagment mass and projectile energy, we have defint a parameter called "inelesticity" as the ratio of the measured $P_{1]}$ value for a given fragment 


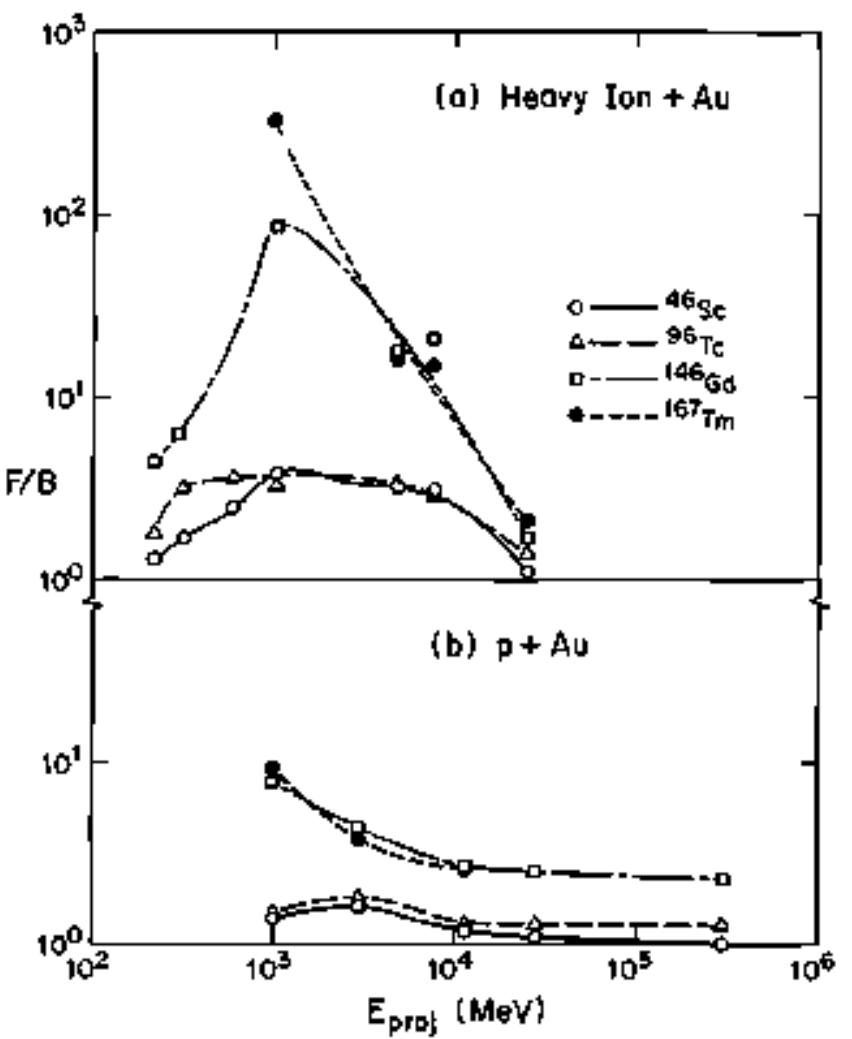

Fig. I. The variation of F/B for selected products from the reaction of (a) heavy ions with ${ }^{197} \mathrm{Au}$; (b) protons with ${ }^{197} \mathrm{Au}$ (Ref. 3). The data for heavy ion projectile energies $\geq 4.8 \mathrm{GeV}$ are from Ref. 1.

(XBL 818-1118)

to the maximum momentum that could be imparted to ibat fragment. The variation of inelasticity with product mass number for varions heavy ion reactions with ${ }^{15} \mathrm{Au}$ is given in Fig. 2. One is immediately struck by the large fractional momentum transfer occurring in the 0.6 and $1.0 \mathrm{GeV}$ ( 50 and $86 \mathrm{MeV} / \mu$ ) ${ }^{12} \mathrm{C}$ induced reactions. The magnitude of these momentum transfers indicates that the generalized critical angular momentum model ${ }^{6}$ does not correctly describe incomplete fusion at these energies because it predicts no significant momentum transfer can occur at energies greater than $\sim 20 \mathrm{MeV} / \mu$. The variation of inelasticily with product mass number appears to be different below $50 \mathrm{MeV} / \mu$, as compared to the higher energies. The fragment inelasticities decrease sInoothly with projectile energies above $\sim 50 \mathrm{MeV} / \mu$, indicating a conlinuous evolution of reaction mechanistr(s).

If one examines the variation of the absolute value of $P_{11}$ with projectile energy, one finds that $\mathbf{P}_{\mathrm{I1}}$ increases from $\sim 10 \mathrm{MeV} / \mu$ to $20 \mathrm{MeV} / \mu$ and decreasits at higher energits. The decrease in $P_{11}$ from $50 \mathrm{MeV} / \mu$ to $2100 \mathrm{MeV} / \mu$ is described by a remarkably simple relationship:

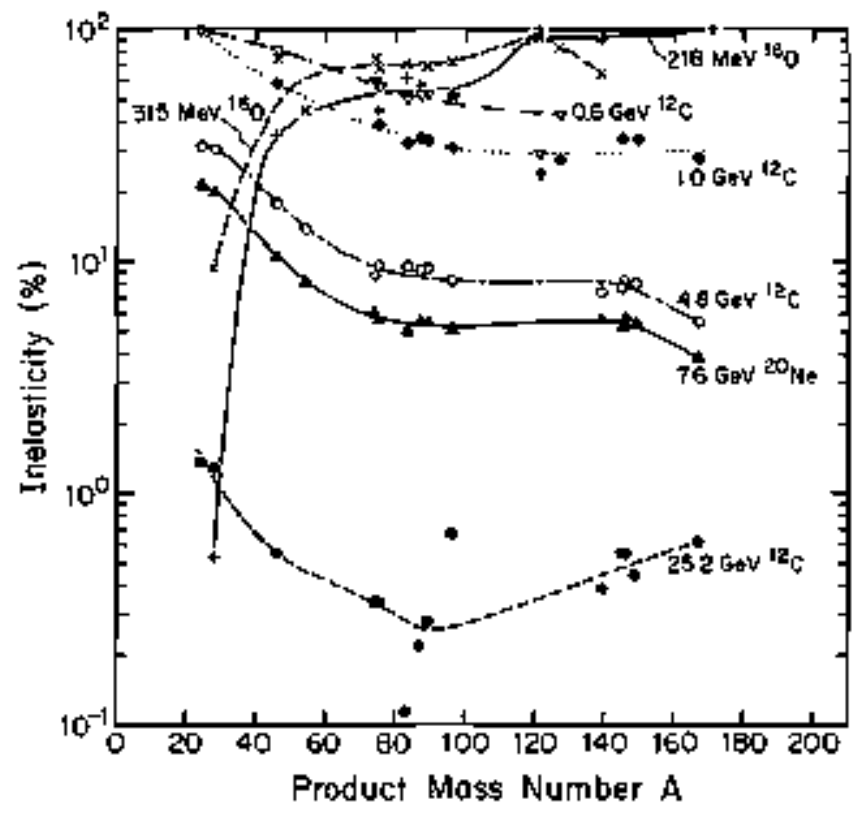

Fig. 2. The pariation of inelasticity with product mass number for the reactions of various heavy jons with ${ }^{197}$ Au. The data for the three highest energies are from Ref. 1 .

(XBL 818-1120)

$$
P_{11}=a+b \sqrt{1-a^{2}},
$$

where $a$ and $b$ are constants and $\rho$ is the projectile velocity in units of $\&$. This relationship is valid for all fragments where data are available at the five projectile energies. The constants $a$ and $b$ smoothly increase and decrease, respectively, with increasing fraginent mass. The reasons for the validity of this simple corrtlation, which encompasses such a wide range of projectile energies and fragment massts, are not clear at this time.

\section{Footnotes and References}

"Radiation Center, Oregon State University, Corvallis, OR 97331.

'Studsvik Science Research Laboratory, S.61I 82 Nyköping Sweden.

I. S.B. Kaufman et al., Phys. Rey. C 22, 1897 (1980).

2. W. Loveland et al., Phys. Rev, C 23, 253 ( 1981 ).

3. S.B. Kaviman, E.P. Steinberg, and M.W. Weisfield, Phys. Rev, C 18, 1349 (1978).

4. See, for example, H. Feshbach, First Worksbop on Ultra-Relativiștic Noclear Collisions, Lawrence Berkẹley Laboratory Report LBL-8957, (1979), p. 261.

5. See, for example, J.M Alexander in Nuclear Chemistry, L. Yaffe, Ed. (Academic, New York, 1968), p. 273.

6. K. Siwek-Wilczynski tt al., Phys. Rev, Lett. 42, 1599 (1979). 


\title{
C. RELATIVISTIC HEAVY IONS
}

\author{
1. Fragmentation Processes
}

\section{Energy Variation of Target Fragment Mass and Charge Distributions in the Interaction of Relativistic Heavy Ions with Ta and $U$}

\author{
W. Loveland," P.L McGatighey, D.J. Morrissey, C. Oertel," \\ LL, Nuntiliey," and G.T. Seaborg
}

The yields of various target fragments of differing atomic mass number produced in relativistic nuclear collisions are measures of some of the detalls of the projectile target interaction. As the velocity of the incident projectile appraaches the speed of light, the projectile-target nuclews jotteraction time approaches a constant value, and experimental product distributions are not expected to change with further increases in projectile energy. This behavior, commonly termed "limiting Iragmentation," appears to dominate the yield distributions observed in the interaction of relativistic heavy ions (RHJs) with low mass targets (e.g., Cu) for projectile tnergies larger than $24 \mathrm{GeV}$. To determine if this effect was predominant with heavy targess, we initiated a program to systematically survey the changes in product yield distributions as a function of projectile energy for Ta and $U$ targets. The use of these targets should make possible the occurrence of more collisions in which the central densities of the two nuclei overlap considerably compared with the light targets.

To date. we have performed experiments to measure these target fragment yield distributions in the reactions of $3.0 \mathrm{GeV}^{12} \mathrm{C}, 5.0 \mathrm{GeV}^{20} \mathrm{Ne}, 8.0 \mathrm{GeV} 20 \mathrm{Ne}, 25 \mathrm{GeV}{ }^{12} \mathrm{C}$, $28 \mathrm{GeV} \mathrm{p}$ and $42 \mathrm{GeV}{ }^{20} \mathrm{Ne}$ with ${ }^{181} \mathrm{Ta}$ and $3.0 \mathrm{GeV}{ }^{12} \mathrm{C}$. $4.8 \mathrm{GeV}^{12} \mathrm{C}, 5.0 \mathrm{GeV}^{20} \mathrm{Ne}, 8.0 \mathrm{GeV}{ }^{20} \mathrm{Ne}, 12 \mathrm{GeV}{ }^{12} \mathrm{C}$, 21 $\mathrm{GeV}{ }^{20 \mathrm{Ne}}, 25 \mathrm{GeV}{ }^{12} \mathrm{C}, 28 \mathrm{GeV}$, and $42 \mathrm{GeV}{ }^{20} \mathrm{Ne}$ with ${ }^{238} \mathrm{U}$. Although the analysis is complete for only a fraction of these experiments, can discuss some of the trends observed in the data. In general, the fragment yields from the interaction of a variety of projectiles of differing energy with ${ }^{181} \mathrm{Ta}$ are similar in magnitude and in variation with fragment mass number, as demonstrated in Fig. 1. The fragment yield distribution from the reaction of $28 \mathrm{GeV}$ protons with ${ }^{1 \mathrm{~B} 1} \mathrm{Ta}$ shows a similar shape to those messured in RHI-induced reactions, but it differs in the magnitude of the yields. The ratio of the total reaction cros sections (as measured by summing the total residue cross sections from $A=40$ to $\mathrm{A}=180$ and correcting for binary fission) for the $25 \mathrm{GeV}{ }^{12} \mathrm{C}$ induced reaction relative to the $28 \mathrm{GeV}$ proton induced reaction was $1.7 \pm 0.3$. which is in moderate agreement with the geometric crosssection ratio of 1.42 . With urantum targets the situation is quite different, as is apparent from an inspection of Fig. 2. In RHI interactions with this target, one notices a dranatic increase in beavy residue yields as the incident projectile entergy increases from 8 to $25 \mathrm{GeV}$. Also, the shape of the

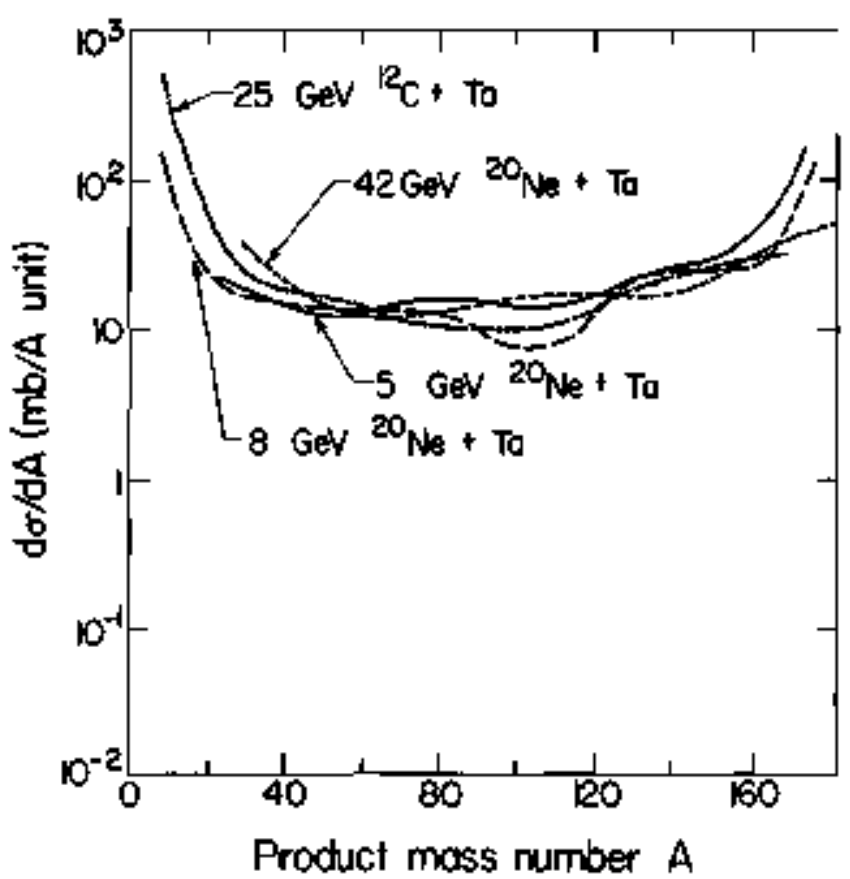

Fig. 1. Target fragment yield distributions for the interaction of $5 \mathrm{GeV} 20 \mathrm{Ne}, 8 \mathrm{GeV}{ }^{20} \mathrm{Ne}, 25 \mathrm{GeV}{ }^{12} \mathrm{C}$, and $42 \mathrm{GeV}{ }^{20} \mathrm{Ne}$ with ${ }^{181} \mathrm{Ta}_{\mathrm{a}}$.

(XBL 818-1124)

yield distribution resulting from the $28 \mathrm{GeV}$ proton induced reaction is quite different from similar distributions in RHI-induced reactions.

We have attermpted to compare thest experimental product yield distributions with the prediction of two cursent models of relativistic nuclear collisions, the intranuclear cascade model ${ }^{2}$ and the muclear frestreak model. ${ }^{3}$ In general, both models are able to predict the approximate shapes and magnitudes of the mass yield distributions and the centers of the isotopic distributions for target fragments heavier than $A=60$. The formation of fragments lighter than this cannot be predicted by conventional statistical de-excitation models; instead, they may require the existence of an "explosion" type mechanism in which the highly excited targets fragment initially into many smallter pieces. Neither model predicts the dramatic increase in heavy fragment production observed in the reaction of $25 \mathrm{GuV}{ }^{12} \mathrm{C}$ with ${ }^{238} \mathrm{U}$. From the observed invariance of 


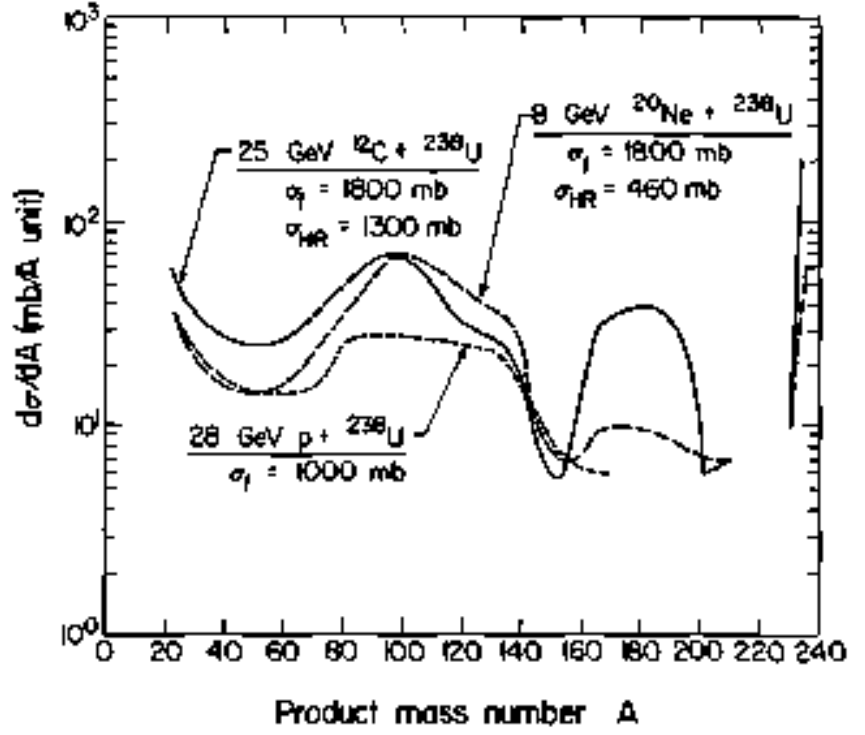

Fig 2. Target fragment yield distributions for the interaction of $25 \mathrm{GeV}{ }^{12} \mathrm{C}, 8 \mathrm{GeV} 20 \mathrm{Ne}$, and $28 \mathrm{GeV}$ proton with ${ }^{238} \mathrm{U}$. the total experimental reaction cross-sections resulting from the interaction of ${ }^{20} \mathrm{Nk}$ with ${ }^{181} \mathrm{Ta}$ at various energiss, we conclude that the nucleus-nucleus reaction cross-section is nearly a constant over this entergy range. Tbis implies that the free nucleon-nucleon scatiering cross-sections, which vary strongly with energy in this energy range may not have a pronounced effect on the total reaction crosssections for RHI reactions with heavy targets, where simple geometry seems to have the predominant effect.

\section{Fooltole and References}

"Radiation Centẹ, Oregon State University, Corvallis, OR 97331.

1. J.B. Cumming. P.E. Haustein, and R.W. Sloenner, Phys. Rev. C 10, 739 (1974); J.B. Cumming, P.E Haustein, T.J. Ruth, and G.J. Virtes, Phys. Rev. C I7, 1632 (1978)

2. Y, Yariv and Z Fraenkel, Phys, Rev. C 20, 2227 (1979).

3. P.L MeGaughey, D.J. Marrissey, and G.T. Seaborg, Nuclear Science Div. Annual Report 1979-1980, LBL IIS88, 159 ( 1981 ).

\title{
Target Fragment Angular Distributions in the Reaction of 3.0 and $12.0 \mathrm{GeV}{ }^{12} \mathrm{C}$ with ${ }^{197} \mathrm{Au}$ and ${ }^{238} \mathrm{U}$
}

\author{
Y. Morita, W. Lovelaud* F.L. McGaughey, and G.T. Seaborg
}

Fragment angular distributions are useful tocts to aid in the uoderstanding of high energy fragmentation reaction mechanisms. Unfortunately, previous experimental studies of heavy-ion induced target fragmentation have only inwolved measurements of $F / B$ satios ${ }^{1,2}$ which are range. weighted measures of the extent of forward peaking of the angular distributions. Therefore, we have undertaken the direst determination of target fragment angular distributions for relativistic heavy-ion reactions. Specifically, we thought it of interest to compare distributious resulting from the interaction of a sub-relativistic heayy jon, $3.0 \mathrm{GeV}{ }^{12} \mathrm{C}$, and a relativistic one, $12.0 \mathrm{GeV}{ }^{12} \mathrm{C}$ with a very fissionable ${ }^{238} \mathrm{U}$ target and the less fissionable ${ }^{197} \mathrm{All}$.

The major diffoulty in the determination of these angular distributions is the relatively low $12 \mathrm{C}$ particle intensity provided by the LBL Beralac; typical intensities are less than $10^{10}$ particles per minute. To orercome this problem, special target-catcher assemblites were employed. Each assembly consisted of 17 jdentical target foils, each backed with a conical-catcher foil assembly in which the fragments recoiling from the target were stopped After irradiation, each conical-catcher foil was cot into four pieces, correspooding to the approximate angular ranges of $0-30^{\circ}, 30-50^{\circ}, 3070^{\circ}$, and $70-90^{\circ}$, with respest to the incident beam direction. Then, the foils belonging to the sarme angular ranges were all combined to form single samples. Gaming-ray spectroscopic techniques were used to assay the relative amounts of different radionuclides present in each sample. ${ }^{3}$

The determination of the effective solid angle subtended by och eatcher foil, the corrections for fragment scattering and absorption, and for widely differing counting efficiencies for the extended sources were a complex matter. A semi-empirical approach to the problem was chosen. First, the relative solid angles subtended by the various woic catcher sections, with respect to extended-arta circular targets, were alcolated numerically. Using the saute method, the average seattering angle of a fragment stopping in each catcher section was evaluated. The next step inwolved the use of a single ${ }^{238} \mathrm{U}$ target-catcher assembly to measure the fission fragment angular distribution produced by $43 \mathrm{MeV}$ alpha priclic induced fission. During this experiment, the alpha particle beam prowided by the LBL 88- Inch Cyclotron was defocused to provide the sarne boam profile used in the Bevalac experiments. The relative activities of the four catcher sections were assayed using the same counting geometry and techniques as employed previously. Relative values of the differential cross-section do/dn (6) were computed for the typical fisston products using the measured activities and numerically calculated 
solid angles. The values of $\mathrm{d} g / \mathrm{d} \Omega(\theta)$ for the observed nuctides were averaged and compared to the known gross fission fragment angular distributions. ${ }^{4}$ This comparison was used to generate a set of correction factors, ranging from 10 to $50 \%$ to correct for the effects of extended counting sources and fragment absorption and scattering.

Despite the measures used to overcoms the problem of Jow beart intensity, only 6 to 8 nuclides were reliably identified in the preliminary antalysis of each experimental set of data, and these all contain appreciable uncertainties. Nevertheless, some useful information may be extracted from trends in the data. In general, roughly isotropic angular distributions are observed for neutron-rich ${ }^{238} \mathrm{U}$ fission products such as ${ }^{97} \mathrm{Zr},{ }^{99} \mathrm{MH}$, and ${ }^{133} \mathrm{I}$ at both beam energies. This is in good agreement with previous determinations which indicated that the low excitation energy fission of ${ }^{238} \mathrm{U}$ induced by relativistic heavy ions results from peripheral collisions with little momentum transfer. Those fragments not normally considered to be ${ }^{23 \mathrm{f}_{\mathrm{U}}}$ fission fragments, such as ${ }^{43} \mathrm{~K},{ }^{72} \mathrm{As},{ }^{149} \mathrm{Gd}$, all show forward peaked distributions with the grteatest degres of peaking being observed in the ${ }^{149} \mathrm{Gd}$ angular distribution. This is in qualitative agroement with the trends of the $F / B$ ratios. $I$ For the fragmentation of ${ }^{197} \mathrm{All}$, all the observed distributions are forward peaked, with maximum peaking observed for fragments with mass number $A$ such that $145 \leq A \leq 155$, which agrees with general trends in their $F / B$ ratios. ${ }^{5}$
It is inleresting to compare the fragment angular distributions measured for this work with similar data measured for the interaction of high energy protons with ${ }^{238} \mathrm{U}^{6}{ }^{6}$ The most dramatic difference between these sets of data is the absence of a sidewise peaked ${ }^{44} \mathrm{Sc}$ fragment distribution in the ${ }^{12} \mathrm{C}$ induced reactions, whereas such a distribution is observed in the interaction of $11.5 \mathrm{GeV}$ protons with ${ }^{238} \mathrm{U}$. If this sidewise peaked light fragment angular distribution seen in proton induced reactions is due to collective effocts such as compreasion of nuclear matter or a shock wave, it is surprising that the effect is not enhanced or even apparent with relativistic beavy ions.

\section{Reference and Foolnotes}

Radiation Center, Oregon State University, Corvallis, OR 97331.

J. W. Loveland et al., LBL-1 I658, (1980).

2. W. Loveland et al., Phys. Rev. C 23, 253 (1981).

3. D.J. Morrissey et al., Nucl. Instrum Meth. 158, 499 (1979).

4. R. Vandenbosch, H. Warhanek, and J.R Huizenga, Phys. Rev. 124, 846 (1\%]).

5. S.B. Kaufmann, E.P. Setinberg, B.D. Wilkins, and D.J. Henderson, Phys. Rev. C 22, 1897 (1980).

6. D.R. Fortney and N.T. Porile, Phys. Lett. B 76, s5 (1978).

\title{
Fragment-Spray Correlations in High Energy Heavy Ion Collisions
}

\author{
AI. Warwick, H.H. Gutbrod, S.B. Kaufman, M.R. Maier, J. Peter, H.G. Ritfer, \\ E.P. Steinberg. ${ }^{*}$ H. Stelzer, F. Weik, H. Mieman, and B.D. Wilkins"
}

We report here the partially analyzed results of an experiment performed at the Bevalac last year, which focused on heavy ion collisions leading to final states with heavy target fragments moving slowly in the laboratory frame. Experimental details were described in last year's annual report' Typically, Iragment energies are in the range $0.3 \mathrm{MeV} / \mathrm{n}$ to $3 \mathrm{MeV} / \mathrm{n}$. Fragments across the entire mass range are meastred.

We particularly wish to investigate the reation mechanism, looking beyond the late stage evaporation kinematics to see features of the fast energy deposition stage.

One of the modts to be considered is a hydrodynamic description of such collisions. This model predicts distinctive "bounce-off" features in which the transverse momentum transfer to the heavy fragment is accomplisbed through the expansion of a pressure zone. ${ }^{2}$ A more traditional approach has been to consider the Coulomb force as the origin of the kinetic energy of the heavy fragment, which, in this model, emerges as an evaporation product or fission fragment. We do observe events for which such a Coulomb description is appropriate there is a wealth of experimental studies of such data. ${ }^{3}$ However, we also observe, due to the exclusive nature of our experiment, a direct correlation between the fast stage of the collision and the fragment enission, in the form of an angular correlation between the numorous fast light charged particles emitted and the fragment itself. This correlation is omipresent but seems to be strongest for the heaviest frag ments, which are considered to be residues of an exaporating system. Thus, by studying the heaviest fragments and their correlation we are looking at the effects of the initial collision, smeared by the random walk of evaporation Jecoil. The properties of the lighter fragments are considered to be dominated by the late-stage evaporation mechanism, although a spray-fragment correlation is observed here too. 
A further observation to be understood within the various models is the fact that the data are dominated by the toral ineident kinetic energy, independent of projectile mass, ${ }^{4}$

Figure I shows the azimuthal correlation betwen the heavy fragionts $M>50$ and the spray of fast charged perticles in the paddle array. The reaction is $400 \mathrm{MeV} / \mathrm{n}$ $\mathrm{Ne}+\mathrm{Av}$ and the fragments are detected at $\Rightarrow 90^{\circ}$, $\Psi=0^{\circ}$. There is a strong azimuthal correlation between the spray and the fragment. Thus, the fast spray plays a role in balancing the transverse momentum of the heavy target remnani. This coupling of the fragment emission 10 the fast stage of the reaction is in direct contradiction to the twro-step interpretation and furthermore is an effect explicitly preticted by the hydrodynamic models. This experimental correlation has been called "bounce-of" but has not been explicitly shown to originate with an expanding pressure zone.

\section{Footnote and References}

*Asgonne National Laboratory, Geseilschaft für Schwerionenforschung, and LBL.

I. Nuclear Science Division Annual Report, LBL-JI588 (1981).

2. H. Stöcker and B. Moller, GS1 Preprint 80-4 (1980).

3. G.D. Westfall et al., Phys. Rev, C 17, 1368 (1978), and references therein.

4. A Sandoval et al., Phys. Rev. C 21, I32l (1980).

W.G. Meyer et al., Phys. Rev. C 22, 179 (1980).

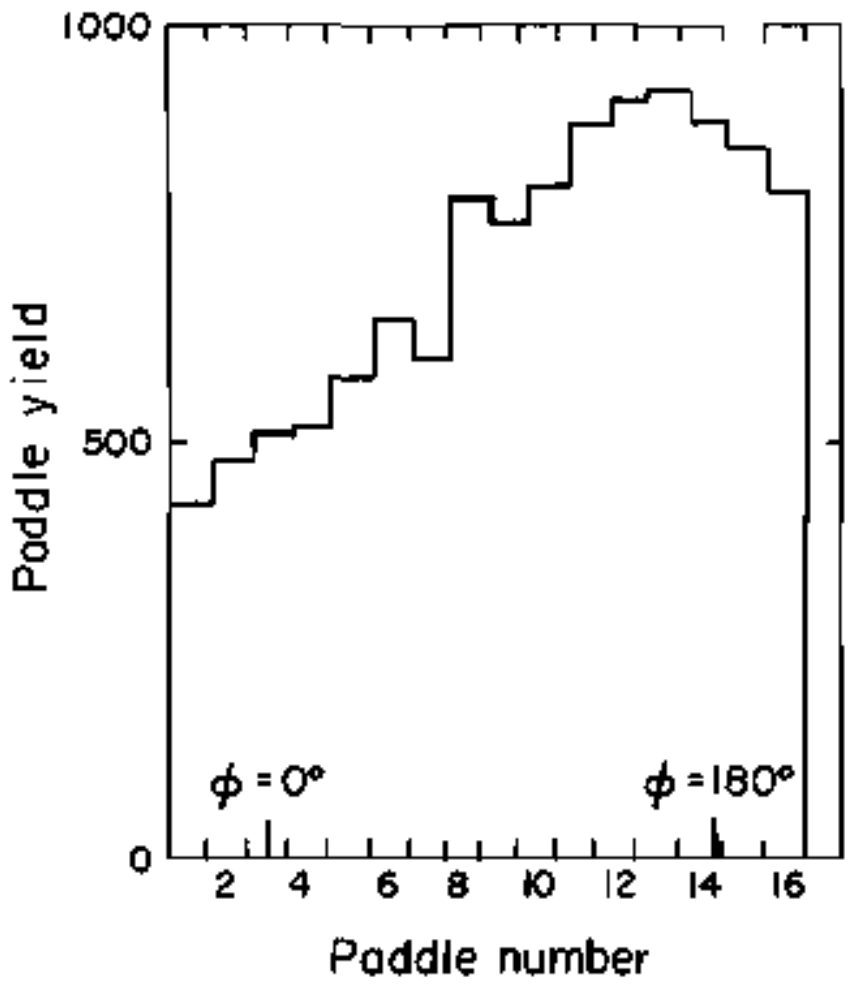

Fig. 1. Yield of fast charged particles in paddle ring $A$ as a function of azimuthal angle, observed in eoinciden $x$ with a heavy fragment $(\mathrm{M}>50)$ at $\theta=90^{\circ}, \psi=0^{\circ}$.

(XBL $8 \mid \$-1155)$

\title{
Comparative Yields of Alkali Elements and Thallium from Uranium Irradiated with $\mathrm{GeV}$ Protons, ${ }^{3} \mathrm{He}$ and ${ }^{12} \mathrm{C}$
}

\author{
T. Björnstad 't H. $\dot{A}$ Gustafsson, B. Jonson, \\ O.C. Jonsson, ${ }^{\$}$. Lindfors. ${ }^{\ddagger}$ S. Matisson, A.M. Poskanzer, \\ H.L. Ravd, and D. Schards
}

Mass-separated ion beams of the alkali elements $\mathrm{Na}$, $\mathrm{K}$, and $\mathrm{F}^{+}$, and of the element $\mathrm{Tl}$, were produced by bombarding a uranium target with $600 \mathrm{MeV}$ protons, $890 \mathrm{MeV}$ ${ }^{3} \mathrm{He}^{2+}$, and $936 \mathrm{MeV}^{12} \mathrm{C}^{4+}$. Isotopic production yields were measured. In the case of the ${ }^{12} \mathrm{C}$ beam, these are thick target yields. Absolute cross-sections for the proton beam data were deduced by pormalizing the delay-time corrected yield curves to metasured cross-sections. For products farthest away from stability, the ${ }^{3} \mathrm{He}^{2+}$ beam gen- erally gives the highest yields.

Footmotes

"Greatly condensed from Z Phys. A 303, 227 (1981).

tDepertment of Nuclear Chemistry, University of Oslo, $N$ 1000 Oslo 3, Norway.

Fepartment of Physics, University of Helsinki, \$F-00]70 Helsinki 17. Finiand.

SThe [SOLDE Collaboration, CERN, Genewa, Switzerland. 


\title{
A Compilation of World Data on "Anomalons"
}

\author{
E.M. Friedlander and Y.J. Karant
}

We present a compilation of world data referring to the observation of relativistic projectile fragments from high energy heavy ion collisions that exhibit an anomalously short mean free path for nuelear interaction ("anomalons"), which were first noticed (1954) by Mitone.'

We use as quanlitative measures for this effect either (1) the ratio $R_{1}$ for the mean (ree path (MFP) observed on projectile fragments to the MFP observed on "primary" nuclei which can be considered "normal," or (2) the ratio $R_{2}$ of the MFPs of projectile fragnents measured at different distances from their points of emission (e.g. . close to the origin and far from it).

$\mathbf{R}_{1}$ implies "external" calibration for theoretical evaluation of the normal MFP, while $R_{2}$ is an "internal standard" comparison and is therefore free of many real or suspected systematic effects.

To assess the statistical significance of deviations of $R_{1}$ or $R_{2}$ from unity (the null hypothesis), we note that $2 n R$ is distributed like $\chi^{2}$ with $2 \pi$ degress of freedion (DOF), where $n$ is the number of nuclear interactions observed. The quantily $R_{2}$ obeys a Fisher variance ratio ( $\sigma F-$ ) distribution with $n_{1}$ and $n_{2} D O F$, where $n_{1}$ and $n_{2}$ are the numbers of collisions observed at the two different distances from the origin. The integral probabilities $P_{1}\left(<R_{1}\right)$ and $P_{2}\left(<R_{2}\right)$ are uniformly distributed between 0 and 1.

The latter property is usefol in combining the results of different experiments to obtain a global level of significance. Indeed, given any set of independent $p_{i}$ values, $(i=1,2, n)$ the quantity $-25 n P_{i}$ is distributed like $\chi^{2}$ with $2 \pi$ DOF. The integral of this $\chi^{2}$ distribution can be used to reduce the confidence level to the more farmiliar number of equivalent standard deviations (ESD) by which the "effect" deviates from "normalcy."

Table 1 shows the results of this kind of analysis performed on
(1) Two cosmic ray emusion experiments measuring $R_{1} \cdot-3$

(2) Three emrulsion experiments measuring $R_{2}$ (Refs. 4 and 5 on 2 ACeV Bevalac beams, Ref. 6 on cosmic rays at hijgher energies), and

(3) A propane bubble chamber (PBC) experiment ${ }^{\dagger}$ using $4 \mathrm{AGeV}$ beams from the Dubna synchrophasotron, measuring $\mathbf{R}_{\mathbf{l}}$.

While the emulsion experiments are directly comparab/e, the PBC experiment (as stated by the authors themselves) lacks the spatial resolution necessary to measure $R_{2}$, i.e., to measure the MFP close to the origin of the projectile fragments.

While all emulsion experiments had this ability (the $R_{\text {I }}$ values reflected the inclusion of the region where most of the efiect is located) the PBC experiment excluded this region; in spite of this systerratic weakening of any possible effect, their $\mathbf{R}_{\mathbf{I}}$ value is systematically lower than unity (for four different charges of projectile fragments by (globally) 2.1 EDS.

Thus, all data available in the world literature consistently show that the MFP of relativistic projoctile fragments are anomalously short immediately after emission (be it by reason of time elapsed or of amount of matter traversed).

Note that all the deviations in Table I are in the same dijection (i.e., all MFPs are shorter then normal or shorter close to the point of emission). For the three emulsion experiments (group 2 in Table 1), the overall probability that this deviation is due to a statistical Huctuation is हूल $\mathrm{IO}^{-7}$.

If the results of all the independent experiments mentioned in Table l are taken together, the level of discrepancy goes up to $>6 \mathrm{SED}$, or, in other words, a probability of $10^{-16}$ for a chance fluctuation!

Table 1. Our statistical evaluation of world data.

\begin{tabular}{|c|c|c|c|c|c|}
\hline Group & Primary beam & $\begin{array}{c}\text { Ratio } \\
\text { measured }\end{array}$ & ESD & Global ESD & Ret. \\
\hline $\mathbf{I}$ & $\begin{array}{l}\text { Cosmic rays } \\
\text { Cosmic rays }\end{array}$ & $\begin{array}{l}R_{1} \\
R_{1}\end{array}$ & $\begin{array}{l}2.8^{\mathrm{a}} \\
3.1\end{array}$ & 2 & 3 \\
\hline 2 & $\begin{array}{l}2 \text { AGeV Fe, O } \\
2 \text { AGeV Fe, Ar } \\
\text { Cosmic rays }\end{array}$ & $\begin{array}{l}\mathbf{R}_{2} \\
\mathbf{R}_{2} \\
\mathbf{R}_{2}\end{array}$ & $\begin{array}{l}3.4 \\
3.2 \\
2.8^{2}\end{array}$ & 5.] & $\begin{array}{l}4 \\
5 \\
6\end{array}$ \\
\hline 3 & $4 \mathrm{AGeV} C$ & $\mathbf{R}_{1}{ }^{b}$ & 21 & & 7 \\
\hline
\end{tabular}

${ }^{8}$ These experiments share, in part, a common data base.

bexcluding the first $7 \mathrm{~cm}$ of track after collision. 
References

1. A Milone, Nuovo Cimento Supple. 12, 353 (1954).

2. T.F. Cleghorn, P.S. Freier, and C.J. Waddington, Can. J. Phys. Suppl. 46, 572 (1968).

3. B. Judek, Can. J. Phys. 50, 2082 (1972).

4. E.M. Friedlander el al., Phys. Rev. Lett. 45, 1084
(1980).

5. P.L. Jain and G. Das, presented at the Sth High Energy Heavy Ion Sumner Study, LBL-126\$2 (1981).

6. H.B. Barber, P.S. Freier, and C.J. Waddington, contribution HE 3.1-6, 16th Cosmic Ray Conference, Ver+ sailles, France.

7. G.M. Agakishyer et al ${ }_{++}$Dubna preprint PI-81-79.

\title{
Further Investigation of Projectile Fragmentation: the Short Mean Free Path Effect
}

\author{
E.M. Friedlander, R.W. Gimpel, H.H. Heckman, Y.J. Karant, \\ B. Judek* and E. Gaussauget
}

Two independent experiments using nuclear emulsion were conducted to examine the interaction characteristics of relativistic projectile fragments (PF), $3 \leq Z \leq 26 .{ }^{l}$ One experiment, scanned and measured at the NRC, Ottara, used $2.1 \mathrm{~A} \mathrm{GeV} 160$ as the incident beam; the other, at LBL, used 1.88 A GeV ${ }^{56} \mathrm{Fe}$.

The principal interaction characteristic examined in these dala was the inclusive reaction mean free path (MFP), symbolized by " $\lambda^{\text {" }}$ "We have measured the MFPs of primary beams, and fit to these primary results a power law, $\lambda=A Z^{-b}$, where " $\Lambda^{\prime \prime}$ is a parameter which is independent of the charge $Z$. For primary beams, $\Lambda=30.4 \mathrm{~cm}$ and $\mathrm{b}=-0.44$. We have also measured the MFP parameter on PFs as a function of distance after emission (Fig. 1). As is readily observable, the MFP parameler $A$ does vary as a function of distance after emission. The solid line in Fig. 1 is the result of a fit with 0.06 of the PFs baving a constant MFP of $2.5 \mathrm{~cm}$. independent of $Z$ (which is a MFP for a nucleus on the order of $\mathrm{Pb}$ ), and the rest of the PFs have the MFP values

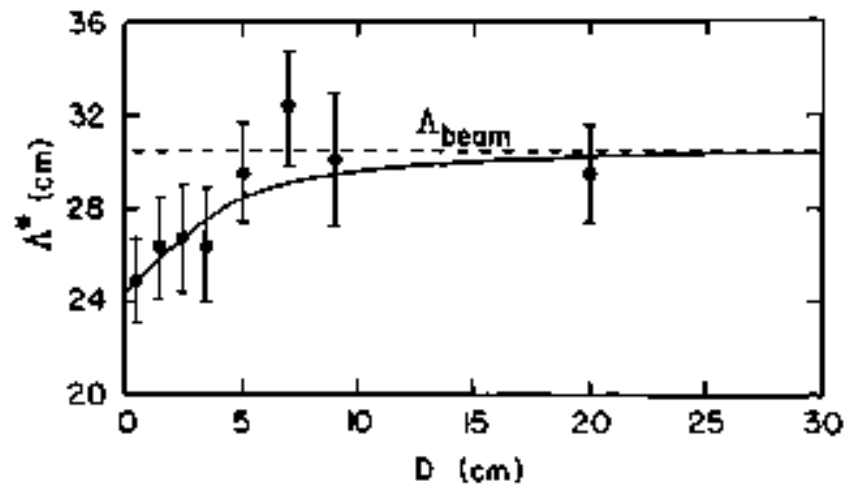

Fig. I. Estimates of $A^{*}$ for the parameter $A$ at different distances D from the origins of PFs: full circles, experiment; dashed line, prediction from $\Lambda$ as fit to primary beams; solid line, fit assuming a 6\% admixure of PFs with a constant $2.5 \mathrm{~cm}$ MFP.

(XBL 808- I1485) obtained from the primary beam fit. This hypothesis of a 6\% adruixture gives a reasonable fit to our observationts. The ratio of any two measured values of the MFP (for particles of the same intrinsic MFP) obeys the Fisher F. variance ratio distribution. The probability of obtaining this ratio or a larger one from a pair of measurements should obey the unjform distribution $\mathrm{U}(0,1)$.

In addition to exarsining the MFP as a function of distance after emission, we also consider the MFP as a function of the generation of the $\mathrm{PF}$; re regard the incident beam nuclei as "primaries," their imunediate PFs as "secondaries," the immediate PFs from secondaries as "tertiaries," etc. Proceeding via the F-test by generation (secondaries versus tertiaries and later generations), we obtain the mean P velue, which is only about 2.1 SD from its expectatjon. However, the largest $P$ value recorded was only 0.778 , and the binomial probability of observing 0 events in the tuniform distribution ost of 29 attenpts with $P \geq 0.778$ is $7\left(10^{-4}\right)$. We would expeet about 6 events in this $\mathbf{P}$ region.

In the above discussion, we have considered total track length $S_{N}$ observed for $N$ interactions, whether or not all particles interacted. We may also limit consideration to those particles which did interact, neglecting those which did not. To investigate further the apparent shortening of the MFP from one generation to the next, we consider the topology in which a secondary interacted and emitted a tertiary that also interacted. Taking into account the expected MFP from the primary beam fit and the distance over which a particle could in fact have been observed to interact within our detectors (the potential path), one may calculare probability values $P_{2}(X)$ and $P_{2}(Y)$ for the secondary and tertiary particles respectively to have interacted within the observed distan $X$, Y, or sborter. These probabilities are independent because of the way in which we have calculated them. Thus, a scatter plot of $P_{2}(X)$ vs $P_{2}(Y)$ should populate uniformly the unil plane. Dividing each axis into two a priori equiprobable bins, we oblain the scaller plot, Fig. 2. We define a quantity $C$ equal 10 the number of counts in quadrant lll minus the number in 


\section{Comment on the Energy Dependence of Target Excitation in RHI Collisions}

Horry H. Heckman

Over the past several years, there have been a variety of radiochemital experiments on the cross sections and recoil properties of target fragmentation products (radionuclides) formed by the reactions of RHI and protons with various target nuclei. Such studies have been found to be particularly sensitive tests of limiting fragmentation as it applies to the asymptotic kinematic limits of the target recoil velocities.

By assuming that the recoil fragments result from a two-step (fast-fow) process, the observations can be inter. preted in terms of the mean forward-directed velocity $\left\langle\beta_{J}\right\rangle$ of the prefragnent produced in the primary projectile-target interaction and the mean Maxwelliandistributed velocity $\langle\phi\rangle$ that arises from the de-excitation of the prefragment to produce the observed residue. Figure I summarizes the dependence of the mean longitudinal recoil velocity of the first step. $\left\langle\beta_{||}\right\rangle$, on the mass number of the detected fragment. These data were $\mathrm{col}$ lected from the works of Loveland and Kaufman ${ }^{1,3}$ and $00-$ workers. 2.4

A most interesting feature of Fig. 1 is that the recoil velocities $\left\langle\beta_{11}\right\rangle$ are inversely proportional to the mass of the fragment, $A_{F}$ partcularly for $A_{F} \leq 100$. This leads

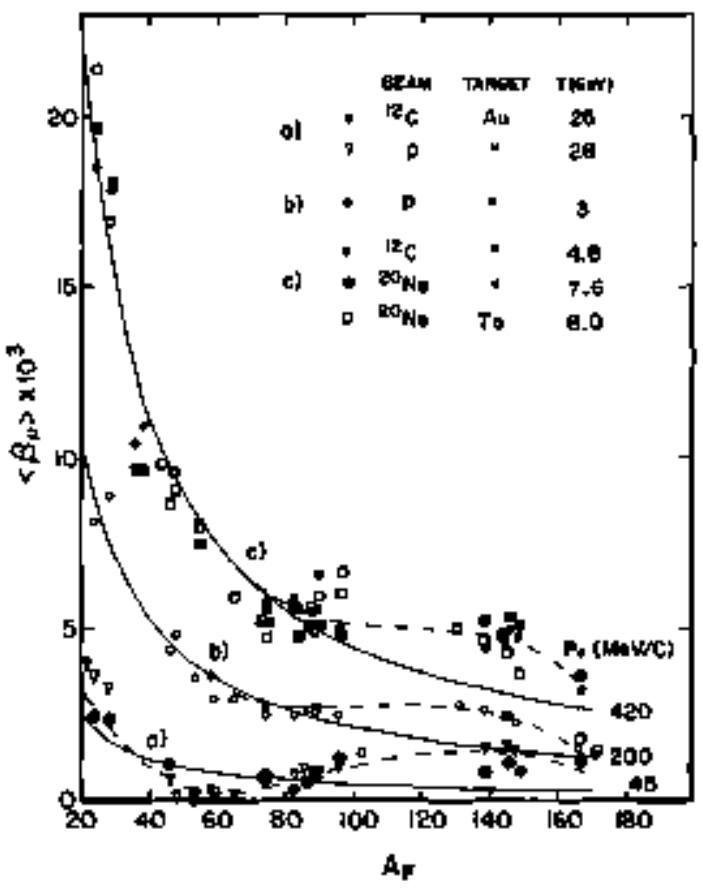

Fig. 1. Longitudinal recoil velocity of fragment of mass number $A_{F}$ as a function of beam and beam energy. Solid curves are $\left\langle\beta_{||}>=P_{0} / 931 A_{F}\right.$ for the values of $P_{0}$ indicated. Dashed lines delineate data points. (XBL 815-9830) to the suggestion that the loci of data points can be expressed rather well in terms of a characteristic mean momentum $P_{0}=97 / A_{F}<\beta_{0}>\mathrm{MeV}$ associated with each plot of $\left\langle\beta_{\mid}>\right.$vs $A_{\text {F }}$. For the data shown, I have evaluatod $P_{p}$ from the unweighted data for $\left\langle\beta_{\mid}\right\rangle$, with $A_{\text {Y }} \leq 100$, and have drawn the resultant curves of the expression $\left\langle\beta_{1}\right\rangle=P_{0} / 971 A_{F}$ where the values of $P_{0}$ corresponding to curves a), b), and c) in Fig. 1 are $420 \pm$ $7 \mathrm{MeV} / \mathrm{c}, 200 \pm 6 \mathrm{MzV} / \mathrm{c}$, and $45 \pm 6 \mathrm{MeV} / \mathrm{c}$. respec1ively. The data are compatible with $A_{F}<\beta_{1}>=$ constant over mast of the range of fragment mass, with the largest deviations occurring at high beam energies (curve c) and, in all cases, for $A_{F} \simeq 140$.

An interesting consequence of this observation comes from considering the Iwo-step kinernatic model proposed by Masuds and Uchiysma, ${ }^{5}$ which describes target fragmenta. tion by the sequence of reactions, i) $\mathrm{B}+\mathrm{T} \rightarrow \mathrm{B}^{*}+\mathrm{T}^{*}$ and ii) $T^{\prime} \rightarrow F+X$, whereby the target (projectile) nucleus $T(B)$ is excited to the state $T^{*}\left(B^{-}\right)$, the decay of which leads to the detected target (projectile) fragment $\mathbf{F}$. Given the approximations that the recoil $<<$ excitation $<<$ nuclear rest energies and that the excitation energies of the target and beam nuclei arc, on the average, equal (considering pionic degrees of freedom, this approximation is applicable to proton beams), the conservation of energy and momentum leads to the following expression for the recoil velocity $\left\langle\beta_{||}\right\rangle$of the target system

$$
\left\langle\beta_{1 \mid}>=\frac{E^{*}}{M_{T}} \sqrt{\frac{\gamma+1}{\gamma-1}},\right.
$$

where $\mathbf{E}^{*}$ is the excitation energy of the larget (projectile), $M_{\mathrm{T}}$ is the mass of the target, and $\gamma$ is the Lorentz factor of the beam nucleus. Given the approximate constancy of the mean momentum $\mathrm{P}_{\mathrm{O}}=\mathrm{M}_{\mathrm{F}}<\beta_{1}>$ of the fragments at beam energy $\mathrm{M}_{\mathrm{B}}(\gamma-\mathrm{l})$ as depicted in Fig. $\mathrm{l}$, the following reaction holds:

$$
\mathrm{M}_{\mathrm{F}} \mathrm{E}^{*}=\mathrm{P}_{0} \mathrm{M}_{\mathrm{T}} \sqrt{\frac{\gamma-1}{\gamma+1}} .
$$

Simple kinematics of the two-step model, incorporating the observation $P_{o}=M_{F}<\beta_{\mid l}>$, thus indicales that the quantity $M_{F} E^{*}$ is a constant for a given beam energy and that $E$ is linearly related to the mean reooil velocity $\angle \beta_{1}>>$ of the fragment $F$, Eq, (1).

In Fig. 2, I have plotted $\mathrm{M}_{F} \mathrm{E}^{*}$ (Eq. (2)) versus bean kinetic energy for a yariety of besn and target nuclei, using the values of $P_{\phi}$ extracted from published $\left\langle\beta_{\|}>\right.$vs A data. 1,23,4 The data are ordered by the kinetic energy of the beam and show that $\mathbf{E}^{*}$ associated with each frag ment mass attains a maximum near $5 \mathrm{GeV}$, decreasing 


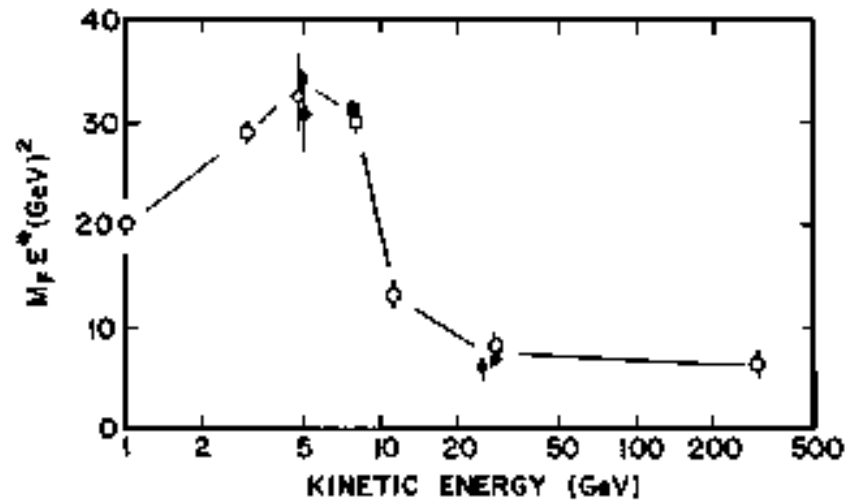

Fig. 2. The product of fragment mass and excitation energy as a function of beam energy and mass. Symbols are: for Aul target, o protons, $12 \mathrm{C},{ }^{20 \mathrm{Ne}}$ for Ta target, $\square^{20} \mathrm{Ne}$, and for $\Psi^{t a r g e t}, \Delta^{12} \mathrm{C}$ and $\nabla^{20} \mathrm{Ne}$. Data are compiled from Refs. 1-4.

(XBL 815-9832)

thereafter with increasing beam energy, approaching an asymptotic value for energies $>25 \mathrm{GeV}$. Within experimental efrors $E^{*}(25 \mathrm{GeV})=\mathbf{E}^{*}(300 \mathrm{GeV})$. Limiting fragonentation is thus satisfoed for the variable $\mathrm{M}_{\mathrm{z}} \mathrm{E}^{*}$ for beam energies $>25 \mathrm{GeV}$. Because $\left\langle\beta_{\mid J}>\infty \mathrm{E}^{2}\right.$ [(Eq. (1)], the recoil velocity $\left\langle\|_{\|}\right\rangle$which is the linematic quantity that controls the forward/backward ratio of fragments, also attains a limiting value at beam energies > $25 \mathrm{GeV}$. Other obvious conclusions from Fig. 2 are: (1) the fragment angoler distributions in the laboratory. because of their dependence on $\left\langle\beta_{1 \mid}\right\rangle$, will exhibit max imum asymmetries in the laboratory at bearn energits $5 \mathrm{GeV}$; (2) limiting fragmentation is clearly not mot for energies $<10 \mathrm{GeV}$; (3) the scaling factor that "universally" interrelates $\left\langle\beta_{\mid}>\right.$and $E^{\prime \prime}$ for beam nuclei $1 \leq A_{B}$ $\leq 20$ for which data are available, is the total kinetic energy of the beam; and (4) the excitation energies, hence $<\beta_{I I}>$, are independent of the projectile mass, i.e., faotorization is valid, as indicated by $\mathrm{Eq}+\mathrm{I}$.

\section{Footmote and References}

'Based on LBL-12656, May $198 \mathrm{I}$.

I. W. Loveland et al., Phys. Rev. C 23, 253 (1981).

2 S.B. Kaufman et al., Phys. Rev. C 22, 1897 (1980).

3. W. Loweland et al., Phys. Rev. C (in press); also LBL I $1658(1980), 38 \mathrm{p}$.

4. S.B. Kaufman, E.P. Steinberg and M.W. Weisfield, Phys. Rer. C 18, 1349 (1978).

5. N. Masuda and F. Lchiyama, Phys. Rev. C 15, 1598 (1977).

6. Derived from relativistic energy conservation with the approximation that neglects all quadratic terus of the recoil momentum and excitation energies. The nonrelativistic expression for the recoil momentum is also used.

\title{
A Measurement of Denteron-Deuteron Elastic Scattering at $5.75 \mathrm{GeV} / \mathrm{c}^{*}$
}

\author{
Edgar T. Whipple, V. Perez-Mendez, A.L. Sagle, R.L. Talaga ${ }^{\dagger}$ F. Zarbakhsh. \\ J.B. Carroll, ${ }^{\ddagger}$ G.J. Igo, J.8. MCClelland, ${ }^{\dagger}$. Bleszynski, ${ }^{\ddagger}$ and $K$ Gatezert
}

The multiple diffraction model of Glanber ${ }^{1}$ successfully explains many of the major features of elastic badror-deuteron scaltering. The model has been extended to systems in which both target and projectile are composite and in which simultaneous multiple collisions can occur in addition to the normal, sequential collisions. ${ }^{2}$ These extra processes introduce more structure into the differential cross section and thus permit new tests of the parameterizations which enter the model. Deuterondeuteron soattering is the simplest system in which such effects can be studied.

This experiment was performed at the LBL Bevatron. A $5.75 \mathrm{GeV} / \mathrm{c}$ deuteron beam was extracted and focused onto a deulerated (about $97 \%$ ) polyethylene target. The three-monentum and time of flight of the forward scattered particle were measured in a magnetic spectrometer. A wire chamber and scintillator determined the position and time of flight of the recoil particle. The elastic signal was identified by selecting events for which the measured vatiables satisfied the four kinematic equations of elastic scattering. A $5.75 \mathrm{GreV} / \mathrm{c}$ proton beam incident on a nor$\mathrm{mgl}$ polyethylene target provided p-p tlastic scattering signal which was used to check the apparatus and analysis procedure.

The results of the experiment are shown in Fig. 1, where only statistical errors are indicated. The t-resolution is always less than the indicated bin width, and the error in the absolute nomalization is estimated to be 10\%. We observe a narrow minimum at $-t=0.18(\mathrm{GeV} / \mathrm{c})^{2}$ and a significant departure from a simple exponential behavigr in the double scattering region. This latter effect has not been mentioned explicily in the literature, although it is implicitly contained in the Glauber model.

At the second maximum $\left[-t \sim 0.3(\mathrm{GeV} / \mathrm{c})^{2}\right]$, the cooss section is dominated by three roughly equal amplitudes, simultaneous and sequential double scattering, and triple scattering. The slope of the sequential double scattering is significantly larger than the almost equal slopes of the other two terms. The phases are such that the 


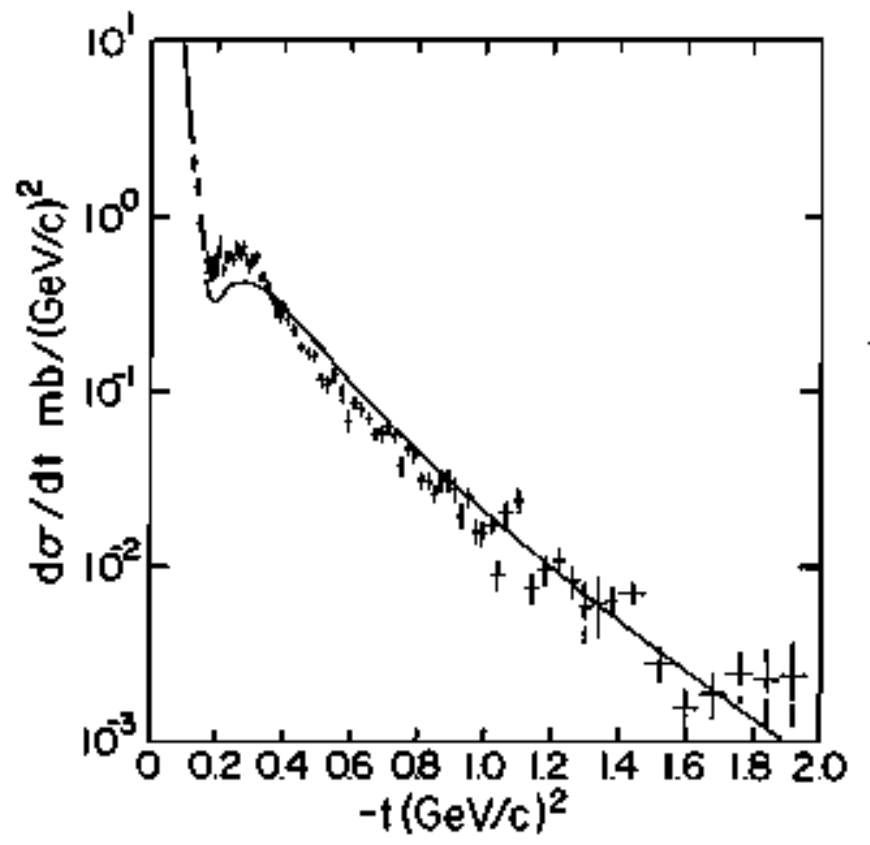

Fig. 1.

(XBL B18-11304) sequential term is approximately paralled to the resultant of the remaining terms. The net effect of the rapidly decreasing sequential amplitude is simply a corresponding rapid decrease in the cross section. When the sequential term becomes smaller than the sum of the other two terms, the cross section assumes the more slowly varying behavior of the simultaneous double and triple amplitudes.

Figure 1 also shows the result of a calculation ${ }^{3}$ in which the d-state of the deuteron was taken into acsount by considering terms in the amplitude proportional both to the square of the quadrupole deformation and to the product of the spit-I operators of the two deuterons.

In conclusion, we have produced the first highstatistics, high-resolution data on deuteron-deuteron scattering in the intermediate energy range. We have observed a well-defined minimum and a hitherto unremarked complex behavior in the double scattering region. We believe that this reflects the oonstructive interference of the simultaneous and sequential double scattering region.

\section{Footnotes and Referentes}

*Condensed from LBL-11966.

'Los Alamos Scientific Laboratory, Los Alamos, NM.

tDepariment of Physios, UCLA

1. R.J. Glauber, in Lectures in Theoretical Phystes, W.E. Brittin et al., eds., Interscience Publishers, Inc., New York (1959), Vol. I, p. 315.

2. V. Franco, Phys. Rev. 175, 1376 (1968).

3. M. Bleszynski and $K$ Ganezer, to be published. 


\title{
2. Pion Production
}

\section{Production of Pions and Light Fragments at Large Angles in High-Energy Nuclear Collisions"}

\author{
S. Nagamiga, M.-C. Lemaire, ${ }^{\dagger} E$. Moeller. ${ }^{\ddagger}$ S. Schmetzer, \\ G. Shapiro, $H$. Steiner, and I. Tomihatof
}

Inclusive cross sections for production of $\pi^{+}, \pi^{-}$, a d. ${ }^{3} \mathrm{H},{ }^{3} \mathrm{He}$, and ${ }^{4} \mathrm{He}$ have been measured at laboratory angles from $10^{\circ}$ to $145^{\circ}$ in nuclear collisions of $\mathrm{Ne}+$ $\mathrm{NaF}, \mathrm{Ne}+\mathrm{Cu}$ and $\mathrm{Ne}+\mathrm{Pb}$ at $400 \mathrm{MeV} / \mathrm{nucl}_{\mathrm{on}} \mathrm{C}+$ $\mathrm{C}, \mathrm{Cl}+\mathrm{Pb}, \mathrm{Ne}+\mathrm{NaF}, \mathrm{Ne}+\mathrm{Cu}, \mathrm{Ne}+\mathrm{Pb}, \mathrm{Ar}+\mathrm{KCl}$ and $\mathrm{Ar}+\mathrm{Pb}$ at $800 \mathrm{MeV} /$ nucleon, and $\mathrm{Ne}+\mathrm{NaF}$ and $\mathrm{Ne}+\mathrm{Pb}$ at $2.1 \mathrm{GeV} /$ nucleon. The production of light fragments in proton-induced collisions at beam energies of $800 \mathrm{MeV}$ and $2.1 \mathrm{GeV}$ has also been measured in order to allow us to compare these processes.

For equal-mass nuclear collisions the total integrated yields of nuclear charges are well explained by a simple participant-spectator model. For $800 \mathrm{MeV} /$ nucleon beams the enesgy spectra of protons at c.m. $90^{\circ}$ are characterized by a "shoulder-arm" type spectrum shape with an exponential fall-off at high entrgies, while those of pions are of a simple exponential type. The inverse of the exponential slope, $\mathbf{E}_{0}$ for protons is systematically larger than that for pions. This $E_{0}$ is larger for heavier-mass projectiles and targets. It also increases monotonically with the beam energy. The angular anisotropy of protons is larger than that of pions. The yield ratio of $\pi^{-}$to total nutelear charge goes up with the beam energy, while the yields of composite fragments decreases. The ratio of low-energy $x^{-}$to $\pi^{+}$as well as that of ${ }^{3} \mathrm{H}$ to ${ }^{3} \mathrm{He}$ are larger than the neutron to proton ratio of the system. The spectrum shape of the composite fragments with mass number $A$ is explained very well by the $A^{\text {th }}$ power of the observed proton spectra. The sizes of the interaction region are evaluated from the observed coalescence coefficients to 3-4 fm. The yield ratio of composite fragments to protons strongly depends on the projectile and target masses and the beam energy, but not on the errission angle of the fragments.

These results have been compared with currently available theoretical models.

\footnotetext{
Footmotes

"Condensed from LBL 12123.

tPresent address: DPhN/ME, CEN de Saclay, $91190 \mathrm{Gif}$ sur-Yvette, France.

tPresent address: Institüt für Theoretische Physik, Freie Universitä́t Berlin, 1000 Berlin 33, West Germany.

SOn leave from Institute for Nuclear Study, Univessity of Tơkyo, Tanasbishi, Tokyo, Japan.
}

\section{Inclusive Single Negative Pion Production at Forward Angles from Collisions of Light Relativistic Nuclei}

\author{
E. Moelier, ${ }^{\dagger}$ W. Brucker, L. Aztderson, S. Nagamiya. S. Nissen-Meyer, ${ }^{\dagger}$ \\ L Schroeder, G. Shapiro, and H. Steiner
}

We have measured single particle inclusive spectra of negative pions produced at angles from $0^{\circ}$ to $12^{\circ}$ (lab) in collisions of 1.05 and $2.1 \mathrm{GeV} / \mathrm{N}$ protons, deuterons, alpha particles, and arbon nuclei with targets of $\mathrm{C}, \mathrm{Cu}, \mathrm{Pb}$, and $\mathrm{H}$ (from a $\mathrm{CH}_{2}$-C subtraction). The detected pions have momenta from $0.25 \mathrm{GeV} / \mathrm{c}$ to $4.25 \mathrm{GeV} / \mathrm{c}$ in the lab frame.

Figure 1 shows the $0^{\circ}$ negative pion spectra for $2.88 \mathrm{GeV} / c / \mathrm{N}$ p. d, $\alpha$ and carbon projectiles on a carbon target. The spectra are seen to fall steeply as a function of utomentum. The proton projectile data show a clear cut-off at the kinematic limit; for pion production from free nucteons, this limit is indicated in Fig. $J$ by an errow. The $d, a$, and $C$ projectiles show considerable production beyond this limit, and it is these fast pions which interest us most. The fact that we sec pions at up to twice the kinetic energy per nucleon of the projectile is a clear indjcation that effects of a nuclear origin are involved. For example, such energetic pions could be produced if the nucleons in the nucleus have sufficiently high monentum or if these are processes involving more than two colliding nucleons. 


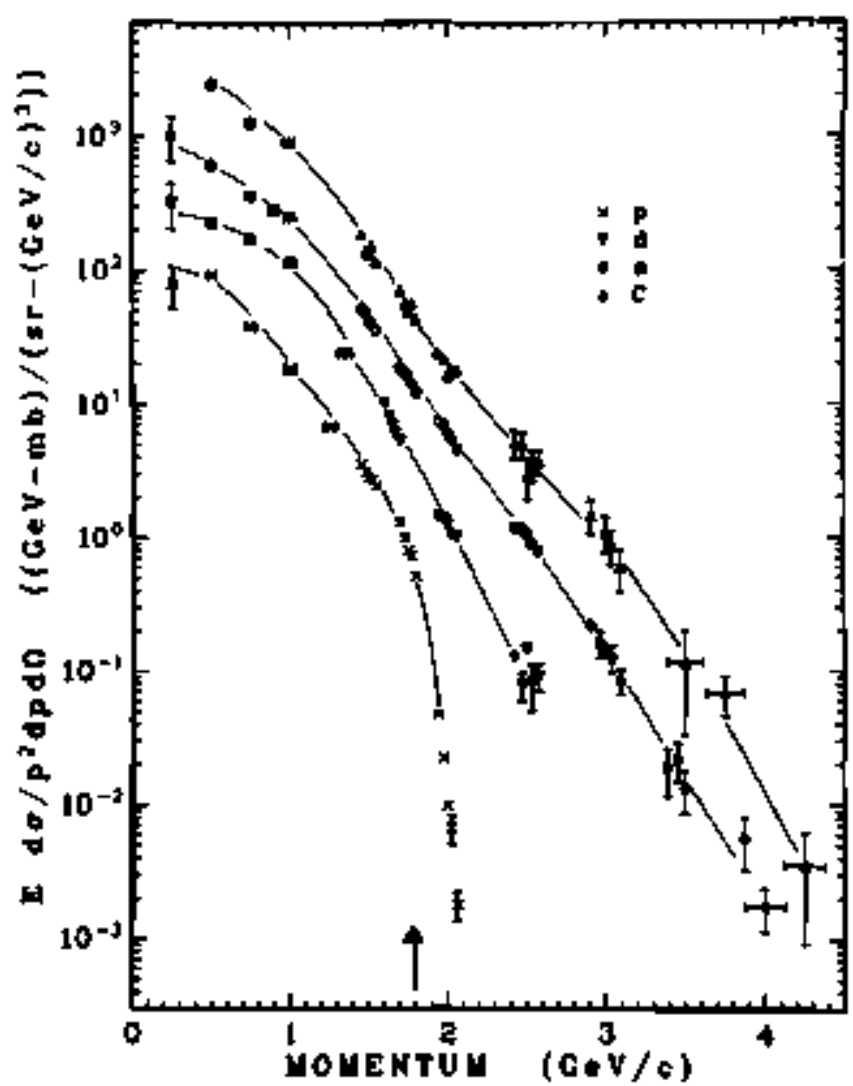

Fig. 1. Lorentz invariant negative pion inclusive cross section vs the (lab) momentum at $0^{\circ}$ for $2.88 \mathrm{GeV} / \mathrm{c} / \mathrm{N}$ protons ( $x$ ), deuterons ( $v$ ), alphas $(O)$, and carbon nuclei $(\Delta)$ interacting with a carbon target. Arrow indicales the kinematical limit for pion production from free nucleons. The curves are drawo to guide the eye.

(XBL 818-I 1 274)

Figure 2 shows the $x^{\prime}$ distribution at $0^{\circ}$ for $2.88 \mathrm{GeV} / c / \mathrm{N} \mathrm{p}_{1} \mathrm{~d}, \alpha$ and $\mathrm{C}$ projectiles on a $\mathrm{C}$ target. Here $\boldsymbol{x}^{\prime}=\mathrm{P}_{\|} / /\left(\mathrm{P}_{\|}\right)_{\max }$ is the Feynmen scaling variable, where $P_{i I}$ is the pion longitudinal momentum in the center of mass and ( $\left.P_{1}\right)$ max is its maximum kinematically allowed value. We see that the fall-off of these distributions with $x^{\prime}$ becomes steeper as the mass of the projectile increases, indicating that complex nuclei tend not to transfer a large fraction of their kinetic energy to individnal pions. The curves in Fig. 2 represent the functional form $\left(1-x^{\prime}\right)^{n}$ with the values $n=3,9,21$, and 69 for $p$, $d, \alpha$ and carbon projectiles, respectively, predicted by the hard scattering model of Schmidt and Blankenbecler.

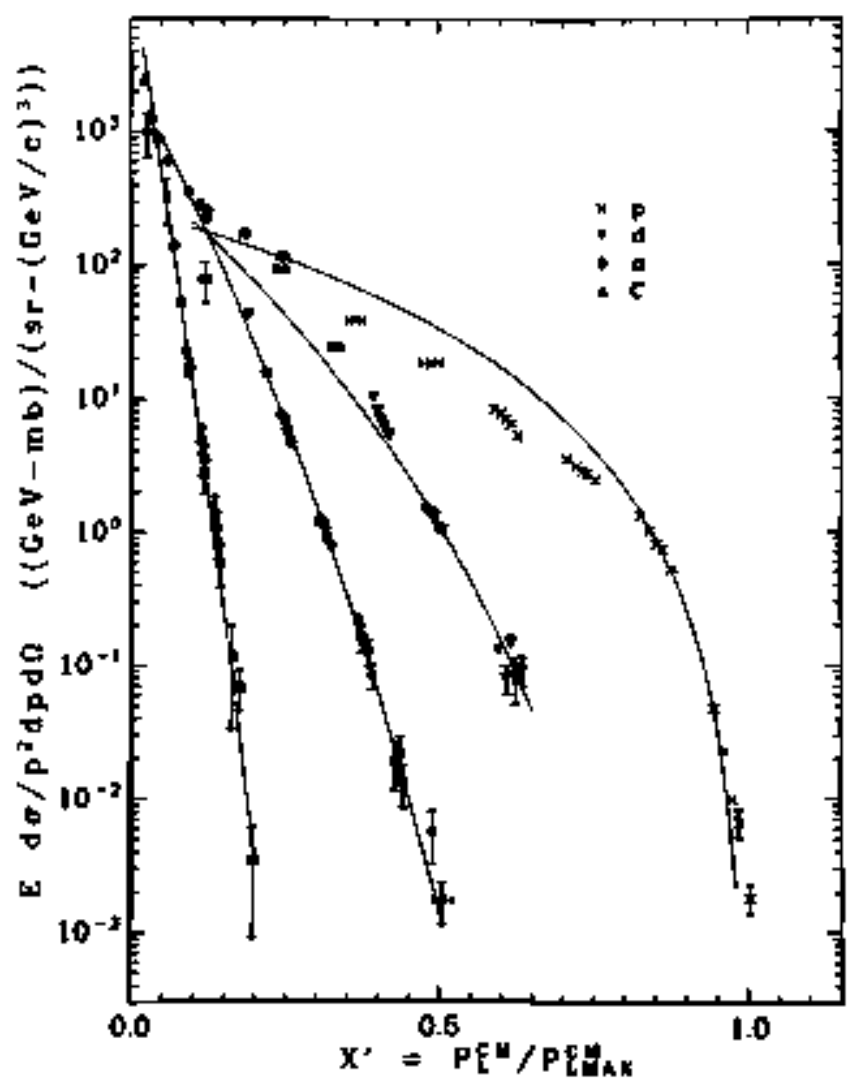

Fig. 2. Comperison of our $2.88 \mathrm{GeV} / \mathrm{c} / \mathrm{N}$ data with the hard scattering model' (see text).

(XBL 818-11275)

This model reproduces the general trend of the data; however, we see deviations from scaling which increase as the mass of the projectile increases.

The dependence of the cross sections on the mass of the target is approximately $A^{4}$ whereas the projectile mass

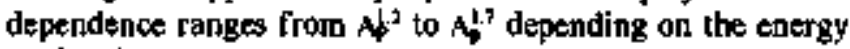
of the pion.

\section{Footnotes and Reference}

"Now at CERN, Geneva, Switzerland,

ton leave from Freie Umiversităt, Berlin, West Germany.

†Now at Simens, Munich, West Germany.

I. 1.A Schmidt and R. Blankenbecler, Phys. Rev. D 15, 3321 (1977). 


\title{
Pion Double Charge Exchange on ${ }^{4} \mathrm{He}$ and Meson Exchange Currents
}

\author{
A Stetz, $L W$. Swenson, ${ }^{*} J$. Davis, ${ }^{\dagger}$ J. Kallne, ${ }^{\dagger}$ R. Mtrehart, ${ }^{\dagger}$ \\ R.R. Whitrey, ${ }^{\dagger}$ V. Perez-Mendez, A. Sagle, J. Carroll ${ }^{\ddagger}$ \\ J. McClelland, and J. Fancert
}

One promising class of reactions for studying meson exchange effects is pion double charge exchange (DCX). Conservation of charge automatically rules out the "irst order" processes that usually mask the weaker exchange efiects. However, there are some DCX transitions between light nuclei and continum states, which are simple enougb for a detailed study of the reaction mechanism as proposed by Germond and Wilkin.'

We have completed an experiment on DCX on ${ }^{3} \mathrm{He}$ and 'He wing the EPICS pion spectroneter at LAMPF, which can be compared directly with the work of Germond and Wilkin as well as with other theoretical reaction mechanisms. 2,3

A substantial number of the low momentum pions decay in the spectrometer. We used two criteria for discriminating between pions and muons, based on trajectory reconstruction and on time of fight.

A typical spectrum is shown in Fig. I. Here we have plotted the differential cross section $\mathrm{d}^{2} \mathrm{~d} / \mathrm{d} \mathrm{d} \mathrm{dT}$ is $\mathrm{T}$ at fixed scattering angle. The energy variable $\mathrm{T}$ is the "missing mass"; this is equivalent to the total kinetic energy of the protons in their own center-of-mass corordinate system. The data in Fig. 1 indude seven pretapping spectrometer settings; to oblain do/d』 we integrated the spectra over $\mathrm{T}$.

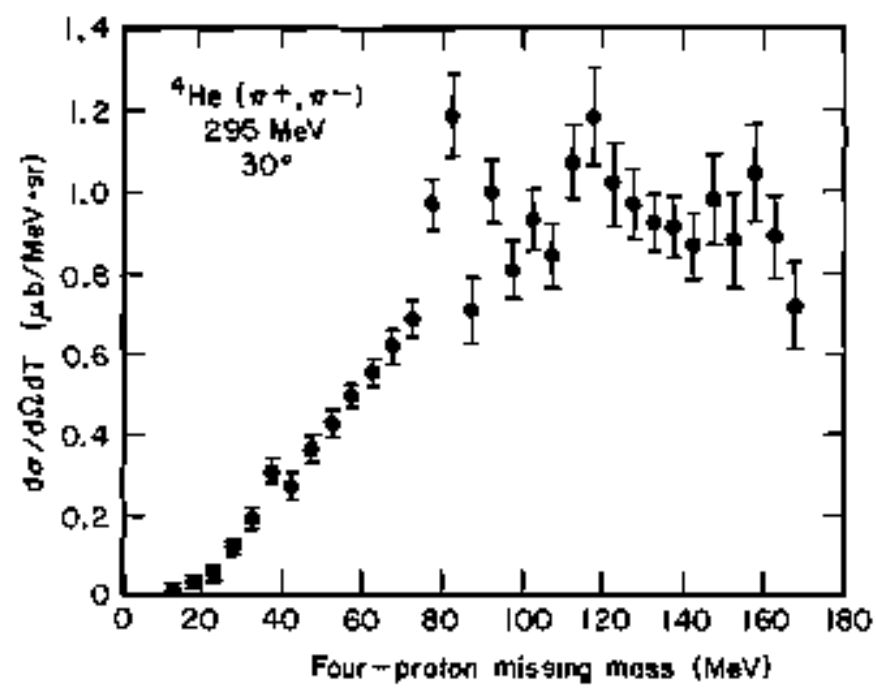

Fig. 1.

(XBL 8111- 1607$)$
In order to obtain the total cross section we fitted the experimentally determined values of $\mathrm{da} / \mathrm{d}$ l with the integrated phase space to oblain or. The fits are shown in Fig. 2. Our cross sections are consistent with the earlier data of Falomkin ${ }^{4}$ and Carayannopoulos. ${ }^{5}$

There are two important mechanisms to consider in calculating double charge exchange al low energies, namely, two sucsessive single charge exchange interactions, and scattering from meson exchange currents. The first reaction mechanism has been discussed by Becker and Schmit ${ }^{2}$ and by Gibbs at al. ${ }^{3}$ these two calculations, while superficially similar, predict total cross sections which differ by three orders of magnitude.

A simple calculation based on pion exchange scattering done by Germond and Wilkin predicts total cross sections that are intermediate between those of Gibbs et al. and Becker and Schmit, and achieves the best overall agreement with experimtents.

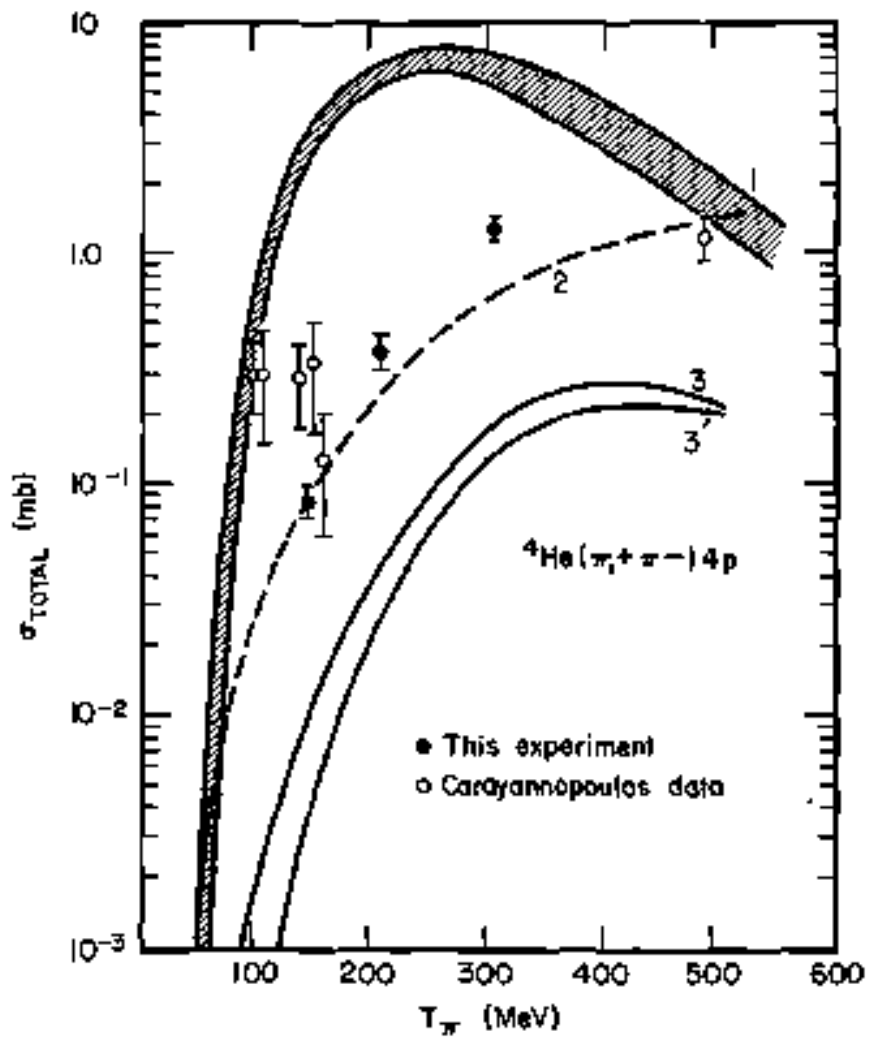

Fig. 2. Experiment $\sigma_{t}$ compared to the theoretical curves of: (1) Becket, Schmidt; (2) Germond, Wilkins; $(3,3)$ Gibbs et al.

(XBL 811 l-1604) 
If these calculations confirm that sequential single charge exchange contributes a relatively small fraction of the observed cross sections, then double charge exchange could become an important probe of mesonic effecss within the nucleos.

\section{Footnotes and References \\ Oregon State University. \\ tUniversity of Virginia.}

"University of California at Los Angeles.

\&University of Oregon.

1. J.F. Germond and C. Wi]kin, Lett. Nwovo Cimento 13, 605 (1975).

2. F. Becker and C. Schmit, Nucl. Phys. B 18, 607 (1970).

3. W.R Gibbs ot al., Phys. Rey. C 15, 1384 (1977).

4. I.V. Falomikin et al., Nuovo Cimento A 22, 333 (1974).

5. N. Carayannopoulos et al., Phys. Rev, Lell. 20, 121S (1968).

\title{
Strong Coulomb Effects on Pions Produced in Heary Ion Collisions"
}

\author{
J.P. Sullivan, J.A. Bistitlich, H.R Bawman, R Bossingham, T. Buttke, \\ K.M. Crowe, K.A. Frankel, C.J. Martoff ${ }^{\dagger}$ J. Miller, ${ }^{\ddagger}$ D.L Murphy. \\ J.O. Rasmussen, W.A. Zaje. O Hashimoto \$ M. Koike, J. Péter \\ W. Benenson," G.M. Crowley," E. Kashy," and J.A. Nolen, J.'
}

Doubly differential cross sections for the production of $x^{+}$and $\pi^{-}$near the velocity of the incident beam for pion lab angles fromt $0^{\circ}$ is $20^{\circ}$ were measured. Beams of ${ }^{20} \mathrm{Ne}$ with $\mathrm{E} / \mathrm{A}=280,380$, and $480 \mathrm{MeV}$ and ${ }^{40} \mathrm{Ar}$ with $\mathrm{E} / \mathrm{A}=535 \mathrm{MeV}$ incident on $\mathrm{C}, \mathrm{NaF}, \mathrm{KCl}, \mathrm{O}$ and $\mathrm{U}$ target were used. A sharp peak in the $\pi^{-}$spectrum and a depression in the $\pi^{+}$spectrum is observed at $0^{\circ}$ near the incident projectile velocity. The effect is explained in terms of Coulomb interactions between pions and fragments of the incident beam. Least squares fits to the data using the Coulomb exrrection formulas of Gyulassy and Kauffmann' and an effective projectile fragment charge have been made.

A sample of the data and the fits is shown in Fig. I which shows the Lorentz invariant cross section cuts for $\mathrm{Ne}+\mathrm{NaF} \rightarrow \pi^{ \pm}$at $\mathrm{E} / \mathrm{A}=380 \mathrm{MeV}$. The left side of the graph shows the cross section vs momentum at $0^{\circ}$ in the lab for $\boldsymbol{r}^{-}$(top) and $\boldsymbol{x}^{+}$(bottom). The right side shows the cross section vs lab angle at a fixed lab momentum near the peak in the $\pi^{-}$spectrum The solid line is from a least squares ft of a function based on the Coulomb correction equations of Gyulassy and Kaufimann. . This solid line has the experimental resolution folded into it. The dashed line is the same Junction before folding with the resolution. The dotted line shows the cross section prodicted by the uncharged pion sourse function to which the Coulormb corrections were applied. The arrows on the left-hand graphs mark the velocity of the incident bearn. The peak in the $\pi^{-}$spectrum and the hole in the $\pi^{+}$speecrum at a pion veiocity slightly below the beam velocity are clearly seen.

For light target-projectile combinations, these Coulomb effects can be explained quantitatively in terms of Coulonb interactions between the pions and cold projectile fragments using Gyulassy and Kauftmann's Coulomb comrection formulas. Our treatment of the Coulomb effects differs Irom that of Gyulassy and Kaufimann in that a different expression bas been used for the uncharged pion sour $\infty$ function. We also reinterpret their formulas for thermal averaging in terms of an average over the velocity dispersion of the projectile fragments. The shift of the peak in the $\pi^{-}$spectra from the incident beam velocity and the approximate width of these peaks are consistent with previously measured projectile fragmentauion data. ${ }^{2-4}$ We have elso sten that the effective cherge of the projectile fragment is less for $\pi^{+}$near bearn velocity than for $\pi^{-}$. This difference is consistent with our expectation that pasitive pions near beam velocity tend to come from more central collisions. Using the same methods, qualjtative agreement is achieved for heavier targets and projectiles. The differences between our filling function and the data are due, at least in part, to an incomplete trealment of impacl parameter averaging.

\section{Foothotes and Referencest}

"Condensed from LBL-1197I, submitted to Phys. Rev. C.

†Boston University, Boston, MA 02215.

†Physik-Institüt der Uniyersităt Zürich, Schōnberggasse 9, 8001, Zürich, Switzerland.

Institute for Nuclear Siudy, University of Tokyo, Tanashi, Tokyo 188, Japan; and LBL,

Institut de Physique Nucleaire, B.P. no. I, 9l406, Orsay, CEDEX, France.

TMichigan State University, East Lansing, M] 48824.

I. M. Gyulassy and \$.K Kaufimann, Nucl. Phys. A 362, 503 (1981).

2. K. Van Bibber et al., Phys. Rev. Lett. 43, 840 (1979); and references therein.

3. D.E Greiner et al., Phys. Rev. Lett. 35, 152 (1975)

4. Y.P. Viyogi et al., Phys. Rev. Lett 42, 33 (1979). 


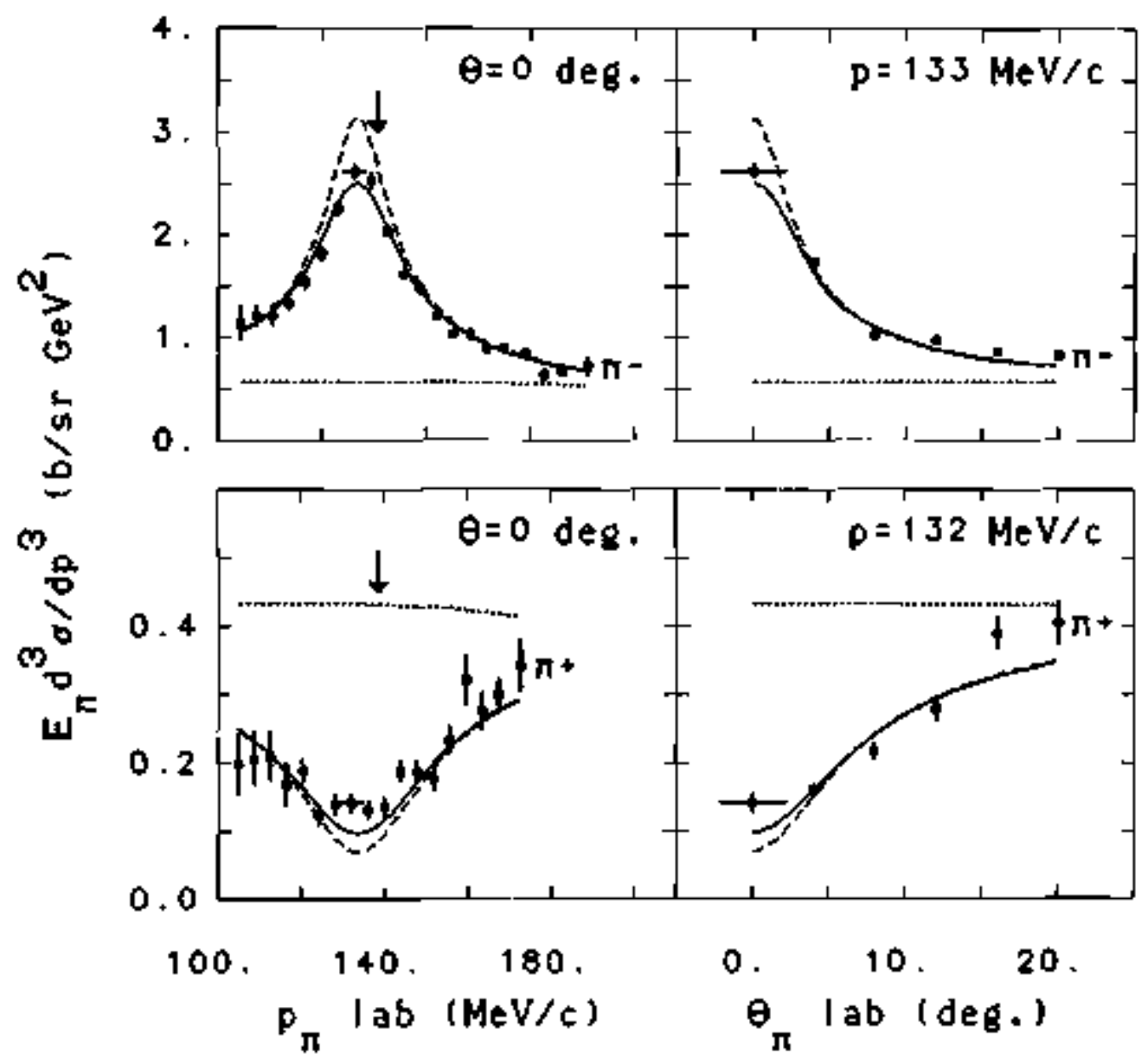

Fig. i. Differential eross seotion ws pion momentum (left side) and vs pion angle (right side) for $\mathrm{Ne}+\mathrm{NaF} \rightarrow \pi^{-}$(10p) and $\pi^{+}$(botlom) at $\mathrm{E} / \mathrm{A}=380 \mathrm{MeV}$. See text for details.

(XBL B16-10183)

\title{
Pions Produced near the Center-of-Mass Velocity in Heary Ion Collisions"
}

\author{
K.A. Franket, J.A. Bistirilch, R. Bassingham, H.R Bowman, X.M. Crowe, C.J. Martoff \\ D.L. Murphy, J.O. Rasmussen, J.P. Sullivan, W.A Zsjc. J.P. Mller, \\ o. Hashimoto, M. Koike, J. Peter, W Wenentson, \\ G.M. Crawley,' E. Kashy,' J.A. Nolen, Jr.' and J. Quebert
}

In a recent experiment by Wolf et al.' a difference between pion production by nucleus-nucleus collisions and production by nucleon-nucleon collisions was noted. The contour plots for the cross section of $\mathrm{E} / \mathrm{A}=1.05 \mathrm{GeV}$ ${ }^{* 0} \mathrm{Ar}$ on ${ }^{40 \mathrm{Ca}}$ sbowed a tidge near $\mathrm{P}_{1}=0.5 \mathrm{~m}_{\mathrm{r}} \mathrm{c}$ and $P_{\text {if }}=0$. Theories by Libbrechl and Koonin, ${ }^{2}$ Gyulassy and Kauftmann, ${ }^{3}$ and Cugnon and Konin ${ }^{4}$ claim that pions ntar $90^{\circ} \mathrm{c} . \mathrm{m}$. would fecl significant Coulomb effects of both target and projectile and particularly of the hot nuclear matter which has been postulated to exist in the
$P_{11}=0$ frame ${ }^{5}$ The present experiment was undertaken to observe the $\pi^{-} / \pi^{+}$ratio $R$ in the center-of-mass region extending from $\mathrm{P}_{\perp}=0$ through the ridge observed by. Wolf et ol. ' A magnetic spectrometer with a $30^{\circ}$ anguler range was set at $15^{4}$ with respect to the beam. The target was $2.0 \mathrm{~g} / \mathrm{cm}^{2}$ of $\mathrm{Ca}$, giving an overall energy resolution of about 2 AeV. A comparison in the right half of Fig. I with the data of Wolf el al. for $\pi^{+}$at $30^{\circ}$ gives good agrement, except for the cases in which the present data were reflected about $\mathrm{P}_{\mathrm{fl}}^{\mathrm{m}}=0$. The data points given by 


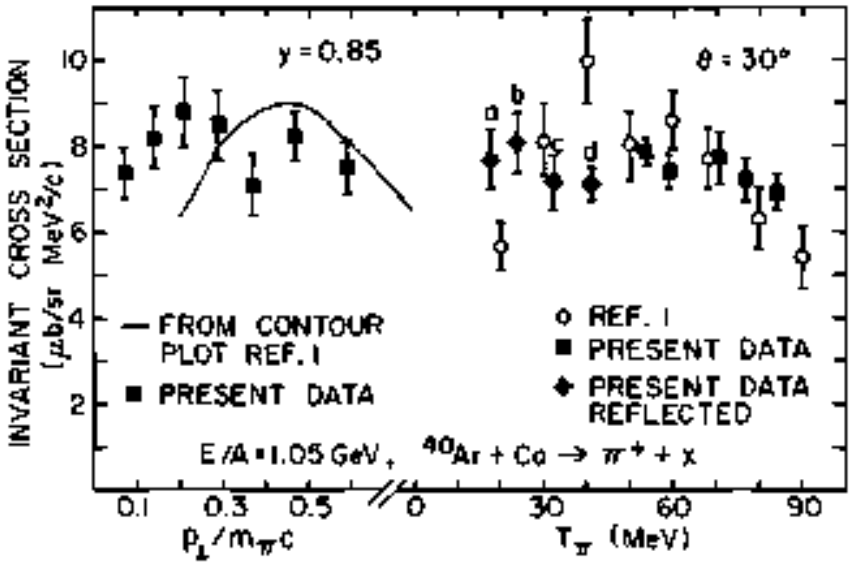

Fig. 1. Comparison of the Wolf et al. data with the present results. In the right side of the Gigure, the diamonds are reflections of present data about the $y_{c m}=0$ axis. In the left side of the figure, our data for $y=0.85$ are compared to such a cul through the contour plot of Wolf et al.

(XBL 815-9920)

closed diamonds in Fig. I are calculated under the assumption of symurnetry of the invariant cross section about the $\mathrm{P}_{\| 1}^{\mathrm{at}}=0$ axis and were taken at the angles and energies in the caption. In the left half of $\mathbf{F}_{i \mathrm{~g}}$. 1 we compare our direct data at $y=0.85$ with a cut through their contour plot at the same rapidity. Our data do net show the rise given in their contour plot from $P_{\perp}=0.2$ to 0.6 $m_{x} c$ at $y=0.85$. This comparison is made on the left half of Fig. I. In this case the plated points are taken directly fron the present date but the curve is from their contour plot, which includes reflections of data about $P^{\mathrm{cm}}=0$. Since $40_{\mathrm{Ar}}+{ }^{40} \mathrm{Ca}$ is only approximately a syumetric system, eaution is in order regarding reflection of data points through the center of mass.

The $\pi^{-} / \pi^{+}$ratio for Ar $+\mathrm{Ca}$ is $1.5 \pm 0.2$ for data taken nearly al rest in the center of mass. Calculations by Cugnon and Koonin ${ }^{4}$ predict the ratio $R$ to be about 5.5 for
Ar $+\mathrm{Ca}$ at this point. On the other hand, if they assume complete iransparency, Libbrecht and Koopin obtain a $\pi^{-} / \pi^{+}$ratio at $c . m$. of $R=1.7$, very close to our experimental results.

Calculations wilh Gyulassy-Kaufimann formulas given $\mathbf{R} \simeq \mathbf{1 . 6}$ for the complete transparency case, ${ }^{6} \mathbf{R}=2.7$ using an impact parameter averaged freball model, and a high degree of transparency at $1 \mathrm{GeV}$ per nucleon. We believe the apparent agreement with the complete transparency limit is fortuitous. Our preliminary calculations show that the Coulomb effects on $x^{-} / \pi^{+}$ratios near the center of mass are small if one does not treat pions as propagating through nuclear matter only under the influence of the Coulomb torces and neglecting charge exchange with nuclear matter. We note that there is a neutron excess in ${ }^{40} \mathrm{Ar}$, so simple counting on $\mathrm{nn}$ to np to pp collision ratios and decay modes of the $\Delta$ intermediate leads to $R=1.15$. Stöcker points out ${ }^{7}$ that thermodynanic considerations in thot nuclear matter allowing for condensation into dusters, such as alpha particles, leads to a primitive $\pi^{-} / \pi^{+}$ratio (1.35) for the ${ }^{40} \mathrm{Ar}$ plus ${ }^{40} \mathrm{Ce}$ sysIem.

Footnotes and References

Partially condensed from LBL-12585.

tDepartment of Physies, Boston University, Boston, MA 02215 .

FInstitute for Nuclear Science, Tokyo, Japan; and LBL.

\$nstitut de Physique Nucléajre, Orsay, France.

'Michigan State University, East Lansing, MI 48824.

Universite de Bordeaux, Le Haut-Vigneau, 33170 Gradignan, France.

1. K.L. Wolf et al., Phys. Rev. Lett. 42, 1448 (1979).

2. KG. Libbrecht and S.E Koonin, Phys. Rev. Lett. 43, 1581 (1979).

3. M. Gyulassy and S.K. Kaufimann, Nucl. Phys. A 362. $503(1981)$,

4. J. Cugnon and S.E. Koonin, Nucl. Phys. A 355, 477 (1981)

5. G.D. Weslfall et al., Phys. Rev. Lett. 37, 1202 (1976).

6. M. Gyulassy, private communication (1981).

7. H. Stöcker, private communication (1981). 


\title{
$\pi^{-}$Temperatures, Sphericity and Thrust for Central Collisions
}

\author{
R. Brockman, "J.W. Harris," A. Sandoval," H. Strobele," R. Stock," M. Maler." \\ R.E. Renfordt, J. Miler, H.G. Pught ${ }^{\ddagger}$ M. Raff ${ }^{\ddagger}$ L.S. Schroeder, F. Riess, \\ K. Wolf' A. Dacal, and M.E. Oriz'
}

\section{Temperature}

Inclusive negative pion spectra from Streamer Chamber data of central collisions of ${ }^{40} \mathrm{Ar}+\mathrm{Ka}$ at $1.8 \mathrm{GeV} / u$ were transformed into the $\mathrm{cm}$. frame of the colliding nuclei. The energy spectra

$$
\frac{\mathrm{I}}{\mathrm{P}_{\mathrm{sn}}} \frac{\mathrm{d} \sigma\left(\mathrm{E}_{\mathrm{c}, \mathrm{m}}, \theta \pm \Delta \theta\right)}{\mathrm{dE}_{\mathrm{c} \text { m }} \mathrm{d}_{\theta}}
$$

were fitied to an exponential distribution $e^{-E / E}$ for every $10^{\circ} \theta_{c m}$ - interval.

In Fig. 1 the obtained slope parameter $\mathrm{E}_{0}$ is plotted as a function of the pion c.m. angle $\theta_{\text {c.m. }}$. One observes a drop in $\mathrm{E}_{0}$ of $20 \mathrm{McV}$ between $20^{\circ}$ and $90^{\circ}$, reflocting a nonthermal distribution. The forward-backward asymmeiry of the slope parameter is mainly due to delector inefficiencies for low energy $\pi$ in the backward direction in the lab frame.

The data are compared with recent calculations of a statistical model. 1 The slope parameter $E_{0}$ of these calcuJatjons is roughly 35\% larger than the data; that means the model gives a relatively larger pion yield at high energies but reproduces the angular dependence of the slope parameter.

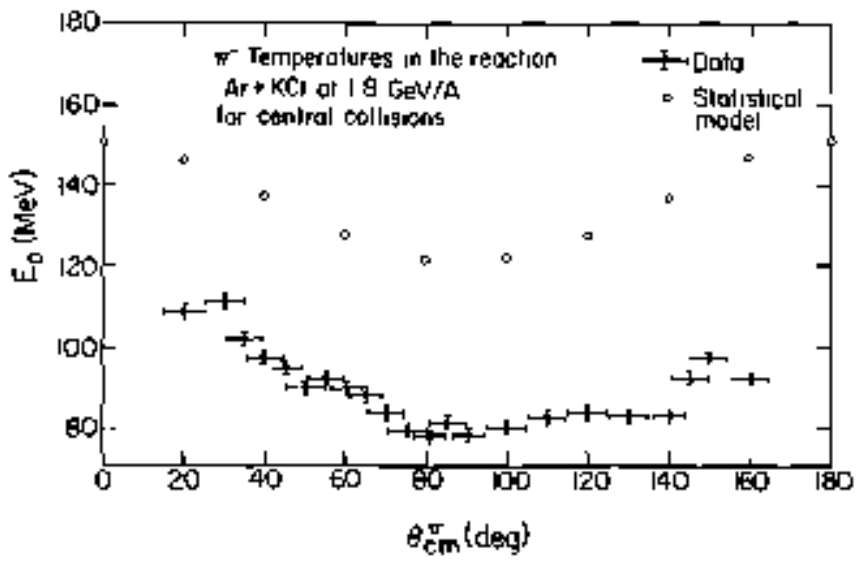

Fig. 1. Slope parameter $E_{a}$ (see texi) as a function of the etrission angle $\theta$ of the negative pions in the NN-c.m.system in the reaction ${ }^{\phi 0} \mathrm{Ar}+\mathrm{KCl} \rightarrow \mathrm{nr} \mathbf{r}^{-}+\mathrm{X}$ for central collisions at $1.8 \mathrm{GeV} / \mathrm{u}$.

(XBL 818-]|SI)

\section{Sphericity and Thrust}

To investigate the question whether the spatial momentum distribution of the nogative pions is spherical or exhibits, for example, a jet-like structure, the variables sphericity and thrust ${ }^{2}$ were calculated in the c.m. frame of the colliding nuclei, in an event-by-event analysis. Figure 2(a) shows the sphericity distribution, and Fig. 3 the frequency distribution of the polar angle of the moin sphericity axis (Q3-axis) relative to the beam axis. A clear forward-backward perak is seen in this distribution.

The sphericity distribution (Fig. 2(a)) was compared with a Monte Carlo calculation. The events in the Monte Carlo calculations were gentrated by randomly selecting a

Ar $+K C l-n \pi^{-}+X$ Centrol collisions $n \geq 6$

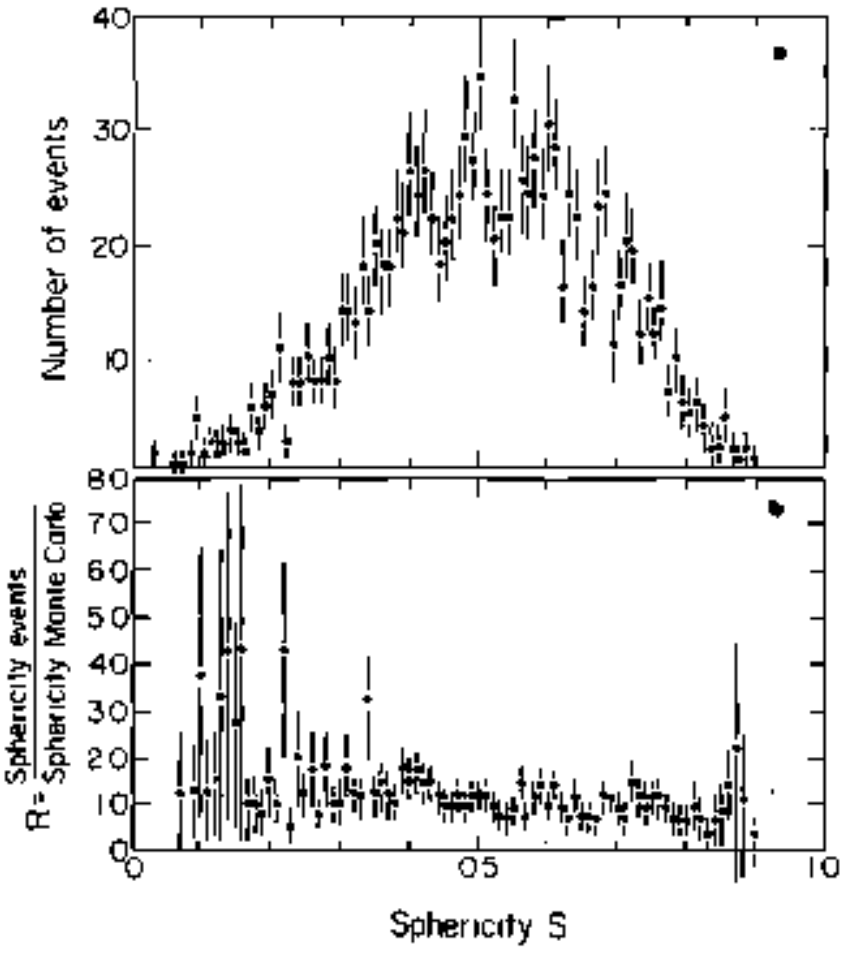

Fig. 2. (a) Frequency distribution of the sphericity $S$ in the regetion ${ }^{40} \mathrm{Ar}+\mathrm{KCl} \rightarrow \mathrm{nx}^{-2}+\mathrm{X}$ for central collisions with $n_{\pi} \geq 6$ at $1.8 \mathrm{GeV} / u$. (b) Ratio $R=$ sphericity of the events/sphericity of a Monte Carlo calculation as a function of sphericity.
(XBL 818-1152) 


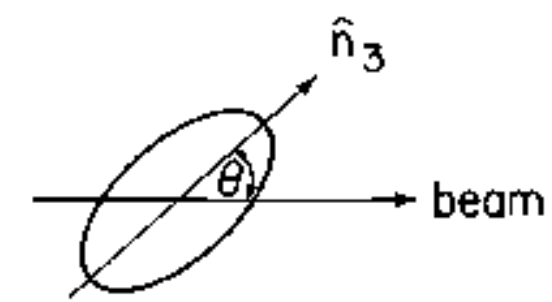

Sphericity orientation

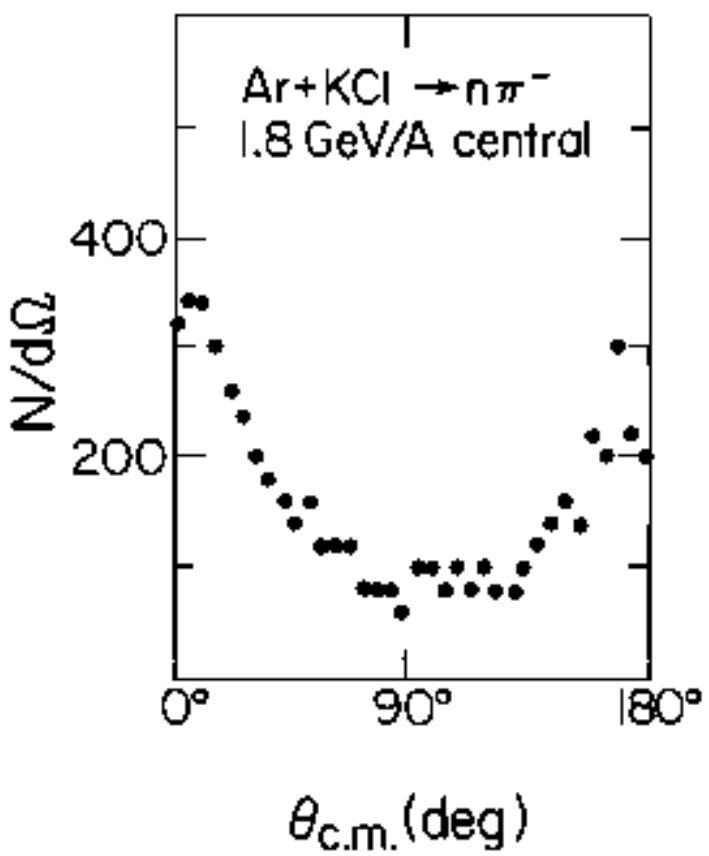

Fig. 3. Frequency distribution of the polar angle $\theta$ of the sphericity axis (Q3 axis) per solid angle interval.

(XBL 818-1153) new direction for the pion momesta within a sniform distribution in the unit sphere. The multiplicity distribulion and the distribution of the pion c.m.-momenta remained the same as in the real events. The streamer chamber efficiencies were taken into account. In the case of spherical events one would expect a mean sphericity $\langle\mathrm{S}\rangle$ close to I, but the Monte Carlo calculation gives a value comparable with experimental data, $\quad \delta S>M C=0.53$, $\langle S\rangle_{\exp }=0.51$. The small $\langle S\rangle_{M C}$ value is due to a finite particle number effect being the $\pi^{-}$multiplicity of $<n_{7}>38$ is not high enowigh to define the shape. Figure 2(b) shows the ratio $R=$ sphericity of the events/sphericity of a Monte Carlo calculation. No clear signal is seen above the statistically distributed events. The same kind of analysis has been cacried out for the variable thrust; again the results are governed by finite particle number effects.

The event shape analysis will be more reasonable when all charged particles are included.

\section{Footnotes and References}

"GSI, Darmstadt, West Germany.

†Universitäl Marburg, West Gentrany.

$\$$ LBL, Berkeley.

Universitäl München, West Germany,

'Argonne National Laboratory.

IIFUNAM, Mexico.

I. S. Bohrmann and J. Knoll, Nucl. Phys. A 356, 498 (1981).

2. S. Brandi and H.D. Dahmen, Z. Phys. C 1, 61 (1979); L Wu and J. Zobernig, Z. Phys. C 2, 107 (1979).

\title{
Charged Particle Exclusive Analysis
}

\author{
R. Brackmann, J.W. Harris, ${ }^{*}$ A Sandowal, H. Strabele, $R$. Stock," M. Maier, \\ R.E. Renfordi, ${ }^{\dagger}$ J. Miller. ${ }^{\ddagger}$ H.G. Pugh ${ }^{\ddagger}$ M. Roff ${ }^{\ddagger}$ L.S. Schroeder, ${ }^{\ddagger}$ F. Riess, \\ X. Wolf, A Dacal, ${ }^{\dagger}$ and M.E. Ortiz
}

The full event analysis of streamer chamber pictures has been started at the Institute for High Energy Physics in Heidelberg using a Frankenstein digitizer and the TVGP reconstruction progiam High multiplícily events have been selected for this first analysis.

Figure I shows the particle trajectories as measured in one of the three views of a $1.8 \mathrm{GeV} / \mathrm{o}{ }^{40} \mathrm{Ar}+\mathrm{KCl}$ interaction. By matching the tracks in the three views, one can reconstruct the trajectory in space and from this its rigidity and emission angle. With mass and charge identification (see below) one obtains the momentum $\vec{p}$ of each particle in the center-of-mass sysiem Figure 2 shows the event of Fig. 1 in the center-of-mass momentum space. Shown here are the momentum per nucleon vectors.
A total of 20 events of the reaction $\mathrm{Ar}+\mathrm{KCl}$ at $1.8 \mathrm{GeV} / \mathrm{u}$ and 38 events of the reaction $\mathrm{Ar}$ on $\mathrm{Pb}$ at $0.8 \mathrm{GeV} / \mathrm{u}$ have been measured and analyzed. The nega. live tracks have been identified as $\pi^{-}$. For the positive tuacks a rough particle identification was performed on a kinematic basis, since identification from track density has not yet been implemented. Protons, deuterons, and tritons have been assigned by making euts in the lab and c.m. momenta corresponding to the maximum momentum a particle with a given mass would have in an elastic scattering taking $200 \mathrm{MeV} / \mathrm{c}$ Fermi motion into account. All particles beyond this linit are tested with the next higher mass assignment.

The positive pions could be partially identified by 


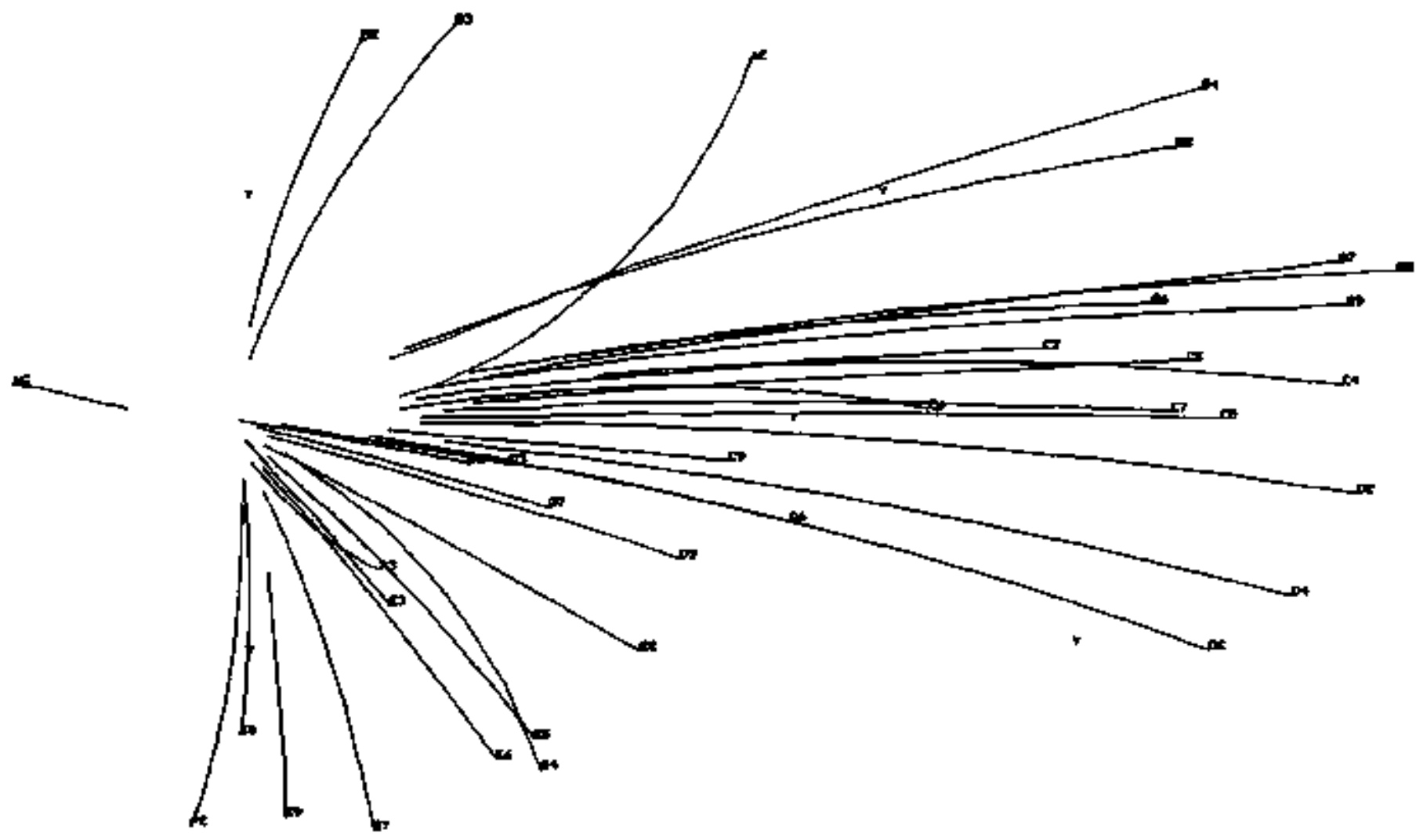

6052702.

Fig. 1. Projection of particle trajeclories produced in the sentral interaction of ${ }^{4} \mathrm{Ar}+\mathrm{KCl}$ at $1.8 \mathrm{GeV} / \mathrm{u}$ as measured from a streamer chamber picture.

(XBL 816-10164)

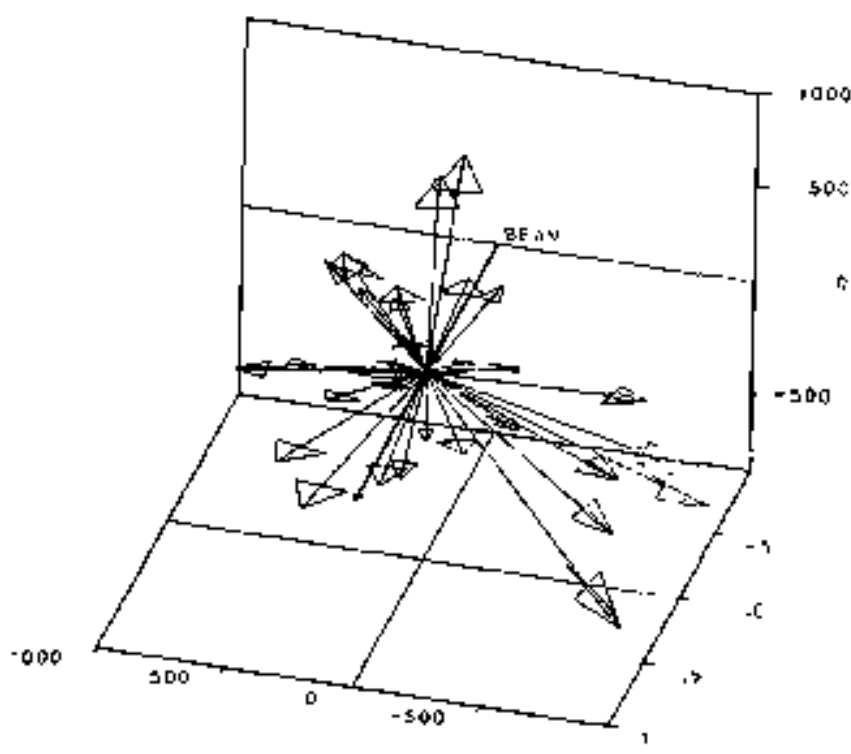

Fig. 2. Center-of-mass momenlum vectors corresponding to the same interaction as shown in Fig. I, In this event 35 charged particles were observed.
(XBL 8IS-11281) comparing the phase space of the $\pi^{-}$in the lab with that of the c.m. system and selecting the regions where they dominate over the protons. This provides a zero order characterization of the charged particle final state in central collisions. The mean multiplicities so extracted are shown in Table 1 together with their estimated uncertainties.

Mean longitudinal and transverse momenta per mucleon per inieraction have been extracted. Figure 3 shows a scatler plot of the $\left\langle p_{\perp}\right\rangle$ us $\Rightarrow p_{\mid} \mid>$per event for both reactions.

\section{Foolnotes}

"GSI, Darmstadt, West Germany.

tUniversität Marburg, West Germany.

*LBL, Berkeley.

SUniversität München, West Germany.

Avgonne National Laboratory.

IIfUNAM, Mexico. 
Table I. Charged particle exclusjve event characlerization of the central oollisions of ${ }^{40} \mathrm{Ar}+\mathrm{KCl}$ at $1.8 \mathrm{GeV} / \mathrm{u}$ and ${ }^{40} \mathrm{Ar}+\mathrm{Pb}$ at $800 \mathrm{MeV} / \mathrm{u}$.

\begin{tabular}{lcccccc}
\hline System & $\left\langle\mathrm{p}_{\text {Charge }}\right\rangle$ & $\left\langle\mathrm{n}_{\pi}\right\rangle$ & $\left\langle\mathrm{n}_{\pi}+\right\rangle$ & $\left\langle\mathrm{n}_{\mathrm{p}}\right\rangle$ & $\left\langle\mathrm{n}_{\mathrm{d}}\right\rangle$ & $\left\langle\mathrm{n}_{\mathrm{t}}\right\rangle$ \\
\hline $\mathrm{AI}+\mathrm{KC}$ & $41 \pm 2$ & $5.9 \pm 0.5$ & $5 \pm 2$ & $24 \pm 4$ & $5 \pm 3$ & $1 \pm 1$ \\
$\mathrm{Ar}+\mathrm{Pb}$ & $45 \pm 2$ & $2.6 \pm 0.3$ & $2.5 \pm 1.5$ & $34 \pm 4$ & $5 \pm 3$ & $1 \pm 1$ \\
\hline
\end{tabular}

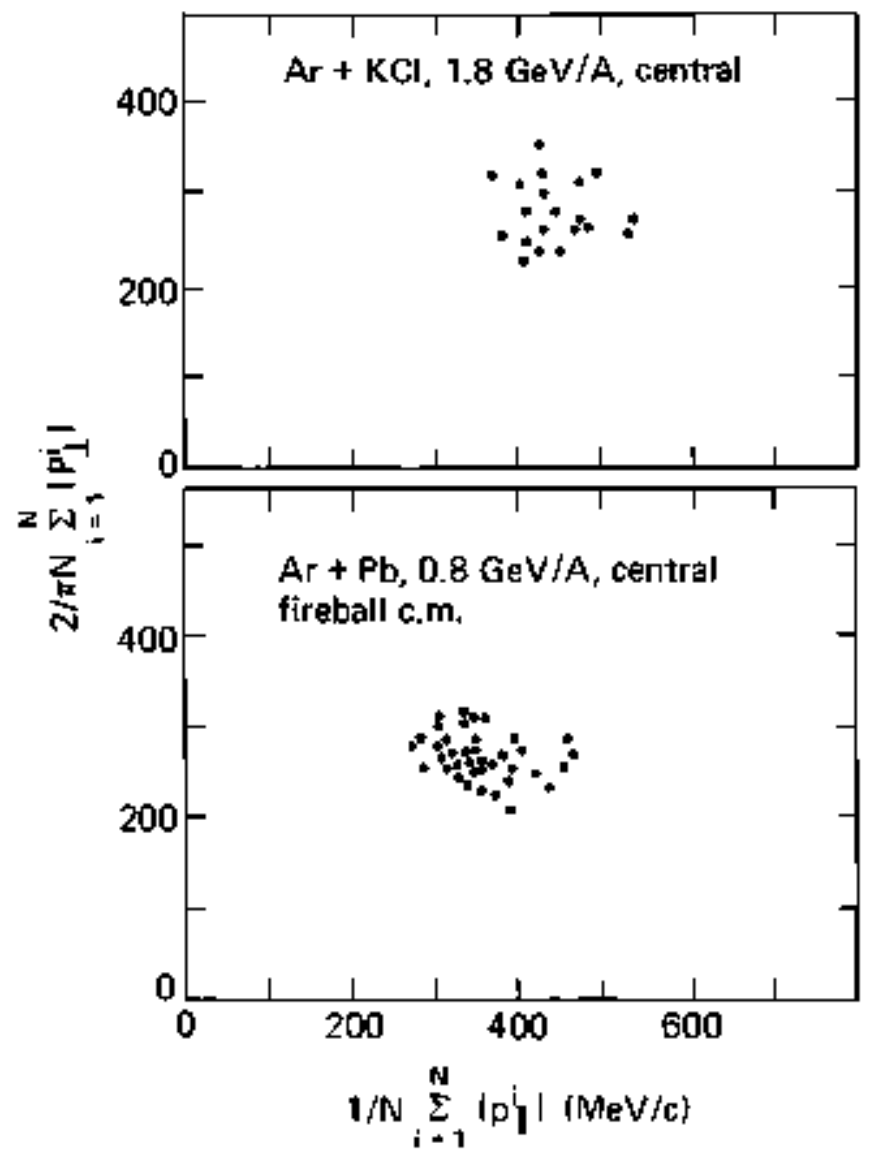

Fig. 3. Scatter plots of the $\left\langle p_{1}>\right.$ vs $\left.\Leftrightarrow 1 p_{1}\right|>$ for the certral collisions of ${ }^{40} \mathrm{Ar}+\mathrm{KCl}$ at $1.8 \mathrm{GeV} / \mathrm{u}$ and ${ }^{40} \mathrm{~A}+\mathrm{Pb}$ at $800 \mathrm{MeV} / \mathrm{u}$, The ordinate has been multiphied by $2 / \pi$ since $a p_{1} \mid>-2 / \pi<p>$ for an isotropic source.

(XBL $818-1254$ ) 


\title{
Coherence and Self-Induced Transparency in High Energy Hadronic Collisions"
}

\author{
G.N. Fowler, E.M. Friedlander, and R.M. Weiner ${ }^{\dagger}$
}

The suppression of cascading in very high energy collisions of hadrons with nuclei was interpreted by Landau and Belenkij ${ }^{1}$ within a hydrodynamical model as a consequence of the formation of a tube in nuclear matter. Alhough the idea of a "coherent" tube has been considered by many workers ${ }^{2}$ up to the present, no explanation or direct experinental confirmation for this "coherent" property was given. With the advent of high+energy accelerator beams the suppression of cascading was confirmed ${ }^{2}$ in the energy range $50-400 \mathrm{GeV}$. Moreover, it was found that the increase of multiplicity with target size is localized in the backward hemisphere and the narne "transparency" was coined ${ }^{3}$ for this effect.

In this article:

1. We intraduce a different (quantum-oplical) concept of cohterence in the physics of proton-nucleus scattering.

2. We show bow this leads to self-induced transparency (SIT) in nuclear matter in close anslogy to the same effect discowered in optios in 1967 by McCall and Hahn. ${ }^{4}$

3. We present the frot direct experimentel evidence for the coherence of meson (pion) fields.

4. We show that in the forward cone a wherent part of the mesonic field is filtered out.

We start with the observation that in a hadron hadron collision a partially coherent mesonic field is created. By coherent field we understand a field the state of which is an eigenslate of the annibilation operator. ${ }^{5}$ So far the existence of this coherent field was "4eriveo" from theoretical arguments (spontaneous breakdown of symmetries, classical solutions in field theory, ete.) and from experimental indications in the Bose-Einstein correlations. In a proton+nucleus collision such a partially oherent field is created in the fust encounter of the projectile with a constituent of the target.

The following conditions are sufficient for SIT:

(1) The coherent pulse is broad enough in the direction of propagation to overlap many scattering centers which remain to be identified.

(2) There exist (at least two) energy levels of the scatier. ing centers in the medium such that the excited levels have lifetimes comparable to or longer than that of the coherent pulse.

(3) The intensity of the pulse is high.

If conditions (1)-(3) are met, the front of the coherent pulse produced in the firsl collision will excite the scatter- ing eenters as it encounters them, and the rear of the (same) pulse will then de-excite them by stimulated emission. This process will continue so that the pulse will eventually leave the medium without cascading.

Possible evidence for coherence was reported ${ }^{6}$ so tar only through the HBT effect, and there the situation is complicated mainly because the second-order correlations are rather insensitive to even large adnuxtures of coherence. There exists, howter, another way to look for wherence, i.e., through the shape of the multiplicity distributions: when the fields are completely cohtrent the mutiplicity distributions have Poisson form.

We now present experimental evidence for "Poissonicity" of the particles emilled into the forward come of high-energy proton-nucleus collisions. The data come from nuclear emulsion exposures to high-energy proton beams. viz., $69 \mathrm{GeV}$ (JHEP-Serpukhov) and 200 and $300 \mathrm{GeV}$ (FNAL-Batavia)

For the purpose of this analysis each event was divided into several pseudorapidity ( $\pi=-\ell \mathrm{n} \tan \theta / 2)$ bins. Each such bin was treated as a "ministar" of multiplicity $n_{i}$, where i denotes the $n$-bin. The ${ }^{4}$ local" multipljcity distribution of these ministars was recorded for each primary energy and $N_{h}$ combination ( $N_{h}$ is the number of target-related heavy prongs, a good measure of target size and (or involvement).

Whereas at low $\eta$ (backward in c.ms.) the "local" multiplicity distributions are close to chaotic, in the forward direction they become purely Poisson, as expected for coherent emission. To illustrate this behavior we define the following measure for the deviation of each local multiplicity distribution from the Poisson law. Sinte for a Poisson distribution

$$
P_{i}(0)=\exp \left(-<n_{1}, 3\right)
$$

the guantity

$$
\Delta_{i}=||\left(\operatorname{tn} P_{j}(0)\left|-\sum_{i}>-\right|\right.
$$

should vanish.

In Fig. 1 we plol estimates for the $\Delta_{i}\left(i={ }^{*}\right.$ l-12) against mseudorapidity. It is obvious that in the "forward" $\eta$-bins the $\Delta$-values are well compatible with zero, while highly significant deviations occur in the Jow-y bins; this effect increases strongly with $\mathrm{N}_{\mathrm{b}}$, i.e., with nuclear size. 


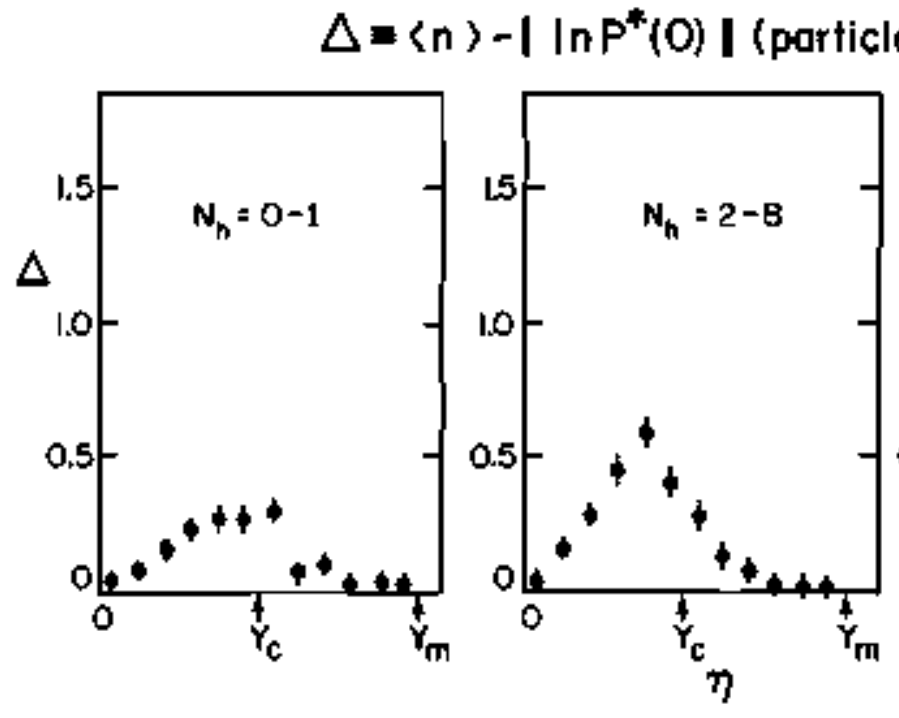

Fig. I.

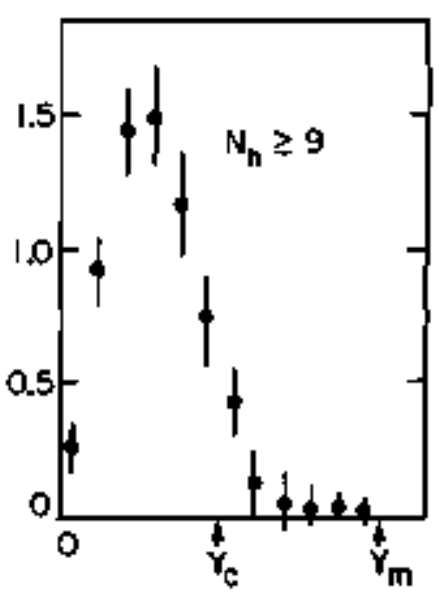

(XBL 816-935)
Foothotes and References

"Condensed from LBL-12279, Phys. Lett. B 104 (1981).

Physics Department, University of Exeter, England.

EPhysics Department, University of Marburg, Germany.

1. S.Z. Belenkii and L.D. Landsu, Usp. Fiz. Nauk 56, 309 (1955).

2. For a review of this subject, see L. Bergström, et al., to appear in Phys. Reports.

3. J. Cohen et al., Lett. Nuovo Cimento 9, 337 (1974).

4. S.L McCall and E.L. Hahn, Phys. Rer, Leth 18, 908 (1967).

5. R. Glauber, Optique et electronique quantique, in Les Houches Lectures, 1964 (Gordon and Breach, New York). 6. G. Fowles and R. Weiner, Phys. Lett. B 70, 201 (1977). 


\title{
3. Strange Particle Production
}

\section{Kaon Production in Relativistic Heavy Ion Collisions}

\author{
S. Schnetzer, M.-C. Lemalre, ${ }^{*}$ R. Lombard, ${ }^{\dagger} E$. Moeller, ${ }^{\ddagger} S$. Naganiyg, \\ G. Shopito. H. Sieiner, and I. Tanihata
}

The inclusive spectra of $\mathrm{K}^{+}$mesons in collisions of $2.1 \mathrm{GeV} / \mathrm{amu}$ nuclei with various targets were measured by means of magnetic spectrometer. The laboratory angles covered were from $15^{\circ}$ to $80^{\circ}$, and the momentum range of

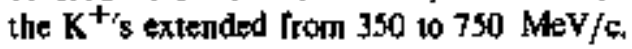

The triple differential cross section, $d^{3} \sigma / d^{3} p$, falls of exponentially with center-ol-mass energy in the aucleonnucleon center-of-mass lrame. As an example, we show the $\mathrm{K}^{+}$c.m. energy distribution for $\mathbf{N e}+\mathrm{NaF}$ in Fig. 1. In

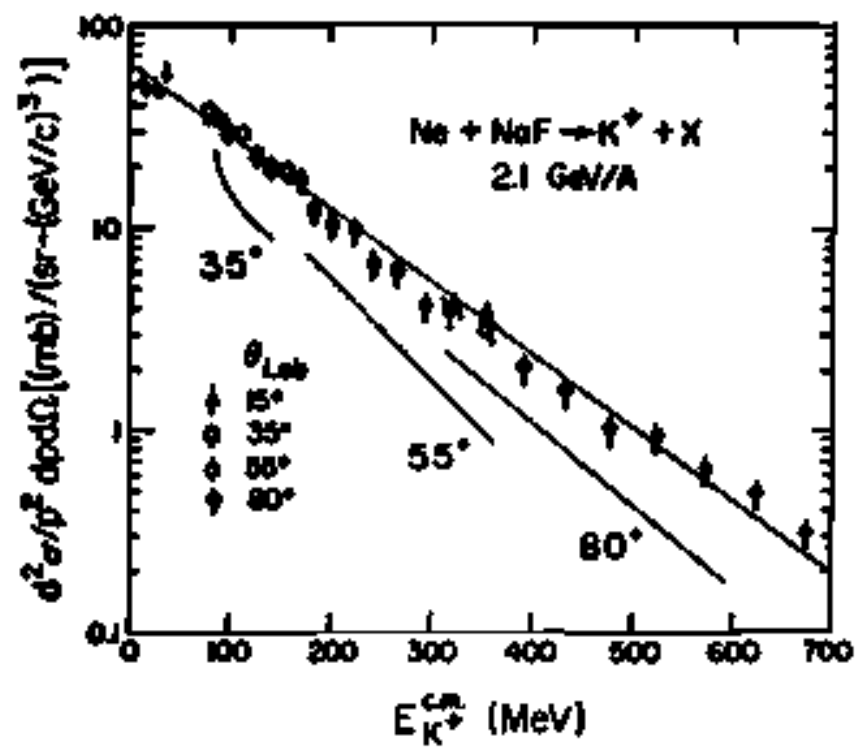

Fig. I. $K^{+}$inclusive c.m. ctoss section $d^{3} \sigma / d^{3} p$ is the kinetic energy of the $\mathrm{K}^{+}$in the c.m. for $2.1 \mathrm{GeV} / \mathrm{A} \mathrm{N}$ e + $\mathrm{NaF}$. Also shown are the row-on-row model predictions for $\theta_{1 \text { ab }}=35^{\circ}, 55^{\circ}$, and $80^{\circ}$.

(XBL 818-11276) addition, the angular distribution of the kenons is nearly isotropic in this frame even for $\mathrm{p}+\mathrm{NaF}$ and $\mathrm{Ne}+\mathrm{Pb}$ collisions.

The data hes been compared with a row-on-row mode] ${ }^{1,2}$ and a thermal model. ${ }^{3}$ Neither are able to explain al] features of the data. The row-on-row does not reproduce the near-isotropy in the NN frame as can be seen in Fig. 1, where the curves represent these predictions for $\theta_{\text {ab }}=35^{\circ}, 55^{\circ}$, and $80^{\circ}$. The thermal model overpredicls the kaon yield by a factor of $=20$.

We have also observed an interesting A-dependence effect. The kaon yield increases by a larger factor when going from $\mathrm{Ne}+\mathrm{NaF}$ to $\mathrm{Ne}+\mathrm{Pb}$ collision than when going from d $+\mathrm{NaF}$ to d + Pb collisions. This is suggestive of some form of collective behavior.

\section{Footmotes and References}

Now at DPhN/ME, CEN Seclay. France.

ton leave from DPhN/ME, CEN Saclay, France.

ton leave from Freie Universität Berlin, Berlin, West Germany.

SOn leave from INS, University of Tokyo, Tokyo, Japan. 1. J. Randrup and C.M. Ko, Nucl. Phys. A 343, 519 (1980).

2. J. Randrup, Phys. Lett. B 76, 547 (1978).

3. F. Asai, H. Sato, and M. Sano, Phys. Lett. B 98, 19 (1981). 


\title{
Observation of Subthreshold $\mathrm{K}^{-}$Production in Relativistic Nuclear Collisions
}

\author{
A Shor, $\mathrm{K}$ Ganezer.* J. Carroll,* G. Iga."J. Geaga," S. Abachi,* \\ A. Sagle, T. Mulera, V. Perez-Mendez, P. Lindstrom, \\ F. Zarbakhsh, and D. Woodwardt
}

The possible ocourrence of exotic nuclear phenomena in relativistic muclear collisions necessitates some degree of thermalization or collective interactions among the collid. ing nucleons. A clear signalure for collective or thermal effects is the production of particles which require significantly more energy than is available in individual nucleon-nucteon collisions. Al $2.1 \mathrm{GeV} /$ nucleon, the threshold for $\mathrm{K}^{-}$production is $140 \mathrm{MeV}$ above the avajabje $\mathrm{N}+\mathrm{N}$ center of mass energy. while antiproton production requires $1 \mathrm{GeV}$ above the available energy.

We have recently conducted a search for $\mathrm{K}^{-}$and $\overline{\mathbf{P}}$ production in the reaction ${ }^{28} \mathrm{Si}+{ }^{28} \mathrm{Si}$ at $2.1 \mathrm{GeV} /$ nucleon. Negative secondaries (with $\mathrm{P}=$ $\hat{c}_{\mathrm{cm}} \gamma_{\mathrm{cm}} \mathrm{M}_{\mathrm{p}}$ ) produced in the collision were transported through a magnetic beam line with detectors at appropriate locations for TOF measurements and pion rejection. The resulting TOF spectrum (Fig. 1) contains a distinct kaon peak Our measured differential cross section for $\mathrm{K}^{-}$produced at $0^{\circ}$ and $\mathrm{l} \mathrm{GeV} / \mathrm{c}$ is $\mathrm{d}^{2} \sigma / \mathrm{dpd} \Omega=1.3 \mathrm{mb} / \mathrm{sr}$ - GeV/e (etror $\cong 50 \%$ ). To compare this result with above threshold $\mathrm{K}^{-}$production, we have also measured the $\mathrm{K}^{-}$rale in the reaction $\mathrm{p}-\mathrm{Cu}$ al $4.8 \mathrm{GeV}$, where for $\mathrm{K}^{-}$ under identical conditions, $d^{2} \sigma / d p d \Omega=0.9 \mathrm{mb} / \mathrm{sr}$ - $\mathrm{GeV} / \mathrm{c}$.

The significance of the subehreshold $\mathrm{K}^{-}$yield is evaluated by comparing the experimestal ratio $\mathbf{R}$. $\mathrm{d} \sigma\left(\mathrm{N}+\mathrm{N}(2.1 \mathrm{GeV} / \pi) \rightarrow \mathrm{K}^{-}\right) / \mathrm{d} \sigma(\mathrm{p}+\mathrm{Cu}(4.8 \mathrm{GeV}) \rightarrow$ $\mathrm{K}^{-}$) with alculations incorporating nuclear Fermi mortentum of the projectile and target nucleons to allow for

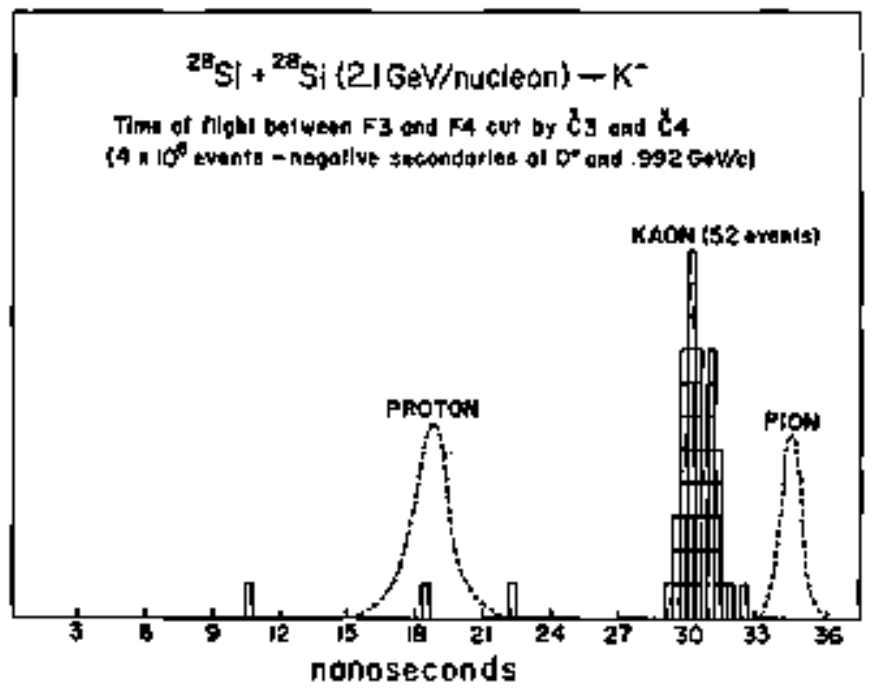

Fig. 1.

(XBL 8111-12545) subthreshold particle production. For the Fermi momentom distribution, applied the double Gaussian reported by Geaga et al.." which also results in a good fit for subthreshold antiproton production in $\mathrm{p}-\mathrm{N}$ collisions, as shown in Fig. 2. These calculations are found to underpredict our subthreshold $\mathrm{K}^{-}$yield by about two orders of magnitude.

We do noc feel we have yet attained the sensitivity required for meaningful antiprolon measurement. How. ever, the large $\mathrm{K}^{-}$rate we have observed is an indication of some interesting phenomena taking place in relativistic nuclear collisions. We plan to pursue the study of sublhreshold particle production as a probe of matter at high energies and densities.

Poothotes and References

"University of California, Los Angeles, CA.

${ }^{\dagger}$ California State Univerșity, Hayward, CA

1. J.V, Geaga et al., Phys. Rev. Lett. 45, 1993 (1980).

2. D.E. Dorfan et al., Phys. Rey. Lett. 14, 995 (1965).

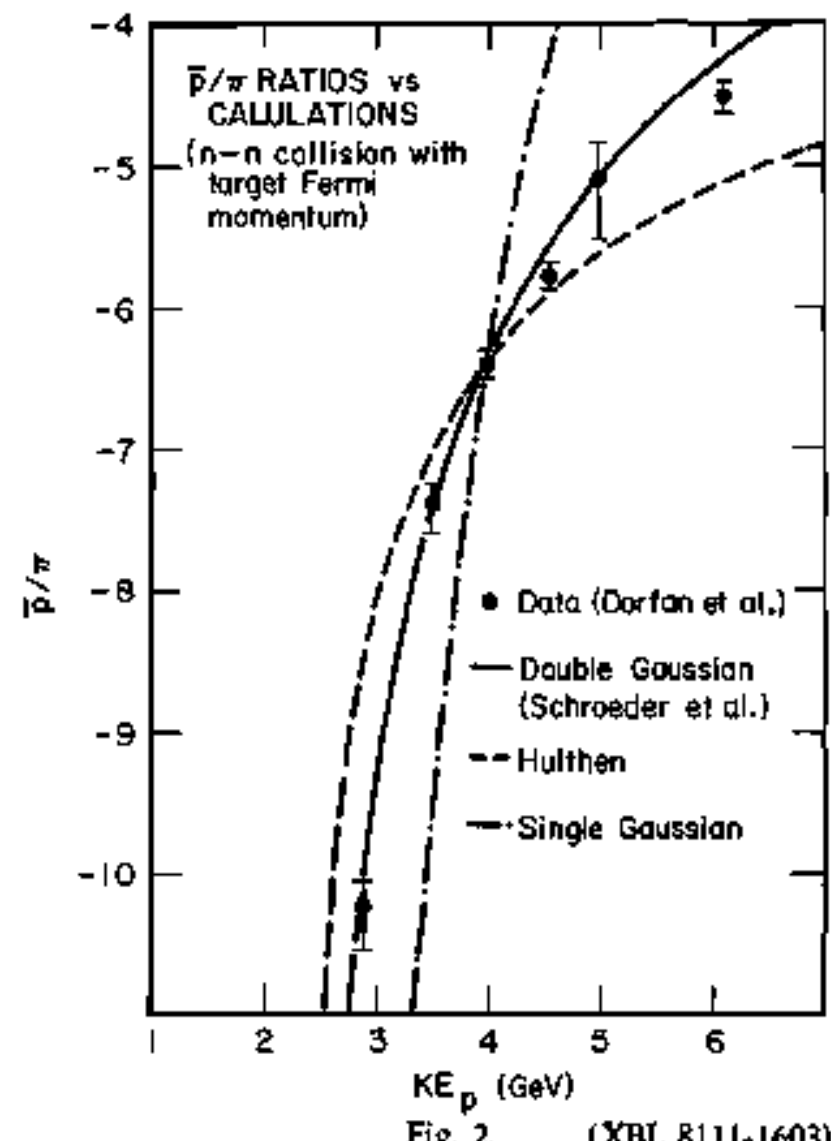

Fig. 2. (XBL $8111-1603$ ) 


\title{
$\Lambda$ Production Near Threshold in Central Nucleus- Nucleus Collisions
}

\author{
R. Brockmann," J.W. Barris, A Sandoval, H. Strobele," R. Stack," M. Maier, \\ R.E. Renford, ${ }^{\dagger}$ J. Miller, H.G. Pugh, ${ }^{\ddagger}$ M.Raff ${ }^{\ddagger}$ LS. Schroeder, ${ }^{\ddagger}$ F. Rless, \\ K. Wolf!' A. Dacal, and M.E. Ortiz"
}

$\Lambda$ production was studied in central ${ }^{40} \mathrm{Ar}+\mathrm{KCl}$ colljsions at $1.8 \mathrm{GeV} / \mathrm{u}$ incident energy using the Streamer Chamber facility at the Bevalac. The streamer chamber, with its $4 \pi$ solid angle capability, was operated in both inelastic and central trigger modes. The central irigger selects events with small total charge in projectile fragments. This trigger, which corresponds to $10 \%$ of the reaction cross section $\left(\sigma_{R}=1.8 b\right)$, is associaled with impact parameters $b<2.4 \mathrm{fm}$ in a geometric model. The open channels for production of strange particles in this energy regime are $\mathrm{NN} \rightarrow A K N(1.58 \mathrm{GeV}), \rightarrow 2 \mathrm{KN}(1.79 \mathrm{GeV})$, and $\rightarrow \mathrm{MN}(1.96 \mathrm{GeV})$. Neutral strange particles were detected by their charged-particle decay in the active volume of the chamber. In order to derive $A$ production cross sections and mọmentum spectra, detection and scanning efficiencies were determined. The neutral strange particles were identifed by a kinematical fit of the decays $\Lambda \rightarrow$ $\mathrm{p} \pi^{-}$and $\mathrm{K} \rightarrow \pi^{+} \pi^{-}$.

The jdentified is are displayed in Fig. la as a function of $p_{\perp}$ and $p_{\|}$in the nucleus-nucleus c.m. system. The solid curve in Fig. la represents the kinematic limit for $A$ momenta produced in the elernentary $\mathrm{NN} \rightarrow \Delta \mathrm{ANN}$ reaction at $1.8 \mathrm{GeV}$. Most of the $A$ are observed beyond the limit indicating the necessity to include Fermi motion and/or collective multi-particle interactions. To investigate further the origin of the observed high $\Delta$ momenta, a Monte Carlo phase space calculation incorporaling Fermi motion was performed. The results after folding in the detection efficiencies are shown in Fig. $1 \mathrm{~b}$. The distribution, which peaks at $\mathbf{p}=0$, is broadened in phase space but still does not adequately describe the observed spectrum.

Inclusion of $A$ rescattering in the nuclear medium was nexi considered. Restattering from the thermal "fireball" distribution (Rsl) bas little effect on the $A$ spectrum. However, rescatlering from a superposition of the colliding initial distributions (Rsll) shown in Fig. le depletes events from the ceniral peak and creates peaks near $p_{u}=$ $\pm 0.8 \mathrm{GeV} / \mathrm{c}$. The backward peak at $\mathbf{p}_{\|}=-0.8 \mathrm{GeV} / \mathrm{c}$ is suppressed by the delection efficiency which is included in the calculation. In addition, the $p_{\perp}$ is boosted in qualjLative agreement with the observations.

A guantitative comparison of the data with results of the Monte Carlo calculations can be found in Table I where the mean and rms values of $p_{\perp}$ and $\mid p_{\mid}{ }^{c m_{I}}$ for the

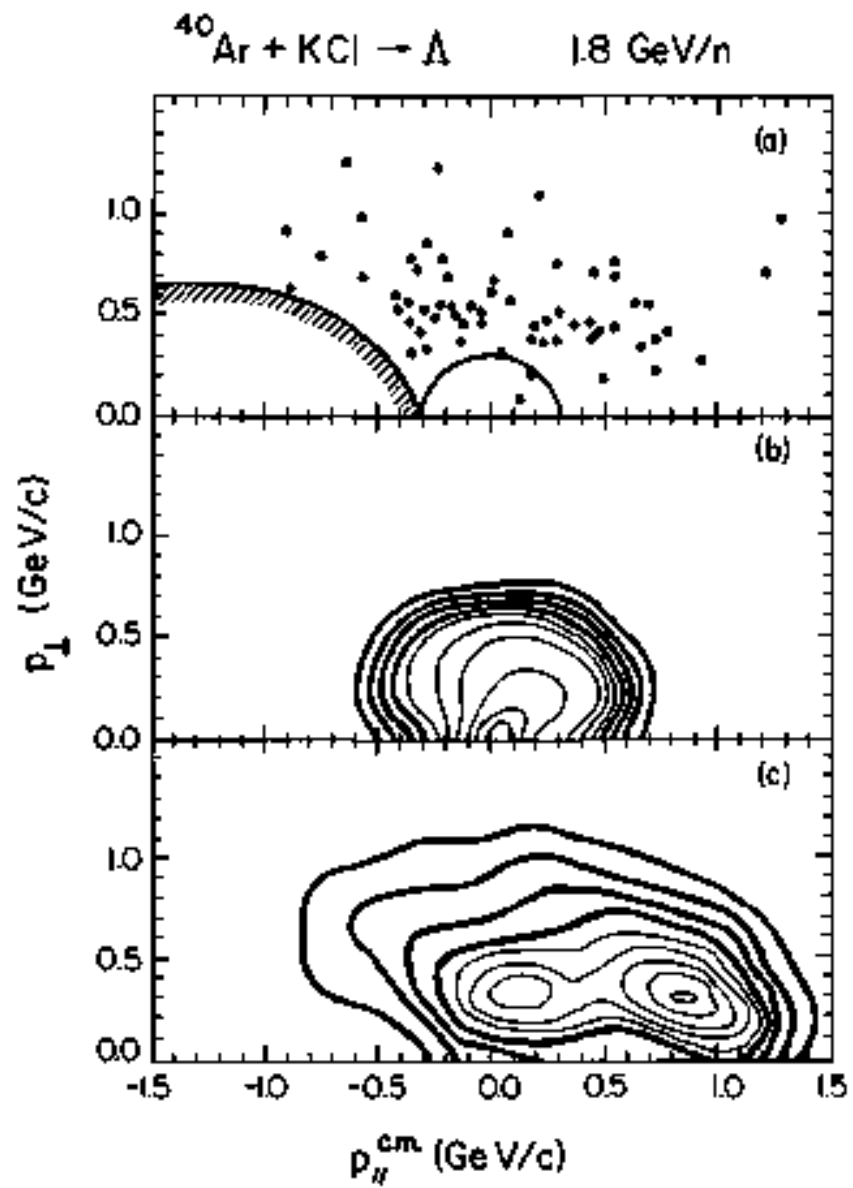

Fig. 1. ${ }^{40} \mathrm{Ar}+\mathrm{KCl} \rightarrow \Lambda$ events as a function of $p_{\perp}$ and $\mathbf{P}_{\text {f }}$ jn the nucleus-nucleus c.m. at $1.8 \mathrm{GeV} / \mathrm{u}$. (a) Scatterplor of the data. The halched curve shows the region where events were excluded due to inefficiencies. The solid curve corresponds to the NN $\rightarrow$ AKN kinematic limit. (b) Linear contour plot of the results for the Monte Carlo calculation AA (Fermi motion only). (c) Linear contour plot of the calculation RsII with subseguent $A$ rescallering (see 1ext).

(XBL 813-437)

invariant $\left(\mathrm{E} / 2 \pi \mathrm{p}_{\perp}\right)\left(\mathrm{d}^{2} \sigma / \mathrm{dp} \mathrm{p}_{\perp} \mathrm{dp}\right)$ distributions are presented. The dala exhibil a much higher $\left\langle p_{\perp}\right\rangle$ than predicted by any of the calculations. 
Table 1. Quantitative comparison of data with results of Monte Carlo calculations.

\begin{tabular}{|c|c|c|c|c|}
\hline & $\begin{array}{c}\left\langle p_{\perp}\right\rangle \\
(G \in V / c)\end{array}$ & $\begin{array}{l}\sigma_{\mathrm{tms}}\left(\hat{P}_{\perp}\right) \\
(\mathrm{GeV} / \mathrm{c})\end{array}$ & $\begin{array}{l}\langle|\mathrm{p} / \mathrm{P}|\rangle \\
(\mathrm{GeV} / \mathrm{c})\end{array}$ & $\begin{array}{c}\sigma_{\text {ras }}\left(\left|\mathrm{Pff}^{\mathrm{m}}\right| \mathrm{l}\right) \\
(\mathrm{GeV} / \mathrm{c})\end{array}$ \\
\hline Data & 0.493 & 0.243 & 0.429 & 0.264 \\
\hline NN & 0.122 & 0.077 & 0.117 & 0.077 \\
\hline $\mathbf{A A}$ & 0.207 & 0.150 & 0.212 & 0.154 \\
\hline RsI & 0.220 & 0.180 & 0.229 & 0.184 \\
\hline Rs! & 0.276 & 0.233 & 0.641 & 0.380 \\
\hline
\end{tabular}

The mean $A$ multiplicity was found to be $\left\langle m_{A}\right\rangle=$ 0.04 for the ceniral trigger. Using the measured cross section for centsal trigger events, $\sigma=180 \mathrm{mb}$, the $A$ cross section in central collisions of ${ }^{40} \mathrm{Ar}+\mathrm{KCl}$ at $\mathrm{I} .8 \mathrm{GeV} / \mathrm{u}$ is $\sigma_{\mathrm{A}}=7.6 \pm 2.2 \mathrm{mb}$.
Since A decay is self-analyzing for polarization in the decay process $\Delta \rightarrow \mathrm{pr}^{-}$, the distribution of decay protons in the A decay frame relative to the $A$ spin direction is $d W / d \theta=(1+\alpha P \cos \theta) / 4 \pi$, where $P$ is the $A$ polariza tion, $\alpha=-0.642$ and $\theta$ is the angle between the dexay proton and the unit vector normal to the plane. Using the relation $\alpha \mathrm{P}=\left\langle\cos \theta>/\left\langle\cos ^{2} \theta>\right.\right.$, the polarization extracted from the present experiment was found to be $P=-0.10 \pm 0.05$

Footnotes and References

"GSI, Darmestadt, West Germany.

†Universität Marburg, West Germany.

柆L, Berkeley.

Universität Mǘnchen, West Germany.

Arsonne National laboratory.

fIFUNAM, Merico.

1. S. Bohrmann and J. Knoll, Nucl. Phys. A 356, 498 (1981).

2. S. Brandt and H.D. Dahmen, Z. Phys, C 1, 61 (1979); L. Wu and J. Zobernig, Z. Phys. C 2. 107 (1979). 


\title{
4. Correlation Studies
}

\section{Measurement of Two-Particle Correlations in $800 \mathrm{MeV}$ pA Collisions"}

\author{
I. Tanihata, Y. Make, H. Hamagaki, S. Kadota, Y. Shida, $\boldsymbol{R}$. Lombard, \\ E. Moeller, S. Nagamiya, S. Schnetzer. and H. Steiner
}

Two-particle ( $p-p, p-d)$ coincidences have been measured in $p+A$ collisions. The production mechanism of bactscatlered protons has been studied by forwardbackward coincidence events. These coincidence spectra are measured in two modes: in-plene coincidence (IPC), $\left(\theta_{1}-15^{\circ}, \theta_{2}=118^{\circ}, \Delta \phi=180^{\circ}\right)$ and out-of-plane coincidence $(O P C),\left(\theta_{1}=15^{\circ}, \theta_{2}=\left[1 B^{\circ}, \Delta \phi=90^{\circ}\right)\right.$.

Figures $1(a),(b)$ show scatter plots of $p-p$ and d-p IPC events in $P+C$ reactions. Solid ourves in the figures show the kintmatics of $p-p$ and $d-p$ quasi-elastic statterings (QES). The kinentatic loci of the breakup of two and three nucleon clusters with zero excitation energy are represented by broken lines. The numbers along the curves indicate the jnitlal momenta of the $\mathrm{p}, \mathrm{d}$, or clusters inside the target nucleus. The IPC events show the clear existence of the $p \mathrm{p}$ and p-d QES which are not seen in OPC. Besides the P-P QES, a broad but definite population is seen in the region of $2 \mathrm{~N}$-cluster breakup. In spite of the large excitation (300-400 MeV) of the remaining system, which includes all the particles other than those delected, the number of $I P C$ events is still larger than that of $O P C$. Figure 2 shows the spectre of the backscattered proton associated with the different components, namely. QES, nor-QES IPC, and OPC. All of the spectra, except d-p $\mathrm{QES}$ are filled well by a function $A \mathrm{e}^{-\mathrm{P} / \mathrm{R}}$, as in the case of the inclusive spectra. The slope factors, $P_{0}$ of the P-p QES and OPC are smallex than those of the inclusive spectra. Because of the fast fall-of of the spectra, these two contponenis cannot contribute significantly to the backscattered high-energy protons. The slope factors $P_{0}$ of in-plane $p-p$ and d-p non-QES are similar to the $P_{0}$ of the inclusive spectra and, therefore, suggest that there is a close rela tionship between these spectra. The similarity of the backscattered proton spestrum of $\mathrm{dp}$ events and the difference spectrum between in-plane and out-of-plane wincidence events on correlated 2N-clusters. However, incoherent multiple scattering processes involving a few nocleons cannot be rejected at this stage of analysis. We need further analysis including angular distributions and also better theoretical calculations to distinguish these components.

\section{Footwote}

"Condensed from LBE-12978.

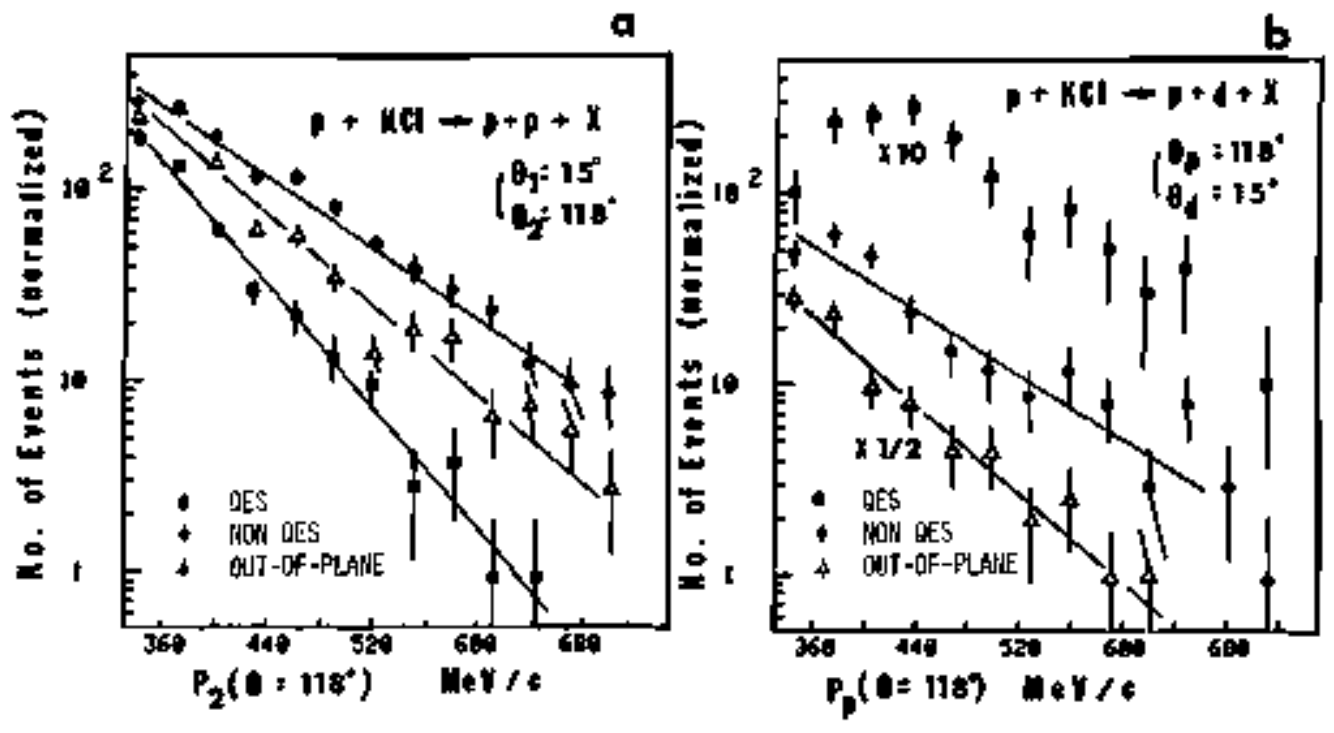

Fig. 1. Momentum-momentum scatter plots of the p-p (a) and p-d (b) events for the in-plane-coincidence mode. The area of circle in the figure is roughly proportional to the number of events.

(XBL 817-]0846) 


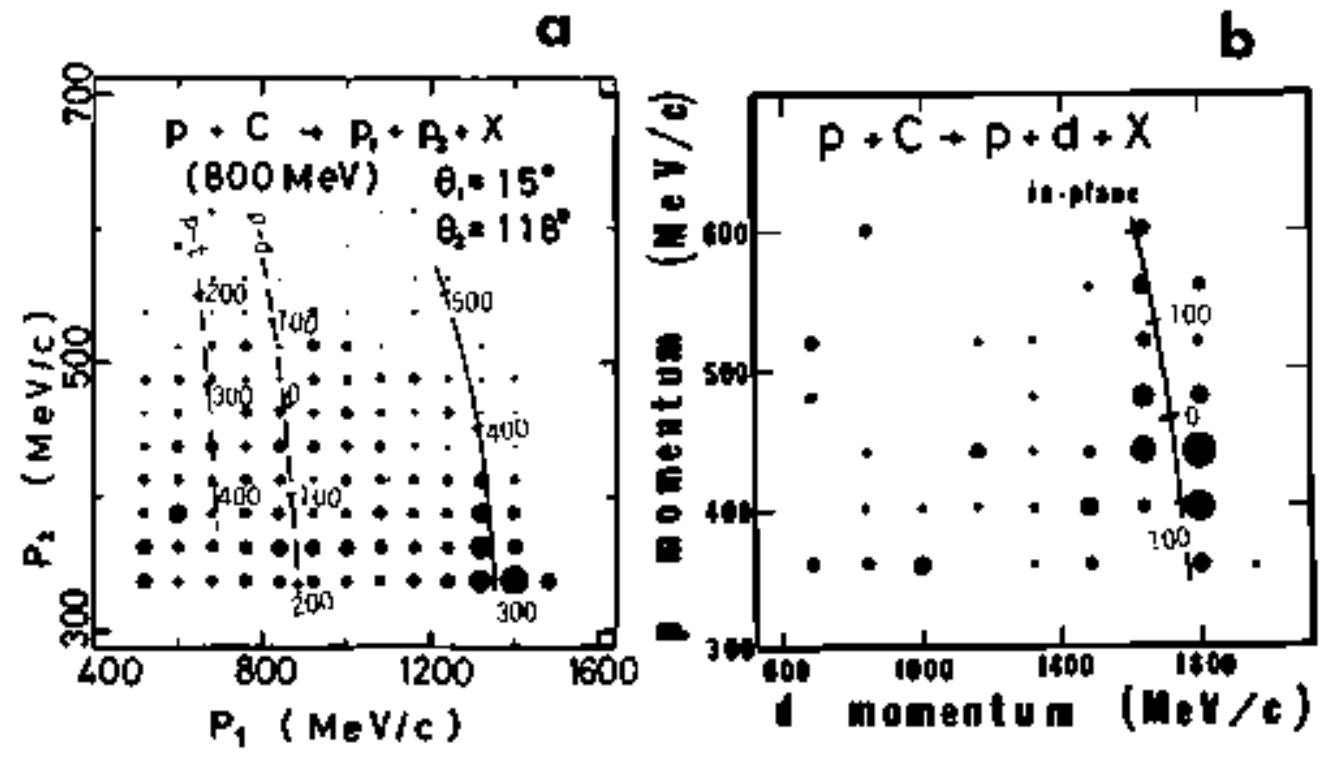

Fig. 2. Spectra of the backscattered protons for different components, Solid lines are exponential fits to the data.

(XBL 817-10847)

\title{
Few Nucleon Interactions and Correlation Studies
}

\author{
R N, Trethoff, J.M. Engelage," J.V, Geaga, J.W, Harris, ${ }^{\dagger}$ \\ P.N. Kirk," R.W. Koontz, H.G. Pugh, G.R Roche, C.L Ruiz, and L.S. Schroeder
}

A principal aim of our research has been to uncover the characteristics of elementary or few-body reactions as they rake place in the nuclear medium. In the study of proton-nucleus eollisions, we pose two questions: (1) Can any of the high mometum back-scatlering of protons on nuclei ${ }^{1,2}$ be attributed to scattering of a small number of correlated nucleons? And if so, (2) can one discover the properties of the proton-correlated group scattering?

In a $(p, 2 p)$ experiment recently done with $T A S S,{ }^{3}$ we concentrated on the possibility of scattering $2.1 \mathrm{GeV}$ incident protons from correlated groups of two nucleons in carbon. The two arms of the spectronneter were set to encompass part of the phase space available to the two outgoing protons in the reaction pd $\rightarrow$ ppn. For example, if the rear arm, which was at $120^{\circ}$ to the beam throughout the experiment, accepted protons in the momentum range 400 to $600 \mathrm{MeV} / \mathrm{c}$, the front arm set at $10.4^{\circ}$ would detect protons from the p-d reaction in two momentum ranges: 400 to $960 \mathrm{MeV} / \mathrm{c}$, and 2170 to $2720 \mathrm{MeV} / \mathrm{c}$. It is expected that Fermi momentum of the two nucleon object in carbon would smear these ranges somewhat.

Figure 1 shows

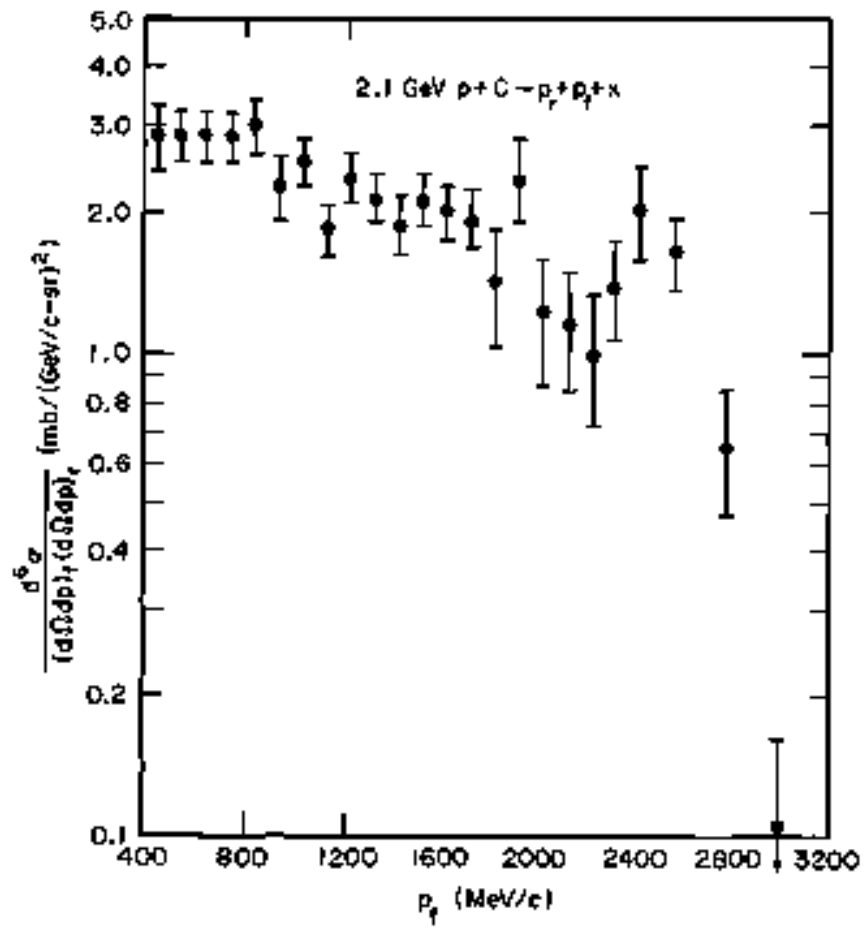

Fig. 1. Preliminary results from $2.1 \mathrm{GeV} p+\mathrm{C} \rightarrow \mathrm{p}+$ $\mathrm{p}+\mathrm{X}$ with $\theta_{\mathrm{f}}=10.4^{\circ}, \theta_{\mathrm{r}}=120^{\circ}, \mathrm{P}_{4}=400-$ $600 \mathrm{MeV} / \mathrm{c}$ (coincidence spectrw).

(XBL B1 J-1606) 
vs $\mathrm{p}_{\mathrm{r}}$ the fortward proton's momentum for the p-arton reaction in the kinematics described above. In contrast to our coincidence dala, Fig. 2 stows the singles spectrum for protons taken by Tanihata et al. at $10^{\circ} .2$ The shapes of the two spectra are different. If the protons observed in the coincidenoe measurement were uncorrelated, we would expect to find that a cut on the backward nomentum vector changed only the scale of the lront momentum spectrum

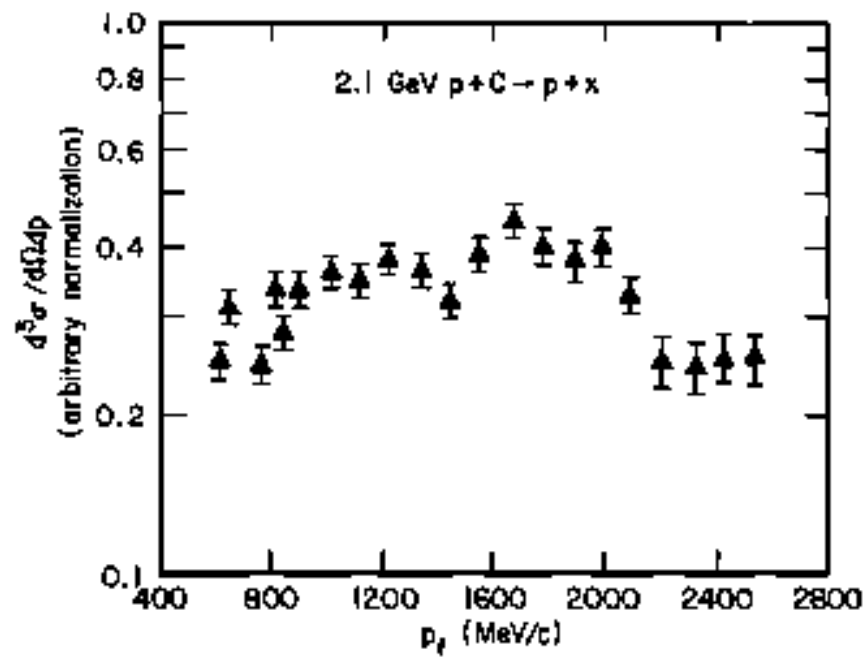

Fig. 2. The $2.1 \mathrm{GeV} \mathrm{p}+\mathrm{C} \rightarrow \mathrm{p}+\mathrm{X}$ with $\partial_{\mathrm{r}}=10^{\circ}$ (single spectrum). and not the shape. After further reduction of the data, we will be able to relate the obstrved correlation to the number of participants in the reaction and make stronger conclusions about the scattering from the 1 wo nucleon clus. ter. We also have data for the same backward kinematics with the forward going proton at $45^{\circ}$ to the beam. In early 1982, we expect to obtain more statistics on a carbon target. Also, for a definitive comparison, we will perform the same set of measurements on a liqpid depterium target.

Viewing the nucleus as an alternative setting for elsmentary interactions is a useful perspective for understanding the behavios of the nuclear participants. This expesin ment will contribute to the description of the nuclear deuteron.

\section{Footnotes and References}

*Department of Physics and Astronomy, Louisiana State Univessity.

tDepartment of Physics, University of California, Los Angeles.

fGSI, Darmstadt, West Germany.

IOn leave from Univessity of Clermont-Ferrand 11, France. Supported in part by a NATO grant.

]. J.V. Geaga et al., Phys, Rey, Lett 45, 1993 (1980).

2. I. Tanibala et al., Lawrence Berkeley Laboratory Report LBL-12978 (1981).

3. Set description of TASS by G.R Roche et al. in this annual report.

\title{
Proton-Emitting Sources in Central Collisions of $\mathrm{Ar}+\mathrm{KC}$ at $1.8 \mathrm{GeV} / \mathrm{N}$
}

\author{
A.L. Sagle, P. Zarbakhsh, F, Brochard, T.A. Mulera, V. Perez-Mendez, \\ R. Talaga, I. Tanthata, J.B. Carroll," X.S. Ganezer," G. Igo," J. Ostens," \\ D. Woodward. ${ }^{\dagger}$ and R. Stutert
}

We have measured the single proton inclusive cross sections, the two-proton correlation funclion, and the associated multiplicity of high $P_{T}$ fragments in collisions of $\mathrm{Ar}+\mathrm{KCl}$ at $1.8 \mathrm{GeV} / \mathrm{n}$. With these measurements, we hope to see if dense nuclear matter can be formed in central relativistic nucleus-nucleus collisions. In particular, we wish to determine if there are enough interactions to slow down and quasi-equilibrate al least some of the nucleons while the nuclei are interpenetrating.

Measurements were made at two different spectrometer settings, $S_{1}$ and $S_{2}$, corresponding to the beam rapidity $Y_{B}$ and to $Y_{B} / 2$, respectively. For projectiles and targets of equal mass, $Y_{\mathrm{B}} / 2$ corresponds to the velocity of the nucleus-aucleus center of mass.

We show in Table 1 the invariant single particle inclusive cross sections $f$ in arbitrary units for settings $\mathbf{S I}$ and $\mathrm{S} 2$. The column labeled $\mathrm{M} \geq 0$ shows the invariant cross section with no mpltiplicity requirements. The column labeled $M>4$ shows the cross section when 5 or more particles are detected by the multiplicity counters. The quantily $C$ (defined as the ratio of $f$ for $M>4$ to $f$ for $M \geq 0$ ) is larger at $Y_{B} / 2$ than $Y_{B}$. The larger value of $C$ at $Y_{\mathrm{B}} / 2$ indicates that this multiplicity requirement is selecting more central collisions sjuce this selection tends to decrease the emission of particles at $Y_{B}$ more than at $Y_{B} / 2$. On the other hand, $f$ is larger for the data at $-Y_{B}$ than at $Y_{B} / 2$ even when the bigh multiplicity requirement is imposed. This implies that even for smaller impact parameter central collisions, most of the nucleons are not thermalized in the Ar- $\mathrm{KCl}$ center of mass.

We now iurn to the results for the correlation function R. Details aboul is definition and experimental determination are given elsewhere.' In Fig. I we show $R$ for data at $Y_{B}$ and $Y_{B} / 2$ when $M \geq 4$. Koonin ${ }^{2}$ has shown that the 
Table 1. Invariant cross sections $\mathrm{E} / \mathrm{P}^{\hat{2}} \mathrm{~d} \sigma / \mathrm{dpd}$ (arbitrary units) for different multiplicity requirements $M$.

\begin{tabular}{lccccc}
\hline \hline Setting & $P$ (lab) & (Lab) & $M \geq 0$ & $M>4$ & $C-M>4 / M \geq 0$ \\
\hline$S 1,\left(Y_{B}\right)$ & 2.4 & 6 & 548 & 37.8 & 0.069 \\
$S 2,\left(Y_{B} / 2\right)$ & 1.0 & 13.5 & 100 & 9.4 & 0.096 \\
\hline
\end{tabular}

peak in $R$ al $\Delta P \simeq 20 \mathrm{MeV} / \mathrm{c}$ decreases with the average separation $\langle\Delta r\rangle$ between the two protons at the time of their emission. The $\langle\Delta y\rangle$ for data at $Y_{\mathbf{B}}$ is larger than for data at $Y_{\mathrm{B}} / 2$. This again supports the idea that most of the projectile and target nucleons are not equilibrated in the $\mathrm{Ar}-\mathrm{KCl}$ center of mass.

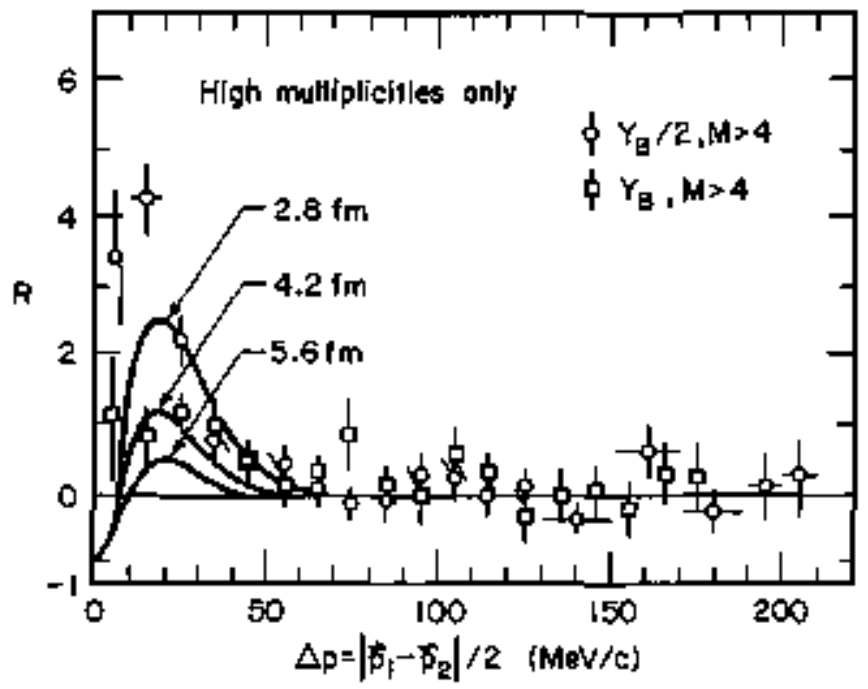

Fig. 1. The correlation function, $\mathrm{R}$, for $\mathrm{Ar}+\mathrm{KCl} \rightarrow \mathrm{p}+$ $p+X$ at $1.8 \mathrm{GeV} / \mathrm{n}$.
(XBL \&1] 1 - 1605)
The $\mathrm{Y}_{\mathrm{f}} / 2$ data do, however, indjcate that a significant number of interactions occur while the projectile and terget are iniepenetraling. The small $\langle\Delta r\rangle$ with $M>4$ excludes the possibility that these mid-rapidity protons are emitted from projectile and target remunants after the colliding nuclei have passed through tach other. If such a process were significant, we would measure a large separation along the beam direction (a large $\langle\Delta r>$ ) since, for equal mass colliding nuclei, any given $\mathrm{Y}_{\mathrm{B}} / 2$ proton could come with equal probability from either the projectile remnant or the larget remnant.

The small $\langle\Delta r>$ is, however, consistent with two other possibilities. The $\mathrm{Y}_{\mathrm{B}} / 2$ protons may come from an isolated low density source at late stages of the collision after most of the projectile and target nucleons bave passed through each other. On the other hand, these mid-rapidity protons may be emitied from a dense non-equilibraled system while the nuclei are still interpenetrating. We plan to better determine the properties of the mid-rapidity proton source by measuring a lerger part of the phase space with the new Hiss facility.

Foofnotes and References

"Universily of California, Los Angeles, CA.

tCalifomia State University, Hayward, CA.

${ }^{t}$ Brookhaven National Laboratory, Upton, L.I., NY.

I. F. Zarbakhsh et al., Phys. Rev. Lett. 48, 1268 (1981).

2. S. Koonin, Phys. Le11. B 70, 43 (1977).

\title{
Two-Pion Correlations in Heavy Ion Collisions*
}

\author{
J.A. Bistirich, R.J. Bossingham, H. R. Bowman, CW. Clawson, K.M. Crowe, \\ K.A. Frankel, O. Hashimoto, J.G. Ingersoll, M. Koike, J.P. Kurck. \\ C.J. Martoff. ${ }^{\ddagger}$ WJ. Macdonald, J.P. Miller. D.L. Murphy. \\ J.O. Rasmussen, J.P. Sullivan, P. Truol.' E. Yoo, and W.A. Zajc
}

The technique of pion interlerometry has been applied to study the reaction $1.8 \mathrm{GeV} / \mathrm{A}{ }^{40} \mathrm{Ar}+\mathrm{KC} \rightarrow$ $2 \mathbf{r}^{ \pm}+\mathrm{X}$ In particular, pion interferometry has been proposed as a ton for measuring the radius and lifetione of the pion source.' This sensitivity to the source size has recently been verifitd in streamer chamber measurements. ${ }^{2}$
In addition, Gyulassy et al. ${ }^{3}$ bave emptasized that the pion-pair correlation function also contains information related to the degree of coherence of the pion field.

Approximately $55002 \pi^{+}$pairs and $45002 x^{-}$pairs with laboratory momentum between 200 and $1000 \mathrm{MeV} / \mathrm{c}$ were analyzed with a magnetic spectrometer system 
situated at $45^{\circ}$ to the beam axis. The measured momenta spectra are in good agreement with the predictions of a Monte Carlo program, which has also been used to calculate the momentum resolution. The Monte Carlo results indicate that the momentum resolution is $\leqslant 4 \%$ for $\mathrm{p} \leqslant$ $250 \mathrm{MeV} / \mathrm{c}$ and falls rapidly to $\sim 2$ 它 for $\mathrm{p} z$ $300 \mathrm{MeV} / \mathrm{c}$.

The measured pion momenta are transformed to the center of mass, where the relative momentum and energy of each pair are calculated, and then used to determine the correlation function

$$
C_{2}\left(q, q_{0}\right)=N(4,90) / B\left(4, q_{0}\right)
$$

Here $q=\left|\vec{p}_{2}-\vec{p}_{1}\right|, q_{0}=\left|E_{2}-E_{1}\right|, N\left(q, q_{0}\right)$ is the number of pions observed with $q$ and $q_{g} . B\left(q, q_{Q}\right)$, the expected background in the absence of Bose-Einstein correJations, is generated by mixing pion momenta from different tvents. (Sec Ref. 4 for [urther details.) The resulting correlation function is then fit to the expression

$$
C_{2}\left(q, q_{0}\right)=I+\lambda \exp \left(-\frac{1}{2} q^{2} R^{2}-\frac{1}{2} q_{0}^{2} T^{2}\right) .
$$

In this expression, $R$ and $T$ are the radius and lifetime of the corresponding spatial distribution function $\rho(r, t)=\exp \left(-r^{2} / R^{2}-t^{2} / T^{2}\right)$. The parameter $\lambda$ is expected to be I for a maximally chaotic pion source. If, however, the pion's wave functions are not mutually incoherent, substantial deviations from 1 may occus.

The resuits of periorming such fits may be found in Table I. In most cases, $T$ has been fixed to $1.5 \mathrm{fm} / \mathrm{c}$, the same value assumed in the analysis of Ref. 2 . This is necessary in most cases because the small number of events, combined with the strong correlation between $q$ and $q_{0}$, often does not permit separate extraction of $R$ and $T$. In the first fit, $\lambda$ is also fixed to the value of one, and no Coulomb corrections have been applied to the data.

This deta and the corresponding fit, assumed over $t_{0}$, are shown in Fig la. The summation over $q_{0}$ defines a spectroneler-ayeraged correlation function

$$
\left\langle c_{2}(q)\right\rangle=\frac{\sum_{q 0} N\left(q, q_{0}\right)}{\sum_{0} B\left(q, q_{0}\right)} .
$$

While no longer a true correlation function, this form is thosen to provide a convenient one-dimensional presentation of the data and the fitted function.

The small value of $R$ implied by the raw data ( $R=$ $1.84 \pm 0.27 \mathrm{fm}$ ) results $\mathrm{from}$ the suppression of the like charge correlation by the Coulonb interaction between the two pions. An event-by-event correction for this effect (Fig. l(b)) leads to $\mathrm{R}=2.98 \pm 0.30 \mathrm{fm}$, again with $\mathrm{A}=$ I. $R$ is largely unchenged when $\lambda$ is left as a free parameter (Fig. l(c)): $R=3.18 \pm 0.33 \mathrm{fm}$, with $\lambda=0.86 \pm$ 0.07 . The $R$ value obtained from the corresponding $2 \pi^{+}$fit (Fig. 1(d)), $3.92 \pm 0.43 \mathrm{fm}$, is somewhat larger, while $\lambda=0.78 \pm 0.07$ is smaller.

It would be premature to conciude that these vajues of $A$ indicate appreciable source coherence. Aside from the question of stetistical significance, $\lambda$ is also affected by factors such as the event geometry, the construction of the background, spatial correlations within the source, etc.

Finally, allowing $T$ to vary in the case of the $2 \pi^{+}$, we

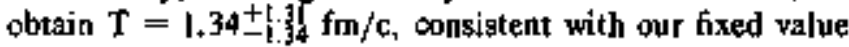
of $1.5 \mathrm{fm} / \mathrm{c}$. At present, the $2 \pi^{-}$data set does not allow separale extraction of $R$ and $T$. However, analysis of added data should produce similar results for these events.

\section{Foonotes and Referentes}

"Condensed from LBL- 12652.

'Institute for Nuclear Studies, University of Tokyo, Tanashi, Japan.

FPresent address: Swiss Institute for Nuclear Research (SIN), Villigen, Switzerland.

The University of A berta, Edmonton, Canada

Boston University, Boston, MA.

"University of Zurich, Zurich, Switzerland.

1. F.B. Yano and S.E. Konstin, Phys. Lett. 41, 1592 ( 1978 ).

2. J.J. Lu et al., Phys. Rev. Lett. 46, 898 (1981).

3. M. Gyulessy et al., Phys. Rev. C 20, 2267 (IS79).

4. J.A. Bistirlich et al., in "High Energy Nuclear Interactions and Properties of Dense Nuclear Matter," Proceedings of the Hakone Seminar, K Nakai and A Goldhaber, eds., Vol. 1, 393 (1980).

Table l. Results from fits to the function $C_{2}\left(q_{1} q_{0}\right)=I+$ $\lambda \exp \left(-q^{2} R^{2} / 2-q^{2} T^{2} / 2\right)$.

\begin{tabular}{cccc}
\hline Description & $\lambda$ & $\mathrm{R}(\mathrm{im})$ & $\chi^{2} / \mathrm{NDF}$ \\
\hline $2 \pi^{-}$, raw & 1 & $1.84 \pm 0.27$ & $114 / 113$ \\
$2 \pi^{-}$, Coulomb Corrected & 1 & $2.98 \pm 0.30$ & $120 / 113$ \\
$2 \pi^{-}$, Coulomb Corrected & $0.86 \pm 0.07$ & $3.12 \pm 0.33$ & $116 / 113$ \\
$2 \pi^{+}$, Coulonb Corrected & $0.78 \pm 0.07$ & $3.92 \pm 0.43$ & $89 / 109$ \\
\hline \hline
\end{tabular}



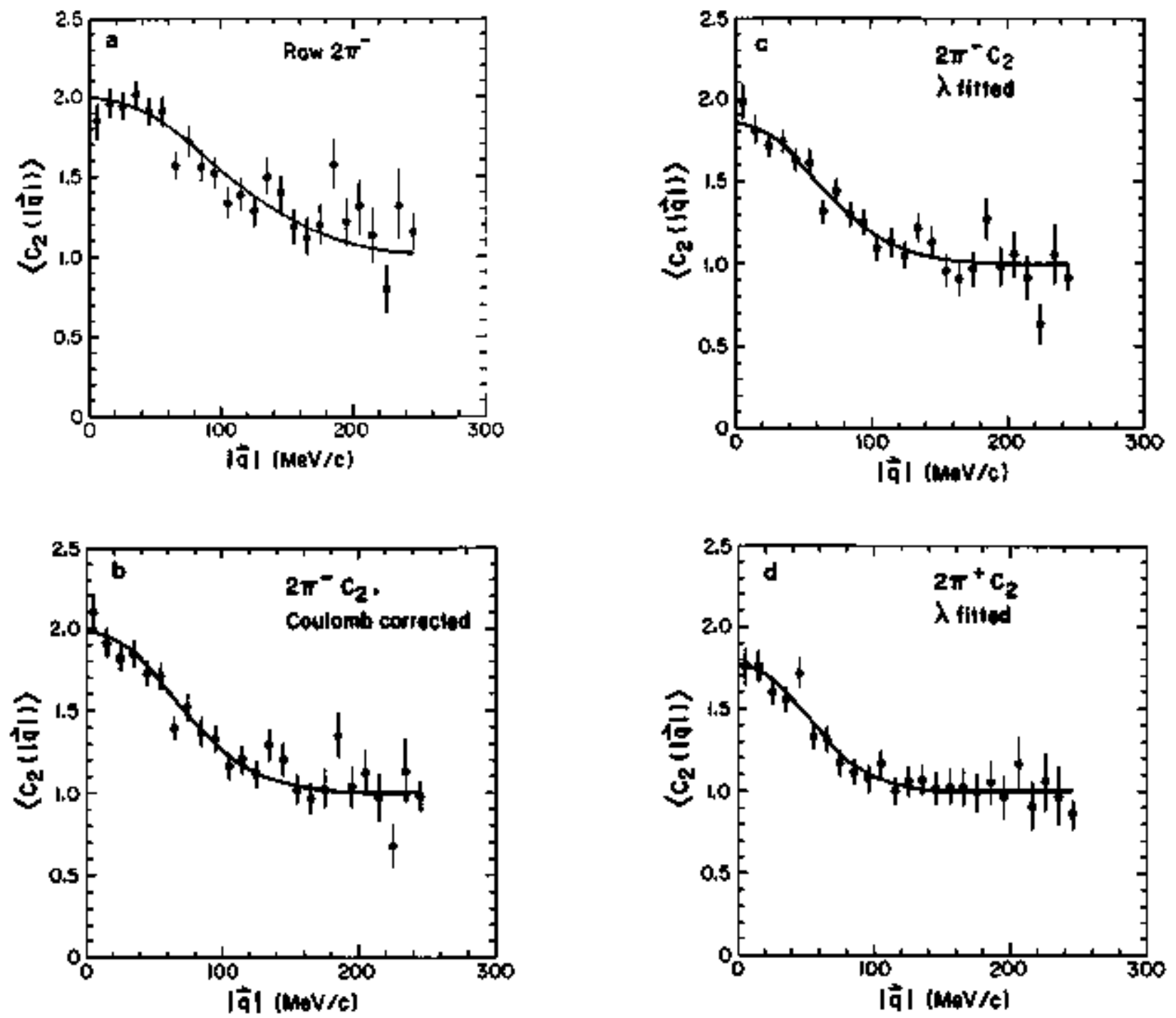

Fig. 1. Correlation Iunctions generated (rom data and fits as deseribed in lext. [(a) XBL 816-3218; (b) 816-3219; (c) 816-3220; (d) 816-3221] 


\title{
Space Structure of a Fireball and the Pion Radius*
}

\author{
T.F. Hoang, Bruce Cork, and H.J. Crawford
}

One of the fundamental problems concerning Landauts hydrodynamical model of meson production ${ }^{1}$ is the spacetime structure of the fireball. That this property can actually be investigated by means of the Hanbury-Brown and Twiss (HBT) effect ${ }^{2}$ has been suggested by several authors. Its application was first made to investigate the diameter of a radiostar and now is used to study the $\pi-\pi$ correlation of multiparticle production in high energy physics. There exists a wealth of data on the measurements of the firebal] dimensions for a variety of reactions covering a wide range of energies, measurements made on the basis of the HBT effect. In this paper, we interpret these messurements in terms of Landau's model and describe a method to estimate the pion radius from the fireball radius.

Our idea is based on the assumption that the fireballs (albeil prematter) produced in, say, high energy collisions undergo a stage of expansion and then break up and that this last stage take place when the volume of a fireball becomes equal to that constituted by the mesons it emits. Acoording to this view, the radius $R$ of a fireball is related to the pion radius $\mathbf{r}_{\mathbf{q}}$ by the simple geonetrical relation:

$$
R=n^{1 / 3} r_{\pi}
$$

n being the number of the emitted mesons, charged as well as neutral.

We have estimated the pion radius using available data on the fireball radius. As our method of estimating $r_{\text {. }}$ is different, it is interesting to compare those measurements with our results. For this purpose, we have plotted in Fig. I our values of $\mathrm{r}_{*}$. The overall average

$$
r_{x}=0.76 \pm 0.20 \mathrm{fm}
$$

is consisient with previous results, within large statistical errors of our estimates. In this regard, it should be mentioned that all the data used in the present analysis, except the case of $\mathrm{pP}$ at $28.5 \mathrm{GeV} / \mathrm{c}$. came from bubble chamber experiments. It would be interesting to have high statistics data in the future so that we can attempt to compare more accurate estimates of $r_{\pi}$ with values determined by other methods, and to investigate if there is actually any dispar. ity of results from different methots as pointed out by Chou. ${ }^{3}$

Bearing in mind that we have applied the method in its simplest form, we mention further refinements to be made, for instance, by talong properly into account the

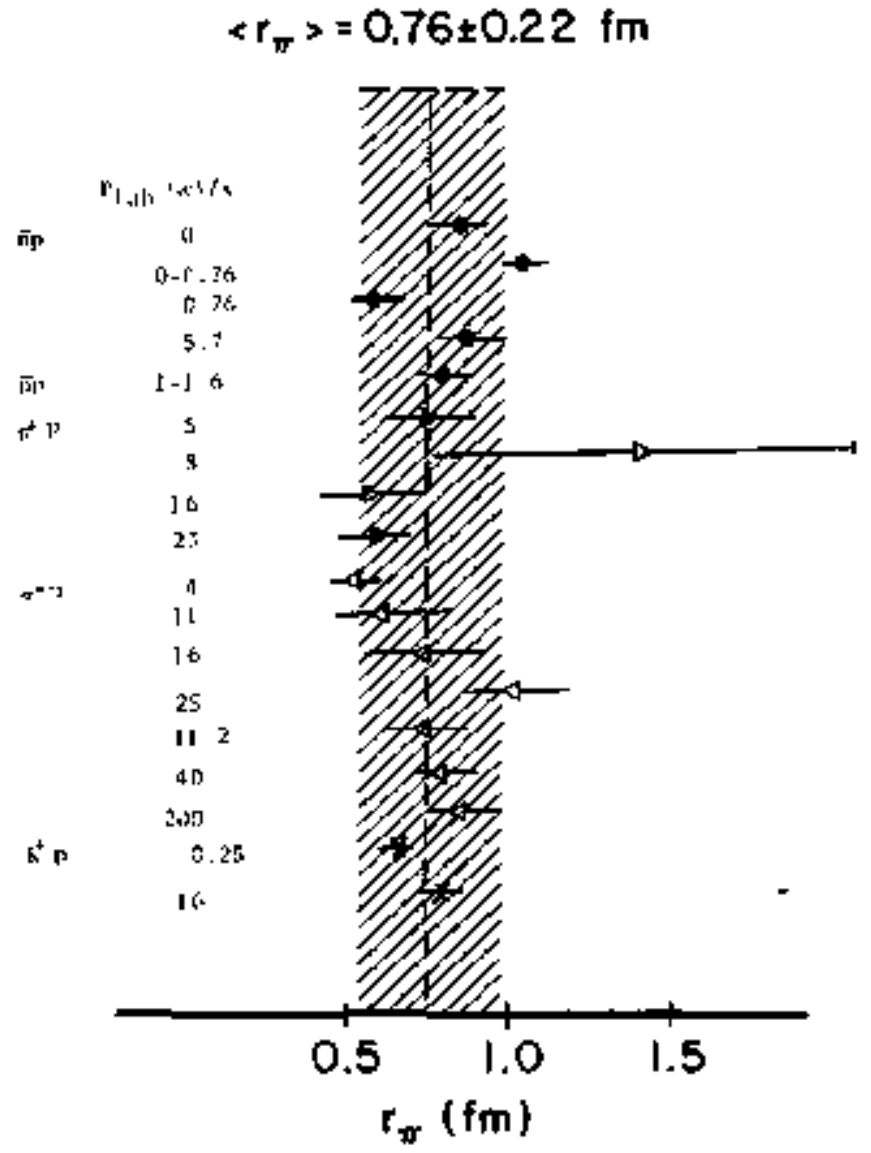

Fig. I.

(XBL 817-10572)

decoherence factor. Furthermore, if events from different channels are used for measuring $R$, accounl should be taken of appropriate weights of these channels in order to estimate the correct number of ins involved in the fireball.

\section{Footmote and References}

"Condensed from LBL 12362.

1. L.D. Landau, Iz*. Akad. Nauk. SSSR, ser. fiz 17, 51 (1953).

2. R. Hanbury-Brown and R.O. Twiss, Phil. Mag. 45, 663 (1954).

3. See, e.g, T.T. Chos, Phys. Rev. D 19, 3327 (1979), $r_{K}=0.54 \pm 0.14 \mathrm{fm}$ and $r_{*}=0.61 \pm 0.03 \mathrm{rm}$. 


\title{
Two-Pion Correlations in Central Collisions of $1.8 \mathrm{GeV} / \mathrm{u}{ }^{40} \mathrm{Ar}$ on $\mathrm{KCl}$
}

\author{
R. Brockmann," J.W. Horris," A. Sondovil, H. Strobele," R Stack, ${ }^{*}$ M. Maier. ${ }^{\dagger}$ \\ R.E. Renfordt, J. Miller, ${ }^{\ddagger}$ H.G. Pugh, M. Raft, LS. Schroeder," P. Riess," \\ K. Wolf, A Dacal, and M.E. Ortiz
}

We have analyzed part of our data of $1.8 \mathrm{GeV} / \mathrm{u}^{40} \mathrm{Ar}$ on $\mathrm{KCl}$ taken at the streamer chamber in terms of intensity interlerometry of the Hanbury+Brown-Twiss type (Rer. 1 and lilerature therein).

The experimental correlation function used is defined as

$$
C_{2}(\mathrm{q}, \Delta E)=\frac{\rho_{2}\left(\mathrm{q}_{1} \Delta \mathrm{E}\right)}{\rho_{1}\left(\overrightarrow{\mathrm{p}}_{1}, \mathrm{E}_{1}\right) \rho_{2}\left(\overrightarrow{\mathrm{p}}_{2}, \mathrm{E}_{2}\right)}
$$

with $q=\left|\bar{\beta}_{1}-\bar{p}_{2}\right|, \Delta E-\left|E_{1}-E_{2}\right|$. The correlated pion pair density $p_{2}(q, \Delta E)$ is directly available from the data, whereas the background of unconrelated pairs (i.e., the product of single particle pion densities) has been constnucted by mixing pions from different events with the same multiplicity, then calculating $\rho_{2}^{\text {back }}(q, \Delta E)$. Only events with $\pi^{-}$multiplicities $n_{-} \geq 4$ have been used in the analysis. The monenta of the correlated pairs have been orrected for their mutual Coulomb repulsion. Figure I shows the two-dimensional correlation function; one sees the enbancement towards $q$ and $\Delta E \rightarrow 0$.

Under the assumption of a Gaussian density distribution in space and time of the pion source, the correlation function can be parameterized as

$$
C_{2}(\mathrm{q}, \Delta \mathrm{E})=1+\operatorname{\beta exp}\left(\mathrm{q}^{2} \mathrm{R}^{2} / 2-\Delta \mathrm{E}^{2} \mathrm{~T}^{2} / 2\right)
$$

with $\mathbf{R}$ giving the space, $\mathbf{T}$ the time dimensions, and $\beta$ the degree of coherence of the source. This function has been fitted to the experimental correlation function in the range $0 \leq \mathrm{q} \leq 150 \mathrm{MeV} / \mathrm{c}$ and $0 \leq \Delta \mathrm{E} \leq 150 \mathrm{MeV}$. A projec- tion of the data and the best fit is shown in Fig. 2. Since half of the $\mathrm{q}-\Delta \mathrm{E}$ space is kinematically forbidden, $\mathrm{i}$.e., $q=0$ implies $\Delta E=0$, the correlation function is very insensitive to the $T$ parameter. The values obtained from the fit are

$$
\begin{aligned}
& R=6.7 \pm 1.5 \mathrm{fm} \\
& T \leq 4 \mathrm{fm} / \mathrm{c} \\
& \beta=1.6 \pm 0.3
\end{aligned}
$$

For comparison the radius of a fused ${ }^{40} \mathrm{Ca}+\mathbf{K}$ aysiem at normal density is $\$ .6 \mathrm{fm}$ in the liquid drop model. The intercept $\mathrm{I}=\mathrm{J}+\beta$ is higher than the value expected for Boston emission by an incoherent source $(I=2)$. However, the present preliminary data are still affected by a systematical error cansed by a mismatch of the tracks in the different viets, an effect that specifically occurs with close tracks (small $4 p$ ) and may account for a $10 \%$ overestimate of $g$. We are now systematically eliminating this error and measuring the pairs of another 3000 events to check conclusively whether or not I exceeds two, a possibility that was recently discussed by Gyulassy. ${ }^{2}$

\section{Footnotes and References}

'GSI, Darmstad, West Germany.

†Universităt Marburg. West Germany.

LBL, Berkeley.

SUniversität München, West Germany.

IArgonne National Laboratory.

IJUNAM, Mexico.

I. J.A. Bistirlich et al., Proceedings of the Hakone School, Vol. I, 343 (1980).

2. M. Gyulassy, LBL-12389 (1981). 


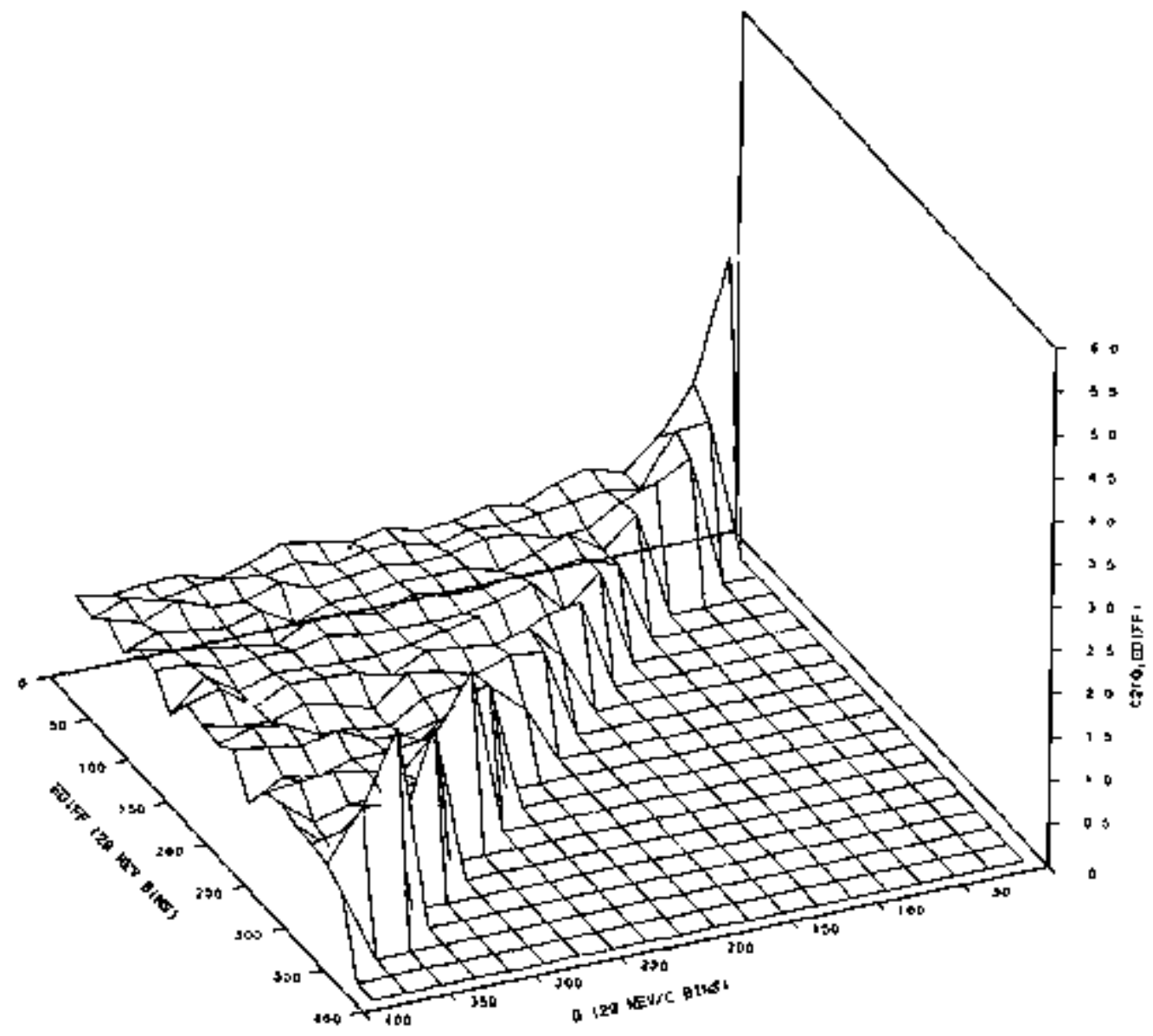

Fig. 1. Two-dimensional correlation function $C_{7}(q, \Delta E)-$ $\rho_{2}^{\text {oft }}(\mathrm{q}, \Delta \mathrm{E}) \rho_{2}^{\text {back }}(\mathrm{q}, \Delta \mathrm{E})$.

(XBL 818-11280)

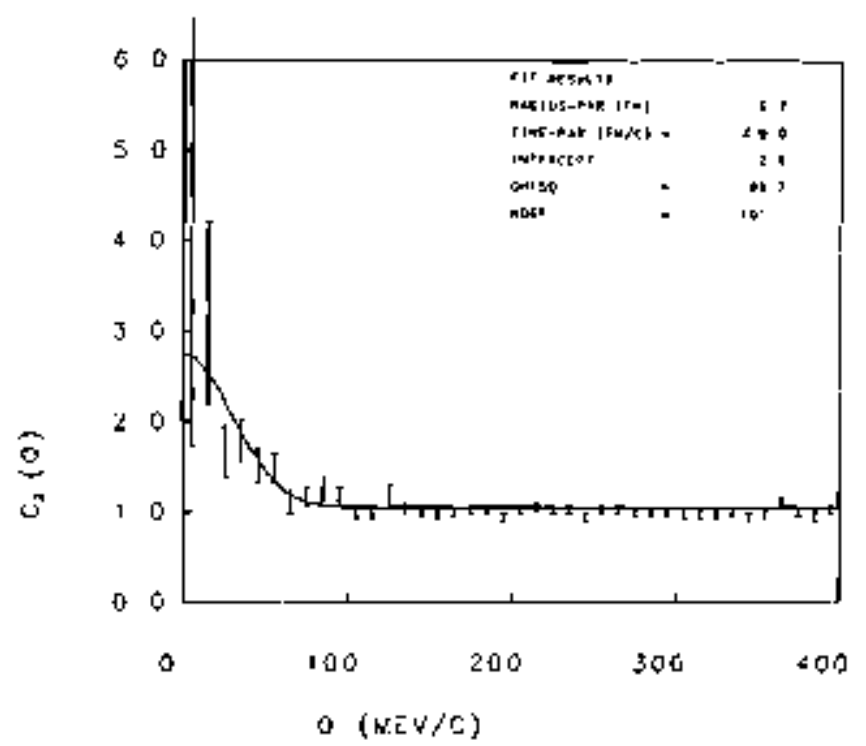

Fig. 2. Projection of $C_{2}(q, \Delta E)$ on the $q$ axis with the maximum likelihood fit. The fit parameters are shown in the insert. (NDGF - number of degres of Jreedom.) 


\title{
Correlations Between Coincident Fragments in Relativistic Heavy-Ion Reactions
}

\author{
E.P. Steinberg. * M.S. Freedman," H.H. Gutbrot, D.J. Henderson," \\ S. B. Kaufmon, ${ }^{*}$ M.R Mater. J. Péter, H.G. Ritfer, ${ }^{\dagger}$ H. Stelzer, ${ }^{\dagger}$ \\ A.I. Worwick, F. Weik, H. Weiman, and B.D. Wlkths"
}

The interactions of $5 \mathrm{CeV}$ prolons and ${ }^{4} \mathrm{He}$ ions, and $5.42 \mathrm{G} \div \mathrm{V} 20 \mathrm{Ne}$ ions with ${ }^{197} \mathrm{Au}$ have been investigated in experiments at the LBL Bevalac. Messes, kinetic energies, and angular correlations between coincident heavy $(A)$ 12) fragments were measured along with the associated multiplicity of fast charged particles.

Two arrays of silicon surface-barrier detectors, one of 12 deloctors, the other of 16 detectors, were mounted ans tither side of the beam to measure the kintic energies and masses of coincident fragments by time of tight. The start signal was providexd by tho gas avalancbe counters. The number and engular distribution of energetic, light charged particles (e.g., $\mathbf{T}^{ \pm}, \mathrm{H}^{+}, \mathrm{He}^{++}$) emitıod al angles greater than $9^{\circ}$ to the beam wore measured in coincidence by an array of 80 plastic scintilators culside a thin-walled, 1meter diameler, spherical chamber. Particles emitted at straller angles were measured by a $2 \mathrm{~m} \times 2 \mathrm{~m}$ plastic wall of 60 pairs of scintillators located about 6 meters downstream of the chamber. Although the analysis of the extensive dat obtained in these experiments is not yet complete, the results to date provide further evidence and delails of an unusual nuclear breakup mechajusm ficst observed with protons at the Argone $Z^{\prime} \mathbf{S}^{\prime}$ and the importance of the total projectile energy, rather than the energy per nucleon, in determining the reaction mechanisms. ${ }^{2}$

The geometrical arrangement of the two arrays of fragment detectors used for mass meesurement covered the region around a correlation angle of $180^{\circ}$, thus emphasizing events with low momentum transfer from the beam. The sum of the two fraginent rasses, $M_{1}=M_{1}+M_{2}$, is a measure of the extent of discuption of the target. The distributions in $\mathrm{M}_{t}$ for projectiles of protons, ${ }^{4} \mathrm{He}$, and ${ }^{20} \mathrm{Ne}$, all of $5 \mathrm{GeV}$ kinetic energy, show a peak at $\mathrm{M}_{1}=$ $160-180^{\circ}$, with a broad distribution down to low masses (Fig. 1). The peak is due to binary fission of the neartarget sesidues which result primarily from peripheral collisions in which litule energy and momentum are transferred. The ovents of much lower total mass probably arise from more nearly central collisions in which extensive disnuption of the target occurs.

The angular correlation between these fragments provides another way to characterize difierent kinds of auclear breakup. As shown in Fig. 2, the correlation is relatively narrow for events with $M_{t}>160^{\circ}$, with a most probable angle a few degrees forward of $180^{\circ}$, as expected for the binary fission mechanjsm. The distribution broadens for lowtr values of $M_{t}$ and is essentially flat for $M_{1}<100$.

For $M_{4}<\rightarrow 150$, a large fraction of the events occur with a total Iragment kinetic energy considerably greater

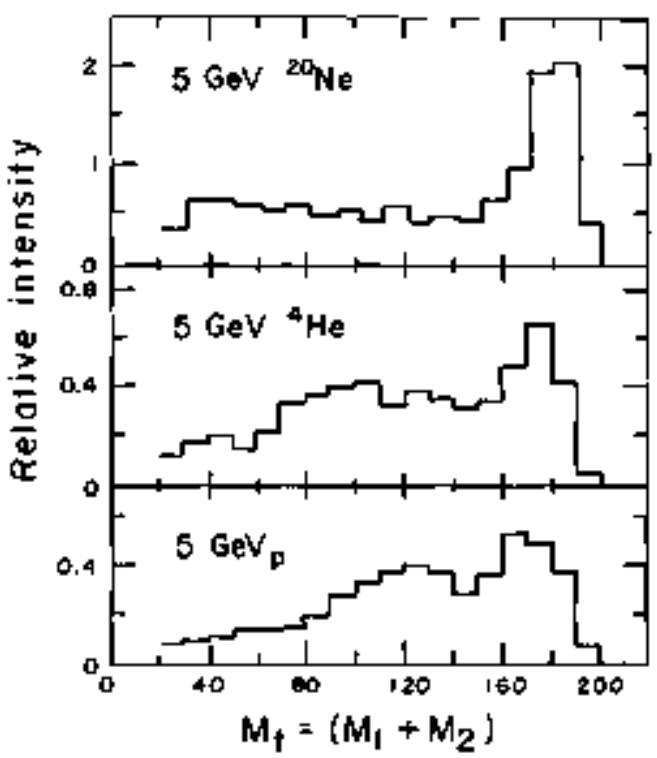

Fig. 1. Coincident fraginent mass distribution.

(XBL 8]8*1154A)

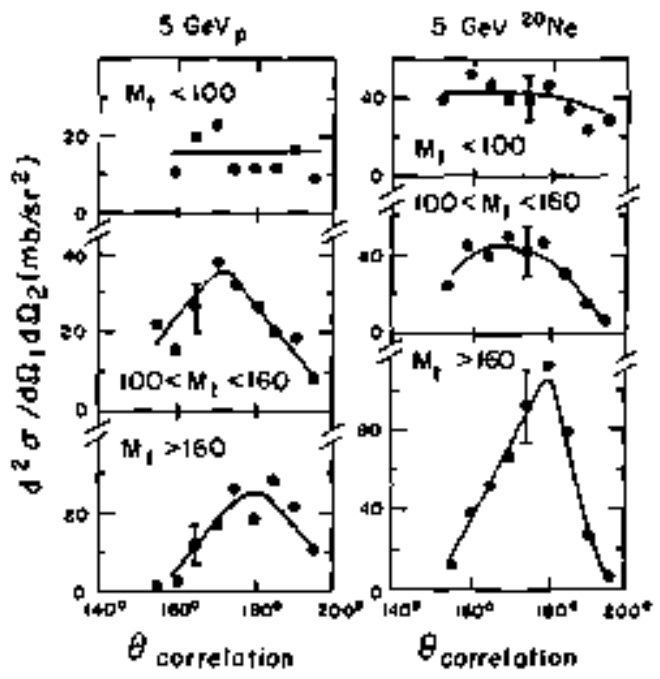

Fig. 2. Angular correlation of coincident fragments.

(XBL 81\&-1 154B) 
than that expected far a fission mechanism. This suggests that the nascent fragments were closer logether at the time of breakup than in a normal, deformed fission configuration.

Correlated light charged perticle multiplicity distributions depend on the total mass of the coincident fragment pairs. For total fragment masses $>160$, the distribution peaks at lon multiplicity, consistent wilh a fission mechantism. For lower $M_{2}$, the distributions show an increasing probability of high multiplicity associated with the binary events. The distributions scale with the total projectile energy rather then the energy per nucleon, a feature cornmon to a number of aspects of these reactions. ${ }^{2}$

These studies have dernonstrated the complexity of the breakup of a heavy nucleus by relativistic projectiles. The features of binary events suggest a new mechanism distinct from fission, but further analysis of the data and further studies are required to establish a detailed interpretation.

\section{Faotnotes and References}

Argonne National Laboratory, Argonne IL 60439. ${ }^{\dagger}$ Gesellschaft fut Schwerionenforschung, Darmstadt, West Germany.

I. B.D Wilkins et al., Phys. Rev. Let1. 43, 1080 (1979).

2. J.B. Cumming el al., Phys. Rev. C 10, 739 (1974), Phys. Rev. C 14, I554 (1976), Phys. Rev. C 17, 1632 (1978); D.J. Morrissey, W. Loweland, and G.T. Seaborg. Z. Phys. Z 28\%, 123 (1978); S.B. Kaufman, E.P. Steinters and B.D. Wilkins, Phys. Rey. Lett. 41, 1359 (1978); W.G. Meyer et al., Phys. Rev. C 22, 179 (1980); S.B. Kaufman et al., Phys. Rev. C 22, 1897 (1980). 


\section{THEORETICAL RESEARCH}




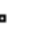




\title{
THEORETICAL RESEARCH
}

\section{Nuclear Deformation Energies}

\author{
W.J. Swiatecki and J. Blackt
}

The binding energy (or mass) of an atomic nucteus may be considered as made up of a "local" parh, akin to the energy of a drop endowed with a surface tensjon, and a "nonlocal" part (Coulomb energy, proximity energy and shell effects-see Ref. 1). The two leading terms in the local (liquid-drop) part are a shape-independent volume energy and a surfece energy proportional to the surface area of the shape in question. Together with the electrostatic energy of a uniform distribution of electric charge, these contributions represent the major part of the potential energy of a nuclear system.

Even though some of the most important features of the deformation energy of a charged drop have been adequately studied and tabulated, there are others that are still not well understood-more than forly years since the introduction of the deformable liqquid-drop model in connection with nuclear fission. The need for a better understanding and a quantitative tabulation of nuclear deformation energies becomes more acute with the increased interest in the physics of nucleus-nucleus collisions, in which a variety of configurations, not encountered in fission, make their appearance. The present work aims at alleviating this need by presenting what amounts to threedimensional tabulations (deformation-energy maps) based ㅁ a careful choice of three deformation degrees of frectom (corresponding to a mass asymmetry variable, and elongation or fragment-distance variable, and a necking or fragronent-deformation variable).

We have finished the production of 665 computerdrawn porential-energy maps togelher with 150 maps of other nuclear properties of interest. Each map refers to a fixod mass asymmetry and displays the property of interest as a function of the elongation/distence and the neck/fragment deformation variables. The maps span the range of virtually all possible systerns accessible through the collision of any two nuclei in the periodic table.

Figure 1 is one of these maps. Contours of equal deformation energy are plotted against the distance or

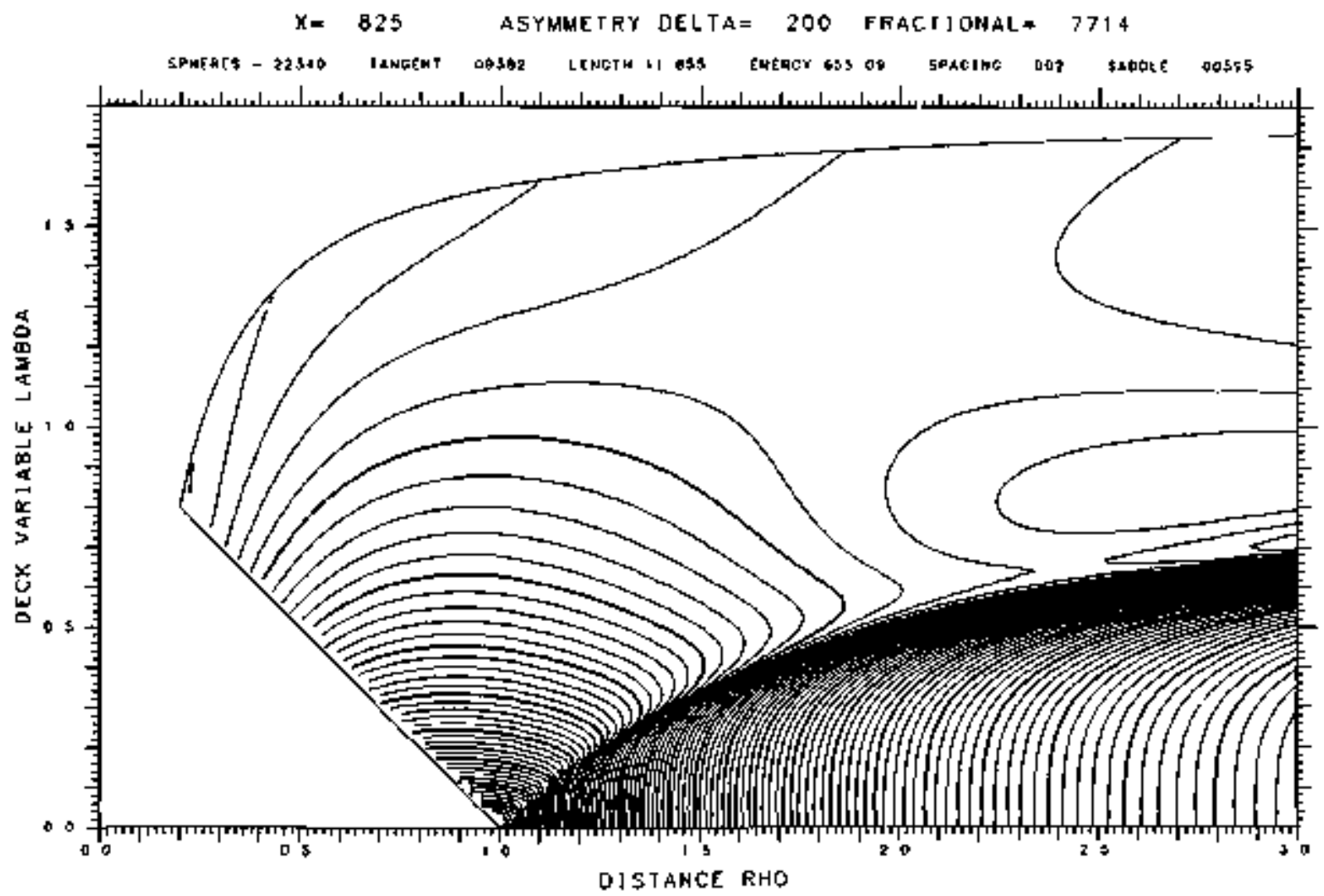

Fig. 1. One of 815 maps of nuciear properties displayed, at fixed asymunetry, as function of a distance variable RHO and a nock variable DECK. See text for a description. 
separation variable "RHO" and the fragment-deformation or neck variable "DECK" The contours are spaced at 0.002 of the surface energy of the sphere $(655.09 \mathrm{MkV}$ in this ease). The map refers 10 a mass asymunetry corresponding to the heavier fragment baving a fraction 0.7714 of the total mass. The total system has a fissility parameter $x=0.825$, corresponding to a nucleus close to ${ }^{252} \mathrm{Cf}$. Configurations of approaching spheres are along the abscissa. The spheres touch at $\mathrm{RHO}=1$. Shapes formed by portions of intersecting spheres are along the edge sloping up at $45^{\circ}$ and end up as a single sptere at the lefthand tip of the map, where the larger sphere has swallowed up the smaller one. The upper curved boundary of the map corresponds to an elongating spheroid with one tip blunted by 8 portion of a sphere. Scission configurations (in the form of two lear-drop fragments with tips is contact) are just below the ridge running from the configuration of tangent spheres at RHO $=1$ to the right-hand edge at $\mathrm{DECK}=0$. ?
An interesting feature of this map is the existence of a conditional minimum in the potential-energy landscape near RHO $=2.7$, DECK $=0.85$, where the (strongly deformed) nucleus would be in equilibrium if the asymmetry were frozen. The unfreezing of the asymmetry des. troys the equilibrium but even so, if a nuclear system could be coaxed to run into the neighborkood of this conditional equilibrium, its dynamical disintegration time might exced $10^{-20} \mathrm{sec}$ - perhaps substantially. Dynamical trajectory calculations are planned which, among other things, should help to optirmize the reaction parameters in a nucleus-nucleus oollision designed to produce rejatively long-lived composite systems.

\section{Footnote and Reference}

"Institute of Nuclear Research, Swierk, Poland.

l. W.J. Swiatecki, Prog. Particle and Nucl. Phys. 4, 383 (1980): Lawrence Berkeley Laboratory preprint LBL 8950 , March 1979.

\title{
The Effect of Deformation and the Neutron Skin on RMS Charge Radii
}

\author{
W.D. Nyers and K. H. Schmidt
}

In 1977, Angeli and Catlos compiled all the data on nuclear RMS charge radii that were available at that tims. They presented these data in a particularly interesting way that setemed to show that there were substential shell effects in the measured quantities.

Our anglysis of these data shows that most (if not all) of the apparent shell effects are due to deformations rather than actual variations in the spatial extent of the nuclear charge distribution. ${ }^{2}$ In order to establish this onjecture we have compared the measured muclear RMS charge radii with droplet model pjedictions, ${ }^{3}$ after first making an approximate correction using calculated values for the nuclear deformation." We have also compared the corrected RMS radii with liquid drop model predictions. Rather large systematic deviations remain in this case. because the model contains no provision for the influence of the neutron skin on the charge radius.

In Fig. I we have plotted the difference between the measured RMS radii and the predictions of the droplet model. Most of the plotted points lie near, but slightly below, the zero line, suggesting that the droplet model nuclear radius constant $t_{0}$ should probably be rednced by about one-half percent. At this stage it is not possible to determine whether the remaining structure is evidence for shell effects or merely due to the approximate nature of the calculated deformation corrections.

In Fig. 2 the same comparison is made, but without the corrections for deformation or for the central depres-

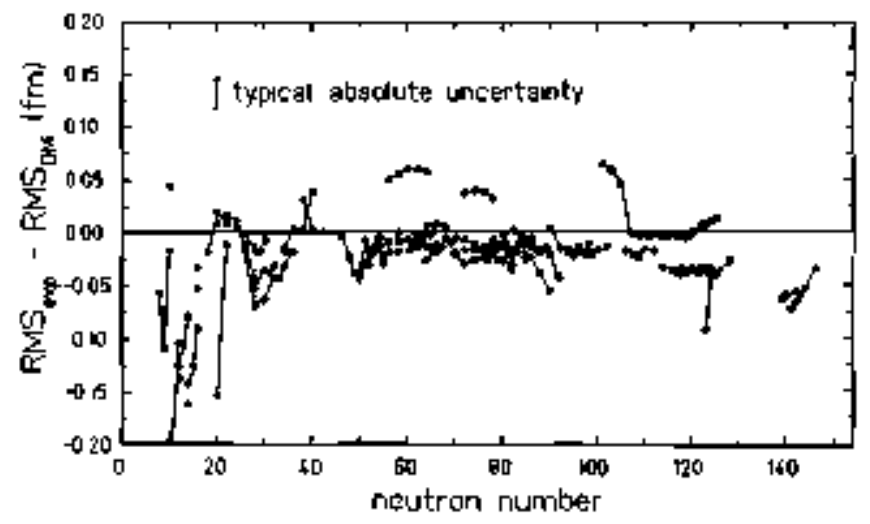

Fig. 1. The difference between measured RMS radii and the droplet model predictions.

(XBL. 815-9566)

sion. The larger mid-shell RMS radius values associated with deformations can be clearly seen. The general upward slope of the points toward heavier nuclei is expected because of the charge redistribution associated with the Conlomb repussion that creates the central depression.

Figure 3 is a comparison of measured and calculated RMS radii similar to Fig. I, except that the liquid drop 


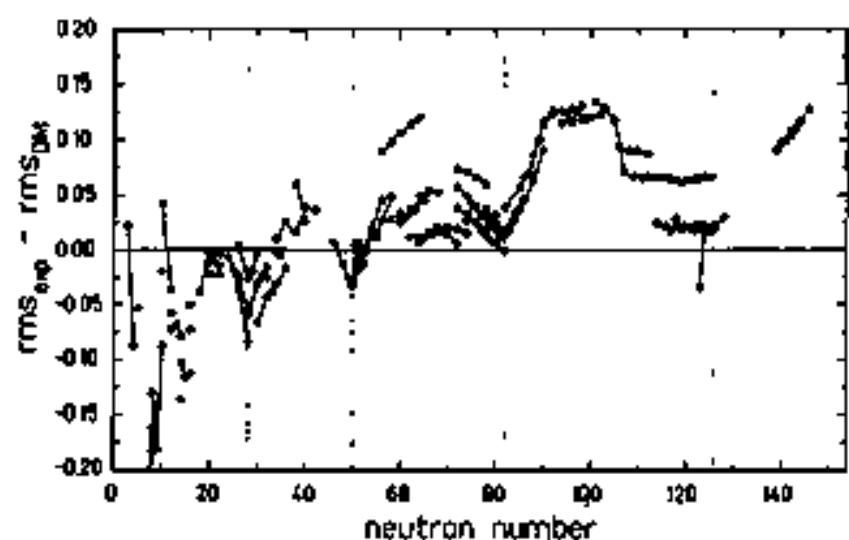

Fig. 2. The same as Fig. I but without the corrections for deformation and redistribution.

(XBL 815-9567)

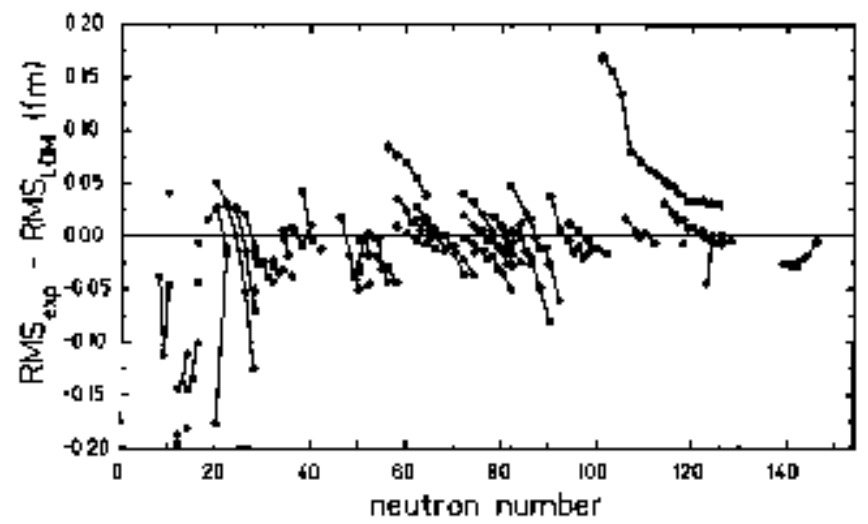

Fig. 3. The same as Fig. 1 but using the liquid model. (XBL 815-9565) model has been used instead of the droplet model. In almost every isotopic sequence the differences plotted slope steeply downward to the right becaluse the eflect of the nettron skin thickness is not included in the liquid drop model.

The three figures above storve to illustrate the impor. tance of deformations and the neutron skin in the calculation of RMS charge radij. Meticulous asce will have to be taken to assess the accuracy of the measured values and the values of the deformations that are used in the calculations before it will be possible to determine whether or not there are volume she]l effects. At present, most of remaining differences lis within the experimental uncertainty.

\section{Foof notes and References}

"Condensed from LBL-12790.

${ }^{\dagger}$ Gesellschaft für Schwerionenforschung D-6300 Darmstadt II, W. Germany

I. $\mathbf{L}$ Angelj and M. Csatlos, Nucl. Phys. A 288, 480 (1977).

2. W.D. Myers, contribution to the "XIX International Winter Meeting on Nuclear Physics," Bormio, Italy, Jan. 1980.

3. W.D. Myers and W.J. Swiatecki, Nucl. Phys. A 336, 267 (1980).

4. P. Möller and J.R. Nix, to be published in Nuct. Phys. A 363.

\title{
Surface-Layer Corrections to the Level-Density Formula for a Diffuse Fermi Gas
}

\author{
W.J. Swiatecki and J. Toket
}

Using the Thomas-Fermi treatment and the leptodermous expansion in powers of $\mathrm{A}^{-1 / 3}$, a formula was derived for the nuclear level-density parameter $a$ which corrects the conventional expression for the presence of a diffuse surface region. This appears to remove the discrepancy (of about a factor of 1.7 ) betwoen the empirical values of a/A and those that would follow from considering the nucleus to be a simple Fermi gas at constant (bulk) density. The formula for $a$ has an appearance quite analogous to the nuclear binding-entergy formula: a volume term, a surfacearea term, and a curvature correction, with a modification for the neutron excess, analogous to the symunetry energy. Thus

$$
\begin{aligned}
a & =\frac{\mathrm{A}}{14.61 \mathrm{MeV}}\left(1+3.114 \mathrm{~A}^{-1 / 3} \mathrm{~F}_{2}\right. \\
& \left.+5.626 \mathrm{~A}^{-2 / 3} \mathrm{~F}_{3} \ldots\right)\left[1-\frac{1}{9}\left(\frac{\mathrm{N}-\mathrm{Z}}{\mathrm{A}}\right)^{2}\right] .
\end{aligned}
$$

where $F$, is the area of the nuclear shape in question in units of the area of the sphere $\left(4 \pi R^{2}\right)_{\text {, and }} F_{3}$ is the integrated curvature, in units of its value for the sphere

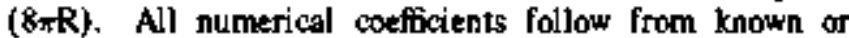
estimated nuclear properties, and agreement with the empirical trend represented by $a \simeq A /(8.5 \mathrm{MeV})$ follows without arbitrary adjustments. Without the surface and 
curvature corrections, the volume term alone would underestimate the values of $a$ by a factor of 1.7. A pocket formula which nocks up the curvature term by a slighl increase in the surface term fand disregards the small neutron-excess correction) is

$$
a=\frac{A}{14.61 \mathrm{MeV}}\left(1+4 A^{-1 / 3} F_{2}\right)
$$

Foolnole

"GSl-24-81 preprint, July 1981, Nucl. Phys. A 372 (1981)

$14 \mathrm{~J}$.

$\mathbf{T}_{\text {GSSI, Darmstadt, West Germany. }}$

\title{
Schematic Calculation of GT Strength in $N=47,49$ Isotones
}

\author{
S.G. Prussin "It Z.M. Olveira, ${ }^{\dagger}$ and J. Randrup
}

Recent masurements of Gamow-Teller (GT) strength distributions via charge exchange reactions on ${ }^{90,91,92,98 \mathrm{Zr}}$ have suggested that an important fraction of the rolal strength lies well below the structureless giant resonance bypothesized by lkeda, Fujii and Fujita. ${ }^{3}$ This Jow-Jying strength will effect substantial modifications to the decay properties of far unstable neutron-rich nuclei as calculated with the gross theory of $\beta$-decay. ${ }^{2}$

The most striking feature of the strength distributions found from the charge exchange reactions is that the envelope of the strength appears to be divided into two rather distinc1 peaks. For ${ }^{\$ 0} \mathrm{Nb}$, data from both $(\mathrm{p}, \mathrm{n})$ and ( ${ }^{3} \mathrm{He}, \mathrm{t}$ ) reactions locate one peak near $8.4 \mathrm{MeV}$ where the GT giant resonance is expecied. The other peak, centered somewhat bejow $2 \mathrm{MeV}$ and containing $20-25 \%$ of the obstrved strength, is found in the energy range where calculations based on the simple shell model with pairing locate low-lying configurations that would be populated by GT decay of ${ }^{9} \mathrm{Zr}$ if it were energetically allowed. Qualita. tively similar results were oblained from the $92,94 \mathrm{Zr}(\mathrm{p}, n)$ reactions. For both cases, the separation in energy of the two peaks was found to be roughly the same as seen in the ${ }^{90} \mathrm{Zr}$ reaction data.

In a previous publication ${ }^{3}$ we showed that these fealures are well reproduced by schemalic RPA calculations using as residual interaction the isospin-flip part of $\vec{x} \vec{\sigma} \cdot \vec{\sigma} \vec{r} \cdot \vec{r}$ to which the GT strength distribution is esper cially sensitive. ${ }^{4}$ The latter, the so-colled GT force, induces correlations amongst configurations formed in varjous $\mathrm{n} \Rightarrow \mathrm{p}$ transitions and for neutron rich nuclei it has the main effect of shifting the $\beta^{-}$-strength to higher energies. From the calculations of GT strength in $90,92,94 \mathrm{Nb}$, we found that in all cases about $25 \%$ of the sum rule strength was located in the lower peak and a principal effect of addition of neutron pairs was to increase the dispersion of strength at lower energies withoul much effect on the splitting between the peak eentroids.

Since, to a first approximation, ${ }^{0} \mathrm{Zr}$ can be considered as a doubly magic nucleus, the insensitivity of the GT strength distribution to addition of neutron pairs beyond the $\mathrm{N}=50$ shell leads naturally to the question of the effects produced by removal of neutron pairs as well as those due to addition or removal of proton pairs from the $Z=40$ core. To examine such effects, in parh, we have calculated the $G T$ strength distributions in the $N=47,49$ jsotones of $\mathrm{Rb}, \mathrm{Y}$ and $\mathrm{Nb}$ that would be probed in charge exchange reactions on the respective $\mathrm{Kr}, \mathrm{Sr}$ and $\mathrm{Zr}$ targets. The ealculation is based on the model developed in Ref. 4.

All of the distributions are remarkably similar and qualitatively the same as found for the heavier $\mathrm{Nb}$ isotopes. ${ }^{3}$ The highest-energy transitions are all located near the strength centroid predicted by the gross theory and are well separated from the remaining strength.

The main part of $\beta$-strength with magnitude corresponding to $\mathrm{Bg}_{9 / 2}$ neutron decay resides in the highenergy peak, as expected, whereas the fraction of strength in the low-energy peak is roughly that associated with 2p neulron decay. This feature can be understood qualitslively from the form of the reduced matrix elements of the GT residual interaction belween the neutron-proton states $|(x v)|^{+}>$and $\left.\mid\left[x^{\prime} v^{\prime}\right)\right]^{+}>$corresponding to configurations formed in GT decay:

$$
\begin{aligned}
& \left\langle\left.\left.(x)\right|^{+}\left|\mathrm{V}_{G T}\right|\left(x^{\prime} v^{\prime}\right)\right|^{+}\right\rangle=
\end{aligned}
$$

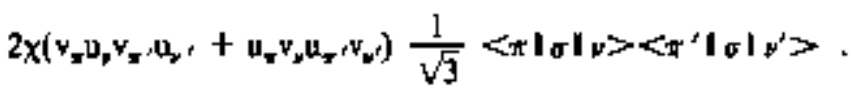

The pairing factors $u$ and $v$ account for the reduction of specific $\pi \leftrightarrow p$ transitions relative to the pure shell model. The reduced single-particle matrix elements of the spin operator are roughly proportional to the square root of the orbital angular momentum of the nucheons involved, $\left\langle\right.$ whely $>\sim e^{1 / 2}$. Consequently, the GT mixing will tend to be strongest between those configurations which contain nucleons with bigh angular momentum, as long as the pairing factors are nol too small. In the shell-model-pluspairing limt, abowt $85 \%$ of the sum rule strength in ${ }^{90} \mathrm{Nb}$ is due to the 1ransitions $\nu \mathrm{gg}_{g / 2} \rightarrow \pi \mathrm{g}_{g / 2}$ and $\nu \mathrm{g}_{/ / 2} \rightarrow \pi \mathrm{g}_{7 / 2}$ while the remainden arises from the decay of 2 p neutrons. In this approximation all transitions lead to $\beta$-strength in the low-lying peak except for the $\mathrm{yg}_{9 / 2} \rightarrow \mathrm{mg}_{7 / 2}$ decay which would correspond to the GT resonance. As a result 
of the residual interaction, the strength in the low-lying peak is substantially reduced and the remainder has a magnitude equivalent to that from pair scaltering plus aboul 1/4 of that from the $\mathrm{rg}_{9 / 2} \rightarrow \mathrm{Ig}_{9 / 2}$ transition. The increase in the low-lying strength as proton pairs art removed fron the $Z=40$ core can thus be traced to the relatively weak interaction between the states formed by p-neutron decay and that corresponding to the GT resonance. On the other hand, the increased total $\beta$-strength as neutron pairs are added to the $d_{5 / 2}$ orbitals in $92,94, \%$ more strongly affected by the residual interaction and leads to the constancy of the fraction of strength seen in the low-lying peak.

Notwithstanding the schematic nature of the calculations, it is expected that the main effects of residual interactions on the $\beta$-strength functions are contained in the $G T$ interaction as the remainder is expected to produce rather uncorrelated effects. Thus, apart Irom the extent of dispersion about centroids, the calculations reported here and in Rer. 3 suggest that the relative weakness of the spin-isospin part of the residual nuclear interaction and the strong spin-orbit splitting of the Ig proton orbitals conspire 1o produce an overall distribution with much more strength in the important low energy region than expected from the simple giant resorance idealization.

\section{Foornotes and References}

"Institut für Kernchemie, Universität Mainz, D-6500 Mainz, West Germany.

tDepartment of Nuclear Enginecring, University of CaljJornia, Berkeley.

1. K. Jkeda, \$. Fujii and J.I. Fujita, Phys. Lell. 3, 271 (1963).

2. K. Takz̧hashi, M. Yamada and T. Kondoh, Atomic Data and Nucl. Data Tables 12, 101 (1973).

3. S.G. Prussin and Z.M. Oliveira, Nucl. Phys. A 339, 503 (I980).

4. J. Randrup. Nucl. Pbys. A 207, 209 (1973).

\title{
Particle Angular Momentum Alignment Effects in Deformed Nuclei ${ }^{*}$
}

\author{
L.K. Peker, ${ }^{\dagger}$ J.O. Rasmussen, ${ }^{\ddagger}$ and J.H. Hamilon
}

Systematic examinations of the angular momentum generated by particle alignment were made for $\mathrm{Dy}$ and $\mathrm{Er}$ isotopes. Marked deviations are observed from the aligned spin values expected for full alienment of $i_{23 / 2}$ (odd A) and $\left(i_{13 / 2}\right)^{2}$ (even $A$ ) particles.

The suddes thanges in the monentum of inertia, back-bending in yrast exscades in eves-tyen rare earth nuclei, are now generally undersiood in terms of the crossing of the ground-rotational band (GRB) with a rotational aligned band built on $a(j \geq 9 / 2)^{2}$ configuration Slockholm band (SB). One of the more important characteristics of this band is the magnitude of the alignment $I_{0}$-the projection of the intrinsic angular momentum of both unpaired aligned particles on the axis of rotation.' Recently Bohr and Motelson ${ }^{2}$ proposed a way to extract the information on $\mathrm{I}_{0}$ from an analysis of experiments dats on the function I - J ( $\omega)$, where the angular frequency how $=\partial \mathrm{E}(\mathrm{J}) / \partial \mathrm{d} \cong 1 / 2 \mathrm{E}_{\gamma}(\mathrm{I}+\mathrm{I} \rightarrow \mathrm{I}-\mathrm{J})$.

Because of the importance of the effect of Coriolis alignment for the understanding of the nature of high spin collective states, we analyze the proposed Bobr and Mottel$\operatorname{son}^{2}$ interpretation of the quantity $i_{\alpha}(\omega)=l_{\mathrm{sp}^{(}}(\omega)-$

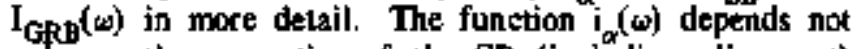
onty on the properties of the SB (including elignment) reflected in the function $\mathbf{I}_{\mathrm{SB}}$ ( $\omega$ ), but by definition depends on the properties of the GRB reflected in the function $\mathrm{I}_{\mathrm{GRB}}(\omega)$ and as well on the strength of coupling of these two bands, $\simeq \mathrm{h} / \Delta E$ (l). In thís paper we consider the possible effects on these last two factors on the magnitude of $i_{\alpha}(\omega)$.
First of all the GRB and the SB have a very different dependence on I. For a more clear qualitative illustration of these differences, we consider another simplified approximation of the expansion, ${ }^{3}$

$$
\mathbf{E}_{\mathrm{ror}}=\sum_{\mathrm{i}} \mathbf{A}_{\mathrm{j}}[\mathbf{R}(\mathbf{R}+\mathbf{l})]^{\mathrm{j}}
$$

where $R=I-I_{b}$, and $I$ is the total angular momentum of the rotational state: which is

$$
E_{\text {tor }}=A_{1}[R(R+1)]-A_{2}[R(R+1)]^{2},
$$

where $A_{2}$ characterizes the softness of a nucleus. From Eq. (2) it follows that the two leading terms of $\mathrm{E}_{\gamma}(\mathrm{I})$ are

$$
E_{\gamma}(1)=4 A_{1} R-8 A_{2} R^{3}+\ldots .
$$

Usually $A_{2} / A_{1}=10^{-2}-10^{-3}$ (Ref. 3) and at low $R$ the influence of the first term is dominant. But with the increasing of $R$, the influence of the second term on $E_{\gamma}$ increases very fast. For the rotational states with the same 1, $R_{\text {GRB }}>>R_{\mathrm{GB}}$ since $R_{\mathrm{GRB}}=\mathrm{l}$ and $\mathrm{R}_{\mathrm{SB}}=\mathrm{I}-\mathrm{I}_{0}$ $\left(I_{0}=12\right.$ for $\left.i=13 / 2\right)$. Therefore, the energies of the GRB at a given I are much mort strongly affocted by the second and highter order terms in Ess. (1) and (2) than are the energies in the SB at the same l. Thus, we can expeet that in the GRB as I increases, $E_{\gamma}$ increases not as fast as 
in the SB, and the I $=\mathrm{I}_{\mathrm{GRB}}(\omega)$ curve up bends faster than the $\mathrm{I}=\mathrm{I}_{\mathrm{SB}}(\omega)$ curve.

Detailed examination of some Dy and $\mathrm{Er}$ isotopes shows that the magnilude of the "aligned angular momentwm," $i_{\alpha}^{c-c}(\omega)$, introduced by Bohr and Motel $\operatorname{son}^{2}$ reflects not only the real alignment in $\mathrm{SB}_{1}$ but also the properties of the GRB and the strength of the mixing of the GRB and $S_{1}$. Thus, $i_{\alpha}$ is not a necasure of the real alignment of the SB.

Footnoter and References

'Condensed from Phys. Lett. B 91, 365 (1980).
${ }^{\dagger}$ NDDC. Brookhaven Netional Laboratory, Upton, LI, NY, USA

tChemistry Department, University of California

PPhysics Department, Vanderbilt University, Nashville, TN, USA.

1. F.S. Siephens and R. Simon, Nucl. Phys. A 183, 257 (1972).

2. A. Boht and B.R. Mottelson, Suppl. J. Phys. Soc. Japan 44, 157 (1978).

3. A. Bohr and B. Mottelson, Nuclear Structure, Vol. 2 (Benjamin, New York, 1975).

\title{
Excitation of Shape-Vibrational Modes in Nuclei by Relativistic Heavy Ions"
}

\author{
J.O. Rasmussen, J.S. Blairt and X.J. Qiu
}

\section{Overview}

Data on excitation of collective $2^{+}$and $3^{-}$states in relativistic $(\geq 250 \mathrm{MeV} / \mathrm{N})$ heayy-ion collisions are reyiewed. Our theoretical approach uses the difluse-edge diffraction model. We use a microscopic model to relare the elemental nucleon-nucleon total cross section to the nuclear transparency perameters for the diffraction model. The integrated inelastic cross sections are approximately inversely proportional to the thickness of the difluse partial transmission regions. Conlonb excitation of giant quadru. pole states in the projectile appears to be negligible in comparison to the excitation produced by nuclear interactions.

\section{Diffraction Model}

Let us now apply the shadow diffraction scattering model of Refs. 1 and 2 . In this model inelastic scattering to isoscalar shape vibrational states increases with the strength function $\delta_{2}^{2}$ (the square of the nuclear radius tims the zero-point amplitude $\beta_{L}$ of the $2^{\mathrm{L}}$ pole vibration). The differential crass section is expressible in terrns of Bessel functions, the period of the diffraction oscillations being inversely proportional to the product of wave number and muclear radius. The fall-of of the envelope with angle is slow for a sharp absorption shadow surface and more rapid the more diffuse the shadow.

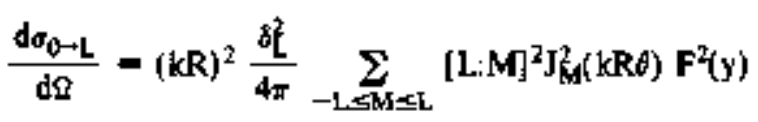

In this formula, $\delta_{2}\left(=\beta_{\mathrm{L}} R\right)$ is the familiar deformation length, $\mathrm{k}$ is the wave number for the relative motion of the projectile and the target, $R$ is the strong absorption radius, and

$$
\begin{aligned}
& {[L+M]=} \\
& \left\{\begin{array}{cl}
i^{L} \frac{[(L+M) !(L-M) !]^{1 / 2}}{(L+M) ! !(L-M) ! !} & , \text { for }(L+M) \text { even } \\
0 & , \text { for }(L+M) \text { odd }
\end{array}\right.
\end{aligned}
$$

For a pertial survival amplitude s(b) of the Fermi form,

$$
\eta_{F}(b)=\left[1+\exp \left(\frac{R-b}{d}\right)\right]^{-1}
$$

so that $d$ parameterizes the difluseness of the shadow, the damping factor $F(y)$ has the form

$$
F(y)=\frac{\pi y}{\sinh x y}
$$

with $y=$ kdo.

This differential cross section can be integrated numerically ower angle to obtain the total inelastic cross section, of for the transition in question. Alternatively, an approximate analytic expression has been found ${ }^{3}$ for $\sigma_{L}$ when the Bessel functions are approximated by their (sinusoidal) asymplotic forms

$$
\theta_{\mathrm{L}}=\frac{\delta_{\mathrm{L}}^{2}}{12} \frac{\mathrm{R}}{\mathrm{d}}
$$

Comparison to values obtained by numerical integration shows thet this formula is amazingly accurate. The exact yalues can be written as the product of the cross section given by $E q$. (3) times a factor $G_{L}$ which is a slowly warying function of the ratio $d / R$ ano $L$ For $d / R=0.05$, a 
value typical of low energy a scattering, $G_{1}$ drops from unity to 0.9 as $L$ varies from 0 to 5 . For the more diffuse shadows, $d / R=0.10$ and $0.15, G_{1}$ varies from unity to 0.7 and 0.5 , respectively, for the sane range of $L$. In sultsequent numerjeal work, the factor $G_{\mathrm{L}}(\mathrm{d} / R)$ is taken to acoount.

Let us examíne diffuseness values frour various inelastic scattering data such as for ${ }^{12} \mathrm{C} \cdot{ }^{40} \mathrm{Ca}$ collisions at $0.4 \mathrm{GeV} / \mathrm{N}$. The inpuls to this latter estifnate have been the observed ${ }^{4}$ cross section, $14.4 \pm 4.4 \mathrm{mb}$, for producing the 3.73.MeV $\gamma$ rays which result from decay of the first $3^{-}$state of ${ }^{40} \mathrm{Ca}$, values of deformation lenglhs for the particle-stable slates in ${ }^{40} \mathrm{Ca}$ oblained from anglysis of lownenergy scatlering experiments, and the $\gamma$-ray branching ratios of the particle-stable states. When the diffraction model is used for the angle-integrated cross sections to the particle stable states, one finds the ratio, $d / R=0.10 \pm$ 0.02 . With a guessed value for the radius parameier, $R=$ $6.0 \mathrm{fm}$, one has $₫=0.6 \mathrm{fm}$. Perhaps more meaningrul is the quantity $3.69 \mathrm{~d}$, the distance over which the transparency changes from 0.1 to 0.9 , which is here estimated to be $2.2 \mathrm{fm}$. A similar analysis of the $\gamma^{\text {say }}$ cross section ${ }^{4}$ for $0.4 \mathrm{GeV} / \mathrm{N} \alpha$ particles on ${ }^{40} \mathrm{Ca}$ yields a value for the retio, $\mathrm{d} / \mathrm{R}=0.16 \pm 0.03$.

There are also available differential cross section data ${ }^{5}$ from the Seturne facility for $0.34 \mathrm{GtV} / \mathrm{N} \alpha$ particles scattered from ${ }^{40} \mathrm{Ca}$ The elastic cross sections are adequately Gited ${ }^{3}$ by the diffraction model with the parameters $R=$ $4.72 \mathrm{fm}$ and $d / R=0.135$ so that $d=0.64 \mathrm{fm}$ Similarly, the elastic differertial cross sections to the lowest $3^{-}$ and $5^{-}$levels are fitted rather well by Eq. (1) with the same values for $\mathbf{R}$ and d and with the deformation lengths, $\delta_{3}=1.14 \mathrm{fm}$ and $\delta_{5}-0.68 \mathrm{fm}$, respectively. Tabie I sumurarizes the deteruination of $(d / R)$ and $d$. sistent.

The derived diffuseness parameters are generally con-

\section{Microscopic Soft-Sphere Model}

Can we stimate by model calculations how the sharpness of the absorplion shadow might change with bombarding energy? Certainly the elemental nucleon-nucleon total cross section changes over the thergy renge of Table 1 . The $\sigma_{\text {tos }}$ (PP) reaches a minimum of $24 \mathrm{mb}$ near $180 \mathrm{MeV}$ and $\sigma_{\text {wo }}(\mathrm{np})$ hes a minimum of $\sim 33 \mathrm{mb}$ near $390 \mathrm{MeV}$.
Both cross sections rapidly rise beyond this energy, reaching a $\sim 40 \mathrm{mb}$ plateau around $1.0 \mathrm{GeV}$.

It is appealing now to make comparison with a microscopic model besed on experimental Pp and np scaltering cross sections, Karol ${ }^{6}$ has used Gaussian forms to approximate nuclear dersity distributions in the surface and has derived simple analytical expressions for the nuclear transparency $T(\mathbf{b})$ as a function of impact parameter $b$. Karol's equation (16) has an apparent misprint in that the left hand side $T(b)$ should actually be $-\ln T\left(b^{\prime}\right)$ and read as follows:

$$
\begin{aligned}
& -\ln T(b)=x^{2}-(E) \rho_{T}(0) \rho_{p}(0) a_{T}^{3} a_{p}^{3}\left(a^{2}+a_{p}^{2}\right)^{-1} \\
& \exp \left[-b^{2} /\left(a_{1}^{2}+a k\right)\right]
\end{aligned}
$$

where target (T) and projectile (P) nuclei have dersities of

$$
\rho_{1}(r)=\rho_{i}(0) \exp \left[-\left(r / a_{i}\right)^{2}\right]
$$

The parameter $\overline{\sigma(E)}$ is a weighted average of $\mathrm{pp}$ and $\mathrm{np}$ cross sections (his $\mathrm{Eq}$. (4)). Karol integrated to get the corresponding reaction cross section in terms of an exponential integral. (Note that factors of ten appear in his equatiens because cross sections are given in millibarns. We use ff $^{2}$ for cross sections.)

Fron the above equations we derive an expression for the diffuseness $d$ of the transmission profile.

$$
d=\frac{a^{2}}{R} \frac{1}{4 \ln 2}=0.3607 \frac{a^{2}}{R}
$$

In terms of the newly defined parameters, $R$ and $d_{r} n(b)$ ean be writlen.

The parameters $R, d$, and $d / R$ given by the "softsphere" model for the heavy ion systems being studied are listed in Table 2. In obtaining these an unnormalized Gaussian density has been adopled for ${ }^{40} \mathrm{Ca}$ which gives a reasonable fit in the surface region to the matter density inferred from elastic electron scatlering. ${ }^{16}$ Nomalized Gaussian densities are chosen for ${ }^{4} \mathrm{He}$, and ${ }^{12} \mathrm{C}$ which give the measured values' of the rms radius of the proton des-

\begin{tabular}{|c|c|c|c|c|c|c|c|c|}
\hline \multirow[b]{2}{*}{$\begin{array}{c}\text { Beam } \\
\text { Energy } \\
(\mathrm{GeV} / \mathrm{N})\end{array}$} & \multirow[b]{2}{*}{$\begin{array}{l}\text { Syatem } \\
\text { Excited }\end{array}$} & \multirow[b]{2}{*}{$\begin{array}{l}\text { Collision } \\
\text { Partner }\end{array}$} & \multicolumn{2}{|c|}{ Exciled State } & \multirow[b]{2}{*}{$\begin{array}{l}\sigma(\mathrm{exp}) \\
(\mathrm{mb})\end{array}$} & \multirow[b]{2}{*}{$\mathrm{d} / \mathrm{R}$} & \multirow[b]{2}{*}{$\begin{array}{c}\text { Diffuseness } \\
\text { Parameter } \\
\text { d(fm) }\end{array}$} & \multirow[b]{2}{*}{ Refs. } \\
\hline & & & $\begin{array}{c}E \\
(\mathrm{MeV})\end{array}$ & $\mathbf{I}_{\pi}$ & & & & \\
\hline 0.40 & ${ }^{40} \mathrm{Ca}$ & ${ }^{12} \mathrm{C}$ & 3.736 & $3^{-}$ & $14.4 \pm 4.4$ & $0.10 \pm 0.2$ & 0.6 & 4 \\
\hline 0.40 & ${ }^{40} \mathrm{Ca}$ & $\alpha$ & 3.736 & $3^{-}$ & $7.2 \pm 2.4$ & $0.16 \pm 0.03$ & 0.76 & 4 \\
\hline 0.34 & ${ }^{40} \mathrm{Ca}$ & $\alpha$ & 3.736 & $3^{-}$ & $\mathrm{d} \sigma / \mathrm{d} \Omega$ & 0.135 & 0.64 & 5 \\
\hline 0.34 & ${ }^{40} \mathrm{Ca}$ & $\alpha$ & 4.4915 & $5^{-}$ & & 0.135 & 0.64 & 5 \\
\hline
\end{tabular}
sily.

Table 1. Shadow diffuseness parameters ( $d / R$ ) and $d$ derived for the difluse-edge shadow diffraction model. 
Table 2. Mieroscopic model values of diffraction model parameiers.

\begin{tabular}{ccccccccccccc}
\hline $\begin{array}{c}\mathrm{E}_{\mathrm{Lat}} \\
(\mathrm{GeV} / \mathrm{N})\end{array}$ & $\mathrm{A}_{\mathrm{P}}$ & $\mathrm{A}_{\mathrm{I}}$ & $\begin{array}{c}\mathrm{a}_{\mathrm{P}} \\
(\mathrm{fm})\end{array}$ & $\begin{array}{c}\mathrm{a}_{\mathrm{T}} \\
(\mathrm{fm})\end{array}$ & $\mathrm{a}^{2}$ & $\begin{array}{c}\mathrm{p}_{\mathrm{f}}(0) \\
\left(\mathrm{fm}^{-3}\right)\end{array}$ & $\begin{array}{c}p_{\mathrm{T}}(0) \\
\left(\mathrm{fm}^{-3}\right)\end{array}$ & $\begin{array}{c}\sigma(\mathrm{E}) \\
(\mathrm{mb})\end{array}$ & $\begin{array}{c}\mathrm{R} \\
(\mathrm{fm})\end{array}$ & $\begin{array}{c}\mathrm{d} \\
(\mathrm{fm})\end{array}$ & $\mathrm{d} / \mathrm{R}$ \\
\hline 0.40 & 12 & 40 & 1.884 & 2.520 & 9.900 & 0.322 & 0.658 & 30 & 67.8 & 6.21 & 0.575 & 0.093 \\
$0.34-0.40$ & 4 & 40 & 1.187 & 2.520 & 7.759 & 0.430 & 0.658 & 30 & 28.9 & 5.45 & 0.576 & 0.119 \\
\hline
\end{tabular}

Comparing the "experimemtal" $d / R$ values of Table 1 to the mieroscopic "soft-sphere" model values of Table 2, we see that the "so[t-sphere" values for collisions with "Ca are of the order of the "experimental" values, though systematically smaller. The narrower transition regions of the "soft-sphere" model may be attributed to two ower simplifications of this model: (a) The unrenormalized Gaussian densily adopted for ${ }^{40} \mathrm{Ca}$ is considerably more demse in the interior than that deduced from the scallering of electrons and protons. (b) The "soft-sphere" model may be viewed as a version of the "optical limil" to the Glauber multiple scattering theory." As such it bears the deficiency of all optical limit treatments of nucleus-nucleus collisions, that to account is taken of shedowing corrections. Both oversimplifications result in greater attenuation for decreasing impact parameter than is predicted by more sophisticated theorits. ${ }^{9}$

Footnotes and References

"Condensed from Proceedings of Nuclear Physics Workshop, Drexel University, September 1980.
'Physies Department, Universily of Washington.

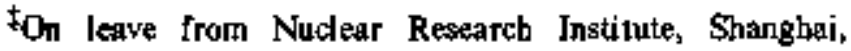
People's Republic of China.

1. J.S. Blair, Leclures in Theoretical Physics, Vol. VIIIc, eds. P.D. Kunz, D.A. Lind, and W.E. Britten (University or Colorado Press, Boulder, 1966), p. 343.

2. N. Austern and J.S. Blaí, Annals of Physics (N.Y.) 33, 15 (1965)+

3. J.S. Blair, Bull. Am1. Phys. Soc. 23, 932 (1978).

4. T. Shibata et al., Nucl. Phys. A 308, 513 (1978).

5. G.D. Alkhazov et al., Nucl. Phys. A 280, 365 (1977).

6. P.J. Karo], Phys. Rev. 11, 1203 (1975).

7. R.C. Barrell and D.F. Jackson, Nuclear Sizes and Structure, (Clarendon Press, Oxford, 1977).

8. W. Czyz, and L.C. Maximon, Ann. Phys. (N.Y.) 52, 59 (1969).

9. G. Fäldt and I. Hulthage, Nucl. Phys. A 316, 253 (1979).

\title{
Calculation of Muon Final Probabilities after Muon-Induced Fission in Four-State Basis*
}

\author{
Mo Zhong-yu, Wh Xi-zhen, Zhong Jing-shong, \\ Zhuo Yizhong, and J.O. Rasmussen
}

Our earliter theorelical work on the relative muon capture between heavy and light fission fragments is extended by including 2por states as well as lso. We calculate about $0.8 \%$ population of the $2 \mathrm{p}$ stale in the beavy fragment, with negligible change from our eajlier two-state basis regarding the ls population of light and heavy fragmenis.

Earlier calculations ${ }^{1,2}$ were made with two basis states, using linear combinations of the is atomic orbitals (LCAO) on the two fragments. In a recent study Maruhn el al, ${ }^{3}$ have made large numerical integrations of the muon wave function in the Coulomb field of spherical uniformly charged fragmenis. They have expended their wave functions to extract p-state as wel] as s-state probabilities.

We have thought it worthwhile to expand the two-slate basis to a four-state basis by including two $2 \mathrm{p}_{\mathbf{z}}$ states. We have investigated the muon dynamics problem in the Coulomb field of two separate exponential charges by the same method as in Ref. 2. The four eigenvalues of the stationary Schroedinger equation al each instant of time are obtained by the LCAO method, and the time-dependent Schroedinger equation is solved by the PSS method (pertosbed stationary states).

We first solve the stationary problem of a muon in the Coulomb field of two centers by the LCAO method. We have chosen four nomorthogonal basis functions, i.e., the wave functions of $1 \mathrm{~s}$ and $2_{\mathrm{p}_{2}}$ states for light and heavy fragments. The Hamiltonian matrix in this asis set can be obtained analyically. Then the generalized eigenvalue problem is solved numerically.

The timo-dependent solution for the moon can be expressed by 


$$
\Psi=\sum_{a=i}^{4} a_{a}(t) \psi_{j} e^{-\frac{i}{h} \int^{\prime} E_{d} d t}
$$

Substitution of Eq. (1) into the time-dependent Schroedinger equation gives the coupled equations,

$$
\begin{gathered}
a_{\alpha}(t)=-\sum_{\beta=1}^{4} a_{\beta}<\psi_{\alpha}\left|\frac{\partial}{\partial t}\right| \psi_{\beta}>e^{-\frac{i}{\hbar} \int^{t}\left(E_{\beta}-E_{\beta}\right) d r} \\
a=1,2,3,4 .
\end{gathered}
$$

The time differential can be expressed as $d / d t=\dot{R} \frac{d}{d R}$, where $\mathbf{R}$ is fragment separation speed.

The results fot the most probable asymmetric case are shown in Fig. 1. The solid line represents the final occupation probability of the muon on the light fragnent; the desh-dot line is for the case of the 2-state basis. The lowest line shows the probability of the moon lying in the ip state of the heavy fragment. It shows that the final occupation probability of the muon so the light fragment is aboul $3.5 \%$ and the probability for the $2 \mathrm{p}$ state of the heavy fragitent is about $0.8 \%$. The population of the $2 p$ level in the light fragment is negligibly small, and the dominant $1 \mathrm{~s} h$ population can be determined by difference.

The results show that the inclusion of the two $2 p$ orbitals along with the ls did not make much change for final is population of fission fragments, compared with the case of two Is states as basis. ${ }^{2}$

We should comment about the oscillation on the $\mathbf{2} \mathbf{p}_{\mathrm{b}}$ probability of large separation distance. This ostillation is not physical and represents a small numerical instability in the calculations giving transitions between Is and $2 p$ stakes in the separated fragment.

Our result of $0.8 \%$ for the heavy fragment $2 \mathrm{p}$ tinal state probability is a bit lower than that of Maruhn tt al., ${ }^{3}$ who found 1.7-2\% probability. Given the many diferent assumptions and methods in our and their calculations, the agreement seems satisfactory. The percentage of $p$ state

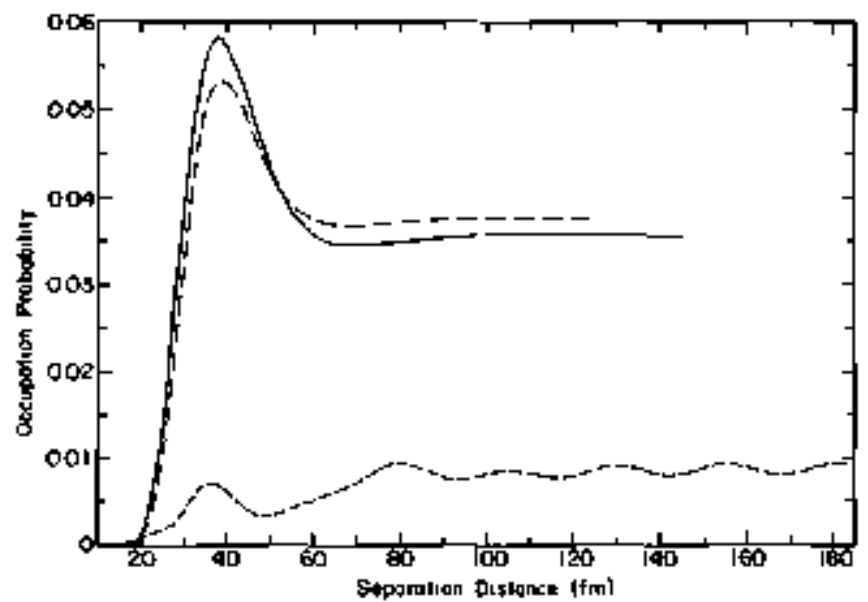

Fig. 1. Calculated muon orbital occupation probabilities as a function of fission fragment separation energies. The upper curves refer to the ls level of the light fragment, the solid curve being present results for the 4-state basis and the dash-do line for the 2-state basis. The lowest (dashed) line is the probability for being in the state that correlates with the $2 p$ level of the heavy fragment. The system shown is ${ }^{98} \mathrm{Zr}+{ }^{140} \mathrm{Te}$.

(XBL 8012-13579)

uxfortunately seems so low as to make experimental detectín unlikely.

This work was pertially supported by the Chinese Acadenty of Science.

\section{Foolnotes and References}

"Condensed from Phys. Lett. B 106, 159 (1981).

'Institute of Atomic Energy, Academia Siniea, P.O. Box 275, Beijing, People's Republic of China.

1. P. Olanders, el al., Phys. Let1. B 90, 193 (1980).

2. J.O. Rasmoussen, el al., Chinese Journal of Nucl. Phys. 2 (1980)(in Chinese); Z.Y. Ma, el al., Nucl. Phys. A 348, 446 (1980).

3. J.A Maruhn, V.E Oheracker, end V. MaruhnRezwani, Phys. Rev. Lett. 44, 1576 (1980).

\title{
Two-Nucleon Transfer Reactions in Deformed Nuclei Using Very Heary Ions*
}

\author{
M.W. Guidry, ${ }^{\dagger} T, L$ NIchols, ${ }^{\dagger}$ RE. Neese ${ }^{\dagger}$ J.O. Rasmussen, \\ L.F. Otiveirat and R. Donongelot
}

Semiclassical methods found to be highly accurale for inelastic scattering are applied to the calculation of rotational population signatures in bescy-ion two-neutron transfer reactions involving highly deformed targets. Basic features to be expected for such reactions are predicted and are shown to have straightforward striclassical interpretations. The rotational population signatures for 2-neulron transfer are shown to be quite different from those expected for the andogous inelastic scattering case. Several calculations were made for $\mathrm{Xe}$ projectiles on rare-earth targets, 


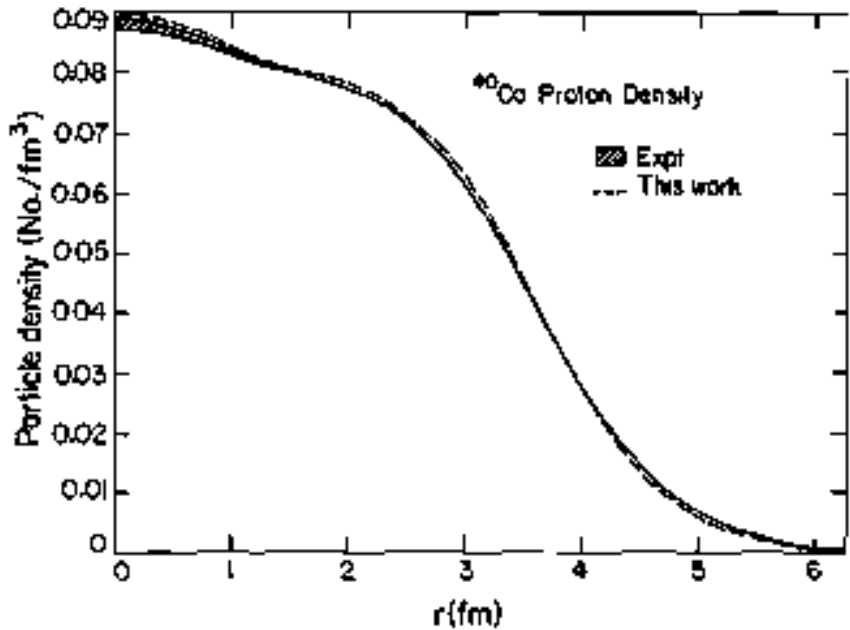

Fig. 1.

XBL $816-1016$

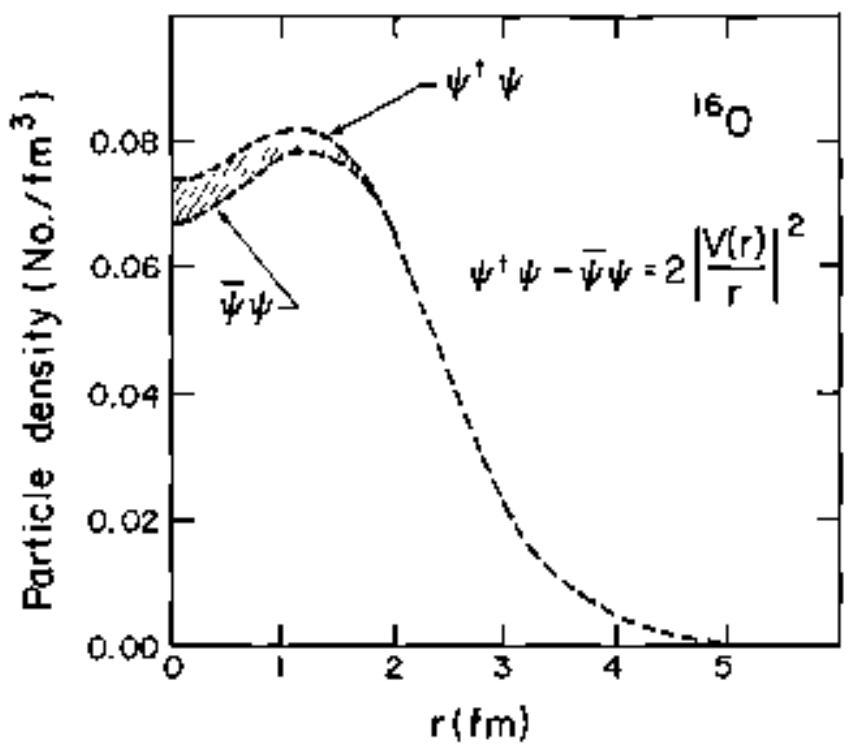

Fig. 2

XBL $\$ 11-25$

\section{Relativistic Quantum Field Theory of $\Delta$ - Hypernuclei*}

\section{Boguta and S. Bohrmant}

That a relativistic model can account for the small spin-orbit splitling in $A$ hypernuclei was first shown by Brockmann and Weise. ${ }^{1}$ Our approach, though relativistic, is essentially different from the above work. The model thal we deal with is a field theory model of nuclear interaclions. This means that the motion of the $A$ inside a nucleus will be completely determined by the existing fields $\sigma(r)$ and $\omega_{0}(r)$ inside the nucleus and the coupling. $g$ asw' $g_{\text {Ahw }}$ wo these fields. The fields $\sigma(\mathrm{s})$ and $\omega_{\mathrm{o}}(\mathrm{s})$ are determined in the Hartree approximation. While Brockmann and Weise had to consider a number of eomplicated intermediate states in the $\mathrm{N}_{\Delta}$ interaction, we describe the $\Delta$ interaction through the fields.

The relativistic quantum field theory of nuclear matter as proposed by Walecka ${ }^{2}$ and extended by Boguta and Bodmer ${ }^{3}$ assumes that the nuelear interactions are mediated by a scalar $\sigma$ and vecler meson fields $w_{\mu}$ and that these mesonic degrees of freedom are essential ingredients in the study of nuclear matter. In the Boguta and Bodmer model, the mesonic degrees of freedom cannol be eliminated in favor of nucleon degrees of freedom, and in general they are necessary to define a systematic progran to compute bighen order eorrections. In the man field approximation, $\sigma \rightarrow$ $\left.\langle\sigma\rangle, \omega \rightarrow t_{00}<\omega_{0}\right\rangle$, these fields ean saturate nuclear matter because of relativistic kinematics. Infinite nuclear matter problem reduces to a simple sel of algebraic equations in terms of $\mathrm{g}_{7} / \mathrm{m}_{\mathrm{s}}, \mathrm{g}_{\mathrm{\gamma}} / \mathrm{m}_{\mathrm{v}}$ (where $\mathrm{g}_{\mathrm{w}} \mathrm{B}_{\mathrm{g}}$ are the cont phing consians of the of and $w_{\mathrm{a}}$ to the nucleon, and $\mathrm{m}_{\mathrm{s}}, \mathrm{m}_{\mathrm{v}}$ are the masses). Since $m_{r}$ is known and $m_{\sigma}$ can be determined by fitting to the surface thickness of a finite nucleus $\left(m_{d}-500 \mathrm{McV}\right)$, all parameters of the model are deter. mined by the gross properties of nuclear matter. We apply this method to the study of $\lambda$ bypernuclei. On the basis of the quark model we will argue that $g_{s} / \mathrm{s}_{\Delta \mathrm{j} \sigma}=$ $\mathrm{g}_{\mathrm{y}} / \mathrm{g}_{\text {Ala }}=\mathrm{x}$, which has to be determined. We need one piece of information about $A$ hypernuclei or $A$ in nuclear matier to detertmine the model completely. We fit to one known neutron-lambda single particle energy difference. 4

The hypernucleus is assumed to consist of a $\Delta$ moving in the mean fields that are created by all the baryons inside the nucleus. The motion of the $\Lambda$ is given by the Dirac equation

$$
\left[-i \vec{\alpha} \cdot \vec{\nabla}+\beta\left(m_{A}+g_{A A_{0} \sigma} \sigma\right)\right] \psi_{\Lambda}=\left(E-g_{\Lambda A} a_{0}\right) \psi_{A} .
$$

The field $\sigma$ and $\omega$ are obtained from the properties of closed shell nuclei in relativistic mean field theory." In Table I we show the predicted energy level differences in $\mathrm{Ca}^{40}$ and $\mathrm{O}^{16}$. The experimental data is also shown. We see that a 
Table 1.

\begin{tabular}{|c|c|c|}
\hline & \multicolumn{2}{|c|}{$\mathrm{A}^{\mathrm{Ca}}$} \\
\hline & Theory (MeV) & $\operatorname{Exp}(\mathrm{MeV})$ \\
\hline$\left(1 d_{5 / 2}, 1 d_{5 / 2}\right)$ & 19.6 & 20 \\
\hline$\left(1 d_{3 / 2}^{-1}, 1 d_{3 / 2}\right)$ & 12.0 & I5 \\
\hline$\left(1 d_{5 / 2}, 1 p_{3 / 2}\right)$ & 12.2 & 10 \\
\hline \multirow[t]{2}{*}{$\left(1 \mathrm{~d}_{3 / 2}^{-1}, \mathrm{p}_{1 / 2}\right)$} & 4.7 & 5 \\
\hline & \multicolumn{2}{|c|}{$A^{0^{16}}$} \\
\hline$\left(P_{3 / 2}^{-1}, P_{3 / 2}\right)$ & 17.6 & 18 \\
\hline$\left(\mathrm{p}_{1 / 2}^{-1}, \mathrm{P}_{1 / 2}\right)$ & 9.9 & 12 \\
\hline$\left(p_{3 / 2}^{-1}, s_{1 / 2}\right)$ & 8.9 & 8 \\
\hline$\left(p_{1 / 2}^{-1}, s_{1} / 2\right)$ & 0.6 & 2 \\
\hline
\end{tabular}

field theoretic approach to the study of hypermuclei can be very sucosessful.

\section{Footnote and References}

"Condensed from LBL-1 1670.

I. R. Brockmann and W. Weise, Phys. Lett. B 69, 167 (1977).

2. J.P. Walecka, Ann. Phys. 83, 491 (1979).

3. J. Boguta and A.R. Bodmer, Nucl. Phys. A 292, 413 (1977).

4. B. Powh, Nocl. Phys. A 335. 233 ( (1980).

5. 1. Boguta, "Gnound State Properties of $\mathrm{O}^{16}, \mathrm{Ca}^{40}$ and $\mathrm{Ca}^{48}$ in a Relativistic Hartree Theory of Nuclear Matter," to be published, LBL-11894.

\title{
Density Dependence of the Single Particle Potential in Nuclear Matter*
}

\author{
J. Boguta
}

A relativistic quanturn field approach to the study of infinite nuclear matter and finite nuclei is a natural idea to pursue. On the basis of sucb an approach Teller, Duerr and Johnson' were able to make reasonable predictions for spin-orbil splitting in fitite nuctei, the well depth of the real part of the optical polential, and its energy dependence. These seemingly unrelated quantities are in fact simple consequence of the Dirac equation for the nucleons, solved in the presence of nuclear interactions of colossal strength. The underlying picture of the model is naively simple. It classifies nuclear interactions according to the irreducible representations of the Lorentz group. Nuclear attraction is generated by a Lorentz scalar mesan $\sigma$ through the interaction $g_{s} \vec{\psi} \psi_{0}$, while the repulsion is given by a Lorentz vector meson $w_{*}$ through as interaction $\mathbf{g}_{4} \bar{\psi}$

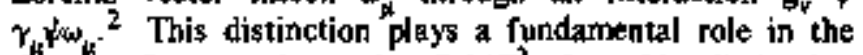
theory. It was shown by Schif $f^{3}$ that this distinction between interactions is sufficient to obtain nuclear matter saturation without introducing hard core repulsion. In the relativistic field approach, nuclear matter saturation is a consequence of the apparent Lorentz covariance of the theory. Nuclear attraction is determined by a Lorentz scalar source term $\rho_{s}$ and repulsion by a Lorentz vector source function $\left(\overline{\mathrm{J}}, \mathrm{i} \hat{\beta}_{v}\right)$.

Recently it was shown that a relativistic mean beld model reproduces reasonably well the real part of the optical potential. ${ }^{4}$ The density dependence of this potential has not been investigated. The purpose of this work is to make such an investigation in a variety of relativistic mean field models. We investigate the Walecka model (W. model), ${ }^{2}$ the Boguta and Bodmer model (BB-model), ${ }^{5}$ and a recent model proposed by the author (B-model). ${ }^{6}$ We show that all these models satisfy the Hugenhol tz-van Hove theorem for an interacting Fermi gas. ${ }^{3}$ A comparison of the predicted single particle potentials at various nuelear densities is made with those of Friedman and Pandharipande (FP-model), who use Fermi-bypernetted and singleoperator-chain summation techniques with a realistic nuclear Hamiltonian. ${ }^{B}$

In Fig. I we show the energy dependence of the optical potential, logether with a compendivom of the available

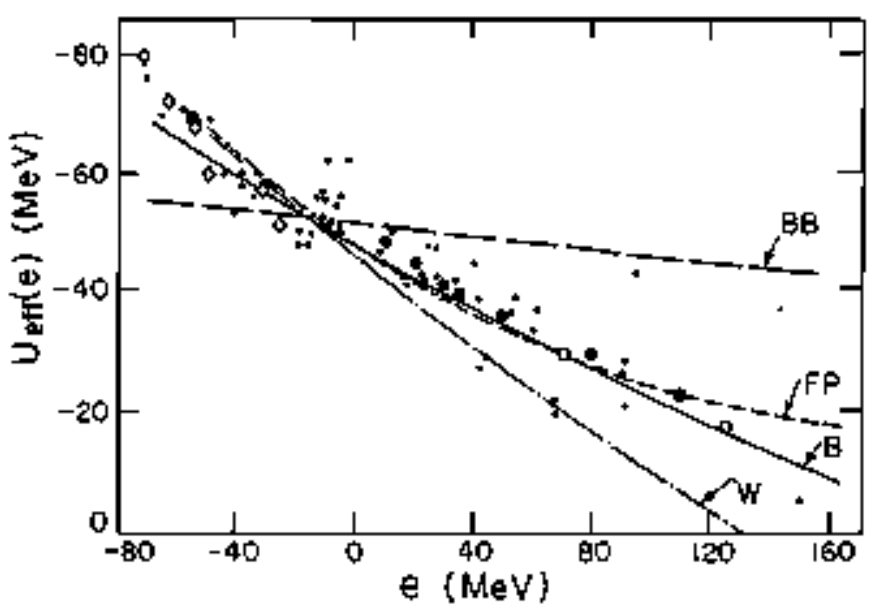

Fig. I. 
data. Clearly the B-model gives a much better description of the data than the Walecka or the Boguta and Bodmer model. In Fig. 2 show the density dependence and the

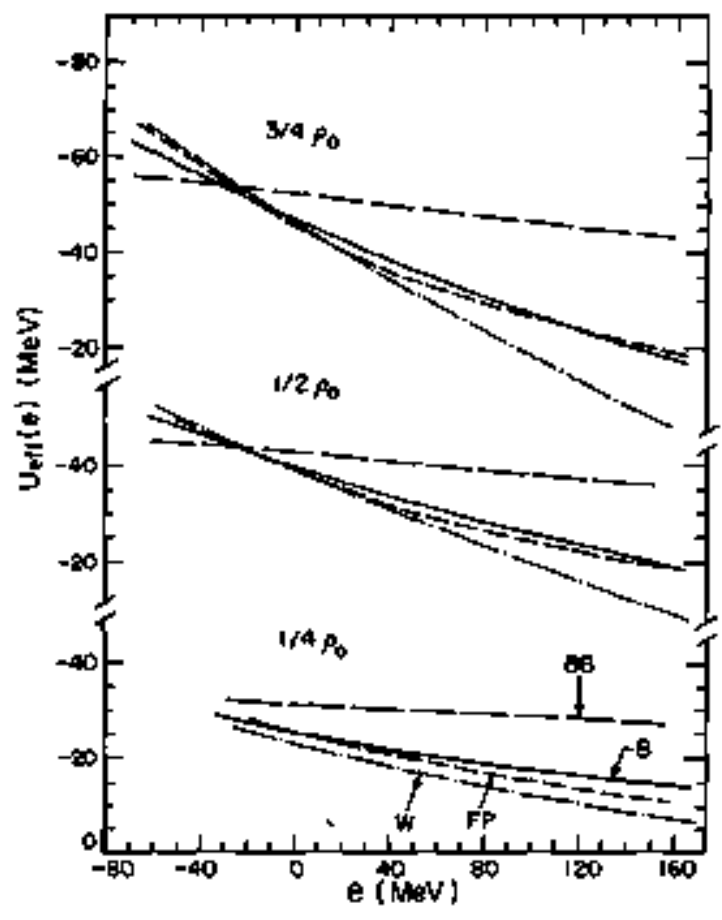

Fig. 2 energy dependence of the optical potential. What is strikingly surprising is that a simple relativistic mean field approach to the study of nuclear mattes can reproduce reasonably well the variational calculation predictions.

\section{Footnote and References}

'Condensed from LBL-12628.

1. M.H. Johnson and E. Teller, Phys. Rev. 98, 783 (1955). H.-P. Duerr, Phys. Rev. 103, 496 (1956).

2. H. Gaus, Zeitschrift für Naturforschung 4a, 721 (1949), J.D. Walecka, Ann. Phys. 83, 491 (1974).

3. L.I. Schiff. Phys. Rev. 84, I (1951); 84, 10 (1951).

4. J. Boguta, "Nucleor-Nucleus Optical Potential in a Relativistic Theory of Nuelear Matter," Lawrence Berkeley Laboratory Report, LBL-11466 (to be published): L.G. Arrold, B.C. Clark and R.L. Mercer. Phys. Rev. C 19, 917 (1979); M. Jaminon, C. Mahaux and P. Rochus, Phys. Rev. Lett. 43, 1097 (1979); L.G. Arnold. B.C. Clark, R.L. Mercer and P. Schwandt, "Dirac Optical Model Analysis of $\mathrm{PCa}^{40}$ Elastic Scattering at $180 \mathrm{MeV}$ and the WintBottle-Botton Shape," OSU-TR-80-35]; M. Jaminon, C. Mahaux and P. Rochus, Phys. Rey. C 22, 2027 (1980).

5. J. Boguta and A.R Bodmer, Nucl. Phys. A 292, 413 (1977).

6. J. Boguta, "A New Mechanism Leading to Density Isomers," Lawrence Berkeley Laboratory Report LBL-12333.

7. N.M. Hugenholtz and L. Van Hove, Physica XXIV, 363 (1958).

8. B. Friedman and Y,R. Pandharipande, "The Single Particle Potential in Nuclear Matter," lLL-(NU)-80-48.

\title{
Dense Neutron Star Matter in the Normal and Pion Condensed State in a Relativistic Field Theory Constrained by Bulk Nuclear Properties
}

\author{
N.K. Glendenting, B. Banterjee and M. Gywlassy
}

This article formulates a theory of dense neutron star matier in a relativistic field theory solved in the mean field approximation. Its special contribution is that the theory is by construction sufficiently rich that it can be constrained to be consistent with the bulk properties of nuclear matter. These important constraints are 1) saturation binding, 2) saturation density of symmperic nucleon matter, 3) compressibility of nuclear maller, and 4) the symmetry energy. In addition the conditions of charge neutrality and $\beta$-siability are imposed. These constraints have never before been imposed on a fietd theoretic modtel of neutron star matter.

In this theory we study the pion condensate solution as well as the normal solution to the field equations. Our goal in this article is to lay the theoretical framework on which more reliable estimates of neutron star properties can be made.

At, and up to a few times normal nuclear density, and below the threshold for quark matter, nuclear forces can be 
described through the exchange of various mesons, the lightest of which are

$$
\sigma, \omega_{\mu}, \pi, p_{\mu}
$$

They are Yukatwa-coupled to the nucleons through the interaction Lagrangian density

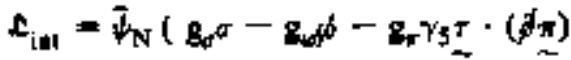

$$
\begin{aligned}
& -\frac{1}{2} B_{0}\left(\psi_{\mu} \cdot \tau^{\mu}\right) \psi_{N}-B_{\nu} p^{\mu} \cdot \pi \partial_{\mu} \pi
\end{aligned}
$$

The pion mean field is assumed to be

$$
x_{ \pm}(x)=\frac{1}{\sqrt{2}} \pi e^{ \pm i k x}
$$

Which is shown to be a self-cossistent solution in the mean feld approximation. We are free to choose the direction of $k$, viz $k_{\mu}=\left(k_{0}, 0,0, k\right)$. Then it can be shown that the Lorentz and iscspin structure of the $p_{M}$ and $\omega_{\mu}$ fields is

$$
\rho_{\mu}=\left(\rho_{0}, 0,0, \rho\right) x(0,0,1), \omega_{\mu} \times\left[\omega_{0}, 0,0, \omega\right)
$$

The eight fitld quantities $\sigma_{,} \overline{\boldsymbol{x}}, \rho_{\mathrm{o}^{\prime}}, \rho, \omega_{\mathrm{o}^{+}}, \omega_{4}, k_{\mathrm{o}}, \mathrm{k}$ are sollutions to the coupled non-linear field equations.

$$
\begin{aligned}
& \left.\left.\mathrm{m}_{\sigma}^{2} \sigma=\mathrm{g}_{\sigma}<\omega \psi\right\rangle \mathrm{dV} / \mathrm{d} \sigma\right\rangle \\
& \mathrm{m}_{\omega}^{2} \omega_{\mu}=\mathrm{B}_{\omega}\left\langle\dot{\psi} \gamma_{\gamma_{\mu}} \psi>\right.
\end{aligned}
$$

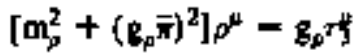

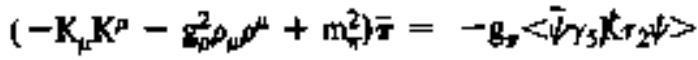

where the nucleom quasiparticle fiteld

$$
\psi=\mathrm{e}^{\frac{i}{2} \mathrm{~lx}^{*},} \psi_{\mathrm{N}}
$$

satisfies

$$
\begin{aligned}
& \left(d-g_{\psi} \phi-m+g_{g} \sigma+\right. \\
& k\left(\frac{1}{2} \tau_{3}+g_{-}-\overline{r g} \tau_{2}\right) \psi=0
\end{aligned}
$$

and

$$
K_{\mu}=k_{p}-g_{p} p_{\mu}
$$

The nucleon quasiparticle propagator and eigenvalue equation and explicit forms for the source currents ate derived.

\title{
The Energy-Dependent Single Nucleon Potential in a Relativistic Field Theory of Nuclear Matter ${ }^{*}$
}

\author{
K.-H. Müler
}

In an attempt to develop a full relativistic description of the many-particle nuclear matter system and to allow explicitly for the mesonic degree of freedorn, Waleckas has proposed a model quantum field theory in which baryons interact with each other via a scalar and vector meson exctange. The single nucteon spectrum that results from this moded determines a momentum $K$ dependent single nucleon mean potential $U_{R}$, which is given in terms of the effective nucleon mass and the repulsive vector meson fitlit. Using the parameters as given by Walecka ${ }^{1}$ the agreernent of $\mathrm{U}_{\mathrm{R}}$ with experiment is excellent as shown in Fig. I.

At nuctear matter densities of $p_{0} / 2$ and $p_{o} / 4$ a cont parison of $\mathrm{U}_{\mathrm{R}}$ is made with results from the nonrelativistic mass-operator theory. Here in the Brückner-Hartreo-Fock approach ${ }^{2}$ the real part of the mean potential $U_{M}$ is determined by a cornplex effective nucleon-nucteon interaction. The momentum dependence of $U_{R}$ in the Walecka model is similar to $U_{M}$ as stown in Fig. 2

A correction to the mean field approxination is the inclusion of the scalar and vector meson exchange contributhons, ${ }^{3}$ which lead to additional momentum dependent

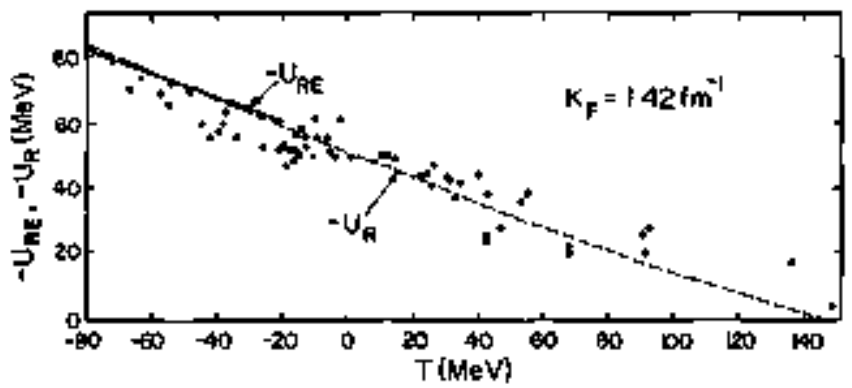

Fig. 1. The single aucleon potential $U_{R}$ without exchange and $\mathrm{U}_{\mathrm{RE}}$ with meson exchange corrections versus. the mucleon enesgy $T$.

(XBL 815-808)

terms in the single nucleon mean potential $\mathrm{U}_{\mathrm{RE}^{*}}$ As we show in Fig. I, the momentum dependence of the potential for bound stales is only slightly changed by the mesom exchange terms. 


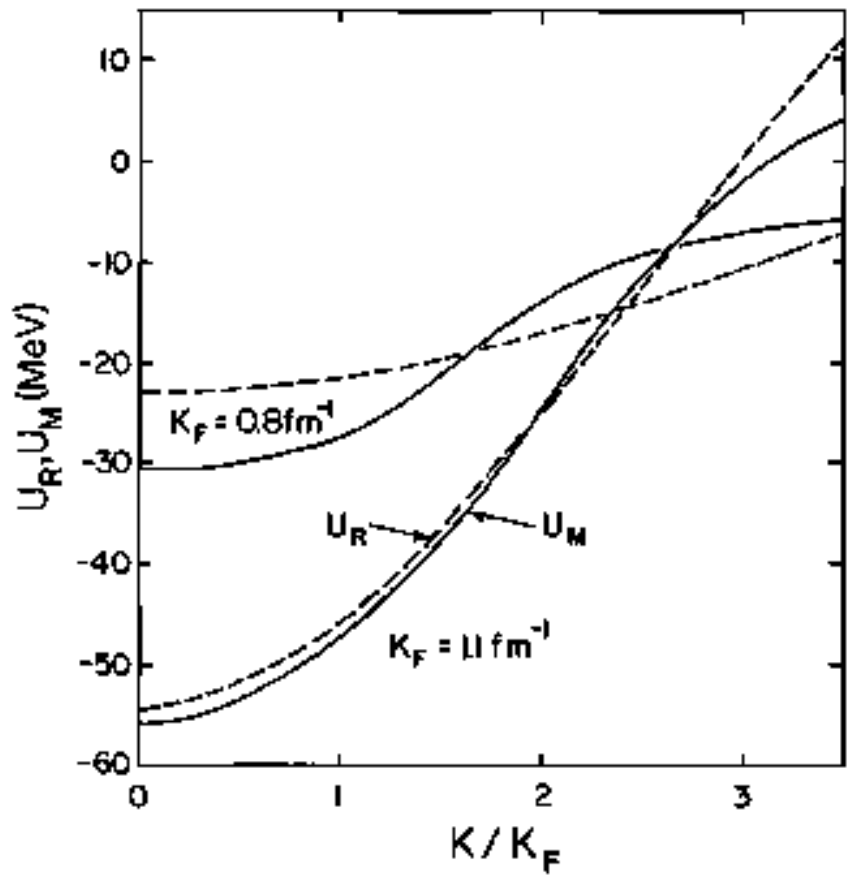

Fig. 2. The single nuclean potential $\mathrm{U}_{\mathrm{R}}$ and the Brīckner-Hartrec.Fock potential $\mathrm{U}_{M}$ versus the momentum ratio $K_{j} / K_{\mathrm{F}}$.
Foothotes and References

"Condensed from LBL-12684. This work was partialty supporled by the Destsche Forschungsgemeinschaft, West Germany.

1. J.D. Walecka, Ann. Phys. 83, 491 (1974).

2. J.P. Jeukenne, A Lejeune and C. Mahaux, Phys. Rev. C 10, I391 (1974).

3. S.A. Chín, Antn. Phys. 108, 301 (1977).

\title{
A Relativistic Quantum Field Theoretical Model for Nuclear Slab Collision Dynamics"
}

\author{
K.-H. Mtiller
}

The rejativistic mean field model proposed by J.D. Walecka' was recently used with great success to describe the properties of nuclear matter as well as the structure of the finile static nuclei. Starting from Walecka's Lagrangian the nucleons are represented by single particle spjnors, which are determined by a Dirac equation containing a repulsive mean neutral vector meson field and an attraotive mean isoscalar meson field. Both fields satisfy KleinGordon equations whose source lerms are again determined by the spinors.

We applied this relativistic mean field model to the dynamics of two colliding nuclear slabs. The slabs are translationally invariant in two transwerse dimensions and consist of spin and isospin symmetric nuclear matter. The appropriate initial conditions for the approaching slabs are constructed by applying inhomogeneous Lorentz transformations to the static relativistic nucleon wave functions, which correspond to the ground state of a single slab at rest.

We solved numerically the conpled system of differential equations of Dirao and Klein-Gordon-Type for different bombarding energies. With the constraint of 1.4 nucleons per unit area for each slab, self-consistency leads to a slab thickness that corresponds to a $\mathrm{A} \simeq 35$ nucleus.

Figure 1 shows the resulting density $\rho_{y}$ during the slab collision at $E_{t a m} / A=100 \mathrm{MeV}$. The maximum density reached is less than $2 p_{\mathrm{o}}$. The dashed curves show the result taking reterdation effects into acount. The rapidly incressing repulsive wector meson field leads to one body emergy dissipation and slows down the colliding slabs. ${ }^{2}$ At low bombarding energies the results are like those from a corresponding TDHF calculation. ${ }^{3}$

\section{Footnotes and References}

Condensed from LBL 12667. This work was partially supported by the Deutsche Forschungsgemeinschaft, West Germany.

1. J.D. Walecka, Ann. Phys. 83, 49! (1974).

2. K-H. Mtiller and S. Bohrmann, Lawrence Berkeley Laboratory Report LBL-12663.

3. P. Bonche, S Koonin and J.W. Negele, Phys. Rev. C 13, 1226 (1976). 


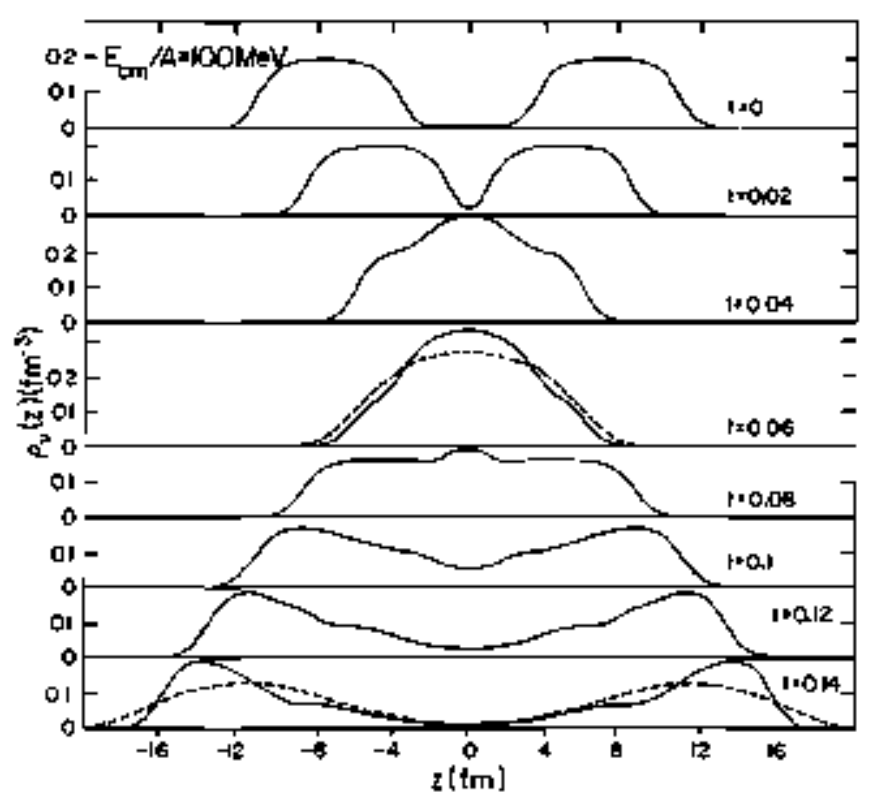

Fig. 1. Density profiles for the $\mathrm{E}_{\mathrm{cm}} / \mathrm{A}=100 \mathrm{MeV}$ slat collision. The time $t$ is in units of $10^{-21} \mathrm{sec}$.

(XBL 814-4570A)

\title{
Interacting Streaming Nuclear Matter Systems in a Relativistic Quantum Field Theory"
}

\author{
$\boldsymbol{K}-\boldsymbol{H}$. Muller and S. Bohrmant
}

We study the energetical behavior of two mutually interacting streaming systoms of spin and isospin sym metric nuclear matter in a relativistic quantum field mode] proposed by J.D. Walecka.' Both matter systems are of infinite size, overlap completely in coordinate space, and strearn in $z$-direction with velocities $V$ and $-V$, respectively. The interaction between the baryons is mediated by an attractive scalar and a repulsive vector-meson field. Beciuse of the symmetry in our problem, thete is no net baryon current in z-direction and therefore all space components of the vector-meson field vanish.

The relativistic many-body baryon state is assumed to be a product of plane wave spinors. The plane wave quantum numbers occupy a Fermi sphere in each respective rest frame. For small flow velocities the Fermi spheres overlap leading to doubly occupied single baryon states. To awoid this, we rearrange the doubly occupied cells. ${ }^{2}$

In order to present the behavior of streaming matter systems transparently and in a way comparable to nonrelativistic many-body theories, we split the total energy into a linetic part $T$ and a potential part. This is sccomplished by identifying each system with a free nucleon gas in an effective spatially constant potential. We assume that before the two systems flowed through each other, both sys- tems existed without any spatial owerlap in their ground states and approached each otber with s.m. energy $T_{c m}$. Total energy conorvation then relates the kinetic enerby $T$ of the interacting system with $T_{c . m}$. Figure 1 shows the ratio $R=T / T_{5, m}$ and the change in flow velocity $\bar{R}=$ $V_{i} / V_{w^{2}}$ where $V_{i}$ and $V_{n}$ are the flow velocities for the interacting and poninteracting systems, respectively.

The conclusion of ov investigation is that for bigh flow velocities the vector meson field evokes a strong repulsion between the streaming systems that slows down the systems strongly. 3

\section{Footnotes and References}

"Condersed Irom LBL-12663. This work was partially supported by the DFG, West Germany, by NATO fellowship, DAAD, West Germany, and by BMFT, West Germany."

'Inst. f. Theor. Phys., Philosophentweg 19, 6900 Heidelberg. West Germany.

1. J.D. Walecks, Ann. Phys. 83, 49J (1974).

2. F, Beck, K.-H. Múller and H.S. Köhler, Phys. Rev. Leit. 40, 837 (1978).

3. K-H. Müler, Lawrence Berkeley Laboratory Report LBL 12667 (submitted to Nucd. Phys.). 


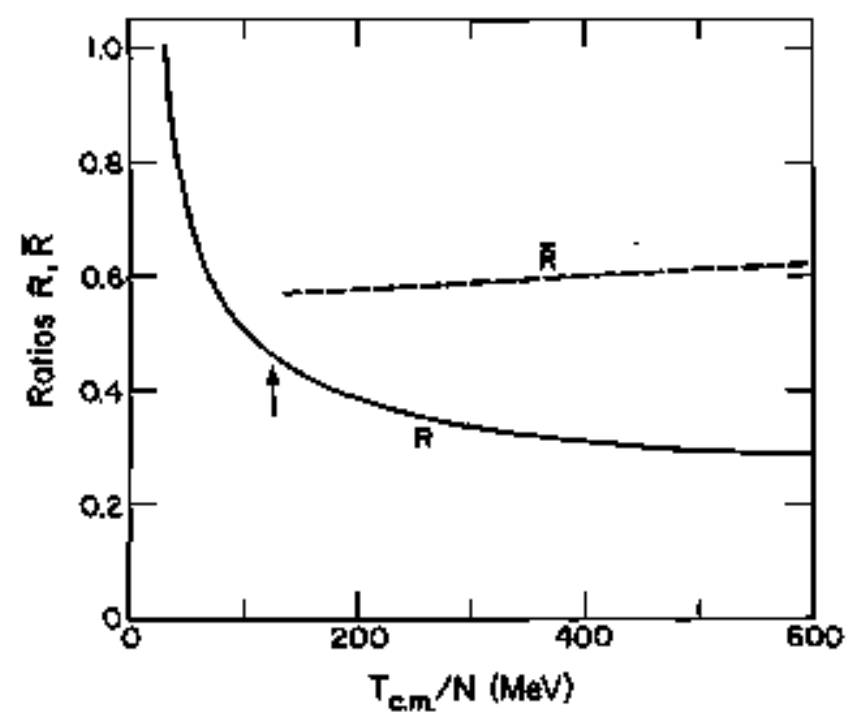

Fig. 1. The relative change $R$ of the kinetic energy and the relative change $\overrightarrow{\mathbf{R}}$ of the flow velocity for systems of normal nuclear matler density.

(XBL 818-1109)

\title{
Theory of Nuclear Dynamics
}

\author{
W.J. Swialeckl
}

The theory of macroscopic nuclear dynamics, based on the concept of a nucleus as a visco-tlastic system ${ }^{1,2}$ has nade appreciable progress in the past year. As is the case with statics, the nature of nuclear dyntamics is profoundly affected by symumetries, associated also with shell effects. There are two limiting cases that are particularly simple. On the one hand, when the system is away from symmetries, the static potential energy is described accurately by a leptodermous (liqujd-drop) type of expression and the dynamics of shape changes is expected to follow a simple equation of motion, based on the one-body dissipation concept ${ }^{3,4}$ The rasulting motion is predicted to be severely overdamped: the nucleus should behave like a viscows finid, with a novel type of viscosity. On the other hand, when the system is close to symmetrical configurations (in the first place a sphere but, to a lesser degree, most groundstate configurations) the single-particle level structure acquires special features (crossings and near-cressings of levels) and the nueleus becomes more like a visco-elastic solid, ${ }^{2}$ in some cases actually dominated by elasticity (e.g., the gisnt quadrupole resonances.

The theoretical exploration of the dissipationdominated regime, initiated half a dozen years ago, $, 3,4$ has made striking contact with recent experiments at GSI, Darmstadt These conjrontations of theory and experiment center around the prediction of an "extra push" necessary to make two sufficiently beavy nuclei fuse, and an "extraexira push" to make a compound nucleus. ${ }^{* 12}$ Mer ely from the conservation of energy and angular momentum it is possible to derive the following extra push theorem, relating the fusion cross section o to the center-of-mass energy E:

"When an extra radial injection energy ower the interaction barriet $B$ is needed to initiate fusion, the square root of the cross-section defect $\Delta$ should be approximately linear in the energy-weighted reduced cross-section $\Sigma$, wiz:

$$
\sqrt{\Delta}=c_{1}+c_{2} \Sigma+\ldots "
$$

In the above

$$
\begin{aligned}
& \Sigma=\mathrm{E} \sigma / \mathrm{\pi r}^{2} \\
& \Delta \equiv \mathrm{E}-\mathrm{B}-\mathrm{E} \sigma / \pi^{2} \\
& r=\text { center-to-center distance at contact. }
\end{aligned}
$$

The parameters $c_{1}, c_{2}$ are constants for a given targetprojectile pair. On dimensional grounds (together with a small neck approximation) the one-body dissipation theory prediets the following dependences of these parameters on the atomic and mass numbers $Z_{1}, Z_{2}, A_{1}, A_{2}$ of the ollid. ing nuclei:

$$
c_{1}=\sqrt{K}\left[\left(Z^{2} / A\right)_{4 f}-\left(Z^{2} / A\right]_{h t r}\right],
$$




$$
c_{2}=\frac{\sqrt{K}}{e^{2} / r_{o}} \frac{8 I^{2}}{A_{1}^{1 / 3} A_{2}^{1 / 3}}
$$

where

$$
\begin{aligned}
K & =\frac{32}{2025}\left(\frac{3}{\pi}\right)^{2 / 3}\left(\frac{e^{2}}{h c}\right)^{2} \\
& \times \pi c^{2} \frac{A_{1}^{1 / 3} A_{2}^{1 / 3}\left(A_{1}^{/ 3}+A_{2}^{1 / 3}\right)^{2}}{A_{1}+A_{2}} a^{2}
\end{aligned}
$$

$r_{0}=$ nuclear radius constant

In $=$ atomic mass unit $=93 \mathrm{~L} .5 \mathrm{MeV} / \mathrm{c}^{2}$.

The effective fissility, defined by

$$
\left(Z^{2} / A\right)_{\text {eI }} \rightleftharpoons \frac{4 Z_{1} Z_{2}}{A_{1}^{1 / 3} A_{2}^{1 / 3}\left(A_{1}^{1 / 3}+A_{2}^{1 / 3}\right)}
$$

appears as a fundamental scaling parameter of fusion dynamics in the regime of necked-in configurations.

There are three parameters (pure numbers) in Egs. (2-4): the threshold fissility $\left(Z^{2} / A\right)_{t b r}$, the "thud wall slope coefficient" $a$ and the "effective angular momenturn fraction" $f$ (see Refs. 9,10 ). They have to be estimated from dynamical model calculations or deduced from fits to experiments. The schematic model of Refs. \& 10 suggested $\left(Z^{2} / A\right)_{\text {thr }}$ somewhat greater than 27 and a considerably greater than 5 . The parameter $f$ should be somewhat less than $\mathrm{I}$.

Figure l shows a comparison of the recent GSI experiments ${ }^{\prime}$ with the above theory. The deduced values of the parameters are: $\left(Z^{2} / A\right)_{\text {th }}=33 \pm \mathrm{l}, a \simeq 12 \pm 2$. $f=3 / 4 \pm 10 \%$.

A recent application of the schematic model of Refs. 8-10 to the clutching stage of nuclent collisions suggests $f \approx 0.85$. (See contribution of $G$. Fai to this anmual report.)

Qurrent dynanical calculations done in collaboration with 5. Blockd and others (at the lnstitute of Nuclear Research, Swierk, Poland, and the Technische Hochschule and GSL, Damstadt), using the model underlying the potential-energy maps deseribed elsewhere in this report, gave $\left(Z^{2} / A\right)_{\text {th }}=33.3$, in agreement with experiment. On the other hand, the same calculations seem to overestimate substantially the value of $a$.

Joint work with S. Bjprnholm (Niels Bohr Institute, Copenhagen), initjated very recently, extends the above consjderations to the extra-extra push necessary to make a compound nucleus. This leads to a semirquantitative survey of nuclear reactions, classified as (a) elsatic (plus quasi-elastic) (b) deep-inelastic, (c) fast-fission and (d) componod-nucleus. It should be possible to provide simple estimates of the associated cross sections. A crucial element in this treatmeat is the discovery of a promising candidate for a scaling parameter for gast-fission and eompound nucleus reactions, namely the (goometrie) mean fissility, defined by

$$
\left(Z^{2} / A\right)_{\operatorname{megn}}=\sqrt{\left(Z^{2} / A\right)_{\text {ff }}\left(Z^{2} / A\right)},
$$

where $Z^{2} / A$ is the ordinary fissility of the whole system.

\section{References}

1. D.L. Hifl and J.A Wheter, Phys, Rev. 89, 1102 (1953).

2. W. Nortenberg, "Memory Efects in the Energy Dissipation for Slow Collective Nuclear Motion," Preprint GSI$81-14$, May 1981 .

3. J. Blocki et al., Annals of Phys. 113, 338 (1978).

4. J. Randrup and W.J. Swiatecki, Annals of Phys. 124. $193(1980)$.

5. G.F. Bertsch, Nuch. Phys. A 249, 253 (1975); J,R Nix and J. Siork, Phys. Rer. C 21, 396 (1980).

6. J.R. Nix and A.J. Sierk, Phys. Rev. C 15, 2072 (1977).

7. W.J. Swiatecki, Prog. Particle and Nuel. Phys. 4, 383 (1980); Lawrence Berkeley Laboratory Report LBL 8950 (1979).

8. W.J. Swiatecki, "The Dynarnics of Nuclear Coelescence of Reseparation," talk presented at the Nobel Symposium on High Spin States, Orenäs, Sweden, June 22-25, 1980, to be published in Physies Scripta, 1981; Lawrence Berkeley Laboratory Report LBL 109]1.

9. W.J. Swiatecki, "Pusion Cross.Sections and the New Dynamics," Lawrence Berkeley Laboratory Report LBL12708 (1981).

10. W.J. Swiatecki, "The Dynamics of the Fusion of Two Nuclei," Lawrence Berkeley Laboratory Report LBL-12642 (1981), to appear in Nucl. Phys. A.

[1. H. Samn et al., "Fusion Reaction Studies of Transuranic Elements," Proc. Int Comf. on Nucl. Physics, Aug. 2430, 1980, Berkeley, California, Lawrence Berkeley Laboratory Report LBL L1118; A Gobbi, Contribution to "GSI Nehrichten" 1-81, p. 4, 1981; H. Sann et al., "Deformability as a Critical Factor in Initiating Fusion between Very High lons." GS1-Preprint 81-6, Feb. 1981; submitted to Phys. Rev. Letters.

12. B. Sikora et al., "Parameter Scaling Test of a Dypenic Nudeus-Nucleus Collision Theory," Lawreske Livermore Laboratory Report UCRL-86277 (1981); submitted to Phys. Rev. Letlers. 


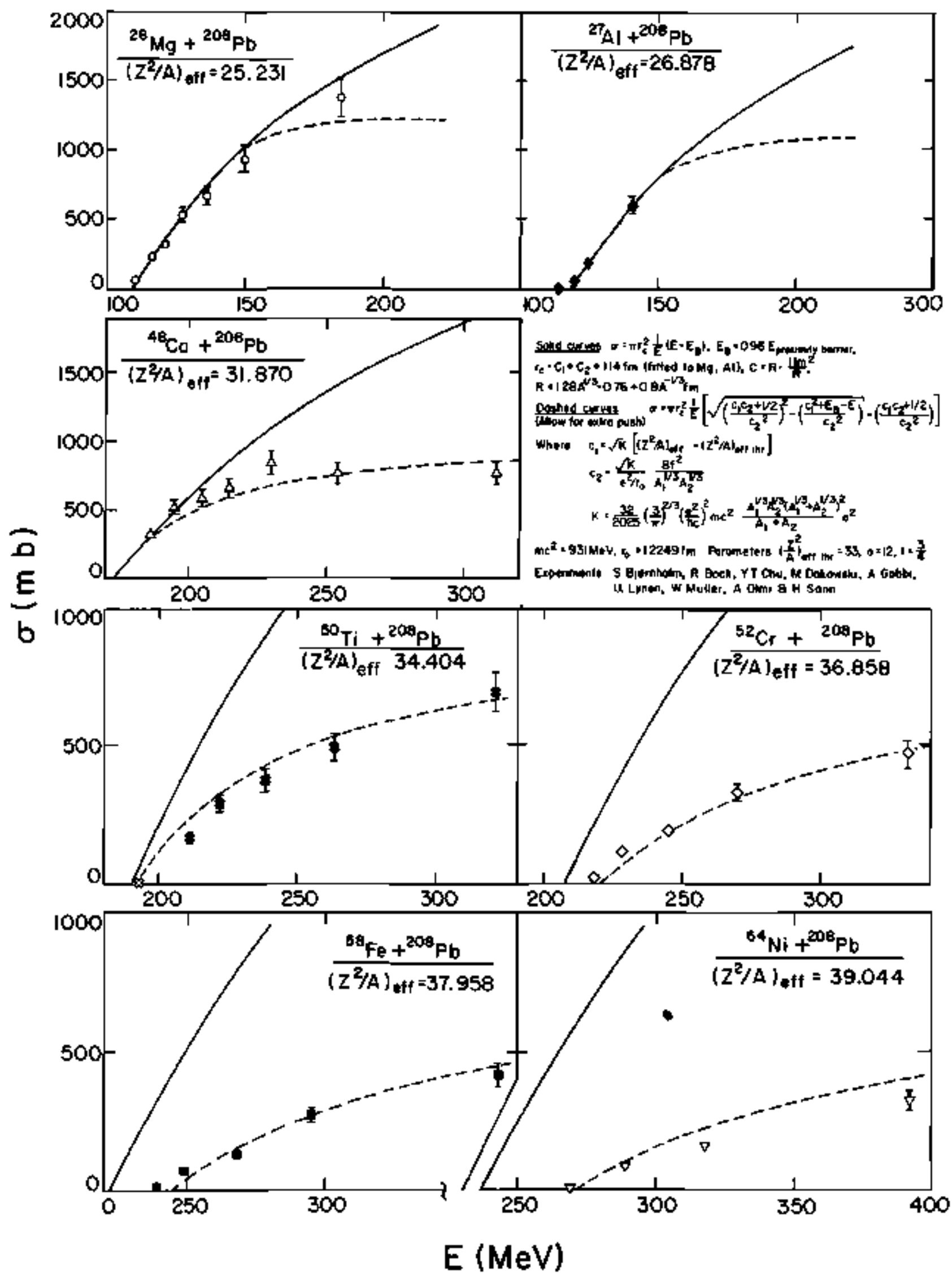

Fig. 1. Comparison of experimental fusion cross-sections (associated with outgoing Iragment masses centered around aymmetry) with theory. The solid curves are conventional reaction eross-section predictions, and the dashed curves incorporate the requirement of an extra push in the approach degree of freedom. The data are from Rer. 9. 


\title{
The Clutching of Two Colliding Nuclei
}

G. Fat

The existing mactoscopic model of nucleat coalescence or reseparation describes head-on collisions. In the present work the treatment is extended to noncentral colljsions by taking into account the relative angular momentum and the rotation of the individual auclei. The relevant degrets of freedost ire the following: asymutrtity, fragment separation, nock size, and the total amount of sliding that has taken place until the two nuclei "clutched." Rolling friction is neglected.

The equations of motion are sufficiently simple that approximate solutions in closed form may be found in several cases of interest. In particular, the theory provides the normal modes of motion around the saddle points of the potential energy surface. For not too light systems the characteristic time of the transition from sliding to rolling appears to be shorter than the characteristic time of any other mode (fission, damping, and oscillation). This can be interpreted as a fast clutching of the colliding auctei.

The dynastics of the collision as described by the present model suggests that centrifugal force to a good epproximation can be traded for electric repulsion via an "angular momentum fraction" as has been done to 6 t recent experimental data. ${ }^{2}$ The model also yields a theoretical prediction for the value of the angular momentum fraction $f$, which turns out to be $f=0.8$, while the best ft to experiments has been obtained using $f=3 / 4 \pm$ $10 \%$ Both of these values are notably far from $f=I$, corresponding to a "mass-points" moment of inertia on one hand, and from I $=0.54$, corresponding to a lirrit of rigidly stuck spheres with a ratio of masses of about 208:50 on the other.

A numerical study of the dynamics of the clutching of two colliding nuclei is under way.

\section{References}

1. W.J. Swiatecki, Lawrence Berkelsy Laboratory Report LBL 10911 ( 1980).

2. W.J. Swiatecki, Lawrence Berkeley Laboratory Report LBL-12642 ( I98I).

\section{Fusion-Fission, Molecular-Capture And Symmetric Fragmentation In 3D-TDHF Calculation of the Reactions ${ }^{238} \mathrm{U},{ }^{2008} \mathrm{~Pb}(4.6-8.0 \mathrm{MeV} / \mathrm{u}){ }^{64} \mathrm{Ni}$, ${ }^{48} \mathrm{Ca} B+\mathrm{G}$}

\author{
H. Stōcker, "t RY. Cusson, ${ }^{*}$ H.J. Lustig. ${ }^{\dagger}$ A. Gobbi, \\ J. Hohn, J.A Aaruhn, and W. Greiner 5
}

Beams of ${ }^{208} \mathrm{~Pb}$ and ${ }^{238} \mathrm{U}$ between 4.6 and $8.0 \mathrm{MeV} / \mathrm{u}$ from the GSI UNILAC have been used recently to bombard light targets such as ${ }^{48} \mathrm{Ca}$ and ${ }^{64} \mathrm{Ni}$. These data show in addition to ordinary deep inelastic processes some broad fusion-fission type mass distributions centered around $\left(A_{T}+A_{p}\right) / 2$; these new effects are the subject of our prusent study using the 3-dimensional time-dependent Hartree-Fock model calculation. ${ }^{2}$

Four distinct reaction types are found in our calculations (see Fig. 1): For impact parameters $b<\mathbf{b}$ grazing. the nuclei develop a neck and the single particle viscosity present in TDHF causes large angular momentum and kinetic entergy losses but the reaction time is too short to cause an appreciable change in fragment mass. Besides these well known deep inelastic collisions, distinguish the following: 1) Capture events with subsequent symmetric fragmentation; the time for mass asymmetry equilibration is approximately $\gtrsim 3 \times 10^{-21} \mathrm{~s}$, while energy and

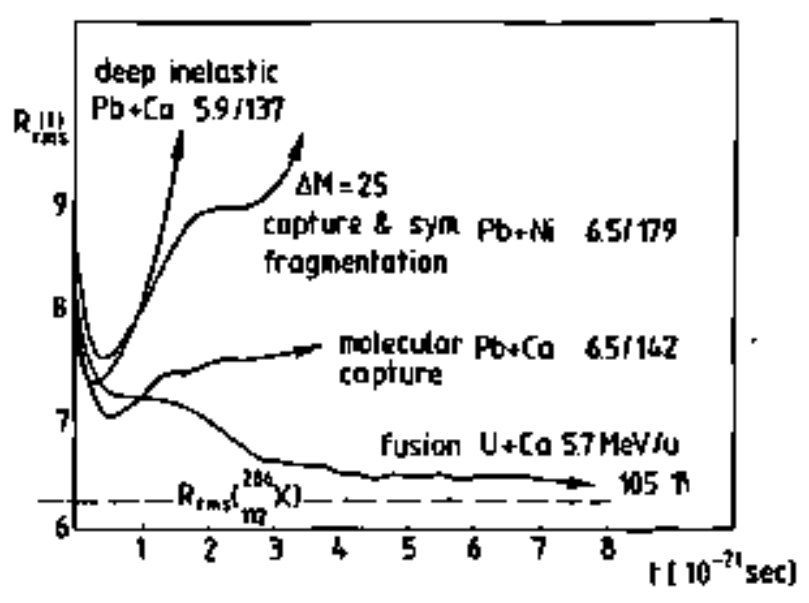

Fig. 1. The rms-radius in fan $*$ sime. (XBL 8110-12]12) 
angular momentum loss is much faster with $\tau=$ 1.1.5 $10^{-21} \mathrm{~s}$. 2) The Tormation of long-lived very heavy nuclear molecules with two-centered shapes, the molecular capture being characterized by nearly stationary, large rms-radii (see Fig. 1). These rotate for $r \geqslant 10^{-21} \mathrm{~s}$ without appreciably changing its shape. 3) Fusion, characterized by an rms-radius of the compound system approaching that of the ground state spherical nucleus after $T$ $10^{-20}$ s. The very beavy composites formed in processes 2 and 3 are likely to fission. Their fission mass distribution ean hardly be distínguished from those of pro cess 1 , capture and symmetric fragmentation.

In order to obtain a rough comparison of our results with the symmetric fragmentation component extracted from the experimental data, ${ }^{1}$ we computed the sum of the capture cross sections (processes $h_{1} 2$, and 3) with a minimum mass drift of 15 mass units, and calculated the capture excitation functions for the system

$$
{ }^{238} \mathrm{U}+{ }^{45} \mathrm{Ca} \rightarrow\left({ }_{11}^{206} \mathrm{X}, \mathrm{SF}\right)
$$

and

$$
{ }^{208} \mathrm{~Pb}+{ }^{48} \mathrm{Ca} \rightarrow\left({ }^{256} \mathrm{X}, \mathrm{S}, \mathrm{SF}\right)
$$

(see Fig. 2). Considering the fact that the theory includes events which art not completely equilibrated in nass asymmetry we find reasonable agreement with the experimental data.

\section{Footmotes and References}

"Presently at LBL

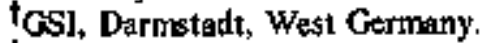

TPermanent address: Physics Department, Duke University, Durbam, NC 27706.

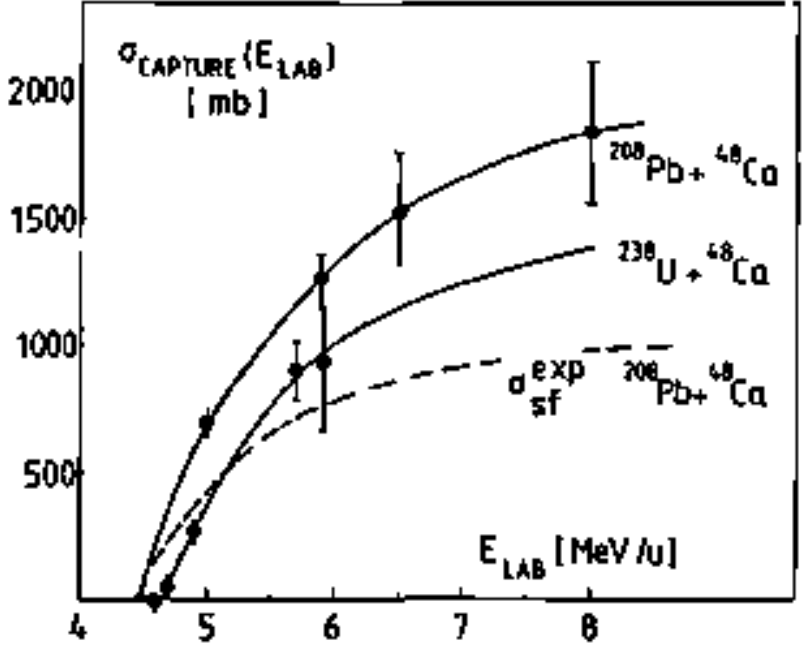

Fig. 2. Excitation function of the calculated capture cross reactions ws $\mathbf{E}_{\text {La }}$ compared to the recent GSI data (Ref. ], dashed line). The bars denote the upper and lower limit for capture, reflecting the fact that only a few impact parameters for each bombarding energy can be compuled. The solid lines are to guide the eyes.

(XBL $8110-12211)$

II Intitut für Theoretische Physik, Universitäl Frankfurt, West Germany.

1. A Olmi th al., "15th Bormio Winter Meeting on Nuclear Physics," Borrio, Italy (1978), p. 724. Also H. Sann et al., to be published.

2. H Stzcker tt al., GSI-preprint 81-3. R.Y. Cusson, J.A Maruhn, and H. Stŏcker, Z. Physik A 294, 257 (1980).

\title{
Quantum Treatment of Charge Equilibration in Fission"
}

\author{
W.D. Myers, G. Mantzourants, and J. Randrup
}

The prospect that originaly motivated this investigation was the bope that the width of the charge distribution in fission would provide a key to differentiating between two substantially different proposals for the path of a fisstioning nucleus on jts way from saddle to scission. In Ref. I two different dyoamical paths are determined for fission that both give the correct value for the asymptotic kinetic energy release. One trajectory is quite rapid and is only weakly damped by an ordinary hydrodynamic viscosity. The final velocity comes from the relative motion at scission and the Coulomb acceleration of the separating fragments. The other trajectory is based on the ont-body damping mechanism and is much slower. The resulting scission shape is more compact and the relative motion is slower. However, the increased Coulomb acceleration after scission resulis in a final binetic energy that is nearly the same as for the trajectory basted on viscous damping.

The final width of the fragment charge distributions depends on the rate at which the neck between the two nuclei clost off at scission. In Fig. I the inverse inass b (in the charge equilibration degree of freedom) is plotted as a function of time for the two different fission trajectories. (See Ref. 2 for details of the calculation,) Since the rate at which b decreases is quite different, we expected that the predicted charge dispersions would also be quite different

Unfortunate]y, the froeze-out of the charge dispersion oocurs so late in the process (see Fig. 2) that there is 


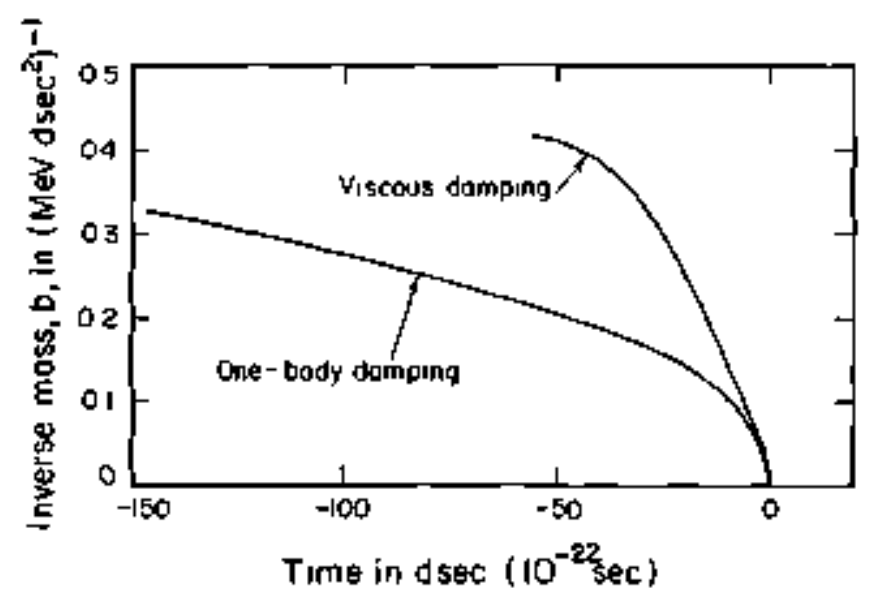

Fig. 1. The inverse mass b associated with the flow of neutrons and protons back and forth through the neck connecting the two halves of the fissioning nucleus ${ }^{236} \mathrm{U}$ is plotted against time.

(XBL 309 -19l5)

almost no difference between the two predictions. In Fig. 2 the last part of Fig. I is shown on an expandod scale. In the lower hall of the figure the quantity $\mathrm{C}$ an adiabaticity variable developed in Ref. 2, is plotted for the case where the fission trajectory was calculated using viscous damping. $C_{\text {attains the critical value of } 1.108 \text { at }}$ $t_{c}=-1.11 \times 10^{-22} \mathrm{sec}$. This corresponds to a predicted charge dispersion of $\mathbf{r}_{\mathrm{r}}=1.38$ which is somewhat smaller than the experimental value of 1.50 . The quantity $C$ for the case of the trajectory associated with one-tody damping is not plotied since it lies ntarly on top of the curve for viscous damping. Since the curves for b are so simjlar when freeze-out oceurs, the predicted charge widths are nearly the same in the two cases. Consequently, we are forced to conclude that these considerations do not provide a means for choosing between the two fission trajectories.

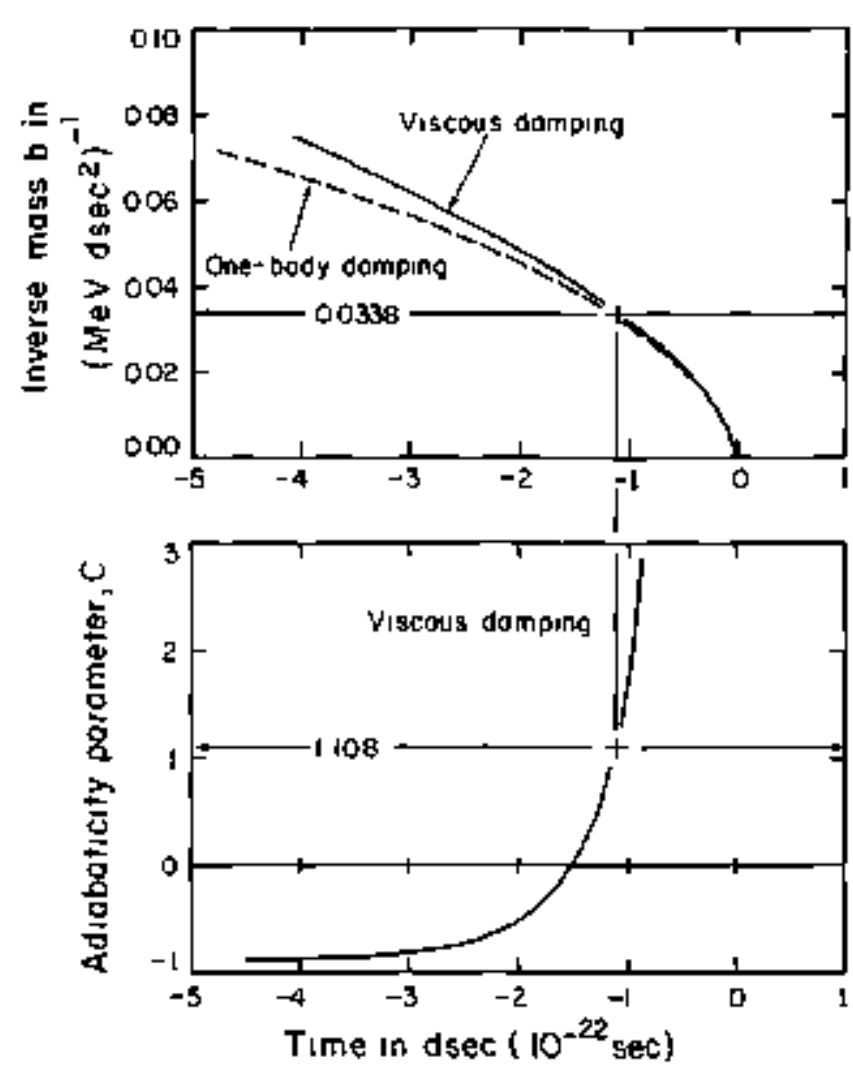

Fig. 2 The same two curves as appear in Fid I are shown here on a greatly expanded scale. In addition the adiabaticity parameter is shown in the lower part of the figure for the viscous damping case.

(XBL 809-1914)

\section{Footnotes and References}

"Condensed from Ref. 2.

${ }^{\dagger}$ Max Planck Institut für Kernphysik, D-6900 Heidelberg, West Gerntany.

1. A.J. Sierk and J.R. Nix, Phys. Rev. C 21, 982 (I980).

2. W.D. Myers, G. Mantzouranis and J. Randrup, Phys. Lett. B 98, 1 (1981).

\title{
Correlated Mass and Charge Transport Induced by Statistical Nucleon Exchange in Damped Nuclear Reactions ${ }^{*}$
}

\author{
W.U. Schrödert, J.R Huizengat and J. Randrtip
}

Extensive studies of damped nucleas reactions at energies a few MeV/u above the barrier bave revealed a reaction mechanism intermediate between those of direct and compound-nucleus processes. Although not all degrees of freedom may reach statistical equilibrium in the course of a damped collision, many reaction features such as fragment distributions in $A, Z$ and energy loss $\left(E_{\text {lose }}\right)$ have been successfully interpreted in terms of chassical or quental tuassport models. Recently, bowever, natrow fragment isotopic mass and isobaric charge distributions have been observed, whose variances $\sigma_{\mathrm{A}}^{2}\left(\mathrm{Z}=\mathrm{const}\right.$ ) and $\sigma_{2}^{2}$ ( $\mathrm{A}=$ const), respectively, salurate after an initial increase with $\mathrm{E}_{\text {Los }}$ ' This feature has been elaimed ${ }^{1}$ to be inconsistent with the picture of statistical exchange of individual 
mucleons and has induced speculations about the existence of a fast collective mode of charge equilitration described in terms of a quantal harmonic oscillator coupled to thermal degrees of Jreedom.

The dominance of such a dinuclear quantal collective mode would call for a significant revision of the interpretation of dampod reaction phenomena previously understood to be due to slatistical processes. However, as shown in this work, the obserwed isobaric and isotopic fragment distributions can actually be quite well accounted for by an independent-particle transport mechanism. There appears to be no need to involve a collective mode of charge equilibration. To demonstrate this important fact, predictions are presented, for the first time, for isobaric and isotopic fraginent distributions that are besed on a model ${ }^{2}$ of stochastic exchange of individual nucleons between the two collision partners.

In a transport approach, a two-dimensional FolckerPlanck equation is expected to govern the dynamical evolution of the probability $P(N, Z ; t)$ for finding $N$ neutrons and $Z$ protons in the projectile-like reaction partner. The physical information on the microscopic transition probabilities at each time $t$ is contained in the transport coefficients $V(N, Z ; t)$ and $D(N, Z ; t)$. Average neutron and proton numbers are predicted to change with rates determined only by the drift coefficients only by the drift coefficients, according to $a \bar{N} / \partial 1 \simeq V_{N}$ and $\partial \bar{Z} / \partial \mathrm{l} \simeq V_{Z}$ whereas the growth of the sorresponding covariances of the probability distribution depends on diftusion and drift coefficients, as well as on the covariances already accumulated. Eyaluated along the mean trajoctory $[\overline{\mathrm{N}}(t), \overline{\mathrm{Z}}(t)]$. these growth rates ean be written as:

$$
\begin{aligned}
& \frac{\partial \sigma_{\mathrm{N}}^{2}}{\partial \mathrm{t}} \simeq 2\left(\mathrm{D}_{\mathrm{NN}}+\sigma_{\mathrm{N}}^{2} \frac{\partial \mathrm{V}_{\mathrm{N}}}{\partial \mathrm{N}}+\sigma_{\mathrm{NZ}} \frac{\partial \mathrm{V}_{\mathrm{N}}}{\partial \mathrm{Z}}\right) . \\
& \frac{\partial \sigma_{\mathrm{N}}^{2}}{\partial t} \simeq 2\left(\mathrm{D}_{\mathrm{ZZ}}+\sigma_{\mathrm{N}}^{2} \frac{\partial \mathrm{V}_{\mathrm{Z}}}{\partial \mathrm{Z}}+\sigma_{\mathrm{NZ}} \frac{\partial \mathrm{V}_{\mathrm{N}}}{\partial \mathrm{Z}}\right) . \\
& \frac{\partial \sigma_{\mathrm{NZ}}}{\partial t}=2 \mathrm{D}_{\mathrm{NZ}}+\sigma_{\mathrm{N}}^{2} \frac{\partial \mathrm{V}_{\mathrm{Z}}}{\partial \mathrm{N}}+\sigma_{\mathrm{Z}} \frac{\partial \mathrm{V}_{\mathrm{N}}}{\partial \mathrm{Z}} \\
&+\sigma_{\mathrm{NZ}}\left(\frac{\partial \mathrm{V}_{\mathrm{N}}}{\partial \mathrm{N}}+\frac{\partial \mathrm{V}_{\mathrm{Z}}}{\partial \mathrm{Z}}\right) .
\end{aligned}
$$

The dispersion $\sigma_{A}^{2}$ of the mass number $A=N+Z$ of the projectile-like fragment can subsequently be obtained from the relation $\sigma_{\mathrm{A}}^{2}=\sigma_{\mathrm{N}}^{2}+\sigma_{\mathrm{Z}}^{2}+2 \sigma_{\mathrm{ZN}}$. In the derivation of Eqs. (1) from the Folkker-Planck equation, acount is given of the confinements impored on the transport processes by the vatiation of the drift coefficients with $\mathbf{N}$ and $Z$. These constraints, mainly due to curvalure and alignment of the underlying time-dependent potential energy surface, are more respective for larger variances and enforce the proper equilibriutn variances.

In order 10 arrive at quantitative predictions for mass and charge distributions of fragments from damped reactions and their variation with $E_{1065}$, the theory of Randrup ${ }^{2}$ is employed in this study. In this model, transport of mass, charge, entrgy, linear and angular momentum in a damped reaction is attributed to the stochastic exchange of individual nucleons between the reaction partners, taking proper account of the Pauli exclusion principle.

The dynamical calculations of mean collision irajectories in the multidimensional coordinate space were performed by solving numerically the Lagrange-Rayleigh equations of motion for 1 wo spherical nuclei joined by a small cylindrical neck.

The predictions of the transport model ${ }^{2}$ based on statistical exchange of individual nucleons are found to be in agreemenl with experimental fragmenl $A$ and $Z$ distributions. They also give a satisfactory account of data on other reaction properties such as energy losses and deflection functions. The observed variations of $\bar{A} / \vec{Z}$ and $\sigma_{\mathrm{A}}^{2} / \sigma_{\mathrm{Z}}^{2}$ ratios with $\mathrm{E}_{\text {lows }}$ are understood to reflect the strong interdependence between neutron and proton exchange processes imposed by the dynamical driving forces. Consistently, after an initial increase with $\mathrm{E}_{\text {loss }}$ predicted isotopic mass and isobaric charge variances exhibji a satura tion behavior in the $\mathrm{E}_{\text {los }}$ range where this correlation is observed to be important for the ratios $\sigma_{\lambda}^{2} / \sigma_{2}^{3}$. The predicted drop in the variances for larger values of $\mathrm{E}_{\text {loss }}$ may not lend itself to a test by the data, before deexcitation processes of the pritrary fragments are more fully understood. The success of the model employed lends strong support to the predominance of incoherent nucleon exchange in the dynamics of incoherent nucleon exchange in the dyntamics of damped nuclear reactions. Although the presence of collective charge equilibration and dissipation mechanisms cannol be exduded on grounds of the present study, no positive evidence in their support is seen in the data.

\section{Foothotes and References}

"Condensed from Phys. Leit. B 98, 355 (1981).

tDepartments of Chemistry and Physies and NSRL, University of Rochester, NY 14627.

I. M. Berlanger et al., Z. Phys. A 291, 133 (1979).

2. J. Randrup. Nucl. Phys. A 307, 319 (1978); A 327, 490 (1979). 


\title{
Correlated Charge And Mass Distributions From Reactions of ${ }^{56} \mathrm{Fe}$ With ${ }^{58} \mathrm{Ni}$ And ${ }^{64} \mathrm{Ni}$
}

\author{
H.C. Brin," B.H. Erkkila," A. Gauron, Y. Patin," R.H. Stokes," M.P. Webb." \\ P.R. Christensen, ${ }^{\dagger} O$. Hansen, ${ }^{\dagger} S$. Pontoppidan, ${ }^{\dagger} F$, Videbaek, ${ }^{\dagger}$ R.L. Ferguson, \\ F. Plasit, ${ }^{\ddagger}$ G.R Young, ${ }^{\ddagger}$ M. Blant, ${ }^{5}$ and $J$. Randrup
}

One of the primary subjects in the study of heavy ion reaction properties has been the attempt to understand the fundemental nature of the energy loss process in deep inelastic collisions. In the past several years a large number of macroscopic and microscopic models have been developed emphasizing radically difterent physical processes which may be important in various stages of the energy loss process. At the extreme of the current microscopic models are those based on transport theories that consider primarily the effects of the exchange of single nucleons between the two beavy jons during the collision process and theories that deal primarily with the damping of energy via an irreversible excitation of various collective surface vibrations or giant resonance modes in the two impinging nuclei. A priori it might be expected that the excitation of collective modes is most important in the early stages of the collision or in the very fast grazing collisions, whereas the nucleon exchange processes would become important at later times in more central collisions. The relative importance of these two energy damping modes bas been a subject of considerable debate, but until recen1ly, most theoretical-experimental comparisons bad been of a qualitative nature and yielded ambiguous onclusions on the fundamestal nature of the energy damping process.

Following the original attempts by Norenberg to apply transport theory to the problem of heavy ion collisions, Randrup ${ }^{1}$ bas recently developed a more realistic transport theory that incorporates energy damping nucleon exchanges in a one-body dissipation epproximation. An important feature of this model is that it incorporates effects due to the blockaing of transfers to state below the Fermi sea because of the Pauli principle. This model has been ormpared to experimental mass and charge variances ${ }^{2-4}$ and yielded good qualitative agreement for relatively heavy systems. In addition, it has given qualitative predictions for average deflection functions and properties of angular momentum transfer to the outgoing particles. ${ }^{5}$ In all cases a reasonably good qualitatiye agreement was obtained between the experimental data and theoretical predictions of average properties of the outgoing particles. In most cases deviations could be at least partially ascribed to the neglect of the deexcitation process for the excited fragments.

We have made a detailed comparison of calculations using this transport theory with experiment for the systems ${ }^{56} \mathrm{Fe}+{ }^{56,64} \mathrm{Ni}$ at bombarding energies of $315 \mathrm{MeV}$ and $464 \mathrm{MeV}$. Moments of the neutron-proton distributions in the projectile-like fragment are compared as functions of kinetic energy loss. Quantitative comparisons afe obtained by using theoretical final fragment distributions determined from the initial theoretical distribution by means of a Monte Carlo evaporation cascade. For energy losses greater than $10 \mathrm{MeV}$ up to the beginning of the completely darnped region the theoretical calculations, with no adjusted parameters, reproduce the measured first and second moments of the projectile-like NZ distributions altrost exactly. This comparison suggests that the singlenucleon exchange mechanism provides an adeculuate framework for describing the damping of kinetic energy in the region between quasielastic and completely damped nuclear reactions. From these results, there seems to be no indication of other important energy damping mechanisms such as the excitation of collective modes. If there were an important mode that involved irreversible energy loss without particle exchange then we would have expected the experimental $\sigma_{\mathrm{A}}$ values to be less than the theoretical transporl model prediclions in some entrgy loss region. If the transfer of large chunks of nuclear matter were important we might have expected the experimental $\sigma_{\mathrm{A}}$ values to be latger.

The excellent agreement between theory and experjment also demionstrates that it is possible to make realistic evaporation corrections even for relatively light higbly excited muclei. However, it is necessary to generate theoretical final fragment distributions from calculated initial distributions rather than trying lo recreale an initial experimental distribution from the measured final distribution.

\section{Footnotes and References}

"Los Alamos National Laboratory, Los Alemos, NM 87545.

${ }^{\dagger}$ Niels Bohr Institute, University of Copenhagen, DK-2100 Copenhagen, Denmark

foak Ridge National Laboratory, Oak Ridge, TN 37830.

Lawrence 1ivermore Laboratory, Livermore, CA 94550.

1. J. Randrup, Nuct. Phys. A 327, 490 (1979).

2. W.U. Schröder, J.R. Birkelund, J.R. Huizenge, W.W. Wilcke and J. Randrup, Pbys. Rev. Lett. 44, 308 (1980).

3. P. Dyer, M.P. Webb, R.J. Puigh, and R. Vandenbosch, Phys. Rev. C 22, 1509 (1980).

4. W.U. Schröder, J.R Huizenga and J. Randrup, Phys. Lett. B 98, 355 (1981).

5. A Lazzarini, V. Metag. AG. Seamster, R. Vandenbosch, and R. Loveman, Phys. Rev, Lett. 46, 988 ( I $98 \mathrm{~L}$ ). 


\title{
Transport of Angular Momentum in Damped Naclear Reactions
}

\author{
Jorgen Randrup
}

During the last decade our understanding of damped nuclear reactions has increased steadily. Many of the characteristic features, such as the defection function, the energy loss, and the angular momentum gain, can be understood by considering the classical motion of two idealized macrosopic nuclei interacting via onstervative and dissipative forces. Furthermore, the accumulation of appreciable dispersions in the various macroscopic observables, such as the binary partition of the total mass and charge and the energies and spins of the emerging fragments, can be described as a tuansport process. Within such a framework the dissipative properties of the system are contained in the cotficients entering in the transport equation. While these transport coefficients con, to a large degret, be determined phenomenologically from data, theit qualitative origin as well as their quantitative calculation still presents a major challenge in nuclear theory.

It is an outstanding feature of damped nuclear reactions that many nucleons are exchanged between the two interacting nucleides in the course of the collision process. (The large dispersions in the mess and charge of the final fragments lestify to this fact.) Elementary kinematical considerations indicate that exchange of nucleons has a substantial effect on the dynamical state of the dinucleus. It is therefore essential to take account of this mechanism in the theoretical description of damped nuclear rections.

Indeed, our estirtates indicate that nucleon exchange plays a major, possibly dominani, role as a dissipative mechanism in damped nuclear reactions. Therefore, we have found it imporitint to explore a model in which the only dissipative coupling between the two interacting nucleides is the exchange of nucleons. This idealization pernits us to go far without introducing arbitrary assumptions and, in fact, enables to to calculate and interrelate a wealth of observables. In consequence, the subsequent comparison with data may lead to relatively unambiguous conclusions concerning the dissipation mechenism.

Towards this end, a general theory was developed for transport induced by nucleon exchange. I' Subsequently, it was demonstrated that the theory provides a good understanding of the relation between energy loss and particle- number dispersion ${ }^{2}$ as well as of the interrelation belween charge and mass exchange. ${ }^{3}$ In view of this success, it is important to broaden the confrontation with experiment by considering other obstervables as well. Therefore, we fockus on the angular-momentuon related observables, which are more complicated but also encompass a richer variely of physical phenomena.

At first, after oullining the description of nuclear dynamies within the iramework of transport theory in general terms, we consider the dynamics of the rotational degrees of freedom in the dinucleus and derive and discuss the appropriate equations of motion. Subsequently some illustrative applications of the theory are made to cases of experimental interest. The characteristic features observed for the spin distributions of the fragments are well reproduced by the model calculations, al though a precise comparison must await the proper inclusion of the deexcitation processes.

The model, being fully dynamical, permits detailed studies of the relaxation phenomena and, in fael, it predicts significant nonequilibrium behavior, particularly for partially damped reactions.

The only dissipative mechanism considered is the exchange of nucleons. In consequence, several observables are fundamentally related. To gain more definjte insight into the reaction mechanisms, it is likely that one must bonsider several observables simultareously. Therefore, more refined experiments are called for. As a specific example, it is anggested that attempis be made to measure the degret of mutual alignment of the two reaction products. Such an experiment would prowide novel information which would help discriminate between different models and thus improve our onderstanding of the reaction mechanism.

\section{Fooinotes and References}

${ }^{*}$ Condensed from LBL-12676; Nucl. Phys. A in press.

I. J. Randrup, Nucl. Phys. A 327, 990 (1979).

2. W.U. Schrỏder et al., Phys. Rev. Lett. 44, 308 (1980),

3. W.U. Schrōder, J.R. Huizenga and J. Randrup, Phys. Lett. B 98, 355 (I981). 


\title{
Equilibrium Statistical Treatment of the Spin Fluctuations Generated in Deep-Inelastic Reactions
}

\author{
A.J. Pacheco, R.P. Schmitt and LG Moretio
}

One of the distinctive leatures of heavy-ion collisions is a large angular-momentum transfer from relative motion to intrinsic degrees of freedom. ${ }^{1}$ It has been found experimentally that in many reactions the rotational degrees of freedom reach complete relaxation, i.e., the disuclest complex rotates rigidly about an axis perpendicular to the line between onters. Recent experiments have also shown that the rigid-rolation limit is but an average regime about which large fluctustions occur, and as a consequence, one observes in the exit channel a probability distribution for both the magnitude and orientation of the spins of the outgoing frapments, tn a recent work Moretto and Schritt ${ }^{2}$ have proposed an equilibrium statistical model to acopunt for the production of random spin components in a system of two equal rigid spheres in contact. The purpose of the present work is to extend this equilibrinm statislical trealment to systems of arbitrary mass asymmetry.

The most general configuration of the system is completely deterwined by the spisis of the heavy and light fsagments $\left(\vec{S}_{H}\right.$ and $\left.\vec{S}_{\mathrm{L}}\right)$ and by the orbital angular momentura (b). In order to describe the norralal modes, it is convenient to define a set of "spin-excess" variables $\Delta \vec{S}_{H}$ and $\Delta \vec{S}_{\mathbf{L}}$ by means of the equations!

$$
\vec{S}_{\mathbf{H}(L)}=\vec{R}_{\mathbf{R}(\mathbf{L})}+\Delta \vec{S}_{\mathbf{H}\{\mathbf{L})}
$$

where $\vec{R}_{y}$ and $\vec{R}_{L}$ are the spins of the fragments arising from rigid rotation about an axis perpendicular to the line of centers and with a total angular momentum $\overrightarrow{\mathrm{I}}=\overrightarrow{\mathrm{S}}_{\mathrm{H}}+\overrightarrow{\mathrm{S}}_{\mathrm{L}}+\ell$. The normal coordinates (iwo for each cartesian axis) are constructed as suitable linear combinalions of $\Delta \vec{S}_{H}$ and $\Delta \vec{S}_{\mathrm{L}}$. Two of these normal modes (tilting and twisting) generate spin components along the direction of symmetry ( $y$-axis). The other four modes (two wriggling and two bending) produce spin components on a plane perpendicular to it ( $x-2$ plane).

The physical interpretation of the modes is given in Fig. I. Curve a) shows the dependence of the ratio

$$
\frac{\left.\Delta \vec{S}_{H}\right\rangle_{12}}{\Delta \vec{S}_{\mathbf{X z}}}
$$

as a function of mass asymunetry for the wriggling mode. In this case both fragments have their spin vectors pointing in the same direction, and as the asymurelry increases the beavy fragment carries an increasing fraction of the angotlar momentum The opposite behavior is predicted for bending as indicated by the fact that the same curve a) represents the ratio

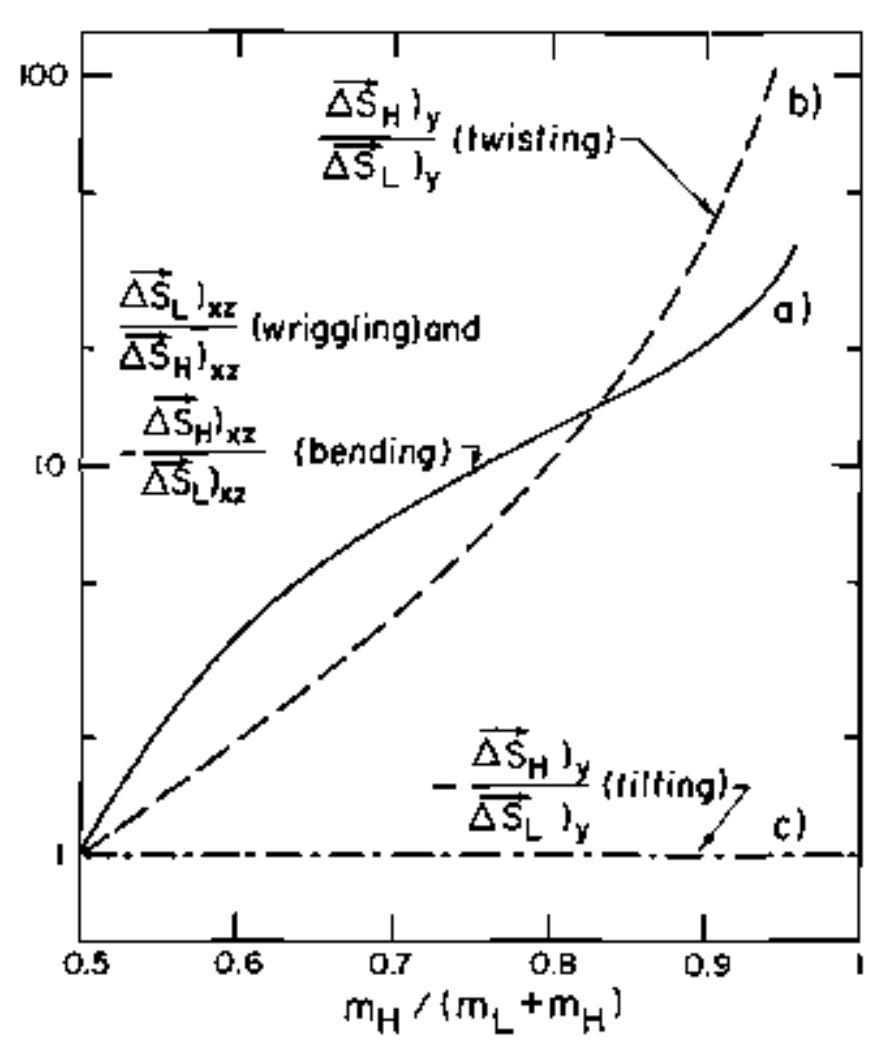

Fig. I. Illustration of the normal modes as a function of mass asymumetry, based on the relative amounts of spirt going into the heavy and light fragments. (XBL 818-2423)

$$
-\frac{\left.\Delta \vec{S}_{t}\right)_{x 2}}{\left.\Delta \vec{S}_{\hat{H}}\right)_{\lambda 2}}
$$

for this riode. The interpretation of the twisting and tilting modes does not differ from that given in Ref. 1 for a symune1ric system

The dependence of the reduced variances of the spin distribution as mass asymmetry is shown, for each mode, in Fig. 2. The overall variances for each carlesian coordinale bave simple analytical expressions in terms of the Jelevant moments of inertia of the system.

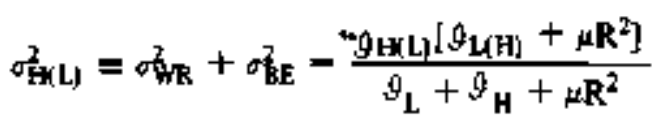

for the $\mathrm{x}$ and $\mathrm{z}$ directions . 


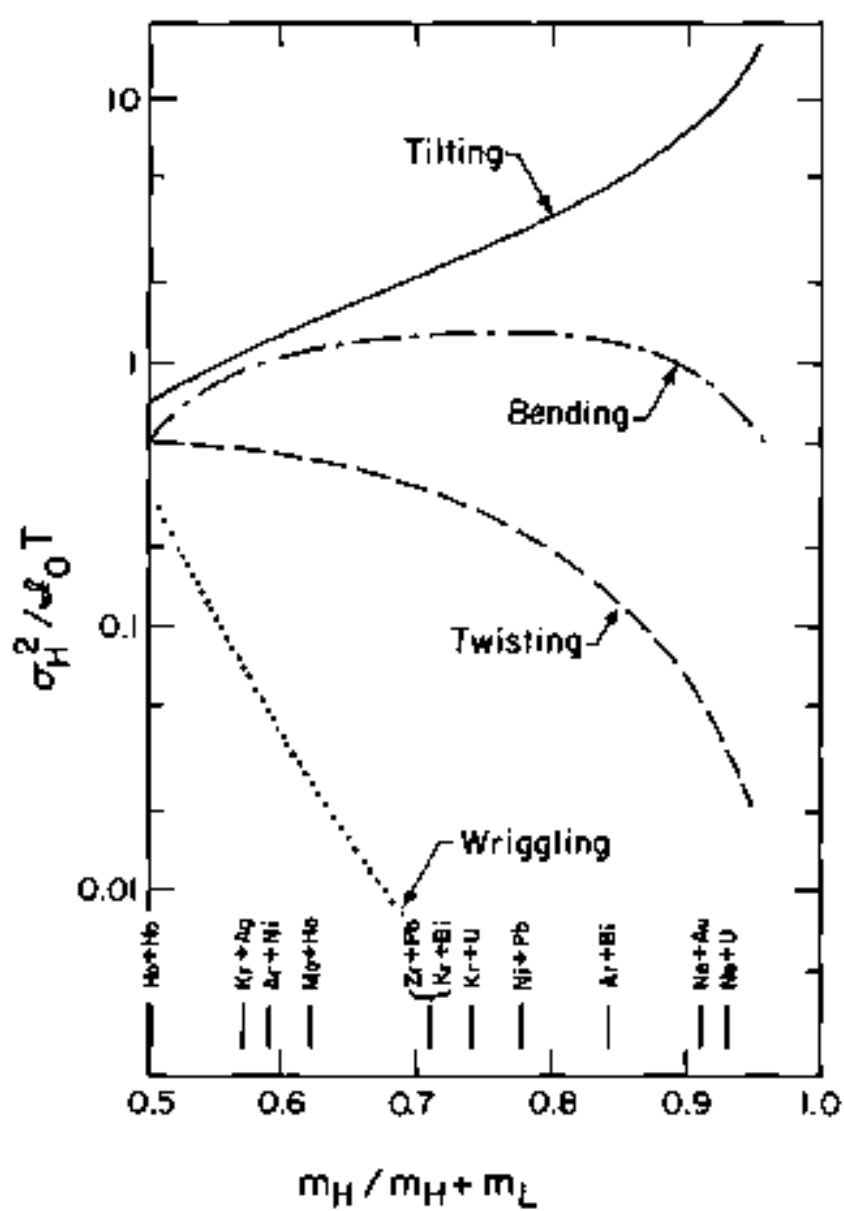

Fig. 2 Contribution from each mode to the reduced variances of the spin distribution in the heavy fragment $g_{0}$ : moment of inertia of a sphere of mass $\left(m_{1}+m_{1}\right) / 2, f$ : nuclear temperature).
(XBL 812-2L14A)

$$
\sigma_{\mathrm{H}(\mathrm{L})}^{2} \equiv \sigma_{\mathrm{fw}}^{2}+\sigma_{\mathrm{f} 1}^{2}=\frac{\vartheta_{\mathrm{H}(\mathbf{L})}\left[\mathfrak{\vartheta}_{\mathrm{H}(\mathbf{L})}+\mu \mathbf{R}^{2}\right]}{\mu \mathbf{R}^{2}}
$$

for the $y$ direction.

Equations (2) and (3) and Fig. 2 indicate that at very large asymmetries the spin fuctuations in the heavy frag. ment occur mainly along the symmetry axis. Such effect bhould be detectable by measuring in-plane angular distribution of the sequential-fission fragnents.

\section{foothotes and References}

"Permanent address: Comisión Nacional de Energía Atónica, Buenos Aires, Argentina.

iPtesent address: Cyclotron Institute and Department of Chemistry, Texas A\&M Unjuersity, College Station, TX 78043.

1. M. Léfort and C. Ngö, Riv. Nuovo Cimento 2, 12 (1979) and references therein.

2. L. G. Moretto and R.P. Schmitt. Phys. Rev. C 21 (1980).

\title{
Systematics of Angular Momentum Transfer in Intermediate Energy Heavy-Ion Reactions"
}

\author{
C.C. Hsu. ${ }^{\dagger}$ D.J. Morrissey. ${ }^{\ddagger}$ LW. Richardson, G.J. Worniak, and LG. Moreno
}

An important question regarding heavy-ion collisions is how the reaction mechanism evolves from the statistical equilibrium observed in the low energy regime to the conpletely nonequilibrium processes observed in the relativistic regime. At bombarding energies of a few $\mathrm{MeV} / \mathrm{A}$ over the Coulomb barrier, the two heavy ions undergo a gentle collision. As the bornbarding energy increases, the oollision becomes harder. At intermediate velocities, new reaction processes may occur which should be amenable to kinematic analysis if the collisions are nol too violenl.

A detailed study of the linear momentum transfer has demonstrated that the ${ }^{16} \mathrm{O}+{ }^{238} \mathrm{U}$ reaction deviates from two-body kinematios al a bombarding energy of
$20 \mathrm{MeV} / \mathrm{A}$. It would be interesting to determine whether similar deviations exist for other reaction systems and the bombarding energy dependence of any deviation. In this article, we present a complementary technique for studying the two-body character of a heavy-ion reaction with nonfissioning products. In this technique we indjrectly delermine the linear morrentum transferred to the product nucleus by measuring the angular momentum converted into intrinsic spin via $\gamma$-ray multiplicity $\left(\mathrm{M}_{\gamma}\right)$ techniques. The connection between the linear and angular momenta transferred in peripheral reactions is straight forward because a localized linear impulse on the periphery of the target nucleuss gives rise to a torque which causes the target nucleus to spin. 
The intrinsic spin can be calculated in a simple model as a function of the detected fragment's mass and energy. This simple model assumes a two-body reaction, a tangential collision, an average impact parameter, and no radial damping. The intrinsic spin, I, that would be created by a highly localized tangential force at the nuclear surface can be estimated rather simply: the torque, $\tau$, is equal to the time integral of the force, $F(t)$ crossed into the radius at which it acts:

$$
\tau=\int R(t) \times F(t) d t .
$$

If the force is tangential and sharply peaked at some radius, $\bar{R}$, then the integral can be replaced by: $\tau=\bar{F}_{t} \vec{R}$, where $\overline{\hat{F}}$ is an average force. The connection to linear momentum balance is now obvious because the linear impulse, $\Delta$ p. can be written as:

$$
\Delta p=\int F(t) d t \text {. }
$$

For a localized force, $\Delta_{\mathrm{p}}=\overline{\mathrm{F}} \mathrm{t}$, the angular mornentum is the change in linear momentum times the average radius at which is it ereated, I $-\Delta p \bar{R}$. A similar analysis with the explicit matching of the entrance channel orbital angular momentum to that in the exit channel has been sug. gested as a tool for the analysis of light-ion reactions. ${ }^{2}$ Assuming that the orbital angular monentern is completely aligned in the entrance and exit channels, we can write a classical expression for the transferred spin: $\mathrm{L}_{\mathrm{j}}=\mathbf{L}_{\mathrm{r}}+$ $\left\langle\mathrm{I}_{\mathrm{TOT} / \mathrm{L}}\right\rangle$ in terms of the orbital angular momenta in the entrance and exit chanmels, $L_{i}$ and $L_{r}$ respectively. These orbital angular momenta ase calculated from the kinetic energies.

The strong as well as the weak points of our picture of the spin transfer can be seen in Fig. I whete the observed value of the total intrinsic spin is plotted against the calculated value. The spin transfer is nearly equal to the simple estimate at low bombarding energies and rougbly only half that at higher energies. Perfect agreement between theory and experiment would, of course, yield a straight line with a slope equal to one and an intercept of $(0,0)$. However, the discrepancies may be more apparent than significant in light of the simplicity of the model. At the lowest energies Fig. I shows a small discrepancy between the model and the data, the slope is -0.8 ratber than 1 . There is a dramatic decrease in the slope at $17 \mathrm{MeV} /$ nucleon which is also observed at higher bombarding entrgies. At 17 $\mathrm{MeV} / \mathrm{A}$ and above, the spin transfer is less than balf that observed at lower energies for producing the same product at the same energy loss at the grazing angle. The

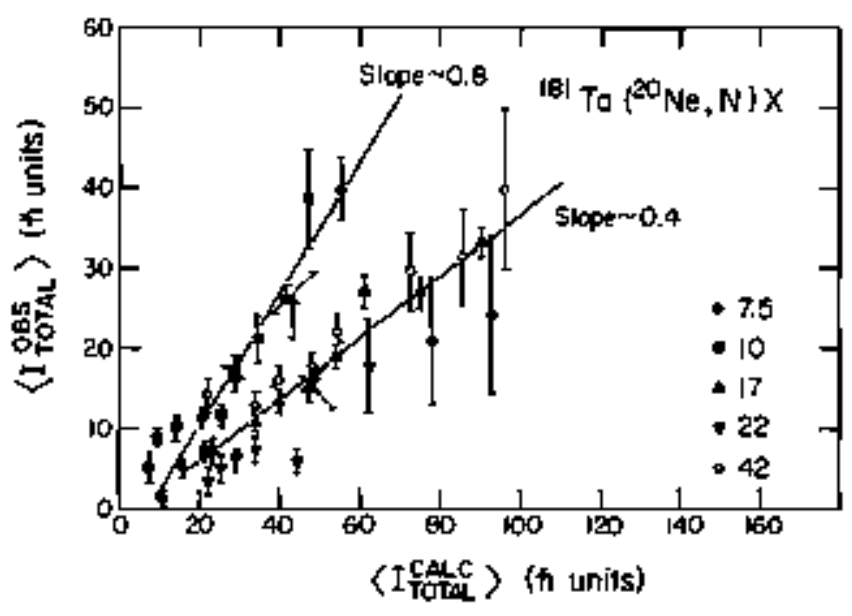

Fig. 1. The colculated total spin trouls is plotted against the observed total spin. Perfect agreement between model and experiment would give a slope of 1 , the grouping of the data by the bombarding energy is discussed in the text.

(XBL. 818-2426)

simple model that we have used is based on momentum balance between the entrance and exit channels where both channels were assumed to have only "two bodies." How* ever, if an excited projectile residue breaks up into two or more bodies then this simple model will overestimate the spin transfer, besalse it assumes all missing momenturn has been captured by the target nucleus. The explanation of the decrease in the abserved spin transfer at high bombarding energies requires a breakdown of the assumption that the exit channel is two body. This is in agrexment with the results of Back et al. on linear momentum transfer at $20 \mathrm{MeV} / \mathrm{A}$ However, it is sornewhat surprising that at $17 \mathrm{MeV} /$ nucleon projoctile bregkup seems to be the predominent process.

\section{Foothotes and References}

"Condensed from LBL 12519, suboritted to Nuclear Physics A.

tPermanent address: Institute of Atomic Energy, Beijing, People's Republic of China.

FPermanent address: Department of Chemistry, Michigan State University, East Lansing, MI 48824

'1. B.B. Back et al., Phys. Rev. C 22, 1927 (1980).

2. D.M. Brink, Phys. Lett. B 40, 37 (1972). 


\title{
Shell Effects on the Angular-Momentum Transfer in Heavy-Ion Reactions
}

\author{
L.G. Moretto and A.J. Pacheco*
}

The measurement of $\gamma$-ray multiplicities in heavy-ion reactions provides information on the sum of the spins generated in both fragments. The dependence of this quantity upon the tolal kinetic energy loss reflects the interplay between the relaxation time of the rotational degrees of freodom and the interaction time associated with a given entrance-channel angular momentum. We present here a very simple model of this process and we use it to calculate the effects that are expected in reactions in which one of the partners is a closed-shell nucleus.

In our model we describe the dinuclear complex by means of two touching spheres with spins $S_{1}, S_{2}$, and orbjtal angular momentom $L$. The relaxation of the rotational modes of the system as a function of interaction time is expressed by: ${ }^{1}$

$$
s_{1}+s_{2}=\frac{\theta_{1}+\theta_{2}}{\eta_{1}+\theta_{1}+\mu R^{2}}\left(1-e^{-1 / n}\right) 1
$$

where $s_{1}$ and $s_{2}$ are the moments of inertis of the nuelei, $\mu$ is the reduced mass of the system, $R$ is the dislance between centers, ${ }^{T} R$ is the relaxation time constant. and $I$ is the total angular moinentum. For the dependence of the interaction time on I. we gasume the following analytical expression:

$$
t=\tau_{1} \ln \left(\frac{l_{\max }}{I}\right)
$$

where $I_{\text {Max }}$ is the maximum angular momentum corresponding to a grazing collision and $t_{l}$ is a constant time From Eqs. (1) and (2) the spin transferred to the fuagments can be related to the total angular momentum:

$$
\frac{\mathrm{J}}{\mathrm{I}_{\mathrm{MAx}}}=\mathrm{I}-\frac{g_{1}+g_{2}+\mu \mathrm{R}^{2}}{g_{1}+g_{2}} \frac{S_{1}+S_{2}}{\mathrm{I}} \tau_{\mathrm{R}} / \tau_{\mathrm{I}}
$$

If we restrict ourselves to the case of short-lived peripheral collișions

$$
\frac{s_{1}+s_{1}}{I}<\frac{\theta_{1}+\theta_{2}}{\mu R^{2}+\theta_{1}+\theta_{2}}
$$

we can find an analytical solution for $\mathbf{l}$, which may in turn be used in the expression of the orbital kinetic energy:

$$
\begin{aligned}
& \frac{E}{E_{M x}}= \\
& \frac{1}{4}\left[1+\sqrt{1-\frac{4\left[g_{1}+g_{2}+\mu R^{2}\right]}{g_{1}+g_{2}} \frac{z_{R}}{\pi_{1}} \frac{s_{1}+s_{2}}{I_{M u x}}}-\frac{2\left[S_{1}+S_{2}\right)}{I_{\max }}\right]^{2}
\end{aligned}
$$

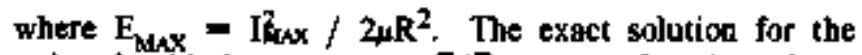
raduced orbital energy $E=E / E_{\text {hax }}$ as a function of the reduced total spin

$$
s=\frac{7}{2} \frac{\left(S_{1}+s_{2}\right)}{l_{\operatorname{sax}}}
$$

is shown in Fig. la for a mass-symmetric system and four different values of the ratio $\tau_{\mathrm{R}} / \tau_{\mathrm{I}}$ Figure $1 \mathrm{~b}$ illustrates the prediction of the orodel for the case in which one of the two fragments is magic and has zero angular monsentum (this is of course an extreme approximation).

We shall now disetss another aspet of the same problem, namely the extent to which shell effects are present in the second moments of the spin distribution. ${ }^{2}$ The dependence of the fluctuations induced in one fragment upon the moment of inertia of the other is shown in Fig. 2. The reduced variance along the symmetry axis is constant as a consequence of an exact cancellation between a stifier twisting mode and a softer tilting mode as one approaches the closed-shell limit

$$
\left(\frac{\eta_{2}}{g_{1}}=0\right) .
$$

Due to a similar effect the fluctuations on a plane perpendicular to the symmetry axis, altbough not constant, are also very insensitive to variations of the ratio $1 / 2$.

\section{Foothote and References}

*Permanent address: Comision Naciontal de Energfa Atómica, Buenos Aires, Argentina.

1. S. Ayik, G. Wolschin and W. Nibremberg, Z. Phys. A 286. 271 (1978).

2. AJ. Pacheco, R.R. Schmitt, and L.G. Moretto, in this annual report. 


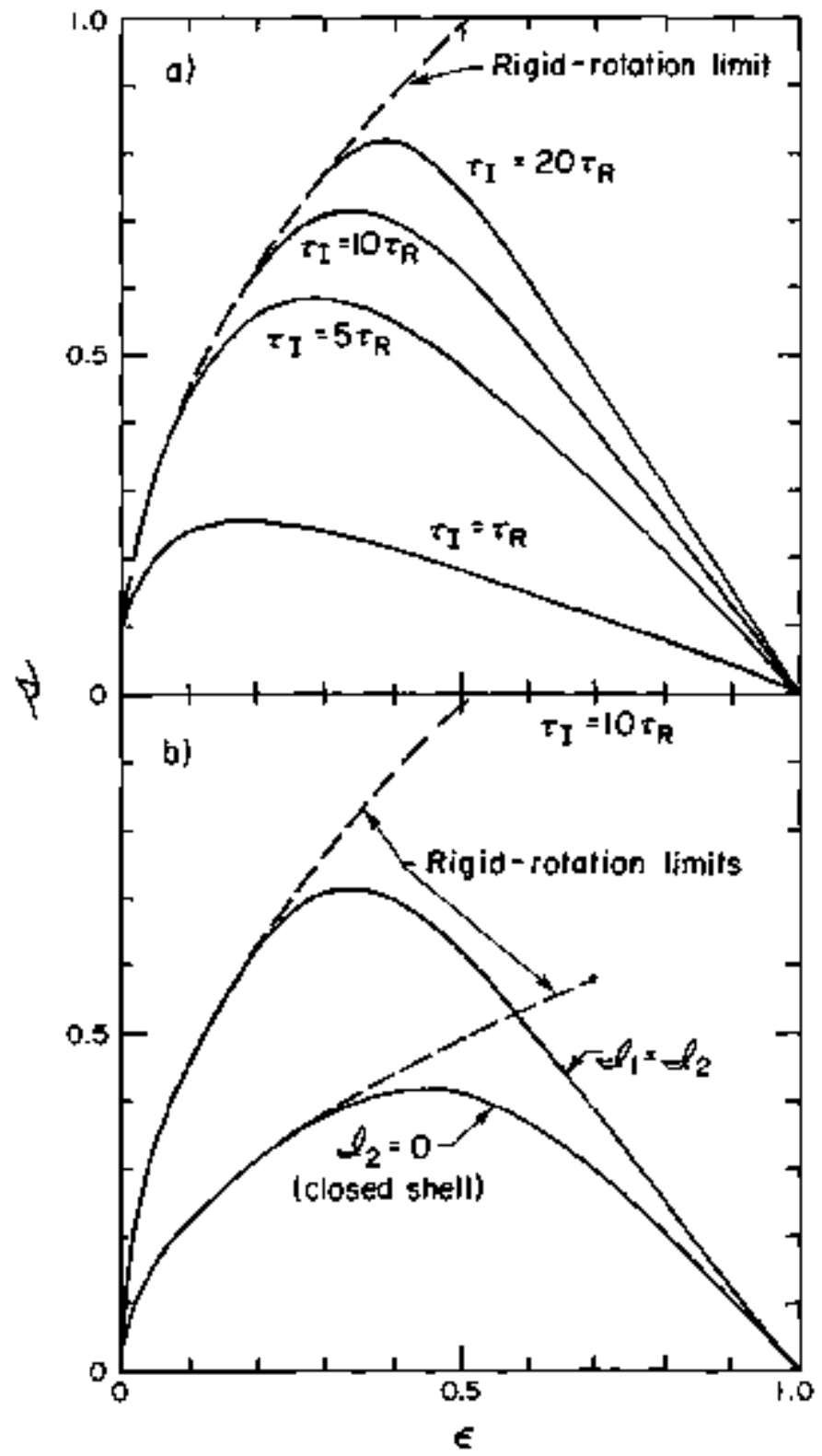

Fig. 1. Reduced orbital kinetic entrgy vs refiuced sum of the spin of a trass-symmetric system, a) for different values of the ratio $\tau_{1} / \tau_{R}$ and $b$ ) for two values of $g_{2} / s_{1}$.

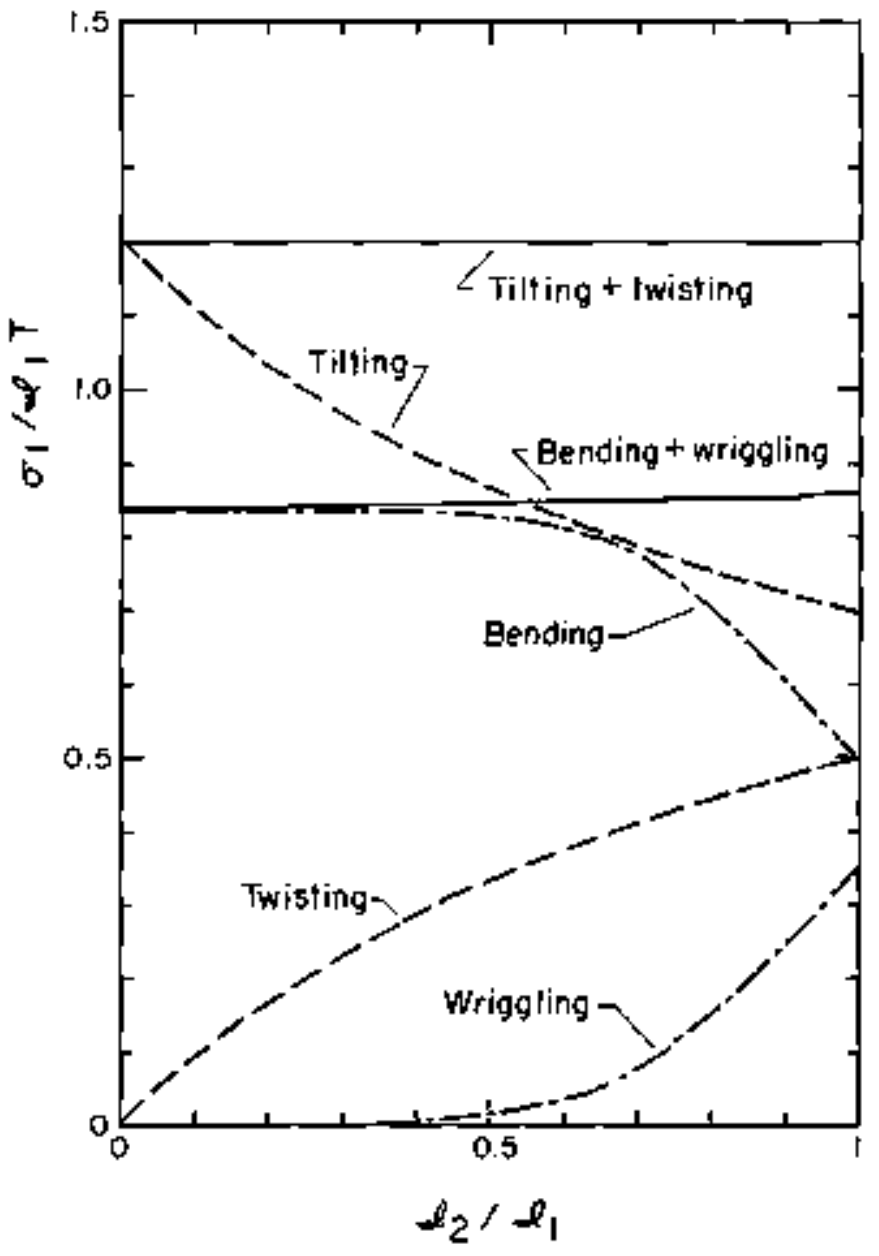

Fig. 2. Reduced varianes of the spin distribution of frag. ment $l$ as a function of the moment of inertia of fragment 2 for a mabs-symmetric system.

(XBL 818.2425) 


\title{
The Influence Of Fluctuations On The Correlation Between Exit-Channel Kinetic Energy And Entrance-Channel Angular Momentum For Heavy Ion Collisions
}

\author{
$L G$ Moretio and LG. Sobatka
}

The role of fuctuations on the relationship between the exit-channel binetic energy and the entrance-channel angular momentum in deep inelastic heavy jon reactions has been studied in the equilibrium limit. Two sources of fluctuations are considered: first, the coupling of the orbstal motion to thermally excited angular-momentumbearing modes, and the second, the effect of random shape fluctuations at scission.

Let us consider the siomple analytical case of two equal touching spheres with one wriggling'mode ${ }^{1}$ coupled to the orbital motion. Using a 2ldI weight, where I is the entrance channel angular momentum, the distributoon function ts

$P(\epsilon, \lambda)$ ded $\alpha \propto$

$$
\frac{\lambda}{\sqrt{\epsilon}} \exp -\left[\frac{7}{2} \epsilon-\sqrt{\frac{5}{2}} \lambda \sqrt{\epsilon}+\frac{5}{2 B} \lambda^{2}\right] \mathrm{ded} \lambda .
$$

Here $t$ and $\lambda$ are reduced variables defined by,$-E / T$ and $\lambda=1 /(\$ T)^{1 / 2}$, where $E$ is the exit channel kinetic energy over the Coulonb barrier, $T$ is the nuclear temperatwre, and 9 is the mownent of inertia of one of the two spheres. The properties of this distribution function can be observed in the two-dimensional plat in Fig. I, where we see that the one to one correspondence between exit chanpel energy and entrance channel angular momentum is substantially perturbed.

Shape fuctuations at scission arise because of the reasonabiy flat dependence of the total polential energy as a function of deformation and of the rather sletp dependence of the two-fragment Coulomb interaction.

Therefore, a small (energy-wise) fluctuation at scission, of the order of $\mathrm{I} / 2 \mathrm{~T}$ in the thermal limit, leads to an amplified fuctuation in the final kinetic energy. ${ }^{2}$

As an example, let us consider the system $\mathrm{Fe}+\mathrm{Fe}$. The overal] features of the probability distribution, $P\left(E_{\text {TKE }} \mathrm{I}\right)$, are shown by the two-dimensional plot in Fig. 2 . While the centroid of the energy distribution at fixed $\ell$ moves towards bigher values with increasing $\ell$, the width also increases, leading to a dramatic swerlap of distributions with widely different \&-values. Most interesting are the entrance-channel angular mornentum distributions for a variety of exit-channel kinetic energies. These distribulions are so broad that at any kinetic energy the whole \&-wave spectrum is substantially sampled Again we see that the correlation between exit-channel kinetic energy and entrance-channel angular momentum is quite probIenatic.

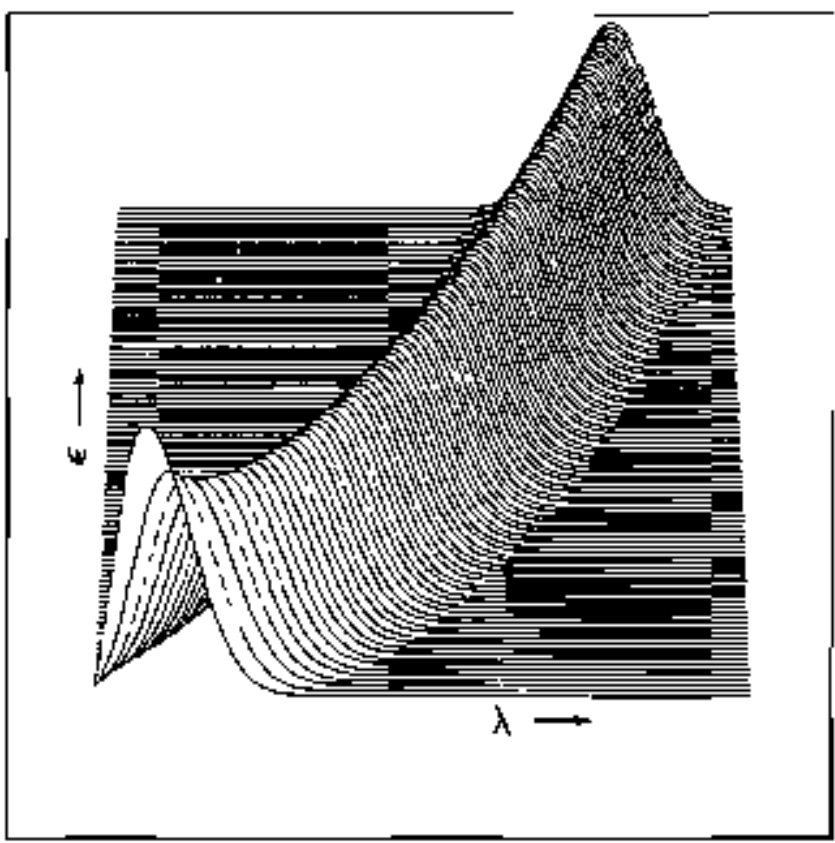

Fig 1 Two-dimensional plot of the distribution Junction given in Eq. (1).

(XBL 815-2268)

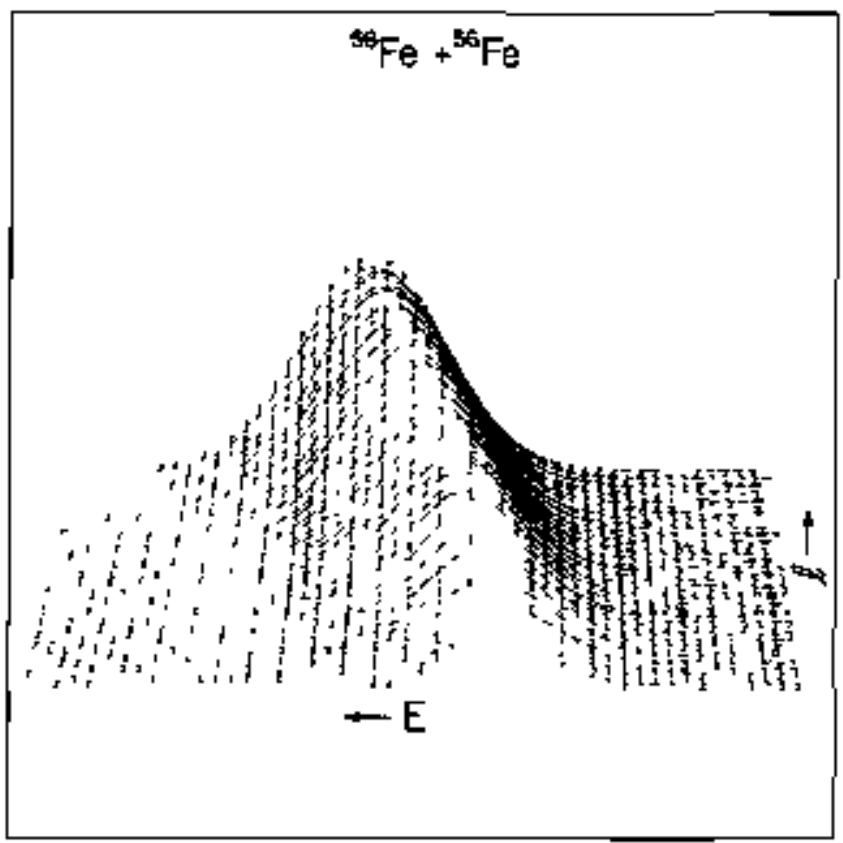

Fig. 2. Two-dimensional plot of the emission probability as a function of entrance-channel angular momenturn and exit-channel kinetic energy.

(XBL 815-2267) 
Foothotes and References

"Condensed from LBL 13007.
1. L.G. Moretto and R.P. Schmitt, Phys. Rev, C 21, 204 $(1980)$.

2. L.G. Moretto, Nucl. Phys. A 247, 2 I (1975).

\title{
On the Use of Isomer Ratios in ${ }^{44} \mathrm{Se}$ for Predicting Spin Populations in High Energy Heary Ion Nuclear Reactions
}

\author{
H. Groening." X.J. Moody and G.T. Seoborg
}

It has been suggested that isomer ratios can be used to determine the spin distributions in the evaporatjon chains of different products formed in spallation reactions. By following the methodology described in Ref. 2 to fit the isomer ratio dependence on energy for the reaction ${ }^{29} \mathrm{Si}\left({ }^{18} \mathrm{O}, \mathrm{p} 2 \mathrm{n}\right){ }^{4} \mathrm{Scm}^{4},{ }^{44} \mathrm{Sc}$, we have also made a fit of the jsomer ratio dependence on energy ${ }^{3}$ for the reaction ${ }^{4} \mathbf{K}(a, n){ }^{4} \mathrm{Sc}^{\mathrm{m}},{ }^{4} \mathrm{Sc}$, using the same set of parameters. Figure 1 shows the positions of the calculated "Se" population averages in the E.J plane for both reactions, joined by "tie" lines that define regions of equal isomer ratios. Our results show that isomer ratios can be used to predict angular mornentum distributions in ${ }^{44} \mathrm{Sc}^{*}$ populations, par- ticularly if such ratios are low. As the ratios become higher, the average energy becomes as important a factor as the average spin, thereby making unique identifications of such properties difficult.

Figure 2 shows in semilog plot the average excitation energy ws average spin for ${ }^{44} \mathrm{St}^{*}$ and also for the ${ }^{47} \mathrm{Ti}$ * precursor. Tie line (AB), which joins two points with equal excitation energies gives rise to a common isoner ratio, suggesting that isomes ratios offer little hope in identifying uniquely spin properties of ${ }^{44} \mathrm{Sc}$ precursors, even if the average energies are known. This situation is expected to deteriorate as such average energies increast.

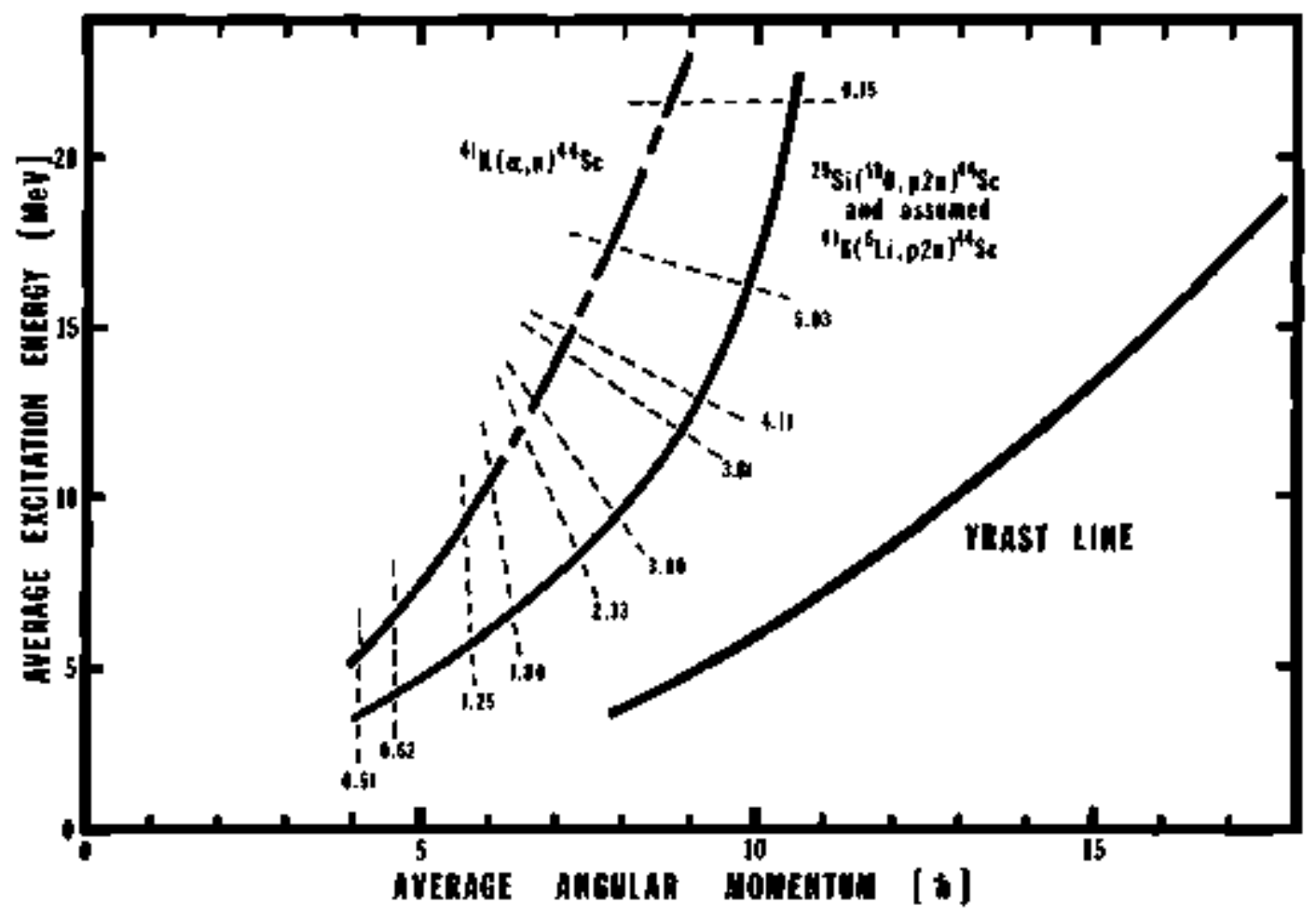

Fig. I. Calculated centroids of population distributions in ${ }^{44} \mathrm{Sc}^{*}$ for the reactions ${ }^{29} \mathrm{Si}\left({ }^{18} \mathrm{O}, \mathrm{p}^{2 n} \mathrm{n}{ }^{44} \mathrm{Sc} \mathrm{c}^{*}\right.$ and ${ }^{41} \mathrm{~K}(\alpha, n){ }^{4} \mathrm{Sc}^{*}$. Dashed lines join regions of popalation averages that give rise to equal isomer ratios.

(XBL 818-I1297) 


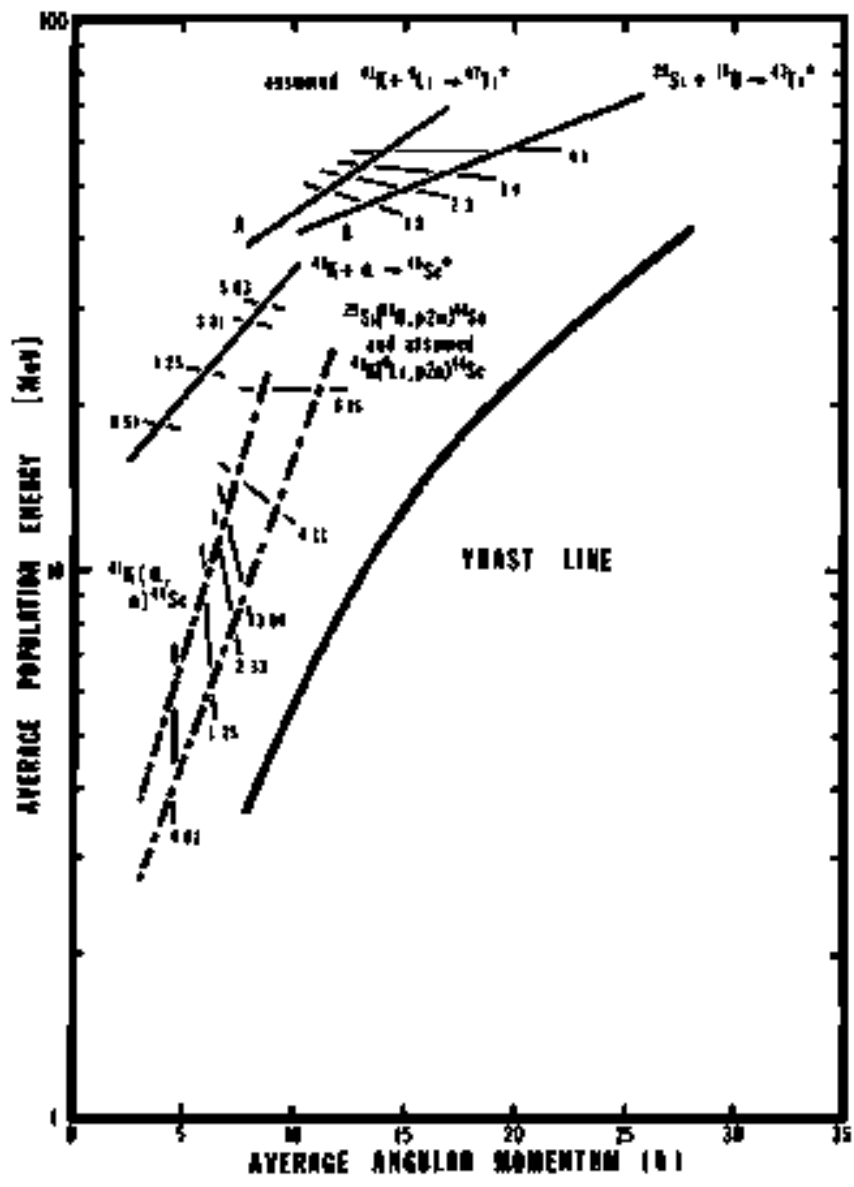

Fig. 2, Sarte as Fig. I except that partial average population regions in ${ }^{47} \mathrm{Tj}^{*}$ that give rise to a common isomer ratio in "Se" are also included. Also shown are partial regions in ${ }^{45} \mathrm{~S} \mathrm{c}^{*}$ that result in given isomer ratios in ${ }^{44} \mathrm{~S} \mathrm{c}^{*}$.

(XBL 818-11294)

\section{Footnote and References}

"On leave from Universidad Simón Bolivar, Caracas, Venezuela.

1. G. Rudstam, Physico Scripta 20, 165 (1979).

2. H. Groening, K. Alektett. K.J. Moody, P.L MoGaughey, W. Loveland and G.T. Segborg, in this annual report.

3. C. Riley, K Ueno, and B. Linder, Phys. Rev, 135 , B1340 (1964).

\title{
Pre-Equilibrium Nucleon Jets in TDHF Calculations of Medium-Energy, Heavy-Ion Collisions
}

\author{
H. Stöcker," R.Y. Cusson, J.A. Marthin, and W. Greiner"
}

Recent experiments with the new $85 \mathrm{MeV} / \mathrm{A}{ }^{12} \mathrm{C}$ beam at $\operatorname{CERN}^{1}$ have further stimulated the current interest in the intermediate bombarding energy range $20 \mathrm{MeV} / \mathrm{A} \leq \mathrm{E}_{\text {ob }} / \mathrm{A}$, $5200 \mathrm{MeV} / \mathrm{A}$ Our study of the ${ }^{12} \mathrm{C}(30 \mathrm{MeV} / \mathrm{A}){ }^{{ }^{p o j}}{ }^{[98} \mathrm{Au}$ reaction shows three interesting regions of impact parameter b (Ref. 2; see Fig. 1): i) deep-inelastic scattering in the grazing region, b $-9 \mathrm{fm}$, ii) complete disintegration of the projectile, which escapes as Jast, forward peaked preequilibrium mucleon jet, and PEP's in the full overlap region $0 \leq \mathbf{b} \leq$ $4.25 \mathrm{fm}$ and iii) the partial overlap region $4.25 \leqslant \mathrm{~b} \leqslant$ $9 \mathrm{fm}$, where several phenomena can occur in varying proportions: a) jet emission at finite angles, b) generally unfocused pre-equilibrium emission, and c) massive transfer, where the remainder of the projectile can orbit around the target. Our results at BS MeV/A (see Fig. 1) are similar to the $30 \mathrm{MeV} / \mathrm{A}$ ones in region i) and $\mathrm{ii)}$. However, in the intermediate region iti), one can no longer distinguish three separate processes. Instead, one observes the pieces of a partially broken up projectile at negative angles, and rempents of both jets and unfocused preequilibrium emission are found.

The formation of a typical nucleon jet is illustrated in Fig. 1 (b - I fm, $\mathrm{E}_{\mathrm{ab}}-30 \mathrm{MeV}$ ): Once the ${ }^{12} \mathrm{C}$ nucleons have entered the target. they expand inside the 


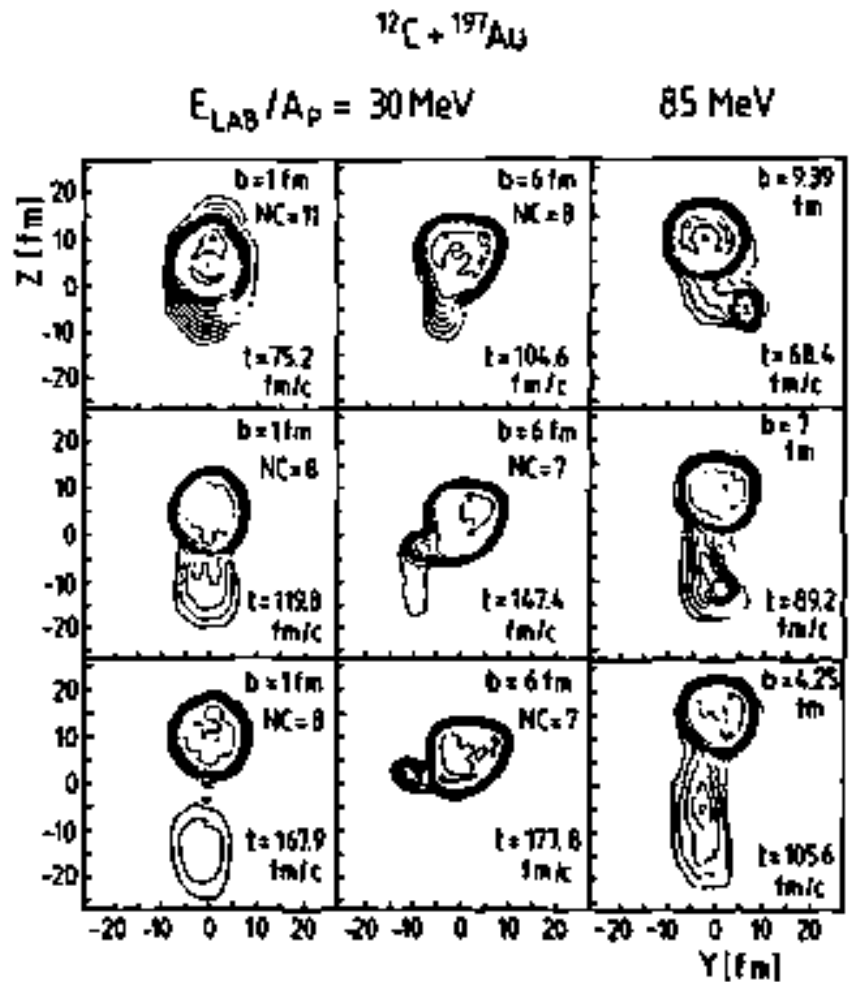

Fig. 1. Contours of constant density in the scattering plane of ${ }^{12} \mathrm{C} \rightarrow{ }^{197}$ All collisjons.

(XBE BI10-12216)

moch larger Au nucteus. This is similar to the spreading of a wave packel in free space. By examining the current distributions, one 6nds that the expension is accompenied by a strong velocity dispersion, where the various com ponents move apart from each other with $V_{\text {proj }}-V_{F} \leqslant V$ ₹5 $V_{\text {PEP }}$ according to their Fermi momenta. First, the fastest parlicles escape with $V_{\text {PEP }} \sim V_{\text {proj }}+V_{F}$ at very low density $p \sim 10^{-3} \hat{\rho}_{8}$ We find only a very small amount of these quasi-free PEP's. At somewhal later time ( $\mathrm{t}=120 \mathrm{fm} / \mathrm{c}$ ) strong preequilibrium jet emission of -8 of the projectile nucloons is observed at $\approx 5 \% \rho_{\circ}$ at smaller velocities (see Fig. 1). The jet should be observable as individual nucleons al forward angles and with a broad energy distribution extending above the thermal eva poration cange. At the intermediate impact parameters, generally unfocused preequilibrium emission acoompanits the jet emission, as well as backward scattered low energy projectile fragments (ste Fig. 1).

The target is not destroyed afuer the emission of the jet, altbough 15-40\% of the incident momentum and all of the incident ${ }^{12} \mathrm{C}$ binding energy are absorbed by the All target. This is not sulficient to prowake complete spallation but may result in copious eyporation of nucleons before fission. The recoil momenta determined from larget Gosion measurements ${ }^{1}$ are larger then the valuts predicted bere. The oorrect values should oome from the including of $n-n$ collisions neglected here. The general piclure emerging is thus that complete fusion at low energy gives way gradually to massive transfer and to the jet process reported here and the number of escaping nucleons rises with $\mathrm{E}_{\mathrm{Lat}}$ until most of the projectile nucleons come out unbound.

\section{Footnotes and References}

"LBL and GSI, Darmstadt, West Germany.

'Permarent address: Department of Physics, Duke University, Durham, NC 27706.

Finstitut itr Theoretioche Physik, Universitäl Frankfurt and Oak Ridge National Laboratory, Oak Rjdge, TN.

l. GSI-Heidelberg-Minster pollaboration, U. Lymen et al.; Marburg-Oslo-CERN collaboration, T. Lund et al.; Grenoble-Lund-CERN oollaboration. H. Niefenecker et al. (all to be published).

2. H. Stöcker et al., Phys. Lett. 1018, 379 (1981).

\title{
Explosion-Evaporation Model for Fragment Production in Intermediate-Energy Nuclear Collisions*
}

\author{
George Fäi and Jörgen Randrup
}

The disassembly of a piece of hot $(20-200$ $\mathrm{MeV} /$ nucleon) nuctear matter is described as a two-stage process. In the primary, fast stage (which is referred to as the explosion) the system quicdy tragments into free nucleons and composite nuclei according to available phase space. (At higher energies pion creation may also be signtificant.) The ejectiles produced in the explosion have to be stable on a time scale characteristic of this violent process. In the present work we include all states having a widih $\Gamma<1 \mathrm{MeV}$.
The metastable products of the explosion will subseguently decay further on a slower time scale in a relaxation process, first by sequential emission of ligbt partictes, later by gamma decay. This secondary stage is referred to as the evaporation.

Following Ref. I, we assume that the explosion populates phase space statistically. Employing the grand canonical approxicnation, we calculate the partition function

$$
Z=\sum_{i} e^{-\rho\left(E_{H_{1}}-\mu A_{r}-\nu T_{t}\right)} .
$$


The sum extends oyer all possible final states I. The Lagrange multipliers, $\beta, \mu, y$ are adjusted to ensure that the ensemble average of the total energy $E_{\text {f }}$, baryon number $A_{f}$, and isospin $T_{f}$ are equal to the prescribed values $\mathrm{E}_{0,} \mathrm{~A}_{0^{2}}$ and $\mathrm{T}_{0^{*}}$

For given values of the avajleble excitation energy per nucleon $\epsilon$, the asymnetry $\mathrm{I}=(\mathrm{N}-\mathrm{Z}) / \mathrm{A}$, and the available volome at disassembly [in units of the reference volume $\left(4 \pi_{0}^{3} A_{0}\right) / 3$ ) ] $x$, the Lagrange multipliers $f, \mu, y$ are determined by solving the three constraint equations. Subsequently, the partition function can be constructed, yielding the information on the system imrnediately after the primary explcsion.

In describing the evaporation stage, we include the most frequently enuitted light particles: nevtrons, protons and alpha particles.

A few instructive results are shown in the figures. In Fig. 1 we compare the relative yields of tinal fragments (full histogram) to those prior to the evaporation stage (dashed histogram) in one particular example.

The change of the relative abundancies of the various fragment species in the eraporation phase significantly affects the fintal deuteron-10-proton yield ratio, $d / p$. This general feature makes it essential to take acoount of the decay of composite fragments before using $d / p$ as a measvere of the entropy of the initial source, as has been atternpted in Ref. 2.

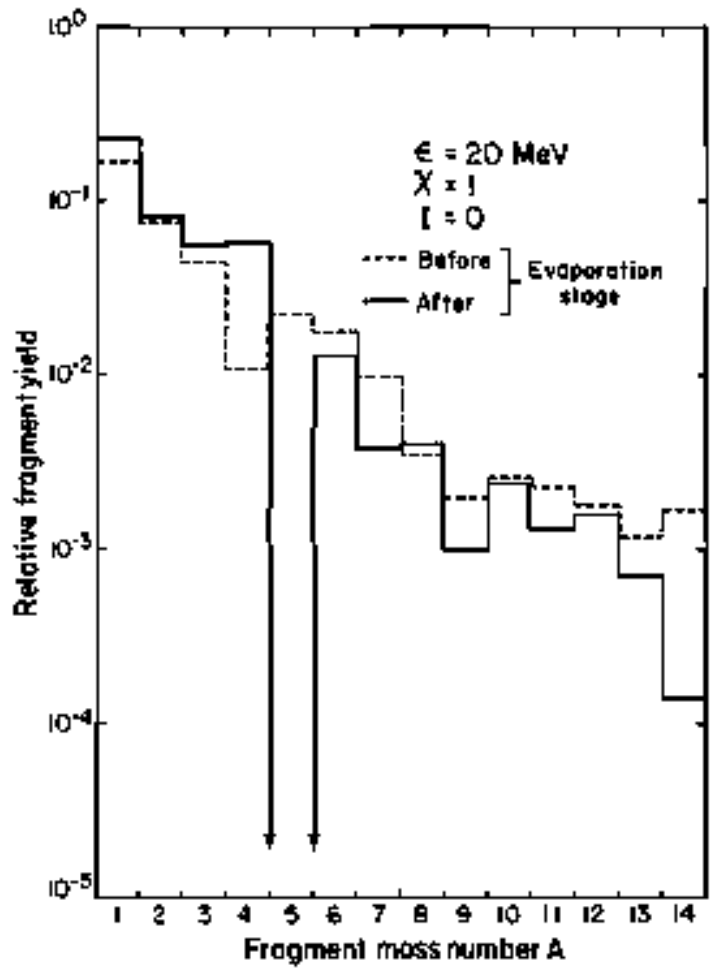

Fig. 1. (XBL 816-3190)
Figure 2 displays the $d / p$ ratio obtained for several values of the parameters $x$ and $l$ in our model as a function of the ayailable excitation energy per participant nucleon $t$. The comparison of our results to the experimental data of Nagamiyg at al. ${ }^{3}$ can only be considered a qualitative one at this point. The source is identified with the participant nucleons and is assumed to consist of projectile and target matter according to the standard sharp sphere geometry; the total center-of-mass energy is assumed to be available for excitation. The data points are included in Fig. 2. The theoretical curves represent expected extreme linits of the parameter range. In our model the tonal relative abundancies are sensitive to the extension of the primary breakup in space and time, and the measurement of composite fragnent yields may therefore elucidate the details of the disassembly process.

While existing dynamical theories, such as the intranuclear cascade and nuclear floid dynamics, only yield a kind of matter distribution emerging from the nuclear collision, the present model incouporates actual physical fragments. So far we have focused on the relative frogment yields but the spectra can also be obtained in a straightforward manner. The model may thus offer a convenient aug. mentation of existing dynamical theories for intermediateenergy nuclear collisions.

\section{Faotnotes and Referentes}

*Condensed from LBL-26261.

1. J. Randrup and S.E. Kornin, Nucl. Phys. A 3\$6, 223 (1981).

2. P.J. Siemens and J. Kapusta, Phys. Rev. Let1. 43, 1486 (1979).

3. S. Nagamiya et al. Lawience Berkeley Laboratory Report LBL-12123 (1981).

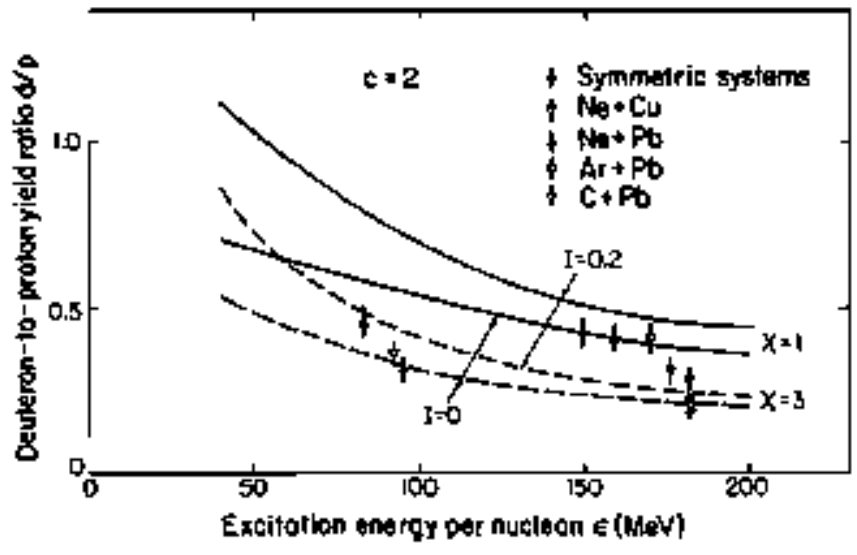

Fig. 2 (XBL 816-3191) 


\section{Pion, Light Fragment, and Entropy Production in Nuclear Collisions}

\section{H. Stöcker}

The production of pions and light fragments in fast muclear collisions is calculated in a relativistic fluid dynamical model. We include viscous effects and a cherrical equilibrium model of particle production. The linear boubarding energy dependence and, in particular, the absolute values of the calculated pion, production, and deu-

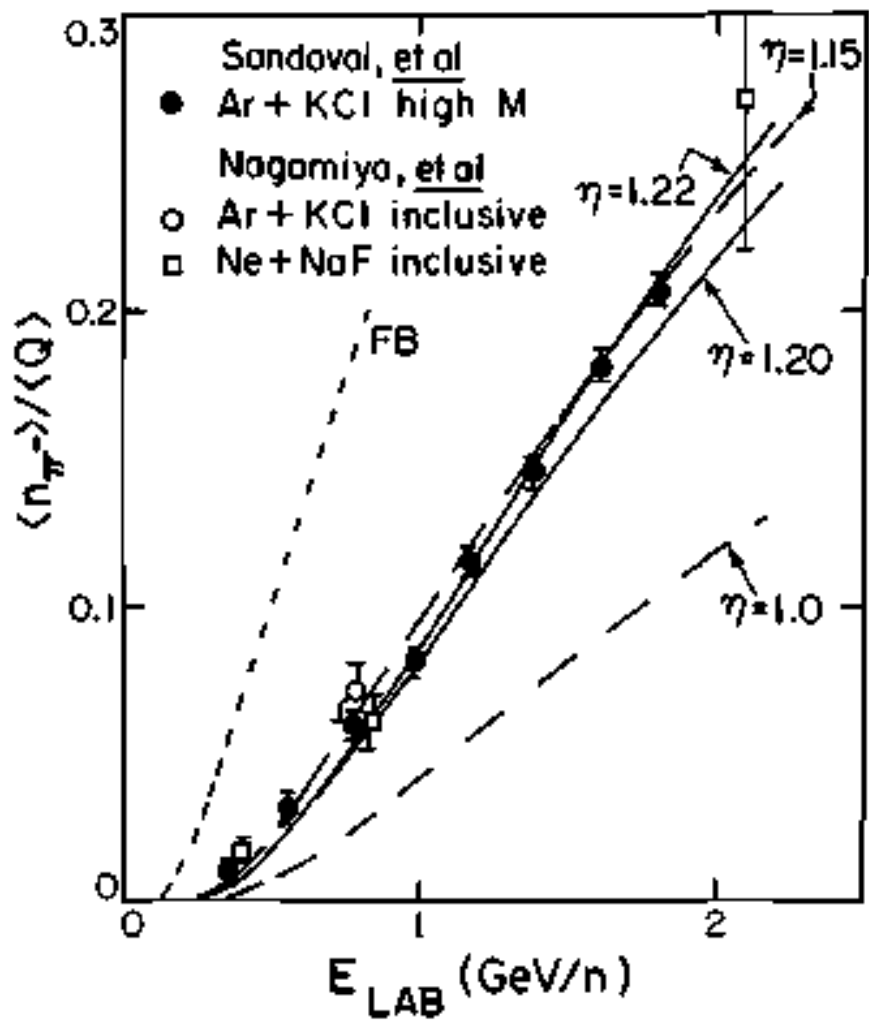

Fig. 1. Bombarding energy dependence of the mean number of pions per nuclear fragment. (XBL 814-652) teron yields are found to be in agreement with the experimental data. The previous discrepancy between the calculated entropy and the measured $d / p$ ration is resolved.

Footnote

"Based on LBL-12302.

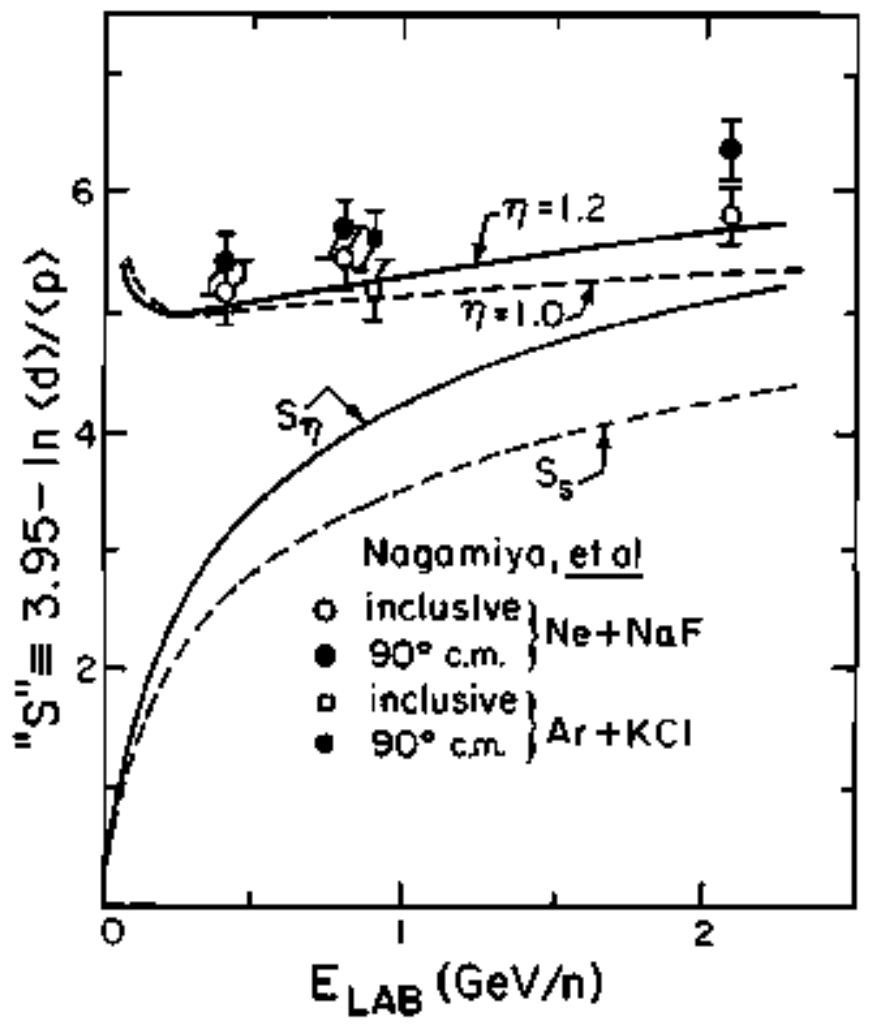

Fig. 2. Connection between the $d / p$ ratio and the entropy.

(XBL 814-651)

\section{Pion Production from Heary Ion Collisions at 90-380 MeV/N*}

\section{P. Heckingt}

Pion multiplicities and pion spectra from heavy-ion collisions with medium bombarding energies of 90 $380 \mathrm{MeV}$ per nucleon are not well understood. The lower the booberding energy, the worse the thermal model performs, indicating that a nonthermal production mechanism is at work, either exclusively or in addition to a thermal component.

For the nonthermal mechanism, the invariant pion production cross section is given by 


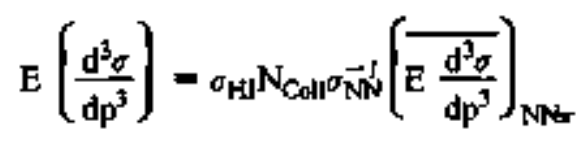

Here, $\sigma_{\mathrm{Hl}}$ is the (geometrical) heavy-jon crass section and $\mathrm{N}_{\mathrm{COll}}$ is the number of initial nucleon-nucleon collisions, taken from a Glauber description.

$$
\left(\overline{E \frac{d^{3} d}{d p^{3}}}\right)_{N N}
$$

is the average pion production eross section per nuctsonnucleon collision (Laken (rom experimental $\mathbf{N N} \pi$ data), averaged over the momentum distribution of the initial nucleans. ${ }^{1}$

$$
\left(\overline{E \frac{d^{3} v}{d p^{3}}}\right)_{N N \pi}
$$

as well as the total mucleon-nucleon cross section onN are rather different from their vacuum values because of the Pauli-blocking of the final state nucleons. The pion angular distribution of the elementary reaction $\mathrm{NN} \rightarrow \mathrm{NN} \pi$ as well as the pion reabsorption is both nuclei are included.

No compression phenomena, shock waves, or col lective effects of any kind are considered within this model. It is assumed that the nuclei simply overlap in coordinate space, the density is the sum of the local densities, and pion production is homogeneous in the overlap region.

Figure 1 shows the importance of the thermal and the proposed nonthermal production mechanism at forward angles the nonthermal comporent is predominant at lowest energies and compeles with the therma] component at higher energies, depending on the pion momentum Pions with large momenta are mostly of nontherutal origin. For pion emission at $90^{\circ}$ (not shown in the figure), the nonthermal production rate is an order of magnitude smaller. and hence the spectrum is more thermal. The angular distribution of the combined thermal and nonthermal production mechanism agrees with the result of Ref. 2.

In conclusion, pion emission at $90-380 \mathrm{MeV}$ per article seems to be well described by a combination of the thermal and a nonthermal mechanism.

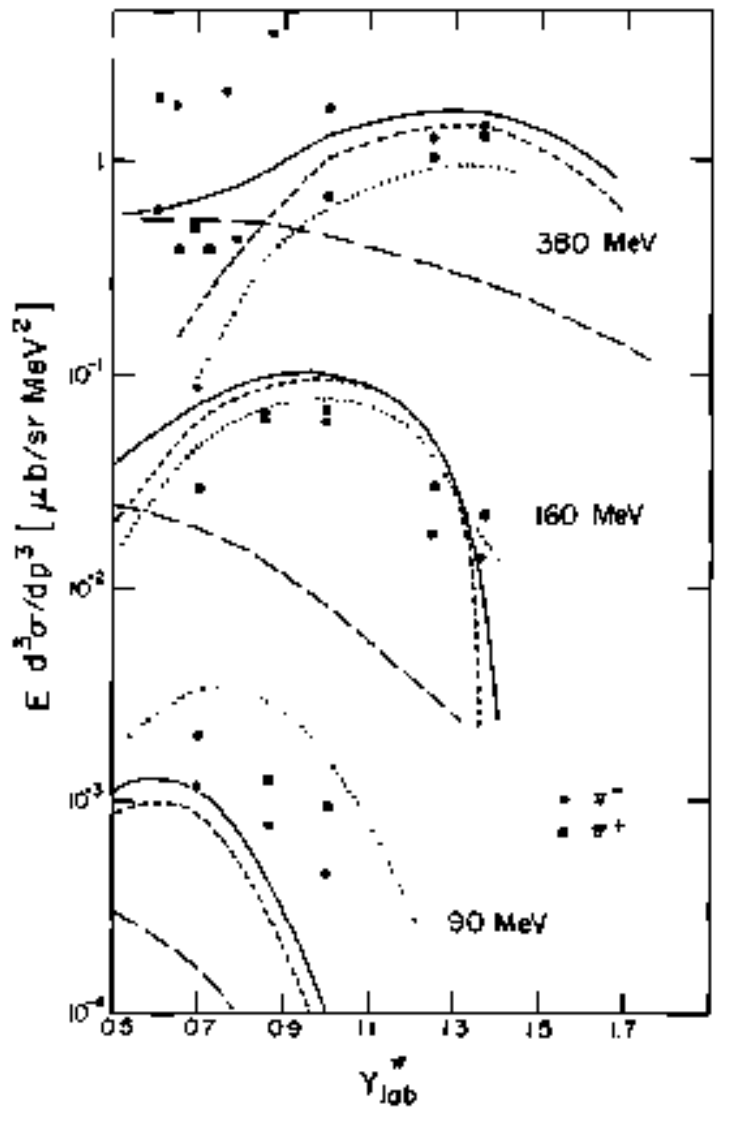

Fig. 1. Triple differential pion production cross section for the reaction $\mathrm{Ne}_{\rightarrow} \mathrm{NaF}$ at three bombarding energies as a function of the laboratory pion rapidity at $0^{\circ}$ emission angle. The dasted curves are the thermal model, and the full curves are sum of the thermal and nonthermal production rate.

(XBL 818-2450)

\title{
Footnotes and Rejerences
}

"Condensed from LBL- 16271.

tWork supported in part by the Deutselne Forschungsgemeinschaft under Contract He 1155/1.

1. G.F. Berlsch, Phys. Rey. C 15, 713 (1979).

2. S. Nagamiya et al., LBL-12123.

\section{Microscopic Theory of Deuteron Formation}

\author{
M. Gyulassy, E. Remier, and X. Frankel
}

We apply the Wigner density formalism of Remler ${ }^{1}$ to the production of deuterons in nuclear collisions. The theory is based on the intranuclear cascade model, which follows the space-monentum trajectories of al] particles. The starting point is the transition rate formula

$$
\Gamma_{12}(t)=-\frac{1}{h} \operatorname{Tr} \rho_{12}\left[U_{12}, p(t)\right]
$$

for particles 1 and 2 to scatter into a bound state characterized by the density matrix, $\rho_{12}$. In Eq. (1) $\rho(\mathrm{t})$ is the 
full A-body density matrix and $U_{12}=Z_{(}\left(V_{1 i}+V_{2 i}\right)$ for $i \geq 3$ is the interaction potential of particles 1 and 2 with the remainder of the system. As are interested in the classical limit, we evaluate the trace in the Wigner representation.

In the classical limit,

$$
\begin{aligned}
& \rho_{W}\left(x, P_{1}, \ldots, x_{A} \rho_{A} ; t\right)= \\
& \quad<\prod_{i=1}^{A}(2 \pi h)^{3} \delta^{3}\left[x_{i}-\bar{x}_{i}(t)\right] \delta^{3}\left[p_{i}-\bar{p}_{i}(t)\right]>
\end{aligned}
$$

in terms of the classical trajectories $\overline{\mathrm{x}}_{\mathrm{i}}(\mathrm{t})$ and $\overline{\mathrm{p}}_{\mathrm{i}}(\mathrm{t})$. The Wigner transform of the commulator in Eq. (1) simplifies in the classical limit to

$$
\begin{aligned}
\lim _{k \rightarrow 0} & \frac{-i}{\hbar}\left[U_{12}, p\right]_{w}= \\
& \sum_{i=1}^{2} \sum_{j=3}^{A} \nabla_{x_{j}} V\left(x_{i}-x_{j}\right)\left(\nabla_{p_{i}}-\nabla_{p_{j}}\right) p w .
\end{aligned}
$$

The final expression for the transition rate is

$$
\begin{aligned}
& \Gamma_{1 z_{A \rightarrow 0}}= \\
& <\sum_{j \geq 3}\left\{F_{1 j} \cdot \nabla_{p}+F_{2 y} \cdot \nabla_{p_{2}}\right\}_{\rho_{12}^{u}}\left(\bar{x}_{1} \overline{\mathbf{p}}_{1}, \overline{\mathbf{x}}_{2} \overline{\bar{p}}_{2}\right)>
\end{aligned}
$$

in terms of the Wigner transform, of matrix and the classical forces $F_{i j}=-\nabla_{x_{i}} V\left(x_{i}-x_{j}\right)$.

Intranuclear cascade provides a particular simple example of Eqs. $(2,4)$. The forces are impulses at discrete limes $\tau_{i}$. The integrated probability for deuteron production is then simply given by ${ }^{1}$

$$
\begin{aligned}
P_{12} & =<\sum_{i=0}^{N} \frac{(2 \pi)^{3}}{V} \delta^{3}\left[P-\bar{p}_{1}(i)\right. \\
& \left.-\tilde{p}_{2}(i)\right]\left\{\rho_{4}[r(i), q(i)]-\rho_{d}[r(i+1), q(i)]\right\}
\end{aligned}
$$

where

$$
\begin{aligned}
& r(i)=\bar{x}_{i}\left(\tau_{i}\right)-\bar{x}_{2}\left(\tau_{j}\right), \\
& q(i)=\bar{p}_{1}\left(\bar{\tau}_{i}+\epsilon\right)-\bar{p}_{2}\left(\tau_{1}+\epsilon\right),
\end{aligned}
$$

and

$$
p_{d}(r, q)=8 \exp \left(-r^{2} / d^{2}-q^{2} d^{2}\right)
$$

for a Gaussian deuteron wave-function $(d \simeq 2.1 \mathrm{fm})$.

The brackets $\langle\ldots\rangle$ denote an ensemble average over many cascade events. We have performed the first calculations based on Eq. (5) using Cugnon's cascade code. The results for $\mathrm{Ne}+\mathrm{U}$ and $\mathrm{Ar}+\mathrm{Ar}$ show that the devteron spectra for lab energies $\geq 50 \mathrm{MeV} / \mathrm{A}$ are well accounted for. For lower energy denterons, further calculations are necessary to estimate higher mass composite yields.

An important qualitative result that was obtained is that the free proton inclusive distributions can be strongly modified from the summed charges inclusive yield due to deuleron production. In particular, for entral collisions, the cascade code predicts a forward enhancement of the total yield. However, in the forward direction the phase space density is high; and, therefore, most nucleons emerge as bound fragments. This nucleosynthesis process depletes the frec proton spectra at forward angles. We are investigating whether the observed forward proton suppression is due to this mechanism as opposed to the hydrodynamic flow mechanism.

\section{References}

I. E. Remler, NSSF-[IP-81-29, preprint (1981).

2. M. Gyulassy, E Remler, K. Frankel, preprint in preparation.

\title{
Coulomb Final State Interactions
}

\author{
M. Gulassy and S.K. Kouffmann
}

Work on Coulomb distortions of inclusive spectra was completed; simple analytical formulas were derived and applied to data. A new analysis of the range of applicability of our formulas was carried oul. In terms of the Coulomb parameters, $\eta_{\text {eff }}{ }^{-}=Z_{N \times c^{\prime}} / K^{\prime}$ and $\rho_{\text {ef }}=k^{\prime} R$, with $\left(\omega^{\prime}, k^{\prime}\right)$ being the thermal averaged energy momentum in the rest frame of a fragment of charge $Z$, radius $R$, and lemperature $T$, our generalized Gamow formulas apply when

$$
\begin{aligned}
& \quad\left|\pi_{\text {eff }}\right|<\rho_{\text {eff }} \\
& \text { or } \quad 2 I \eta_{\text {eff }} \mid<\left(1-\rho_{\text {eff }}^{2}\right) / \rho_{\text {eff }} .
\end{aligned}
$$

For the $\mathrm{Ne}+\mathrm{NaF} \rightarrow \pi$ data, Eq. (I) is satisfied. However, for reactions with $Z \geq 40$, nonperturbattve corrections must be calculated. 
In addition, we investigated the impact parameter dependence of the Coulornb effects. We found that the impact parameter $b^{*}$ that contributes most $10 \pi^{+}, x^{-}, x^{\circ}$ yields differ due to Coulomb eflocts. For example, at the projectile rapidity $b^{\circ}=3.09,2.44,1.47$ fm for $\pi^{-}, \pi^{\circ}$, $\pi^{+}$respectively. Therefore, in this kinematic range, the $x^{-}$prefer more peripheral collisions because of the large Coulomb enhancernent near the projectile. On the other hand, $x^{+}$prefer to corne from more central collisions at this rapidity due to the large Coulomb suppression due to projectile remnants in peripheral eollisions. The important lesson here is that the inclusive $\pi^{+}$and $\pi^{-}$yields sample different impact parameters and, therefore, the ratio $\pi^{+} / \pi^{-}$mixes different dynamical mechanisms. As shown in Ref. 1 , the $\pi^{+} / \pi^{-}$ratio depends sensitively on tight physical parameters of the nuclear system. Hence, Coulomb effects eannot be easily disentangled nor effectively used as a diagnostic tool of nuclear colligions.

\section{Reference}

J. M. Gyulassy, S.K. Kaufimann, Nucl. Phys. A 362, 503 (1981).

\title{
Formulation Of The Coulomb Effects Of Spectator Fragments On Pions From Heary Ion Collisions"
}

\author{
Hafez M.A. Radi, J.O. Rasmussen, J.P. Sulilvan, K.A. Frankel, and O. Hashimoto
}

Coulomb eflects on heavy-ion pion production cross sections are formulated in terms of weighted averaging over various projectile fragments. Satisfactory fis to zerodegree pion data are found for $\mathrm{Ar}+\mathrm{C}$ and $\mathrm{Ne}+\mathrm{C}$ sys. 1ems. The fragment distributions of exciter compound nuclei, before nucleon evaporation, must be used; and the fragment velocity dispersion parameter needed is smaller than that measured for fragments after nucleon evaporatín. Average charge $Z_{\text {eff }}$ values are delermined and cortpared with those from an experimental paper. In the heavy-ion entergy range of the $O^{\circ}$ pion studies (300 MeV $<E / A<600 \mathrm{MeV}$ ), it is inferred that target-projectile factorization for fragmentation cross sections does not hold.

We take the velocity distribution of projectile fragments as Gaussian, with velocity dispersion width $\alpha_{F}$ given as

$$
\alpha_{F}=\sigma_{0} c /\left(m_{N} c^{2}\right) \sqrt{\left(A_{0}-A_{F}\right) /\left[A_{F}\left(A_{Q}-1\right)\right]} .
$$

In Eq. (1) the constant $\sigma_{0}$ has been found to be about $86 \mathrm{MeV} / \mathrm{c}$ by experiment: $\mathrm{m}_{\mathrm{N}} \mathrm{c}^{2}$ is the nucleon resl mass energy constant; $A_{b}$ is the projectile mass; and $A_{F}$ is the mass of fragment under consideration.

Furthermore, the averaging technique requires weighting with respect to the cross section of forming a fragment of mass $A_{F}$ and charge $Z_{F}, \sigma\left(A_{F}, Z_{F}\right)$ before evaporation of protons and neutrons, and also with tesper to the differential cross section (before Coulomb correction) of forming a pion simultanecisly with a fragment of mass $A_{F}$ near beam velocity, which is assumed to be proportional to the mass loss ( $\left.A_{0}-A_{F}\right)$. o $\left(A_{F}, Z_{F}\right)$ is calculated by the firestreak computer code of P. McGaughey and D. Morrissey. Figure I shows comparison of their code with data. ${ }^{2}$

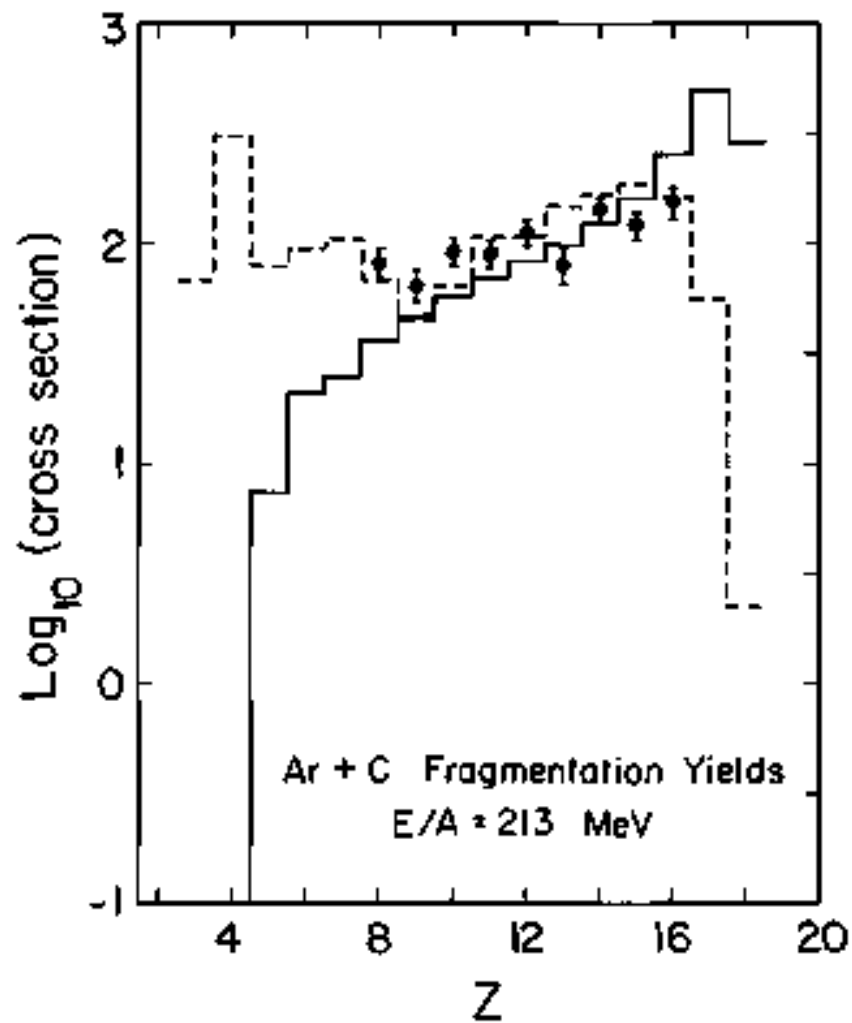

Fig. 1. Comparison of ${ }^{40} \mathrm{Ar}$ fragment yields for given $\mathrm{Z}$ between theory and experiment. Data points are from Symons et al ${ }^{2}$ at $\mathrm{E} / \mathrm{A}=213 \mathrm{MeV}$ on a carbon target. The solid (dashed) histogram is the theoretical distribution before (after) nucleon evaporation according to the firestreak code of McCaughey. The summed cross sections are in millibarns.

(XBL 816- 10169)

The \$ommerfeld parameler is averaged over the velocity dispersion to give 


$$
\left\langle n_{ \pm}\right\rangle_{\beta_{\varphi}}= \pm\left(\frac{Z_{F} \kappa^{2}}{h c}\right) \frac{\operatorname{erf}\left[\beta_{r \pm} /\left(\sqrt{2} \alpha_{F}\right)\right]}{\beta_{\pi \pm}}
$$

which replacts the average over $\beta_{F}$ in the pion cross secion. The grand average of the pion cross section becomes
Proceedings of 5th High Energy Heavy lon Study, May 18-2I, 1981, LAL-12652 (1981).

tPhysics Department, Kuwait University, Kuwait.

1. T.J.M. Symons tt a1., Phys. Rev. Lett. 42, 40 (1979).

2. J. Sullivan el al., Lawrence Berkeley Laboratory Report LBL-11971 (1980), Phys. Rev. C 25. (Feb. 1982).

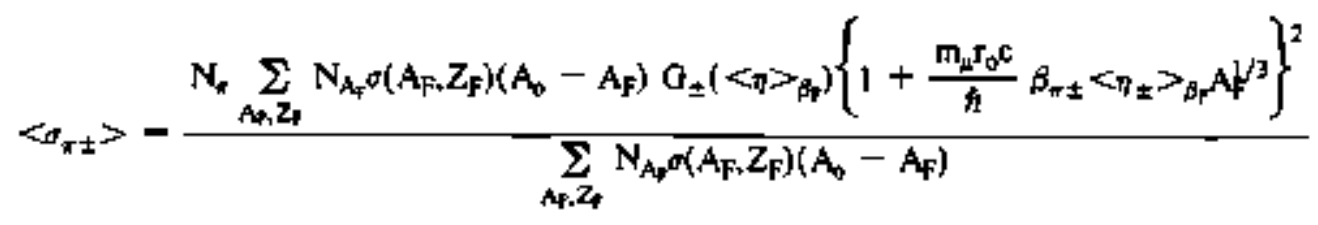

where

$$
N_{A_{F}}=\sqrt{2 \pi} \text { 蜘 }
$$

and

$$
G_{ \pm}(x)=2 \pi x[\exp (2 \pi x)-1]^{-1} .
$$

Since the pions should move out many nuclear diameters during a mean life of compound nuclti, it stems more correct to use the primary fragment distribution rather than the fintal distribution for our pion Coulomb code. Fig. ure 2 shows the results of our theoretical code compared to ${ }^{49} \mathrm{Ar}+\mathrm{C}$ experimental pion data of Sullivan et al. The firestreak code fragment yields at $533 \mathrm{MeV} / \mathrm{N}$, s]ightly broader than those of Fig. 1, were used as input Here, we made the simplest assumption for the pion production factor, namely, purely linear dependence on fragment mass loss $\left(A-A_{F}\right)+A$ momentum dispersion constant of $\sigma_{g}=$ $60 \mathrm{MeV} / \mathrm{c}$ and a radius consiant $\mathrm{r}_{0}=1.5 \mathrm{fm}$ were used in Eq. (3). The theoretical curve was corrected by folding in the experimental resolution. The agreement seems quite satisifactory.

\title{
Foornotes and References
}

"Condensed from Lawrence Berkeley Laboratery Report LBL-12591, Phys. Rev. C 25, (Feb. 1982); and from

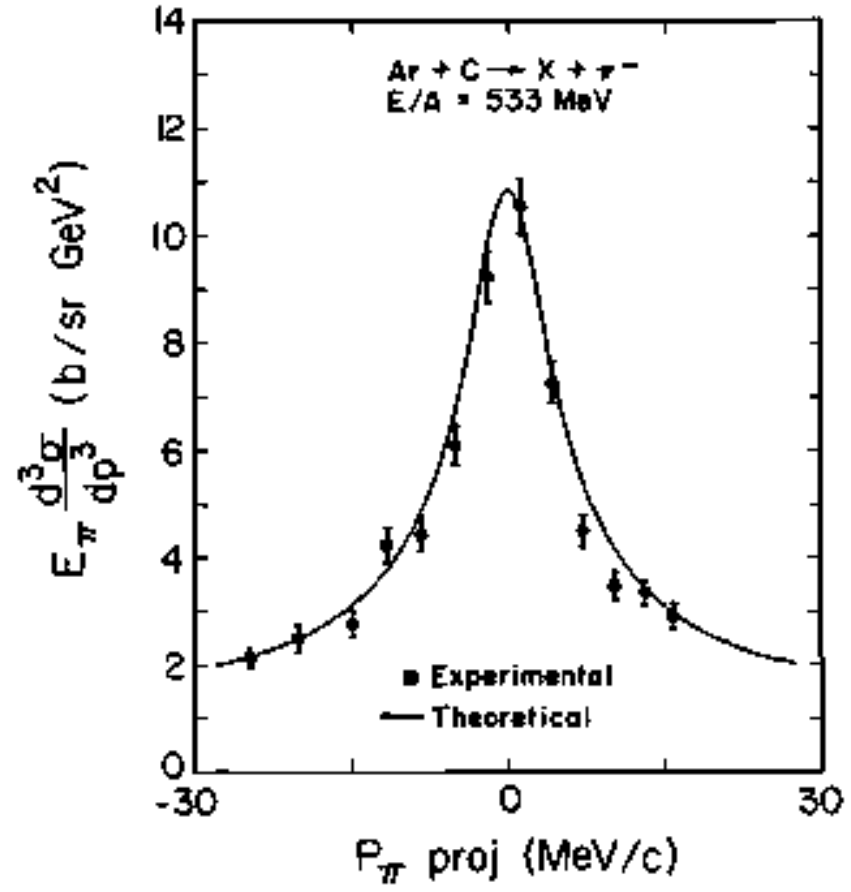

Fig. 2. The $\boldsymbol{x}^{-}$spectrum at $0^{\circ}$ calculated by Eq. (3) and compared to data of Sullivan et al. ${ }^{2}$ The solid curve is the theory after folding with experimental resolution. The abscissa is pion momentum in the projectile frame.

(XBL 816-10170)

\section{Dynamies vs Symmetrization in Hadron Interferometry}

\author{
M. Gyulessy
}

The famous Hanbury-Brown and Twiss effect leads to the expectation that the small angle correlation function $\mathbf{R}_{12}$ of identical pions $\left(\pi^{-} \pi^{-}\right.$) showild reveal the space-time geometry of the source

$$
R_{12}-I+\left|\rho\left(k_{1}-k_{2}\right)\right|^{2}
$$

where $\rho(q)$ is the Fourier transform of $\rho(x, t)$, the pion pro- 
duction region. However, in Ref. I, partially coherent pion fields were shown to modify Eq. (1) as

$$
\begin{aligned}
R & =1+\left|\rho\left(k_{1}-k_{2}\right)\right|^{2}\left(1-D_{1}\right)\left(1-D_{2}\right) \\
& +2 \rho\left(k_{1}-k_{2}\right)\left[D_{1}\left(1-D_{1}\right) D_{2}\left(1-D_{2}\right)\right]^{1 / 2}
\end{aligned}
$$

where $D_{i}=D\left(k_{i j}\right)$ is the degree of coherence of the field.

In this work, ${ }^{2}$ a new correlation source was found that is specific to hadron interactions. I call these ensemble correlations. The idea is that indusive cross sections implícitly average over an ensemble of final configurations. If the two partictes are correlated dynanically with the unobserved ones, then that ensemble average ean induce a large modification of Eq. (2).

Two examples were considered: final state nonisotropic shadowing and coherent jet production. The result is ${ }^{2}$

$$
\begin{aligned}
R_{12}- & R_{12}^{\text {ahs }}\left\{1+\left|\rho\left(k_{1}-k_{2}\right)\right|^{2}\left(1-D_{1}\right)\left(1-D_{2}\right)\right. \\
& \left.+2 R_{120\left(k_{1}\right.}-k_{2}\right)\left[D_{1}\left(1-D_{1}\right) D_{2}\left(1-D_{2}\right)\right]^{1 / 2} \\
& \left.+\left(R_{12}^{\text {ah }}-1\right) D_{1} D_{2}\right\},
\end{aligned}
$$

where $\mathrm{R}^{\mathrm{ch}}$ is the coherent jet entienbite correlation function and $R^{l}$ is as interference factor due to colherent ensemble effects. $R_{12}^{\text {ath }}$ is an absorption factor. Detailed formulas for these Rs are found in Ref. 2. As an illustration of Eq. (3), the form of $R_{12}$ appropriate for an almost chaotic field with $D<t$ was derived

$$
R_{12} \simeq 1+e^{-q^{2} R^{2}}+X_{j e t} e^{-q^{2} t_{j=1}^{2}}
$$

where $x_{j e t}$ specifies the colerent jet ensemble and $r_{j e}^{2}$ measures the momentum spread of that jet. For $A \sim 100$, $D=0.1$ we estimate $X_{\text {jet }}=1$.

Equation (3) liberates us from the bias that the intercept of the correlation function should be less than 2 . In particular, $R_{11}=R_{l \mid}^{\text {abs }}\left(2+X_{\text {jet }}\right)$, which could be arbitrarily large. It shows that the canonical HanburyBrown Twiss form, Eq. (1), cannot be taken for granted. When interesting dynamical correlations exist, these can in general overwhelm the correlations due to Bose or Fermi statístics.

\section{References}

1. M. Gyulassy, S.K. Kaufimann, L.W. Wilson, Phys. Rev. C 20, 2267 (1979).

2. M. Gynlassy, LBL-12389 (1981).

\title{
The Quasi-Elastic Component in High-Energy Nuclear Collisions
}

\author{
Bernd Schürmannt and Jorgen Randrup
}

The reaction mechanisms acting in higb-energy nuclear collisions have been the subject of intensive studies in recent years. Although purely thermal models have been quite sucoessful in explaining early measurentents of inclusive spectra, recent, more accurate measurements at forward angles indicate the presence of a strong quasielastic component. Simplified multiple-collision models transport theory, micro-canonical, and the two-component model direct-plus-thermal contain the quasi-elastic scattering mechanism in one way or another. The discussion of a nonequilibrated component in the inclusive spectra of high-energy nucleons has been carried to the extreme in Ref. 1, where it was assurned that the knack-out mechanjsm (i.e., a "cold" projectile nucleon collides with a "cold" target nucleori and both subsequently leave the interaction region) is the dominent reaction mechanism at forward angles. Meanwhile, it has become clear, however. that such a knock-out process has a quite small cross section, if properly normalized. By contrast, the properly weighted single-collitsion process contributes significantly. In the single-collision mechanism the observed nucleon sufiers only one collision, while its collision partner may have undergone previous scatterings. Guided by experiment, it appears a reasonable approximation to replace the mormentun-space part of the single-collision contribstion by the knock-out distribution. This approximated singlecollision contribution is denoted the direct component. It is the aim of this note to phrase the notions of a "knockout," a "single collision," and a "direct" component in a quantitative way and, in particular, to compare the direct and the single-collision components.

The tool for our study is the rows-on-rows model, ${ }^{2}$ in which the one-particle inclusive invariant cross section is an incoherent sum of susltiple-scattering contributions,

$$
\mathrm{E} \frac{\mathrm{d} a}{\mathrm{dp}}=\sum_{k=1}^{\infty} \mathrm{E} \frac{\mathrm{d} \sigma^{(\mathrm{k})}}{\mathrm{d} \bar{p}} \text {. }
$$

The contribution from nucleons which have suflered exactly $k$ collisions can be written as 


$$
\begin{aligned}
E \frac{d \sigma^{(k)}}{d \bar{p}}= & \sum_{M} \sigma_{A B}(M, k) \sum_{m=1}^{M} W_{m k}^{A}(\bar{p}) \\
& +\sum_{N} \sigma_{A B}(k, N) \sum_{n=1}^{N} W_{k n}^{B}(\vec{p})
\end{aligned}
$$

Here, the indices $A$ and $B$ refer to the projectile and target, respectively. The quantity $\sigma_{A B}(m, n)$ is the cross stetion for the scattering of a row consisting of $m$ projectile nucleons with a row consisting of $n$ target nucleons. The spectral functions

$$
\sum_{m=1}^{M} w_{m k}^{A} \text { and } \sum_{n=1}^{N} w_{k n}^{B}
$$

afe normalized to $M$ and $N$, respectively. In the rows-onrows model, the cross sections $\sigma_{A B}(m, n)$ are calculated in the eikonal approximation. From their explicit form (cf, e.g., Ref. 2), one easily derives the sum rules

$$
\begin{aligned}
& \sum_{M} M \sigma_{A B}(M, k)=A \sigma_{B}(k) \\
& \sum N \sigma_{A B}(k, N)=B \sigma_{A}(k)
\end{aligned}
$$

with

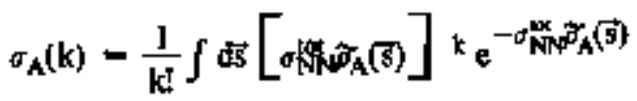

and analogously for $\sigma_{\mathrm{g}}(\mathbf{k})$. Here $\sigma_{\text {㑇 }}$ is the free nucleonnucleon total cross section, and $\partial_{\mathrm{A}}$ is the z-integrated nucleon density of nacleus $A$ (normalized to the total nucleon number also denoted by A). It follows that the contribution to the total inclusive cross section from nucleons which have collided $\mathrm{k}$ times is given by

$\sigma^{(k)}=\int E \frac{d \sigma^{(k)}}{d p} \frac{d \vec{g}}{E}=A \sigma_{B}(k)+B \sigma_{A}(k)$.

A recent calculation ${ }^{3}$ of these quantities in a full threedimensional caseade model yields results which are nearly identical to those of the rows-on-rows model given in Rel. 2. This fact lends additional support to the utility of the eikonal approximation.

In the following, focus on the single-collision term of Eq. (4) and its approximstions. The direct term in the transport model $l^{4}$ and the two-component model ${ }^{5}$ is obtained [rown Eqs. (4) and (S) by approximating $P_{M}^{A}$ by $P_{1]}^{A}$ and $\mathrm{P}_{\mathrm{N}}^{\mathrm{N}}$ by $\mathrm{P}_{11}^{\mathrm{G}}$; i.c.,

$\mathrm{E} \frac{\mathrm{d}_{\sigma^{\text {direca }}}}{\mathrm{d} \overrightarrow{\mathrm{p}}}=\mathrm{A} \sigma_{\mathrm{B}}(1) \mathrm{P}_{1}^{\mathrm{A}}(\overrightarrow{\mathrm{P}})+\mathrm{B} \sigma_{\mathrm{A}}(1) \mathrm{P}_{\mathrm{II}}^{\mathrm{B}}(\overrightarrow{\mathrm{p}})$

The knock-out component is obviously given by
$E \frac{d \sigma^{k o}}{d p}=\sigma_{A B}(1,1)\left[P_{11}^{A}(\vec{p})+P_{1}^{A}(\vec{p})\right]$.

One finds that $\sigma_{A B}(1,1) \ll A \sigma_{B}(1), B_{A_{A}}(1)$, (when $A, B$ 3 1, as is usually the case). Therefore, the pure knockout process is only a small fraction of the total direct component. For the symmetric system $\mathrm{Ne}$ on $\mathrm{NaF}$ at $800 \mathrm{MeV} / \mathrm{N}$, which we shall use as an illustration, one finds typically $\mathrm{d} \sigma^{\mathrm{k} 0} / \mathrm{d}_{\sigma}$ direct $\simeq 0.2$ This is the reason why the knock-out component will not be visible in the inclusive cross section.

After having clarified the relation between the singlecollision, the direct, and the knock-out components, it remains to be seen whether

$$
\text { E } \frac{d \sigma^{d i r e a}}{d p}
$$

is a good approximation to

$$
E \frac{d \sigma^{(l)}}{d \vec{p}}
$$

To elucidate this question we have calculated the corresponding spoctra in the rows-on-rows model, ${ }^{2}$ using Saxon-Woods nuclear density distributions with the paranteters specified in Ref. 2), Gaussian momentym distribstions with equivalent Fermi momenta $P_{F}=230 \mathrm{MeV} / \mathrm{c}$, a total nucleon-nucleon cross section of $\mathrm{N}_{\mathrm{N}}=\mathbf{4 0} \mathrm{mb}_{1}$ and differential cross sections $d \sigma / d 1 \sim \exp \left(\mathrm{t} / 2 \mathrm{q}^{2}\right)$ with $\mathrm{q}=$ $350 \mathrm{MeV} / \mathrm{c}$. Figure 1 shows the results for $800 \mathrm{MeV} / \mathrm{N}$

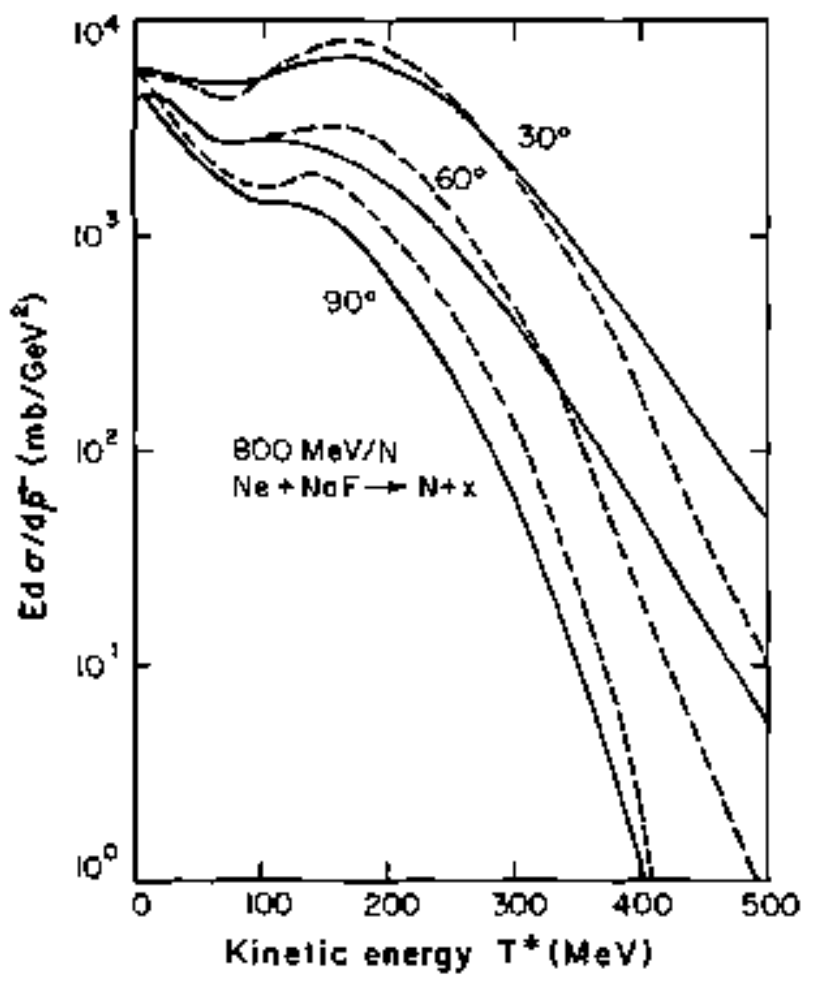

Fig. J.

(XBL 8010-2253) 
Ne on $\mathrm{NaF}$. The inclusive nucleon spectra are shown in the mid-rapidity frame at angles $30^{\circ}, 60^{\circ}, 90^{\circ}$. The correspondence between the single-collision spectrum (solid curves) and the direct spectrum Eq. (8) (dashed curves) is rather good over a wide kinematical range. (The agreement is better than a factor of two for proton energies between $50 \mathrm{MeV}$ and $450 \mathrm{MeV}$.) The main reason for this striking result is that the leading corrections $P_{21}^{A}$ and $P_{12}^{B}$ are still rather similar to $P_{1 j}$, as can be jnferred from the oollision kinematics. Although the deviations are larger at the bigh-energy end, this is of litlle consequence since the multiple-scattering component of the nucleon inclusive cross section is dominant in this regime.

To summarize, we have elucidated the relation between the notions of a single, direct, and knock-out conponent in the nucleon inclusive cross section. A significan1 outcome of ow investigation is the good agreement between the single-collision and the direst component over a wide kinematical region. Since the latter has a far simpler structure than the former, it permits an easy and fairly reliable estimate of the single-collision term As a conse- quence, given the experimental inclusive cross section, it is relatively easy to disentangle single from multiple scattering contributions and study their competition in various kinematical regions. Such investigations are adding greatly to our general understanding of the reaction mechanism in high-energy nuclear collisions.

\section{Foothotes and References}

"Based on Fhys. Rev, C 23, 2766 (1981) (LBL-1 1954). This work was partially supported by the Bundesministerium for Forschung and Technologie, West Germany.

${ }^{\dagger}$ Tochnische Universität Mitnchen, Physik Departrnesit, $D$ 8046 Garching, Wesl Germany.

1. S.E. Koonin, Phys. Rev. Lell. 39, 680 (1977).

2. J. Knoll and J. Randrup, Nocl. Phys. A 324, 445 (1979).

3. J. Cugnon, Caltech preprint MAP-1S (1980).

4. H.J. Pimer and B. Schürmann, Nucl. Phys. A 316, 461 ( 1979$)$.

5. M. Chemtob and B. Schïrmann, Nucl. Phys. A 336, 508 (1980); Z. Phys. A 294, 371 (1980).

\title{
Consequences of Impact Parameter Restrictions in Relativistic Nuclear Collisions
}

\author{
Steffen Bahrmannt
}

The statistical model of high energy nuclear collisions ${ }^{1}$ has proven to be a valuable tool for studying the high rtomentum parts of inclusive spectra. Its main ingredients are:

1. Geomelrically defined groups of nucleons interact independenily from each other, and

2. Momentum distributions within one group are determined by phase space.

Comparison of predictions of this model with observed spectra of protons from relativistic nuclear collisions at $180^{\circ}$ leads 10 two important conclusions:

1. The two-component structure of the spectra is due to the different decay schemes of participants and spectators; the statistical model, a model for participants only, reproduces the high momentum component only.

2. The shape of the high momentum component is closely related to the average group size (number of nucleons contained in a groupl.

The model also predicts that restricling the impact parameter 10 small values (i.e., going ower to central collisions) has practically no influence on the spectral shape. This means that the average group size is rather insensitive to impact parameter restrictions. It can be shown that, although the distribution of group sizes chaniges considerably, the average group size remains practically constant (Fig. 1). This finding is valid for any combination of pro-

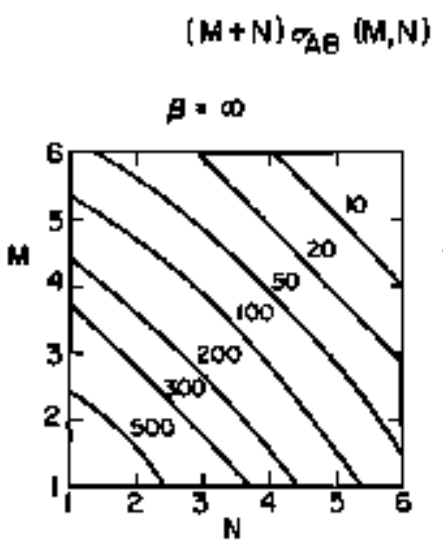

$$
{ }^{12} \mathrm{C}+{ }^{12} \mathrm{C}
$$

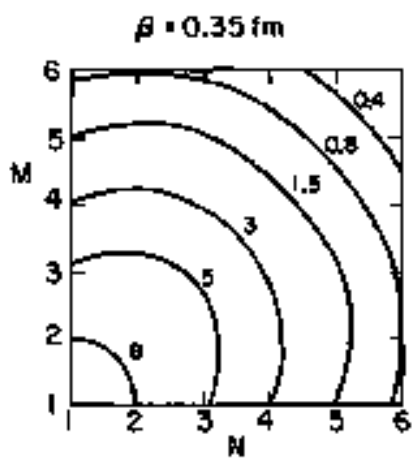

Fig. 1. The weight $(M+N) \sigma_{B}\left(M_{1} N\right)$ of different combinations of projectile and taget mucleon numbers (M and $\mathrm{N}$, respectively) in the inclusive cross section in millibarns. Left: impact parameter averaged; average group size is $\langle\mathrm{M}+\mathrm{N}\rangle=$ 3.8. Right: central collision; $\langle\mathrm{M}+\mathrm{N}\rangle=$ 4.2. The parameter $\beta$ is the width of a Gaussian weight for the impact parameler.

(XBL 8143757)

jectile and target masses and is supporled by recent results of three-dimensional cascade calculations. ${ }^{3}$ If one assumes that the probability for observing "collective effects" is, in a way, proportional to the number of mutually interacting particles, the above result implies that the restriction to 
central collisions does not necessarily increase the chance for such observations.

\section{Foothotes and References}

"Condensed from LBL-12928. Work supported by NATO fellowship, DAAD, W.-Germany, and by BMFT, W.-
Germany.

${ }^{4}$ Inst. f. Theor. Phys., Pbilosophenweg 19, 6900 Heide]-

berg, W-Germany.

J. J. Knoll, Phys. Rev. C 20, 773 (1979).

2. J.V. Geaga, et al., Phys. Rev. Le1t: 4S, 1993 (1980).

3. J. Cugnon, et al., Nucl. Phys. A 363, 444 (1981).

\title{
Recent Results of Statistical Model Calculations for Relativistic Nuclear Collisions
}

\author{
Stefen Bohrmannt and Jörn Knollt
}

The applicability of the statistical model of high energy nuclear collisions ${ }^{1}$ to a wide range of recent experimental data has been investigated. The main results are:

1. The model gives reliable predictions for the bigh momentum parts of inclusive spectra ower a wide range of projectile energies from $\leqslant 100 \mathrm{MeV} / \mathrm{A}$ 1o $>1000 \mathrm{MeV} / \mathrm{A}$.

2. The model gives reliable prediclions for almost any combination of projectile and target masses.

3. The model gives reliable predictions for the shapes of inclusive nucleon as well as pion spectra, but not for pion multiplicities (Ref. 2). The observed slope difference between nucleon and pion spectra from the same reaction is reproduced. It follows from the concept of independently interacting nucleon groups, which is basic to the model; the majoxity of nucleons comes from ensemb]es in which no pions have been produced, while the pions come from groups that have been "oooled" by the pion production process.

4. The model predicts the observed "scaling" behavior with respect to bean energy and target mass for inclusive pion specira at $0^{\circ}$ plotted versus the Feynman scaling variab]e $X_{F}$ (cf. Ref. 2).

5. Above $=1000 \mathrm{MeV} / \mathrm{A}$, the observed spectra tend to be steeper than the calculated spectra. This indicates that all the avajlable energy and momentum is no longer distributed evenly over the members of a group, as is assumed in the model. One might describe this as an "onset of transparency."

The wide range of applicability of the statistical model with respect to projectile energies, projectile and target masses, and type of the observed particles indicates that any more detailed dynamical model of high energy nuclear collisions must incorporate the ingredients of the statistical model: small numbers of mulually interacting particles and even oocupation of the available phase space, especially close to kinematical limits. These are serious restrictions on possible reaction dynamics.

\section{Foonores and References}

*To be published. Work supported by NATO-fellowship. DAAD, West Germany, and by BMFT, West Germany

Inst. f. Theor. Phys.+ Pbilosophenweg 19, 6900 Heidelberg, West Germany.

FGesellschalt fur Schwerionenforschung, Postfach 11054I,

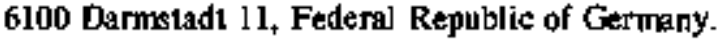

1. J. Knoll, Phys. Rev. C 20, 773 (1979).

2. S. Bohrmann and J. Knoll, Nucl. Phys. A 356, 498 (1981).

\section{Cascade Calculations of Relativistic Nuclear Collisions}

\author{
J.D. Sievenson"
}

Cascade calculations have been surprisingly successful in explaining the details of relativistic heavy ion collisions. In my work, ${ }^{1,2}$ I have emphasized that cascades predicl within experimental errors the inclusive proton and neutron cross sections. 1 also demonstrated that an apparent ano- maly ${ }^{3}$ in the neutron-to-proton ratio for $\simeq 400 \mathrm{MeV} / \mathrm{N}$ $(\mathrm{Ne}+\mathrm{U})$ was, in fact, explained by my cascade model when formalion of light nuclej was taken into account using the coalescence model. Figure 1 is a comparison of the nentron-to-proton ratio calculations with experimental 


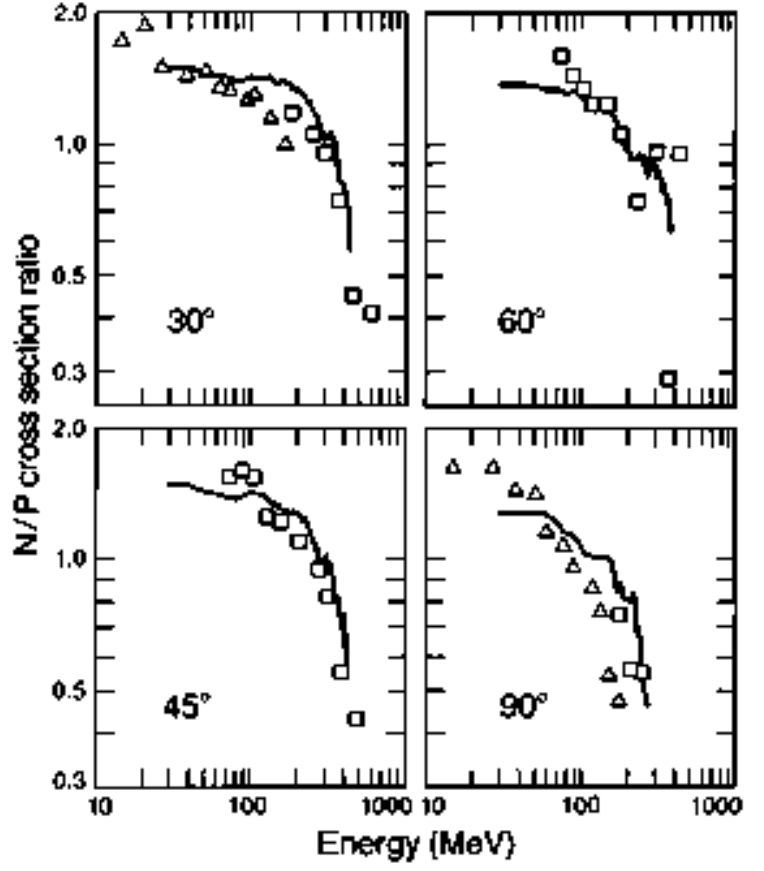

Fig. I. Neutron-to-proton ratio. Symbols are data from Ref. 3. The solid line is calculated frotn the cascade model discussed in the text.

(XBL g09-11909) results. Figure 2 compares my calculation with the neutrón spectra of Rer'. 3.

The mosi important unanswered question regarding cascade calculations concerns their límits of validity: where do they fall? My current plans include conparing caseade results to the data from CERN on 56 and $84 \mathrm{MeV} / \mathrm{N}^{12} \mathrm{C}$-induced reactions. I an also interested in comparing cascades to new, exclusive reaclion dala from the HISS and Ball-Wall detectors.

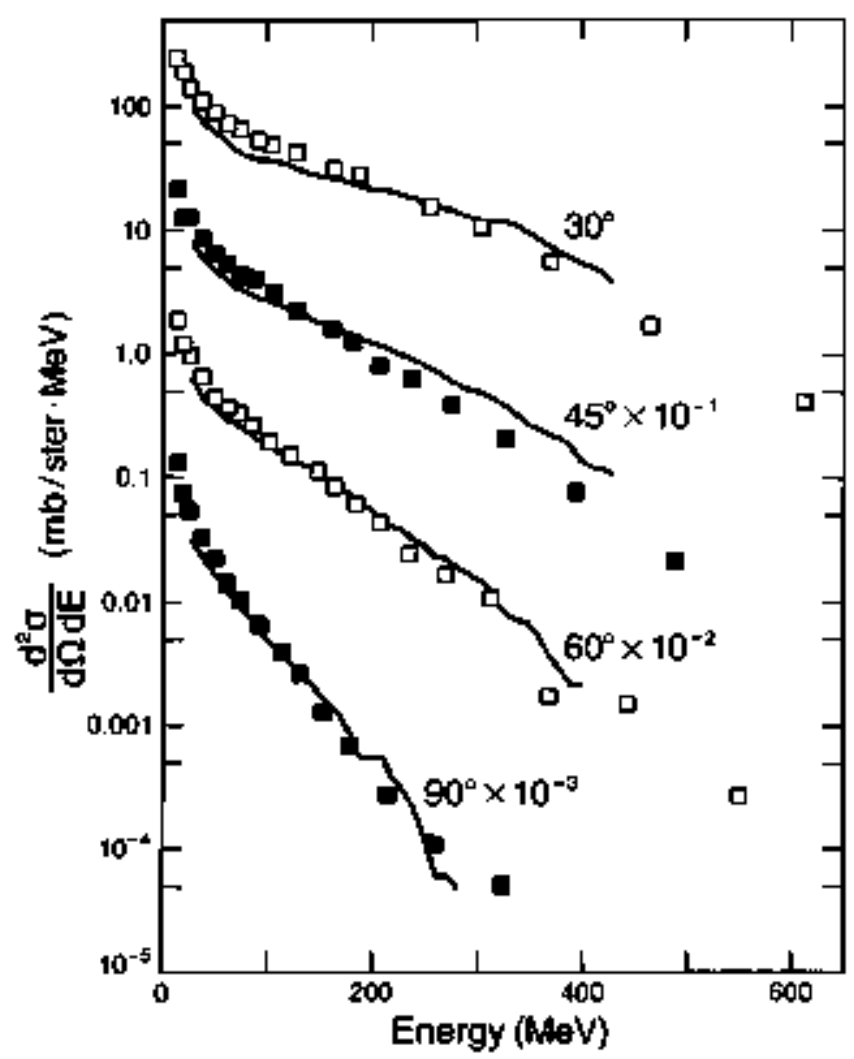

Fig. 2. The square symbols are the experimental neutron cross sections corrected to the time before coelescence. The soljd line is calculated from the cascade model.

(XBL 809-1 1910)

\section{Footnotes and Referentes}

*Also at Space Sciences Laboratory.

1. J.D. Stevenson, Phys. Rev. Lett, 4L 1702 (1978).

2. J.D. Stevenson, Phys. Rev. Lett. 45, 1773 (1980).

3. W. Schimmerling et al., Phys. Rev. Lett. 43, 1985 (1979).

4. H.H. Gutbrod el al., Phys. Rev. Lelt. 37, 667 (1976).

\section{Kaon Rescattering in Relativistic Nuclear Collisions*}

\section{J. Randrup}

Inclusive spectra of kaons produced in relativistic nuclear collisions have recently been measured.' In conjunction with the experiment, a theoretical study was made of the kaon production. ${ }^{2}$ This study pictured the nucleusnucleus collision as a sequence of elementary baryonbaryon collisions. The corresponding multiple-collision problem was solved in the linear eascade model, "rows on rows." In each elementary collision there is a small, $-10^{-3}$, branching ratio for kaon production which can therefore be included perturbatively. The required diflerential cross sections were obtained by generalizing the scarce NN data by help of the one-pion exchange model. In this way, the spectra of the produced kaons were calculated for a number of experimentally interesting cases.

Correspondence of this reference alculation with the data should only be expected if the kaons were to propagate through the surrounding matter without suffering any disturbances on their way. Because, in the energy region considered, the average kaon-nucleon cross section, which is 
nearly 1olally elastic, is $\sigma_{\mathrm{KN}}=9 \mathrm{mb}$, the kaons do in fact have a fairly good chance of escaping undisturbed. How. ever, simple estimates indicate that the average number of collisions suffered by a produced kaon is around one and rescattering therefore may not be entirely negligible. The purpose of the present work is to elucidate the role that the kaon rescattering mechanism has in bringing the calculation into good agrement with the preliminary and yet unpublished data

The first step towards understanding the importance of the rescattering mechanism is to estimate the number, $x$, of elastic collisions a produced kaon is expected to suffer with the surrounding matter. We arrive at the following rough estimate

$$
\kappa=0.7\left(\mathrm{R}_{\mathrm{A}}+\mathrm{R}_{\mathrm{f}}\right){ }^{\rho \sigma} \mathrm{KN}=\left(\mathrm{A}^{1 / 3}+\mathrm{B}^{1 / 3}\right) / 8
$$

where $A$ and $B$ are the nuclear mass numbers: we have used $1.15 \mathrm{fm}$ for the nuclear radius constant, $\rho=$ $0.17 / \mathrm{fm}^{3}$ for the nucleon density, and ${ }^{a} \mathrm{KN}=9 \mathrm{mb}^{4}$ (Table 1 shows the collision number $x$ for some cases under experimental investigation.) Therefore, on the average, a produced kemon is expected to suffer, at most, one rescatlering. Nevertheless, the appearence of the spectral distribution can be significantly altered.

To elucidate quantitatively the effect of kaon rescattering we have made the following augmentation of the model of Ref. 2: each kaon produced is allowed to suffer isotropic tlastic collisions with the baryons before escaping from the interaction zone. The number of actual collisions suffered by a given kaon is governed by a Poisson distribution, $\mathbf{P}(\mathbf{k})=\kappa^{k} \exp (-\alpha) / k$ !, characterized by the specified mean value $x$. The baryon collision partner (either a nucleon or a delta) is assumed to have a momentum distribution eorresponding to the one-particle inclusive cross section. This calculational simplification is not expected to be important for the ensuing conclusions.

To illusirate the effect of rescallering, the resulting observable inclusive kaon cross sections are displayed in Fig. 1 in the form of contour plots in the rapidity plane.

Table I. The stimated mean kacm collision number $k$ for a number of experimentally interesting combinations of projectiles and targets.

\begin{tabular}{llll}
\hline$\kappa$ & $\mathrm{NaF}$ & $\mathrm{KCl}$ & $\mathrm{Pb}$ \\
\hline $\mathrm{p}$ & 0.46 & 0.55 & 0.67 \\
$\mathrm{~d}$ & 0.50 & 0.58 & 0.90 \\
$\mathrm{Ne}$ & 0.68 & 0.77 & 1.08 \\
$\mathrm{Ar}$ & 0.77 & 0.85 & 1.17 \\
\hline
\end{tabular}

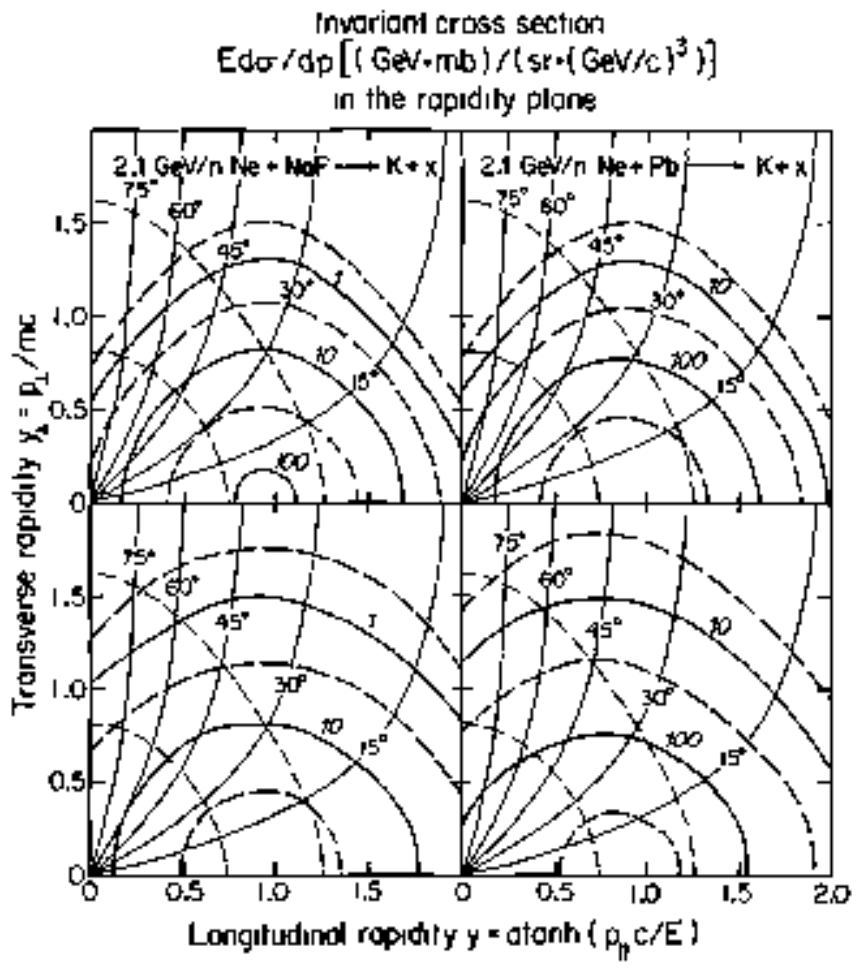

Fig. 1. Contour pot in the rapidity plane of the invariant differential cross section for observing a kaon, whether it be a $\mathrm{K}^{+}$or a $\mathrm{K}^{\circ}$. Upper portion; primary spectra as calculated in Ref. 2; lower portion: fingl spectre after rescatiering using the $x$-values in Table $I$. Grids of fixed laboralory angles and fixed laboratory momenta (of $\mathbf{4 0 0}$ and $B 00 \mathrm{MeV} / \mathrm{c}$ ) are superimposed to aid the comparisons.

(XRL 809-1953)

The cases shown are $2.1 \mathrm{GeV} / \mathrm{n} \mathrm{Ne}$ on $\mathrm{NaF}$ and $\mathrm{Pb}$. The upper portion shows the primary kaon distributions as calculated in Ref. 2. Initially, the kaons are nearly isotopic in the mid-rapidity frame and fall off relatively slesply. Their appearance after the rescattering is stown in the lower portion. The rescatteving process spreads the distribution over a wider domain in rapidity space and produces a market elongation in the beam direction, caused by the predominantly forward-backward ditected motion of the baryons. In the asymmetric case, a tilt develop because of the larger abundance of target participanis. Al large laboratory angles, this evolution significantly alters the differential spectra; they become much larger and fall off wonsiderably gentler. The overall kaon multiplicity is of course unaffected, since the kaons suffer only elastic scattering. The approximate scaling of the primary differential spectra ${ }^{2}$ is thus broken and the final distributions appear more like what would result in a thermel calculation.

The effect of rescattering on the angular distributions is only moderate at forward angles, but the effect is dramaic at sidewerds angles. In the typical kinematic region around $\mathrm{P}_{\mathrm{K}}=500 \mathrm{MeV} / \mathrm{c}$, the kaon yield is easily increased by an order of magnitude; and the slopes are halved. 
The results demonstrate that the scattering of kaons after their initial creation can substantially affect their spectral distributions. In the presented calculations, we have employed the $n$-values given in Table 1 which represent only a rough guess. One should therefore not expect immediate agreement with the data, particularly not in the large-angle region where the dependence on $x$ is most delicate. Rather, by firtue of this very sensitivity. one may hope that the large angle kaon spectra can eventually be exploited to provide information on the actual number of rescatterings and, therefore, yield new insight into the collision dynamics.

\section{Foolnole and References}

"Based on LaL-11508: Phys. Lett. B 99, 9 (1981).

1. S. Schnetzer et al., Proceedings of the International Conference on Nuclear Physics, Berkeley, 2430 Augusi 1980, p. 619, and to be published.

2. J. Randrup and C.M Ko, Nucl. Phys. A 343, 590 (1980).

3. J. Knoll and J. Randrup, Nuct. Phys. A 324, 445 (1979).

\title{
Central Collisions of Heavy Ions - Indication for Fluid Dynamical Behavior?*
}

\author{
H. Stöcker, ${ }^{\dagger}$ C Riedel, ${ }^{\dagger}$ G. Buchwald, ${ }^{\dagger}$ LP. Csernal, ${ }^{\ddagger}$ G. Graebner $_{r}^{\ddagger}$ \\ W. Greiner, ${ }^{\ddagger}$ J.A Maruhn, P. Subramanian, ${ }^{\ddagger}$ Y. Yoriv, ${ }^{\natural}$ K. Frankel, M. Gytlassy, \\ J. Stephenson, D. Strotiman," J.R. Mix $_{r}^{\mathbf{T 1}}$ B. Schtirmanin "-
}

The first detailed comparison of a variety of models with high multiplicity selteced proton sross sections is presented. The reaction studied is $\mathrm{Ne}(393 \mathrm{MeV} / \mathrm{n})+$ $\mathrm{U} \rightarrow \mathrm{p}+\mathrm{X}$ (high associated multiplicities). The observed preferential sidewards emission of the matter is reproduced by a hydrodynamical calculation with inal evaporation of protons, but the intranuclear cascade models and the two component (phase space) model fail in reproducing the data. The results seem to indicate that collective suclear flow, as described by nuclear thuid dynamics, does occor in nuclear collision. The forward peaking of the protons predicted by the cascade calculations, on the other hand, prowides an example of a qualitative failure of such models. which are based on subsequent independent nucleon-nucleon collisions. While previous data in $\mathrm{AgCl}$ detectors have also indicated sidewards peaking of aparticles, more precise and detailed data on central collisions will be necessary to extract information on the muclear equation of state.

\section{Foothotes}

*Based on LBL-12634.

tGSI, Darmstadt, West Germany.

fUniversität Frankfurt, West Germany.

TWeizniann Institute, Israel.

Los Alarno National Scientife Laboratory.

"Universituät München, West Germany.

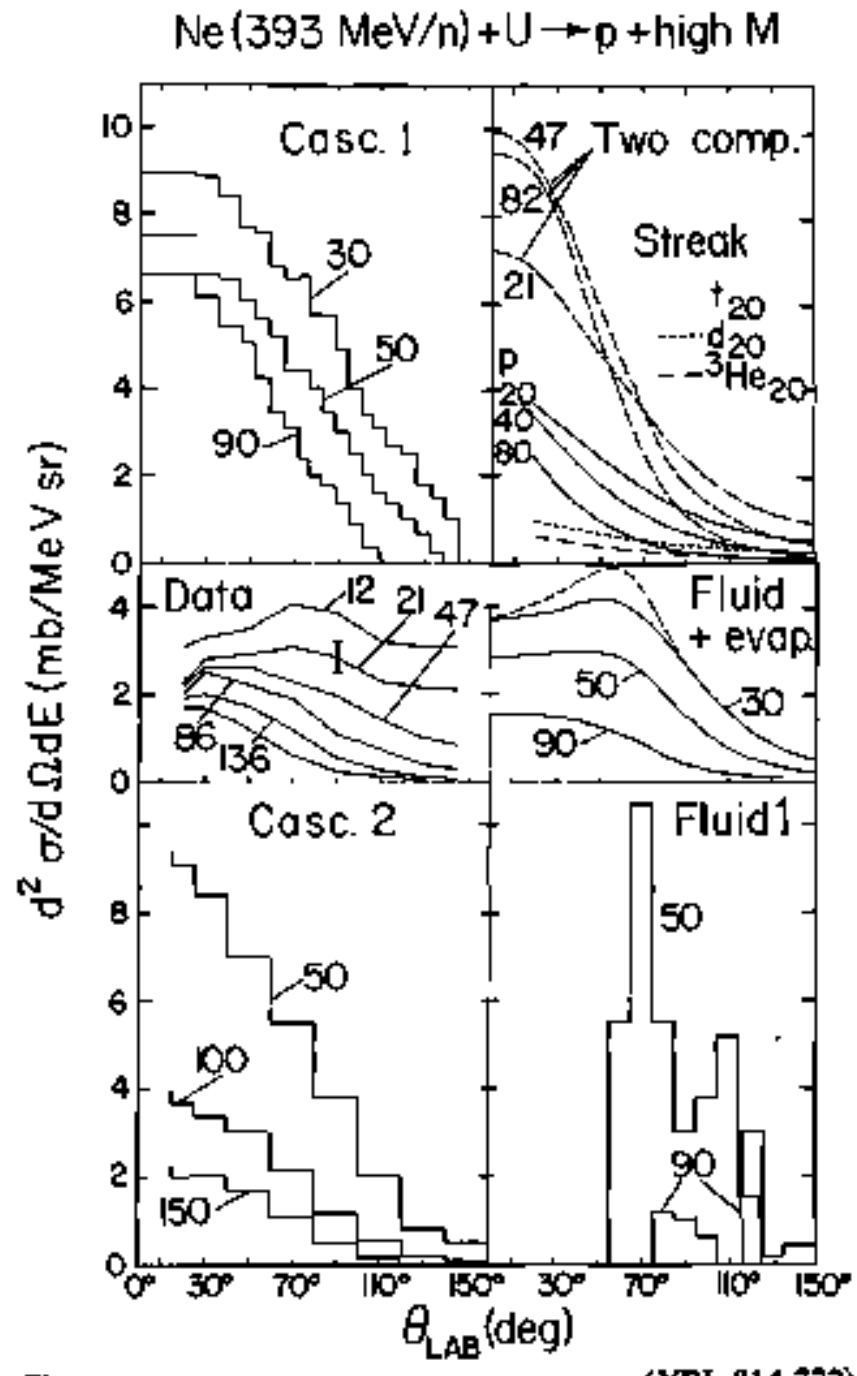

Fig. 1 . 


\title{
Two Particle Correlations Cansed by Collective Fluid - Dynamical Flow
}

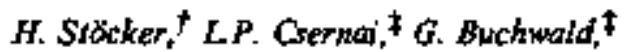 \\ G. Graebner, ${ }^{\ddagger}$ J.A. Maruhn ${ }^{\dagger}$ and W. Greiner ${ }^{\ddagger}$
}

Three-dimensionat fluid dynamical calculations with the description of final evaporation wese perfosmed for $\mathrm{Ar}+\mathrm{Pb}$ and $\mathrm{Ne}+\mathrm{U}$ at $\mathrm{E}_{\mathrm{Lab}}-400$ and $800 \mathrm{MeV} / \mathrm{n}$. The triple differential cross sections show the signs of the bounce-off effect, an azimuthal anticorrelation between the projectile and target fragments. The correlation function calculated from this triple differential cross section shows a good agreement with experiments on: (1) correlations between light and heavy fragments (see left side of the figure). and (2) two nucleon correlations (right). Thus, our calculations confirm that correlations in collisions of heavy ions are mainly determined by the collective flow of the nuchear matter and microscopic correlations play only a minor role for collisions of very beavy systems.
The figure shows the aximuthal dependence of the triple differential cross section for the system $\mathrm{Ne}+\mathrm{U}$ (upper left) at $\mathrm{E}_{\mathrm{L}} \mathrm{b}-393 \mathrm{MeV} / \mathrm{n}$ and the corresponding correlation function for lights to heavy fraguents (lowter lefi), logether with the data. The right hand side of the figure shows the calculated (upper right) and measured two nucleon correlation function (lower right) for the sysien $\mathrm{Ar}+\mathrm{Pb}$ at $\mathrm{E}_{\mathrm{ssb}}=800 \mathrm{MeV} / \mathrm{n}$.

\section{Foornotes}

"Hased on LBL- 12660.

†OSI, Darmstalt, West Germany.

tUniversität Frankfurt, West Germany.
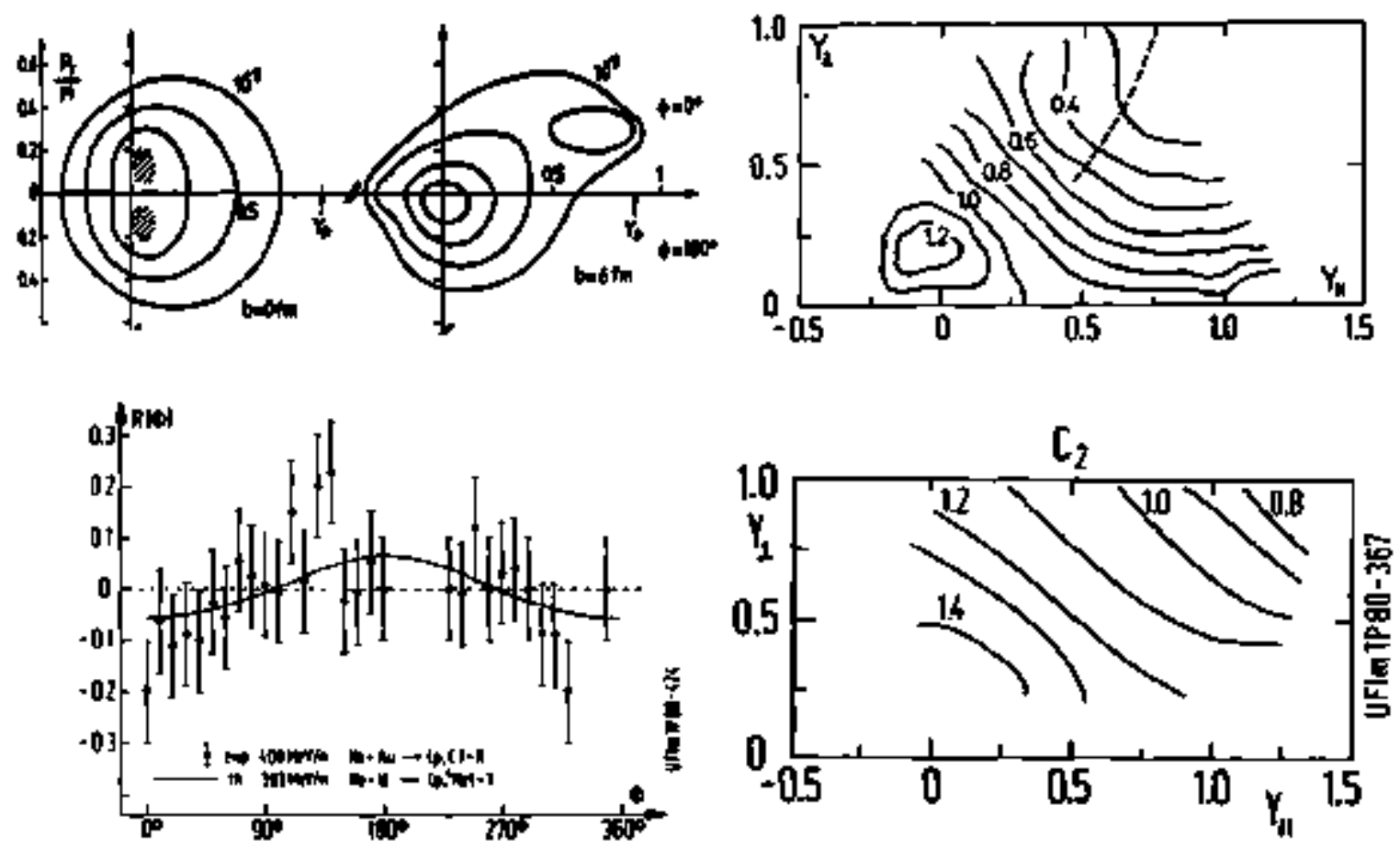

Fig. 1. Data on left from $\mathbf{H}$ Gutbrod et al., on right from I. Tanilata et al.

(XBL 8110-12213) 


\title{
Jets of Nuclear Matter from High Energy Heavy Ion Collisions
}

\author{
H. Stöcker, ${ }^{\dagger}$ L.P. Csernai, ${ }^{\ddagger}$ G. Graebner, \\ G. Buchwald, ${ }^{\ddagger}$ H. Kruse, ${ }^{\ddagger}$ R.X. Cussom ${ }^{\ddagger}$ J.A. Maruhn $\ddagger$ and W. Greiner $\ddagger$
}

The nuclear fluid dynamical model with finel thermal breakup is used to study the reaction ${ }^{20} \mathrm{Ne}+{ }^{238} \mathrm{U}$ and ${ }^{40 \mathrm{Ar}}+{ }^{40} \mathrm{Ca}$ at $\mathrm{E}_{\mathrm{Lab}}=390 \mathrm{MeV} / \mathrm{n}$. The calculated doulble diferential eross seclions $\mathrm{d}^{2}$ o/d d dE are in agreement with recent experimental data However, it is shown that the azimuthal dependence of the eriple differential distribultions, $\mathrm{d}^{3} \sigma / \mathrm{dEd} \cos \theta \mathrm{d} \phi$, to be obtained from $4 \pi$ exclusive experiments with single event analysis, can yield considerably deeper insight into the collision process and allow for snapshots for the reactions. Sirongly correlated jets of nuclear matter are predicted. The je1 angle, $\theta_{\text {jet }}$ relative to the beam axis increases from $0^{\circ}$ for peripherál collisions $1090^{\circ}$ for sentral collisions. A transition is prodicted from a $180^{\circ}$ azimuthally correlated two jet structure at intermediate impact parameters to a doughnut-shaped azimuthally symmetric distribution for central collisions. The figure shows the azimuthal dependence of the triple differential rapidity distribution (left) and the impact parameter dependence of the angle of the jet axis, $\theta_{j \text { jot }}$ relative to the beam axis.

\section{Foothotes}

"Based on LBL-1 1774.

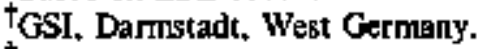

‡Universität Frankfurt, West Germany.

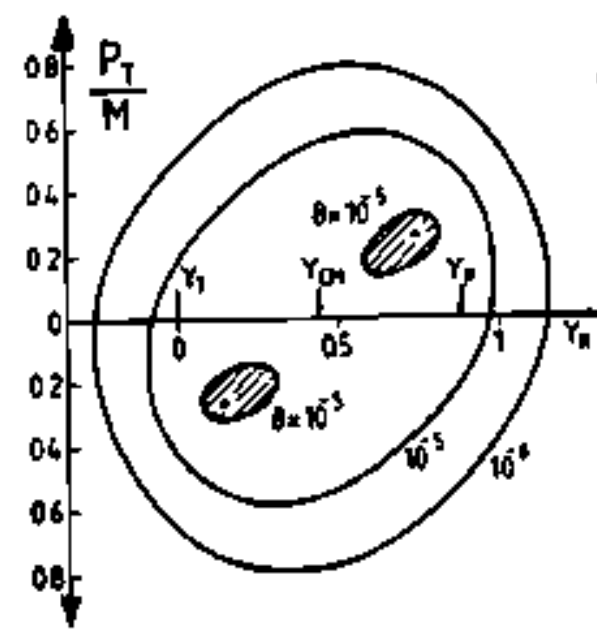

$4+0^{*}$

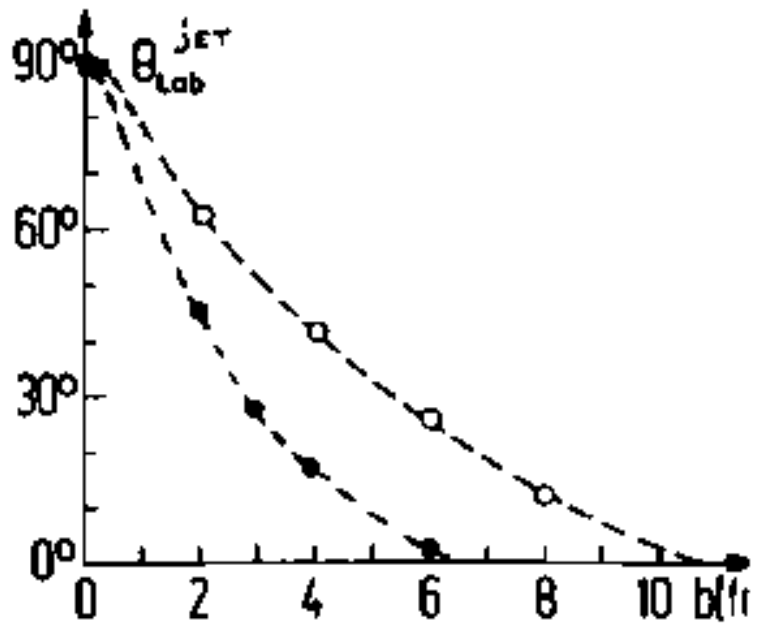

(XBL 8110-12214)

\section{The Bounce-Off Effect as a Barometer for Hot, Dense Matter in High Energy Nuclear Collisions"}

\author{
Horst Stöcket and Bernd Mtillert
}

The observed large collective transverse momentum transfer ${ }^{3}$ in high energy nuclear collisions is studied in a simple bounce-of model. The calculated mornentum transfers are in agreement with the experimental data. The bounce-off process is shown to be sensitive to the nuclear equation of state. The transverse momentum transfer can be estímated via

$$
\Delta \mathrm{PT}_{\mathrm{T}}=\mathrm{P}(\rho, \mathrm{T}) \mathrm{G}(\mathrm{b})
$$

where $P(\rho, T)$ is the pressure in the high energy, high tert perature zone and $G(b)$ is a geometrical function which depends on the impact parameter only. We suggest using this for a systematic experimental investigation of $\mathrm{P}(p, \mathrm{~T})$ 
in high energy nuclear collisions. Such measurements could provide evidence for phase transitions into abnormal states in dense nuclear maller and into quark matler. Above all, the scattered fragments are direct witnesses of the initial, hot, and dense parts of the reaction and as such they deserve much future attention.
Footnotes and References

'Condensed from LBL-1247].

'Depariment of Physics and Astronomy, Nuclear Physics Division, Vanderbilt University, Nashville, TN 37203.

l. H.H. Gutbrod, LBL-11123 and A Wartick et al., LBL- 12180 to be published.

\title{
Probing Dense Nuclear Matter via Nuclear Collisions"
}

\author{
H. Stäcker, M. Gyulassy, and J. Boguta
}

We study the sensitivity of nuclear hydrodynamics to the equation of state of nuclear matter at high densities and temperatures. The relativistic Rankine Hugoniot equation is used to calculate the pressure, P, and entropy, $\mathbf{S}$, generated in nuclear collisions between $\mathrm{E}_{\text {Lab }}=50$ and $200 \mathrm{MeV} / \mathrm{n}$ in a variety of models for the equation of state (see Fig. l), P(E) and $S(E)$ can be directly related to experimental stservables. The pressure, as the driving force for the fluid motion, determines the mean collective fow behavior, especially the iranswerse momentum transfer, while the entropy produced determines the hadron production rates and chemical composition of light nuclear fragments.

We find both $P(E)$ and $S(E)$ to be most sensitive on a $550 \%$ level to the compression part of the equation of state at intermediate bombarding energies, $\mathrm{E}_{\text {Lab }}<$ $250 \mathrm{MeV} / \mathrm{n}$. At high energies, $\mathrm{E}_{\mathrm{b}}>1 \mathrm{GeV} / \mathrm{n}, \mathrm{P}(\mathrm{E})$, and $S(E)$ are sensitive (on a $\leqslant 25 \%$ leve) to the thermal part of the equation of state.

Hence, high precision is required in the combined measurement of P(E) and $S(E)$ via detailed jel analysis and composition analysis, which promistes, in fact, the best prospects for deteruining the equation of state ultimately from nuclear collision data.

\section{Footmote}

"Condensed from LBL-12095.

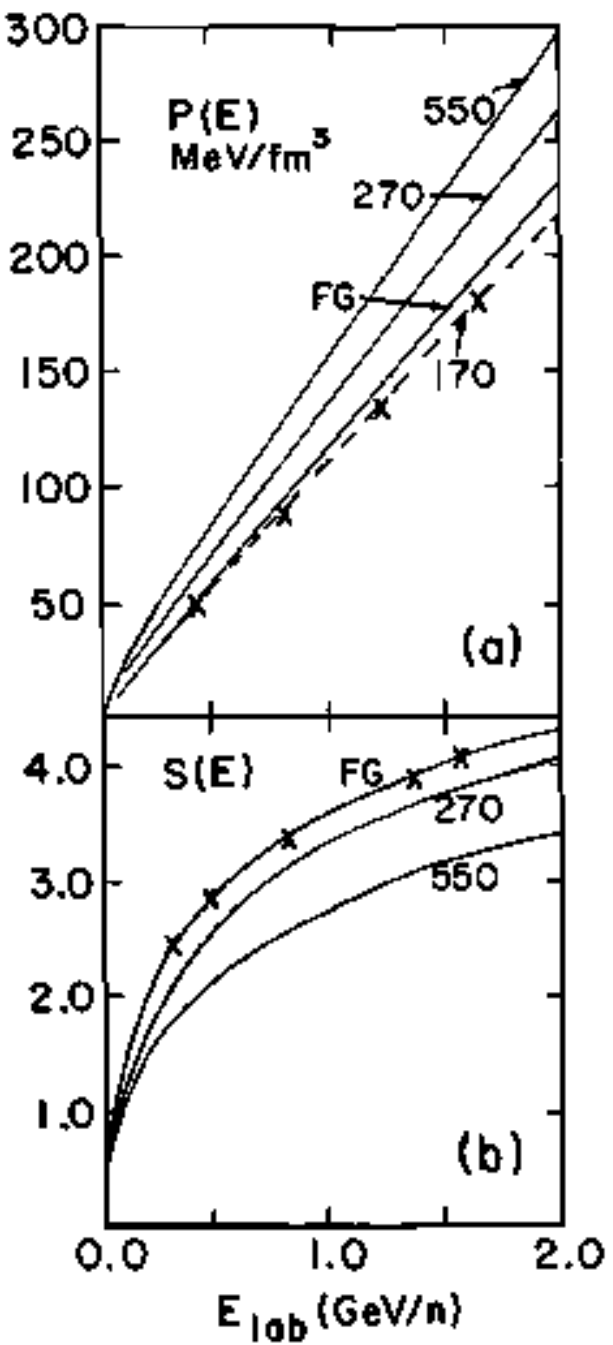

Fig. 1. (a) Total pressure P(E) vs $\mathbf{E}$ for Fermi gas (solid line) compared with neean field theory, $K=550, K=$ 270 , and $K=170$ (marked with $X$ ). (b) Entropy per baryon $S(E)$ vs $E$ for equation of state in (a). $S_{\mathrm{TG}}$ is the exact Fermi gas result.

(XBL 813-8436A) 


\title{
Optical Model Solutions for Pions in Nuclear Matter
}

\author{
P. Hecking
}

Pionic modes and pion propagation in baryonic matter are of considerable interest concerning pion production in nuclei and heavy-ion collisions and more exotic phenomena such as pion condensation. The purpose of this work is to point out the extent that information about propagation and attenuation of the "pionic" branch of the pion spectrum in nuclear matter, in contrast to the $\Delta$-isobar branch or the acoustical branch, can be deduced from optical model parameters which are compiled from scaltering and pionic atom data.

The (complex) pion momentum $k=k_{1}+i k_{2}$ in the modium is related to the (also complex) optical potential $\mathrm{U}_{\text {opt }}(k, \omega)$ by the expression

$$
k^{2}-k_{\delta}^{2}=-2 \omega U_{\text {opr }}(k, \omega)
$$

where $k_{0}^{2}=w^{2}-m_{2}^{2}$ is the pion mornentum. The optical potential $\mathbf{U}_{\text {opt }}$ can be parameterized in the usual manлer with the complex quantities $b_{0}, c_{0,}, B_{0,}$ and $C_{0}$ and with Landay Fermi-Jiquid parameter $g^{\prime}$, which describes the short-range correlation. These five quantities have been determined from pion scattering and pionic atom data. ${ }^{2}$

The imaginary part $k_{2}$ (of $k$ ) is connected with the pion mean free path by

$$
\lambda=\frac{1}{2 k_{2}}=\frac{k_{1}}{20 \mathrm{Im} U_{o p p}}
$$

Table 1 gives the result for the "Belf-contistent" solution of $\mathrm{Eq}$. (1) with $\mathrm{U}_{\mathrm{og}}(\mathbf{k}, \mathrm{\omega})$. The mean free path $\lambda$ is rather flat for $0.8 \mathrm{~m}_{x} \mathrm{c}^{2} \neq k_{0} \neq 2.4 \mathrm{~m}_{x^{\mathrm{c}}}{ }^{-1}$, although one should expect a pronounced minimum at the resongnce. Also for $\rho=2 \rho_{0}$ (not shown in Table 1), the mean free path is larger than at $\rho=\rho_{0}$, which is rather unteasoneble. Furthermore, a $\lambda$ between 0.6 and $1 \mathrm{fm}$ setms extraordinerily small.

A different approach would be the application of the free pion monentum $k_{0}$ in $U_{o p}\left(k_{0}, \omega\right)$ instead of $k$. A distinct minimum at the resonanee is obtained, fitting well to the estimate $\left(\lambda \sim \rho^{-1} \sigma_{\pi N}^{-1}\right)$ with $\sigma_{\pi \mathrm{N}}=140 \mathrm{mb}$; for $\rho=$ $2 \rho_{o}$; the mean free path is smaller throughoul than for $p=$ $\rho_{0^{+}}$and the result is in good agreement with the $\Delta$-isobar model (in the resonence region). Also the pion spectrum in medium energy heavy-ion collisions is highly nonthermal,
Table 1. The solutions for $\mathrm{U}_{\mathrm{opt}}(\mathrm{k}, \omega)$ and $U_{\text {opt }}\left(k_{0}, \omega\right)$ in Eq. (l) for the optical model parameters of Refs. 1 and 2 at $p=p_{p}=0.17$

\begin{tabular}{|c|c|c|c|c|}
\hline \multirow[b]{2}{*}{$\begin{array}{c}k_{0} \\
{\left[m_{k} c^{-I}\right]}\end{array}$} & \multicolumn{2}{|c|}{$U_{o p t}(k, \omega)$} & \multicolumn{2}{|c|}{$U_{o x}\left(k_{0}, \omega\right)$} \\
\hline & {$\left[\begin{array}{c}k_{1} \\
{\left[m_{x} c^{-l}\right]}\end{array}\right.$} & $\begin{array}{c}\lambda \\
{[\mathrm{fm}]}\end{array}$ & {$\left[\begin{array}{c}k_{1} \\
{\left[m_{\mathbf{s}} c^{-l}\right]}\end{array}\right.$} & $\begin{array}{c}\lambda \\
{[\mathbf{f m}]}\end{array}$ \\
\hline 0 & -0.18 & 0.68 & 0.14 & 1.47 \\
\hline 0.4 & -0.42 & 1.09 & 0.39 & 293 \\
\hline 0.8 & 0.19 & 0.75 & 1.06 & 3.92 \\
\hline 1.2 & 0.76 & 0.57 & 1.65 & 2.59 \\
\hline 1.6 & 1.13 & 0.61 & 2.16 & 1.36 \\
\hline 2.0 & 1.25 & 0.70 & 2.60 & 0.70 \\
\hline 2.4 & 1.60 & 0.97 & 2.45 & 0.51 \\
\hline
\end{tabular}
$\mathrm{fm}^{-3}$

which adwoleates a rather large mean free path. The mean free path $\lambda$ bas also boen determined ${ }^{3}$ for parameters $c_{g}$, $B_{a^{\prime}}$ and $C_{\mathrm{O}}$ which had been obtained from nuclear matter calculations. ${ }^{4}$ The results agree well with those for empirical values of $\mathrm{c}_{\alpha^{*}} \mathrm{~B}_{0^{*}}$ and $\mathrm{C}_{p^{*}}$

In conclusion, a "self-consistent" solution of the pion momentum $k$ in the medium turns out to be unsatisfactory in comparison with the approximation $k=k_{0}$ in the optical potential $U_{o p p}$ if the parameters for $U_{o p t}$ are deduced from experimental data. The reason for this is not entirely clear, probably the abilities of the optical model are overstressed

\section{Footnotes and Referentes}

Condensed Irom LBL-12911.

tWork supported in part by the Deutsche Forschungsgemeinschaft under contract He 1155/1.

1. K. Stricker, H. McManus and J.A Carr, Phys. Rev. C 19. 929 (1978).

2. J. Hüfner, Phys. Rep. C 21, 1 (1975).

3. P. Hecking, Phys. Lett. 103B, 401 (1981).

4. J. Chai and D.O. Riska, Nucl. Phys. A 329, 429 (1979). 


\title{
Finite Temperature Pion Condensation
}

\author{
N.K Glendenning and A. Lumbroso"
}

In a related work, pion condenstion in zero temperature motter was investigated in a relativistic field theory solved in the mean field approximation. ${ }^{1}$ Unlike earlier work on the subject, the theory was constrained to possess the known saturation properties of nuclear matter. H\$re, we investigate matler in the same theory at finite temperature. Finite temperatures are interesting becsuse pion condensation is believed to be an important mechanism involved in the cooling of neutron stars. Moreover, nuclear collisions at high energy, if they produce dense matter, wertainly produce it at finite temperatore.

The properties of the normal state can be used to determine all parameters of the theory except for the effective pion-nucleon coupling, $\mathrm{g}_{\mathbf{T}}$. This is a very important parameter of the theory, one which we take great care in determining. It would be unacceptable to simply use the vacuum value. This would greatly overestimate the importance of the condensate state. Through the derivative cour pling in our Lagrangian, ${ }^{1}$ the pions interact with nucleons dominantly in the p-wave state as it should be. We can represent this by the diagram

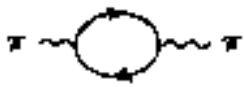

corresponding to pion absorption on a particle in the Fermi sea exciting a particle-hole state and the subsequent re-emission of the pion. In addition, other processes are bejieved to be important which are not explicit in our theory. One of these is the absorption of a pion to create a $\Delta$ isobar;<smiles>[3H]C1C2CC3CC(C2)CC1([3H])C3</smiles>

where the double line represents the jsobar. This process encourages condensation and is very important because it is not inhibited by the Pauli-exclusion principle. Acting in the opposite direction are the repeated scatterings of the particle-hole excitations by the nucleon-nudeon interaction. In the particle-hole state, which bas quantum numbers of the pion, this can be discussed in terms of the Landav parameter $\mathrm{g}^{\gamma}$. The above two diagrams should be replaced by the renormalized p-wave interaction defined by

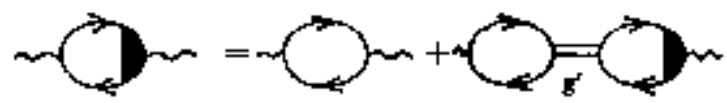

where the particle state can be either nucleon or isobar. Wile these additional processes would be extremely difficult to incorporate in a theory of the fully developed condensate state such es ours, they are relatively easy to incorporate in the pion self-energy at the pion condensation threshold. The reason is that, at threshold, the infinitesimal pion field does not affect the nucleon states so that the abowe contributions can be calculated on the unperturbed basis. Such a calculation of the pion condensation threshold has been carried out by many authors, injtially by Migdal ${ }^{2}$ and Sawyer and Scalapino. ${ }^{3}$ The finite temperature propagetor was first calculated by Ruck. Gyulassy, and Greiner, ${ }^{4}$ and more recently by Hecking, weise. and Aktoury. 5 We make use of this most recent calculation of the condersate critical density as a function of temr perature.

Our strategy in brief is to remormalize the pionnueleon coupling gr in our theory to such a value as to reproduce, at each temperature, the critical density as calculated by Hecking et al. ${ }^{5}$ In this way we constrain our theory to the bulk properties of the normal state and the best estimates, to date, of the pion condensation threshold density.

We make a remarkable observation concening the pion condensed state, which is illustrated in Fig. 1. The binding energy per nucleon as a function of density at various temperatures between 0 and $100 \mathrm{MeV}$ is shown. At low temperature, the condensate makes very little contribution to the energy, when the theory is constrained by the bulk properties of nuclear matter, as was found in our earlier work.' But at higher temperatures, although the critical density increases, the condensate makes an increasingly important contribution to the energy. This dramatic softening in the equation of state will strongly influence the hydrodynarnical flow of hot dense matter if a transition to the condensed state occurs. This is likely in the region

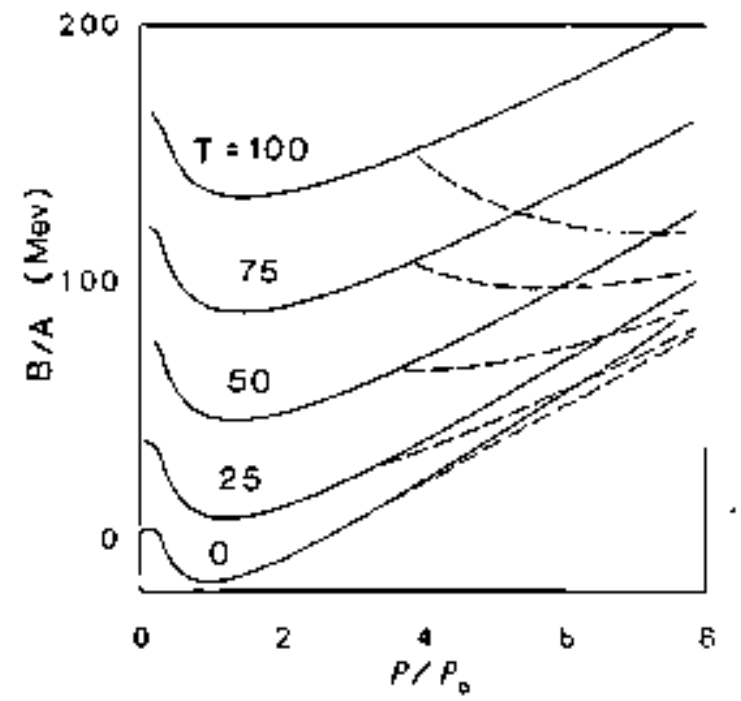

Fig. I. Binding energy for asymmetric muclear matter. Solid lines are normal and deshed pion condensate state. The critical density follows the trajectory in the $\rho$-T plane calculated in Ref. 5 for $g^{\prime}=0.6$.

(XBL 811-7534) 
above the eritical density, because the free energy in the pion pondensed state is lower than in the normal state at the same energy and baryon densities. Moreover, because the temperature is much higher in the condensed state, subsequent iransition to a quark matter phase may be facilitated, if the density is high enough.

Foornote and References

On leave from CEN, Saclay. France.
1. B. Banerjee, N.K Glendenning, M. Gyulassy, LBL 10979 and Nucl. Phys. A in press.

2. A. B. Migdal, Phys, Rev. Lelt. 31, 247 (1973).

3. R.F. Sawyet and D.J. Sealapino, Phys, Rev. D 7, 953 (1973).

4. V. Ruck, M. Gyulassy and W. Greiner, Z. Physisk A 277, 391 (1976).

5. P. Hecking, W, Weise and R. Akhoury, Preprint.

\title{
Pion Condensation Threshold in Nuclear Matter and Thermal $\Delta$-Isobars"
}

\author{
P. Hecking
}

Pion condensation in nucleat and neutron matter at zero, as well as finite temperalure, has been investigaled in recenl years with the hope of finding some evidence of it in heary-ion collisions and for the properties of neutson stars. Pion condensation occurs at the lowest baryen density, $p_{\mathrm{B}}$, for which a solution of the pion dispersion relation

$$
\omega^{2}=k^{2}+m^{2}+\Pi\left(k, \omega, \hat{B}_{B}, T\right)
$$

exisls. The (negative) pion self-energy in the medium depends on $\rho_{\mathrm{B}}$, the pion four-mornentum $\mathrm{q}=(\omega, \mathrm{k})$, and the temperature $T$ with $\omega=0$ for isospin-symmetsic ( $N=$ Z) nuclear matter.

An important part of the pion self-energy $\Pi$ results from the $\pi \mathrm{N} \Delta$ and $\pi \Delta \Delta$ interaction wilh the $\Delta(1232)$ isobar. At finite temperature, there are already purely thermal $\Delta$-isobars present, even in the absence of a pion condensate. Consequently, the pion self-energy II results in part from the interaction of the pion with a Fermi-sea of $\Delta$-isobars. The density $\rho_{\mathrm{A}}$ is obtained from the chervical equilibrium $\mu_{N}(T)=H_{A}(T)$ of the reaction $N+N-$ $N+\Delta$. The p-wave part of the pion self-energy II is given by the response of a Fermi-sea of nucleons (or $\Delta$. isobars). Short-range repulsive correlations, given by the Landau Fermi-liquid parameter $\mathbf{g}^{\prime}$, an effective nucleon mass $m^{*}<m$ and finite range $\pi$-baryon vertices with a cutoff $\Lambda$, are taken into account along the lines of Refs. I and 2.

The cutofi $\Lambda$ is rather well determined in the range l$1.4 \mathrm{GeV}$ and is not very density-dependent. At nuclear deasity $p_{0}=0.17 \mathrm{fm}^{-3}$, the Fermi-liquid parameter $\mathrm{g}^{\prime}$ has velues of $0.6-0.7$ and the effective mass $\mathrm{m}^{*}$ is $-0.8 \mathrm{~m}$, but their values, especially that of $\mathrm{m}^{*}$, remain uncertain for densities larger than $p_{0}$. In order to cope with that, several values fot $\mathrm{g}^{\prime}$ are taken; and for $\mathrm{m}^{*}$ two choices ate made: a density independent $\mathrm{m}^{*}=0.8 \mathrm{~m}$ and a density. dependent $m^{*}(\rho)=m\left[1-k_{F}(\rho) k_{F}^{-1}\left(\rho_{\alpha}\right) 0.2\right]$ with the Fermi-momentum $\mathrm{k}_{\mathrm{F}}$.
Figure 1 shows the result for the stitieal baryon density $\rho_{\mathrm{c}^{\prime}}$ One obvious feature is the ratber "parallel" behavior of the phase boundaries with increasing temperature for various values of $\mathbf{A}$ and $\mathrm{g}^{+}$. The Fermi-sea of thes. mally exciled $\Delta$-isobars is of importance only above $k_{B} T$ $50 \mathrm{MeV}$. Below this temperature, $\rho_{\Delta}$ approaches zero exponentially. Even at $k_{B} T-100 \mathrm{MeV}$, the influence on $\rho_{\mathrm{f}}$ is a (less than) $20 \%$ effect. The temperature $k_{B} T$ $180 \mathrm{MeV}$ is obtained in a typical central heavy-ion oollj. sion with a bombarding energy of $1 \mathrm{GeV} / \mathrm{N}$, provided com. plete thermalization is obtained.

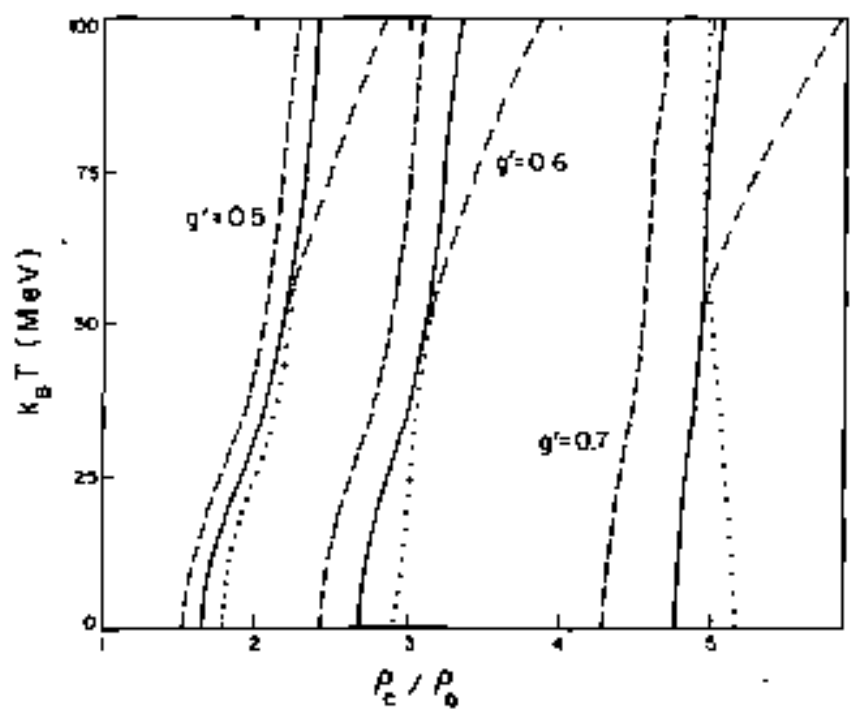

Fig. 1. Critical beryon density $p_{c}(\boldsymbol{T})$ as function of the temperature and various parameters. The pion-condensed phase is to the right of the curves. Without $\Delta$-Fermi-sea: fuhl curves: $\boldsymbol{\lambda}=1.2 \mathrm{GeV}$ and $\mathrm{m}^{*}=0.8$; dashed corves: $\Lambda=1.4 \mathrm{GeV}$ and $\mathrm{m}^{*}=0.8 ;$ dolled curves $\Lambda=1.2 \mathrm{GeV}$ and $\mathrm{m}^{*}=\mathrm{m}(\rho)$. With $\Delta$.Ferni-sea: deshed-dotted curves: $\Lambda=1.2 \mathrm{GeV}$ and $\mathrm{m}^{*}=0.8$. 
It turns out that a Fermi-sea of thermally excited $\Delta$ isobars is of equal or less importance for the critical effective nucleon mass $\mathrm{m}^{*}$, and the short-range correlation parameter $\mathrm{g}^{\prime}$. The latter still remains the most decisive and, unfortunately (especially at densities larger than $\rho_{\mathrm{o}}$ ), a]so the least well known.
Footnotes and References

- Condensed from LBL-12254. This work was supported in part by the Deutsche Forschungsgemeinschafl under contract He $1155 / 1$.

1. P. Hecking, Nucl, Phys. A 348, 493 (1980).

2. H.J. Pirner, M Rho, $K$ Yazaki and D. Bonche, Nucl. Phys. A 329, 491 (1979).

\title{
Test for Pion Condensation in Nuclear Collisions
}

\author{
M. Gyulassy
}

If pionic instabilities occur in nuclear collisions, a macrosoopic spintisospin current, $\mathbf{3}_{4} 5(x, t)$, would grow in time in the nucles volume. Since $\mathbb{S}_{\mu 5}$ is a source of pions

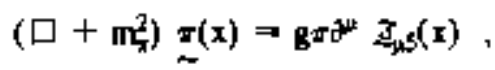

a time-dependent $\boldsymbol{J}_{\text {s }}$ will radiate pions. Furthermore, that sadiation is in the form of a colerent state. The idea is therefore to calculate the pion spectrum that would be generated by $\mathfrak{z}_{\mu \mathrm{s}}$ and compare it with data.

Based on fuean field oalculations, ${ }^{2}$ we expect a large amplitude spin-isospin wave associated with pion condensation

$$
g_{x} \partial^{\mu} \quad J_{\mu 5}(x) \underset{1 \rightarrow \infty}{\rightarrow} g_{n} k_{c} a_{5 \rho} e^{-i k_{a} x}
$$

with

$$
\mathrm{g}=\mathrm{m}_{\mathrm{r}}^{-1}, \mathrm{k}_{\mathrm{c}}=2 \mathrm{~m}_{\mathrm{r}}, \alpha \sim 1 / 2, p=\mathrm{m}_{\mathrm{g}}^{3} .
$$

As a guess of the small time behavior, we combine the results of growth rate calculations ${ }^{3}$ with Eq. (2) to estimate

$$
\begin{aligned}
& g_{x}{ }^{2} J_{\mu 5}(x)= \\
& \theta(t) \gamma\left(k_{c}\right) 1 g_{2} k_{q} g p e^{-i k_{x} x} e^{-x_{2} / 2 R^{2}} e^{-1 / \Delta}
\end{aligned}
$$

where the growth rate is $\gamma=0.2 \mathrm{~m}_{-}^{-1}$ and $\gamma(\mathbf{k})$ is peaked for $\mathrm{k}_{\mathrm{c}} \sim 2 \mathrm{~m}_{\pi} \hat{e}_{\perp}$, i.e., perpendicular to the bean axis. Taking the modulus square of the spacetime Fourier transform of $\mathrm{Eq}$. (3) gives the single pion inclusive distribution. That invarient distribution has the form

$$
\omega \frac{d_{3} \sigma}{d^{3}}=\sigma_{0} n_{0} \frac{\gamma^{3}(k)}{\langle\gamma\rangle}
$$

where

$$
\left\langle\gamma>0=\int d^{3} k \gamma(k) / \omega_{k} .\right.
$$

and

$$
\frac{n_{0}}{A}=B_{s}^{2} k_{c}^{2} \alpha^{2} \rho \gamma^{2}\left(k_{c}\right) /\left(\sqrt{2} \omega_{k s}\right)^{5} \sim 10^{-4}
$$

is the average number of coherent pions radiated by $\tau_{\mu 5}$ per nueleorn. Note that $n_{0} / A<C(n / A)_{\exp } \sim 10^{-2}$.

Even though $n_{0}$ is so small, the important observation is that $\omega^{3}{ }^{3} \sigma / \mathrm{dk}^{3}$ is peaked at $\theta_{\mathrm{cm}}=90^{\circ}, \mathrm{k}_{\mathrm{em}}-2 \mathrm{~m}$, due to the peculiar dependence of $r(\mathbf{k})$ on $\mathrm{k}^{3}$ The chlaotic source of pions produces, on the other hand, an approximately thermal spectrem that is peaked at $k_{c m} \sim 0$ for $\left.\mathrm{k}_{\mathrm{cm}} \sim 2 \mathrm{~m}_{x}, \omega^{\frac{1}{3}} \sigma / \mathrm{dk}^{3}\right)_{\text {chaotic }} \propto \exp \left(-2 \mathrm{~m}_{\mathrm{n}} / \mathrm{m}\right)$. For low enough bombarding energies, the slope $T \& 2 \mathrm{~m}$, and the chaotic yield are strongly suppressed. Therefore, the small coberent component could even becomse dominant for $\mathrm{k}_{\mathrm{cm}} \sim 2 \mathrm{~m}_{\mathrm{x}}, \theta_{\mathrm{cm}} \sim 90^{\circ}$ when the bonberding energy is low enough. Estimates in Ref. 1 indicate that $200 \mathrm{MeV} / \mathrm{A}$ is the ideal bombarding energy to reach for this ooherent component. The signature of pionic instabilities would be a break in the $90^{\circ}$ slope parameter at $\mathrm{k}_{\mathrm{cm}} \sim 2 \mathrm{~m}_{\text {x }}$.

\section{References}

1. M. Gyulassy, Nucl. Phys. A 354, 395c (1981).

2. B. Banerjec, N.K. Glendenning, M. Gyulassy, Nucl. Phys. A 361, 326 (1981).

3. M. Gyulassy, W. Greiner, Ann. Phys. 109, 485 (1977). 


\title{
Significance of Temperature Measurement in Relativistic Nuclear Collisions
}

\author{
H. Stöcker, A. A. Ogloblint, W. Greinget
}

We point cut the importance of temperature measurements in fast nuclear collisions. Recent data ${ }^{\text {' }}$ indicale values for $T_{0}$ at $3.6 \mathrm{GeV} / \mathrm{n}$ which conne close to the limiting temperature $T^{\max }=m_{1} C^{2}$ which has been postulated for a badronic mass spectum that increases exponentíally. The bombarding energy dependence of the temperature is calculated in the fluid dynamical model for a pure nuclear ges and for an exponentially increasing hadronic mass spectrum and compared to the data (left figure). The spectra of pions, protons, and desterons are calculated using an

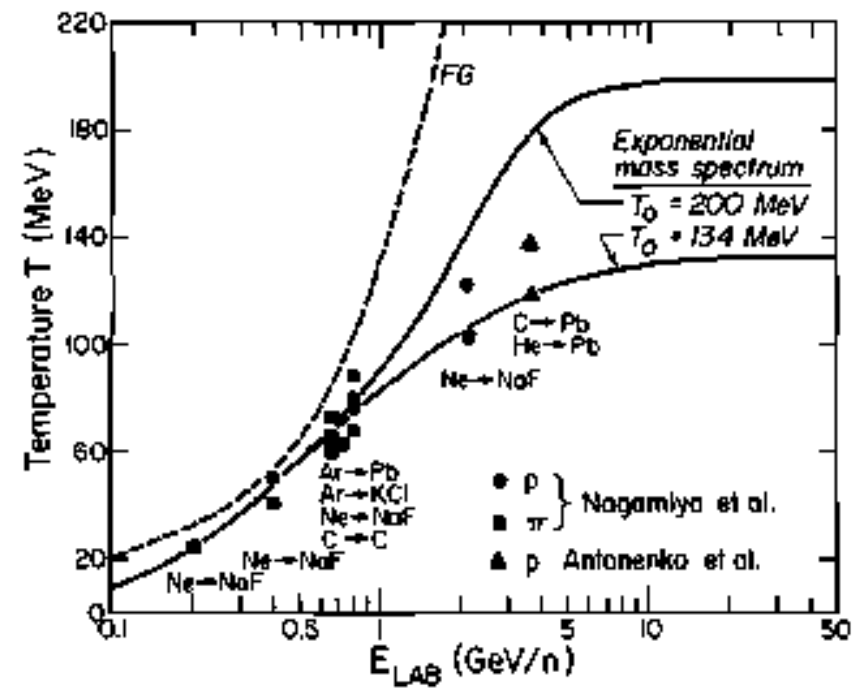

XBL 817-1049 isotropic expansion model (right figure). It is interesting to note that the apparent temperatures after the expansion are close to the initial temperatures.

\section{Foothotes and Reference}

"Condensed from LBL 12971

†Kurchatow Institnte, Moscow

Inst. f. Theor. Physik, Frankfurt

1. V. G. Antonenko to al., Pisma Jetp 28, 609 (1978); and S. Nagamíya, tt à., LBL-12123.

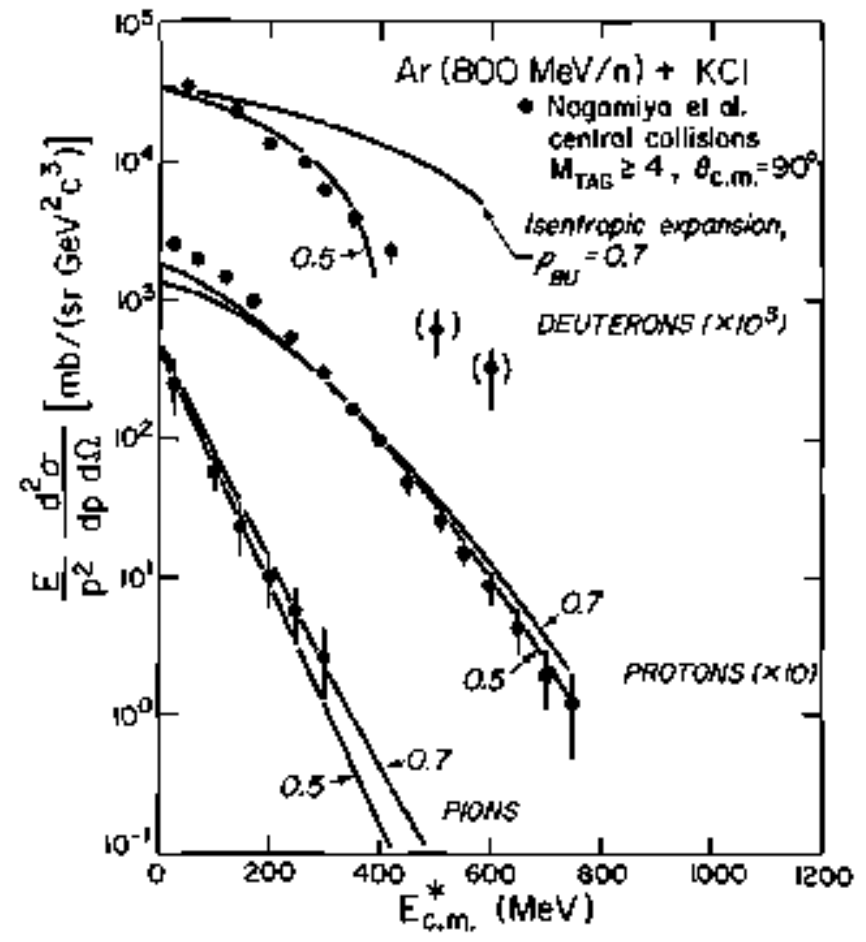

XBL $817-1046$

Fig. 1. 


\title{
On the Significance of Temperature Measurements of Nuclear Fireballs
}

\author{
Norman $\boldsymbol{K}$ Giendenning
}

Several experiments, involving the collision of highenergy particles with nuclei and performed over a range of energies, have been interpreted as measuring the temperatures of nuclear fireballs. The extracted temperatures have the appearance of approaching an asymptotic value, and this has given rise to the thought that the ultimale temperature of hadronic matter predicted by Hagadorn' has actually been observed. The purpose of this note is to suggest that the interpretation of the data as indicating an asymplotic temperature is probably not correct.

The first remark is that if a dense high energy fireball is focmed in a nuclear collision, the hreball subsequently must expand because it is unbound. Depending on the injthal densily of the areball, the expansion may entail a manyfold expansion unti] a [rezzout density is reached. Although evaporated particles that escape during the expansion carry information about the conditions at the instant of escape, they are indistinguishable from others ersitted earlier or later. Meanwhile, the temperature of the fireball falls during the expansion, and the populations of the various hadronic species readjust. The bulk of the observable particles are released at the freezout point in the evolution, and they therefore obscoure the information aboul the hotiest stages carried by those particles that were emitted early in the life of the fireball. The conclusion to be drawn from the discussion up to this point is that the spectra of particles enitted by nuclear fireballs are dom. inated by particles released at the freezout stage ${ }^{2}$, and therefore the temperature deduced from the slope of spectra will be the final fireball temperature. To learn about the conditions in the initial fireball requires mbre elaborate experimentation and analysis than the observation of spectra $^{2}$

The question remains whether the final fireball terr peratures that have boen dednced carry interesting information about the underlying mass spectrum of hadrons or the existence of an ultimate temperature. To address this question, we must first admit that we do not know the precise conditions that prevail when thermal contact of the particles is lost. Presumably the hadronic density has fa]+ len to normal nuctear density or less. Adopting as a nominal freezout condition that the toial hadron density including all baryons, antibaryons, and mesons is $0.17 \mathrm{fm}^{-3}$, the density of normal nuclej (Fig. I), shows the fireball terrperature at freezout as a function of C.M. energy per net baryon. The thermodynamics of a relativistic free gas of hadrons is assumed. ${ }^{2}$ If all of the initial bonbarding energy remains in the fireball until frozzout, then these two energies are oqual. If the spectrum of tradrons is an exponentially increasing function of their mass, the temperature of the fireball is bounded by $T_{0}=134 \mathrm{MeV}$. For energies above $\sim 5 \mathrm{GeV}$, the energy is stored in

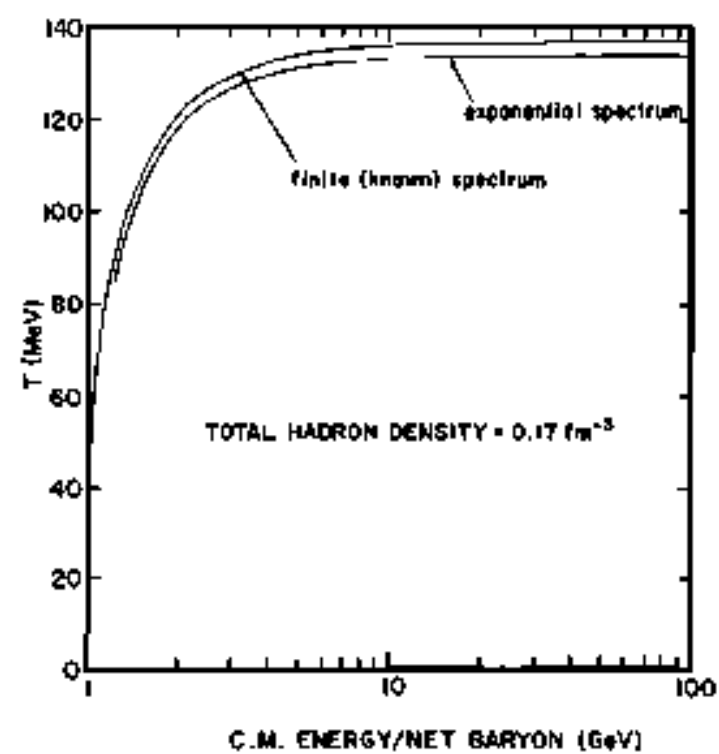

Fig. 1. Fireball terryerature at txed total hadronic density $=0.17 \mathrm{Im}^{-3}$ as a function of total c.m. energy per net baryon for two extreme forms of the hadronic mass spectrum.

(XBL 818-1147])

massive resonances to such an extent that the temperature remains saturated near this asymptote down to the freezout density. ${ }^{2}$ However, as the other curve indicates, if the spectrum of hadronic resonantes is bourded and lirrited to those already known, the opposite extreme from an exponential spectrum, the temperature rises so slowly with energy above $\sim 5 \mathrm{GeV}$ that it appears to be asyurptotic even though it is unbounded Moreover, it is indistinguishably close to the temperature curve corresponding to the exponential hadronic spectrum! Only if $\mathbf{T}_{\mathrm{o}}$ were much less than $\mathrm{m}_{\mathrm{r}}$ would the asymptotic temperature of an exponential spectrum be distinguishable from the temperature corresponding to a finite hadronic spectrum. But $T_{p}$ is not expected to differ appreciably from $\mathrm{m}_{\mathrm{r}}$

The reason that the temperature at freezout density rises so slowly with energy is that mesons are copiously produced. The flatness of $T$ at a value near $m_{\pi}$ is parely eoincidental for the finite spectnum. In fact, $T$ is unkounded. Doubling $m$ changes $T$ by only several $\mathrm{MeV}$. The erolution of temperature and particle populations for a $20 \mathrm{GtV} /$ nucleocn fireball was shown in Ref. 2, Figs. 5 and 6.

The conclusion is clear. Measurement of the final fireball temperature cannol be used to infer the form of the badronic mass spectrum nor the existence of an asymptotic 
temperature unless it is much less than $T \simeq 130 \mathrm{MeV}$. This conclusion was reached under the assumption of a freezoust density of $0.17 \mathrm{fm}^{-3}$. It is unreasonable to expect it to be larges than this, but it could be less. In this case. the curves in the figure would be even closer than they are.

\section{References}

1. R Hagadorn, in Cargese Lectwes in Physics, od. by E. Schatzman, Gordon and Breach. New York, 1973, Vol. V.

2. N. K. Glendenning and Y. J, Karant, Phys. Rev, C 21, 1501, (1980).

\section{Nuclear Physics from Subhadronic Structure-a Toy Model}

Herbert M. Ruck

In a mathematical model in $1+1$ dimensions (one time, one space dimension), we mirnic some properties related to (i) the structure of single particles (hadrons), (2) two particle interactions (nuclear potential), and (3) multiparticle states (muclei and nuclear mitter), as known from nuclear physics in 3+1 dimensions. The model consists of a set of classical field equations that describe two fermionic felds, "quarks," interacting wilh two scaler fields, "gluons." The number of felds involved and the structure of the interactions are determined by the internal cyclic symmetry group $Z(3)$. The sejf-interaclion of the scalar fields is cubic and quartic and is essential to the entire calculation.

It can be shown in $1+1$ dimensions as well as in $3+1$ dimensions, that as long as the internal aymmetry $Z(3)$ is preserved the fermions cannot be separated from each other and ase therefore cousined. Aside from this general result the calculations are done in the special case of $1+1$ dimensions, where the soliton solutions of nonlinear equations occur readily, and by negleeting the feedback of the ferIrions onto the scalar fields. Partial results about lifting this approximation are reported at the end of this note.

l. For single particles we obtain a compositeextended structure, with the ground-state of mass $\mathrm{M}=10$, for particular values of the three fres parameters in the model. Time ascillations of the fields generate a resontince state with $\mathrm{M}^{*}=15.56$. If the internal symmetry can be violated in a collision by removing one fermion, a system analog to the colored state in QCD result with the mass $\mathrm{Mc}^{\mathrm{c}}=21.68$. A system without fermions, "glueball, ${ }^{,+}$has the highest mass $\mathrm{M}^{\mathbf{B}}=23.31$.

2. The two porticle interaction potential between two ground-states has a shallow attraction of -10 percent of $M$ near the touching point of the particles and a bard core repulsion of $185 \%$ of $\mathbf{M}$ at complete owerlap. The interaction between "colored" states or "glueballs" is strongly attractive.

3. The consiruction of miltiparticle states shows the saturation phenomenon of multiparticle forces. Converting the data of the binding energy as a function of the distance into a function of the number density, we obtain the equatiom of state.

Recently, taking the feedback of the ferrrions onto the scalar fields into acoount, we solved iteratively the basic feld equations. The effect of the new solutions is a shift in the mass spectrum which, for the same parameters as above, is now $\mathrm{M}=17.03, \mathrm{M}^{*}=22.69, \mathrm{M}^{2}=22.04$, and $M^{B}=23.31$. The hard core of the isteraction potential for two ground-states is no longer present.

\section{Faonole}

"Condensed from H. Ruck, Lawrence Berkeley Laboratory Reports LBL-12316, -12479, -12872 , and -12852 . 


\title{
Percolation versus Gibbs Equilibrium - the Phase Transition from Hadron Matter to Quark Matter
}

\section{Horst Stăcker}

The phase transition from hadron matter to quark matter is investigated in two distinct models: the percolation approach and the Gibbs phase equilibrium. We find a strong dependence of the onsel of the percolation phase transition on the internal excitation energy $E^{*}$ of the matter; for $E^{*} \simeq 3 \mathrm{GeV}$ per baryon, the percolation sets in already at the ground state dessily of nuclear matter, $p_{0} \simeq 0.17 \mathrm{fm}^{-3}$. The implications of our results for relativistic nuclear collisions are discussed. In the hydrodynamical model, the onset of the hadron-quark matter phase transition is predicled al bomberding energies $\mathrm{E}_{\mathrm{Lab}} \supsetneq \mathrm{J} \mathrm{GeV/n}$ for central collisions of massive nuclei.

\section{Foornote}

"Based on LBL-12633.

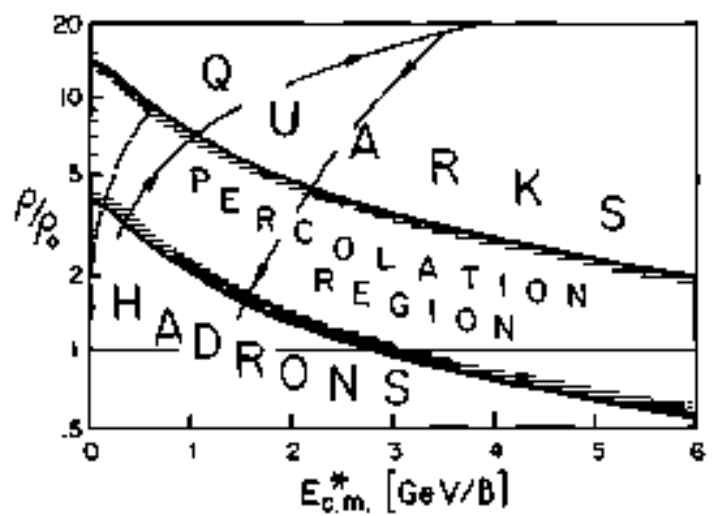

Fig. 1 .

\section{Strange Baryon Fraction from Quark- Gluon Plasma}

\author{
N. K. Glendenaing, M. Gywlassy, $R$. Anishetty, $P$. Koehler, and L. McLerrant
}

We have calculated the fraction, $f_{s}$, of baryons that have non zero strangeness emerging from very high energy nuclear collisions. If a quark-gluon plasina is formed, then that fraction can be calculated perturbatively from $Q C D$. Asymptotically, $f_{2} \rightarrow 1-(2 / 3)^{3}=0.7$ because $(2 / 3)^{3}$ is the a priori probability that all three quarks are nonstrange. As a function of energy per baryon, $E^{*} / A$, in the rest system of the plasma, we found $f_{s}=0.45,0.5,0.55$, 0.6 for $E^{*} / A=9,11,14,20 \mathrm{GeV} / A$ Below $E^{*} / A$ $\leqslant 7 \mathrm{GeV} / \mathrm{A}$, perturbation theory is unreliable.

In conurast, $f$ for a Hagadorn gas of hadrons reaches for $\mathrm{E}^{*} / \mathrm{A} \geqslant 4 \mathrm{GC}$, the asymptotic value $f_{\mathrm{g}} \neq 0.34,0.35$, $0.37,0.38$, for $\mathrm{E}^{*} / \mathrm{A}=9,11,14,20 \mathrm{GeV} / \mathrm{A}$

Therefore, if large enough energy densities $t=\mathrm{E}^{*} / \mathrm{A} \rho \geqslant 10 \mathrm{Gev}\left(0.15 \mathrm{fm}^{-3}\right) \sim 1.2 \mathrm{GrV} / \mathrm{fm}^{3} \mathrm{can}$ be equilibrated in a nuclear collision, $f_{s}$ could possibly serve as a signature of a quark-gluon plasme phase transition.

\section{Non-Linear Vacuum Polarization in Strong Fields}

\author{
M. Gyulassy
}

Based on the Wichmonn-Kroll formalism, the effect of non-linear $\alpha(Z \alpha)^{\text {ñy }}$ vacuum polatization was reviewed for very large $\mathbf{Z}$ systems, $\mathbf{Z} \rightarrow \mathbf{1 8 0}$. The question is whecher QED effects could prevent the $1 S_{1 / 2}$ electronic orbitals from reaching a binding energy $B \stackrel{1 / 2}{=} 2 \mathrm{~m}_{c^{\prime}}$ Beyond that point, the vacuum decays to a state with two fret positrons and twa bound electrons. The non-linear vacuum polariza- tion density $\rho^{9+}$ is calculated assuning that the $\mathbf{j}=1 / 2$ states dominate. The besic formula is

$$
\begin{aligned}
\rho^{3+}(r)= & \frac{1 \mathrm{e} \mid}{2 x} \int \frac{\mathrm{d} \omega}{2 \pi i} \operatorname{Tr}\left[G_{1}(r, r ; \omega)\right. \\
& \left.+G_{-1}(r, r, \omega)-2 Z \alpha G \mid(r, r ; \omega)\right]
\end{aligned}
$$


in lerms of the radial Green's functions $G_{k}$ and the first order piece $G$. The results of these calculations were published in Ref. 1.

The new observation ${ }^{2}$ is that an effective screening charge can be defined fromn the energy shifts due to $\mathrm{Eq}+$ (1) as

$$
\Delta \mathrm{E}_{3}+=\mathrm{Q}_{\mathrm{E}}^{\mathrm{H}}<\alpha / \mathrm{r}>1 \mathrm{~s}_{1 / 2}
$$

where $\langle 1 / \mathrm{t}\rangle$ are calculated from the finite radius electron wavefunctions. Similarly the screening charge due to third order $\alpha(Z \alpha)^{3}$ can be defined. These quantities are convenient because they can be readily compared to the point nucleus screening charged computed by Wichrnann and Kroll. The results are shown in Fig. J. The remarkable result is that $Q_{\text {en }}(Z)$ seems to be just a shifted version of the Wichmann-Kroll charge $Q_{w k}(Z)$ such that

$$
Q_{\text {eff }}(Z)=Q_{u k}\left(Z-Z_{\varphi}+137\right)
$$

where $Z_{c} \approx 175$ is the critical charge for a finite radius nucleus. Figure 1 shows directly that $Q_{\text {eff }}<Z$ even for $Z \rightarrow Z_{c}$ and therefore diving $\left(B=2 m_{e}\right)$ is not prevented by vacuum polarization.

\section{References}

1. M. Gyulassy, Nud. Phys, A 244, 49 ? (1975).

2.M. Gyulassy, Lawrence Berkeley Laboratory Report LBL 12972 (1981).

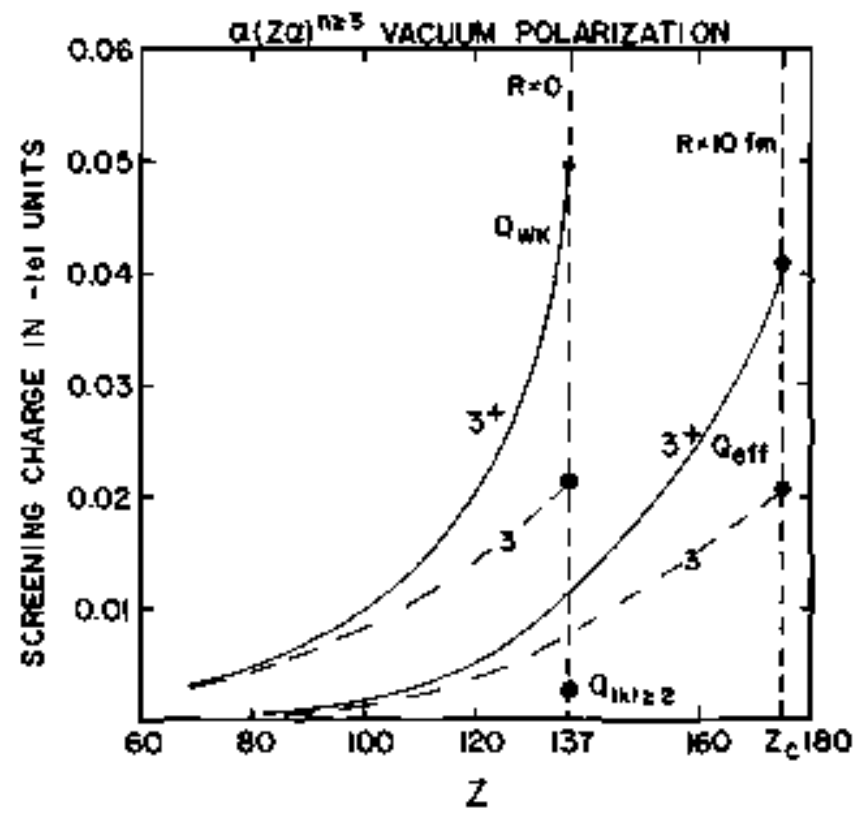

Fig. 1. Effective non-linear screeting for orders $a(Z \alpha)^{n>3}$ labeled $3^{+}$and order $\alpha(Z \alpha)^{3}$, labeled 3. (XBL 817-10647) 


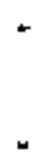

$เ$ 


\title{
INSTRUMENTATION
}

\author{
A. Spectrometers
}

HISS Detector Systems

Hank Crowford"

Experiments at the HISS facility require analysis of multiple particles from each event to investigate the energy transfer spectrum, the production of very high transverse momentum light and heavy fragments, the role of electromagnetic excitation in nuclear fragmentation, and the size of the interaction region in relativistic nuclets-nucleus reactions. In this report. I describe the detector system which, in conjunction with the HISS dipole, must provide the charge, mass, and momentum of 10 or more fragments from each interaction.

The Phase 1 detector system consists of three major subsystems. To measure rigidity, we use two large $(I \times 2$ m) drift chambers in conjunction with the HISS magnetic field ( $\int$ Bdl $=75 \mathrm{kG}-\mathrm{m}$ ). To measure charge for light fragments ( $\mathrm{Zf} \leq 6)$, we pulse-height analyze both the timo-of-fight scintillators and a subset of drift chamber wires. To measure charge for $\mathrm{Zf}>6$, we use a $1 \times 1 \times 5$ m multíple sampling xenon filled ionization chamber. To measure velocity, we use both a scintillator array (30 pieces, each $200 \mathrm{~cm} \times 10 \mathrm{~cm} \times 2 \mathrm{~cm}$ ) for time of flight and a silica aerogel Cherenkov bodoscope ( 10 pleces ach $100 \times 10 \times 2 \mathrm{~cm})$. In addition, we will employ two position sensitive detectors to define the incident perticie vector. With the system configured as shown in Fig. I, we expect to attain charge resolution of $\mathrm{dZ}<0.0 \mathrm{Z}$, mass resolution of $<0.3 \mathrm{amu}$ at $A=60$, and momentum resolution of $d p<0.001 P$ for each frag. ment.

The first generation of experiments proposed for the HISS facility required construction of drift chambers for operation $2 \mathrm{~m}$ and $3 \mathrm{~m}$ downstream of the center of the dipole as shown in Fig. ${ }^{1}{ }^{1}$ The alternation of anode and cathode wires in successive planes removes left-right ambjguity, improves particle peir resolution, and allows construction of a track vector from a single chamber.

The electronics for these chambers were designed to provide both position and pulse height information for each Iragment passing through them. This pulse height is to distinguish charges $I, 2$, and greater than 2 to augment the charge information attained in the TOF wall and MUSIC detector and to aid in unscrambling multiple fragment events. Each chamber, being $\sim 10 \mathrm{~cm}$ thick, can provide a rough track vector which will point to the second chamber location. This allows straightforward unscrambling of even high multiplicity events.

Charge for light fragments $(Z I \leq 6)$ is determined by pulse-height analysis of the TroF wall scintiltators and by pulse-beight analysis of a subset of the drift chamber
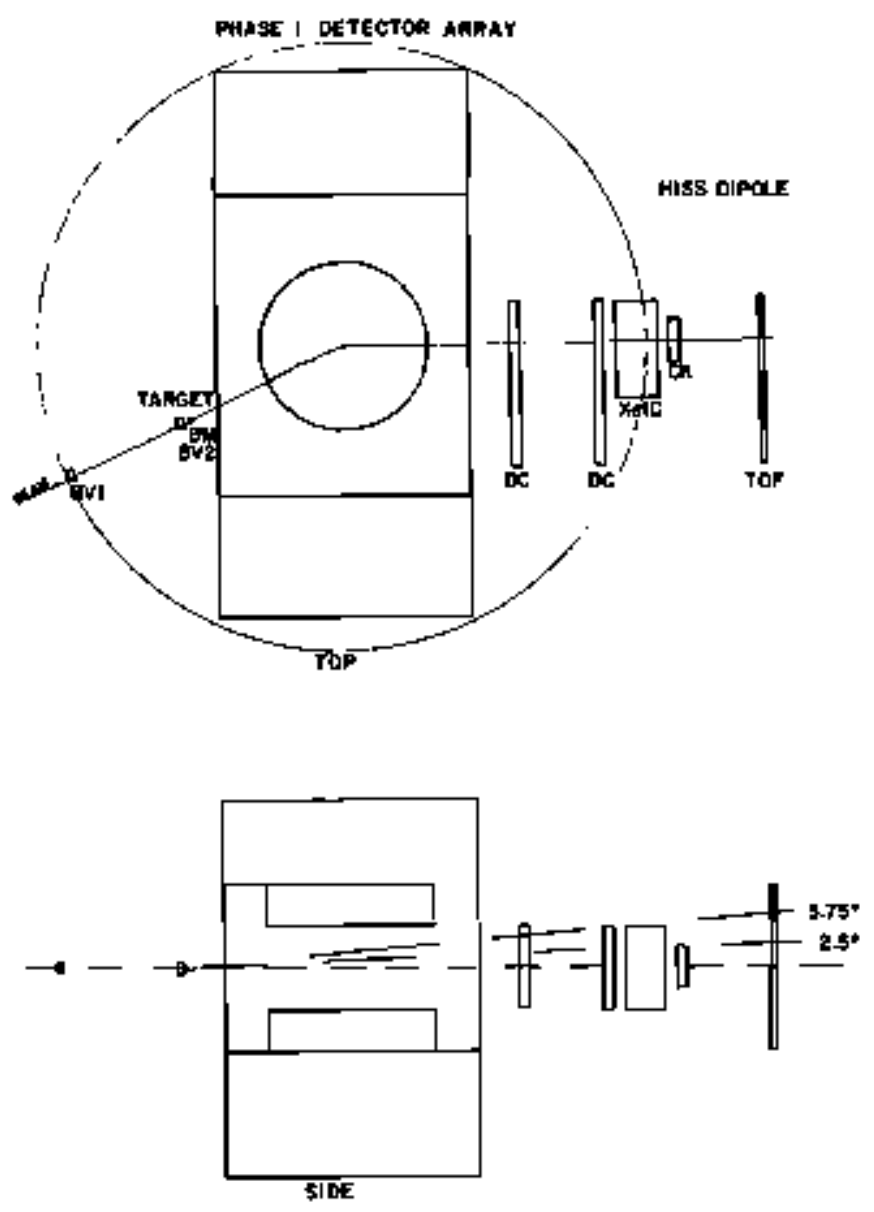

Fig. 1.

(XBL 807-10739)

wires. Fragment charge for heavy fragments is determined primarily by a new type of large area high resolution detec. tor, a Multiple Sampling Ionization Chamber (MUSIC). High charge resolution is attained in the MUSIC detector by measuring many samples of the ionization produced along the path of a particle as it traverses $50 \mathrm{~cm}$ of Xe gas. ${ }^{2}$

A measurement of fragment velocity is required to convert rigidity and charge measurements to mornenturm, charge, and mass. Velocities of light fragments will be determined by time of flight from the target to the scintil. lators of the time-of-fight wall. Velocities of heavy fragments (Af > 10) will be determíned by their signal levels in a silica aerogel Cherenkov detector array. ${ }^{3}$ 
As designed, the detector system is modular to accommodate the range of experiments envisioned. The charge, mass, and momentum resolution are sufficient to provide high quality data ower a brodd range of new physics from high energy heavy ion interactions.
Foothote and References

"UC Space Sciences Laboratory.

1. D.E Greiner th al., IEEE Trans. Geosci. Elec. 16. 163 (1978).

2. G. Igo and R.M. Eisberg, Rev. Sci. Instrum. 25, 450 (1954).

3. M. Cantin et al. Nucl. Instrum. and Methods 118, 177 (1974).

\title{
Monitoring the HISS Magnet
}

\author{
F. Bieser and C. McPorland"
}

The recently ampleted HISS superconducting dipole has been outfitted with nearly 100 sensors to prowide diagnostic and control feedback information about the electrical, mechanical, and cryogenic systems. Thirly-two strain gages are mounted around the support rings, which must withstand over one million pounds of thrust as the magnet is energized. Messuremeats are made with a resolution of 1 , 2, of 4 microstrain with full scale limits of plos or minus 2000,4000 , or 8000 ustrain. Nineteen thermistors are located on the cryostats and transfer lines to monitor the temperatures of the coil bobbins and helium gas during cool-down. Measurements cover the range of $293 \mathrm{~K}$ (room temperature) to $4 \mathrm{~K}$ with a resolution of $0.1 \mathrm{~K}$.

Solid state pressure transducers and liquid level sensors monitor the pressure and level of the liquid helium. Isolation amplifiers art used to monitor various voltages while the magaet is energized. Forty-eight channels of relay input registers monitor the power supply and quench protection chains.

All parameters are read into a PDP $11 / 34$ computer vie CAMAC once every second. A variety of pictorial displays are available on viden monilors for real-time mon. itoring. The data is compressed and archived on the VAX $11 / 780$ where it can be accessed at any future time for tJend analysis, event jeconstruction, elc.

Dipole paramelers, read once a second, are archived into five separate files. These files contain date from the moot current hour, day, week, month, and year. Sirce all five files are of identical length, each file's time resolution is different. Thus, interesting events (dipole current charges, loss at coolant, etc.) can be examined immediately with one-second resolution between data points. As time passes, this same data propagates through each file, losing some time resolution between each file. The above system, combined with the MULT analysis program, enable graphics display and analysis of all dipole engineering data.

Footmole

"UC Space Sciences Laboratory.

\section{Program Needs for a High-B Field Facility for Visual Detectors at the Bevalac}

\author{
Horry H. Heckman
}

The corrent tread in Bevalec research roquires the ust of $4 \pi$, high-spatial-resolution, visual track detectors. In addition to nuclear emulsions, such detectors include plasties and $\mathrm{AgCl}$ crystals. Future visual-detector experiments of bigh priority will be those to determine: (1) the properties of "anomaloms," i.e., projecti]e [ragments that have anomalously short meant froe paths, and (2) the mutiplicities, charge, and energy spectra of projectile and target fragments produced in relativistic uranium-nucleus collisions. We cite these two experiments, not only for their scientific interes1, but also to demonstrate how the use of nuclear enulsions (in particular), when exposed in a highintensity magnetic fejd of 0.3-0.5 megagauss (MG), wil] tnable us to obtain the following exciusive charged particle data: (1) the total multiplicity of charged partictes and theit emission angles, and (2) the rigidity $R$ (hence momenta $P=2 R$ when charge $Z$ is measured) of all charged particles that are emitted within approximately $I$ $30^{\circ}$ of the plane norme] to B (i.e., $0=2 \pi$ sterad). These measurements can be performed under the highest conceiv- 
abie multiplicities, $M$, expected from $\mathrm{U}_{92}+\mathrm{Ag}_{47}$ collisions at I $A$ GeV, e.g., $M=150-300$. Furthermore, the charge and rigidity measurements can be made to accuracies $\sigma_{z}<0.5$ unit and $\Delta R / R \leqslant \pm 5 \%$, respoctively, in track lengths of $1 \mathrm{~cm}$, or less, a feature crucial to our planned experiments on anomalons. Measurements of ionization $(\mathrm{dE} / \mathrm{dx})$ and rigidity will) lead to the mass identification of $\pi, K$, and nuclei $Z \leqslant 5$ over a significant range in phase space.

In anticipation of the applicability of a high-B field facility for visual detector to Bevalac research, Heckman and Herlach exposed a small $\left(\sim 1 \mathrm{~cm}^{3}\right)$ emulsion stack to $19 \mathrm{GeV}$ electrons at SLAC while placed in an exploding single-turn, field-producing coil at B $=0.92 \mathrm{MG}^{1}{ }^{1}$ At $\sim 1$ MG, magnetic defection dominates multiple scattering and the measurements of rigidity can be made to an accuracy of several percent. It was found that: (1) no deleterious effects occur to nuclear emulsion, physically or photographically, when subjected to pulsed magnetic fields as bigh as I MG, and (2) the < l micron spatial resclution of emulsion tnables precise curvature measurements of a particle track irrespective of accompanying particle background and/or multiplicities.

As an example of a specific experimental application of a high-B facility, we consider the case of the calastrophic destruction of a 1 A $\mathrm{GeV}^{238} \mathrm{U}$ mucleus in a headon collision with an emoulsion nucleus where all the enritted particle have $Z=I$ and include $\pi, K, P$, d, and $t, F i g-$ wre $I$ is a plot of rigidity ws ionization, expressed as grain density relative to minimum grain density, for each particle. The rigidities of the and $K$ mesons are plotted to arbitrarily high values, whereas the $p, d$, and $t$ are limited to velocities $\beta \equiv p_{\text {beam. }}$. The open rectangles indicate the Fermi widibs of projectile fragments. The small bars underneath the rectangles show the errors of rigidity measvrement ( $t=I \mathrm{~cm}, \mathrm{~B}=0.5 \mathrm{MG}$ ) at beam velocity. The vertical and borizontal error bars drawn on the d-curve of $\mathrm{R}$ vs $\mathrm{g} / \mathrm{g}_{\mathrm{maj}}$ represent a $\pm 5 \%$ error in the measurement of $\mathrm{g} / \mathrm{g}$, and a $\pm 6 \%$ error in $R$, respectively. The obvious conclusion from this figure is that the $p$, $d$, and $t$ are fully resolved over all allowed rigidjties. With the expectation that the pion spectra are predominantly $<1 \mathrm{OV}$ and the production of kaons will be small, corr-

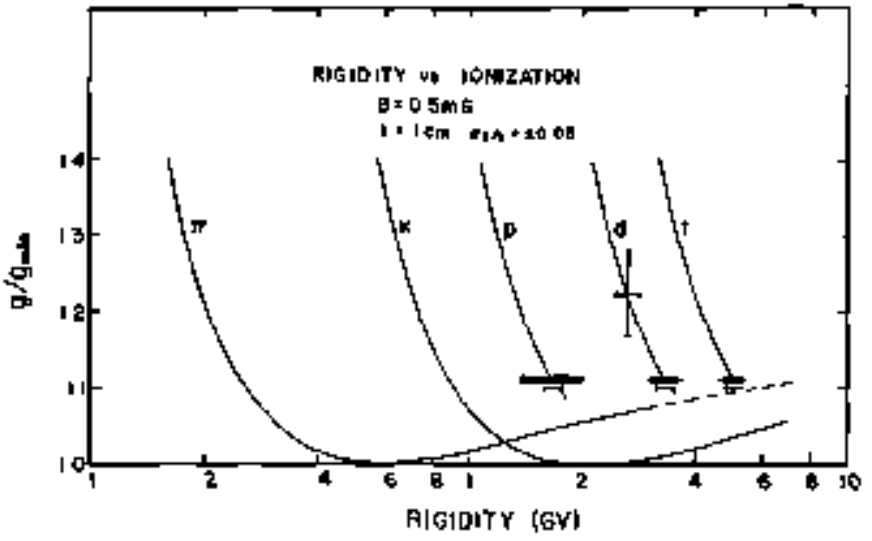

Fig. 1. Rigidity versus normalized grain density for $Z=1$ mesons and nuclè.

(XBL 8111-12618)

plete mass separation of all $\mathrm{Z}=1$ particles can be expected in actual practice.

Basic guidelines for the design of a high-B feld faclity for the Bevalac that we propose are:

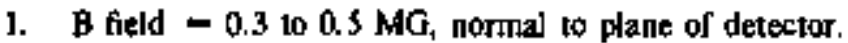
Field volume at leasi 5 con diameter $\times I \mathrm{~cm}$.

2. Pulse duration should be order of $1-10$ msec, with the requirement that the field be maintained to witbin a few percent of peak field during beam arrival. Comr paribility with beam extraction is essential.

3. Polse rates of the magnet may be as low as $1 / \mathrm{min}$; with coil lifetime $=1000$ pulses.

4. The coil design should j) acoommodate the entrance (and exit) of the beam ( $-1 \mathrm{~cm}$ diam.) without intercepting the ooil or otber structures and ii) allow for easy mounting of the detectors in the field volume.

5. The $\mathrm{B}$ field over the area of the detector should be uniform to $10^{\text {W }}$. Field structure (calibration) can be determined by curvature measurements of beam tracks of known rigidity.

\section{Reference}

1. H.H. Hockman and F. Herlach. Nocl. Instrum. Meth. 106, 269 (1973).

\title{
TASS - Two-Arm Spectrometer System
}

\author{
G.R. Roche, J.A. Engelage, J,V. Geaga, J.W. Harris, P.N. Kirkt \\ R.W. Koontz, H.G. Pugh C.L Rutz, LS. Schroeder, and R.N. Trethof
}

The construction of the Tw0*Arm Spectroneter System (referred to as TASS) was fist motivated by a backward-forward particle correlation study in p-nucleus and nucleus-nucleus reactions. ${ }^{1}$ The design for TASS was started in 1979, with installation completed by July 1980. Recently, a stcond-generation experiment to study $x^{ \pm}$pro- duction with high statistics has been started. ${ }^{1}$ and several proposals for future study are being prepared.

TASS consists of two C-magnets mounted on top of seperate air pads which allow the independent angular positoning of the arms. Figure 1 shows a diagram of the 


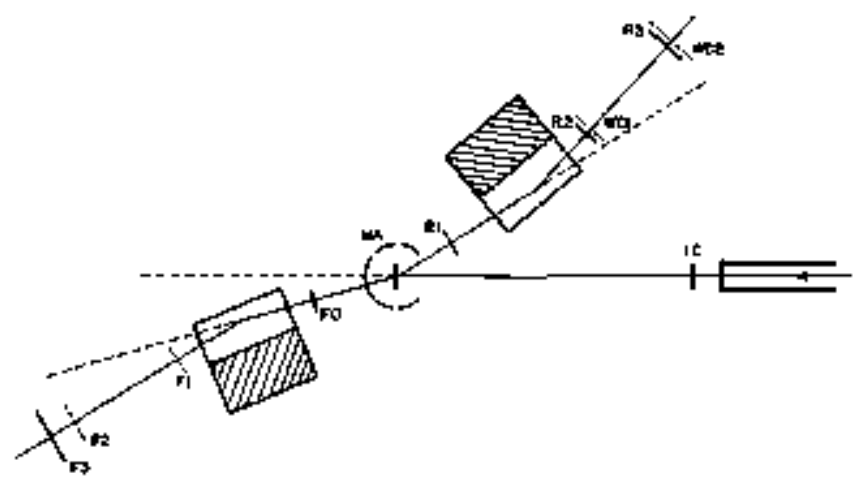

Fig. 1. Diagram of the Two-Arm Spectrometer Systert

$\begin{array}{ll}\text { RJ: } & \text { 3-element scintillator hodoscope } \\ \text { R2,R3: } & \text { scintillater gating counters } \\ \text { WC1,WC2: } & \text { multiwire proportional chembers } \\ \text { F0,F3: } & \text { scintillator gating counters } \\ \text { FI,F2: } & \text { 16-element scintillator hodoscopes } \\ \text { MA: } & \text { 16-element multiplicity array } \\ \text { IC } & \text { ion chamber }\end{array}$

(XBL 8110-1610)

apperatus. In jis present configuration, the rear arm consists of both scintillation counters and wire chambers: these are used with the known magnetic field of the spectrometer to provide the momentum, angle, and identity of a charged particle traversing the systerm. The forward arm consists primarily of two 16-element scintillation hodoscopes, which allow us to measure the forward particte's momentum, angle, and identity, using the beam center al the target location or the interaction point coordinates given by the rear artol. Extensive wire orbiting of both spectrometer magnets has been done.

Table 1 gives the train characteristics of TASS.
TASS is equipped with electronic eircuitry and a PDP-1l compuler, which at present enables the following or-line analyses to be made: time-of-flight and amplitude distributions for each scintillation counter, real to random signal for each arm and both arms in coincidence, momentum and angle distributions, and particle identification. The data-taking rate can be of the order of 200 events per spill, with a dead time of about $30 \%$.

As an example of TASS performance, Fig. 2 shows the ability of the system to identify particles. The figure is a scatter-gram of the particle masses determined when the two astrs of TASS were operated in a coincidence mode.

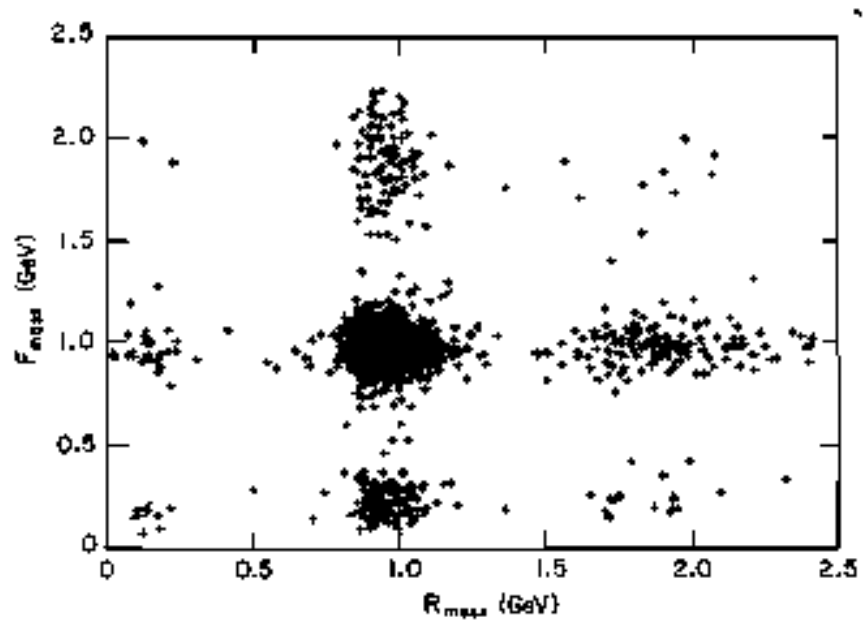

Fig. 2. Scatter-gram of the particle masses determined when the two arms of TASS were operated in a coincidence mode. FMASS and RMASS are the masses of the particles in the forward and rear arms, respectively.

(XBL 8110-1611)

Table 1. Characteristics of TASS.

\begin{tabular}{|c|c|c|}
\hline & Rear arm & Front erm \\
\hline Central mamentum range & 0 to $2150 \mathrm{MeV} / \mathrm{c}$ & $0102150 \mathrm{MeV} / \mathrm{c}$ \\
\hline $\begin{array}{l}\text { Mornentum acceptance: } \Delta \mathrm{p} / \mathrm{p}_{0} \\
\text { (tof of the central comenturn } \mathrm{p}_{0} \text { ) }\end{array}$ & -35 to $+50 \%$ & -35 to $+500 \%$ \\
\hline $\begin{array}{l}\text { Angular range } \\
\text { (minimum angle between both arms }-70^{\circ} \text { ) }\end{array}$ & 60 to $180^{\circ}$ & 1 to 60 \\
\hline $\begin{array}{l}\text { Angular acceptance: } \Delta \Omega \\
\text { (sleradian) }\end{array}$ & $3.26^{-10^{-3}}$ & $2.01 \cdot 10^{-3}$ \\
\hline Horizontal angulat acceptance & $2.77^{\circ}$ & $2.71^{\circ}$ \\
\hline Vertical angular acceptance & $3.86^{\circ}$ & $2.43^{\circ}$ \\
\hline$\frac{\Delta p}{p_{0}} \cdot \Delta Q($ steradian $)$ & $2.77 \cdot 10^{-3}$ & $1.71 \cdot 10^{-3}$ \\
\hline Momentum resolution & $2.0 \%$ & $4.4 \%$ \\
\hline Angular resolution & $0.25^{\circ}$ & $0.33^{\circ}$ \\
\hline
\end{tabular}


This data sample was taken with the rear arm at $\theta_{b}=120^{\circ} . P_{b}=400 \mathrm{MeV} / \mathrm{c}$, and the forward arm at $\theta_{\mathrm{f}}=45^{\circ}, \mathrm{Pr}=400 \mathrm{MeV} / \mathrm{c}$ for $2.1 \mathrm{GeV}$ protons on carbon. The data are seen to produce a clustering of points at the expecied masses for pions, protons, and deutercins. Thus, we observe clear coincidence signals for proton forward/proton backward, pion forward/proton backward, etc.

By early 1982. TASS wil be improved by the addition of wire chambers to the front arm, so that both arms will be identically instrumented. The orientation of the rear magnet is also being sotated by $180^{\circ}$ to entale both sms to be used in the forward direction; the minimum angle between the arms will then be reduced to $45^{\circ}$. We antici- pate a full program on TASS using the up-graded Bevalac in 1982 and beyond.

\section{Footnotes and Reference}

"On leave from University of Cermont-Ferrand II, France. supported in part by a NATO grant.

tDepartment of Physics and Astromomy, Louisiana State University.

FDepartment of Physics, University of Califomita-Los Angeles.

Gesellschaft für Schwerionenforschung, Darmstadt, West Germeny.

1. See R.N. Treuhaft et al., "Few Nucleon Interactioms and Correlation Studies," this annual teport.

\title{
Streamer Chamber Instrumentation
}

\author{
R. Brockmant," J.W. Harris," A. Sandowat, H. Strobele, $R$ Stock." M. Moler," \\ R.E. Renfordt, J. Miller ${ }_{s}^{\ddagger}$ H.G. Pugh, ${ }^{\dagger}$ M. Raff. L.S. Schroeder. ${ }^{\ddagger}$ F. Riess \\ K. Wotf', A. Dacel, and M.E. Oriz"
}

During the year, improvements were made in the trigger system, the targets, the image quality, and the data and image acquisition. In the trigger system, the beam defining scintillation counters were replaced by two $\mathbf{1 5 0}$ micrometer $300 \mathrm{~mm}^{2}$ \$i surface barrier detectors with a fast time pickoff. In addition to reducing the overall mass of the trigger system and therefore the probability of interactions in the trigger counters, the energy resolution permitted discrimination between fragmented beams and interactions in the detectors.

Metallic targets were used inside the chamber for the first time. Thin $\mathrm{Ca}$ and $\mathrm{Pb}$ targets inside $9-\mu \mathrm{rn}$ plastic bags in air or encapsulated in an atmosphere of $\mathbf{S F _ { 6 }}$ were used. For the Jow-energy runs a slack of six thin $\mathrm{BaJ}_{2}$ tergets (25 m/ $/ \mathrm{cm}^{2}$ ) was constructed and used successfully.

Several changes were made to improve the quality of the strearner chamber photographs. The Sprague soljd capacitors were replaced with oil-filled, $0.1 \mu \mathrm{F}$ Maxwell capacitors in the Marx gesserator, improwing the stability and reliability of the high-vollage system (Fig. 1). A better system for moniloring Marx generator high voltage and time jitter was installed.

The streamer chamber gas mixture was modified by the addition of isobutane in a concentration of several ppm. The result was more condensed flares and brighter, better defined tracks.
The camera system was moditied by the addition of image intensifiers, although the utility of these devices was found to be compromised by a still-unresolved problem of ghost jimages (For details see separate report in this section). A charge coupled device (CCD) TV camera was installed to monitor the events, providing directly digitized images (See separate report in this section). Downstream of the chamber, the two walls of plastic scintillator, used for pulse height and time-of-flight analysts of fragments, were ftted with LEDs for purposes of uniform calibration.

Extensive use of computers was made. An LSI-1]/23, running under RT- $I]$, controlled the $\mathrm{CDD}$ electronics. A PDP I1/34, running QDA/MULT under RSX-I]M, took data from a CAMAC crate monitoring the scintillator hodoscopes and the beam and trigger counters, providing immediate feedback on the bearn (une, the trigger electronics, and the high-voltage pulse.

\section{Footnotes}

"GSI, Dasmstadt, West Germany.

Universităt Marburg, West Germany.

${ }^{4}$ LL, Berkeley.

\$Universităt München, West Germany.

'Argonne National Laboratory.

IIFUNAM, Mexico. 


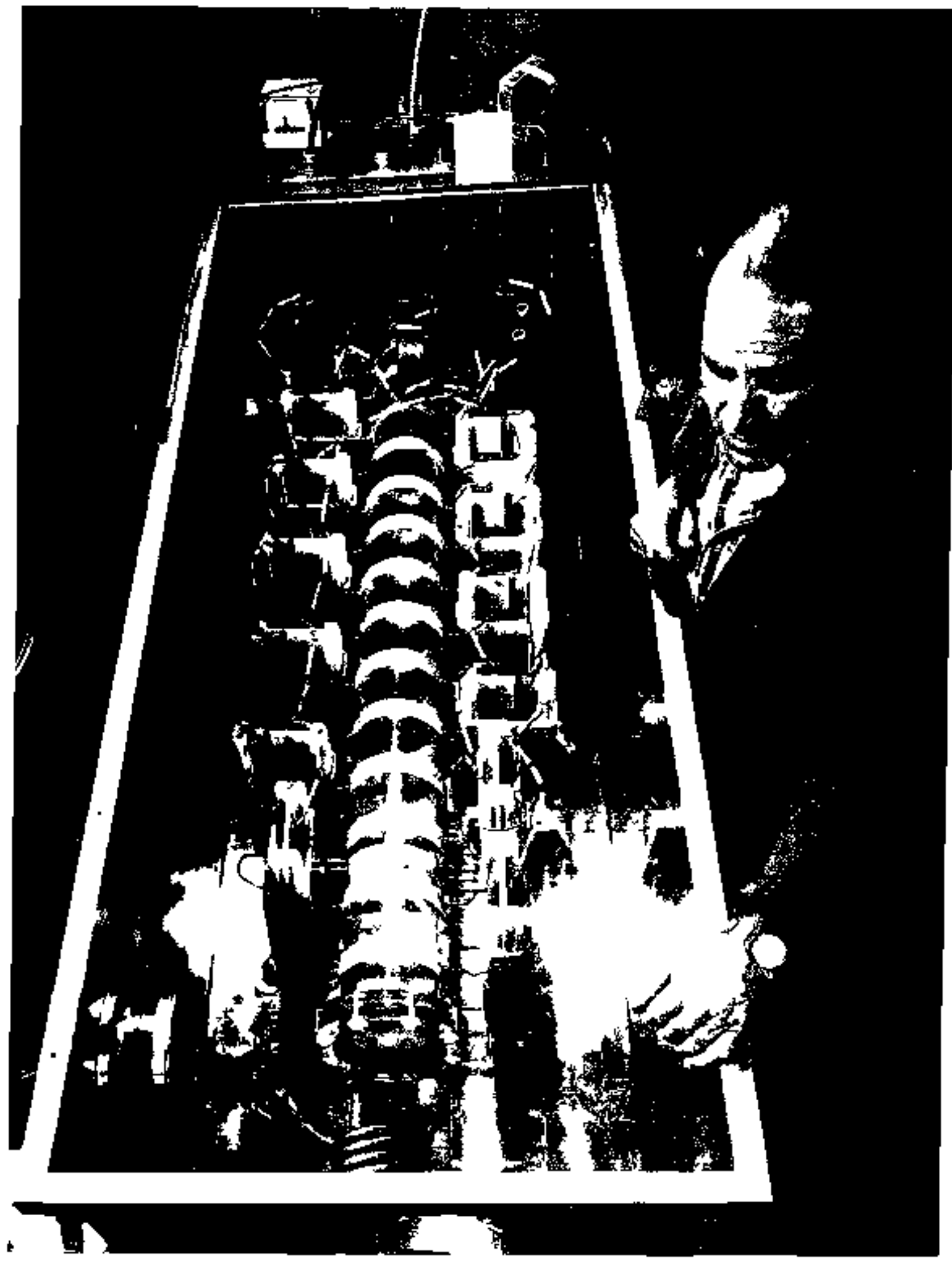

Fig ] View of the Marx generator with the new set of capacitors The capacitors wuth a voltage difference of $60 \mathrm{kV}$, are charged in serjes and discharged in parallel through the spark gaps in the columer producing a $720 \mathrm{kV}$ pulse that, after shaping is applied to the streamer chamber (CBB 8]7.6558) 


\title{
Use of a Directly Digitized CCD Camera
}

\author{
R. Brockmann," J.W. Harts," A. Sandoval," H. Sirobele, R Stock, \\ M. Mater, R.E. Renfordt, J. MHer, ${ }^{\ddagger}$ H.G. Pught ${ }^{\ddagger}$ M. Raff: \\ L.S. Schroeder, F. Riess, ${ }^{\prime}, K$. Wolf', A. Dacal,' and M.E. Ottiz'
}

Since the introduction of the streamer chamber in the early 1970s, there have beon several studies of the use of directly digitized pictures. Thest usually employ very setsitive TV tubes ${ }^{1}$ or solid state cameras. ${ }^{2}$ In the past, tecknological reasons have made it unfeasible to supplant the film with directly digitized images for data acquisition. The recent availability of large atrays of chatge-coupled devices (CCD) with high quantum efficiencies as TV camera sensors, the development of fast (5-20 MHz) ADCs, and the availability of relatively inexpensive large memory modules enable reevaluation of their use for streamer chamber event recording.

We have obtained a CCD camera, digitizer, and memory developed by Lawrence Livermore National Lab, based on an RCA-SID 5250] CCD with $\$ 25$ by 320 sensitjve elements (Fig. 1). Each sensitive element is $30 \mu \mathrm{m} \times 30 \mu \mathrm{m}$ in size, with a linear response and a quantum efficiency of $56 \%$ at $736 \mathrm{~nm}$ (color of the streamers in $\mathrm{HeNk}$ ). With the camera enabled, it can be triggered by an external pulse and the image is read out after $8 \mathrm{~ms}$ of integration and digitized pixel by pixel into 8 bit words. The data are stored in a $160 \mathrm{~K}$ of RAM memory, which can be directly accessed by an LSI 11-23 computer. The digitized dala in the mernory can also be converted to analog and stored in the CCD for viewing on a TV monitor.

Software has been developed to enable picture holdand-store replay, obtain slices of the picture or relief maps of the intensity, and do background subtraction and curve fitting. The carnera has been tested in several of the tecent experiments. In Fig. 2 the interaction of a $2.1 \mathrm{GeV} / \mathrm{A}$ Ar on $\mathrm{Pb}$ is shown as recorded on film. Fig. 3 shows the intensity ontonir of the same event recorded by the CCD eameta. Depending on the particle emission angle, one obtains between 100 and 300 samples of the light intensity per track giving enough spatial resolution for a track reconstruction equal to that of the film. Howtever, the two track resolution is worse than on film, being about $1,2 \mathrm{~cm}$ in streamer chamber space if the whole chamber is imaged onto one CCD sompared to $0.5 \mathrm{~cm}$ if film is used. This can be improved by using larger or multiple CCDs. At present the min problem is the enormous amount of information that must be processed and stored. With the LSI-I I, one frame can be written onto a I 600 bpi magnetic
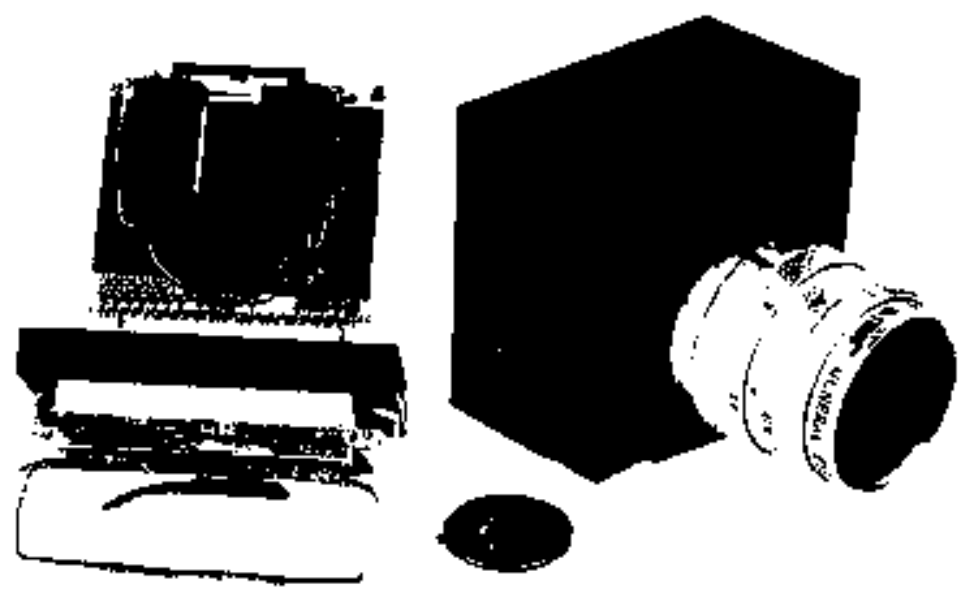

Fig. 1. CCD camera head. The $7.3 \times 9.8 \mathrm{~mm}^{2}$ image sensor, mounted on the board at the left of the picture contains an array of $320 \times 525$ light sensitive dements. A 25-cm CCTV lens was used to innage the streamer chamber on the array.

(CBB 817-6556) 


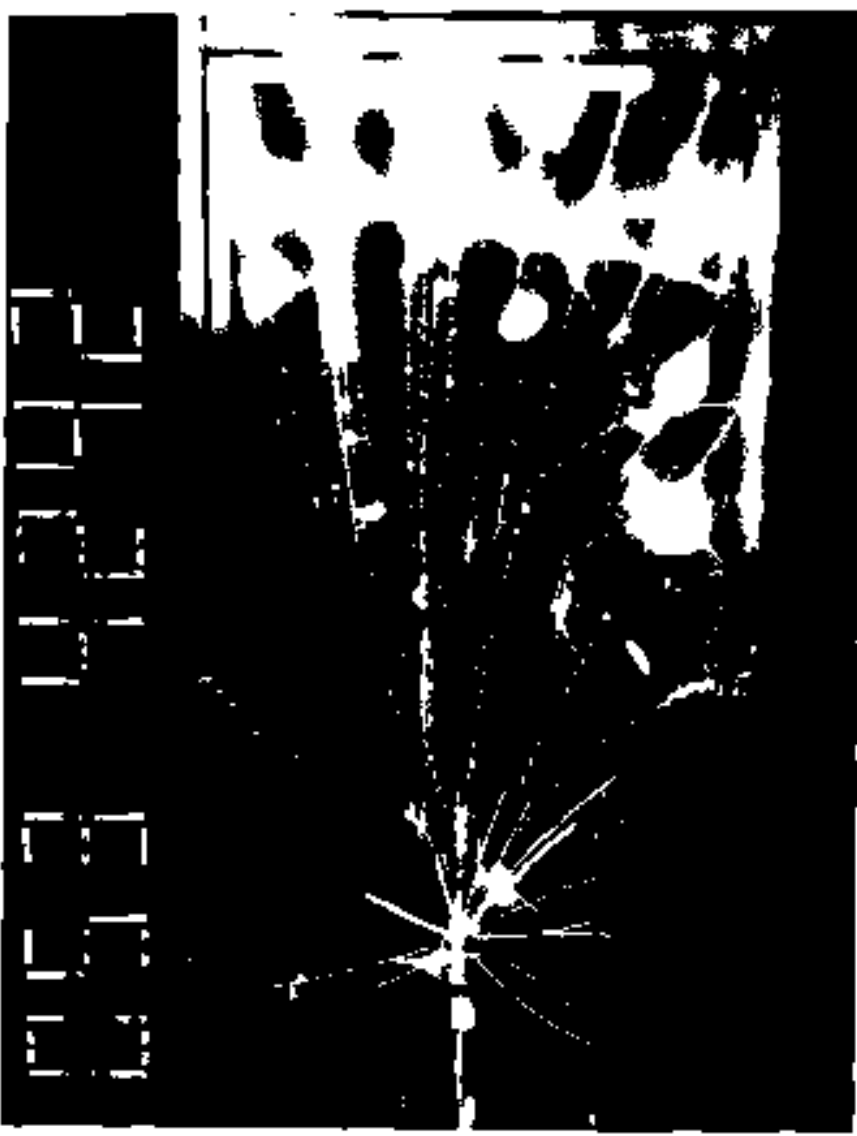

Fig. 2. Interaction of a $2,1 \mathrm{GeV} / \mathrm{A}$ - 0 Ca on $\mathrm{Pb}$ recorded on film. This picture was selected ror comparison with the directly digitized CCD picture (Fig. 3) because of its large multiplicity and large range of brighiness between the beam, flare, and sparking tracks on one side and minimum ionizing pions and electrons on the other. In this case the beam enters from below.

(XBB 818-806L)

lape in 4 seconds; a tape can hold only 100 frames. Data comparison and use of faster and larger storage devices are being investigated.

\section{Footmotes and References}

"GSi, Darmstadi, West Germany.

†'Universităt Marburg West Germany.

†BL, Berkeley.

SUniversitāt München, West Germany.

Argonne National Laboralory.

IFUNAM, Mexico.

1. M. Sevei, Proceedings of the Frascati Conference on Instrumentation 1973.

2. F. Villa and L.C. Wang. Nucl, Instrum. \& Methods 144, $533(1977)$.

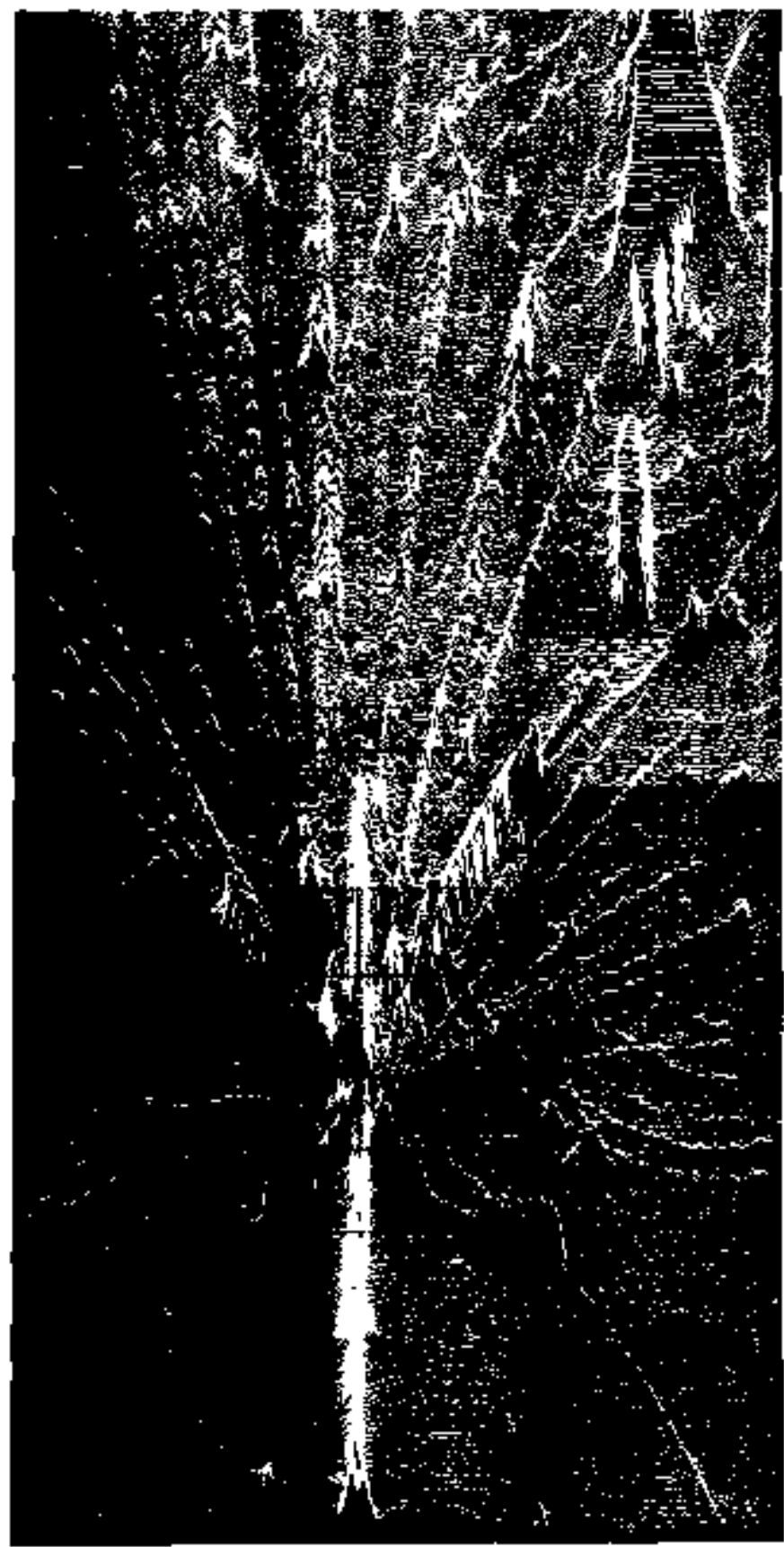

Fig. 3. Intensity contours of the directly digItIzed CCD image of the same event as Fig. 2 Each CCD poxel has been digitized into eight bits and shown here as linear spectra. The minimum ionizing Isacks ase just above background. Tracks well separated from each olher are perfectly resolved. In the high track densjly reglons, as for example, just downstream of the targel, one sees that the minimum distance for two track resolutions is a factor of 2 to 3 larger than on film.

(XBB 817-6698) 


\title{
Image Intensifier Cameras for Event Recording at the Streamer Chamber
}

\author{
R. Brockmann," J.W. Harris," A. Sandoval, H. Strobele," R. Stock, \\ M. Maier, ${ }^{\dagger}$ RE. Renfordt, J, Miller, ${ }^{\ddagger}$ H.G. Pugh, ${ }^{\ddagger}$ M. Raff, \\ LS. Schroeder, F. Riess, ${ }^{\dagger} K$. Wolf." A. Dacal. ' and M.E. Ontiz"
}

To directly photograph streamer chamber events, the primary ionization produced by charged particles in the gas has to be amplified by at least a factor of $10^{8}$. This is done by applying an electric field with a strength of $\sim 30 \mathrm{kV} / \mathrm{cm}$ for about $15 \mathrm{ng}$. In the Bevalac Streamer Chamber, a $400 \mathrm{kV}$ pulse at the central electrode is required to generate this field strength. It is desirable to run the chamber at lower field gradients, which provides betier stability and longer lifetime for the high-voltage Marx generator. This can be acoomplished by photograpting the fainter tracks with image-intensified cameras.

We have acquitred four single stage, electrostatically focused Varo image inlensifiers (Model 1248-1) with a 1/2-inch optical-fiber outpot coupling to the film. The imege intensifiers have an S-20 photocathode and a P-20 phosphor with a gain of 140 at $15 \mathrm{kV}$ and a resolution of 30 line pairs/rom. There is an inherent pincushion distortion on the intensified image that must be corrected in addition to the usual leas distortions.

The lens support on the flight research cameras were moditied to acoommodate the image intensifiers. The imag. ing on film is done by pressing the film with air against the optical fiber outpul of the proximity-focused image intensifiers. The stray magnetic field of the streamer chamber was reduced from 200 gauss to I gauss to enable the imtige intensifiers to operate properly. This was accomplished by using a Helmboltz wil to cancel the feld.

The blooming of extremely bright tracks was reduced due to proximity foessing of the image intentifiers. This will be useful in the particle identification project that is being implemented at ANL for the analysis of these data.

An unexpocted problem arose for sparking tracks which are about 1000 tirnes brighter than the streamers. The slow component of the P2O phosphor produces a ghos1 image of sparking tracks in the next frame after the film has advanced (approximately $300 \mathrm{msec}$ later). Fortunately, in this run, because such sparks were rare and also because the use of a 7-target stack greatly reduced the probability of sucesssive interactions in the same target, there was very little confosion. Nevertheless, for high energy runs with a single target, the use of these image intensifiers is severely compromised due to the ghosi

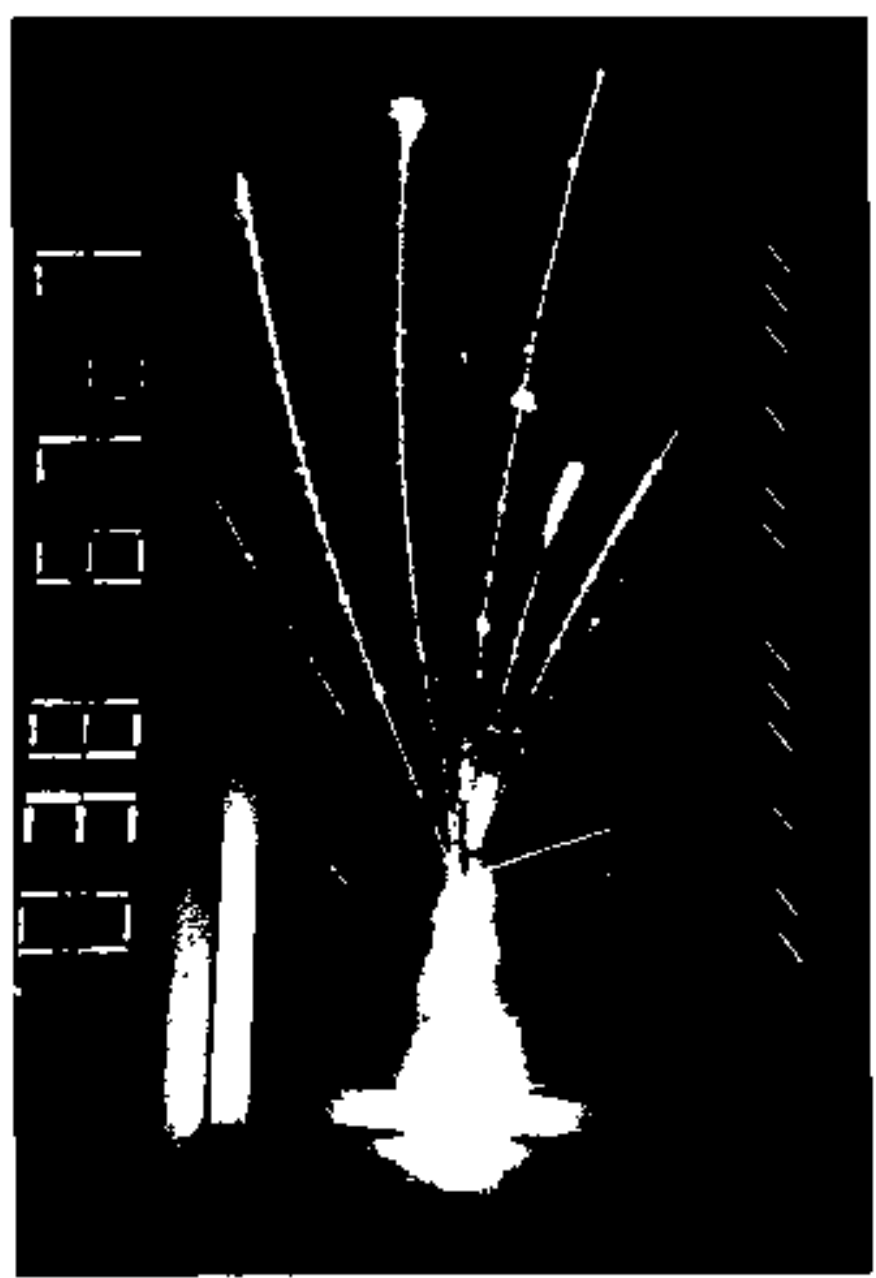

Fig. 1. Streamer chamber picture of the interaction of a $50 \mathrm{MeV} / \mathrm{a}^{{ }^{40} \mathrm{Ar}}$ on $\mathrm{BeI}{ }_{2}$ taken with the image intensifier oquipped cameras. Several of the particles stop in the gas, producing a Bragg peak. One sees differences in ionization densities for different tracks.

(XBB $814-3521$ )

images in the $\mathrm{P} 2 \mathrm{O}$ phosphor. The use of faster phosphors is being investigared. 


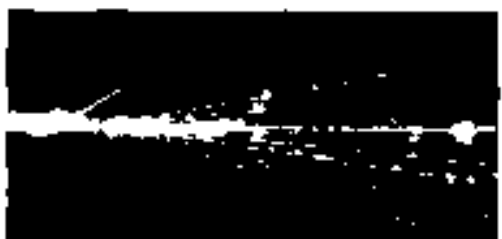

Fig. 2. Interaction of a $90 \mathrm{MeV} / \mathrm{u}{ }^{40} \mathrm{Ar}$ on $\mathrm{KCl}$ with a ghost track produced by a very bright track of the previous event.

(XA1 812-8062)
Foormores

"GSI, Darmstadi, West Germany.

Universität Marburg, Wesı Germeny.

${ }^{\ddagger}$ LBL, Berkeley.

\$ัUniversität München, West Germany.

IArgonne National Laboratory.

IIFUNAM, Mexico.

\title{
The Plastic Ball Spectrometer
}

\author{
A Baden, H.H. Gusbrod, H. Lähner, M.R Maler, A.M. Poskenzer, \\ H. Riedesel, H.G. Rirter, H. Spieler, A.I. Warwick, F. Weik, \\ $H$, Weman, and $K . L$ Wolf
}

The Plastic Ball was constructed for the sludy of relativistic nuclear reactions at the Bevalac. ${ }^{1}$ It consists of $815 \Delta \mathrm{E}-\mathrm{E}$ detectors covering a solid angle of $96 \%$ of $4 \pi$. Each module is capable of identifying the hydrogen and helium isotopes and the positive pions. The low energy pion channel (LEP) at LAMPF was used to determine the energy response and efficiency of several prototype modules for positive pions and protons at yarious energies. ${ }^{2}$ Resolution and efficiency were found to be independent of the point at which the particle entered the detector. Scatlered out particle events cosld be reconstructed by including neighboring detectors in the analysis.

Aftex assembly of the modules, each counter was tested and calibraied at the 184 [nch Cyctotron with $\alpha$ particles of 400 and $800 \mathrm{MeV}$. In this procedure, the voltage of the pbotonultiplier tube was first adjusted in order to obtain approximattly the same pulse height for all modules. This measurement yielded calibration factors to take into account difierences in the $\mathrm{E}$ and $\Delta \mathrm{E}$ response. For 36 modules ( 2 of each type), the dependence of the pulse height on the energy of the incident hydrogen and belium isotopes was obtained by looking at the fragmentation products emitted by a thick target in the $800 \mathrm{MeV} \alpha$ bearm and by measuring the time of flight of those products.

After the measurement with the beam, each module was calibrated with a staudard light source (xetion flash monitored by a pholodiode) that was couplad to the module via a light fiber cabie. During the experiment, this light source is regularly fired and serves to survey the stability of the detectors.

After assembly the Plastic Ball was installed on the Bewatron floor and operated successfully during two weekends in June. A total of two million events (minimum bias and central trigger) were taken in this first experiment for the following projectile-target combinations: 400 and $1000 \mathrm{MeV} / \mathrm{u}$ calcium on calcium, 400 and $1000 \mathrm{MeV} / \mathrm{u}$ argon on lead, and $800 \mathrm{MeV} / \mathrm{u}$ neon on lead.
Analysis of the data is in progress, and preliminary results show that, after applying known correction factors, the response of all modules is identical. The quality of the p,d,t-separation for $\mathbf{2 0}$ modules added is shown in Fig. 1 . The $x$-axis represenls the projection of a band in the $\Delta \mathrm{E}$-E plane on an axis perpendicular 10 the particle identification lines and the yaxis the number of events.

\section{References}

1. M.R. Maier, H.G. Ritter, and H.H. Gutbrod, IEEE Trajs. Nuct. Sci. NS27 No. ] (1980).

2. H.H. Gulbrod al al., IEEE Trans. Nucl. Sci. NS-28 No. I (198]).

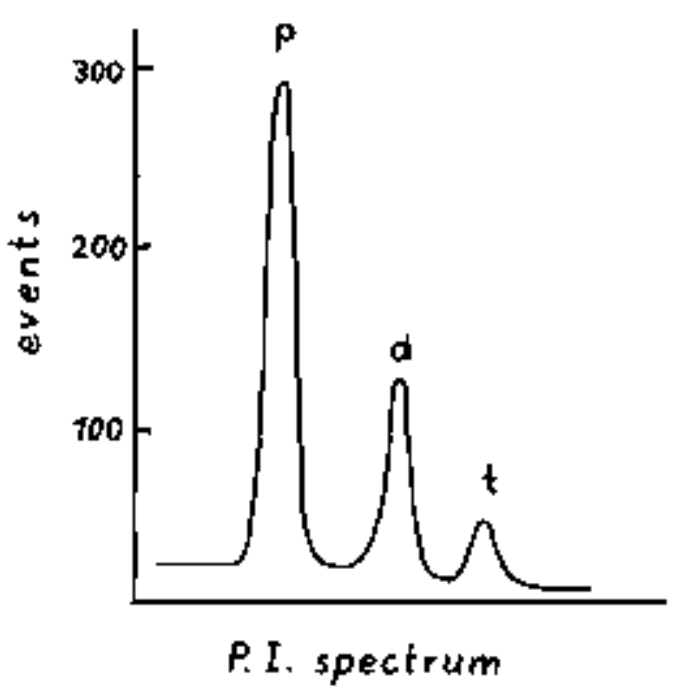

Fig. 1. The proton, deuteron, and triton separation achieved with the Plastic Ball modules. 


\title{
New Developments at OASIS
}

\author{
J.M. Nitsthke and J.D. Molitoris
}

Several new developnents and improvements of the On-line Apparalus for SuperHILAC Isotope Separation (OASIS) occurted during the last year. Major changes in the targel/ion-source region will be covered in a separate section of this annual report. The ion source is being operated in the electron bombardment/plesme and surface ionization modes. As has been reported pteviously, several raze earth elements, as well as $\mathrm{Na}, \mathrm{Fr}, \mathrm{Ra}$, and $\mathrm{Ac}$, have been separated using surface ionization. In addition, we bave now obleined In isotopes from ${ }^{107}$ [n to ${ }^{104}$ [n. Plasma ionization has been used for $\mathrm{Cd}$, Az, Se, and Br. All these nuclé were produced in compound nucleus reactions, mostly with parasitic ${ }^{20} \mathrm{Ne}$ beams), with the target located about $4 \mathrm{~cm}$ from the ion saurce.

A modification of the ion source, however, enables us to now produce neutron-rich isotopes via deep jnelastic reactions. The Ta larget in this case is loctated aboul $1 \mathrm{~mm}$ from the jonization region and an acceptance angle of nearly $2 \pi$ sr for the targel recoils is achieved. With ${ }^{40} \mathrm{Ar}$ beams on ${ }^{181} \mathrm{Ta}$, the following isolopes were identified: ${ }^{39} \mathrm{Cl}^{34}{ }^{34} \mathrm{~S},{ }^{36} \mathrm{P}$, and ${ }^{39} \mathrm{~S}$. With ${ }^{20,22} \mathrm{Ne}$ beams on ${ }^{181} \mathrm{Ta}$, we have separated $19,20,2 \mathrm{I}_{\mathrm{O}}$ and $20,21,22 \mathrm{~F}$. The oxygen isolopes were also separated as carbon monoxides to avoid comlamination from fluorine.

For the identification of neutron-rich light $\beta$-emitters, a cooled $\left(B 0^{\circ} \mathrm{K}\right) \mathrm{Si} \triangle \mathrm{E}-\mathrm{E} \beta$-telescope was built which permits on-line counting inside the cave. Because of severe background problems, highest priority at the moment is given to an isetope bean transport line which will guide isotopically pure beams to a shielded room located above the separator. Ion optical calculations (Fig. 1) show that this can be accomplished with an electrostatic mirror and two quadrupole triplets, $\mathrm{Q}_{2}$ and $\mathrm{Q}_{3}$. The transport line terminates in a tape system which will permut conduct of low backgzound studies of isolopes far from the line of $\beta$ stability. The tape system, which is presently under development, is based on an IBM 729 1ape drive with a tape speed of $3 \mathrm{~m} / \mathrm{s}$. Assurging a distance of $10 \mathrm{~cm}$ between collection and counting position, half-lives below $100 \mathrm{~ms}$ can be

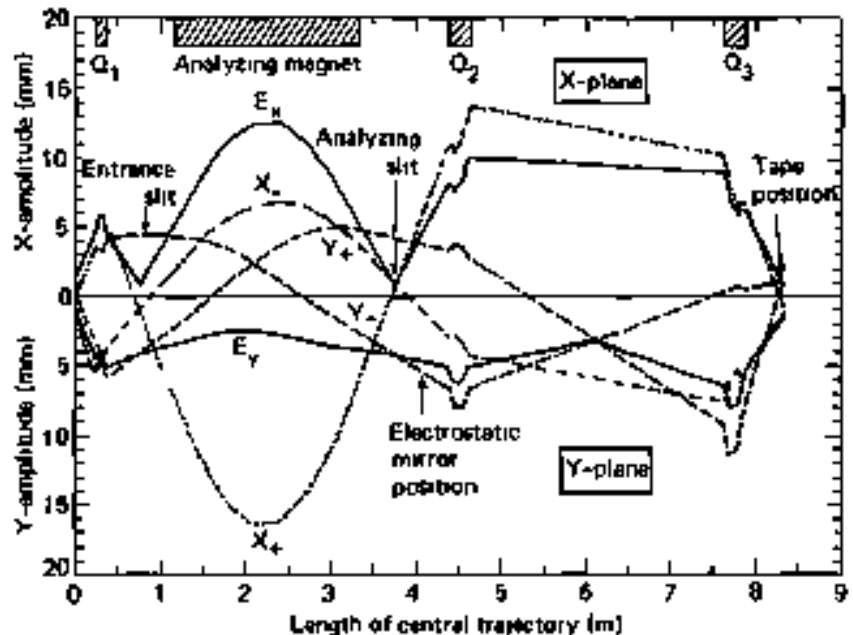

Fig. 1. First order ion optics for the on-line isotope separator OASIS including the jsotope transport line. The analyzing magnet is an $180^{\circ}, n=1 / 2$ dipole, $Q_{1}, Q_{2}$, and $Q_{3}$ are electrastatic quadrupole triplets, and $x_{ \pm}, y_{ \pm}$are positive and negalive $x$ and $y$ particle vectors, respectively. $E_{x}$ and $E_{y}$ represent the beam envelope along the central trajectory.

(XBL B18-1 192)

studied if the release time of the ion source is of this order of magnitude. The detection equiprnent initially associated with the lape \$ystem will consist of a large volume, bigh efficiency Ge $\gamma$-delectot, a $S i$ delector for $\beta$ particles.

The entire separator is monitored by a PDP- $11 / 10$ process control computer which displays and prints ont all major parameters, calculates masses from NMR measurements, and stabilizes the main high-voltage power supply. In the future, it will also be used to stabilize critical ion source parameters which are important in maintaining bigh ionization efficiencies during prolonged on-line runs.

\section{A Versatile, High Temperature, High Efficiency Ion Source for the On-Line Isotope Separator OASIS}

\author{
J.M. Nitschke
}

The theart of any online isolope separator is the ion source. Considerable effort was therefore put into the development of a source for the On-line Apparatus for SuperHILAC lsotope Separation facility (OASIS) which would meet the following requirements:
a. high efficiency,
b. short delay time,
c. good beim quality,
d. versatility, and
e. long life tume. 
Most of these goals have been achieved with the design shown in Fig. 1.

The operating principle of the source can be described as follows: target recoils are embedded in the anode end plate (catcher) and, because of its high temperature, diffuse rapidly into the ionization plasma region which is surrounded by the anode grid. This grid extracts electrons from a concentric cathode which, in turn, is heated by electron boubardment. The electrons oscillate on radial paths through the ionization volume, creating positive ions with the bighest density on the axis of the ion source where the ions are extracted and accelerated towards the isotope separator.

The above-mentioned design goals are accomplished in the following ways.

a High teficiency (n) is achieved dut to the ascillation of the electrons and a high electron temperature and density $\left(\eta_{\mathrm{KG}} \simeq 34 \%\right.$ ).

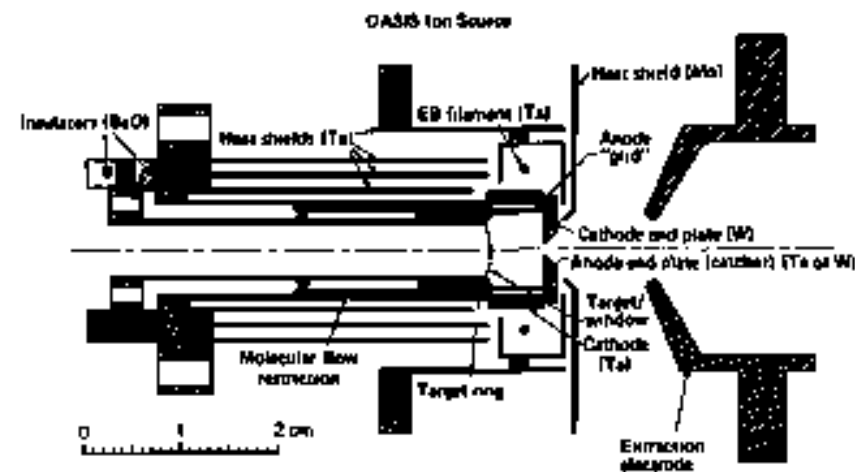

Fig. I. Schematic representation of the OASIS ion source in its DIC version for the study of nuclei produced in deep inelastic reactions. b. Short delay times are obtained because of the high calcher temperature $\left(2800^{\circ} \mathrm{C}\right.$ for Ta), the small volume of the souree (typically $0.26 \mathrm{~cm}^{3}$ ), and the large exit opening (typically $1.5 \mathrm{~mm}$ diameter).

c. The resolution of the separator is betier than $1000(\mathrm{~m} / \Delta \mathrm{m})$ for $390 \%$ transmission, which indicales a good beam qualíty. This can be attributed to the fact that there is no magnetic field present in the ion source (the field of the filament cancels on the axis), and that the tota] extracted ion current is low (typically $\leq 20 \mu \mathrm{A}$ ).

d. The source is quite versatile and, apart from the DIC version shown in Fig. I, can be converted for the study of CN reactions and operated in the surface ionization mode.

e. The lifetime of the source (typically 10 to $>100$ hours) is limited by the evaporation of the tantalum or tungsten anode end plate. The usual insulation problems of high temperature ion sources are absent because all insulators are at low temperature $\left(\leq 1500^{\circ} \mathrm{C}\right)$.

The source is sealed by small baps (typically $0.25 \mathrm{~mm}$ ) which act as moleculat flow restrictions, and the entire ionization region is kept al a temperature of $\sim 2500^{\circ} \mathrm{C}$ which avoids condensation of most tements. The operating parameters are usually kept within the following ranges:

anode potential:

120 to $160 \mathrm{~V}$

anode current:

0.6 to $1.5 \mathrm{~A}$

electron bombardment power: $\quad 400$ to $600 \mathrm{~W}$

extraction and acceleration potential: $\quad 50 \mathrm{kV}$ 


\title{
B. Detectors
}

\section{A Glow Memory Chamber for Use in the Measurement of High Multiplicity Events*}

\author{
M.A Elola, T.A. Mulera, V. Perez-Mendez, and P.E. Wiedenteck
}

One characteristic of relativistic heavy ion collisions is the bigh multiplicity (50-100 secondary particies) of a "typical" event. Information regarding the multiplicity, both its magnitude and any directional information, is desirable as an indicator of the violence of the collision and in the study of such phenomena as jet formation, hydrodynanic splash or other forms of collective behavior. We have continued our development of a detector capable of providing such information for use as a target area detector in the Heavy Ion Spectromete System (HISS) at the Bevalac.

Conventional wire chamber readout tochniques are plagued by $x$-y cortelation ambiguities when mote than one particle is registered in the chamber. In fact, to completely resolve all such ambiguities for $n$ particles requires the reading out of $n+1$ separale coordinates. Clearly, resolution of the ambiguities invalved in an event of multiplicity 50, say, would be prohibitively expensive by this method. The reading out of each separate $x-y$ coordinate in the detector via charge coupled devices, for example, is likewise an expensive solution to the problem. In our scherre, the $x-y$ ambiguities are resolved in an inexpensive fashion by employing the detector itself as a correlated event memiory, a method firsl suggested by Neurnann and Sherrard. 1

The passage of a charged particle through our apparatus is used to ignite a glow discharge at the point of passage. This ignition may be achieved through the use of pulsed spark chamber lechniques or by some less eata. clysmic avalanche method. Once ignited, the glow discharge may be maintained by a DC potential of several hundred volts for an indefinite period of time. This "membrization" of the position of the event allows the $x-y$ correlation ambiguity to be resolved as follows. Each wire of one coordinate, say $x$, which registers a hit, is successively interrogated by the superposition of a voltage pulse over the DC glow maintenance voltage. The wires of the $y$-coordinate are then examined for the transmission of this pulse through the conductive glow. In this way, the $x-y$ pasitions of the glows, and thus of the bits, are detemined in an unambiguous way.

A large effort has been expended in studying the glow discharge mechanism and in prototyping glow discharge memory gaps. A "honeycomb" cell structure has been developed which gives good glow stability and multiple hit efficiency. and which also prevents the ignition of spurious glows in cells neighboring the location of the desired glow. Development of a readout system to locate these glows is being carriad out with the help of the Physics Instrument Sysiems Group.

Ignition of glow dischanges may be achieved by placing a conventional spark chamber gap atop the glow memory gap. Residual ions and ultravioiel light from the spark serye to ignite a glow discharge in the cell nearest the sperk. Because of the traditional problems of long dead times and high radiofrequency noise levels associated with the use of spark chambers, alternative ignition methods are being investigated. Two such melhods are ignitiont by utraviolet light produced in gas scintillations, and ignition by the production and multiplication of electront-ion pairs in an avalanche less cataclysmic than a spark discharge, i.e., an avalanche taking place in the proportionsl or Geiger-Muller regime.

The final version of the multiplicity detector will surround the HISS rarget and will bave an active area subtending as much of $4 \pi$ steradians as is compatible with consiruction requirements.

A prototype device including a readout system (developed with the belp of the Physics Insirument Systems Group) is being assembled. A novel feature is the ignition scherne, which consists of a multi-step aralanche chamber operating with pulsed high voltage 2 Tests performed with this device will determine its single and multiple track efficiency, spatial and temporal resolution, and count rate capability. If the performance of this chamber proves satisfactory, i1 may be employed as an end cap in the HISS target area detector.

\section{Footnote and References}

"Condensed from a paper in preparation for the IEEE 1981 Nuclear Scitentce \$ymposium

1. M.J. Neumans and T.H. Sherrard, IEEE N-12, 379 (I\$65). An elaboration of this idea may be found in H. Kobayakawa and T. Yamaki, International Conference on Instrumentation for High Energy Physios, Frascati (1973).

2. G. Roux et al., IEEE NS-15, 67 (1968). 


\title{
Development of a Calorimeter for Relativistic Heavy Ions
}

\author{
J.D. Stevenson," J. Martinis" and P.B. Price
}

Calorimeters have been used extensively in highenergy physics. They provide a relatively compact, lowcost detector with a large dynamic range.

We have boilt a small calorimeter and tested its responsel to heavy ions at the Bevalac. Figure l shows the average pulse height in the calorimeter for various 2.1 GeV/N ions with charge-to-mass ratio of $1 / 2$. The response of the calorimeter is quite linear.

Our primary interest in using the calorimeter was originally to starch for exotic, high-mass, neutral particles such as polyntutrom nuclei or hyperstrange nuclei among projectile fragments. We have recently found another interesting application ${ }^{2}$ of the calorimeter. We have measured the total mass of the projectile residue on an event-

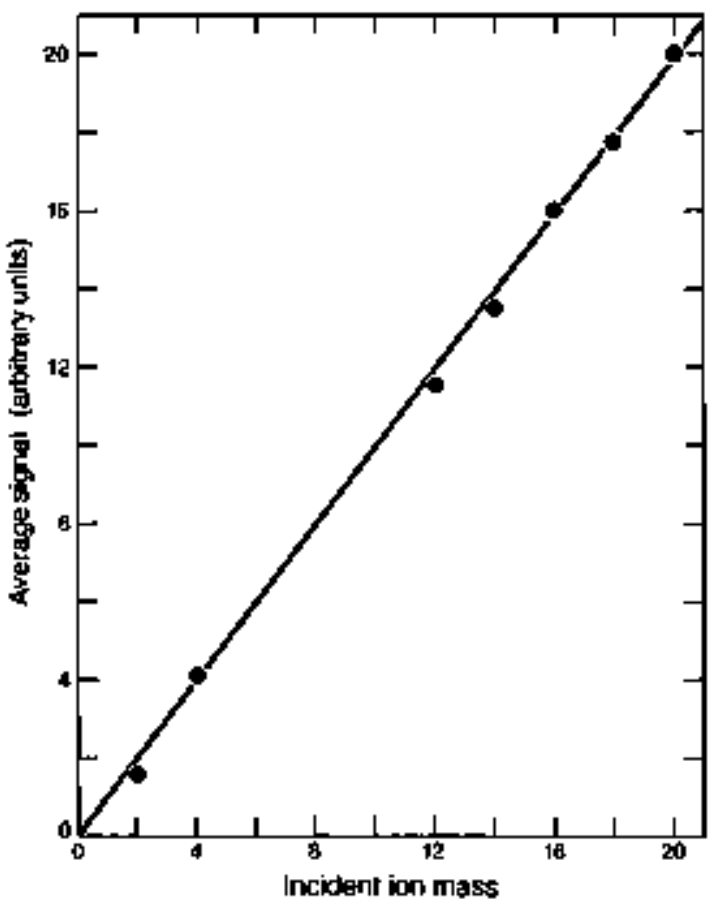

Fig. 1. Plot of the average signal in the calorimeter for various 2.1 GeV/nucleon ions as a function of ion mass.

(XBL 81]-7694) by-event basis for $2.1 \mathrm{GeV} / \mathrm{N} 2 \mathrm{Ne}_{\mathrm{Ne}}+\mathrm{C}$ and ${ }^{20}$ Ne + Mo. Unlike previous measurements of cross sections for individual projectile fragments, the measurements are quite sensitive to the size of the target nucleus. The carbon target data (Fig. 2) are depleted of low-mass projectile residues by about a factor of 20 as predicted from a simple geometrical abrasion model (solid curvi). The results provide the first conclusive evidence for the validity of the abrasion model. The portion of the curve at masses higher than that of the beam is due to the finite instrument tal resolution of the calorimeter.

\section{Footmote and References}

"Also at Space Sciences Laboratory.

I. J. Stevenson, J. Martinis and P.B. Price, Nucl. Instrum. and Meth. (1981) in press; also LBL-12264.

2. J. Stevenson, J. Martinis and P.B. Price, submitted to Phys. Rev. Lett. (1981).

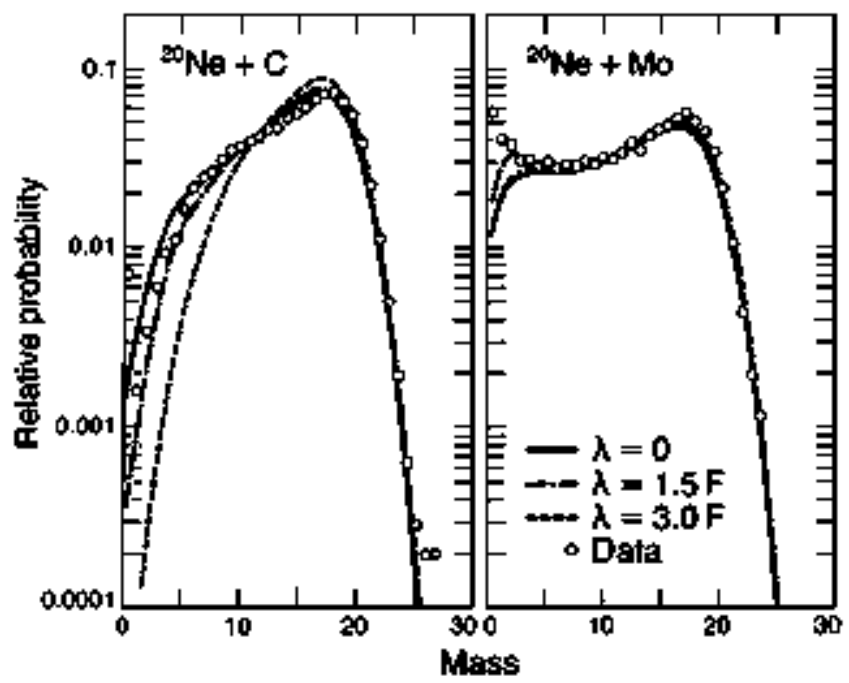

Fig. 2. Plots of the distribution of sumumed projectile masses for $2.1 \mathrm{GeV} / \mathrm{nucl}$ eor $20 \mathrm{Ne}+\mathrm{C}$ and $20 \mathrm{Ne}+\mathrm{Mo}$. Masses beyond 20 are due to spreading of instrumental resolution. The curves are based on abrasion calculations.

(XBL $8110 \cdot 12205$ ) 


\title{
A Lead-Glass Gamma-Ray Detector for Relativistic Heavy Ion Collisions
}

\author{
M.P. Budiansky, S.P. Ahlen, tand G. Tarlét
}

We have developed a lead-glass detector for highenergy ( $\sim 20-400 \mathrm{MeV}$ ) gamula rays resulting from relativistic heavy ion collisions. This detector has achieved an energy resolution signiticantly superior to any previously reported for lead glass.

Our detector has been used in an experiment at the LBL Bevalac. Our interest has been in measuring garmma rays from such sources as nucleus-nucleus brentsstrablung, $\pi^{\circ}$ decay, and decay of the excited nucleon state $\Delta(1236)$. For this purpose we required a detector sensitive to gamma rays of energy $\approx 20 \mathrm{MeV}$, and preferably insensitive to lower-energy gamma rays from deexcitation of nuclear lev. els. In addition, the detector bad to be able to reject the large number of neutrons produced by high-energy ( $\sim 1.8 \mathrm{GeV} /$ nucleon) collisions of ions as heavy as argon.

The basic detector scheme has been used by others ${ }^{1,2}$ and is shown in the right half of Fig. I. The photon to be detected interacts in lead sheet $\mathrm{C}$. where it converts to an electron-positron pair. These two particles produce signals in scintillator $S$ and plastic Cerenkov detector $C K$ and then depasit the rest of their energy in the array of lead-glass blocks ( $30 \times 30 \times 30 \mathrm{~cm}$ of SFs lead glass). A valid photon event is determined by the presence in $S$ and $C K$ of signals corresponding to two relativistic singly-charged particles. We have calibrated our detector in a series of experiments at the electron linac at the Lawrence Livermore National Laboratory. We sbtained electrons of energy $30,50,70$, and $94 \mathrm{MeV}$, and we obtained calibration photoms from the electron bean by using the method ${ }^{3}$ shown in Fig. $I^{3}{ }^{3} S 2, S 1$, and A are scintillator paddles, and $T$ is a lead target: the delectors to the right of the dashed line comprise the gamma-ray detector to be lested. An electron passing through the target hes a certain proba. bility of stopping in the terget and transferring almost all

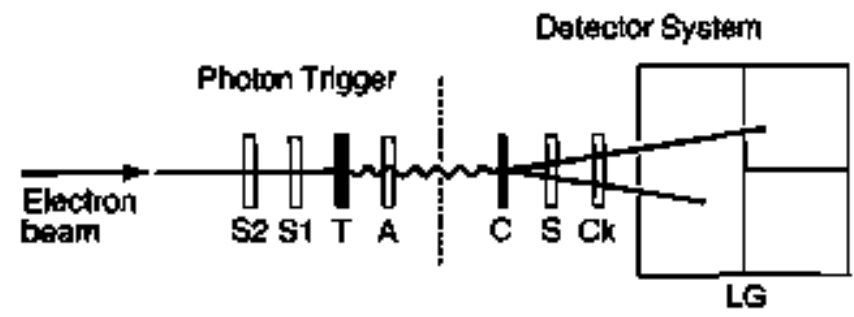

Fig. 1. Left balf of figure shows scherne used at LNL to obtain calibration photons. Right half shows main components of photon detector.

(XBL 801]-12206) of its kinetic energy to à single bremsstrahlung photoo The production of such a bremsstrahlung photon is identified by having signals in $\$ 1$ and $\$ 2$, and no signa] in $\mathbf{A}$

By using RCA's "teacup dynode" phototubes and good light collection, we have achieved excellent energy resolution. Figure 2 shows the full width at half-manimum for Gaussian curves fitled to the lead-glass signtal distriburtions for electrons of various energies. The FWHM is fit approximately by the relationship:

$$
\mathrm{FWHM}=5.7 \% / \sqrt{\mathrm{EGeV})} .
$$

This is a factor of 2 better than the typical values given in the Particle Properties Data Bookles.

\section{Foonotes and References}

"Condensed from paper subrritted to Nucl. Instrum. and Merbods.

†also at Space Sciences Laboratory.

1. A.F. Dunaitsev and Yu.D. Prokoshkin, Nucl. Phys. 56, 300 (1964).

2. G. Neuhofer et al., Phys. Letis. B 37, 438 (1971),

3. D.J. Thompson, J. Gecphys. Res. 79, 1309 (1974).

4. Particle Data Group, Kelly et al., Rev. Mod, Phys. 52, SI (1980).

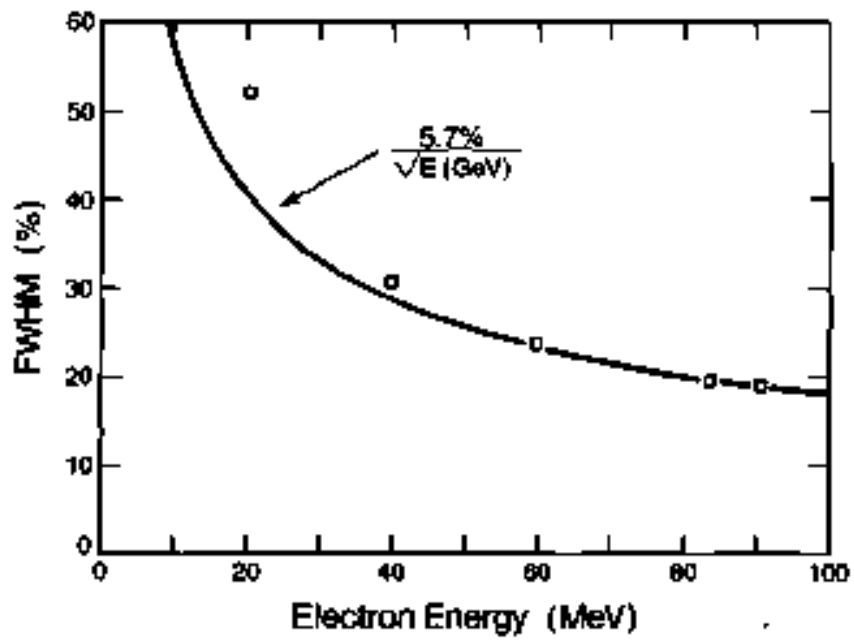

Fig. 2. Lead-glass energy resolution for electrons of various energies.

(XBL 8010-12207) 


\title{
Response of Organic and Inorganic Scintillators to Relativistic Heavy Ions"
}

\author{
M.H. Salamon and S.P. Ahten
}

We have studied the response of inorganic [Nal(Tl) $]^{1}$ and organic (Pjlot $Y$, Pílot B, Pilot $F$ and NEJ 10) scintillators ${ }^{2}$ to the ions ${ }^{20} \mathrm{Ne}^{4},{ }^{40} \mathrm{Ar}$ and ${ }^{56} \mathrm{Fe}$ from 50 to $500 \mathrm{MeV} / \mathrm{amu}$. Scintillator response is most conveniently characterized as the scintillation efficiency, dL/dE (light output per unit energy deposited) as a function of stopping power, $d E / d x$. Data for $\mathrm{NaI}(\mathrm{Tl})$ are shown in Fig. 1, while those for Pilot $\mathrm{Y}$ are in Fig. 2 (similar results were found for the other plastic scintillators).

Aiso shown in the figures are the results of calculations of response based on different scintillation mechasisms. For $\mathrm{NaI}(\mathrm{Tl})$ we adopted a diffusion model whereby deposited energy is free to migrate in the form of excitons. The saturation of scintillation light (observed as a reduc tion in $\mathrm{dL} / \mathrm{dE}$ with increased $\mathrm{dE} / \mathrm{dx}$ ) is accounted for in the model by allowing for a bimolecular exciton-exciton quenching mechanism. The separation of efficiency curves of difterent ions for a given $\mathrm{dE} / \mathrm{dx}$ reflects the dependence of dose profile on projectile velocily. Our model for NaJ explains a-posteriori, for the first time, why there is no observed dependence of relative saturation behavior as a function of $\mathrm{n}$ concentration. Rather than depleting the fluorescent $\mathrm{T}$ sites, high energy densities are effectively reduced due to intense non-radiative exciton-exciton annihilations. Thus, the quenching process is a property of the Nal host itself.

A very simple model has been used to acconst for the Pilot $Y$ data. Because of the inefficient molecular energy

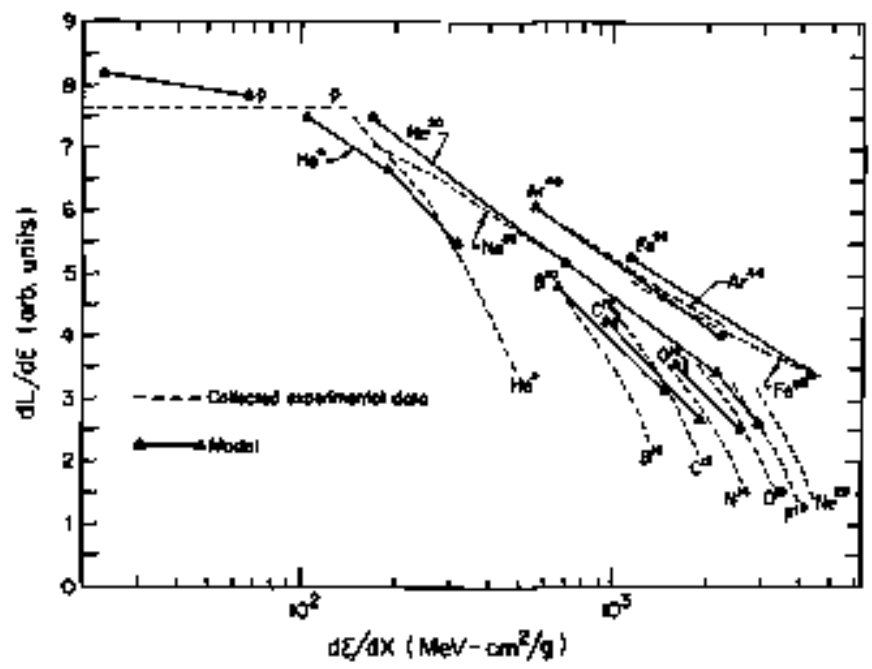

Fig. 1. Nal data and results of calculations from model.

(XBL 801 I-12802)

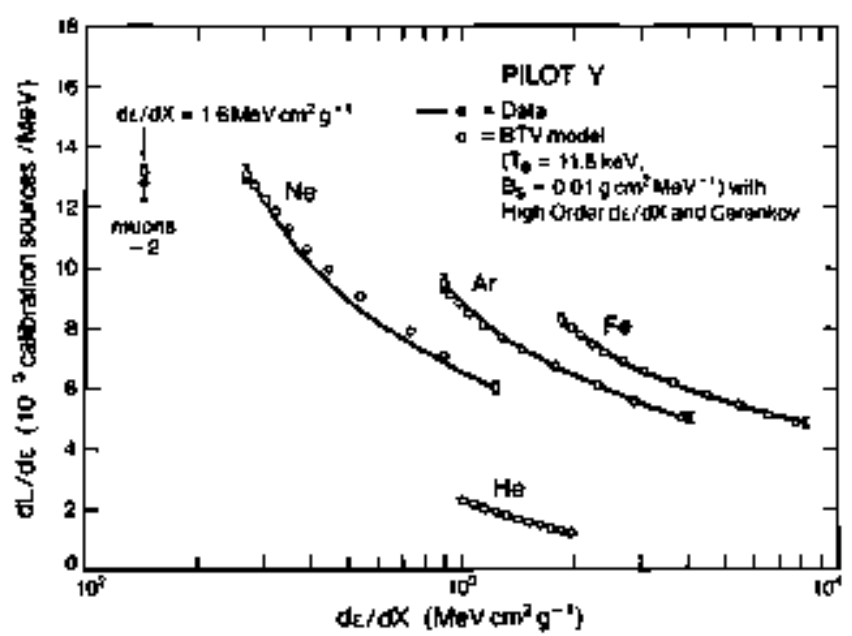

Fig. 2 Pilot $Y$ data and results of calculation from model. Contributions due to Cerenkow radiation have been included.

(XBL $8010-12208)$

migration processes in plastic, as compared with NaI scintillators, there is a much stronger delia-ray tffect, which entances the separation of efficiency curves for Pilot $Y$ compared to $\mathrm{NaI}$. It is adequate to consider two distinct physical regions: the core, which is the region quite close to the trajectory, and the halo, which is outside the core. Quenching of scintillation light accurs within the core sccording to the Birks model: ${ }^{2} \mathrm{dL} / \mathrm{dE} \propto \mathrm{l} /(1+$ $B_{s}(d E / d x) d$, where $(d E / d x)_{s}$ is the energy deposited per unit length in the core and $\mathrm{B}_{\mathrm{s}}$ is the quenching paraneter. Energy deposited ontsjde the core by delta rays is not subject to saturation. By incorporating higher-order ontrections, the core and balo stopping powers can be calculated. The distinction is provided by the parameter $\mathrm{T}_{\sigma}$, which is the minimum delta-ray energy corresponding to the halo. The calculated points in Fig. 2 were obtained by determining the overall normalization from the muon point and the B parameter from low-energy alpha data. The best-fit value of $T_{0}$ was $11.5 \mathrm{keV}$. The strong dependence of plasij scintillators on delta-ray energies makes these detectors ideal for the task of searching for antimatter in cosmjc rays by taking advantige of the charge sign asymumetry of the Mott cross section.

\section{Footnotes and References}

"Condensed from Refs. 1 and 2.

Also at Spate Stíences Laboratory.

I. M.H. Salamon and S.P. Ahlen, Phys. Rev. B (1981), to be publisted.

2. MH Salamon and S.P. Ahlen, submitted to Nud. Instrum. and Methods (1981). 


\title{
Calculation of the Relativistic Bloch Correlation to Stopping Power
}

\author{
S.P. Ahient
}

Previous workl done at the Bevalac with relativistic ${ }^{20} \mathrm{Ne},{ }^{40} \mathrm{Ar}$ and ${ }^{56} \mathrm{Fe}$ ions in $\mathrm{Al}, \mathrm{Ca}$ and $\mathrm{Pb}$ absorbers has clearly demonstrated the existence of a substantial deviation of particle stopping power from that predicted by the standard Bethe theory. Although much of the diserepancy could be acoounted for by inclusion of Mott cross section corrections and Bloch's nonrelativistic wave packet coirtection, there was a slight residual discrepancy. It was suspected that the discrepancy was due to the inadequacy of Bloch's nontelativistic treatment. Recently. ${ }^{2}$ this calcalation has been extended to arbitrary particle velocity by the incorporation of the third-order Born approximation to the Dirac wavefunction of an electron scaltering of of a hesvy nucleus. By summing over momenturn states, a relativistic wave packet was constructed which was then allowed to scatler off the nucleas. In this manger, the close-collision energy loss of a heavy nucleus passing through matter could be calculated. Comperison of the results of this calculation with particle range data and with the Bethe theory is shown in Fig. I. The abscissa is the charge of $600 \mathrm{M}=\mathrm{V} / \mathrm{amu}$ ions utilized and the ordinate is the discrepancy of calculated entrance energies with those obtained from time-of-flight (TOF) measurements. The dashed lines correspond to standard deviations in the TOF measurement, while error bars include uncertainties in range measurements and in the values of the adjusted mean ionization potential. It is seen that the calculations which include all corrections are in excellent agreement with data. The same is clearly not the case for the Bethe theory.

\section{Foothotes and References}

Condensed from Ref. 2.

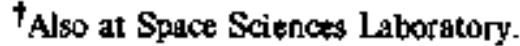

1. M.H. Salamon, S.P. Alten, G. Tarlé and K.C. Crebbin, Phys. Rev, A 23, 73 (1981).

2. S.P. Ahlen, submitled to Phys. Rev. A (1981).

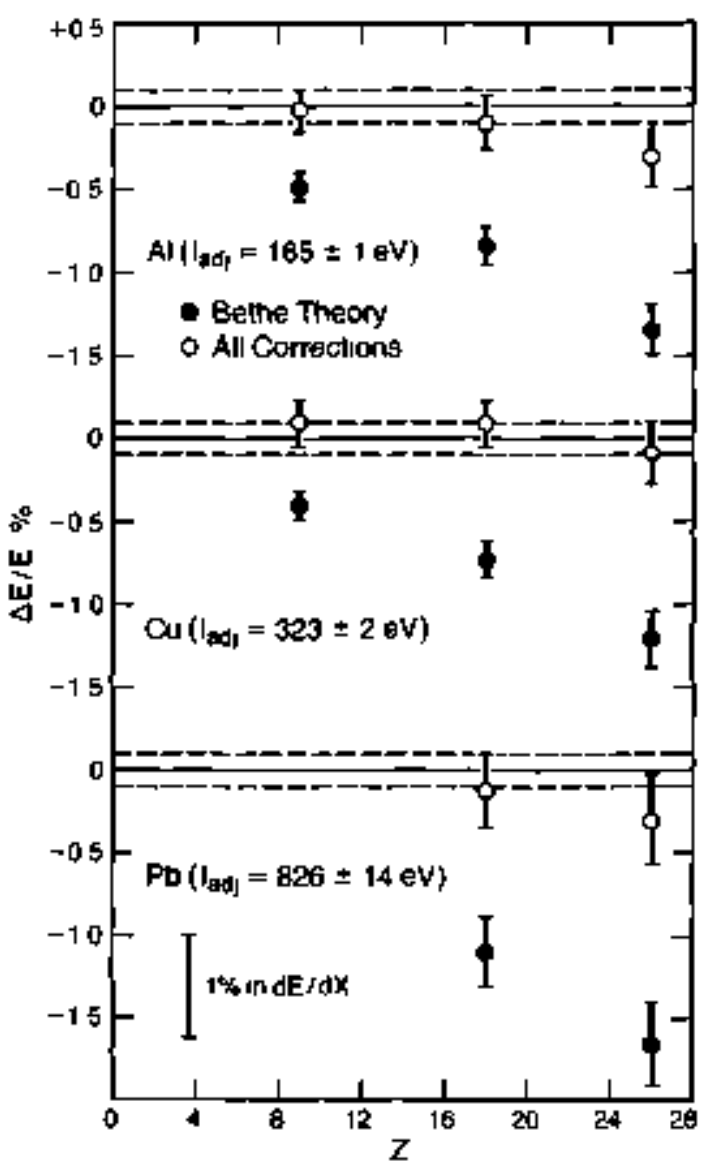

Fig. 1. Comparison of the Bethe theory with particle range data and with calculations which include all corrections.

(XBL $\$ 110-12209$ )

\section{A Search for New Nuclei Far from the Valley of Stability}

\author{
J.D. Stevenson, ${ }^{*}$ P.B. Price and J, Musser
}

Fragmentation of relativistic heavy ions has proved to be a powerful technique ${ }^{1,2}$ for studying nuelei far from the valley of stability.

In our previous work with a beam of $213 \mathrm{MeV} / \mathrm{n}^{48} \mathrm{Ca}$ we observerd ${ }^{20} \mathrm{C}$ and ${ }^{27} \mathrm{~F}$ for the first tirne (see Fig. 1).
We are now building a new detector which will allow fragments with energies from 300 to $600 \mathrm{MeV} / \mathrm{n}$ to be isocope-identified. Using these higher energies will result in factors of $\sim 20$ to 30 improvement in sensitivity for rare isotopes. This impsovement is pribnarily duc to use of 


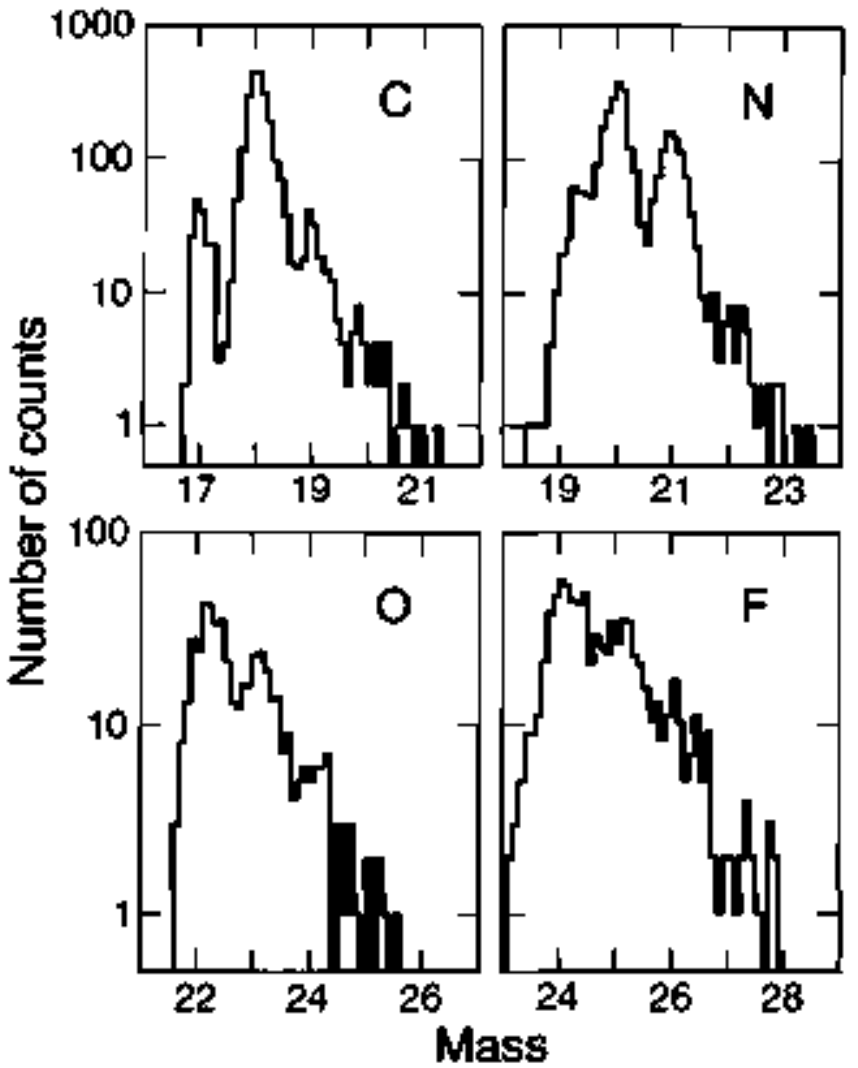

Fig. 1. Mass histograns for carbon, nitrogen, oxygen, and fluorine fragments from $213 \mathrm{MeV} /$ nucleon ${ }^{48} \mathrm{Ca}$ projectiles. The isotopes ${ }^{20} \mathrm{C}$ and ${ }^{27} \mathrm{~F}$ are clearly observed.

(XBL 8110-12210) thicker targets and improwed transport efficiency through the bearm-40 spectrometer. With the higher beam intensities and heavier ions soon to become avalable, it should be possible, with this system, to determine the limits of particle stability at least up to neon.

The detector uses velocity vs rigidity to separate isotopes. The velocity measurement is done with a Cerenkoy detector rather than time of flight. A Cerenkov detector provides a much better velocity measurement for heary ions in this energy range than any practical time-of-filight systen. The mass tesolution is expected to be in the range $\sigma_{\mathrm{m}}=0.2$ to 0.3 for masses less than aboul 50.

\section{Footnote and References}

*Also at Space Sciences Laboratory.

1. G.D. Westfall et al., Phys. Rev. Lett. 43, 1859 (1979).

2. J.D. Stevenson and P.B. Price, LBL-11737 and Phys. Rev. C (198L), in press.

\title{
Radiation Damage in Si(Au) Surface Barrier Detectors Produced by Energetic Heavy Ions
}

\author{
LG. Sobotka, G.J. Wozniak, R.J. McLonald, and LG. Moretio
}

During the past year we have done experiments at the SuperHILAC using bearns of ${ }^{46} \mathrm{Ar},{ }^{84} \mathrm{KJ}$, ${ }^{160} \mathrm{Xe}$, and ${ }^{165} \mathrm{Ho}$. Typically the beam-like particle (after elastic, quasielastic, or decply inelastic seattering) was detected in a partially depleted Ortec detector of type A-023-150-300. In the course of an experiment these detectors are generally exposed 10 approximately $10^{B}$ particles $/ \mathrm{cm}^{2}$.

While no significant radiation dariage was observed in the Ar' runs, the heavier ions produced significant damage in the $\mathrm{Si}$ detectors. One of the most obvious signs of this damage is the loss of pulse height as a function of dost. This ourcelation for a representative sample of detectors exposed to $\mathrm{Kr}, \mathrm{X}_{e}$ and $\mathrm{Ho}$ ions is shown in Fig. 1 . If the applied voltages are similar, an increase in the rate of pulse height loss is observed as the mass of the ion increases.

Perhaps more interesting is the correlation of the rate of pulse beight loss with applied voltage. This correlation is shown in Table I for seven detectors exposed to Xe ions. In fact, it seems that the signs of damage (pulse height loss) diminish as the applied bias is increased. This is probably the result of an increase in charge collection efficiency in the damaged regions as the fild strength increases.

These results indicate that large energy corrections as a function of dose are required for experiments where partially depleted $\mathrm{Si}$ detectors are exposed to $>10^{7}$ very beavy ions. These corrections are likely to become prohibj. tively large for experiments using the intense beams of very heavy ions that will shortly becoine available at the SuperHILAC Totally depleted Si detectors with large overbiases may be an acceptable alternative due to the high field strength throughout the bulk of the detector. In addition, p-type Si has been reported to be more resistant to radiation damage than $n$-type. ${ }^{1}$ Therefore, for experiments involving very heavy ions it may be preferable to use ptype Si, even though the dead layers are larger. 


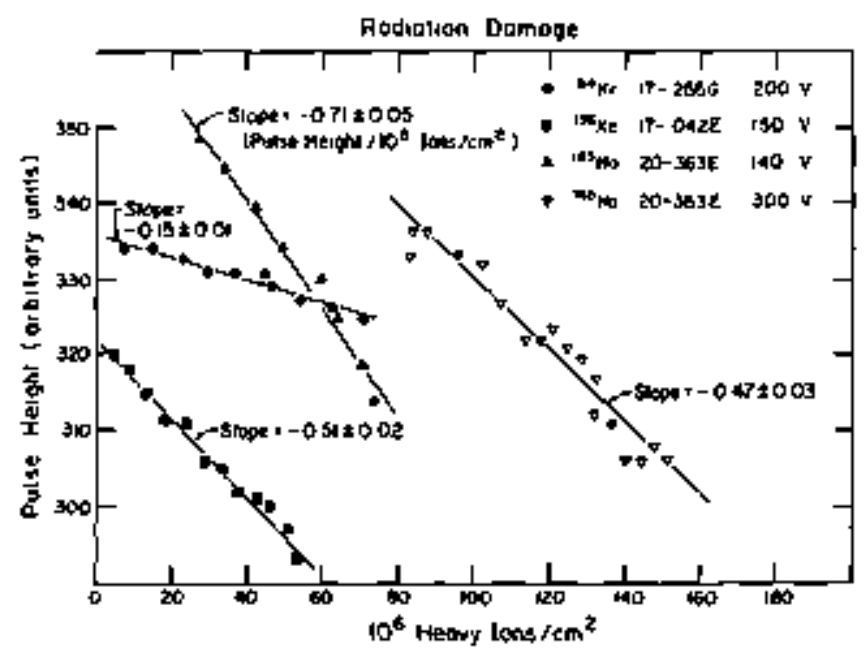

Fig. 1. Radiation damage in Si(Aus) detectors produced by various beavy ions. Detector number, voltige and least squares fit of rate of pulse height loss is indicated in (pulse height $/ 10^{6}$ ions $/ \mathrm{cm}^{2}$ ).
(XBL 818.2453)
Table I. Correlation of rate of pulse height loss with applied voltege.

\begin{tabular}{llll}
\hline Detector & Beam & Bias & Slope $\frac{\text { Pplse height }}{10^{5} \mathrm{HJ} / \mathrm{cm}^{2}}$ \\
& & $(\mathrm{~V})$ & \\
\hline $15-450 \mathrm{C}$ & $\mathrm{Xe}_{e}$ & 105 & $-0.64 \pm 0.02$ \\
$15-441 \mathrm{~J}$ & $\mathrm{Xe}_{e}$ & 105 & $-0.57 \pm 0.03$ \\
$15-329 \mathrm{D}$ & $\mathrm{X}_{e}$ & 125 & $-0.61 \pm 0.05$ \\
$17-042 \mathrm{E}$ & $\mathrm{Xe}_{e}$ & 150 & $-0.51 \pm 0.02$ \\
$15-596 \mathrm{C}$ & $\mathrm{Xe}_{e}$ & 225 & $-0.32 \pm 0.02$ \\
$15-596 \mathrm{~B}$ & $\mathrm{Xe}_{e}$ & 250 & $-0.29 \pm 0.02$ \\
$15-248 \mathrm{G}$ & $\mathrm{Xe}_{e}$ & 265 & $-0.25 \pm 0.02$ \\
\hline
\end{tabular}

\section{Reference}

I. V.V, Avdeichikow, Nacl. Instrum, and Methods 155, 125 (1978).

\title{
Development of a Beta Detector and Computer Analysis System for the On-Line Mass Separator, RAMA
}

\author{
J.M. Wotters, H.M. Thierens," M.D. Cable, R.F. Parry, and Joseph Cerny
}

The RAMA on-line mass separater has, until recently, been used predorninantly for studies of $\beta$-delayed proton enitters. A system consisting of a fast tape drive, a plastic scintillator telescope, a $\mathbf{G e}(\mathbf{L}$ i) detector, and a computer analysis program, has now been added to permit the determination of $\beta$-endpoints to $\sim 120 \mathrm{keV}$ accuracy via $\beta-\gamma$ coincidence spectroscopy. Our first results using this sys$1 \in m$, the $\beta$-endpoint measurements of ${ }^{103-105}$ In, are discussed elsewhere in this annual report.

The first component of this system, a fast tape drive, transports the activity collected on the focal plane of RAMA $18 \mathrm{~cm}$ to the detector station. Sirce the tape drive's initial developtment. ${ }^{\text {, }}$ the detector station has been repositioned (see Fig. 1) and a newer capstan motor has been added to reduce the transport time of radicactive sources to 120 milliseconds. Recently, an automatic tape rewind teature has been included for shorh-lived activities that require rapid cycling of the tape. This feature allows the MODACS III computer, which monitors RAMA, to rewind the tape with minimum downtime.

The s-telescope consists of a 10 -mm diameler and $\mathrm{J}$ $\mathrm{mm}$ thick NE102 plastic scintillator as a $\triangle \mathrm{E}$ detector (for $\gamma$-ray rejection) and a large cylindrical NE102 plastic scisttillator, $11.4 \mathrm{~cm}$ in both diarneter and lengh as an E deleter. The $\gamma$-ray detector is located on the opposite side of the tape in close proximity to the $\triangle E$ scintillator for $\$-\gamma$ coincidence spectroscopy. Standard fast-slow coincidence

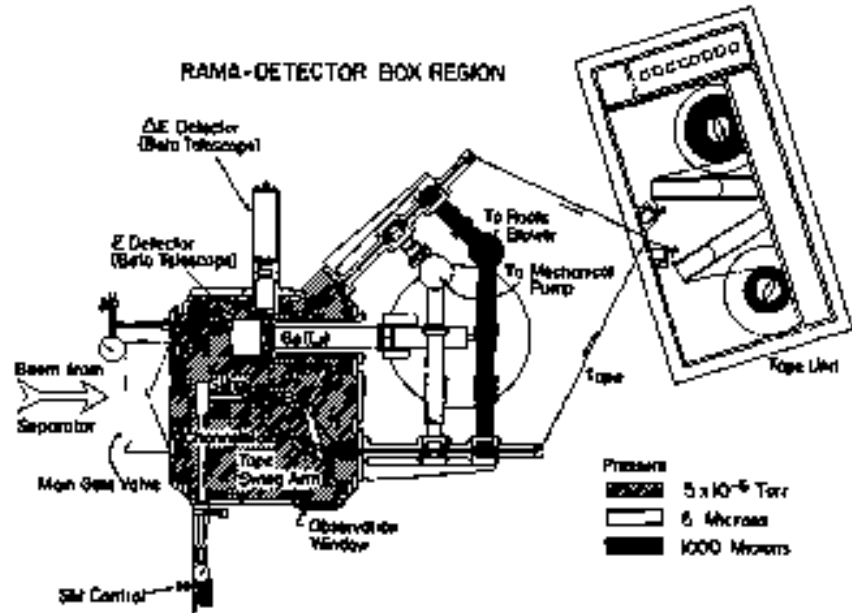

Fig. 1. Schematic view of the detector box region of the on-line nass separator RAMA.

(XBL 817+1034)

networks are used between all three delectors with a coinciderce timing of 5 ns (FWHM) between the two scintillators and $20 \mathrm{~ns}$ (FWHM) between the fi-E scintillator and the $\mathrm{Ge}(\mathrm{Li})$ detector.

The finsl spectra obtained with the above system need to be corrected for the finite energy resolution of the $\mathrm{E}$ 
scintillator. ${ }^{2}$ The program SPECTR performs this correction by assuming a theoretical shape, $T(E)$, for the $\beta$ spectrum with endpoint energy $E_{a}$ and distoring it using a semi-empirically derived response function, $R\left(E, E^{3}\right)$ for the $\mathrm{E}$ detector. This procedure yields an energy-dependent correction factor, K(E), by which the measured $\beta$-specirum is multiplied. A Gaussian response function is employed with a $\sqrt{E}$ dependence for the FWHM:

$$
K(E)=T(E) \quad\left[\begin{array}{ll}
E_{0} & \\
\int_{0}^{\prime}\left(E^{\prime}\right) R\left(E, E^{\prime}\right) \partial E^{\prime}
\end{array}\right] .
$$

where

$$
R\left(E, E^{\prime}\right)=\frac{1}{\sigma_{1} \sqrt{2 \pi E^{\prime}}} e^{-\left(E-E^{\prime}\right)^{1} / 2 \sigma E^{\prime}}
$$

The endpoint is then calculated by SPECTR, which performs a weighted linear leasi squares fit on a Fermi-Kurie plol of the data. Finally, the erjor is calculated in a two step procedure. First, the statistical error associated with the linear least squares fil, weighted by of $=4 p_{i} F_{i} E_{i}$, is delerrinined:

$$
\sigma_{\mathrm{E}_{0}}^{2}=\frac{1}{\mathrm{~A}^{2} \Delta} \sum_{i} \frac{\left(\mathrm{E}_{\mathrm{i}}-\mathrm{E}_{\mathrm{v}}\right)^{2}}{\sigma_{\mathrm{i}}^{2}},
$$

with

$$
\Delta=\sum_{i} \frac{1}{\sigma_{i}^{2}} \underset{i}{\sum} \frac{E_{i}^{2}}{\sigma_{i}^{2}}-\left(\sum_{i} \frac{E_{i}}{\sigma_{i}^{2}}\right)^{2} .
$$

In this expression $A$ is the slope of the Fermi-Kurie plat, $F_{i}$ is the Fermi function, $E_{0}$ is the endpoint, and the energy corresponding to channel $i, E_{j}$ is summed over the energy limits of the fit. This statistical error is then added quadratically to the error due to the energy calibration:

$$
\begin{aligned}
& \sigma_{\mathrm{E}_{\text {eatitration }}^{2}}= \\
& \sum_{\mathrm{i}}^{\mathrm{N}}\left[\sigma_{\mathrm{it}}\left(\mathrm{X}_{\mathrm{o}} \frac{\delta f_{\mathrm{A}}}{\partial \mathrm{E}_{\mathrm{l}}}+\frac{\delta \mathrm{f}_{\mathrm{B}}}{\delta \mathrm{E}_{\mathrm{i}}}\right)^{2}+\sigma_{\mathrm{FK}}^{2}\left(\mathrm{X}_{\mathrm{o}} \frac{\delta \mathrm{f}_{\mathrm{A}}}{\delta \mathrm{X}_{\mathrm{j}}}+\frac{\delta \mathrm{f}_{\mathrm{B}}}{\partial \mathrm{X}_{\mathrm{i}}}\right)^{2}\right],
\end{aligned}
$$

where the slope $f_{A}$ and the intercept $f_{B}$ of the calibration function depend on the literature values for the endpoints of the calibralion nuclei $\mathrm{E}_{\mathrm{i}}$ with uncertainties $\sigma_{L i t}$ and the corresponding measured channel number $X_{i}$ with errors $\sigma^{\sigma_{F}}$ ' $^{\prime}$

Five known endpoints were measured to calibrate the spectrometer and as an overall test of the system. The linearily of the final energy calibration (see Fig. 2) demonstrates the accuracy of this approach.

\section{Footnote and References}

'On leave from the University of $G$ ent, Belgium.

1. Nuclear Science Annual Report for 1978-1979, LBL 9711, p. 227.

2. P.C. Ragers and G.E. Gordon, Nucl. Instrum and Methods 37, 259 (1965).

3. David M. Rehfield, Nucl. Instrum. and Methods 157, 351 (1978).

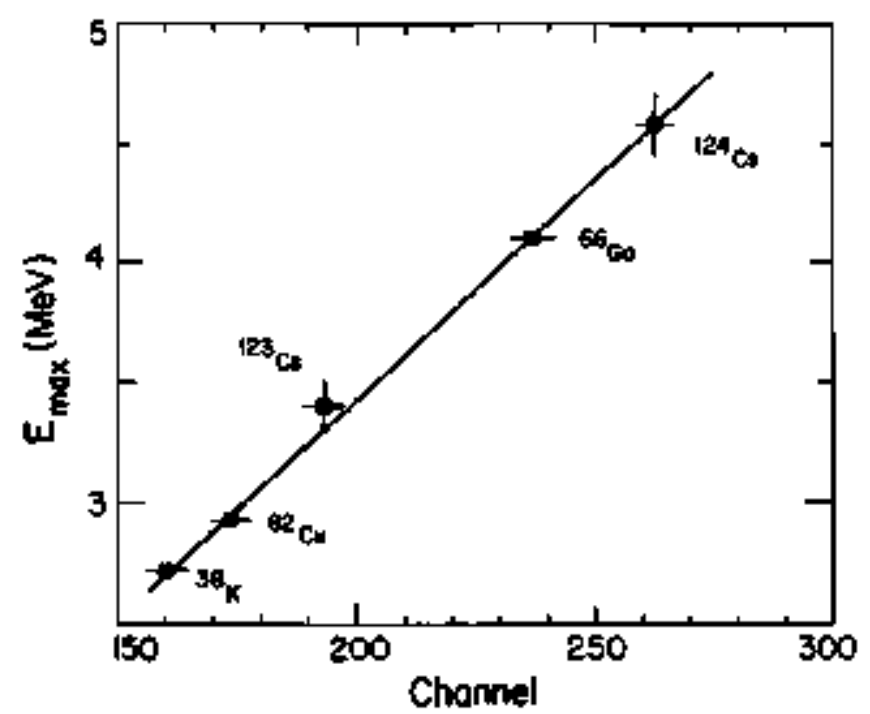

Fig. 2. Energy caljbration of the $\beta$ spectrometer using the nuclei ${ }^{38} \mathrm{~K},{ }^{62} \mathrm{Cu},{ }^{66} \mathrm{Ga},{ }^{123} \mathrm{Cs}$, and ${ }^{124} \mathrm{Cs}$.

(XBL 815-820) 


\title{
C. Emulsions and Plastic Track Detectors
}

\section{Demonstration of a Detector with Unprecedented Charge Resolution"}

\author{
S.P. Ahlen, ${ }^{\dagger}$ P.B. Price, ${ }^{\prime}$ G. Tarle, ${ }^{\prime}$ and $M$. Tincknell
}

The most fundamental limitation of detectors of all types used in the transmission mode is the fluctuation of deposited energy. By deliberately madifying the polymerization procedure of the commercial plastic, CR.39, we have produced a material which, when used as a nuclear track detector, has a charge resolution better than would be predicled on the basis of fiuctuations in total deposited energy. This is demonstrated in Fig. 1. As illustrated in the inset, I.86 GeV/amu ${ }^{56} \mathrm{Fe}$ nuelei were incident on a

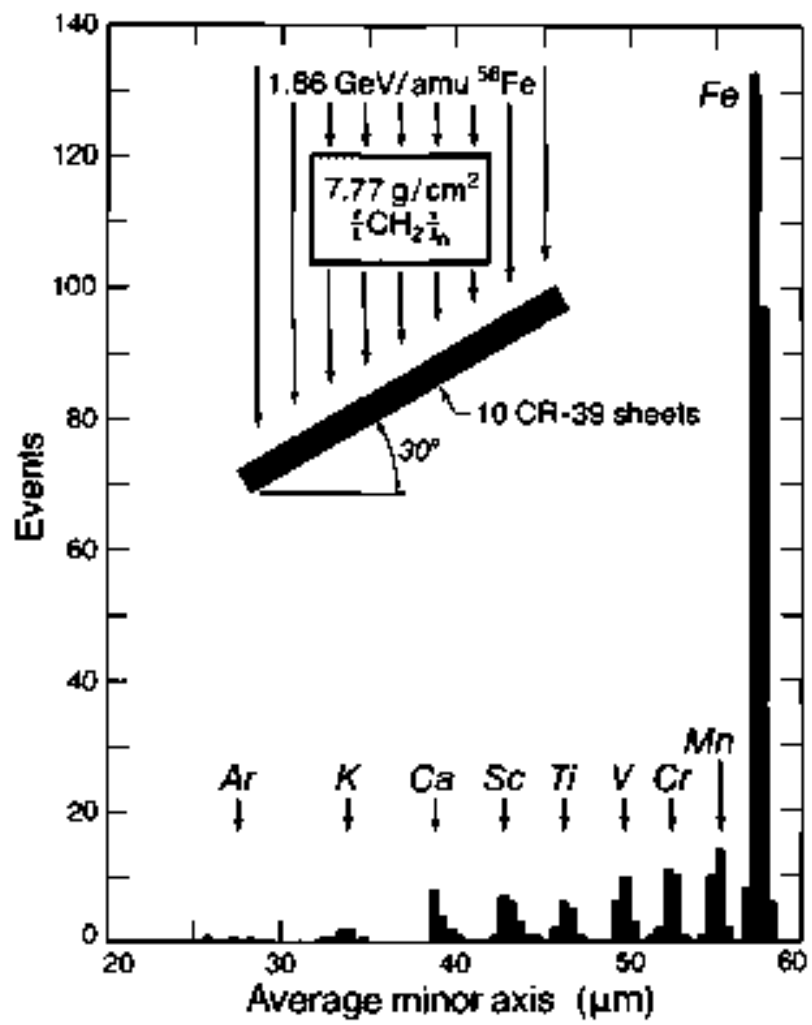

Fig. 1. Histogram of the average of the minor axes of four track mouths for the particles emerging from the poiyethylene indicated in the insel.

(XBL $8110-12262)$ slab of polyethylene. The oulgoing partides penelrated a stack of ten sheets of the modified CR-39, two of which were used in the analysis of the charge of the emxrging parlicles. The analysis involved the measurement of the minor axes of four consecutive tracks of each particle. The average value of the minor axis was then tabulated in the histogram. The resulting charge resolution at $\mathrm{Ft}$ is $0.12 \mathrm{t}$. For comparison, the tharge resolution at a $3 \mathrm{~mm} S \mathrm{si}$ detector in a similar configuration has been measured to be $0.17 e^{2}$ Since the effective thickness of the CR-39 delectors used to obtain the data in Fig. I was only 190 mm, it is apparent that track detectors have a substantially enhanced intrinsic resolution compared to lotal energy loss detectors such as silicon semi-conductor diodes. This is due to the complete insensitivity of track delectors to delta rays which are cbiefy responsible for energy straggling.

As one particularly suitable epplication of these new detectors, we have recently exposed several slacks of pure CR-39 and of altemating CR-39/lead assemblies to $1.8 \mathrm{GeV} / \mathrm{arnu}{ }^{40} \mathrm{Ar}$ in the hopes of furthering the understanding of the so-called "anomalons." With the use of a semi-automated measuring system curtenty under construction, we will be able to measure the charge of a million particle tracks in the spece of one year. Thus, statistically meaning[ul information will be obtained in a shorter period of time then is possible with nuclear emulsion. In addition, the use of the track detectors allow for greater flexibility in target choice.

\section{Footnotes and References}

Condensed lrom Ref. 1.

†Aso at Space Sijences laboratory

1. G. Tarle, S.P. Ahlen and P.B. Price, Nature (1981), in press.

2. G.D. Westfall, et al., Phys. Rev, C 19, 1309 (1979). 


\title{
Identification and Imaging of Nuclear Particles Using Etched Solids*
}

\author{
S.P. Ahlen ${ }^{\dagger}$ P.B. Price. ${ }^{\dagger}$ and $G$. Tarlet
}

\begin{abstract}
Although media capable of recording visible light inages were developed only within the last century, it is a fascinating fact, with widespread consequences, that media capable of recording permanent nuclear track images are as old as the solar system. In fact, when a highly charged particle penetrates any nonconducting solid, it creates a subnicroscopic trail of radiation-damaged material that can be chenically amplified, by the so-called track-etch process, into a track large enough in diameler to be measured in an optical microscope.

This arlicle reviews track production mechanisms and the track-etch technique and then discusses several recent advances, especially in high-resolution particle identification and imaging. The major topics are extraterres. trial tracks; fission track dating; space physics; nuclear physics; technological uses; the CR-39 polymeric detector; the use of dopants to improve the resolution and intage quality of the CR-39 detector; the bigh charge resolution of doped CR-39; applications of doped CR-39 to cosmic rays, antimatter searches, searches for nonintegrally charged nuclei and monopoles; and advances in nuclear imaging with CR-39, including heavy-ion radiography, microscopic mapping of trace element distributions, lensless heavy-jon microscopy, and coded imaging of leser fusion reactions.
\end{abstract}

\section{Foormotes}

"Condensed froun a paper in the September, 1981 jssue of Physics Today.

†Also at Space Sciences Laboratory.

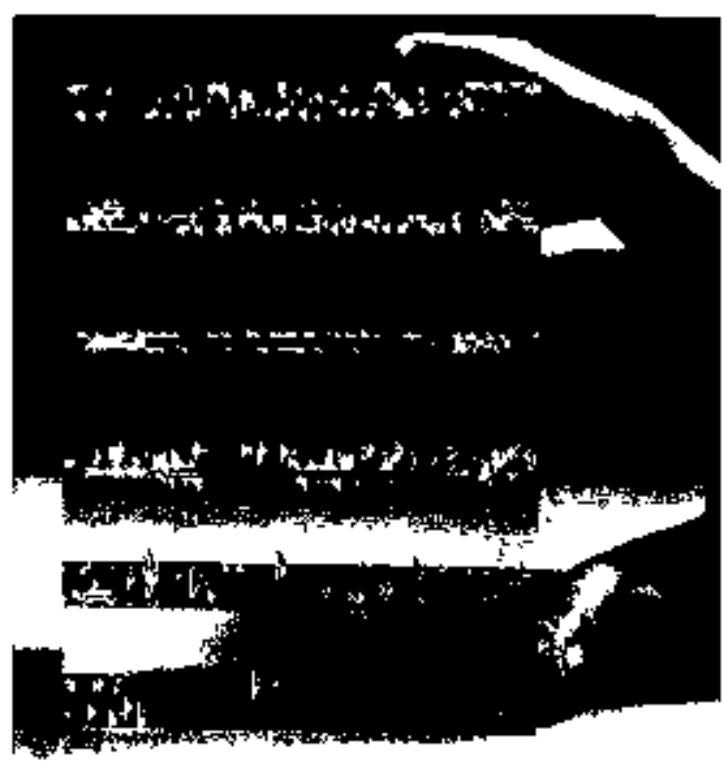

Fig. 1. Macroscopic etched tracks of a $100 \mathrm{GeV}$ beam of ${ }^{56}{ }^{5}$ e nuclei brought to rest in a stack of five slabs of CR39 doped with $1 \%$ of dioctyl phthalate. Careful examination of the actual slabs shows numerous examples of nuclear fragmentations. Etching for 1 month in 6.25 notmal sodium hydroxide solution at $70^{\circ} \mathrm{C}$ has removed $\sim 0.75 \mathrm{~mm}$ of material from the surfaces and caused the cone-shaped track etch pits to develop.

(CBB 813-2393)

\section{Applications of Nuclear Track-Recording Solids to High-Energy Phenomena"}

\author{
P.B. Pricet
}

As part of a Festschrift for Nicolas Cabrera, this paper introduces solid state physicists to some of the ways in which the track-etch process enables problems in cosmic ray astrophysics, particle physics, and relativistic heavy ion physics to be studied.

The dersily of defects along the track left by a nuclear particle in a nonconducting solid provides a permanent record of the particle's velocity, charge, and mass. The record can be deciphered by etching the solid in a suitable reagent and measuring the sizes of the etch pits at points of intersection of the track with the surfaces. Useful solids include minerals and glasses, which are sensitive only to particles with charge/velocity ratio $\mathrm{Z} / \beta>150$, and to polymers, the most sensitive of which delects particles with $Z / \beta$ as low as 6 .

A tion in inorganic solids (via a s0-called ion-explosion spike) and in polymers (via scission of long-chain molecules), I summarize the present status of the track-etch technigue by which our group has obiained charge-resolution unequalled by any other transmission detectors of equivalent thickness. I then describe brieffy a few applications.

\section{Relativistic Nucleus-Nucleus Collisions}


a. Negative results of searches for products with charge grtater than projectile charge by more than $2 \mathrm{e}$ in reactions such as $1.8 \mathrm{GeV} / \mathrm{N}{ }^{40} \mathrm{Ar}+\mathrm{Pb}$. New experi. ments in 1982 will use ions having $Z \geq 36$ in the hope of seeing density isomers.

b. Evidence for nonthermal emission of fragments with $Z=3$ to 12 in such reactions as $0.5 \mathrm{GeV} / \mathrm{N}$ $40 \mathrm{Ar}+\mathrm{Aul}$.

c. Production of new nuclides ${ }^{20} \mathrm{C}$ and ${ }^{27} \mathrm{~F}$ in projec tile fragmentation.

d. An experiment with tigh statistics $\left(>10^{5}\right.$ secondary interactions) to characterize the properties of anomalons. Three stacks with different combinations of CR-39 detectors and targets were successfully expased to 1.86 GeV/N ${ }^{40} \mathrm{Ar}$ jons in June 1981. Among the results we should be able to obtain will be a rigorous deterntination of the fraction of secondary interaction produets with norintegral chargen

\section{Particle Physics}

a. A search for highly ionizing parlicles produced at PEP in $15 \mathrm{GeV} \mathrm{e}^{+}+15 \mathrm{GeV} \mathrm{e}^{-}$collisions. Our present limit ( $95 \% \mathrm{CL}$ ) on the crass section for production of monopoles with $M \neq 14 \mathrm{GeV} / c^{2}$ and $\beta>0.01$ is $\sigma<1$ pb. The same limit applies to electrically charged particles with $Z / \beta>16$ and a range greater than $\sim 0.5$ inm of plastic.

b. A search for highly ionizing particles that survive tratersal through $600 \mathrm{~B} / \mathrm{cm}^{2}$ of air to reach large arrays of plastic (CR-39) at the sumnit of White Mountair, California Two specific classes of particles are being someht: Grand-Unification Momopoles with mass $\sim 10^{15 \pm 1} \mathrm{GeV} / \mathrm{c}^{2}$ and quark globs (the possible precursors of Centauro-lype interactions). Despite negative results to date (flux $<0.4$ $\mathrm{m}^{-2} \mathrm{y}^{-1}$ at $95 \% \mathrm{Cl}$ ), we have succeeded in measuring, for the first time, the energy spectra of $\mathrm{He}$ and $\mathrm{Li}$ al nowntain altitude, and we have detected nuclei with $Z$ up to $\sim 15$ at an altitude of 41,000 feet, in accord with our calculated fuxes of surviving cosmic ray fragments.

\section{High-Emergy Particles in Space}

a. Discovery of preferential emission of heavy nudei in solar flares.

b. Measurement of charge distribution of ultraheavy cosmic rays, up to $Z \simeq 96$, in a 250-day exposure of $1.2 \mathrm{~m}^{2}$ plastic stack on Skylab.

c. Discovery of intense fluxes of heavy ( $Z$ up to 26), muli- $-\mathrm{MeV} / \mathrm{N}$ jons in the inser magnetosphere, using a delector outside the Skylab.

d. Collaborative experiment with the University of Chicago, using transition-radiation detectors plus CR-39 to measure accurately charge distributions of cosmic rays al Lorent2 factors up to $\sim 2000$. This experiment is scheduled for the second Spacelab fight, some time in 1984.

c. A planned experiment using CR-39, plastic scinti]lators, and plastic Cerenkov detectors, to search for an anti-jion/iron ratio as low as $3 \times 10^{3}$ in the cossmic rays. The experiment could be flown either in a balloon or on the shuitle and could also study anomalons at energies well above those attainable at the Bevalac.

Needless to say, this work was and is being done in collaboration with senior colleagues, graduate students and undergraduates.

\section{Foothotes}

"Condensed from paper to appear in January, 1982 issue of Pbilosophical Magazine.

${ }^{\dagger}$ Also at UC Space Sciences Laboratory.

\title{
On the Possibility of Detecting Fractionally Charged Relativistic Projectile Fragments in Nuclear Emulsion
}

\author{
Harry H. Heckman
}

One of the speculations on the "anomalon" effect observed in empulsion studies on the interaction properties of projectife fragments in $\mathbf{R H I}$ collisions is that they may indicate the existence of fractionally charged projectile fragments. With this speculation in mind. I have shown that charge measurements on relativistic nuclei can be made in nuclear emulsion to an accuracy $\leqslant \pm 0.05$ e, provided: (1) the lacunarity (L $-\sum$ gap lengths/track Itength) is $\mathrm{L}>0.10$, and (2) track length $\ell \geqslant 2 \mathrm{~mm}$.

As discussed by Barkas ', the experimental observation that the distribution of gap lengths in an ionization track is exponentially distributed leads to the following expression which relates the lacunarity to the grain density $g$ of the track:

$$
L=e^{-\alpha z} \text {, }
$$

where $\alpha$ is the average developed gratin size in the emulsion. Assuming $\mathrm{g}$ is proportional to $\mathrm{DE} / \mathrm{dx}=\mathrm{Z}^{2} \mathrm{~F}(j)$, we have at relativistic entergies, eg., $T=2 \mathrm{~A} \mathrm{GeV}$, the ele. mentary result that $Z^{2}=-k$ en determined by calibration with beam nuclei of known 
charge. A measurement of $L$ of a relativistic track thus leads to an estimate of its tharge:

$$
Z=\sqrt{k \ln \mathbf{L}},
$$

with an erroc in Z

$$
\sigma_{Z}=\frac{k}{2 Z} \frac{\sigma_{L}}{L}
$$

A good approximation of the fractional error in $L$ is given by

$$
\frac{\sigma_{1}}{\mathrm{~L}}=\left[\frac{2 \alpha}{\ell}\left(\frac{1-\mathrm{L}}{\mathrm{L} \mathbf{L}^{-1}}-\mathrm{l}\right)\right]^{1 / 2} .
$$

where $\ell$ is the track length ower which the lacunarity $L$ is measured.

From Eqs. ( 3 and 4) one finds, quite astonishingly, that one needs only to measure $L$ over distances $\ell=$ 1-3 III of track length to obtain a measure of $Z$ to an accuracy of \pm 0.05 unils. Taking a value of $k=9.62$ (appropriate to our emulsions currently bejng scanned), Table 1 gives the predicted values of $\&$ nectessary to obtain a $\sigma_{z}=0.05$.

We bave experimentally verified the predicted accuracies of charge measurement by the lacunarity method for charges $Z=1,2$ and 3. The measurements on $\mathbf{Z}=2$ nuclei over a I mon track length are shown in Fig. I. Figure la shows the measurements on ${ }^{3} \mathrm{He}$ beam nuclei (1.9 A GeV) and Fig. 1 b shows the measurements of $Z$ - 2 projectile fragments from $1.9 \mathrm{~A} \mathrm{GeV}{ }^{56} \mathrm{Fe}$ interaction in emulsion.

The observed ms width of the distributions of 0.04 . 0.05 charge units are in agreement with the simple theoresical model of track structure described above. With the demonstrated ability to measure the cherges of RHI to accuracies $\sim 0.05 \mathrm{e}$, one clearly has the capability of searching for fractionally charged ions in multiples as small as $1 / 3 e$.

\section{Reference}

I. W.H. Barkas, Nuctear Research Emtalsions, Academic Press, New York (1963).
Table 1. Track length $\ell$ necessary to determine charge to $\sigma_{\mathrm{Z}}= \pm 0,05$. L is the lacunarity of RHI tracks, charge $\mathrm{Z}$, at energy $\sim 2 \mathrm{~A}$ GeV. Saturation effects will limit applications to $Z \leq 4$.

\begin{tabular}{lll}
\hline $\mathrm{Z}$ & $\mathbf{L}$ & $(\mathrm{mm})$ \\
\hline 1 & 0.912 & 0.73 \\
2 & 0.693 & 0.79 \\
3 & 0.438 & 0.94 \\
4 & 0.231 & 1.18 \\
\hline
\end{tabular}

(a)

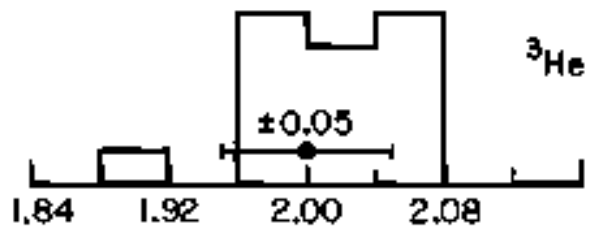

(b)

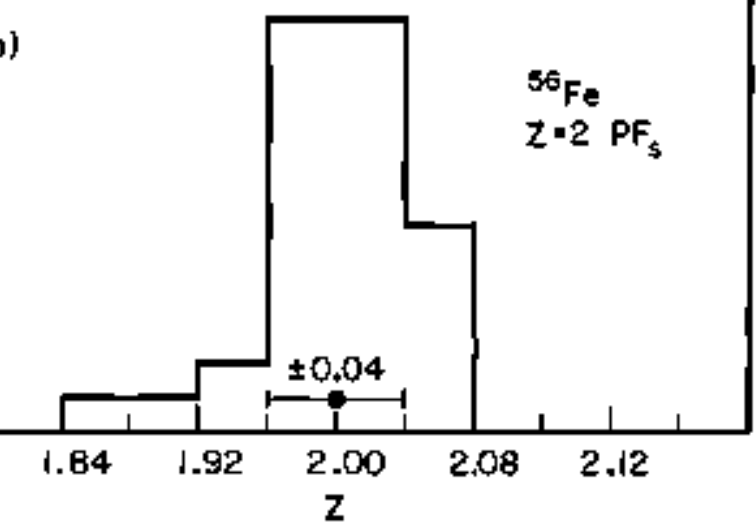

Fig. I. Measured yalues of charge $Z$ for (a) ${ }^{3} \mathrm{He}$ beam muclei and (b) $Z=2$ projectile fragments of ${ }^{56} \mathrm{Fe}$. Beam energies are $1.9 \mathrm{~A} \mathrm{GeV}$. Measurements were made over $1 \mathrm{~min}$ track length.

(XBL $8111-1609$ ) 


\title{
ICAMS: A System for Computer Assisted Nuclear Microscopy
}

\author{
E.M. Friedlander, A.H. Heckman, Y.J. Koront
}

We are in the process of installing the first prototype of a new system for emuision daca analysis and reduction. the ICAMS (Interactive Compuler Assisted Measurement System). JCAMS is composed of two basic parts: a central compuler and individual ODS (Optical Data Stations). The entral corriputer (PDP.11) is responsible for overall system control and data reduction for physics interpretation. The system operates under the standard multj-task RSX-11M operating environment, to which has been added an additional packsge, [CMON (]Cams MONitor system), to perform ODS control on a sysiem basis.

Each ODS has a local microprocessor (6502) with a 9611 arithmelic processor. This combination enables local rapid computalion for the control of the actual stage molion, thereby permitting each ODS to respond quickly to the individual operator. Eacb ODS independently:

1. Prompts the operator to perform measurement functions.

2. Responds to operator data entry commands.

3. Avtomatically records $(x, y, x)$ position information to 1 micron accuracy.

4. Upon command, automatically follows a given trajoctory and automatically selurns to any vertex in the emulsion.

5. Verifies certain track parameters (such as fit of a trajectory to a specified hypothesis) to provide on-line data quality control. Figure 1 is a block diagram of the basic components of the ICAMS.

Since the data are immediately available in the central computer, we can fully eliminate many of the traditional slow steps of visual data reduction. such as sean sheets, compulet data entry forms, and handwritten computer data validation forms. Instead, all of the information important to an operator appears on the individual ODS consoles, while data from all the ODS also is accessible to the physicist for immediate review.

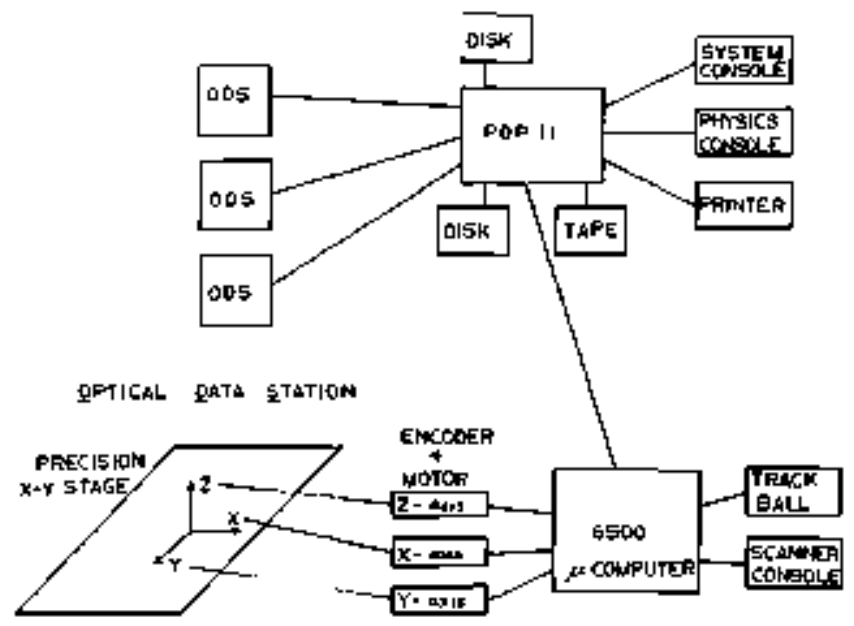

Fig. 1. System layout of ICAMS, illustrating the central PDP II computer and peripherals, and one of the ODS attached to ICAMS. Each ODS has its own microcomputtr with its own peripherals. The link between each ODS and ICAMS is directly on the PDP I U UNJUS.

(XBL $\$ 110-11749)$

\section{Study of Highly Ionizing Particles at Mountain Altitude ${ }^{*}$}

\author{
K. Kinoshile and P.B. Pricet
}

The inspiration for this experiment was the widely acknowledged peculiar nature of the "Centauro" class of ultrahigh-energy interactions discovered at Mt. Chacaltaya about a decade ago by a Brazil-Japan collaboration. The rate of Centauro events is quite low, $-0.02 \mathrm{~m}^{-2} \mathrm{yr}^{-1}$. Among the proposed explanations is the possibility that they might be initiated by a highly charged primary particle. To look for such particles, we deployed a series of CR-39 plastic track detectors with large collecting power at the summit of White Morntain, California $\left(603 \mathrm{~g} / \mathrm{cm}^{2}\right)$. Two experiments heve been completed and analyzed: a single layer with a $10 \mathrm{~m}^{2}$ yr exposure and three coincident layers with a $0.025 \mathrm{~m}^{2}$ yr exposure. In the first experiment, the single layer was adopied to maximize the collecting area at soone sacrifice in velocity information. "The results demonstrate the superiority of CR-39 as a detector of highly ionizing particies $(Z / \beta \geqslant 30)$ and its particular suitability as a collector of very rare particles. The performance of the plastic was evaluated by examining the bigh density of tracks due to slow, light ions. The low-energy spectra of auclei with $Z<3$ have been measured and are found to be consistent with spectra calculated from a model 
where the source of jons is almospheric collisions of ener. getic hadrons. This is the first time that energetic Li has been identified at mountain altitude and that enough He has been seen to permit measurements of its energy spectrum. A density $=10 \mathrm{~m}^{-2}$ of fast particles with $Z \geq 4$ found in individual layers is consistent with the exposure received in a l0-hour commercial jet fligbt. A fast scanning method was used to examine the entire single-layer array. In the interval $30 \leqslant \mathrm{Z} / \beta \leqslant 100$ no events were found, from which infer an upper limit of $0.4 \mathrm{~m}^{-2}$ $\mathrm{yT}^{-1}$ (95\% CL) on the flux of electrically charged particles with $Z / \beta=30$ to 100 and on the flux of superheavy magnetic unonopoles with $\beta>0.02$. Two objects were located which, if they are indeed tracks, would cortespond to particles with $\mathrm{Z} / \beta>100$, with abundagce $\sim 0.2 \mathrm{~m}^{-2} \mathrm{yr}^{-1}$. Wilhout sbeets in coincidence, it is impossible to distinguish these objects trom certain flaw's in the plastic. A new delector, with three layers interleaved with absorbers and with an area of $20 \mathrm{~m}^{2}$, will cortect this shortcorring and be able 10 measure the spectra of light elements to higher energies as well.

\section{Footnotes}

*Abstrace of paper in Phys. Rev. D.

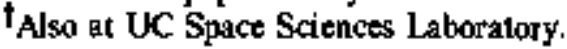




\title{
D. Computers
}

\section{The Design of MIDAS-A Modular Interactive Data Analysis System*}

\author{
Oreve Maples, Whllam Rathbun, Daniel Weaver, and John Meng
}

A specialized, multi-user computer facility utilizing a parallel processor architecture to achieve high-spoed, interactive reduction and analysis of experimental data is currently under development. ' Data analysis is currently restricted to either a large central computer system (with the advantage of high computational speed and large mass storage capacity but typically batch-oriented and with limt ited interactive graphics capability) or to a smaller and more dedicated computer system (often offering a highly developed jnteractive graphics capability and permitting more flexible user contsol of the hasdwart environment but with slowtr calculational speed). Because of the bigh level of interactive graphics required, the necessity of continuously dealing with large data bases (from 200 to 5000 Mbytes per user), and the potentially significant amount of processing required per data packet (event), neither typical small nor large compuier environiments appear ideally svited to the requirements of data analysis.

The design objective is an environment that permits the user to interact with and guide the analysis process and to optimize conditions in order to best utilize the user's time. To be an effective interactive tool, the proposed system must be able to operate on large amounts of data and produce results, in terms of graphic displays within human time frames of seconds to minutes. The two main difficulties which must be addressed to achieve the desired performance are $\mathrm{CPU}$ and $\mathrm{J} / \mathrm{O}$ bandwidth limitations.

Assuming that the data to be analyzed consist of rela. tjuely discrete and independent packets of information, the process of data analysis lends itself extremely well to the techniques of paralltl processing. By creating an environment where data packets may tow ibrough the system in parallel rather than serially, as is currently required, considerable improvement in processing speed and throughput is possible. The proposed system, although passessing all the attributes of a conventional mid-range computer, is not designed as a general purpose computational facility, but instead is to specifically handle froblems associated with high-speed, interactive data analysis. To this end, certain very general assumptions are made concerning the structure of data and the process of analysis. These assumptions are:

1. The data base pro-exils.

2. The data base consists of packets of information (events).

3. Fach event packet is ssentially independent of other events.

4. Processing for each event packet is well defined and essentially repetitive for a given event type.
The general architecture of the system shown in Fig. 1, consists of five subsystems, which are described below.

1. The Primary Computer System is designed to bandie user requests and to allocate resources of the system. It is the only processor that directly deals with user's requests, and it has absolute control in allocating physical resources and determiaing system priorities. It is designed to respond to requests within human or interactive time scales and, therefore, will not diresly handle CPU intensive problems such as analysis. The design is a distributed environment, not a network, operaing as a master-slave hierarchy in which the Primary Computer functions as the master.

2. The Interoctive System consists of the processors and associated hardware necessary to support the interactive needs of the users. These elentents provide communication only with the Primary Computer. Basically, each

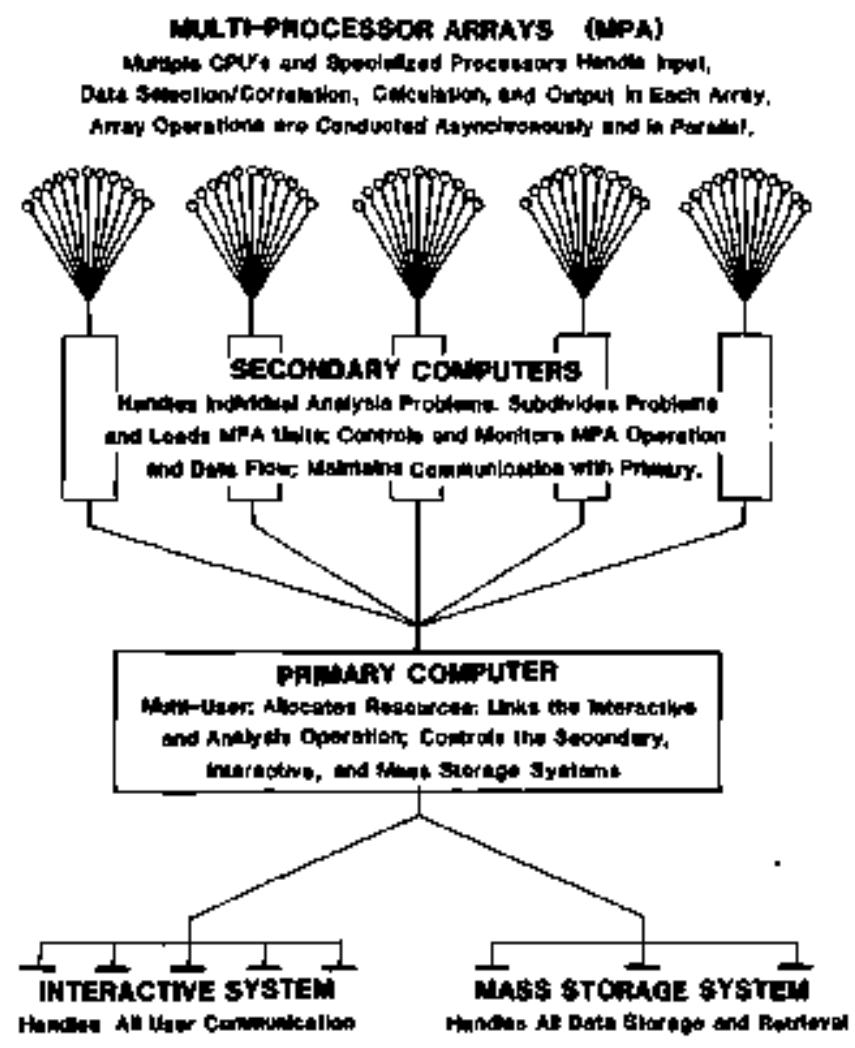

Fit 1. General architectural design of the MDDS system.

(XBL 815-9786) 
section of the lnieractive System consists of a high-speed, high-resolution graptics terminal and a dedicated hardcopy device capable of providing a copy of the graphics serten and functioning as a printer and rough plotter. The terninals are a very importent aspect of the system since they provide the interactive communication channel between the user and the entice system. Each would be equipped with a processor to handle simple graphics manipulation and screen updales and also with special inleracive devices such as programuable switches, track balls, touch panels, etc.

3. The Mass Storage System is involved with the storage and retrieval of experimental data or resulis. Separate disc and tape drives are maintained by the Primary Computer for the storage and handling of user programs, files, elc. The Mass Storage System is controlled directly and solely by the Primary Computer and is functionally divided into three sections: magnetic tape control, disc control, and random-access bulk menory control, each of which is governed by Multi-ported Programunable Controllers (MPC). These controllers are specialized CPUs whose function is to control data flow 10 and from the specific device. Utilization of such devices permits the complete overlapping of data retrieval and transmission with analysis and processing. The magnetic tape and disc sysiems provide high-speed sequential access to stored information, typically raw or transformed data High speed, random-acoess storage, for which discs are not suitable, is supplied by external bolk memory. Each memory unit will have two addressing modes: Direct Memory Access (DMA) and Direct Mernory Incrementation (DMI). The DMA mode gives standard read or write access to any memory location, and the DMI capability is useful in the creation of large nnulti-dimensional histogram arrays from analyzed dela.

4. The Secondary Computer System will handle CPU-intensive problems delegated to it by the Primary (e.g., the analysis of data according to sonte spectified critertia). The Secondery communicates with, and is controlled by, the Primary Computer and, in turn, communicates with and controls a Multi-Processor Array which is described below. Although the Secondary Computer is a general parpose CPU and is potentially capable of dewoting all its resources to the solution of a particular problem, it is, in general, too slow to be direcily effective. Its normal function is thus to prepare the high-speed, parallel units of the Multi-Processor Array to handle the particular analysis problem specified by the user. Once the setup and inituali. zation is complete, the Secondary Compuler allows the data to flow into the parallel system and continuously monitors the processing of the dati. Although the Secondary is not directly involved in the analysis or data transfer through the system, it can control all active elements of the MultiProcessor Array.

5. A Multi-Processor Array (MPA) is associaled with and controlled by each Seoondary Connputer. An MPA consists of an input port, an outpul port, and a number of CPUs and specialized devices to permit bighspeed, asyachronious, parallel operations on the incoming data stream. The general function of of these devices and their interrelationship are as follows:

a) The Inptut Formatter is a parallel pipelined device that receives raw data Irom the input port. Is function is to "read" and unpack the data according to inforuation supplied by the Secondary, for example, separating nondata information (such as header records, directories, or end-of-files) from data, recognizing a separate event or data packet, uncompressing events if necessary, and performing simple logical operations on the data stream. Since the Input Formatter is a pipeline processor, data passes through it at $\mathrm{I} / \mathrm{O}$ bus speeds and each pipeline stage operates in parallel on a different data word. Its ultimate objective is to place a formatted event (or multiple events) into a Data Stack.

b) Each MPA contains a mumber of independent Data or Memory \$tacks (currently 16). Each memory stack conlains its own 32-bí L/O bus, 16 Kbytes or $200 \mathrm{~ns}$ solid state momory, and special memory zeroing hardware. The stacks are designed to hold formalted data or events and each stack may be dynamically swilched to any processor in the MPA.

c) The Programmable Arithmetic Modules (PAM) are, in effect, commercial, high-level CPU and EAU (Exiended Ajithmetic Unit) boards. Each PAM contains integer and floating point hardware, multiple register banks, hardware mapping, and will support pipeline mode operation. In order to maximize throughpul, these CPIS do not "read" $\propto$ Iransfer data into memory; instead, any Data Stack, containing data to be processed, may be switched to any PAM unit. The altached Mernory Slack, from the perspective of the associated PAM, is operationally indistinguishable from dedicaled mernory. Multiple PAMs may thus process data asynchronously and in parallel. PAM units are initially down-loaded with userspecified analysis code by the Secondary. The analysis code obtains data from a dedicated common block. This common block (actually a Memory Stack) may also be used to store analysis resulis. Al the completion of the analysis of one data block, the program exits, a new block is switched in, and the analysis restarts on new data The entire switching process should require, at the most, a few microseconds.

d) The Conductor is the hardware unit that controls the dynamic switching of Data Stack buses to appropriate active processors. The Conductor is intialized by the Secondary with a prestelected switching sequence. This specifies which processocs will be utilized in the subseguent analysis and the order in which the data will be presented to the processors. When an active processor signals completion, its current Data \$lack is switched to the next available class of processor in the sequence. The next unattached Data Stack, which is available for that class processor, is switched in and the processor restarted. Itera tive operations between processors are also permilted within this framework. It is important to entphasize that data itself is not being transferred in these operations, but rather that memory blocks containing data are being 
switched. When all processing of the data in a stack is completed, the Conductor can actiyate stack hardware that will automatically zero the memory. At this point, the stack is available for new data and the switching sequence is restarted.

e) The Programmable Sorting Module (PSM) is a rather simple processor which was designed to permit a Data Stack to be examined rapidly for user-specified patterns or correlations (loaded into it by the Stocondary Computer). The module utilizes special hardware to perform masking, gating and logical tests at very high speeds. The information generated can then be transferred to a Data Stack (for use by any other processor), to the Storage Memory, or to the Output Port. Although a PAM could be programined to carry out similar operations, this type of data examination is suficiently common and time consuming to warrant a specialized hardware unit.

5) The Output Formatter is essentially the inverse of the Input Formatter. It is a pipelined processor designed to take selected information from a Data Stack, format. compress it if desired, and transmit the results either to the local Storage Mentory or the Output Port. Information in a Data Stack may be utilized to determine the exact processing necessary.

g) The Storage Memory is a dual-ported bulk memory onit designed for local storage of analysis results. One port prowides standard DMA read/write access and is connected directly to the Secondary Computer. This link can supply interim as well as final results to the Secondary (for transmission to the Primary) independent of the operation of the MPA. The second port may be utilized for either sequential or incremental memory access. In the latter case. the content of the transmitted menory address is incremented. The initial design specifies that this unit contains at least $128 \mathrm{~K}$ of 32 -bit memory, with a meximum storage capacity of 8 Mbytes. There is one such storage memory in each MPA.

The fondamental objectives of the MIDAS facility are to provide:

l. high-speed data processing

2 optìnized data transmission rates.

3. high interactive capability,

4. modular construction, and

5. ease of usage.

The architecture described here provides a framework in which these goals appear achievable. The besic design was presented to and approved by a special workshop consisting of hardware and software representatives from varjous National Laboratories, universities, and private industry. The construction and development is planned in four stages, each leading to a viable and stable configuration, as well as serving to test certain basic architectural aspects.

\section{Foothote and Reference}

"Condensed from LBL-12504.

1. Creve Maples et al., IEEE Trans. Nucl. \$ci. N\$-3764, (1981).

\title{
Development of a MIDAS Prototype ${ }^{*}$
}

\author{
Creve Maples, Mlitam Rothbut, Dantel Weaver, and John Meng
}

In 1979 , a proposat ${ }^{1}$ was made to deveiop a Modular Interactive Data Analysis System (MIDAS), that utilized a parallel provessor architecture to achieve high-speed, interactive reduction and analysis of experimental data. In February 1980, after the completion of a detailed design document, a workshop (attended by scientists, electronic engineers and designers, computer scientists, and software systems specialists from several universities and National Laboratorim) was held to discuss and dissect the detailed proposal. A number of design modifications and enhancements resulting from this workshop were subsequently incorporated into the basic architecture of the system Software models, emulating the architecture of the system, were constructed to predict the effect of various hardware design considerations on the overall system throughput. Theoretical determinations of the critical paths and operational performance constraints are, however, very difficult in such a highly parallel and asynchronous environment.

The development of the proposed architecture into a working facility, MIDAS, was planned in four separate phases: I) a procotype, 2) a working model, 3) a corrpiete system (3 to 5 users), and 4) Juture extensions. Each phase was designed to provide particular capabilities and to test the viability of different aspects of the architecture. These discrete development phases would, furthermore, permit detailed evaluation of the project with respect to estimated cost. manpower, development time, and perfor* mance. The goals of each phase are well defined and each represents a working system. This report deals with the first or prototype phase, a schematic outline of which is shown in Fig. I.

Hardware construction of the basic prototype began in May 1980. In terms of the previously defined architecture, ${ }^{2}$ the prototype is an abbreviated version of a single Distributed System-a Secondary Computer and a simplified Multi-Processor Array (MPA) - plus one element of the Mass Storage System, a disc with a Multi-ported Programmable Controller (MPC). The purpose of Phase I is to lest the performance and control of the MPA. This unil contains all of the high-performance design features 


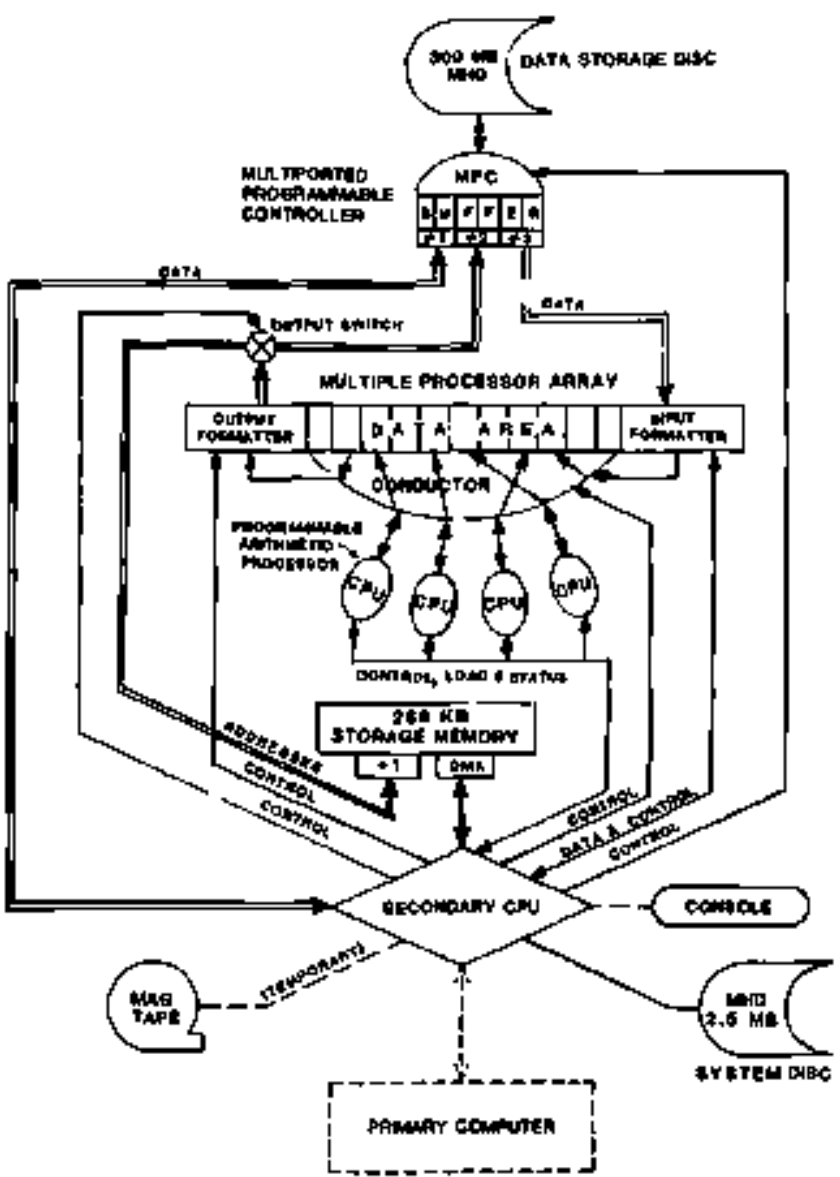

Fig. 1. Schematic Layoul of MIDAS Phast $\downarrow$ Prototype.

(XBL. 815-9787)

that are essential to the architecture: asynchronous paralle] processors and parallel multi-bus operation. Due to the absence of a Primary Computer in this development phase, the Secondary has been given more capability (memory, peripherals, etc.) and responsibility than would nomally be required. For example, in this prototype it is responsible for loading data onto the disc through the MPC and for direetly controlling the MPC. Such direct connections between a Secondary Computer and the Mass Storage System will nol exist in the general design.

The Phase 1 prototype of the Multi-Processor Array contains: two I/O processors (the Input and Output For- matters); four general purpose, parallel CPU's (Programmable Arithmetic Modules, each with 128 Kbytes of memory); the bigh-speed bus switching controller (the Conductor); tight Data Stacks (each with independent buses and $16 \mathrm{Kbytes}$ of memory); and a dual-ported Storage Memory (with $\$ 12$ Kbytes of memory). The development of a PSM ${ }^{2}$ (intended to perform mesking, gating and logical tests at very high speeds) is not essential to the basic design or operation, and it is therefore not included in the Phase I development. The goal of highspeed data processing is achieved by utilizing multiple, high-level CPUs operating asynchronously and in parallel on the data stream. The transmission of data, or $1 / 0$ throughput, is optimized by utilizing multipte $1 / O$ buses operating in parallel, by minimizing the sctual movement of data, and by completely overlapping I/O and CPU operations. Both of these bjectives ase tested in the Phase 1 developrnent.

This protocype is scheduled for completion in surnmer 1981. At this time, benchmark tests will be run on the model to compare processing capability, both with curtent computers and with the performance predicted by our software models. The latter is an important comparison since knowing the accuracy of the software architectural model makes possible more precise predictions of the capabilities of the future development stages. The comparison tesis will be pertorcued as far as possible, by analyzing real experimental data, which utilizes identical soltware on both the Phase I prototype and existing computers. Because performence is potentialy sensitive to the dependence of a particular analysis on J/O versus CPU utilization, tests will be conducted on both CPU and [/O-intensive problems. Although generalized predictions are difficult, the results of model calculations suggess that the Phase 1 protoxype should give a factor of 6 to 12 increase in throughput compared to our current (ModComp JV-25) computers. The hardware cost of the Phase I system is expected to be approximately $\$ 110 \mathrm{~K}$ and is estimated to require 4 man-years of hardware effort and 2 of soltware effort.

\section{Footnote and References}

"Condensed from LBL 12504.

1. Creve C. Maples, IEEE Trans. Nucl. Sci. Ns-26, 4580 (1979).

2. Creve Maples et al., JEEE Trans. in Nuct, Sci. N-28, 3746 (1981). 


\title{
The Utilization of Parallel Processors in a Data Analysis Environment
}

\author{
Creve Maples, Daniel Weaver, Wilfiam Rathbun, and John Meng
}

In many research environments, data are accurmulated as a series of information packets or events. These events consist of discrete pieces of information, or parameters, which are essociated with the detection of the event. Recording these events as they occur in real-time permits later reconstruction of the data off-line and allows more detailed examination and evaluation of the information. In nuclear and particle physies, the pest decade has witnessed an explesion in the volume and complexity of recorded experimental data. The complexity of modern experiments is, in lasge part, caused by the increasing rarity or subtlety of the physical processes under examination. This in turn leads to an increasing complexity in the data reduction and analysis. It is not uncommon for basic data analysis to require many days of computer processing, in some cases as much as 10 times longer than the data collection process.

In 1979 , a proposal was made to develop a specialized computer facility specifically designed to deal with the rapidly escalating problems associaled with interactive data reduction and analysis. This propased design utilized a bierarchy of multiple, higb-level processors to permit high-speed parallel analysis of data. The besic siructure of data (discrete event packets) makes its analysis ideally suited to paraltel processing. In this framework, ewents are analyzed asynchronously and in parallel by multiple CPUS as opposed wo the serial one-event-at-a-time appoach of conventional computers. Such a design is now practical because high-level CPUs are comrikrcially available at a relatively modest cost. Construction of the proposed Modular Interactive Data Analysis System (MIDAS) was begun in May 1980.

Essentially all the processors employed in the MIDAS design are full, mid-range compuiers, not microprocessors. This level of processor is necessary to maintain overall flexibility, provide the necessary cornguting power, and permit parallel otilization of standard user code. Although MIDAS is generally designed to deal with problems astociated with interactive data processing, the actual analysis programs typically will be user generated and will vary widely in terms of their application and the dernands placed on the facility. It is therefore essential that the system be capable of employing code written in essentially standard high-level languages (e.g., FORTRAN), in the multipłe-processor environment and in a manner basically transparent to the uses.

The architecture adopted for MIDAS is a distributed processor environment organized in a "vertical tree" structure. This structure utilizes a master-slave hierarchy for control and cornmunication. In this environrnent, processors at each Jevel of the "tree" control the processors in the branch directly above and report to the processor directly below. There is essentially no direct communication between processors at the same level. Such an architecture greatly simplifies operation and comununication in a highly parallel, asynchronous environment and avoids many dificulties encountered in more traditiona] computer networks. Detailed information on the MPDAS erchitecture, design considerations, and dewelopenent plans are presented elsewhere. ${ }^{23}$

The processors are organized into three functionally distinct systems controlled by a central computer referred to as the "Primary." Each system consists of a number of processors wjth specjfic responsibilities. The following is an outline of the control structure:

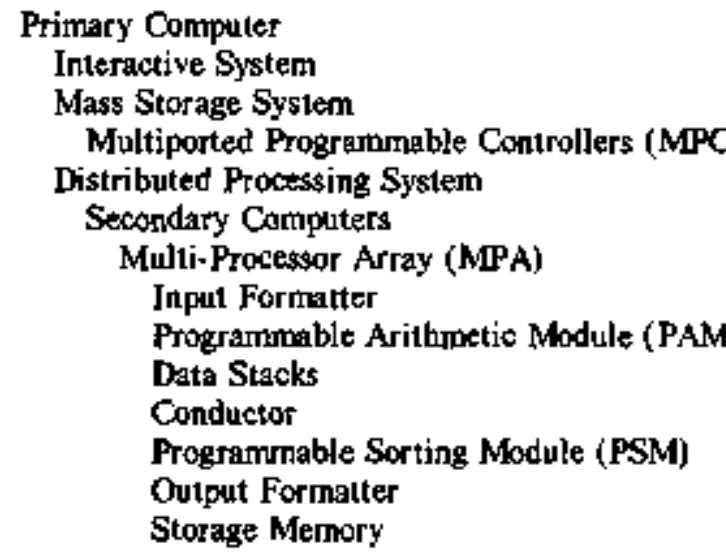

A schematic illustration of the organization of the major processing elements is provided in Fig. 1. A rather high degree of parallel operation is inherent in this structure. The Primary Computet and the three systems it controls - Interactive, Mass Storase, and Distributed - all operate in parallel. The Interactive System in Fig. I consists of five independent processors while the Mass Storage System utilizes three classes of processing units (for disc, lape and bulk memory) involving five Multiported Pro. graminable Controllers. The Distributed Processing System consists of five Distributed Subsystems, also illustrated in Fig. 1. Each Subsystem (a Secondary Compuier and Multi-Processing Array) operates in parallel, both externally, with respeet to other \$ubsystems, and internally with respect to the Secondary Computer and the Multi-Processor Array. The Multi-Processor Array consists of multiple processing units which operate asynchronously and in parallel.

Figh-speed data processing is the specific respontsibility of the Distributed Subsystems. Each of these consists of two processing levels, a Secondary Computer and a Multi-Processor Array. The Secondary is responsible for the particular single analysis problem delegated 10 it by the Primary: and it, in 1urn, controls a Muli-Processor Array. Although the actual configuration of this arcay is flexible, for the purpose of discussion it will be assumed to consist of 11 programmable processors, two logic units ssociated 


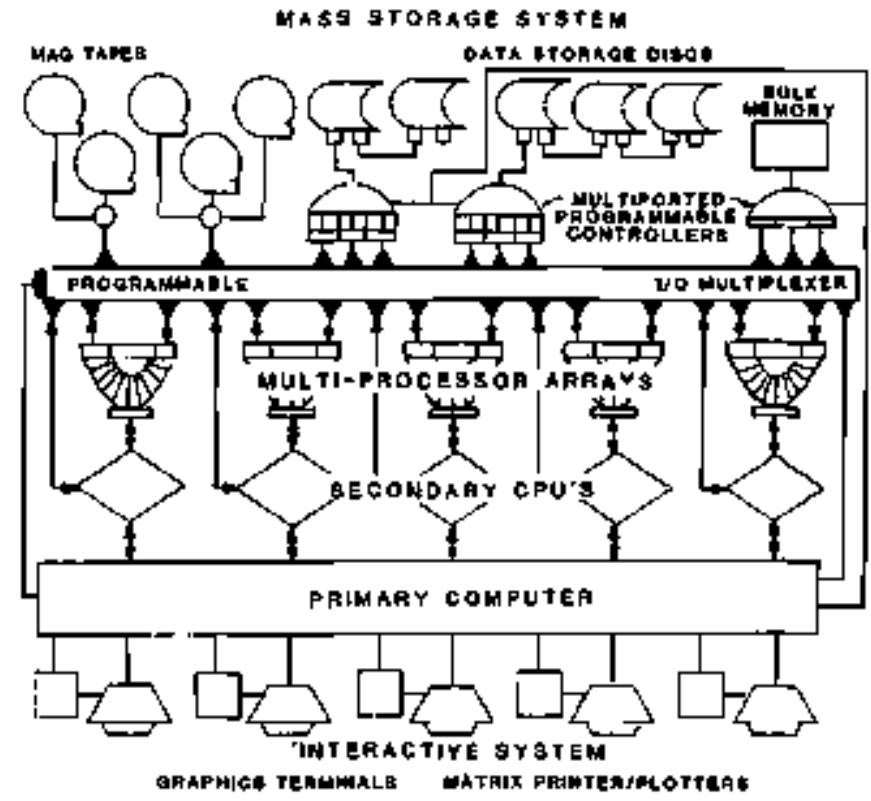

Fig. 1. Organization of mejor processing elements in the MIDAS design.

(XBL 858-11277)

with memory (or bus) switching, and a local memory for storing results. The 11 processors include 8 general purpose CPUs and three specialized units. Two of the specialized units are pipelined $1 / 0$ processors' and the third is designed for high-speed data pattern recognition. The function, operation, and control of the various processors, utilized within the MIDAS design to achieve high-speed data analysis, are reviewed in Ref. 2.

The process of analysis may be divided into four general stages:

1. problem definition,

2. setup and initialization.

3. data reduction and analysis, and

4. presentation of results.

The general application of parallel processing to each of these stages is oullined below (more detailed information is contained in Ref. 4). In defining the problem, a user would typically be involved with two processors, a dedicated unit of the Interactive System and the Primary Computer. Al this slage of operation, the functions of the Primary Computer are to respond to user requests for information and to store information from the user pertaining to specific analysis requirements. Once the problem is specified, the Primary will initialize the necessary units in the Mass Storage System and will allocate a Distributed Subsystem to perform the actual analysis. During initialization, the Primary would use the I/O Multiplexer to connect the input and output channels of the selected MultiProcessor Array with the appropriate Mass Storage ports. The analysis specifications are then transmitted by the Primary Computer to the selocted Secondary. These specifications might consist of a list of data transformations or actual analysis code, an array defining sets of sorting conditions or logical correlations which are of interest, an artay describing the desired formatting of the resuits, and information on the structure of raw data. The analysis code will be compiled and linked, if necessary, to form an analysis task. This task will typically then be loaded by the Secondary into each of the PAM (Programmable Arithmetic Module) CPUs. Each PAM unit is a high level combmercial computer, stripped of everytbing but processor and memory. These modules will execute the down-losided tasks directly with essentially no operating system, but with certain error traps operational.

The actual analysis operation is carried out in a Multi-Processor Array under the supervision and control of a Secondary Computer. After the Secondary Computer has completed initialization of the MPA it begins the analysis operation by starting the Input Formatter which is directly connected to a porl in the 1/O Multiplexer. The Distributed Subsystem need not know what device will be transmitting information to this port because all transmissions across the $1 / 0$ Multiplexer follow the same protocol. A virtual disc port could, for example, be supplying date to the Input Formatter. Since the disc MPC was initially supplied with a list of the requisite data, the first 32 Kbytes of information have been retrieved from disc and are stored in memory buffers of the virtual port. When the lnput Formatker acknowledges the data-ready flag, a direct-memory transfer of data begits.

The presentation of analysis results to the user should be considered in two time frames, preliminary results during analysis and final resuits at the conclusion of analysis. During the anslysis process, a user may wish to receive various information on its progress. Specific requests for information are received by the Primary Compuler which normally transiers them to the Secondary. The Storage Memory, where local results are stored, has a direct DMA connection to the Secondary Computer. At approprjate times the Secondary, if requested, will obtain a copy of the current results from this memory and transfer them to the Primary. Once in the Primary, this preliminary information may be manipulated and examined at will by the user through the Interactive System. In this manner, continuously updated results may be supplied to the user without interfering in the analysis process. If these preliminary results are not satisfactory, a user may immediately abort the analysis rather than waiting for its completion.

The goal of the MIDAS design is to provide an individual user with high-speed analysis capability. Sixteen processors, operating asynchronously and in parallel, are dedicated to each user during antalysis. This uilizes only one Distributed Subsystem of the five shown in the basic design. The MIDAS structure shown in Fig. I has " pro cessors and can handle five simultaneous analysis problems. Because of the architecture, these independent analysis problems can be run at the same time and with essentially no interference; that is, each analysis will be carried out at the same speed whether or ato the others are present.

The operating structure of MIDAS supports a considerable number of processing units, independent $1 / O$ buses, mass storage devices, and a substantial amount of memory and is reasonably tolerant of hardware failures. The functional segregation of operations, the duplication of 
hardware, and the dynamic ability to allocate resources stould greatly reduce the seriousness of most hardware failures and generally permit quick secovery and continued operation. The flexible, modular framework of the MIDAS system permits the simultaneous exceution of different analysis problens, each of which vtilizes multiple processors. The structure further permits the dymamic allocation of resources according to the particular needs of each problem Finally, the modular nature of the design and the independent asynchronous operation of each processor should permit the relatively simple incorporation of new technology in the future.
Footnote and References

"Condensed from LBL 12505.

1. Crew C. Maples, IEEE Trans. Nucl. Sci. MS-26, 4580 (1979).

2. Creve Maples et al., IEEE Trans. Nucl. Sci. NS-28. 3746 (1981).

3. W. Rathbun et al., IEEE Traps. Nucl. Sci. NS-28, 3875 (1981).

4. C. Maples e1 al. IEEE Trans. Nucl. Sci. NS-28, 3880 (1981).

\title{
A Fast, Time-Sliced, Multiple Data Bus Structure for Overlapping I/O and CPU Operations
}

\author{
W. Rathbun, C Maples, J. Meng, and D. Weaver
}

\begin{abstract}
Within the Nuclear Science Division of LBL, a specialized Modular Interactive Data Analysis Systern (MIDAS), designed specifically to reduce the time requised for interactive dats analysis, is being constructed. The general architecture of this system has been discussed in detail elsewhere. ${ }^{1 \cdot 3}$ Basically, analysis time is reduced by minimizing the movement of data and maximizing the use of parallel operations in both data transfer $(1 / O)$ and data processing. Through the multiple data bus structure described in the following paragraphs, several independent processors operate asynchyonously and in parallel on large blocks of event-structured data loaded into sixtoten indepen dent memories or data stacks. This structure allows large blocks of data to be rapidly switched among various processors, in contrast to conventional word-by-word bus movement of data.
\end{abstract}

The Data Stacks provide a key element in the MIDAS system's high-speed parallel operation. Various processors comprising the Multi-Processor Array can be electronically connected to the Data Stacks in a predelermined sequence. One of these processors, the Input Formatter, is a specialized hardware device developed to unpack an input stream of event-structured data and fill any stack requesting new data. A second lype of processor consists of a strippeddowa version of a comnercially-produced $C P U$ with a fast arithmetic processor. As many as eight of these CPU-iype processors, Programmable Arithmetic Modwles can operate in paraljel on data in a given user's data stacks. A third type of processor, the Output Formatler, unpacks results stored in the ata stacks and passes them to another processor, ultimately to be graphically communicated to the user. Other processors bave been conceived and can be added to the parallel system as the applicalion may dictate. When one processor has completed its work on a given stack, another processor can be rapidly connected to the stack to perform additional operations as required. These operations may include calculations or data transforma- tions, may serve to create an augmented data base upon which future calculations can be performed, or may provide for the tabulation of results for ulimate prisentation in a manner selected by the user. The following sections detail the switching and control logic which manages the communication with the Data Stacks.

Event-structured data loaded into the Dala Stacks can be accessed by any of several classes of processors in a programnlable manner. These processors, discussed in detail elsewhere, 2,3 an operate simultanecusly and asynchronously in a sequence determined by the user or the analysis program Hardware logic known as the Conductor handles this dyoamic interconnection sequence.

Current hardware permits as many as eight CPU's and four other processors to be simultaneously connected to separate Data Stacks. Each processor operates on its currently assigned stack asynchronously and in parallel with all other system operations. Each Data Stack appears as a dedicated memory block to the currently-attached processor. When a processor has cormpleted worlong on a given set of data, it signals the Conductor to 5 witch the assecialed Data Stack to the next proctessor type in a userdetermined sequence. Several types of processors ean be sequentially mapped to a given stack.

The key to this switching process is a simple Connect Module illustrated in Fig. 1. Each device capable of passing data into and out of the Data Stacks has a dedicated Connect Module comsisting of an Input F1FO, a Connect Register, and an Output FIFO. The Input FIFO holds up to sixtoen 4-bit stack numbers, specifying the list of Data Stacks 10 be worked on by the processor. The Connect Register holds the current 4-bit stack number (connect code), which serves to gate the data bus of the associated processor into its currently dedicaled Data Stack The Ouiput FlFO holds the connect code of the stack just processed so that it can be passed into the lnput FIFO of the 


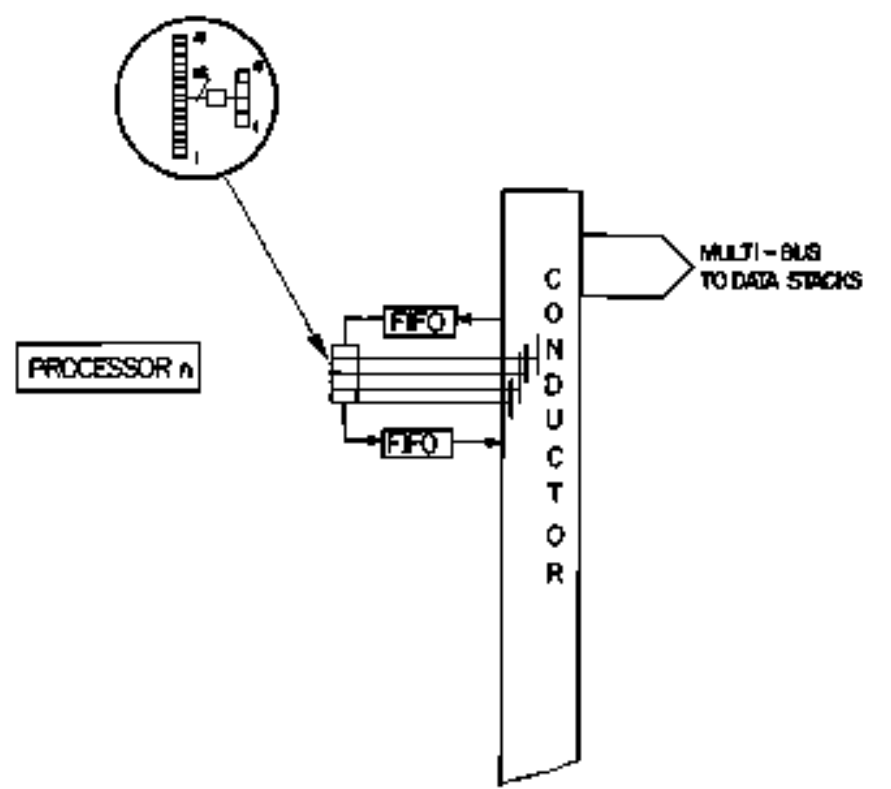

Fig. J. Details of a Connect Module, which interfaces a Data Stack to a selected processor. The inset illustrates how a four-bit connect code is decoded to enable one of sixteen sets of gates.

(XBL 816n]0208)

next processor of the pre-loaded sequence. Associated with each Data Stack is a control block, which contains the order in which the processors are to be attached to that stack. This sequence is initially downloaded by a Secondary Computer that oversees the operation of the parallel proctssors.

To reduce the large number of interconnections potentially possible in the Multi-Processor Array (up to 12 active processors connected to eny of 16 Date Stacks), two classes of data buses have been implemented: (a) a timesliced bus through which CPU-type processors can access their respective Data Stacks and (b) standard $\mathrm{H} / \mathrm{O}$ buses that are connected to all other processors of the MultiProcessor Array. Figure 2 shows how all processors are connected to the read and wite gates of all stacks through Stack Gates. When a processor has completed its operations on a given stack, it signals "done" to the Conductor; this causes the next connect oxde in the processor's lnput FIFO to be shifted into the Connect Register, enabling the set of gates connecting this processor with the desired stack. These gates stay enabled until the current processor has signaled that it has finished with the stack, except for the time-sliced gates connecting the CPUs to the Date Stacks. These time-sliced gates are reloaded at each phase of the time-slice, as deternnined by the contents of the Connect Register of the associated CPU. This gate switching can be accomplished in approximately thirtetn nanoseconds. In the time-sliced environment, the eight CPU-type processors of the Multi-Processor Array share a common set of gates into the Data Stacks. A time-slicer scans all CPUs every J00 namoseconds. Any CPU requesting a data transfer into or out of a stack is allocited an

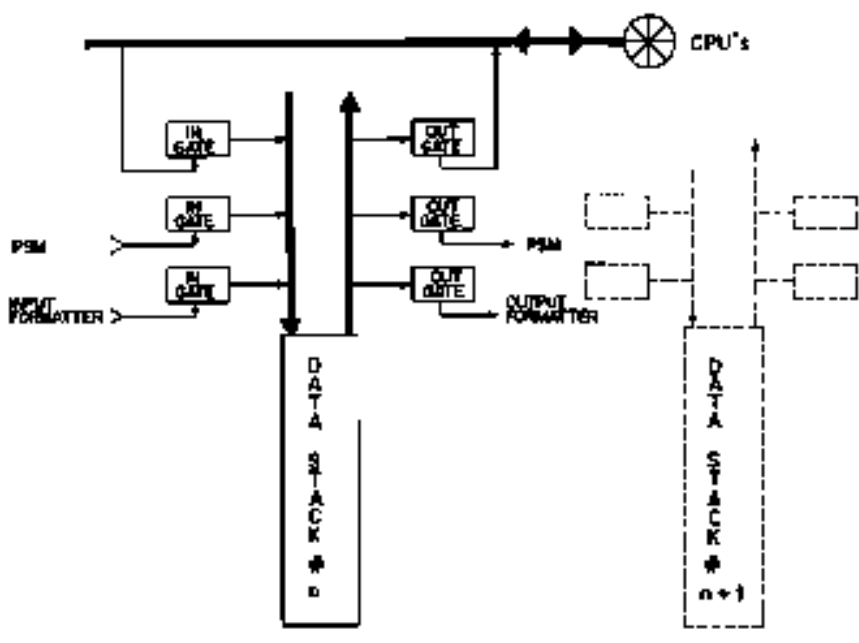

Fig. 2. The Stack Gates which connect all processors of the Multi-Ptocessor Array with all Data Stacks.

(XBL 816-10207)

extended cycle of approximately 50 nanoseconds. A write operation can be accomplished in a singlo cycle, but a read operation requires two cycles: the first cycle strobes the address and the data recquested are available by the next cycle. At each phase of the time-slice, the Connect Regis. ter of the associated CPU, if active, is decoded into appropriate stack-enable gates which connect the processor to the associated stack. When a CPU has finished processing a stack, it generates a signal that causes the next stack number (comnect oode) in the associated Input FIFO to be shifted into the Connect Register, enabling the read and write gates that connect the processor to the proper stack.

The goral of the MDAS system is to provide a highly effective tool for the interactive analysis of experimental data. The huge volume of data to be processed necessitates a high degree of parallelism, both in 1/O and CPU. intensive operations. The use of multiple data buses that connect independent Data Stacks to selected parallel processors provides the framework to attain the desired throughput. While the MIDAS system is designed specifically to facilitate the analysis of data from experimental physics, the erchitecture should adapt well to a wide variety of analysis problems, including medical and geophysical applications. Additionally, the modular strueture allows processors or peripherals to be upgraded or replaced as advancing technology dictates.

\section{Footnote and References}

"Condensed from LBL-12498.

1. Creve C. Maples, IEEE Trans. Nucl. Sei. NS-26, 4580 (1979).

2. Creve Maples et al., IEEE Trans. Nucl. Sci. N's-28, 3746 (1981).

3. Creve Maples et al., IEEE Trans. Nucl. Sci. NS-28, 3880 (1981). 


\title{
Multidimensional Numerical Integration*
}

\author{
R.W. Koontz
}

Computer trodels of experimental apparatus and of particle interactions are an essential part of modern nuclear scitence. For many applications, this modeling process reduces, at least in part. to numerical evaluation of rmultidimensional integrals. Nomerical evaluation of sucb integrals, however, is generally not a simple task and of tep iowolves montbs of program desigm and testing. Thertfore, there is considerable opportunity for resource saving by consiructing a general purpose program that does multidimensional integration in an arbitrary number of dimensions.

I bave constructed a multidimensional nomertcal integration program called "INTGRL" that has been tested on integrals with dimensions up to ten with good accuracy. An 8-point Gavss quadrature rule, exact up to polynomials of order 17 in one dimension, is used as the basic panel rule. A complete description of the program may be found in Ref. 1.

\section{Footnote and Reference}

*Is preparation for publication in Nuclear Instruments and Methods.

1. R.W. Kooptz, Lawrence Berkeley Laboratory Report LBL-12892 (1981).

\section{DATACQ, A New Data Acquisition Program}

\author{
Friedemsnn Welk"
}

Most data acquisition installations at the Bevalac use $\mathrm{QDA}^{1}$ to transfer the data from CAMAC via a micro programened branchdriver (MBD) into a DEC PDP-1]/34 (or 11/45) and from there to magtape. However, if the size of an event is of the same order as the buffersize, as can be the case at the Plasticball ${ }^{2}$ or HISS, ${ }^{3}$ the restriction to fixed size buffers and a synchronous consumication with the MBD may waste a lot of memory and lead to increased deadime.

To handle those data toote efficiently, a new data acquisition program DATACQ, Was developed. Since its overall structure is not too different from QDA, modifications to the user-part of existing MBD-code are minimal. The use of conditional assembly directives can make them run in both systems.

The handling of variable size boffers is dome in the following way. Injtially, DATACQ hands as much contigucas memory to the MBD as possible, plus a value indicating the "wanted buffer size." On an intersapt from CAMAC (LAM), the MBD obtains from a specific userentry the length of the new event and checks if this (pending) event fits into the remaining memory and if the curreat buffer size plus the length of this event are smaller than the "wanted buffer size." If both checks are valid, the event is stored in the buffer. If the second condition fails, the current buffer is marked as a complete buffer by interrupting the computer and sending the correlated control ioformation. Since the pending event would fit into the remaining memory, the beginoing of a new buffer is marked by inserting a 6-word header, and the event is stored in the new bufler without waiting for the acknowledgment of the internupt. A wait is only imposed if there is not enough memory for the pending tvent. As soon as at least the memory needed for the pending event is available, the MBD continues. If, on the other hand, a memory block, which is contiguous to the one known by MBD, becomes available, it is handed to the MBD and is seen there with the next event.

DATACQ handles the end of buffer interrupt from the MBD by updating, appending, or inserting into its table of the currently defined bufters. At that time, the new boffer is marked for transfer to magtape, to disk, or into anotber much larger buffer area. The transfer of the variable size buffer is asyochronous and is handled on a first-in first-out mode.

A preliminary version of DATACQ, with parallel transfer to disk and magtape but without the transfer to a larger buffer, has been successfully used with HISS.

\section{Foomote and References}

"Gesellschaft fuer Schwerionenforschung D-6100 Darmstadt, West Germany.

1. E. Harvey, JEEE Trans, Nuct. Sci. NS 26, 4633 (1979).

2. A. Baden $t$ al., contribution to this annual report.

3. C. McParland et al., contribution to this annual report. 


\title{
An X-Y Histogramming Package Added to MULTI
}

\author{
Friedemann Weik
}

The current version of MULTI ${ }^{1}$ is use at the Bevalac data acquisition computers (PDP 11/34 or 11/45) normally utilizes only one graphics display (Tektronix 4012) at a time. To get X-Y information, one has to tie up this graphics display with a scatterplot; the storage screen of the display is the only medium that can hold this information.

To improve this situation, a package to create, store, and display two-dimensional histograms was added to MULTI. The peckage itself consists of several programs, which reside in $3-9$ tasks outside of MULTI, which has the ability to control those tasks and send dala to them for storage. Many of the programs are written in MACROassembler to optimize speed and minimize the memory needs. Communication relies heavily on the SEND/RECEIVE DATA and the mapping facilities of the RSX11M operating systern.

Instead of reserving one place for each passible $\mathrm{X}-\mathrm{Y}$ combination, only the points with actual counts are stored in an "ordered" way. This will be explained in the following exantele: A AE-E bistogram for the Plastic Ball module \#586 was defined to aceepe for $E$ (DA803) and $\Delta E$ (DAl\$1]) the channel range from 10 - 2000 with every 10 channels contracted to $\mathrm{l}$. The storage area should not exceed 8192 words and the histogram was named MOD586. The definition command in MULT] (written as one line) for that was:

\section{H2 DA803 DAI811 MOD586 \$192 10 $2000 \quad 10 \quad 10200010$}

To store the "used" points in 8192 words instead of 40,000 , the following scheme was used. For each Y-Jow with al least one count, there is a word with the Y-value of that row plus another word to tell how many X-points of that row contain counts. There is ont word for each of these X-points with the (contracted) X-coordinate informs tion stored in their lower bits. The remaining bits give the number of counts in the bin. Higher $X$-resolution means fewer bits available for counting contents before overflow is reached, In this example, $X$ janges from 1 to 200, requiring 8 bits. This leaves 7 bits $(=127)$ for contents and one bit for the sign indicating a bin overflow.

Every new point has to be inserted by mowing al] higher points one place up in memory until all avajlable space is used. In case of a space overflow a message is printed and no new points are inserted after that printout.

The display of the histograms is done by another task, which gets its commands from MULTJ and which maps to the selected date aree. There are three display modes: a simple isometric display (Fig. 1), a scatterplol with randomization around the contracted ares to stow the intensity (Fig. 2), and a contour plot (Fig. 3). The randomization can be switched of to speed up the display and to show the actual location.

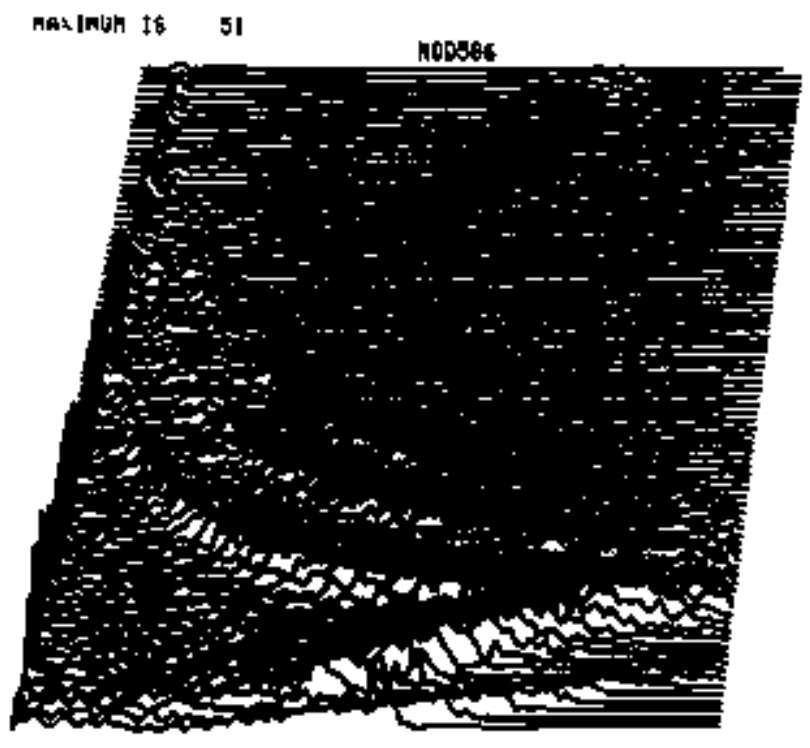

Fig. I. Isometric display.

(XBB 818-7793)

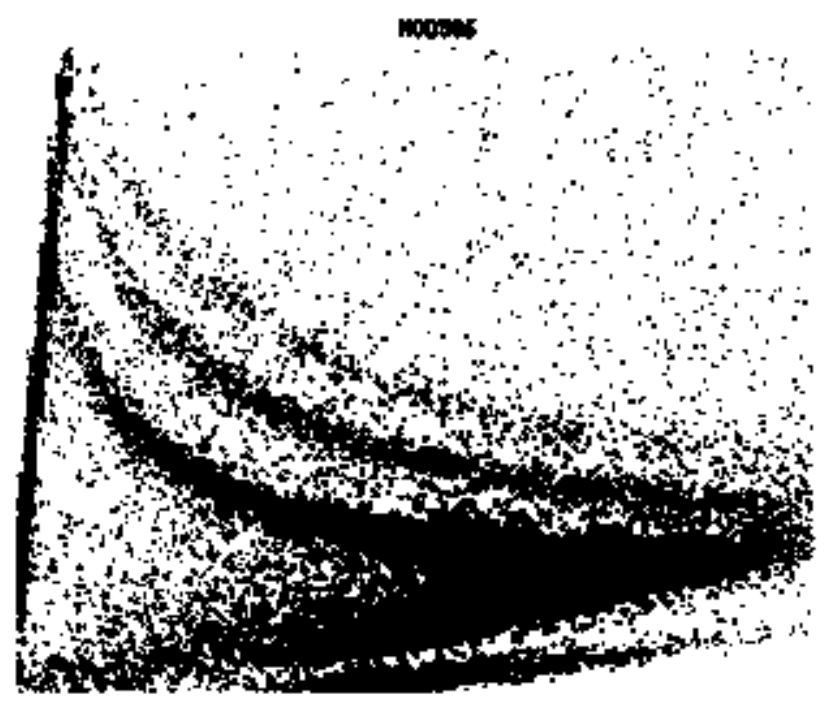

Fig. 2. Scalter plot.

(XBB 818.7792) 


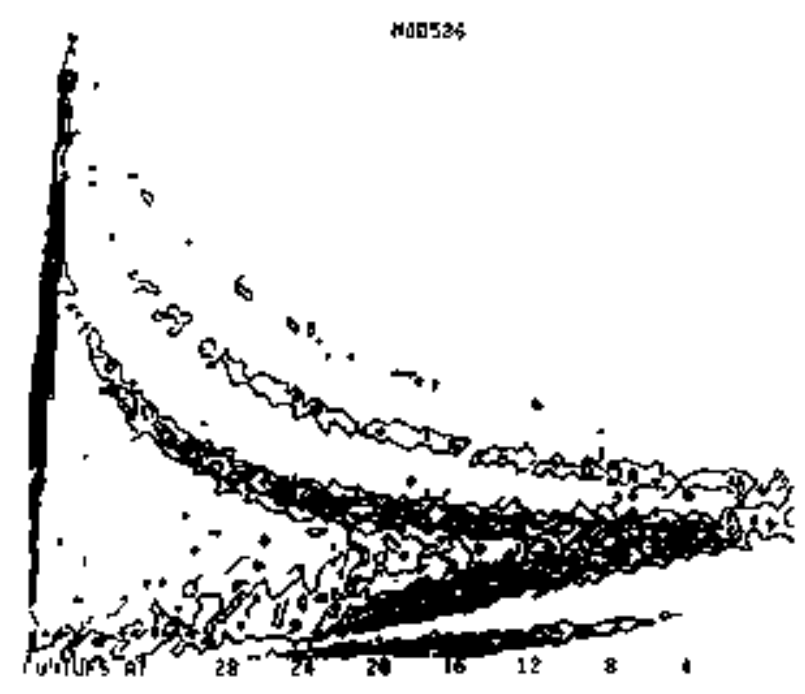

Fig. 3. Contour plot.
Footnote and Reference

"Gesellschaft fuer Schwerionenforschung D.6100 Darmstadt, West Germany.

I. J.F. Bartlett et al., FERMILAB Report PN-97.4.

\title{
Data Acquisition at the HISS Facility
}

\author{
Charles McParland"
}

Experimental data at the HISS facility has been acquired and analyzed with a system of loosely coupled computers comnected by a shared mass storage disk. Data from various detector systems are routed vja CAMAC and an intelligent branch driver (MBD) to one of three Irontend PDP-1l computers. These machines acquire variable length data events and log them to local maguetic tape. Displays of spill-by-spill diagnoslic information as well as current data set statistios are compiled and displayed in the experimental area.

In addition to tape logging, data are written to a shared 80-megabyte disk sysiem This disk contains three large data fles, one for each front-end computer. These files are organized as circular buffers and always contain the "most recent" experimental data. Each front-end computer has write access 10 its data file; all other accesses are read only. Thus, each front-end data file on the shared disk appears similar in both content and formal to the most recent section of experimental data tape (aboul 20 megabytes).

Coupled to this shared disk system is a VAX $780 \mathrm{com}$ puter. Although this general purpose machine is not directly involved in experimental data acquisition, it does have acoess to the "most recent" experimental data.

A collection of subroutines provides a simple user interface to data files. Users can connect to any front-end data file and access data Irom any location within these files.

One unique feature of this user interlace is the ability to access any source of experimental data, either current "live" data or previously tecorded data residing on disk or 1ape. Users can easily select any source of dato for their analysis programs. This enables one set of programs to be developed for both on-line data analysis and off-line data reduction. This feature is expected to reduce the development time for future on-line analysis programs and greatly improve the quality of experimental diagnostic tools.

\section{Footnote and Reference}

"Also Space Science Laboratory. l. See contribution of $\mathrm{F}$. Weik in this annual report. 


\title{
Data Acquisition Setup for the Plastic Ball (488H)
}

\author{
Friedemann Weik
}

Since a previous experiment $(489 \mathrm{H})^{1}$ of moderate size with 455 datawords/event touched the limits of the standard data acquisition system in use at the Bevalac, ${ }^{2}$ a more powerful system had to be used for the Plastic Ball experiment $(488 \mathrm{H})^{3}$ which is capable of generating up to 3673 data-words/event. Since an improvement in memory size and in computing power was needed, a solution with more than one computer was sought. To keep the additional effort for system development and management to a minimum, a second computer of DEC's PDP II-series $(11 / 44)$, which uses the same operating system as the existing $1 \mathrm{l} / 50$, was selected.

The resulting layout of the data accuuisition setup is shown in Fig. 1. The experimental data are collected in CAMAC ADCs and TDCs. Each ADC-crate has its own processor (LeCroy 2280) to subtract pedestal values of 'the ADCs and to suppress empty channels. An equivalent gystem (LeCroy 4290) is used for the long range (10 us) TDCs. Since standard eight-fold TDCs had to be used for the TOF-measurement in the Plastic Ball, the data ompression is done by the micro programmed branch driver (MBD) during the transfer from CAMAC into the memory of the PDP 11/50.

The layout for the PDP 11/50 follows the general rules for the Bevalac data acquisition system (248 kB memory, MBD, 2 magtape drives, $10 \mathrm{MB}$ disk capacity, RSXI IM operating system) with which an adjusted version of the QDA / MULTI system could be used. The QDA / MULTI system has a clear cut between data acquisitiont (QDA) and monitoring (MULTI). This cut was used for the implementation into a two-computer system. having the PDP 11/50 to deal only with data-taking and the PDP $11 / 44$ with monitoring osing its increased power.

The original transfer task between QDA and MULTI (MULQDA) was broken up into two tasks, one for each machine. A relative fast link (DAl 1B) supported by the operating system couples the two computers. The PDP 1]/44 with an $80 \mathrm{MB}$ disk, a cache memory, and $248 \mathrm{kB}$ memory is connected to three Tektronix graphics terminals, all of which can be used simultaneously by MULTI. Additional standard terminals and the line-printer are used during program development. The $80 \mathrm{MP}$ disk is dual-ported ( $\$$ [9400 controller, DM980 drive) and can be accessed by the PDP 11/50. This connection, however, should only be used to transfer files to the PDP 11/50 and to backup the disk to magtape.

To supplement the hardware improvements, a new data acquisition program is developed wjth variabie blocksize and follow-up buffering (DATACQ) ${ }^{4}$ A preliminary version of this program (including disk-spooling) has successfully been used during HISS-experiments. ${ }^{4}$ MULTI has been broken into about 20 tasks to overcome the sizelimitations and to imptement new features. ${ }^{6}$ Further specialized monitors and display programs are planned.

Footnote and References

'Gesellschaft fuer Schwerionenforschung D-6100 Darmstadt, West Germany.

1. A. Baden et al., contribution to this annual report

2. C. McParland, Lawrence Berkeley Laboratory Nuclear Physics Division Annugl Report 1979-1980, LBL-11588, p. 200 .

3. A Baden et al., see "DATACQ, a New Data Acquisition," in this ansual report.

4. F. Weik, contribution to this annual report.

5. C. McParland at al., contribution to this annual report.

6. F. Weik, ste "An X-Y Histogramring Package Added to MULTI," in this annual report.

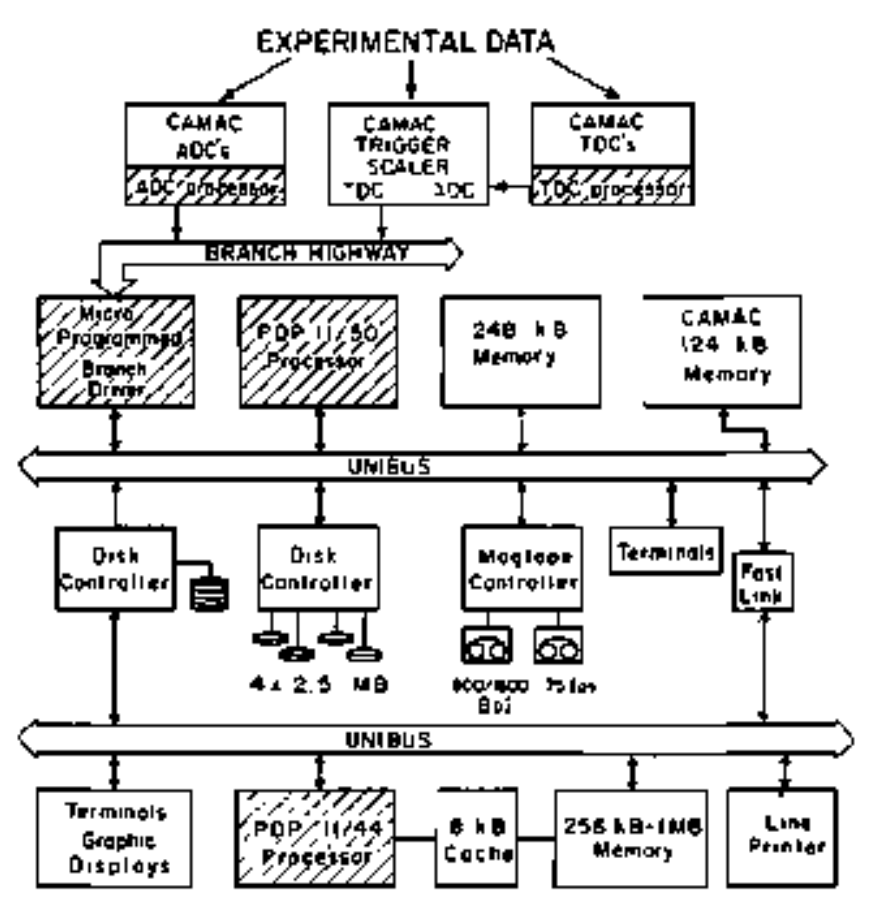

Fig. 1.

(XBL 809-1959) 
IV. ACCELERATORS 


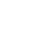

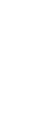

4 .

- 


\title{
Accelerators
}

\section{8-Inch Cyclotron Operation}

\author{
D.J. Clark. C.M. Lymeis, D. Elo. L Glosgow, $R$ Lam \\ T. Mast, $R$ Muller, P. Tans, and J. Welch
}

The 88-Inch Cyclotron, operated by the Nuclear Science Division, provides a large fraction of the beam time that is used by Division scjentists. Variable energy, highresolution beams from bydrogen through argon are produced and used for studies of nuclear stricture and nuclear reaction mechanisms. The 88-Inch Cyclotion is also the Laboratory's major source of medical isotopes and its only source of polarizod proton and deuteron beants.

The large energy constant, $K$, recently increased to 160 , permits the acceiteration of ions as heavy as ${ }^{40} \mathrm{Ar}^{+}$to energies useful for nuclear physics. The lighter heavy ions reach energies up $1030 \mathrm{MeV} / \mathrm{u}$. This cyclotron is one of the few sources in the world of variable energy heavy ions in the important transition region between low and high energies: $10-30 \mathrm{MeV} / \mathrm{u}$.

These beans, as well as the polarized proton beams, ate extensively used by outside groups from many institutions in the U.S. and abroad. In recent years, about $25 \%$ of the operating time has been taken up by outside users. Proposals are made by fetter and are informally reviewed by two oulside consuliants. Approval can be made within two weeks, and the experiment can be scheduled wthin two weeks thereafter. This exonomical and flexible system is much appreciated by the users.

\section{Operations}

In the year onding June 30, 1981, the 88-Inch Cyclotron was schoduled for 20 eight-hour shifts per week for experinents in muclear science, isotope production, and machise development. One eight-bour period per week was scheduled for routine maintenance. In addition, there were 6ye werks of shutdown for general mainlenance and machine improvements, and because of budgetary restric tions. Accelerator time distribution is shown in Table 1. The list of beams avajlable is shown in Table 2, while Fig. 1 shows the particle distribution of light and heavy ion beams over the past 13 years. The ratio of beavy-ion to light-jon beam time has stabilized at $2: 1$ in the last several years.

Upgrading the cyclolon is an ongoing process. Some modifieations are directed at immediately improving the efficiency apd reliability of the cyclotron. Longer-range improveusent projects are directed towerd extending the capabilities of the cyclotron into new regions such as development of new bearrs, highter energies, or increased currents. Both immodiate and loug-range improvements are crucial to the continued successful operation of the cyclotron.
During the past year a nurnber of modifications were made on the magnet power supplies and the rf system. A new regulator was installed on the $\mathrm{M}_{4} \mathrm{l}_{1} \mathrm{M} 42$ anlalyzer magnels, raising the maximum current from $1000 \mu \mathrm{A}$ to $1160 \mu \mathrm{A}$, and we expect to increase the current to $1250 \mu \mathrm{A}$ by a minor modification of the linear transformer. This will allow the magnets to be used on the high-energy os particle and heavy-ion beams resulting from the new higher $K$ operation. A new regulator was added to the switching magnet power supply for the high resolution beam line. Regulation was added to the valiey coil no. 5 power supply to improve the stability of bearn extraction from the cyclotron. To reduce power usage and tube replacement costs, two new $300 \mathrm{~W}$ wide-band amplifiers are now ready for installation in place of the final if driver nbe. A new fauit detector for the modulator was instalied, which will better protect the final of amplifier tube from inadvertent damage.

Extensive modifications were done on the components used in the polarized source and axial injection system. A new ionizer was installed on the polarized source and will be ready for testing in late 1981; it is expected 10 improve the output of polarized ions by a factor of 10 . The installation of the new ionizer required modification of the axial injection system because of physical size changes. While the system was disassembled, the high-voltage vacuum feedthroughs, which had been a source of vacuum leaks, were replaced. A cryopumping head was added to replace

Table 1. Operating and maintenance time distribution of the 88-Inch Cyclotron, including total calendar time except holidays $(7 / 80-6 / 81)$.

\begin{tabular}{lrr}
\hline & Hours & \% \\
\hline Operating Time & & \\
Experimental program & 6,312 & 74.9 \\
Beam development & 340 & 4.1 \\
$\quad$ Subtotal: & 6,652 & 79.0 \\
Maintenance Time & & 6 \\
Routine & 512 & 6.0 \\
Scheduled shutdown & 818 & 9.8 \\
Unscheduled shutdown & 442 & 5.2 \\
$\quad$ Subtotal: & 1,772 & 21.0 \\
$\quad$ Grand Total: & 8,424 & 100.0 \\
\hline
\end{tabular}


Tabie 2. 88-Inch Cyclotron beam list.

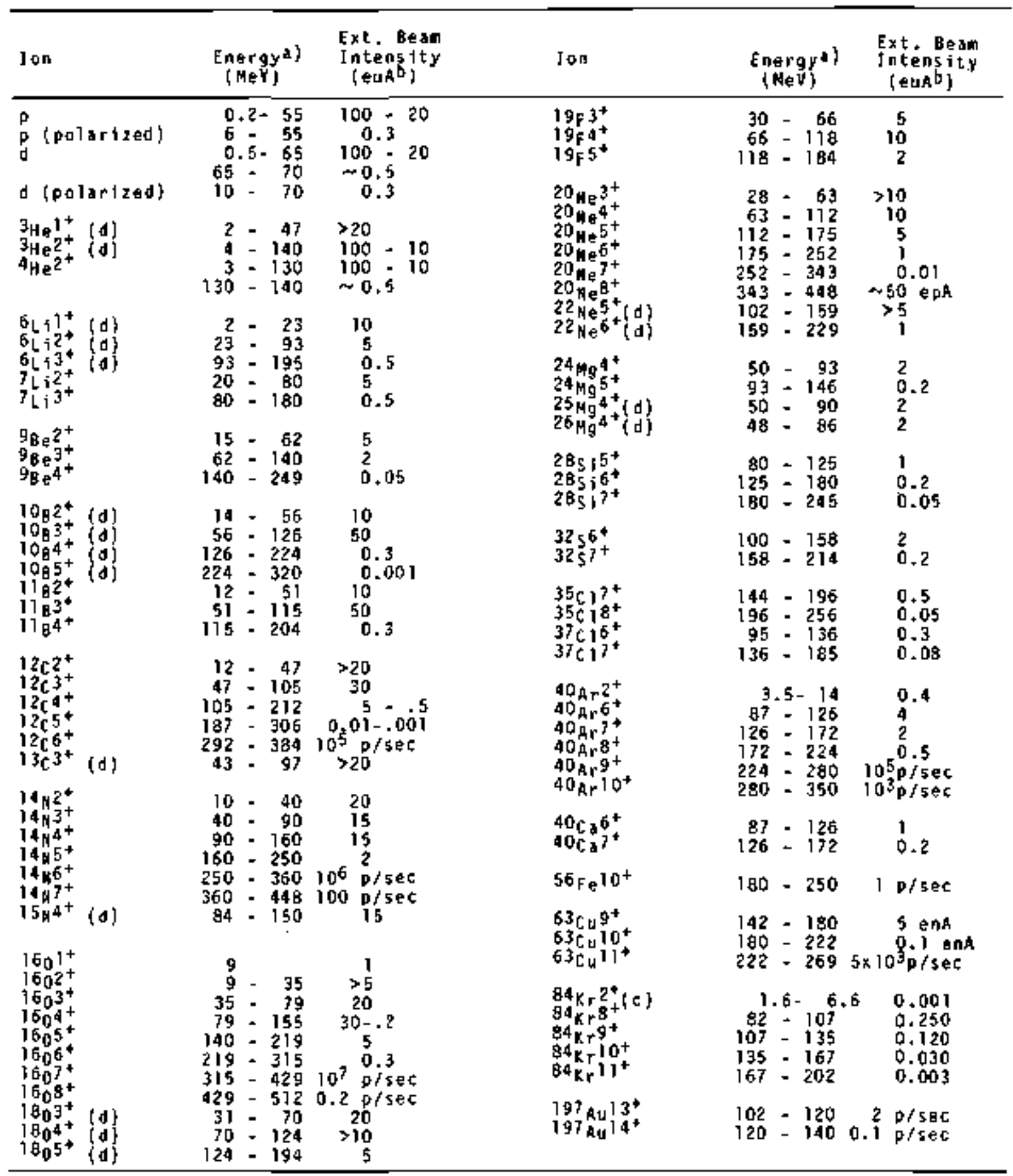

a) Heary ion energy range shows nodinal naximum energy for a particular charge state dorn to the energy which can be raached by the next towast charge state. Beams can also be run at energies belon I Mev/nucleon.
D) Electrical aicroamperes except as moted.

c) 15th haraonic

d) Isotopicatly enriched source foed 


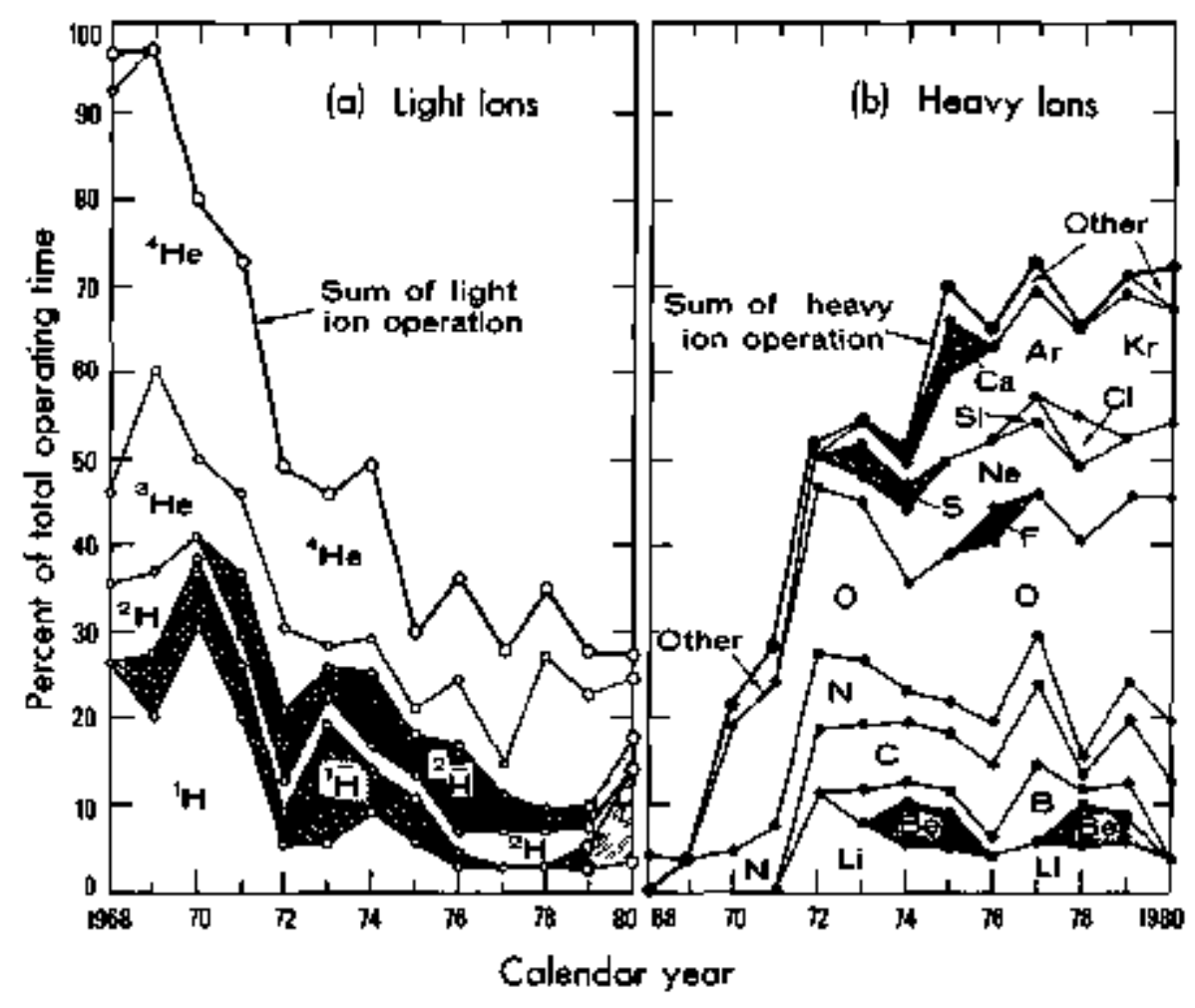

Fig. 1. Distribution of light and heavy ion beams, as percent of cyctotron operating time, 1968. 1980.

(XBL 809.1999A)

a diffusion pump. The improved vacuum should benefit both polarized and heavy ion beams.

The external heavy-ion PIG source and its beam line to the axial injection beam line were eompleted, tested, and used during this report period. Ions from the new source are transported horizontally before being deflected vertically into the existing axial injection column that is also used for polarized protons. The final assembly and testing of the new heavy ion facility were done in collaboration with the Muller astrophysics group. which proposes to use the source for sample injection in their radioisotope detection progrem. Initial tests of the source emphesized injec tion of ${ }^{12} \mathrm{C}^{3+}$ and ${ }^{14} \mathrm{~N}^{3+}$ at energies of about $7 \mathrm{kV}$ with acceleration to about $40 \mathrm{MeV}$. A series of tests made steady improvement in the jon source output, transport efficiency, and extracted current Irom the uachine. The best transmission from source to syelotron external beam was $2 \frac{5}{5}$ at $12 \mathrm{kV}$ injection, with a first harmonic buncher being used. losses include charge exchange in the injection line and cyciotron phase acceptance and emittance matching. The addition of cryopurmps in the injection line and the cyctotron is expected to improve the transmission. Typical currents at the exit of the cyclotron were about I HA A source specifically designed for sputtering has been built and will be tested soon.

The south port cryopump was installed and tested. The tests showed that a modification to allow temperature control of the cold head is required. Without a temperature control, the device can cause the hydrogen coolant to freeze, which in turn decouples the refrigerator from the cryopanels. A temperature controller will be installed shortly, allowing the south port cryopump to become operational. This additional pumping will inoprove the vacuum in the tank and thus the heary ion performance of the machine.

The passive magnetic deflection channel will provide radial focusing and greater defection strength for all cyclo. tron beams. The radial focusing will increase the beam transmission to experimental targets. The greater defiection strength will provide efficient extraction of the high-energy $\alpha$-particle and beavy ion beams accelerated. using the new higher $K$ operation. The magnetic chennel will replace the third element of the electrostatic deflector. The magnetic design js complett. Final orbit calculations of phase space ellipses through the channel will be done as a joint projot with the Cyclotron Institute Texas A\&M University. Mechanical design and fabrication will then be completed.

Planning is underway for an advanced heavy ion source for the cyclotron. The goal is to provide useful beam intensities of $10^{9}-10^{10}$ particles/sec on target with energies of 2 to 3 times those of present heavy ion beams with ousses between nitrogen and zenon. The energy would be up to $40 \mathrm{MeV} / \mathrm{s}$ for the lower misses. The charge states would be, for example, $\mathbf{N}^{7+}$ and Arl418+. The two leading candidates for sources art the Electron Beam Ion Source (EBIS) and the Electron Cyclotron Resonance (ECR) source.

Research and development to build a test bench EBIS 
were begun in January 1980, under the direction of the plasma group in the LBL Accelerator Division. The goal is to reproduce the high electron-beam densities observed by the Orsay group. This requires careful aligpment of the electron gus and magnet system, and high-vacuum techno]ogy. The progress during the past year includes the successful production of an extremely well-aligned magnetic field and electron beam-both straight to within $0.05 \mathrm{~mm}$ along the $60-\mathrm{cm}$ solenoid length. Detailed metsurements have been made on the relation of electron beam profile to magaetic field and injection voltage. Study of the ion beam is now underway. This test bench will give valuable information on the requirements for high-charge-state production. If the tests indicate that the intensity, charge state, and duty factor requirements can be met, a full-scale EBIS will be built for installation on the axial injection system of the cyclotron.

\title{
SuperHILAC Operations and Research
}

\author{
M.S. Zisman and R.M. Diamond
}

The SuperHILAC furnishes beams from ${ }^{4} \mathrm{He}$ to ${ }^{208} \mathrm{~Pb}$ (and $\operatorname{soon}{ }^{238} \mathrm{U}$ ) with energies variable from 1.2 to 8.5 MeV/nucleon. It is the only accelerator in the United States currently able to provide very-heavy-ion beams at energies above the Coulomb barrier, and will continue to be the prime source of such projectiles in this country for the next several years.

As a result of its unique capabilities, the SuperHILAC has been made a national facility available to qualified scientists in nuclear and atonic physics throughout the United States, and to collaborating groups in Canada and Europe. At present, more than half of the operatiog time is given to user groups from outside the Laboratory. Proposals are submitted twice a year, and beam time is allocated by a Program Advisory Comrittee made up of experts in heavy-ion physics who may be inwolved in experiments at the SuperHILAC, but are not major users there. In addition, a User's Executive Comrnittee, made up principally of major users elected by the whole user community, helps advise the Nuclear Science and the Accelerator and Fusion Research Divisions on the operation of the machine and the needs of the users. The SuperHILAC runs 20 shifts a week, 7 to 8 months a year.

On the wholt, machine performance bas been quite smooth and reliable during the past year; experimenters have bad beam ayailable to them about $75 \%$ of the scheduled time. Improwement of the accelerator is an ongoing process, and the major development of the past few years is the construction and bringing into operation of the third injector, ABEL. This is an injector for relatively high-intensity, very heavy-ion beams. It consists of a twin high-power PIG source, a 750-kV Cockcroft-Walton accelerator, and a Wideroe linac. Between the CockcroftWalton and the Wideroe, a $90^{\circ}$ bendjing mognet provides isotope separation with a resolution of 1 part in 390 . This new injector was brought jnto ser vice in June, just bejore the annual budgetary shutdown, and should now powide the users with more reliable and more intense beams of rare-earth ions on up to uranium Anticipated ions from the ABEL injector, for fall of FY 1982, along with hopedfor intensities, ace given in Table 1.
A featore nocessary for the simultaneous operation of the \$uperHILAC and the Bevalac was the development of timesharing the beam. The macrostructure of the beam out of the SuperHILAC is 36 pulses/sec of variabie width, 1 to 9 msec long. These pulses may be divided between different beams from the injectors, at different energies, in almost any manner desired. A typical operation is $\mathbf{3 2}$ pulses/sec of a very heavy ion to the main SuperHILAC user, 2 pulses/sec of a ljghter ion to the Bevalac, and 2 pulses/sec of either beam (at a different energy if so desired) to the next SuperHILAC user to test his equipment of to do a parasitic experiment. This scheme has been very successful. Work is continuing in order to increase the duty cycle from the present $25 \%$ for "ligh" heavy jons and $10 \%$ for $\mathrm{Pb}$ to $\sim 35$ \% and $20 \%$ respectively, and to construct a buncher cavity of a new design to prowide beam bunches in a particular tine of less than 500 psec FWHM.

The construction of an on-line isotope separator, mentioned in last year's report, has been completed, and this instrument has been successfolly used. Efforts are now underway to provide a laboratory above the separator along with a tape transport system to allow low-background $p$. and $\gamma$-spectroscopic studies on the seperated products.

Table I. ABEL Ions Available In FY 1982.

\begin{tabular}{lc}
\hline & Post-exit pnA (avg. at 36 pps) \\
\hline${ }^{84} \mathrm{Kr}\left(22^{+}\right)$ & $\sim 75$ \\
${ }^{129} \mathrm{X}_{e}\left(29^{+}\right)$ & $\sim 40$ \\
${ }^{197} \mathrm{Au}\left(37^{+}\right)$ & $\sim 5$ \\
${ }^{208} \mathrm{~Pb}\left(39^{+}\right)$ & -5 \\
${ }^{238} \mathrm{U}\left(42^{+}\right)$ & $\sim 5$ \\
\hline
\end{tabular}

${ }^{3}$ Not expected before beginning of FY 1982. 
The research program at the SuperHILAC is varied. but can be grouped into four main categories. Roughly $50 \%$ of the beam time is devoted to the study of heavy-ion reaction manisms, with five outside groups and one from LBL participating. A second topic is nuclear structure studies; these are carried out primarily by an LBL group that collaborates with three major outside groups. A third calegory is the study of exotic nuclej-the production of new isotopes of known elements and of new chentical elements. Two LBL groups and one from outside carry on the bulk of these studies; they have largely focused on the very heavy elements, but with the availability of the new isotope separator, more wark will take place over the whole periodic table. The fourth type of study is heary-ion atomic physics, which is carried oul by two LBL groups (outside the Nuclear Science Division) and three outside groups.

The studies at the SuperHILAC by groups in the Nudear Science Division ase described in the various group reports in this annual report. 


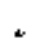

.

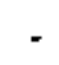

. 
PART 3. APPENDICES 


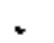

.

.

. 


\title{
Thesis Abstract
}

\section{Effects of Particle Evaporation on the Angular Momentum of the Emitting Nucleus for Deep Inelastic and Compound Nuclear Reactions}

\author{
S.K. BitaN
}

A model is developed which allows one to calculate analytically the angular momentum removed, and the angular momentum misalignment created by the evaporation of light particles from an excited nucleus. The mass, tem- perature, and angular momentum of the enitting nucleus are explicitly considered. The formalism applies exvally well to heavy ion and compound nucleas reactions.

\section{Nuclear Science Division Seminars}

July 24,1980

Aug 11, 1980

Aug 14, 1980

Aug 19,1980

Aug. 29, 1980

Sept. 8, 1980

Sept 15, 1980

Sept. 29,1980
Dr. Hans Sann

J. de Swast

Nijmegen, Holland

Itshalk Tserruya

Weizmann Institute Israel

Gabi Goldring Weizmann Instítute Israel

Yuri T.S. Oganessian

Uli Lynen GSI, Darmstadt West Germany

W.J. Swiatecli LBL

John O. Newton Australian National University, Cassbersa
Symmetric Mass Yields and the Reaction $\mathrm{U}+\mathrm{Ca}$

Dibaryon Resonances and Moltiquark States

Nevtron Emission from $12 \mathrm{MeV} / \mathrm{n}$ $\mathbf{K r}+\mathbf{E r}$ - a Nonequuilibrium Component?

Tilted Foi] Hyperfine Interactions

Transuranium Element Research at Dubna

Reactions with 86-MeV/a Carbon Beams

The Dynamics of Nuclear Cualescence or Reseparation and Dynamical Limits on Superheavy Element Formation

Limits to Evaporation Residue Cros Sections from Fission and Particle Decay at High Angular Monentum 
Oct. 20, 1980

Nov. 3, 1980

Nor. 10,1980

Dec. I, 1980

Dec. 8,1980

Dec. 15. 1980

Dec. 22,1980

Jan. 12, 1981

Jan. 19,1981

Jan. 26, 1981

Feb. 17, 1981

Feb. 23, 1981

Mar. 2, 198]
Grant J. Mtrthews

Kelloge Radiation

Laboratory, Caltech

Kurt Kilian

MPI, Heidelberg

West Germany

Ken Moody

LBL

\section{Ed Ludwig University of North Carolina and TUNL}

Gerhard Sofi Frankfurt

West Germany

R.J. McDomald LBL

Sigurd Köhler University of Arizona

R.W. Koontz LBL

H. Zankel University of Graz Austria

Achim Weiguny
LASL and Munster
Umiyersity
West Germany

Jarres Symons

LBL

\section{Georg Leander Lund University Sweden}

Al Stetz
Oregon State
University
Current Topies in Nuclear Synthesis

Anti-Protons at LEAR
Actinide Production in the Reaction of ${ }^{136} \mathrm{Xe}$ and ${ }^{86} \mathrm{Kr}$ with ${ }^{248} \mathrm{Cm}$

The Interesting Systematics Observed in Studies of Isospin Forbidden Resonances
Atomic Clock for Nucletar Collisions
Fragment Spin Orientationn in Deep Inelastic Reaction from Anisotropy Measurements of Continuum $\gamma$-Rays
Heavy Ion Collisions with Two-Body Dissipation
Fragmentation of 4 He at $35 \mathrm{MeV} / \mathrm{n}$ : Correlated Fragments and "Impulsive Absorption"

Approximate Coulomb Corrections in Light Nuclear Systems
Quasi-Molecular and Surface Wave Resonances in Heayy. lon Scattering

$\alpha \alpha$ and $P \alpha$ Collisions in the CERN Intersecting Storage Rings

\section{Theoretical Description of Quasi-Contínumm Gamama Rays}

Double Charge Exchange Reactions on Helium Isotopes 
Mar. 9, 1981

Mar. 16, 198l

Mar. 23, 1981

Mar. 30, 1981

Apr. 3, 1981

Apr. 6, 1981

Apr. 13,1981

Apr. 27, 1981

May 4, 1981

May 11, 1981

June 1, 198I

June 15,1981

June 17, 1981

June 29, 198I
Jobn Calarco Stanford Unjversity

Phil J. Siemens

Texas A\&M

University

Arturo Menchaca-Rocha University of

Mexìno

Hiroski Toki
Michigan State
University

Ginther Rosner Max Panck Institut Heidelberg

West Germany

Norman K. Glendenning LBL

\section{Thomas Dpssing NORDITA \\ Copenhagen}

Charles R. Gruhn LBL

Rudolph C. Hwa University of Oregon

Rosemarie McFarland

LBL

Jan Wouters

LBL

Sam Austin
Michigan State
University

L.G. Amold Ohto State University

Bo Jakobsson University of Lund Sweden

Loon Van Hove CERN and JTP, Santa Barbara
Excitation and Decay of

Giant Resonances

Matter at $10^{12} \mathrm{~K}$ : Theory versus Experiment

On the Time Evolution of ${ }^{16}$ O-Induced Direct Reactions

Pionic Mode of Excitation in Nuclei
Deep Inelastic and Fusion Processes in the Reactions ${ }^{32} \mathrm{~S}+{ }^{2} \mathrm{AJ}$ at $\mathrm{E} / \mathrm{A} \leq 10 \mathrm{MeV}$

\section{Pion Condensation Gravitational Waves and the Evolution of Neutron Stars}

\section{Angular Correlations and Spin Alignment in Deep Inelastic Nuclear Reactions}

Bragg Curve Spectroscopy

From Parton to Nuclei

\author{
Heavy Ion Direct Transfer \\ Reactions in the Actinide Region \\ Mass Measurements of the Neutron \\ Deficient Indium Isotopes
}

The Giant Isovector Resonances -A Hadronic View
Relativistic Noclear Optical Model: Nucteons on Nuclei or Bags on Pokes

Production of Light Particles and Beam Fragmentation in 58 and $86 \mathrm{MeV}$ A Heavy Ion Reactions

What Happens to Quarks and Gluons in Hadronic Collisions? 


\section{Nuclear Theory Meetings}

July 23,1980

July 30, 1980

July 3], 1980

Aug. 7, 1980

Aug. 21, 1980

Sept. 10,1980

Oe1. 22, 1980

Oct. 29,1980

Nov. 12, 1980

Jan. 14, 1981

Feb. 4,1981

Mar. 1 ], 1981

Mar. 18,1981

Apr. 1, 1981
Eugene Marshalek Notre Dame University

$$
\begin{aligned}
& \text { Alan Goodman } \\
& \text { Tulane University }
\end{aligned}
$$

Jgal Talmi Weizmann Institute Israel

Robert Vinh Mau Paris

Sven Bjornbolm Niels Bobr Institute Copenhagen

\section{Csernai}

\section{B.R. Mottelson NORDITA \\ Copentaigen}

J. Boguta

LBL

Herbert Ruck LBL

T. Matsui Stanford University

P. Hecking LBL

Berndt Müller Frankfust University West Germany

Dr. H. Orland Saclay, France

\section{E.A. Remlar Institule for Theoretical Physics Santa Barbara}

Hextert Ruck LBL

\section{Hiroski Toki} Michigan State University
Review of Microscopic Boson Expansion

Finite Temperature HFB Theory

Interacting Boson Model for Nuclei

Recent Progress with the Paris Nucleon-Nucleon Potential.

On the Dividing Line Between Heavy Ion Scattering and Capture Reactions

\section{Realistic Two and Three Dimensional Fluid Dynamical Calculations with a Description of Final Evaporation}

Alignment Effects for Particles and Pairs (Analysis of Some Features of the Interacting Boson Model)

Relativity and Nuclear Structure

Polynomial Quantum Chromodynamics

Structure of Pion Condensation

Abnormal Matter and Pion Condensation

Hot Color Phase Transitions of the QCD Vacuum at High Temperatures

Funetional Integral Methods

Composite Particle Production in Heavy Ion Collisions

From Quarks to Nuclear Matter

Short-Range Part of NN Interaction within the Non-Relativistic Quark Model 
May 13, 1981

May 27, 1981

July 22, 1981

July 27,1981

July 29, 1981
Kej-Fei Liv

University of Kentucky

W. Norenberg GS]

Peter Moller

Lund University, Sweden

J.P. Blaizot CEN, Saclay, France

Xin-Hua Yane
Quarks in Nuclei

Memory Efiects in the Energy Dissipation for Slow Collective Motion

Nuclear Shapes

Time Dependent Yariational Principles and Semiclassical Bound States

Many Body Theocy of Confined Quarks and Gluons and the Calculation of the Exchange and Self-Energy 


\section{Papers Published And LBL Reports Issued}

\section{0-81}

ALBRECHT, K. (See DIEBEL, M., LBL-11522)

ALEKLETT, K, D.J. Morrissey, W. Loveland, P.L. McGuaghey, and G.T. Seaborg.

The Entrgy Dependence of ${ }^{203}$ Bi Fragmentation in Relativistic Nuclear Collisions, LBL-] 1273, July 1980, Phys. Rev, C 23, 1044 (1981).

ALEKIETT, $\mathrm{K}$ (SeE LOVELAND, W., LBL 10010)

ARYAEINEJAD, R., R.B. Firestone, W.H. Bentley and William C. McHarris

In-Beam $\gamma$-Ray Spectroseopy of Excited States in ${ }^{143} \mathrm{Ey}$ Phys. Rev. C 23, 194 (1981).

AWES, T.C. (Se BACK, B.B., LBL-11852)

AYYSTÖ, J., M.D. Cable, R.F. Parry, J.M. Wọters, D.M. Moltz, and Joseph Cerny

Decays of the $\mathrm{T}_{2}=-2$ Nuclei ${ }^{20} \mathrm{Mg}$. ${ }^{24} \mathrm{Si}$ and ${ }^{36} \mathrm{Ca}$, LBL-1] 193, June [980, Phys. Rev, C 23, 879 (198]).

ÄYSTÖ, J, (See CERNY, J., LBL 12853)

ÄYSTÖ, J. (S*e MOLTZ, D.M., LBL-] 1427)

BACK, B.B., AC Shotter, T.J.M. Symons, A Bice, C.K. Gelbike, T.C. Awes, and D.K. Scott

Fission of ${ }^{238} \mathrm{U}$ Induced by Inelastic Scattering of I20 MeV $\alpha$-Particles, LBL-1]852, September 1980, Phys.

Rev, C 23, 1105 (1981).

BAER, H.W. (See MARTOFF, C.1., LBL-1 1914)

BANERJEE, B., NK Glendenning and M. Gyulassy

Fion Condensation in a Field Theory Consistent with Bulk Properties of Nuclear Matter, LBL-10979. May 1980, Nud. Phys. A 361, 326 (1981).

BEMIS, C.E, Jr. (See NITSCHKE, J.M., LBL-10]73)

BENTLEY, W.H. (SEe ARYAEINEJAD, R.)

BENTLEY W. H. (SeE WALKER, P.M.)

BERMAN, B.L. (See OLSON, D.L. LBL 12292)

BICE A.N., A.C. Shotter, D.P. Stahel and Joseph Cerry Investigation of the $\left({ }^{10} \mathrm{~B},{ }^{6} \mathrm{Lj}^{*}\left(3^{+}, 2.18 \mathrm{MEV}\right)\right.$ Reaction as a Method for a-Cluster Transfer Studies, LBL11194, July 1980, Phys. Letts. B 101, 27 (1981).
BICE, A. (Søe BACK, B.B, LBL-1 1852)

BICE, A.N, (See SHOTTER, A.C., LBL-11375)

BICE, AN, (See SHOTTER, A.C., LBL-11376)

BICE, AN, (See SHOTTER, AC., LBL 11458)

BIRCHALl, J., W.T,H van Oers, H.E. Conzett, P. won Rossen, R.M. Layimer, J. Wateon, and R.E. Brown Anlyzing Powrers of ${ }^{3} \mathrm{He}(p, p)^{3} \mathrm{He}$ Elastic Scattering between 30 and $50 \mathrm{MeV}$, LBL L1 I21, Jume 1980, presented al the sth International Symposium on Polarization in Nucl. Phys., Santa Ft, NM, Augusl 11-15, 1980.

BISTIRLJCH, J.A. (See MARTOFF, C.J., LBL-11914)

BLAU, S.K, L.G. Moretto

Effects of Particle Evaporation on the Angular Monenturn of the Emitting Nucletus for Deep Inelastic and Compound Nuclear Reactions, LBL 10926, Seplember 1980, Nucl. Phys. A 359, 477 (1981).

BLAU, S. (See MOREJTO, L.G., LBL-10805)

BLOCKI, J., W.J. Swiatecki

A Genteralization of the Proxinuty Force Theorem, LBL9574, Annais of Physics 132, 53 (198i).

BOGUTA, J.

Remarks on the Beta Stability in Neutron Stars, LBL 11465, August 1980, submitted to Phys. Letts. B.

BOGUTA, J.

Nuxleon-Nucleus Optical Potential in a Relativistic Theory of Nuclear Matter, LBL-11466, Augast 1980, subnitted to Phys. Letts. B.

BOGUTA, J.

Relativistic Quantum Field Theory of Finite Nuclei, LBL 1 1467, August 1980, submitted to Phys. Letts. B.

BOGUTA, J.

Propetties of $\mathrm{T} \neq \mathbf{0}$ Nuclei in a Relativistic Field Theory of Nuclear Matter, LBL-1]468, August 1980, submitted to Phys. Letts. B.

BOGUTA, J.

Ground State Properties of $0^{16} \mathrm{Ca}^{40}$ and $\mathrm{Ca}^{48}$ In a Rela tivistic Hartree Theory of Nucles Matter, LBL I 1894, Decenter 1980, submitted to Nucl. Phys. A. 
BOGUTA, J.

A New Mechanism Leading to Density Isomers, LBL [2333, April 198], subritted to Phys. Letts.

BOGUTA, J.

Density Dependence of the Single Particle Potential in Nuclear Matler. LBL-12628, April 1981, submitted to Ptys. Letts.

BOGUTA, J., S. Bohrmann

Relativistic Quantum Field Theory of a Hypernuclei. LBL 11670, October 1980, submitted to Phys. Letts. B.

BOGUTA, J. (Sø STÖCKER, H., LBL-12095)

BOHRMAN, S. and Jörn Knoll

Finite Particle Number Effects in High-Energy Nuclear Collisions: Implications on Pion Spectra, LBL-10970, May 1980, Nucl. Phys. A 356, 498 (1981).

BOHRMANN, S. (See LBL- I 1670, BOGUTA, J.)

BOHRMANN, S. (See KO, Che Ming, LBL-1104L)

BOLOTIN, H.H. (See McDONALD, R.J., LBL-L ]608)

BROWN, R.E. (See J. BIRCHALL, LBL-1I12I)

BUCHWALD, G. (Seะ STÖCKER, H., LBL-1 I774)

CABLE, M.D. (See ÄYSTÖ. J., LBL-11193)

CABLE, M.D. (See CERNY, JOSEPH, LBL-12853)

CABLE, M.D. (See MOLTZ, D.M., LBL 1 1427)

CERNY, J., J. ̈̈ystö, M.D. Cable. P.E. Haustein, R.F. Parry, H.M. Thierens, and J.M. Wouters

A Study of the Beta-Decay Energies of Highly NeutronDeficient Indium Isotopes, LBL-12853, June I98I, presented at the Fourth Jnternationsl Conference on Nuclei Far from Stability, Helsingotr, Denmark, July $7.13,1981$.

CERNY, J. (Șet ÄYSTÖ, J., LBL-I ] 193)

CERNY, J. (Set BICE, A.N., LBL- III94)

CERNY, J. (See MOLTZ, D.M., LBL-11427)

CERNY, J. (SEe SHOTTER, AC., LBL-11375)

CERNY, J. (See SHOOTTER, AC., LBL-11376)

CERNY, JOSEPH (SEe SHOTTER, A.C, LBL-11458)

CHESSIN, S.A. (See GEAGA, J.V., LBL-10265)

CHIOU, J.H. (See MA, A.Y., LBL-9689)

CHO. Y.C. (SEe MA, AY., LBL-9689)
COLE, A.J. (See RAE, W.D., LBL-12568)

CONZETT, H. E.

Concerning Tests of Time Reversal Invariance Via the Polarization-Asalyzing Power Equality, LBL-11087, June 1980, presented at the International Conference on Polarization Phenomena in Nuclear Physies, Santa Fe, NM, Augusi 11-15, 1980.

CONZETT, H.E.

Polarization Effects in Light Nuclei, LBL-11545, September 1980, Rapporteur's Report, presented at the Fifth International Symposium on Polarization Ptenomena in Nuclear Physics, Santa Fe, NM, Angust I1-15, 1980.

CONZETT, H.E.

Large Deviations Irom the Polarization-Analyzing Power Equality and Implied Breakdown of Time Reversal Invariance, LBL-11546, Seplember 1980, Invited paper presented at the Fifth International Symposium on Polarization Phenomena in Nuclear Physics, Santa Fe, NM, August ]l-15, 1980.

CONZETT, H.E., P. von Rossen, F. Hinterberger, R.J. Slobodrian, C. Rioux, and R. Ray

Large Deviations from the Polarization-Analyzing Power Eqquality and Implied Breakdown of Tỉme Reversal Invariance, LBL-11576, September 1980, Invited paper presented at the 1980 International Symposium on High Energy Phy. sics with Polarized Beams and Polarized Targets, Lausanne, Switzerland, September 25 - October I, 1980.

CONZETT, H.E. (See BIRCHALL, J, LBL-] 1121)

CONZETT, H.E. (Sce SLOBODRLAN, R.J., LBL-1]372)

CRAMER, J.G. (See ZISMAN, M.S., LBL-I0204)

CRAWFORD, H.J. (See OLSON, D.L., LBL- 12292)

CROWE, K.M. (SeE MARTOFF, C.J., LBL-11914)

CSERNAI, L.P. (Søe STÖCKKER, H., LBL-11774)

CUGNON, J., J. Knoll, and J. Randrup

Participant Intimacy - A Cluster Analysis of the Intranuclear Cascade, LBL-1130l, August 1980. Nucl. Phys. A 360, 444 (1981).

CUMMING, J.B. (SoE LOVELAND, W, LBL-10010)

CUSSON, R.Y. (SEe STÖCKER, H., LBL 11774)

DACAL, A. (Se HARRIS, J.W., LBL-12334)

DACAL, A (See RAE W.D., LBL-11135)

DACAL, A. (See RAE, W.D., LBL-12\$68)

DEVRIES, R.M. (SEe Z1SMAN, M.S., LBL- 10204) 
DIAMOND, R.M. and F.S. STEPHENE

Nuclei at Higb Angolar Montentom, LBI-10325, June 1980, Ann. Rev, Nuci. Part. Sci. 1980 30:85-157.

DIAMOND, RM.

Nuclear States and Sbapes at High Spin, LBL-11436, Augost 1980, presented at the XII Summer School in Nuclear Physics, Mikolajkj, Poland, September 1-13, 1980.

DJAMOND, RM.

Continutu Gamma-Ray Spectroscopy, LBL-12792, June 1981, presented at the Divisional Conference of the European Physics Society on Nuclear and Atomic Physics witb Heavy Ions, Bucharest, Romania, June 9-12, 1981.

DLAMOND, R.M. (See MeDONALD, R.J., LBL-11608)

DIAMOND, R.M. (Se NEWTON, J.O., LBL-12261)

DAMOND, RM. (S* SIE, S.H., LBL 11352)

DAMOND, RM. (Sec WOZNIAK, G.J., LBL-1 I196)

DIEBEL, M. Klabs A J brecht and Rainer W. Hasse

Microscopic Calculations of Fission Barriers and Critical Angular Momenta for Excited Heavy Nuclear Systems, LBL-11522, September 1980, submitted to Nucl. Phys.

DINES, E.L (Ste NEWTON, J.O., LBL ]2261)

DRAPER, J.E. (See NEWTON, J.O., LBL 12261)

EGGERS, R.C., L.P. Somerville

The Maximum Likelihood Method Applied to the Poblem of the Multi-Component Decay, LBL 12060, January I981, submitted to Nucl. Inst. Meth.

ESKOLA, P. (Se NITSCHKE, J.M., LBL 10173 )

FABER, S.R (See WALKER, P.M.)

FIRESTONE, R.B., R.C. Pardo, RA Warner, WmC. McHarris, and W.H. Kelly

The $\epsilon / \beta^{+}$Deay of ${ }^{145} \mathrm{Gd}$ : Resolution of $\epsilon / \beta^{+}$Decay

Branching Ratio Anomalies and Evidence for Pronounced Structures in the B-Decay Strength, LBL-12424, March 1981, submitted to Physical Review C.

FIRESTONE, R.B. (Set ARYAEINEJAD, R)

FIRESTONE, RB. (Sø MATSUSHITA, N.)

FIRESTONE, R.B. (See SCHUBERT, K.R.)

FIRESTONE, R.B. (SEe WALKER, P.M.)

FOWLER, M. (S $₫$ NITSCHKE, J.M., LBL-10173)
FRIEDLANDER, E.M, RW. Gimpt, H.H. Heckran, Y.J. Karant, B. Judek, and E. Gansstatge

Evidence for Anomalous Nuclei among Relativistic Projeotile Fragments from Heavy lon Collisions at Bevalac Energies, LBL-11136, Junt 1980, Phys. Rev. Lett. 45, 1084 (1980).

GANSSAUGE, E. (See FRIEDLANDER, E.M., LBL11(36)

GEAGA, J. V., S.A. Chessin, J.Y. Grossiord, J.W. Harris, D.L Hendrie, L.S. Schroeder, R.N. Treuhaft and K. Van Bibber

Otservation of High Momentum Protons From Limiting Target Fragmentation, LBL-10265, April 1980, Phys. Rev. Letts. 45, 1993 (1981).

GEAGA, J.V. (See HARRIS, J.W., LBL 12334)

GELAKE, C.K. (See BACK, B.B., LBL-11852)

GHIORSO, A (See LEINO, ME, LBL-12816)

GHIORSO, A (S NTSCHKE, J.M., LBL-10173)

GIMPEL, R.W. (SEE FRIEDLANDER, E.M., LBl-11136)

GLENDENNING, N.K.

Hyper-Strange Hedronic Matter, LBL-11538, November 1980, Pbys. Rey. C 23, 2757 (1981).

GLENDENNING, N.K

Pion Condensation and the Evolution of Neutron Stars, LBL-12427, March 1981, submitted to Phys. Rev. Letts.

GLENDENNING, N.K, A Lumbroso

Pion Condensation, Density Jsomers and Anisotropic Pressures, LBL-12108, January 1981, Reported at the interwatichal Workshop IX on Gross Properties of Nuclei and Nuclear Excitations, Hirschegg, Alustria, January 1981.

GLENDENNING, N.K. (See BANERJEE, B., LBL-10979)

GOLDBERG, D.A (See ZISMAN, M.S, LBL- 10204)

GOODMAN, A.L

Finite-Temperature HFB Theory, LBL-11151, July 1980 , submitted to Phys. Rev. C.

GOODMAN, AL

The Two-Lewel Model at Finite-Temperature, LBL-11 171, July 1980, subsitited to Nucl. Phys. A

GRAERNER, G. (SeE STÖCKER, H., [BL-]1774)

GREINER, W. (See STÖCKER, H., LBL 11774) 
GREJNER, D.

The HISS Spectrometer at LBL, LBL-11818, November 1980, Invited speaker at the Workshop on "Future Relativistic Heavy Ion Experiments," CSI Darmstadt, West Germany, October 7.10, 1980.

GREINER, D.E (See OLSON, D.L, LBL-12292)

GROSSIORD, J.Y. (See GEAGA, J.V., LBL-10265)

GRUHN, C.R.

Brage Curve Spectroscopy, LBL-12678, May 1981, Invited talk presented at the INS Intemational Conference on Radiation Detectors, Tokjo, Japan, March 23-28, 198 I.

GUJBROD, H.H., M.R. Majer, H.G. Ritter, A. Warwick, F. Weik, H. Wieman, and K.L Wolf

Chargod Particle Identification with Modules of the Plastic Ball, presented at the IEEE Nuclear Science Symposium, Orlando, Florida, November 5-7, 1980, LBL 11797. October 1980, IEEE Transections on Nucl. Science N\$28, 451 (1981).

GYULASSY, M.

Relativistic Nuclear Collisions: Theory, LBL-11040, Jvly 1980. Invited lecture presented at the INS Kikuchi Summer School on Nuclear Physies at High Energies, FijiYostida, Japan, July 1-4, 1980.

GYULASSY, M.

Current Topics in Relativistic Nuclear Collisions, LBL1 1556, August 1980, presented at the International Conference on Nhrelear Physics, Lawrence Berkeley Laboratory. Berkeley, CA, Auggust 24-30, 1980; and published in the Proceedings.

GYULASSY, $M$.

Relativistic Nuclear Collisions in Perspective, LBL-11790, November 1980, submitted to Comments on Particle \& Nuclear Physics.

GYULASSY, M. and S.K. Kauftomann

Coulomb Effects in Relativistic Nuclear Collisions, LBL 10279. March 1980, Nucl. Phys. A 362, 503 (1981).

GYULASSY + M (SEe BANERJEE, B., LBL-]0979.

GYULASSY, M. (Ser NOACK, C.C., LBL 11053)

GYULASSY, M. (See STÖCKER, H., LBL-12095)

HARRIS, J.W., A Sandoval, R. Stock, H. Stroebele. R.E. Renfordt, J.V. Geaga, H.G. Pugb, LS. Schroeder, K.L. Wolf, and A. Dacal

Production Near Threshold in Central Nucleus-Nucleus Collisions, LBL-12334, April 1981, submitted to Phys. Rev, Letts.

HARRIS, J.W. (See GEAGA, J.V., LBL 10265)
HAR VEY B.G.

Small Mornenturn Widths in Heavy-Ion Fragmentation at $20 \mathrm{MeV} / \mathrm{anu}$ and Below, LBL-12787, Mtay 1981, submitted for publication in Phys. Rev. Letts.: Corrutents.

HARVEY, B.G. (See RAE, W.D., LBL-11135)

HARVEY, B.G. (See RAE, W.D., LBL 12568)

HASSE, R.W. (See DJEBEL, M., LBL-1522)

HAUSTEIN, P.E. (Ste CERNY, J., LBL-12853)

HAUSTEIN, P.E. (See LOVELAND, W., LBL- ]0010)

HAUSTEIN, P.E. (See MOLTZ, D.M. LBL-11427)

HECKJNG, $P$.

Pion Condensation Threshold in Noclear Matter and Thermal $\Delta$-Isobars, LBL 12254, February 1981, submitted to Physical Review C.

HECKMAN, H.H.

Evidence for Anomalous Nuclei Among Relativistic Projectile Fragments at Bevalac Energies, LBL 12120, January 1981, presented at the Workshop on Futore Relativistic Heavy lon Experiments Gesellschaft för Schwerionenforschung, Darmstadt, W. Germany, October 7+10, 1980.

HECKMAN, H.H.

Experiments with Relativistic Heavy Ions: A Polpourri of Chemistry, Canis Majoris and Grains of Silver, LBL21656, May 1981, presented at the Third Adriatic Europhysics Study Conference on the Dynanics of Heavy-ion Collisions, Hras, Croatia, Yugoslavia, May 25-30, 1981.

HECKMAN, H.H. (See FRIEDLANDER, E.M., LBL 11136)

HECKMAN, H.H. (See OLSON, D.L, LBL-12292)

HENDRIE, D.L (See GEAGA, J.V., LAL-10265)

HERNANDEZ, E.S., G. Mantzouranis

Markovian and Non-Markovian Effects in Heavy-Ion Reactions at $10-200 \mathrm{MeV}$ per Nucleon, L8L-9568, August 1979, Phys. Rev. C 22, 575 (1980).

HERNANDEZ, E.S., W.D. Myers, J. Randrup, and B. Remaud

Quantal Dynarrics of Charge Equilibration in Damperd Nuclear Collisions, LBL-9761, October 1979, Nucl. Phys. A 361, 483 (1981).

HERSKIND, B. (See NEWTON, J.O, LBL-12261)

HINTERBERGER, F. (See CONZETT, H.E., LBL-11576) 
HINTERBERGER, F. (SE SLOBODRIAN, R.J., LBL 11372)

HSEUH, H.C. (Se LOVELAND, W., LBL-10010)

HSU, C.C. (See MORRISSEY, D.J., LBL ] 2]81)

HSU, C.C. (See SOBOTKA, L.G., LBL L 1 1 48)

HSU, C.C. (See SOBƠTKA, L.G., LBL-11840)

HSU, C.C. (Ste WOZNIAK, G.J., LBL-11 I\%)

HULET, E.K. (See NITSCHKE, J.M., LBL-10173)

JING-SHANG, Zhang, (See ZHONG-YU, MA, LBL 11893)

JUDEK, B. (See FRIEDLANDER, E.M., LBL-1I136)

KARANT, Y.J. (See FRIEDLANDER, E.M, LBL-II 136)

KASAGl, J. (See MATSUSHITA, N.)

KAUFFMANN, S.K (See GYULASSY, M., LBL 10279 )

KAUFFMANN, S.K. (See NOACK, C.C., LBL-1 J053)

KAUFMAN, S.B. (SEe LONELAND, W., LBL- 10010)

KELLY, W.H. (Søe FIRESTONE, R.B., LBL-12424)

KELLY, W.H. (See MATSUSHITA, N.)

KLUGE, H. (See WOZNIAK, G.J., LBL-11196)

KNOLL, J., J. Randrup

Proton-Proton Corrtations in High-Entrgy Nuclear Collisions, LBL-1 1418, Augus1 1980, submitted to Phys. Letts.

KNOLL J. (See BOHRMAN, S., LBL. 10970)

KNOLL, J. (S.e CUGNON, J., LBL-11301)

KO, Che Ming, and S. Bohrmann

A Model for Pion Absorption in Nuclei, LBL-11041, June 1980, Phys. Letts. B 97, 188 (1980).

KOIKE, M (See MARTOFF, C.J., LBL 1 19]4)

KOKER, G.T. (See SCHUBERT, K.R.)

KOONIN, S.E. (See RANDRUP, J., LBL-10959)

KRUSE, H (Seะ STÖCKER, H. LBL-11774)

LANDRUM, J.H. (See NITSCHKE, J.M., LBL-10173)

LARIMER, R.M. (See BIRCHALL, J., LBL.11121)

LEBER, R.E. (\$ee NITSCHKE, J.M., LBL-10L73)
LEDERER, C. $M$

Jsotopes, LBL- 10124, October 1980, an abridged version of this report will be published in the Encyclopedia of Chemical Technology, eds. M. Grayson and D. Eckroth, John Wiley and Sons, Inc., New York (to be published in 198I).

LEDERER, C. M.

El Transition Probabilities from $\mathrm{K}^{\pi}=0^{-}$and $K^{*}=1^{-}$ States of ${ }^{233}$ Py. LBL-12075, January 1981, submilled to Phys. Rev, C.

LEGRAJN, R. (See RAE, W.D., LBL-II135)

LEGRAIN, R. (Ste RAE, W.D., LBL-12568)

LEIGH, J.R. (Soe \$lE, S.H., LBL-I 1352)

LEINO, M.E., S. Yashita, and A Ghiorso Alpha Decay of Neutron Deficient Polonium and Bismuth Isotopes, LBL 12816, May 1981, submitted to Phys. Rev. C.

LEMAIRE, M.C. (SeE NAGAMIYA, S., LBL-12]23)

LINDENBERGER, K.H. (Set NEWTON, J.O., LBL 12261)

LINDSTROM, P.J. (See OLSON, D.L., LBL-12292)

LlU, Yuan-fang, Cheng Luo, Hans $R$ von Guten, and Glean T. Seaborg

A Procedure for a Fast Separation of Berkelium and Cerium, LBL-12\%09. April 1981, to be published in Inorganic and Nucl, Chem. Letts.

LOUGHEED, R.W. (SeE NITSCHKE, J.M., LBL 10173).

LOVELAND, W., Cheng Luo, P.L McGaughey, D.J. Morrissey and G.T. Seaborg

Target Fragment Energies and Momenta in the Reaction of $4.8 \mathrm{GeV}{ }^{12} \mathrm{C}$ and $5.0 \mathrm{GeV}{ }^{20} \mathrm{Ne}$ with ${ }^{238} \mathrm{U}$, LBL 11658 , Oetober 1980, submitted to Phys. Rev. C.

LOVELAND, W., D.J. Morrissey, K. Ajeklett, G.T. Seaborg, S.B. Kaufmen, E.P. Steinberg, B.D. Wilkins, J.B. Cumming, P.E. Haustein, and H.C. Hseuh

Target Residue Recoil Properties in the Interaction of $8.0 \mathrm{GeV}{ }^{20} \mathrm{Ne}$ with ${ }^{181} \mathrm{Ta}$, LBL- 10010 , May 1980 , Phys. Rev, C. 23, 253 (1981).

LOVELAND, W. (SEe ALEKLETT, K, LBL-11273)

LUMBROSO, A (Ste GLENDENNJNG, N.K., LBL. 12108)

LUO, C. (See LIU, YUANFANG, LBL 12909)

LLO, C. (See LOVELAND, W., LBL ]1658

MA, A.Y., X.Z. Wu, G.S. Zhang, Y.C. Cho, Y.S. Wang, J.H. Chiou, S.T. Sen, F.C. Yang, and J.O. Rasmussen 
Calculation of Muon Final State Probabilities After Muon-Induced Fission, LBL 9689, Angust 1979, Nud. Phys. A 348, 446 (1980).

MAHONEY, J. (SE RAE, W.D., LBL-11135)

MAHONEY, J. (SOE RAE, W.D., LBL-12568)

MAIER, M.R. (See GUIBROD, H.H., LBL- 1]797)

MAJER, M.R. (SEe RICHARDSON, L.W., LBL-10146)

MAJER, M.R. (See WOZNIAK, G.J., LBL-1 1493)

MANTZOURANIS, G. (See HERNANDEZ, E.S., LBL9568)

MANTZOURANIS, G. (See MYERS, W.D., LBL 11542)

MARTINIS, J, (See STEVENSON, J,, LBL-12264)

MARTOFF, C.J., J.A Bistitlich, K.M. Crowe, A. Koike, J.P. Miller, S.S. Rosenblum, W.A. Zajc, H.W. Baer, A.H. Wapstra, G. Strassner, and P. Truol

Orbital Recoupling Dominance in the A - 20 Isovector Giant M1 Transition, LBL-11914, December 1980, submitted for publication.

MARUHN, J.A (See STÖCKER, H., LBL 11774)

MAST, T.S. (SE MULJER, RM., LBL 12797)

MATHEWS, G.J. (See SCHMITT, R.P., LBL-9512)

McHARRIS, W.C. (See ARYAEINEJAD, R.)

McHARRIS, W.C. (See MATSUSHITA, N.)

MatSUSHITA N., W.C. McHarris, R.B. Firestone, J. Kasagi, and W.H. Kelly

On Improving Ge Detector Energy Resolution and Peakto-Complon Ratios by Pulse-Shape Discrimination, Nucl. lnstr. Meth. 179, 119 (1980).

McDONALD, R.J., A.J. Pacheco, G.J. Wozniak, H.H. Bolotin, LG. Moretto, C. Schilck, S. Shih, R.M. Diamond, and F.S. Stephens

Evidence for Spin Fluctuations in the Deep Inelastic Reac tion ${ }^{165} \mathrm{Ho}+{ }^{165} \mathrm{Ho}$ at $8.5 \mathrm{MeV} / \mathrm{amu}$, LBL-II608. May 1981، subritted to Nuclear Physics.

McDONALD, R.J. (See MORRISSEY, D.J., LBL-12181).

MeDONALD, R. J. (See SOBOTKA, LG., LBLII ]48).

McDONALD, R.J. (See WOZNIAK, G.J., LBL-11196).

McGAUGHEY, P.L. (See ALEKLETT, K, LBL-11273).

McGAUGHEY, P.L. (See LOVELAND, W, LBL-I1658).
MCHARRIS, Wm. C. (SEe FIRESTONE, R.B., LBL12424)

MILLER, J.P. (See MARTOFF, C.J., LBL-11914)

MOELLER, E (See NAGAMTYA, S., LBL-12123)

MOLITORIS, J.D., J.A. Nitschke

Evaluation of Thin Metal Foils as Targets and Windows for Heavy-Ion Experiments, LBL 9725 , January 1981, subruitted to Nuclear Instruments and Methods.

MOLTZ, D.M., J. Äysiö, M.D. Cable, R.F. Parry, P.E. Haustein, J.M. Wouters, and J. Cerny

Advances in the Helium-Jel Coupled On-Line Mass Separator RAMA, LBL-11427, September 1980, presented at the Electrontignetic Isotope Separalor-10 Conference, Zinal, Switzerland, September 1-6, 1980, Nuclear Instruments and Akthods 186, 141 (1981).

MOLTZ, D.M. (See ÄYSTÖ. J., LBL-I l 193)

MORETTO, L.G.

Spin and Iscspin Fluctuations in Heavy Ion Collisions and Their Dependence Upon the Shape of the Dinuclear Complex, LBL- I 1428, August 1980, Lectures given at the XIII Masurian School of Nuclear Physies, "Nuclear Structure Study by Means of Nuclear Reactions," Mikolejki, Poland, September 1+13, 1980.

MORETTO, L.G.

Angular Distributions of Sequentially Enitted Particles and Garmine Rays in Deep Inelastic Processes, LBL-11975, January 1981, presented at the Fourth Oaxtepec Symposium in Nuclear Physics, Oaxtepec, Mexico, January 1981.

MORETTO, L.G., S. Blau, and A. Pacheco

Angular Momentum Misalignment in Deep Intlastic Processes and Angular Distribution of Sequentially Emitted Particles and Gamma Rays, LBL-1080S, December 1980, Nucl. Phys. A 364, 125 (1981).

MORETTO, L.G.

Experimental Evidence and Theoretical Implications of Fluctuations in Deep Inelastic Heavy lon Collisions, LBL 12596, April 1981, presented at the Europhysics Conf. on Nucl. Physices, Huar, Croalia, Yugoslavia, June 29.July 3 1981.

MORETTO, L.G., (See BLAU, S.K., LBL-10926)

MORETTO, LG. (SE MCDONALD, RJ., LBL-11608)

MORETTO, LG. (Se MORRISSEY, D.J., L.FL-L ]49])

MORETTO, LG. (See AORRISSEY, D.J., LBL-12181)

MORETTO, LG. (See RICHARDSON, L.W., LBL-10146)

MORETTO, L.G. (See SCHMITT, R.P., LBL-9512) 
MORET1O, LG. (See SOBOTKA, L.G., LBL 11148)

MORETTO, L.G. (See SOBOTKA, L.G., LBL-11840)

MORETIO, L.G. (See WOZNIAK, G.J., LBL-111\%)

MORRISSSEY, D.J., LG. Moretto

Manifestations of Excitation Energy Equilibrium in DexpInelastic Collisions, LBL-1149I, September 1980, Phys. Rev, C 23, 1835 (1981).

MORRISSEY, D.J., G.J. Wozniak, L.G. Sobotka, A.J. Pacheoo, C.C. Hsu, R.J. McDonald, and L.G. Mortto Angular Momentum, Siatistical Equilibrium and Sequential Fission in Very Asymmetric Systems, LBL 12181, March 1981, submitted to Phys. Rev. Letts.

MORRISSEY, D.J. (See ALEKLETT, K, LBL-11273)

MORRISSEY, D.J. (SeE LOVELAND, W., LBL-100LO)

MORRISSEY, D.J. (SEe LOVELAND, W., LBL-1 l658)

MORRISSEY, D.J. (See SOBOTKA, L.G., LBL 11840)

MORRISSEY, D.J. (See WOZNIAK, G.J., LBL-J1196)

MULLER, B. (Seе STÖCKER, H., LBL-12471)

MULLER, R.M., P.P. Tans, T.S. Mast, and J.J. Welch Mass Specirometry with a Very Small Cyclotron, LBL. 12797, May 1981, presented at the Argonne Symposium on High Energy Spectrometry, Argonne National Lab. Argonne, IL, May II-13, 1981.

MURPHY, M.J. (Set RAE, W.D., LBL-IJ135)

MURPHY, M.J. (SeE RAE, W.D., LBL-12568)

MYERS, W.D.

The Width of the Charge Distribution in Fission, LBL 12072, January 1981, to be presented at the Conference on Gross Peoperties of Nuclei and Nuclear Matter, Hirschegg, Austria, January 19-23, 1981.

MYERS, W.D.

The Dependence of RMS Cherge Radii on the Neutron Stin and on Deformation, LBL-12073, January 1981, presented at the Bormio Winter Meeting, Bormio, Jtaly, Janpary 26-30, 1981.

MYERS, W.D., G. Mantzouranis, J. Randrup Adiabalicity Criterion for Charge Equilibration with Application to Fission, LBL-11542, September 1980, Phys. Letis. B 9,1 ( 1981$)$.

MYERS, W.D., K.H. Schmidt

The Efect of Deformation and the Neutron Skin on RMS Charge Radii, LBL-12790. May 1981, presented at the Fourth International Conference on Nuclti Far From Stability, Helsing $r$, Dentrark.
MYERS, W.D. (See HERNAINDEZ, E.S., LBL.9761)

NAGAMIYA, S., M.C. Lemaire, E. Moeller, S. Schuetzer, G. Shapiro. H. Steiner, and I. Tanihata Production of Pions and Light Fragments at Large Angles in High-Energy Nuclear Coflisions, LBL-12123, March I981, submitted to Phys. Rev. C.

NAGAMIYA, S. (SEe TANIHATA, I., LBL-11488)

NEIMAN, M

SUSIE: A Program For Asalysis of 1 Dimensional Spectra; Version: 20 February [98], PUB 3014, February 1981.

NEWTON, J.O., B. Herskind, R.M. Dianond, E.L Dines, J.E. Draper, K.H. Lindenberger, S. Shih, C. Schück, F.S. Stephens.

Observation of Giant Dipole Resonances Built on \$tates of High Energy and Spin, LBL 12261, February 1981, Phys.

Rev. Letts. 46, 1383 (1981).

NEWTON, J.O. (See SIE, S.H., LAL.11352)

NITSCHKE, J.M.

Synthesis and Investigation of Neutron-Rich Transuranium lsotopes, LBL-11712, September [980, Presented at the International Symposium on the Synthesis and Properties of New Elements, Dubnt, U.S.S.R.

NITSCHKE, J.M., M. Fowler, A Ghiorso, R.E. Leber, M.J. Nurmia, L.P. Somerville, K.E. Williams, E.K. Hulet, J.H. Landrum, R.W. Lougheed, J.F. Wild, C.E. Bemis, Jr., R.J. Silva, and P. Eskola

Search for an 80-ms Spontaneous Fission Activity in Bonbardments of ${ }^{249} \mathrm{Bk}$ with ${ }^{15} \mathrm{~N}$, LBL-10173, January 1980 , Nucl. Phys. A 352, 138.146 (1981).

NITSCHKE, J.M. (See MOLITORIS, J.D., LBL.9725)

NOACK, C.C., M. Gyolasby, and S.K. Kauffmann

Test of Square Law for Deuteron Formation in Relativistic Nuclear Collisions, LBL-1 1053, August 1980, submitted to Phys. Rev, C.

NUCLEAR SCIENCE DIVISION

Nuelear Seience Division Amual Report 1979-1980, LBL. 11588, UC-34, March 1981.

NURMIA, M.J. (See NITSCHKE, J.M., LBL-10173)

OLSON, D.L, B.L Berman, D.E. Greiner, H.H. Heckman, P.J. Lindstrom, G.D. Westrall, and H.J. Crawford Electromagnetic Dissociation of Relativistic ${ }^{1 B_{0}}$ Nuclei, LBL-12292, February 1981, submitted to Phys. Rev. C.

PACHECO, A.J. (See McDONALD, R.J., LBL-1]608)

PACHECO, A.J. (Set MORETTO, LG., LBL- 10805)

PACHECO, A.J. (SEe MORRISSEY, D. J., LBL 12181) 
PACHECO, A J. (See SOBOTKA, L.G., LBL ] 1 ]48)

PACHECO, AJ, (See WOZNIAK, G.J., LBL-11196)

PARDO, R.C. (Ste FIRESTONE, R., LBL-12424)

PARRY, R.F. (SEE ÄYSTŌ, J., LBL-11193)

PARRY, R.F. (Se CERNY, J., LBL_]2853)

PARRY, R.F. (See MOLTZ, D.M. LBL, 11427)

PRICE, P.B. (Se STEVENSON, J.D., LBL- 1 1737)

PRICE, P.B. (See STEVENSON, J., LBL-12264)

PLGH, HG.

Future Relativistic Heavy lon Experiments, LBL 11974, December 1980, Introductory Remarks presented at the Workshop held at Gesellschaft for Schwerionenforschung, Darmstadt, West Germany, October 7.10, 1980.

PUGH, H.G.

Ultrarelativistic Heavy Ions, LBL 12053, December 1980, Presented at the Fourteenth Los Alamos Meson Physics Facility Users Group Meting, Los Alarnos, NM, October 27-28, 1980.

PUGH, H.G. (See HARRIS, J.W., LBL-12334)

RAE, W.D., A.J. Cole, A Dacal, R. Legrain, B.G. Harvey, J. Mahoney, MJ. Murphy, R.G. Stokstad, and J. Tserruya,

Coherent and incoherent Processes in Projectile Fragmientation, LEL-12568, April 1981, submittad to Phys. Rev. Letts.

RAE, W.D., R.G. Stokstad, B.G. Harvey, A Dacal, R. LeGrain, J. Mahoney, M.J. Murphy, and T.J.M. Symons

Molocular Resonances and the Production of Fast Particles in the Reaction of 160 with $12,13 \mathrm{C}$ Nuelei, LBL11135, June 1980, Phys. Rev. Letts. 45, 884 (1981).

RAE, W.D. (SeE SHOTTER, A.C, LAL-11458)

RANDRUP, J.

Kaon Rescattering in Relativistic Nuclear Collisions, LBL L 1 508, September 1980, Phys. Letts. B 99, 9 (1981).

RANDRUP, J., and S.E. Koonin

The Disassembly of Nuclear Matter, LBL-10959, August 1980. Nuclear Physies A 366, 223 (1981).

RANDRUP, J, (See HERMANDEZ, E.S., LBL 9761)

RANDRUP, J. (SEe KNOLL, J. LBL-1 1418)

RANDRUP, J. (SEe MYERS, W.D., LBL 11542)

RANDRUP, J. (See CUGNON, J., LBL-]1301)
RANDRUP, J. (See SCHURMANN, B, LBL- I 1964)

RASMLSSEN, J.O. (SE MA, A.Y., LBL9689)

RASMUSSEN, J.O. (See ZHONG-YU, MA, LBL-11893)

RATTAZZI, G.U. (Se SOBOTKA, LG., LBL 11148)

RATTAZZI, G.U. (S:e SCHMITT, R.P., LBL-9512)

REGIMBART, R, (\$et SCHMTT, R.P., LBL-9512)

REMAUD, B. (Se HERNANDEZ, E.S., LBL-4761)

RENFORDT, R.E. (Se* HARRIS, J.W., LBL-12334)

RJCHARDSON, L.W., G.J. Wozniak, M.R. Maier and LG. Moretto

Neutron-Gamma Discrimination Using Nal(TI) Scintilla tors with Application for Measurement of the Gamma-Ray Multiplicity Distribution in Heavy-Ion Reactions, LBL 10146, Nowember 1979, Nucl. Inst. and Meth. 173 (1980).

RJCHARDSON, LW. (See WOZMAK, G.J, LBL 11493)

RHOUX, C (SeE CONZETT, H.E., LBL-11576)

RIOUX, C. (See SLODODRIAN, R.J., LBL 11372)

RITTER, H.G. (See GUTBROD, H.H., LBL-11797)

RONNINGEN, R.M. (SEE WALKER, P.M.)

ROSENBUUM, S.S. (Søe MARTOFF, C.J,, LBL- 11914)

ROY, R. (See CONZETT, H.E., LBL-11576)

ROY, R (SeE SLOBODRIAN, R J., LBL-11372)

RUCK, H.M.

Polynomial Chromodynamics in $1+1$ Dimension I. Confinement of Quarks and the Structure of Composite Particles, LBL-12316, February 1981, submitted to Nocl. Phys.

RUCK, H.M

Polynomial Chromodycargics in 1 + I Dimensions, LBL12479, Janpary 198I, submitted for publication.

SANDOVAl, A (See HARRIS, J,W., LBL-12334)

SCHMIDT, K-H. (Set MYERS, W.D., LBL-12790)

SCHMITT, R.P., G.J. Wozniak, G.U. Rattazzi, G.J. Mathews, R. Regimbart and L.G. Moretio

Fast Particle Emission in the Deep Inelastic Reaction ${ }^{n a} \mathrm{Co}+20 \mathrm{~N}$ at $12.6 \mathrm{MoV} /$ Nucleon, LBL-9512. January 1980, Phys. Rev. Letts. 46, 522 (1981).

SCHNETZER, S. (Se NAGAMTYA, S., LBL-12123) 
SCHNETZER, S. (See TANIHATA, I., LBL-11488)

SCHROEDER, L.S.

Pion Production in Nucleus-Nucleus Collisions Below A Few GeV/Nucleon - Past, Present and Future LBL10899, July 1980, Presented at the Seminar on High Energy Nuclear Interactions and Properties of Very Dense Matter, Hakone, Japan, July 7-11, 1.980.

SCHROEDER, L.S.

High Energy Nuclear Collisions in the Few GeV/Nucleon Region - Projectile and Target Fragmentation

LBL-11102, June 1980, inviled talk presented at the 1980 Institute for Nucleer Studies, Kikuchi \$ummer School on Nuclear Physics at High Energies, Fuji-Yoshida, Japan, July 1-4, 1980.

SCHROEDER, L.S. (See GEAGA, J.V., LBL 10265)

SCHROEDER, LS. (SEe HARRIS, J, W., LBL-12334)

SCHUBERT, K.R, G.T. Koker and R.B. Firestone Alnus Glufinosa and Glycine Max: Shor-tern Stodies Using $\left[{ }^{13} \mathrm{~N}\right]$ Ammonium, Plant Physiology 67, 662 (1981).

SCHÜCK, C. (See McDONALD, R.J., LBL IL608)

SCHÜCK, C. (SEe NEWTON, J.O., LBL-12261)

SCHǗCK, C (Ste WOZNIAK, G.J., LBL-11196)

SCHURMANN, B, J. Randrup

The Quasj-Elastic Component in High-Energy Nuctear Collisions, December 1980, LBL-1 1964, submitied to Phys. Rev. C.

SCOTT, D.K. (See BACK, B.B., LBL-11852)

SEABORG, G.T. (Scientific Editor)

Proceeding of the Symposium Commenorating the 25th Anniversary of the Discovery of Mendelevium, LBL I1599, March 28, 1980 .

SEABORG, G.T.

Charting the New Elements, LBL 11610 (1980), SCIQUEST, 1980, 53, 7(1980).

SEABORG, G.T. (See ALEKLETT, K, LBL-11273)

SEABORG, G.T. (See LIU, Yuan-Fang, LBL-12909)

SEABORG, G.T. (See LOVELAND W., LBL 10010 )

SEABORG, G.T. (See LOVELAND W., LBL-1 1658)

SEN, S.T. (See MA, A.,. LBL-9689)

SHIPIRO, G. (See NAGAMIYA, S., LBL-12123)
SHIH, \$. (See McDONALD, R.J., LBL-11608)

SHIH, S. (Sec NEWTON, J,O., LBL-12261)

SHIH, S. (See WOZNJAK, G.J., LBL-11196)

SHOTTER, AC., AN. Bice and J. Cerny

An Aralytical Expression for the Effective Solid Angle of a Rectangular Collimator for the Passage of Fragments Jrom Breakup Projectiles, LBL-11375, August 1980, Nucl. Inst. and Methods. 189, 201-203 (1981).

SHOTIER, AC., A.N. Bice, D.P. Stahel, and J. Cerny The Quasielastic Breakup of $187 \mathrm{MeV}{ }^{12} \mathrm{C}$ lons on ${ }^{208} \mathrm{~Pb}$, LBL-1 1376, July 1980, subnitted to Phys. Letts.

SHOTtER, AC. AN, Bice, J.M Wouters, W.D. Rec. and J. Cerny

Otservation of the Direct and Sequential Breakup of ${ }^{\top} \mathbf{L}$ from ${ }^{12} \mathrm{C}$ and ${ }^{200} \mathrm{~Pb}$ Targets at $70 \mathrm{MeV}$, LBL II 458 , September 1980, Phys. Rev. Lets. 46, 12 (1981).

SHOTTER, AC. (Set BACK, B.B., LBL-11852)

SHOTTER, AC. (See BJCE, A.N., LBL-11194)

SIE, S.H, J.O. Newton, J.R. Leigh, and RM. Diamond Multiplicity of the Statistical $r$ Rays Following $(H I, x n)$ Reactions, LBL-11352, August 1980, Phys. Rev. Letts. 46, $405(1981)$.

SILVA, R.J. (See NITSCHKE, J.M., LBL-10173)

SLOBODRIAN, R.J., C. Rioux, R. Roy, H.E. Conzett, P. won Rossen, and F. Hinlerberger

Breakdown of Time Reversal Invariance in the lnteraction of Nucltar Particles, LBL-11372, August 1980, submitied to Phys. Rev. Letts.

SLOBODRIAN, R.J. (See CONZETT, H.E., LBL-11576)

SOBOTKA, L.G., C.C. Hsu, G.J. Wozpiak, D.J. Morrissey and L.G. Moretto

Angular Mornenturn Transfer and Partition in the DetpInelastic Reaction: $664 \mathrm{MeV}{ }^{84} \mathrm{Kr}+{ }^{\mathrm{Nal}} \mathrm{Ag}$, LBL-11840, April 1981, submitted to Nucl. Phys.

SOBOTKA, LG, C.C. Hsu, G.J. Wozniak, G.U. Ratiazzi, R.J. McDonald, A.J. Pacheco and L.G. Morelto

Evidente for Rigid Rotation and Large Deformations in the Deep Inelestic Reaction: $664 \mathrm{MeV}{ }^{84} \mathrm{Kr}+{ }^{\mathrm{ns}} \mathrm{A}_{\mathrm{Ag}}$, LBL 11 148, Augus1 1980, Phys. Rev. Letts. 46, 887 (1981).

SOBOTKA, L.G. (SEe MORRISSEY, D.J., LBL-12181).

SOBOTKA, L.G. (SE WOZNAK, G.J., LBL-L1196).

SOMERVILLE, L. P. (Ste EGGERS, R.C., LBL-12060)

SOMERVILLE, L.P. (See NTTSCHKE, J.M., LBL L0173) 
STAHEL, D.P. (See BICE, AN, LBL-I194)

STAHEL, D.P. (Ste \$HOTTER, A.C, LBL-1]376)

STEINBERG, E.P. (SEe LOVELAND, W., LBL 10010)

STEINER, H (See NAGAMIYA, S., LBL 12123)

STEINER, H. (See TANIHATA, I., LBL-1 1488)

STELZER, H.

Some Recent Developments in Nuclear Charged Particle Detectors, LBL- 11366, Angust 1980, presented at the International Conference on Nuclear Physics Lawrence Berkejey Laboratory, Berkeley CA, August 24-30, 1980.

STEPHENS, F.S.

Phenomena at Very High Spins, LBL-10645, March 1980. presented at the Conference on Band Structure and Nuclear Dynanics, New Orleans, LA, February 77 - March I. 1980, Nucl. Phys. A 347 (1980) 275-286.

STEPHENS, F.S.

Properties of Nuclei at Very High Spin, presented at the International Conference on Nuclear Physics, Lawrence Berkeley Laboratory, Berkeley, CA, August 24.30, 1980. LBL 11620 , September 1980.

STEPHENS, F.S. (SEe DIAMOND, R.M., LBL-10325)

STEPHENS, F.S. (\$Ee MCDONALD, R.J., LBL-II608)

STEPHENS, F.S. (So NEWTON, J.O., LBL 12261)

STEPHENS, F.S. (See WOZNIAK, G.J., LBL-11 196)

STEVENSON, J.D.

Neutron Emission in Relativistic Nuclear Collisions, LBLII736, December 1980, submitted to Phys. Rev. Lett.

SIEVENSON, J., J. Martinis, and P.B. Price A Calorimeter for High-Energy Hetavy lons, LBL-12264, Jamuary 1981, submitted to Nucl. Instr. \& Meth.

STEVENSON, J.D., P.B. Price

Production of the Neutron-Rich Nuclides ${ }^{20} \mathrm{C}$ and ${ }^{27} \mathrm{~F}$ by Fragmentation of $213 \mathrm{MeV} /$ Nucleon ${ }^{48} \mathrm{Ck}$, LBL-11737, December 1980, submitted to Phys. Rev, lett.

STOCK, R (S૯E HARRIS, J.W., LBL-12334)

STÖCKER, $\mathrm{H}$.

Pion, Light Fragment and Entropy Production in Nuclear Collisions, LBL 12302, April 1981, submitled to Phys. Rev, Letts.

STÖCKER, H., L.P. Cseraai, G. Graebner, G. Buchwald, H. Kruse, R.Y. Cusson, J.A. Maruhn, and W. Greiner Jets of Nuclear Matter from High Energy Heavy Ion Collisions, LBL-11774, November 1980. (LBL-11774 revised February (981), submitied to Phys. Rev. Letts.
STÖCKER, H., M. Gyulassy, and J. Boguta

Probing Dense Nuclear Matter Via Nudear Collisions, LBL-12095, January 1981, subrnitted to Phys. Letts.

STÖCKER, H., and B. Míller

The Bounce of Effect as a Barometer for Hox, Dense Matter in Fast Nuclear Collisions, LBL-12471, March 1981, submitted to Phys. Letts.

STOKSTAD, R.G.

Noclear Struchure and Heavy-Ion Fusion, LBL-11664, Otober 1930, presented at the XIIJ International Summer School in Nuclear Physics, Mikolajkj, Poland, September $[-I 3,1980$; and to be published in Nukleonikg.

STOKSTAD, R.G. (See RAE, W.D., LBL-11135)

STOKSTAD, R.G. (Sec RAE, W.D., LBL 12568)

STRASSNER, G. (See MARTOFF, C.J., LBL-1 1914)

STROEBELE, H. (See HARRIS, J.W, LBL- 12334)

SWIATECKI, W.J.

The Dynamics of the Fusion of Two Nuclei, LBL-12642, April 1981, submitted to Nucl. Phys. A.

SWIATECK, W.J.

Fusion Cross-Sections and the New Dymamics, LBL-12708, May 1981, presented at the 4th International Conference on Nuclei Far from Stability, Helsingorr, Denmark, June $7-13,1981$.

SWIATECKI, W.J. (See BLOCKI, J., LBL-9574)

SYMONS, T.J.M. (See BACK, B.B., LBL-11852)

SYMONS, T.J.M. (See RAE, W.D., LBL-1]135)

TANIHATA, $\mathrm{I}$.

Two-Particle Correlations in Nuclear Collisions, LBLI 1320, July 1980, presented at the INS Kulkuchi Summer School on Nuctear Physics at High Energies, Fuji-Yoshida, Japen, July 1-4, and the Hakone Seninas on High Energy Nuclear Interactions and Properties of Dense Nuclear Matter, Hakone, Japan, July 7-1 I, 1980.

TANIHATA, I, S. Nagamiya, S. Schnetzer, and H. Steistr

One- and Two-Ptoton Inclusive Spectra in $800 \mathrm{MkV}$ Proton-Nucleus Collisions and the Mean Free Path of Protons in Nuclei, LBL-11488, October 1980, submitted to Phys. Letts. B.

TANIHATA, I, (See NAGAMIYA, LBL-12123)

TANS, P.P. (SEe MUL.LER, R.M. LBL-12797)

THIERENS, H.M. (See CERNY, J., LBL.]2853)

TREUHAFT, R.N. (See GEAGA, J.V, LBL, 10265) 
TRUOL, P. (See MARTOFF, C.J., LBL- 1914)

TSERRUYA, I. (See RAE, W.D., LEL-12568)

VAN BIBBER, K (See GEAGA, J.V., LBL-10265)

VAN OERS, W.T.H. (See J. BIRCHALL, LBL-11121)

VON ROSSEN, P. (See J. BJRCHALL, LBL-[II2J)

VON GUNTEN, H.R (SeE LIU, YUAN-FAN, LBL12909)

VON ROSSEN, P. (See CONZETT, H.E, LEL 11576)

VON ROSSEN, P. (See SLOEODRLAN, R.J., LBL-11372)

WALKER, P.M., S.R. Faber, W.H. Beptley, R.M. Ronningen, and R.B. Firestone

A Study of $172 \mathrm{Yb}$ Following the ${ }^{170} \mathrm{Er}(\alpha, 2 \mathrm{n})$ Reaction, Nucl. Phys. A 343, 45 (1980).

WANG, Y.S. (See MA. A.Y., LBL-9689)

WAPSTRA, AH, (See MARTOFF, C.J., LBL-11914)

WARNER, R.A (Set FIRESTONE, R.B., LBL-12424)

WARWICK, A. (See GUTBROD, H.H. LBL-1 1797)

WATSON, J. (See BIRCHALL, J., LBL- I1121)

WATSON, J.W, (See ZISMAN, M.S., LBL-10204)

WEIK, F. (Se GUTEROD, H.H., LBL-11797)

WELCH. J.J. (Se MULLER, R.M., LBL-12797)

WESTFALL, G.D. (See OLSON, B.L., LBL- 12292)

WEDMAN, H. (See GUTBROD, H.H., LBL-11797)

WLD, J.F. (See NITSCHKE, J.M., LAL- 10173)

WLKINS, B.D. (See LOVELAND, W, LBL IOOL)

WLLIAMS, K.E. (See NITSCHKE, J.M., LBL-10173)

WOLF, K.K. (SUTBROD, H.H, LBL-11797)

WOLF, K.L. (See HARRIS, J.W., LBL- 12334)

WOUTERS, J.M. (Sєe ÄYSTÖ, J., LAL-1 1193)

WOUTERS, J.M. (Sœ CERNY, J., LBL-12853)
WOUTERS, J.M. (See MOLTZ, D.M., LBL 1 1427)

WOUTERS, J.M. (See SHOTTER, AC, LBL 11458)

WOZNIAK, G.J., R.J. McDonald, AJ. Pacleco, C.C. Hsw, D.J. Moritissty, L.G. Sobotka, L.G. Moretto, S. Shib, C. Schuck, R.M. Diamond, H. Kluge, and F.S. Stephents The Rise and Fall of the Spin Alignment in Deep-Inelastic Reactions, LBL 11196, July 1980, Phys. Rev. Lets. 4s, 1081 (1980).

WOZNIAK, G.J., L.W. Richardson and M.R. Maier Time-Walk Characteristics of an Improsed Constant Fraction Discriminator

LBL-11493, Sepiember 1980, Nucl Instr. and Meth. 180, 509 (1981).

WOZNIAK, G.J. (See McDONALD, R.J., LBL.11608)

WOZNIAK, G.J. (SE MORRISSEY, D.J., LBL-12181)

WOZNIAK, G.J, (See RICHARDSON, L.W., LBL- 10146)

WOZINIAK, G.J. (SEE SCHMITT, R.P. LBL-9512)

WOZNIAK, G.J. (SeE SOBOTKA, L.G., LBL.11148)

WOZNMAK, G.J. (Sœ SOBOTKA, L.G., LBL-11840)

WU, X.Z. (See MA. A.Y., LBL-9689)

XI-ZHEN, Wu (Set ZHONG-YU, MA, LBL-11893)

YANG, F.C. (SEe MA, A.Y., LBL-9689)

YASHITA, S. (SeE LEINO, M.E., LBL-12816)

Yi-ZHONG, Zhuo (See ZHONG-YY, MA, LBL, 1893)

ZAJC, W.A (SeE MARTOFF, C.J., LBL-JI914)

ZHANG, G.S. (See MA, AY., LBL-9689)

ZHONG YU, MA, Wu Xi-zhen, Zhang Jing-Shang, Zbuo Yi-zhoog, and J.O. Rasmussen

Calculation of Muon Final Probabilities Aiter MuonInduced Fission in Four-State Basis, LBL-11983, May 198 I, submitted to Phys. Letts.

ZISMAN, M.S., J.G. Cramer, D.A. Goldberg, J.W. Watson, and R.M. Dewries

Dominance of Strong Absorption in ${ }^{9} \mathrm{Be}+{ }^{28} \mathrm{Si}$ Elastic Seattering. LBL. 10204, December 1979, Phys. Rev. C 21, 2398 (1980). 
Author Index

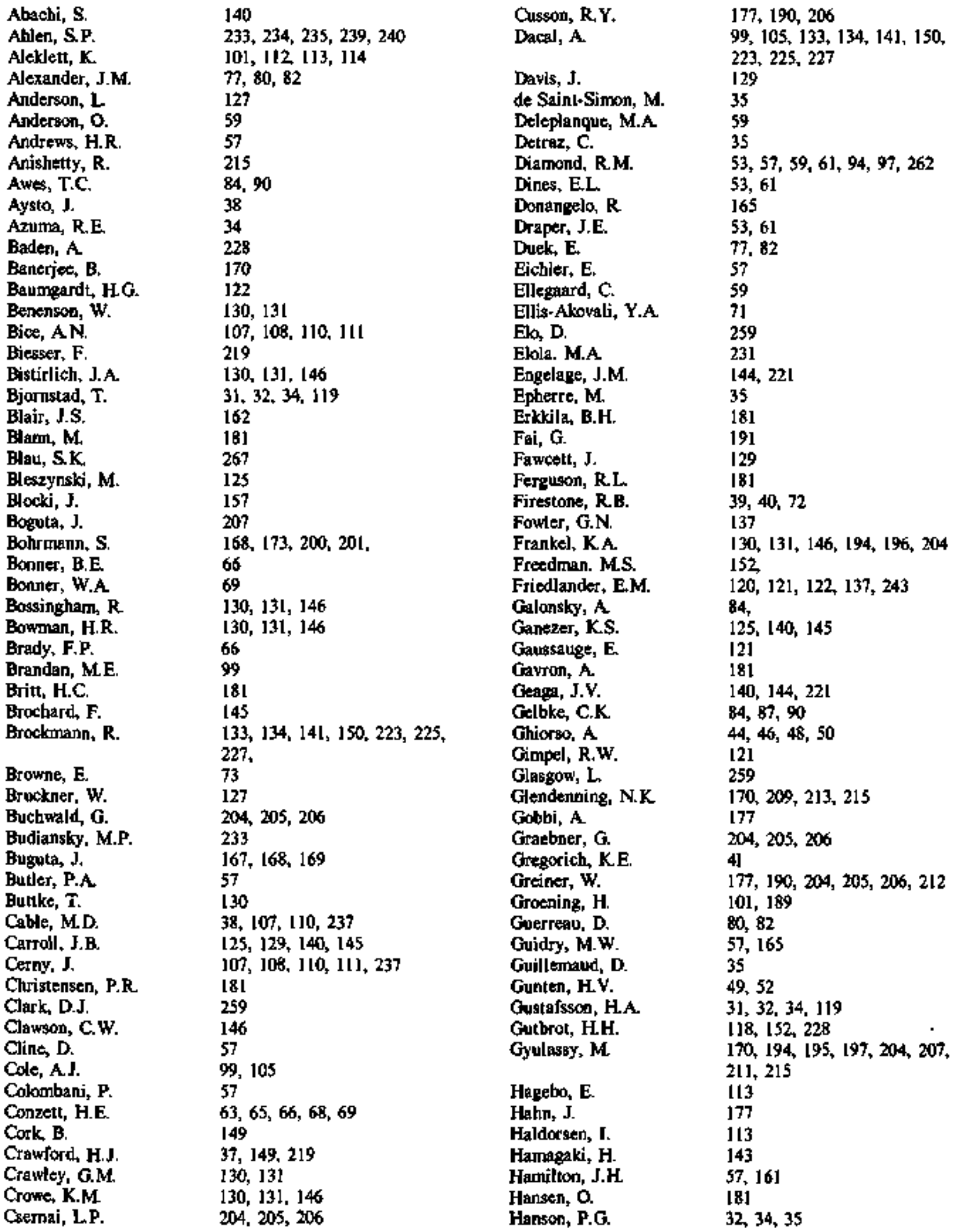


Harris, J.W.

Harvey, B.G.

Hashimoto, 0 .

Hausser, $O$.

Hauslein, P.E.

Hecking, P.

Heckman, HH.

Henderson, D.J.

Hendrie, D.L.

Herskind, B.

Hichwa, RD.

Hinterberger, $F$.

Hoang, T.F.

Hofiman, D.C.

Hogen, J.J.

Hsu, C.C.

Huizenga, J.R.

Hulet, E.K.

Igo, G.J.

Ingersoll, J.G.

Johnson, N.R.

Jolmsson, P.

Jonson, B.

Jonssign, O.C.

Judek, B.

Kadota, S.

Kahler, A.C.

Kallne, J.

Kaplan, M.

Karant, Y.J.

Kasagi, J.

Kashy, E.

Kauffmann, S.K.

Kaufrman, S.B.

Keinonen, J.

Kelly, W.H.

Kildir, M.

King, N.S.P.

Kinoshita, K.

Kirk, P.N.

Klapisch, R.

Kuge. $\mathrm{H}$.

Knoll, J.

Koehler, P.

Koike, M.

Kontz, R.W.

Kraus, Jr., R.H.

Kruse, $\mathbf{H}$.

Kurck, J.P.

Lam, $R$.

Langevin, $M$.

Larsson, P.O.

Lederex, C.M.

Lee, D.

Let, I.Y.

Legrain, R.L.

Leino. M.

Lemaire, M.-C.

Lemmon, R.M.

Lindenberger, K.H.
$133,134,141,144,150,221$,

$223,225,227$

$99,103,104,105$

$130,131,146,196$

57

38

193, 208, 210

121, $124,220,241,243$

152

103

59,61

5 ?

65

149

49

42

$96,97,184$

179

46

$125,140,145$

146

57

[12

$31,32,34,35,119$

I19

121

143

57 ,

129

$77,80,82$

120, 121, 243

84.

130, 131

195

118,152

57

39

80

66

243

144, 221

35

59

201

215

130, 131, 146

$144,221,253$

113, 114

206

146

259

35

31,32

74

49

57

$84,90,99,105$

44

127,137

69

$53,61,94$
Lindfors, $\mathrm{V}$

Lindstrorn, $P$.

Liu, Y.-F.

Logan, D.

Lohner, H.

Lombard, $R$.

Lougleed, R.

Loveland, W.

Lumbroso, A

Lond, $T$.

Lo, Cheng

Lustig, H.J.

Lynch, W.G.

Lyneis, C.M.

Ma, Z. $-y$.

Mahoney, J.

Maier, $\mathbf{M}$.

Mantzouranis, $G$.

Maples. C.

Martinis, J.

Martoff, C.J.

Maruhn, J.A

Mast, T.

Mattsson, $\mathrm{S}$.

MoClelland, J.B.

McDonald, R.J.

MacDoneld, W.J.

McFarland, RM.

McGaughey, P.L.

Mchlarris, Wm. C.

McLerran, L.

McNaughton, MW.

McParland, C.

Meng, $J$.

Miake, Y.

Miller, J.

Minehart

Moeller, E.

Molitoris, J.D.

Molzahn, D.

Moody, K.J.

Moretto, LG.

Morita, Y.

Morrissey, D.J.

Mulera, T.A.

Muller, B.

Muller, K.H.

Muller, $R$.

Murphy, D.L

Musser, J.

Myers, W.D.

Nagamiya, $S$.

Naulin, F.

Neese, R.E.

Neiman, $M$.

Newton, J.O.

Nichols, T.L.

Nissen-Meyer, S.
$31,32,119$

140

49,52

$77,80,82$

228

137, 143

46

$101,112,113,114,116,117$

209

113

52

177

87

259

164 ,

103. 105

$1] 8,133,134,141,150,152$,

$223,225,227,228$

178

$245,247,249,251$

232

[30, 131, 146

$177,190,204,205,206$

259

$31,32,34,35,118$

125, 129

$53,76,94,96,97,236$

146

$46,50,114$

$101,112,113,114,116,117$

39

215

66

220,255

$246,247,249,251$

143

130, I31, I33, 134, 14I, 146.

$150,233,225,227$

129

$127,127,137,143$

229

113

41, 42, 101, 112, 114, 189

$53,76,94,96,97,183,184$,

186, 188,236

113,117

$53,76,94,96,97,116,184$

140, 145, 231

206

$171,172,173$

259

$37,99,103,105,130,131_{+}, 146$

235

158,178

$127,137,143$

35

165

57

$53,61,94$

165

127 


\begin{tabular}{|c|c|c|c|}
\hline $\begin{array}{l}\text { Nitschke, J.M } \\
\text { Nix, J.R. } \\
\text { No]=n, J, J.A }\end{array}$ & $\begin{array}{l}48,229 \\
204 \\
130,131\end{array}$ & $\begin{array}{l}\text { Ruix, C.L. } \\
\text { Sagle, A.L. } \\
\text { Saini, S. }\end{array}$ & $\begin{array}{l}144,221 \\
66,125,129,140,145 \\
84,90\end{array}$ \\
\hline Nurnelley, L.l. & 93,116 & Salamon, M.H. & 234 \\
\hline $\begin{array}{l}\text { Nurmìa, M.J. } \\
\text { Nyman, } \mathbf{G} .\end{array}$ & $\begin{array}{l}45,48,49,50 \\
31,34,35\end{array}$ & Sandoval, A & $\begin{array}{l}133,134,141,150,223,225, \\
227\end{array}$ \\
\hline Dertel, C. & 116 & Schardt, D. & 31,119 \\
\hline Ogloblin, A.A. & 212 & Schmidt, K-H. & 158 \\
\hline Oliveira, L.F. & 165 & Schmitt, RP. & 183 \\
\hline Oliveira, Z.M. & 160 & Schnetzer, S. & $127,137,143$ \\
\hline Oostens, J. & 145 & Schopper, E. & 122 \\
\hline Ortiz, M.E. & $\begin{array}{l}133,134,141,150,223,225 \text {, } \\
227\end{array}$ & Schroder, W.U. & $\begin{array}{llllll}179 & & & & & \\
127 & 133 & 134 & 141 & 144 & 150\end{array}$ \\
\hline Pacheco, A.J. & $53,76,94,96,97,183,186$ & & $221,223,225,227$ \\
\hline Pang, W. & 103 & Schuck, $\mathrm{C}$ & $53,59,61,94$ \\
\hline Parda, RC. & 39 & Schurmann, B. & 198,204 \\
\hline Par'ry, R.F. & 38,237 & Scott, D.K & 84 \\
\hline Patin, $Y$. & 181 & Seaborg, G.T. & $41,49,52,101,112,113$ \\
\hline Peker, L.K. & 161 & & $114,116,117,127,189$ \\
\hline Perez-Mendez, $\vee$. & $125,129,140,145,231$ & Shapíco, G. & 127,137 \\
\hline Peter, J. & $118,130,131,152$ & Shida, Y. & 143 \\
\hline Plesil, F. & 181 & Shib, S. & $53,59,61,94,97$ \\
\hline Poggi, G. & 84,90 & Shirley, V.S. & 7 \\
\hline Pontoppidan, S. & 181 & Shor, A & 140 \\
\hline Poskanzer, A.M. & $31,32,34,35,119,228$ & Shotter, AC. & $107,108,110,111$ \\
\hline Price, P.B. & $232,235,239,240,243$ & Simon, R.S. & 57 \\
\hline Prussin, S.G. & 160 & Singh, $\mathbf{B}$. & 72 \\
\hline Pugh, H.G. & $\begin{array}{l}133,134,141,144,150,221 \text {, } \\
223,225,227\end{array}$ & $\begin{array}{l}\text { Slobodrian, R.J. } \\
\text { Sobotka, LG. }\end{array}$ & $\begin{array}{l}65 \\
53,76,94,96,97,188,236\end{array}$ \\
\hline Puigh, R.J. & 93 & Somerville, L.P. & $45,46,48,50$ \\
\hline Qiu. X.J. & 162 & Spieler, H. & 228 \\
\hline Quebert, J. & 131 & Steinberg, E.P. & 118,152 \\
\hline Radi, H.M.A. & 196 & Steiner, H. & $127,137,143$ \\
\hline Rac, W.D.M. & $99,105,108$ & Stelzer, H. & I]8, 152, \\
\hline Raff, M. & $\begin{array}{l}133,134,141,150,223,225 \\
227,\end{array}$ & $\begin{array}{l}\text { Stephens, F.S. } \\
\text { Stephenson, J. }\end{array}$ & $\begin{array}{l}53,54,55,57,59,61,94,97 \\
204\end{array}$ \\
\hline Randrup, J. & $160,178,179,181,182,191$, & Stetz, A & 129 \\
\hline & 198,202 & Stevenson, J.D. & 20l, 232, 235, \\
\hline Rasmussen, J.0. & $\begin{array}{l}130,13],[46,16], 162,164, \\
165,196\end{array}$ & Stock, R. & $\begin{array}{l}133,134,141,150,223,225 \text {, } \\
227\end{array}$ \\
\hline $\begin{array}{l}\text { Rathbun, W. } \\
\text { Ravn, H.L }\end{array}$ & $\begin{array}{l}245,247,249,25] \\
31,32,34,35,119\end{array}$ & Stocker, H. & $\begin{array}{l}177,190,193,204,206,207, \\
212,215\end{array}$ \\
\hline Remier, E & 194 & Stokes, R.H. & 181 \\
\hline Renfordt, R.E. & $\begin{array}{l}133,134,141,150,223,225 \text {, } \\
227,\end{array}$ & $\begin{array}{l}\text { Stolostad, RG. } \\
\text { Strobete, H. }\end{array}$ & $\begin{array}{l}99,105 \\
133,134,141,150,223,225\end{array}$ \\
\hline Richardson, L W. & 87,184 & & 227 \\
\hline Riedel, C. & 204 & Strottman, D. & 204 \\
\hline Riedesel, H. & 228 & Subramanian, P. & 204 \\
\hline Riedinger, L. L. & 57 & Sullivan, J.P. & $130,131,146,196$ \\
\hline Riess, F. & $\begin{array}{l}133,134,141,150,223,225 \\
227\end{array}$ & $\begin{array}{l}\text { Sutter, R. } \\
\text { Swenson, L.W. }\end{array}$ & $\begin{array}{l}145 \\
129\end{array}$ \\
\hline Rioux, C. & 65 & Swiateclj, W.J. & $157,159,174$ \\
\hline Ritter, H.G. & $118,152,228$ & Symons, T.J.M. & 37 \\
\hline Rivet, M.F. & 77 & Takahashi, K & 35 \\
\hline Robinson, E.L. & 57 & Talaga, RL. & 125.145 \\
\hline Roche, G.R. & 144,221 & Tanihata, I. & $127,137,143,145$ \\
\hline Romero, J.L. & 66 & Tans, $P$. & 259 \\
\hline Ronningtn, R.M. & 57 & Taras, P. & 57 \\
\hline Roy, R. & 65 & Tarle, G. & $233,239,240$ \\
\hline Ruck, H.M. & 214 & Thibault, $C$ & 35 \\
\hline Rud, N. & 57 & Thiterens, HM. & 38,237 \\
\hline
\end{tabular}


Thorras, T.D. 93

Timkknell, M. 239

Toke, J. 159

Touchard, F. $\quad 35$

Treuhaft, R.N. 144, 221

Truol, $\mathbf{P}$.

Tsang, M.B.

Tserruya, 1.

Van Bibber, $\mathrm{K}$.

Vandenbosch, $\mathbf{R}$.

Vaz, L.C.

Videbaek, $F$.

Viggers, D.

won Rossen, P.

Ward, D.

Warnet, R.A.

Warner, R.E.

Warwick, A.J.

Weaver, D.

Webb, M.P.

Weik, F.

Weiman, $H$.

Weiner, R.M.

239
59
35
144,221
146
87
105
103
93
80
181
72
65
57
39
87
$118,152,228$
$245,247,249,251$
181
$18,152,228,253,254,256$
152
137

Weich, $\mathbf{J}$.

Westfal, G.D.

Whipple, E.T.

Whitney, R.R.

Wiedenbeck, P.E.

Wieman, $\mathbf{H}$.

Wilkíns, B.D

Wolf, $\mathrm{K}$.

Woodwatd, D.

Wouters, J.M.

Wozniak, G.J.

Wu, X.-z.

Yariv, $\mathbf{Y}$.

Yashita, S

Yates, S.W.

Yoo, $\mathrm{E}$

Young, G.R.

Zajc, W.A.

Zarbakhsh, F.

Zhang, J.-s.

Zhuo, Y.-Z.

Zisman, M.S.
259

37.90

125

129

231

118,228

118,152

$84,133,134,141,150,223$,

$225,227,228$

140,145

$38,108,237$

$53,76,94,96,97,184,236$

164

204

44

57

146

1B1

$130,131,146$

$125,140,145$

164

164

$77,80,82,93,262$ 